\title{
SPECTRAL INVARIANTS WITH BULK, QUASI-MORPHISMS AND LAGRANGIAN FLOER THEORY
}

\author{
KENJI FUKAYA, YONG-GEUN OH, HIROSHI OHTA, KAORU ONO
}

\begin{abstract}
In this paper we first develop various enhancements of the theory of spectral invariants of Hamiltonian Floer homology and of Entov-Polterovich theory of spectral symplectic quasi-states and quasi-morphisms by incorporating bulk deformations, i.e., deformations by ambient cycles of symplectic manifolds, of the Floer homology and quantum cohomology. Essentially the same kind of construction is independently carried out by Usher Us4 in a slightly less general context. Then we explore various applications of these enhancements to the symplectic topology, especially new construction of symplectic quasi-states, quasi-morphisms and new Lagrangian intersection results on toric and non-toric manifolds

The most novel part of this paper is to use open-closed Gromov-WittenFloer theory (operator $\mathfrak{q}$ in FOOO1] and its variant involving closed orbits of periodic Hamiltonian system) to connect spectral invariants (with bulk deformation), symplectic quasi-states, quasi-morphism to the Lagrangian Floer theory (with bulk deformation).

We use this open-closed Gromov-Witten-Floer theory to produce new examples. Especially using the calculation of Lagrangian Floer cohomology with bulk deformation in [FOOO3, FOOO4, FOOO7, we produce examples of compact symplectic manifolds $(M, \omega)$ which admits uncountably many independent quasi-morphisms $\widetilde{\operatorname{Ham}}(M, \omega) \rightarrow \mathbb{R}$. We also obtain a new intersection result for the Lagrangian submanifold in $S^{2} \times S^{2}$ discovered in [FOOO6].

Many of these applications were announced in [FOOO3, FOOO4, FOOO6].
\end{abstract}

Date: October 19, 2016.

2000 Mathematics Subject Classification. Primary 53D40, 53D12, 53D45; Secondary 53D20, 14M25, 20F65.

Key words and phrases. Floer homology, Lagrangian submanifolds, Hamiltonian dynamics, bulk deformations, spectral invariants, partial symplectic quasi-states, quasi-morphisms, quantum cohomology, toric manifold, open-closed Gromov-Witten theory.

Kenji Fukaya is supported partially by JSPS Grant-in-Aid for Scientific Research No. 23224002, NSF Grant No. 1406423, and Simons Collaboration on Homological Mirror Symmetry. YongGeun Oh is supported by the IBS project IBS-R003-D1. Hiroshi Ohta is supported by JSPS Grant-in-Aid for Scientific Research Nos. 23340015, 15H02054. Kaoru Ono is supported by JSPS Grant-in-Aid for Scientific Research, Nos. 18340014, 21244002, 26247006, 23224001. 


\section{CONTEnTs}

1. Introduction

1.1. Introduction

1.2. Notations and Conventions

1.3. Difference between Entov-Polterovich's convention and ours 18

Part 1. Review of spectral invariants $\quad 20$

2. Hamiltonian Floer-Novikov complex $\quad 20$

3. Floer boundary map 23

4. Spectral invariants 31

Part 2. Bulk deformations of Hamiltonian Floer homology and spectral invariants

5. Big quantum cohomology ring: review

6. Hamiltonian Floer homology with bulk deformations 40

7. Spectral invariants with bulk deformation 46

8. Proof of the spectrality axiom $\quad 48$

8.1. Usher's spectrality lemma 49

8.2. Proof of nondegenerate spectrality $\quad 52$

9. Proof of $C^{0}$-Hamiltonian continuity $\quad 52$

10. Proof of homotopy invariance $\quad 57$

11. Proof of the triangle inequality 59

11.1. Pants products $\quad 59$

11.2. Multiplicative property of Piunikhin isomorphism 65

11.3. Wrap-up of the proof of triangle inequality $\quad 70$

12. Proofs of other axioms 71

Part 3. Quasi-states and quasi-morphisms via spectral invariants with bulk

13. Partial symplectic quasi-states

14. Construction by spectral invariant with bulk $\quad 75$

14.1. Existence of the limit $\quad 76$

14.2. Partial quasi-morphism property of $\mu_{e}^{\mathfrak{b}} \quad 79$

14.3. Partial symplectic quasi-state property of $\zeta_{e}^{\mathfrak{b}} 80$

15. Poincaré duality and spectral invariant 82

15.1. Statement of the result

15.2. Algebraic preliminary

15.3. Duality between Floer homologies 83

15.4. Duality and Piunikhin isomorphism

15.5. Proof of Theorem 15.1 86

16. Construction of quasi-morphisms via spectral invariant with bulk 87

Part 4. Spectral invariants and Lagrangian Floer theory 90

17. Operator q; review $\quad 90$

18. Criterion for heaviness of Lagrangian submanifolds $\quad 97$

18.1. Statement of the results 97

18.2. Floer homologies of periodic Hamiltonians and of Lagrangian submanifolds 
18.3. Filtration and the map $\mathfrak{I}_{(H, J)}^{\mathbf{b}, \mathfrak{b}} \quad 105$

18.4. Identity $\mathfrak{I}_{(H, J)}^{\mathbf{b}, \mathfrak{b}, *} \circ \mathcal{P}_{\left(H_{\chi}, J\right), *}^{\mathfrak{b}}=i_{\mathrm{qm}, \mathbf{b}}^{*}$

18.5. Heaviness of $L \quad 109$

19. Linear independence of quasi-morphisms. 110

Part 5. Applications 111

20. Lagrangian Floer theory of toric fibers: review 111

20.1. Toric manifolds: review 111

20.2. Review of Floer cohomology of toric fiber 112

20.3. Relationship with the Floer cohomology in Section 17

20.4. Properties of Floer cohomology $\operatorname{HF}_{T}((L, \mathbf{b}) ; \Lambda)$ : review 116

21. Spectral invariants and quasi-morphisms for toric manifolds 123

21.1. $\mu_{e}^{\mathfrak{b}}$-heaviness of the Lagrangian fibers in toric manifolds. 123

21.2. Calculation of the leading order term of the potential function in the toric case: review.

21.3. Existence of Calabi quasi-morphism on toric manifolds. 125

21.4. Defect estimate of a quasi-morphism $\mu_{e}^{\mathfrak{b}}$.

22. Lagrangian tori in $k$-points blow up of $\mathbb{C} P^{2}(k \geq 2)$. 127

23. Lagrangian tori in $S^{2} \times S^{2} \quad 128$

23.1. Review of the construction from [FOOO6] 128

23.2. Superheaviness of $T(\rho)$. 129

23.3. Critical values and eigenvalues of $c_{1}(M)$. 134

24. Lagrangian tori in the cubic surface 137

25. Detecting spectral invariant via Hochschild cohomology 145

25.1. Hochschild cohomology of filtered $A_{\infty}$ algebra: review 146

25.2. From quantum cohomology to Hochschild cohomology 1

25.3. Proof of Theorem 25.1 148

25.4. A remark 150

Part 6. Appendix 151

26. $\mathcal{P}_{\left(H_{\chi}, J_{\chi}\right), *}^{\mathfrak{b}}$ is an isomorphism 151

27. Independence on the de Rham representative of $\mathfrak{b}$. 158

28. Proof of Proposition 20.7. 162

28.1. Pseudo-isotopy of filtered $A_{\infty}$ algebra 162

28.2. Difference between $\mathfrak{m}^{T}$ and $\mathfrak{m}$.

28.3. Smoothing $T^{n}$-invariant chains. 164

28.4. Wrap-up of the proof of Proposition 20.7. 167

28.5. Proof of Lemma 20.9 172

29. Seidel homomorphism with bulk 172

29.1. Seidel homomorphism with bulk 173

29.2. Proof of Theorem 29.13 180

29.3. Proof of Theorem 29.9 186

30. Spectral invariants and Seidel homomorphism 186

30.1. Valuations and spectral invariants 186

30.2. The toric case 189

Part 7. Kuranishi structure and its CF-perturbation: summary 192

31. Kuranishi structure and good coordinate system 192 
31.1. Orbifold 192

31.2. Kuranishi structure 193

32. Strongly smooth map and fiber product $\quad 196$

32.1. Strongly smooth map $\quad 196$

32.2. Fiber product 196

33. CF perturbation and integration along the fiber 197

33.1. Differential form on the space with Kuranishi structure 197

33.2. CF-perturbation 198

33.3. Integration along the fiber 201

34. Stokes' theorem 203

34.1. Normalized boundary 203

34.2. Statement of Stokes' theorem 205

35. Composition formula 206

\begin{tabular}{|l|l|}
35.1. & Smooth correspondence and its perturbation \\
\hline
\end{tabular}

35.2. Composition of smooth correspondences 207

35.3. Statement of Composition formula 208

References 209

Index 213 


\section{Preface}

Floer theory is an important tool in the study of symplectic topology and in the study of mirror symmetry phenomenon of string theory in physics (and also in low dimensional topology, which is not touched upon in this paper). Since the theory was invented by Floer in the late 80 's, it has gone through the period of technical underpinning and theoretical enhancement, especially manifested in the Lagrangian Floer theory. Now the period of theoretical and technical enhancement of the Gromov-Witten-Floer theory has passed and the time for applying this enhanced theory as a major tool comes for studying symplectic topology and its related areas.

Before these theoretical enhancements took place, the so called, spectral invariants, were introduced by the second named author in the context of Lagrangian submanifolds of the cotangent bundle. This construction adapts the classical RabinowitzHofer-Zehnder's mini-max theory of the action functional and Viterbo's stable Morse theory of generating functions to the mini-max theory for the chain level Floer homology in the construction of symplectic invariants. This construction was subsequently applied to the Hamiltonian Floer theory by Schwarz in the aspherical case and then by the second named author in general. The construction in the nonexact case involves the usage of a Novikov ring and non-Archimedean analysis in a significant manner. These spectral invariants have been used by Entov-Polterovich in their remarkable construction of symplectic quasi-states and quasi-morphisms, which starts to unveil the true symplectic nature of the analytical construction of Floer homology and spectral invariants.

In this memoir, we develop various enhancements of the theory of spectral invariants of Hamiltonian Floer homology and of Entov-Polterovich theory of spectral symplectic quasi-states and quasi-morphisms by incorporating the above mentioned theoretical amplification and also non-Archimedean analysis into the theory. More specifically, we incorporate bulk deformations introduced in [FOOO1, i.e., the deformations by ambient cycles of symplectic manifolds, of Floer homology and of quantum cohomology into the construction of spectral invariants. Essentially the same kind of construction is independently carried out by Usher [Us4] in a slightly less general context. Then we explore various applications of these enhancements to the symplectic topology, especially a new construction of symplectic quasi-states, quasi-morphisms and new Lagrangian intersection results for toric and non-toric manifolds by exploiting various explicit calculations involving the potential function that the authors made in a series of recent papers on the Lagrangian Floer theory of toric manifolds [FOOO3, FOOO4, [FOOO7].

The most novel part of this memoir is our usage of the open-closed GromovWitten-Floer theory (more specifically, the usage of operator $\mathfrak{q}$, which is a morphism between the quantum cohomology of $(M, \omega)$ and the Hochschild cohomology of Lagrangian Floer cohomology in FOOO1 and its variant involving closed orbits of periodic Hamiltonian system), which connect spectral invariants (with bulk deformation), symplectic quasi-states, quasi-morphism to the Lagrangian Floer theory (with bulk deformation) and open-closed Gromov-Witten theory.

We use this open-closed Gromov-Witten-Floer theory to produce new examples. Especially, using the calculation of Lagrangian Floer cohomology and the critical point theory of the potential function with a bulk deformation, we produce examples of compact symplectic manifolds $(M, \omega)$ that admit a continuum of linearly independent quasi-morphisms $\widetilde{\operatorname{Ham}}(M, \omega) \rightarrow \mathbb{R}$. 
We also prove a new intersection result for the Lagrangian submanifold in $S^{2} \times S^{2}$ discovered in [FOOO6].

Many of these applications were announced in [FOOO3, FOOO4, FOOO6].

On the technical side, we exclusively use the de Rham version of various constructions and calculations performed in the relevant Gromov-Witten-Floer theory. We adopt the de Rham version in this memoir partly to make the exposition consistent with the series of our papers studying toric manifolds [FOOO3, FOOO4, FOOO7. Another reason is that many proofs become simpler when we use the de Rham version of the constructions. We have no doubt that all the results can be proved by using singular homology. Then we can use $\mathbb{Q}$ instead of $\mathbb{R}$ as the underlying ground field.

November 2011

Fukaya, Oh, Ohta, Ono 


\section{INTRODUCTION}

1.1. Introduction. Let $(M, \omega)$ be a compact symplectic manifold. We consider one-periodic nondegenerate Hamiltonians $H: S^{1} \times M \rightarrow \mathbb{R}$, not necessarily normalized, and one-periodic family $J=\left\{J_{t}\right\}_{t \in S^{1}}$ of almost complex structures compatible with $\omega$. To each given such pair $(H, J)$, we can associate the Floer homology $H F(H, J)$ by considering the perturbed Cauchy-Riemann equation

$$
\frac{\partial u}{\partial \tau}+J_{t}\left(\frac{\partial u}{\partial t}-X_{H_{t}}(u)\right)=0
$$

where $H_{t}(x)=H(t, x)$ and $X_{H_{t}}$ is the Hamiltonian vector field associated to $H_{t} \in$ $C^{\infty}(M)$. The associated chain complex $\left(C F(M, H), \partial_{(H, J)}\right)$ is generated by certain equivalence classes of the pairs $[\gamma, w]$ where $\gamma$ is a loop satisfying $\dot{\gamma}(t)=X_{H_{t}}(\gamma)$, $w: D^{2} \rightarrow M$ is a disc with $\left.w\right|_{\partial D^{2}}=\gamma$ and $[\gamma, w]$ is the homotopy class relative to the boundary $\gamma$. See Definitions 2.3 and 4.9. The boundary operator $\partial_{(H, J)}$ is defined by an appropriate weighted count of the solution set of the equation (1.1). This chain complex carries a natural downward filtration provided by the action functional

$$
\mathcal{A}_{H}([\gamma, w])=-\int w^{*} \omega-\int_{0}^{1} H(t, \gamma(t)) d t
$$

since (1.1) is the negative $L^{2}$-gradient flow of $\mathcal{A}_{H}$ with respect to the $L^{2}$-metric on $\mathcal{L}(M)$.

The homology group of $\left(C F(M, H), \partial_{(H, J)}\right)$ is the Floer homology $H F_{*}(M ; H, J)$ associated to the one-periodic Hamiltonian $H . H F_{*}(M ; H, J)$ is known to be isomorphic to the ordinary homology $H_{*}(M)$ of $M$ with appropriate Novikov field as its coefficient ring. $([\mathrm{Fl}])$. The isomorphism is given by the Piunikhin isomorphism Piu

$$
\mathcal{P}_{H}: H_{*}(M) \rightarrow H F_{*}(M ; H, J) .
$$

We use it to transfer a quantum cohomology class $a \in Q H^{*}(M) \cong H^{*}(M)$ to a Floer homology class via the map $a \mapsto a_{H}^{b}:=\mathcal{P}_{H} \circ b(a)$ where $b$ is the Poincaré duality: $Q H^{*}(M) \rightarrow H_{*}(M)$.

The spectral invariants constructed by the second named author in Oh4 for the general non-exact case are defined as follows. (See Vi1, Oh1, Sc2 for the earlier related works for the exact case.) For a given quantum cohomology class $a$, we consider Floer cycles $\alpha$ representing the associated Floer homology class $a_{H}^{b}$. Then we take the mini-max value

$$
\begin{aligned}
\lambda_{H}(\alpha) & =\max \left\{\mathcal{A}_{H}\left(\left[\gamma_{i}, w_{i}\right]\right) \mid \alpha=\sum a_{i}\left[\gamma_{i}, w_{i}\right], a_{i} \in \mathbb{C} \backslash\{0\}\right\} \\
\rho(H ; a) & =\inf \left\{\lambda_{H}(\alpha) \mid \partial_{(H, J)}(\alpha)=0,[\alpha]=a_{H}^{b}\right\}
\end{aligned}
$$

We can show that the right hand side of (1.5) is independent on the choice of $J$. So $J$ is not included in the notation $\rho(H ; a)$. Via the $C^{0}$-continuity of the function $H \mapsto \rho(H ; a)$, the function extends continuously to arbitrary continuous function $H$.

It induces an invariant of an element of the universal cover of the group of Hamiltonian diffeomorphisms as follows. We denote by $\operatorname{Ham}(M, \omega)$ the group of Hamiltonian diffeomorphisms of $M$ and by $\widehat{\operatorname{Ham}}(M, \omega)$ its universal cover and by 
$\phi_{H}: t \mapsto \phi_{H}^{t}$ the Hamiltonian path (based at the identity) generated by the (timedependent) Hamiltonian $H$ and its time one map by $\psi_{H}=\phi_{H}^{1} \in \operatorname{Ham}(M, \omega)$. Each Hamiltonian $H$ generates the Hamiltonian path $\phi_{H}$ which in turn determines an element $\widetilde{\psi}_{H}=\left[\phi_{H}\right] \in \widetilde{\operatorname{Ham}}(M, \omega)$. Conversely, each smooth Hamiltonian path $[0,1] \rightarrow \operatorname{Ham}(M, \omega)$ based at the identity is generated by a unique normalized Hamiltonian $H$, i.e., $H$ satisfying

$$
\int_{M} H_{t} \omega^{n}=0
$$

It is proved in Oh4, Oh7 that $\rho(H ; a)$ for normalized Hamiltonians $H$ depends only on the homotopy class $\widetilde{\psi}=\widetilde{\psi}_{H}$ of the path $\phi_{H}$ and $a$, which we denote by

$$
\rho(\widetilde{\psi} ; a):=\rho(H ; a) .
$$

This homotopy invariance is proved for the rational $(M, \omega)$ in Oh4 and for the irrational case in Oh7, Us1 respectively.

In a series of papers EP1, EP2, EP3, Entov and Polterovich discovered remarkable applications of these spectral invariants to the theory of symplectic intersections and to the study of $\operatorname{Ham}(M, \omega)$ by combining ideas from dynamical systems, function theory and quantum cohomology. We briefly summarize their construction now. (As we explain in Subsection 1.3 we adopt conventions slightly different from Entov and Polterovich's below. However there is no essential mathematical difference.)

Let $Q H^{*}(M ; \Lambda)$ be the quantum cohomology ring of $M$. (Here $\Lambda$ is the (universal) Novikov field. See Notation and Convention (16).) They considered idempotent elements $e$ of $Q H^{*}(M ; \Lambda)$, i.e., those satisfying $e^{2}=e$ and defined the function $\zeta_{e}: C^{0}(M) \rightarrow \mathbb{R}$ by

$$
\zeta_{e}(H):=\lim _{n \rightarrow \infty} \frac{\rho(n H ; e)}{n}
$$

for autonomous $C^{\infty}$ functions $H$ and then extended its definition to $C^{0}(M)$ by continuity.

It is proved in EP1, EP2, EP3 that $\zeta_{e}$ satisfies most of the properties of quasistates introduced by Aarnes $\mathrm{Aa}$ and introduced the notion of a partial symplectic quasi-state. They also formulated the notions of heavy and super-heavy subsets of symplectic manifolds. Entov and Polterovich also considered the map $\mu_{e}: \widetilde{\operatorname{Ham}}(M, \omega) \rightarrow \mathbb{R}$ by

$$
\mu_{e}(\widetilde{\psi})=-\operatorname{vol}_{\omega}(M) \lim _{n \rightarrow \infty} \frac{\rho\left(\widetilde{\psi}^{n} ; e\right)}{n} .
$$

Whenever $e$ is the unit of a direct factor of $Q H^{*}(M ; \Lambda)$, which is a field, $\mu_{e}$ becomes a homogeneous quasi-morphism. Namely it satisfies

$$
\left|\mu_{e}\left(\widetilde{\psi}_{1}\right)+\mu_{e}\left(\widetilde{\psi}_{2}\right)-\mu_{e}\left(\widetilde{\psi}_{1} \widetilde{\psi}_{2}\right)\right|<C,
$$

for some constant $C$ independent of $\widetilde{\psi}_{1}, \widetilde{\psi}_{2}$ and

$$
\mu_{e}\left(\widetilde{\psi}^{n}\right)=n \mu_{e}(\widetilde{\psi}), \quad \text { for } n \in \mathbb{Z} .
$$

These facts were proved by Entov-Polterovich [EP1] in case $Q H^{*}(M ; \Lambda)$ is semisimple and $M$ is monotone. The monotonicity assumption was improved by Ostrover Os2. It was observed by McDuff that instead of the semi-simplicity assumption one has only to assume that $e$ is the unit of a field factor of $Q H^{*}(M ; \Lambda)$. In fact, 
Entov and Polterovich prove several other symplectic properties of $\mu_{e}$, and call them Calabi quasi-morphisms.

One can generalize the constructions mentioned above by involving bulk deformations. Here bulk deformation means a deformation of various Floer theories and others by using a cohomology class $\mathfrak{b}$ of the ambient symplectic manifold. We call $\mathfrak{b}$ a bulk cycle and denote the corresponding deformed Floer homology by $H F^{\mathfrak{b}}(M ; H, J)$ which we call a Floer homology with bulk. (We will define it in Part 2.) Actually as in the case of $\operatorname{HF}(M ; H, J)$, the Floer homology with bulk $H F^{\mathfrak{b}}(M ; H, J)$ as a $\Lambda$ module is also isomorphic to the ordinary homology group $H(M ; \Lambda)$ of $M$. The generalization is straightforward way except the following point:

We note that for the construction of partial quasi-states and quasi-morphism the following triangle inequality of spectral invariant plays an important role.

$$
\rho\left(\widetilde{\psi}_{1} \circ \widetilde{\psi}_{2}, a \cup_{Q} b\right) \leq \rho\left(\widetilde{\psi}_{1}, a\right)+\rho\left(\widetilde{\psi}_{2}, b\right),
$$

here $\cup_{Q}$ is the product of the small quantum cohomology ring $Q H^{*}(M ; \Lambda)$ and $\rho$ is the spectral invariant (1.7) (without bulk deformation).

We generalize the definitions of (1.3), (1.5), (1.7) to obtain corresponding ones $\mathcal{P}_{H}^{\mathfrak{b}}, \rho^{\mathfrak{b}}(H ; a), \rho^{\mathfrak{b}}(\widetilde{\psi} ; a)$, respectively. Then (1.12) becomes

$$
\rho^{\mathfrak{b}}\left(\widetilde{\psi}_{1} \circ \widetilde{\psi}_{2}, a \cup^{\mathfrak{b}} b\right) \leq \rho^{\mathfrak{b}}\left(\widetilde{\psi}_{1}, a\right)+\rho^{\mathfrak{b}}\left(\widetilde{\psi}_{2}, b\right),
$$

where $\cup^{\mathfrak{b}}$ is the deformed cup product by $\mathfrak{b}$. (See Definition 5.1 for its definition.) Thus in place of the small quantum cohomology ring $Q H^{*}(M ; \Lambda)$ the $\mathfrak{b}$-deformed quantum cohomology ring (which we denote by $Q H_{\mathfrak{b}}^{*}(M ; \Lambda)$ ) plays an important role here.

Whenever $e \in Q H_{\mathfrak{b}}^{*}(M ; \Lambda)$ is an idempotent, we define

$$
\zeta_{e}^{\mathfrak{b}}(H)=-\lim _{n \rightarrow \infty} \frac{\rho^{\mathfrak{b}}(n H ; e)}{n}
$$

for the autonomous function $H=H(x) \in C^{\infty}(M)$ which in turn defines a partial symplectic quasi-state on $C^{0}(M)$. See Definition 13.6 and Theorem 14.1. Similarly we can define $\mu_{e}^{\mathfrak{b}}: \widehat{\operatorname{Ham}}(M, \omega) \rightarrow \mathbb{R}$. We will call any such partial quasi-state or quasi-morphism obtained from the spectral invariants a spectral partial quasi-state or a spectral quasi-morphism respectively.

Theorem 1.1. Let $\Lambda e \cong \Lambda$ be a direct factor of $Q H_{\mathfrak{b}}^{*}(M ; \Lambda)$ and e its unit. Then

$$
\mu_{e}^{\mathfrak{b}}: \widetilde{\operatorname{Ham}}(M, \omega) \rightarrow \mathbb{R}
$$

is a homogeneous Calabi quasi-morphism.

Theorem 1.1 is proved in Section 16 In particular, combined with the study of big quantum cohomology of toric manifolds [FOOO7, this implies the following: (The proof is completed in Subsection 21.3.)

Corollary 1.2. For any compact toric manifold $(M, \omega)$, there exists a nontrivial homogeneous Calabi quasi-morphism

$$
\mu_{e}^{\mathfrak{b}}: \widetilde{\operatorname{Ham}}(M, \omega) \rightarrow \mathbb{R} .
$$

We say that a quasi-morphism is nontrivial if it is not bounded. Corollary 1.2 is also proved independently by Usher Us4]. 
It is in general very hard to calculate spectral invariants and partial quasi-states or quasi-morphisms obtained therefrom. In Part 4 of this paper we provide a means of estimating them in certain cases. We recall the following definition:

Definition 1.3. (Entov-Polterovich EP3]) Let $\zeta: C^{0}(M) \rightarrow \mathbb{R}$ be any partial quasi-state. A closed subset $Y \subset X$ is called $\zeta$-heavy if

$$
\zeta(H) \leq \sup \{H(p) \mid p \in Y\}
$$

for any $H \in C^{0}(X)$. $Y \subset X$ is called $\zeta$-superheavy if

$$
\zeta(H) \geq \inf \{H(p) \mid p \in Y\}
$$

for any $H \in C^{0}(X)$.

Remark 1.4. (1) Entov-Polterovich proved in EP3, Theorem 1.4 (i)] that superheaviness implies heaviness for $\zeta_{e}$. The same can be proved for $\zeta_{e}^{\mathfrak{b}}$ by the same way.

(2) Actually in Definition 1.3 we use a different sign convention from that of Entov-Polterovich. See Remark 1.14.

We can also define similar notions $\mu$-heaviness and $\mu$-superheaviness, for $\mu$ : $\widehat{\operatorname{Ham}}(M, \omega) \rightarrow \mathbb{R}$, instead of $\zeta: C^{0}(M) \rightarrow \mathbb{R}$. See Definition 18.5. In Part 4 of this paper we provide a way to use Lagrangian Floer theory to show certain Lagrangian submanifold is $\mu$-heavy or $\mu$-superheavy for $\mu=\mu_{e}$ or $\mu_{e}^{\mathfrak{b}}$.

Let $L$ be a relatively spin Lagrangian submanifold of $M$. In FOOO1] we associated to $L$ a set $\mathcal{M}_{\text {weak,def }}\left(L ; \Lambda_{+}\right)$, which we call the Maurer-Cartan moduli space. (See also Definition 17.11)

Remark 1.5. The Maurer-Cartan moduli space that appears in [FOOO1 uses the Novikov ring $\Lambda_{+}$. A technical enhancement to its $\Lambda_{0}$-version was performed in FOOO4, Fu3 using the idea of Cho Cho, which is used in this paper. In this introduction, however, we state only the $\Lambda_{+}$-version for the simplicity of exposition.

The Maurer-Cartan moduli space comes with a map

$$
\pi_{\text {bulk }}: \mathcal{M}_{\text {weak,def }}\left(L ; \Lambda_{+}\right) \rightarrow \bigoplus_{k} H^{2 k}\left(M ; \Lambda_{+}\right)
$$

For each $\mathbf{b} \in \mathcal{M}_{\text {weak, def }}\left(L ; \Lambda_{+}\right)$, the Floer cohomology $H F^{*}\left((L, \mathbf{b}),(L, \mathbf{b}) ; \Lambda_{0}\right)$ deformed by $\mathbf{b}$ is defined in [FOOO1, Definition 3.8.61]. (See Definition[17.13.) Moreover the open-closed map

$$
i_{\mathrm{qm}, \mathbf{b}}^{*}: H^{*}\left(M ; \Lambda_{0}\right) \rightarrow H F^{*}\left((L, \mathbf{b}),(L, \mathbf{b}) ; \Lambda_{0}\right)
$$

is constructed in [FOOO1, Theorem 3.8.62]. (See (17.17).) Utilizing this map $i_{\mathrm{qm}, \mathbf{b}}^{*}$, we can locate $\mu_{e}^{\mathfrak{b}}$-superheavy Lagrangian submanifolds in several circumstances.

Theorem 1.6. Consider a pair $(\mathbf{b}, \mathfrak{b})$ with $\mathbf{b} \in \mathcal{M}_{\text {weak, def }}\left(L ; \Lambda_{+}\right)$and $\pi_{\text {bulk }}(\mathbf{b})=\mathfrak{b}$. Let $e$ be an idempotent of $Q H_{\mathfrak{b}}^{*}(M ; \Lambda)$ such that

$$
i_{\mathrm{qm}, \mathbf{b}}^{*}(e) \neq 0 \in H F^{*}((L, \mathbf{b}),(L, \mathbf{b}) ; \Lambda) .
$$

Then $L$ is $\zeta_{e}^{\mathfrak{b}}$-heavy and $\mu_{e}^{\mathfrak{b}}$-heavy.

If $e$ is a unit of a field factor of $Q H_{\mathfrak{b}}^{*}(M ; \Lambda)$ in addition, then $L$ is $\zeta_{e}^{\mathfrak{b}}$-superheavy and $\mu_{e}^{\mathfrak{b}}$-superheavy.

See Definition 18.5 for the definitions of $\mu_{e}^{\mathfrak{b}}$-heavy and $\mu_{e}^{\mathfrak{b}}$-superheavy sets. Theorem 1.6 (Theorem 18.8) is proved in Section 18. 
Remark 1.7. (1) Theorem 1.6 gives rise to a proof of a conjecture made in [FOOO3, Remark 1.7].

(2) Theorem [1.6 is closely related to [EP3, Theorem 1.20].

Theorem 1.6] also proves linear independence of some spectral Calabi quasimorphisms in the following sense.

Definition 1.8. Let

$$
\mu_{j}: \widetilde{\operatorname{Ham}}(M, \omega) \rightarrow \mathbb{R}
$$

be homogeneous Calabi-quasi-morphisms for $j=1, \ldots, N$. We say that they are linearly independent if there exists a subgroup $\cong \mathbb{Z}^{N}$ of $\widetilde{\operatorname{Ham}}(M, \omega)$ such that the restriction of $\left(\mu_{1}, \ldots, \mu_{N}\right): \widetilde{\operatorname{Ham}}(M, \omega) \rightarrow \mathbb{R}^{N}$ to this subgroup is an isomorphism to a lattice in $\mathbb{R}^{N}$. A (possibly infinite) set of quasi-morphisms of $\widetilde{\operatorname{Ham}}(M, \omega)$ is said to be linearly independent if any of its finite subset is linearly independent in the above sense. The case of $\operatorname{Ham}(M, \omega)$ can be defined in the same way.

Corollary 1.9. Let $L_{j}$ be mutually disjoint relatively spin Lagrangian submanifolds. $(j=1, \ldots, N$. $)$ Let $\mathfrak{b}_{j} \in H^{\text {even }}\left(M ; \Lambda_{+}\right)$and $\mathbf{b}_{j} \in \mathcal{M}_{\text {weak,def }}\left(L_{j} ; \Lambda_{+}\right)$with $\pi_{\text {bulk }}\left(\mathbf{b}_{j}\right)=\mathfrak{b}_{j}$. Let $e_{j}$ be the unit of a field factor of $Q H_{\mathfrak{b}_{j}}^{*}(M, \Lambda)$ such that

$$
i_{\mathrm{qm}, \mathbf{b}_{j}}^{*}\left(e_{j}\right) \neq 0 \in H F^{*}\left(\left(L_{j}, \mathbf{b}_{j}\right),\left(L_{j}, \mathbf{b}_{j}\right) ; \Lambda\right), \quad j=1, \ldots, N .
$$

Then $\mu_{e_{j}}^{\mathfrak{b}_{j}}(j=1, \ldots, N)$ are linearly independent.

This corollary follows from Theorem 1.6 mentioned above and EP3, Theorem 8.2]. (See also Section 19 of this paper.)

The study of toric manifolds FOOO4 and deformations of some toric orbifolds FOOO6 provides examples for which the hypothesis of Corollary 1.9 is satisfied. This study gives rise to the following theorem

Theorem 1.10. Let $M$ be one of the following three kinds of symplectic manifolds:

(1) $S^{2} \times S^{2}$ with monotone toric symplectic structure,

(2) Cubic surface,

(3) $k$ points blow up of $\mathbb{C} P^{2}$ with a certain toric symplectic structure, where $k \geq 2$.

Then $(M, \omega)$ carries a collection $\left\{\mu_{a}\right\}_{a \in \mathfrak{A}}$ of linearly independent Calabi quasimorphisms

$$
\mu_{a}: \widetilde{\operatorname{Ham}}(M, \omega) \rightarrow \mathbb{R}
$$

for an uncountable indexing set $\mathfrak{A}$.

Remark 1.11. (1) In the case of $(M, \omega)=S^{2} \times S^{2}$, we have quasi-morphisms $\mu_{a}: \operatorname{Ham}(M, \omega) \rightarrow \mathbb{R}$ in place of $\mu_{a}: \widehat{\operatorname{Ham}}(M, \omega) \rightarrow \mathbb{R}$. See Corollary 23.6

(2) We can explicitly specify the symplectic structure used in Theorem 1.10 (3). See Section 23 ,

(3) We can also construct an example of the symplectic manifold that admits an uncountable set of linearly independent quasi-morphisms in higher dimension by the similar way. (For example we can take direct product with $S^{2}$ of the symplectic manifolds in Theorem 1.10)

(4) Theorem 1.10 for $S^{2} \times S^{2}$ was announced in [FOOO6, Remark 7.1], and for the case of $k$-points $(k \geq 2)$ blow up of $\mathbb{C} P^{2}$ in [FOOO4, Remark 1.2 (3)], respectively. 
(5) Biran-Entov-Polterovich constructed an uncountable family of linearly independent Calabi quasi-morphisms for the case of the $\operatorname{group} \operatorname{Ham}\left(B^{2 n}(1) ; \omega\right)$ of compactly supported Hamiltonian diffeomorphisms of balls with $n \geq 2$ in BEP. Theorem 1.10 provides the first example of closed $M$ with such property.

(6) Existence of infinitely many linearly independent homogeneous Calabi quasimorphisms on $\operatorname{Ham}(M, \omega)$ is still an open problem for the case of $M=\mathbb{C} P^{2}$ as well as $\mathbb{C} P^{n}$ for general $n \geq 2$.

(7) Theorem 1.10 implies that the second bounded cohomology of $\widetilde{\operatorname{Ham}}(M ; \omega)$ is of infinite rank for $(M, \omega)$ appearing in Theorem 1.10. More precisely, the defect Def $_{a}$ defined by

$$
\operatorname{Def}_{a}(\phi, \psi):=\mu_{a}(\phi)+\mu_{a}(\psi)-\mu_{a}(\phi \psi)
$$

defines a bounded two-cocycle. It follows from the perfectness of the group $\operatorname{Ham}(M ; \omega)$ [Ba2, Corollary 4.3.2 (1)] that the set of cohomology classes of $\left\{\right.$ Def $\left._{a}\right\}$ is linearly independent in the 2 nd bounded cohomology group of $\widetilde{\operatorname{Ham}}(M, \omega)$. In fact, if otherwise, we can find a nonzero linear combination of elements from $\left\{\operatorname{Def}_{a}\right\}$ which is a coboundary. Namely we have

$$
\sum c_{i} \operatorname{Def}_{a_{i}}(\phi, \psi)=\rho(\phi)+\rho(\psi)-\rho(\phi \psi)
$$

where $\rho: \widetilde{\operatorname{Ham}}(M ; \omega) \rightarrow \mathbb{R}$ is bounded and $c_{i} \in \mathbb{R}$. Therefore

$$
\sum c_{i} \mu_{a_{i}}-\rho: \widetilde{\operatorname{Ham}}(M ; \omega) \rightarrow \mathbb{R}
$$

is a homomorphism. The linear independence of $\mu_{a_{i}}$ implies that $\sum c_{i} \mu_{a_{i}}$ is unbounded. Therefore (1.18) defines a nontrivial homomorphism, which contradicts the perfectness of $\widetilde{\operatorname{Ham}}(M, \omega)$.

(8) At the final stage of completing this paper, a paper Bor appears in the arXiv which discusses a result related to Theorem 1.10 (3) using [AM].

Another corollary of Theorem [1.6] combined with Theorem 1.4 (iii), [EP3, Theorem 1.8] is the following intersection result of the exotic Lagrangian tori discovered in $\mathrm{FOOO6}$.

Theorem 1.12. Let $T(u) \subset S^{2}(1) \times S^{2}(1)$ for $0<u \leq 1 / 2$ be the tori from FOOO6. Then we have

$$
\psi(T(u)) \cap\left(S_{\text {eq }}^{1} \times S_{\text {eq }}^{1}\right) \neq \emptyset
$$

for any symplectic diffeomorphism $\psi$ of $S^{2}(1) \times S^{2}(1)$.

Remark 1.13. Theorem 1.12 was announced in the introduction of [FOOO6]. The proof is given in Subsection 23.2

A brief outline of the content of the paper is now in order. The present paper consist of 7 parts. Part 1 is a review. In Part 2, we first enhance the Hamiltonian Floer theory by involving its deformations by ambient cohomology classes, which we call bulk deformations. In this paper, we use de Rham (co)cycles instead of singular cycles as in [FOOO4, FOOO7]. After this enhancement, we generalize construction of spectral invariants in Oh4 involving bulk deformations and define spectral invariants with bulk. Part 3 then generalizes construction [EP2, EP1] of 
symplectic partial quasi-states and Calabi quasi-morphisms by replacing the spectral invariants defined in [Oh4] by these spectral invariants with bulk.

In the course of carrying out these enhancements, we also unify, clarify and enhance many known constructions in Hamiltonian Floer theory in the framework of virtual fundamental chain technique. (We use its version based on Kuranishi structures and accompanied abstract perturbation theory originally established in $[\mathrm{FO}$ and further enhanced in FOOO2, Appendix A.2].) Variants of virtual fundamental chain technique, some of which we use in this paper, are given in FOOO4, FOOO7, Fu3. Explanation of further detail of virtual fundamental chain technique is provided in FOOO10, FOOO11. Systematic application of virtual technique is needed particularly because many constructions related to the study of spectral invariants (with bulk) have to be done in the chain level, not just in homology. Examples of such enhancement include construction of pants product [Sc1] and Piunikhin isomorphism whose construction was outlined in [Piu, RT, PSS]. We give a complete construction of both of these in general compact symplectic manifolds without assuming any conditions on $(M, \omega)$ such as semi-positivity or rationality.

In Part 4, we connect the study of spectral invariants to the Lagrangian Floer theory developed in FOOO1, FOOO2. The main construction in the study is based on open-closed Gromov-Witten theory developed in [FOOO1, Section 3.8], which induces a map from the quantum cohomology of the ambient symplectic manifolds to the Hochschild cohomology of $A_{\infty}$ algebra (or more generally that of Fukaya category of $(M, \omega)$ ). This map was defined in FOOO1 and further studied in [FOOO4, FOOO7, Section 2.6] and etc.. This part borrows much from [FOOO1, FOOO2, FOOO7] in its exposition. The main new ingredient is a construction of a map from Floer homology of periodic Hamiltonians to Floer cohomology of Lagrangian submanifold, through which the map from quantum cohomology to Floer cohomology of Lagrangian submanifold factors (Subsection 18.4). We also study its properties especially those related to the filtration. A similar construction was used by Albers $\mathrm{Al}$ and also by Biran-Cornea $\mathrm{BC}$ in the monotone context, which has been exploited by Entov-Polterovich [EP3 in their study of symplectic intersections.

In Part 5, we combine the results obtained in the previous parts together with the results on the Lagrangian Floer theory of toric manifolds obtained in the series of our previous papers [FOOO3, FOOO4, FOOO6, FOOO7, give various new constructions of Calabi quasi-morphisms and new Lagrangian intersections results on toric manifolds and other Kähler surfaces. These results are obtained by detecting the heaviness of Lagrangian submanifolds in the sense of Entov-Polterovich EP3 in terms of spectral invariants, critical point theory of potential functions and also the closed-open map from quantum cohomology to Hochschild cohomology of $A_{\infty}$-algebra of Lagrangian submanifolds.

Finally in Part 6, we prove various technical results necessary to complete the constructions carried out in the previous parts. For example, we establish the isomorphism property of the Piunikhin map with bulk. We give the construction of Seidel homomorphism with bulk extending the results of [Se] and generalize the McDuff-Tolman's representation of quantum cohomology ring of toric manifolds in terms of Seidel elements [MT] to that of big quantum cohomology ring. 
Some portion of the present paper is devoted to proving various results in Hamiltonian Floer theory, spectral invariants, Entov-Polterovich theory of symplectic quasi-states and others for arbitrary compact symplectic manifolds. These proofs are often similar to those in the literature except we apply virtual fundamental chain technique (via the Kuranishi structure) systematically. In fact, most of the literature assume semi-positivity since they do not use virtual fundamental chain technique. Even when virtual fundamental chain technique is used, not enough details on the way how to apply the technique are provided. Because of these reasons, for readers's convenience and for the completeness' sake, we provide a fair amount of these details on the proofs in the literature in a unified and coherent fashion in the most general context using the framework of Kuranishi structure and virtual fundamental chain technique, without imposing any restrictions on the ambient symplectic manifold $(M, \omega)$. We include a brief summary of the theory of Kuranishi structure and its perturbation as Part 7 for reader's convenience.

On the other hand, the most novel part of this paper is to combine the story of spectral invariants and Entov-Polterovich theory with that of bulk deformations, Lagrangian Floer theory and the calculation of them in the toric case to obtain new examples, especially those appearing in Theorem 1.10

1.2. Notations and Conventions. We follow the conventions of Oh4, Oh7, Oh8 for the definition of Hamiltonian vector fields and action functional and others appearing in the Hamiltonian Floer theory and in the construction of spectral invariants and Entov-Polterovich's Calabi quasi-morphisms. There are differences from e.g., those used in EP1, EP2, EP3] one way or the other. (See Subsection 1.3 for the explanation of the differences.)

(1) The Hamiltonian vector field $X_{H}$ is defined by $d H=\omega\left(X_{H}, \cdot\right)$.

(2) The flow of $X_{H}$ is denoted by $\phi_{H}: t \mapsto \phi_{H}^{t}$ and its time-one map by $\psi_{H}=\phi_{H}^{1} \in \operatorname{Ham}(M, \omega)$. We say that $H$ or its associated map $\psi_{H}$ is nondegenerate if at $p \in \operatorname{Fix} \psi_{H}$, the differential $d_{p} \psi_{H}: T_{p} M \rightarrow T_{p} M$ does not have eigenvalue 1 . We denote by $\operatorname{Ham}_{\text {nd }}(M, \omega)$ the subset of $\operatorname{Ham}(M, \omega)$ consisting of nondegenerate $\psi_{H}$ 's.

(3) We denote by $\left[\phi_{H}\right]$ the path homotopy class of $\phi_{H}:[0,1] \rightarrow \operatorname{Ham}(M, \omega)$ relative to the ends which we generally denote $\widetilde{\psi}_{H}=\left[\phi_{H}\right]$. We denote by $z_{H}^{p}(t)=\phi_{H}^{t}(p)$ the solution associated to a fixed point $p$ of $\psi_{H}=\phi_{H}^{1}$.

(4) $\widetilde{H}(t, x)=-H(1-t, x)$ is the time-reversal Hamiltonian generating $\phi_{H}^{1-t} \phi_{H}^{-1}$.

(5) We denote by $H_{1} * H_{2}$ the Hamiltonian generating the concatenation of the two Hamiltonian paths $\phi_{H_{1}}$ followed by $\phi_{H_{2}}^{t}$. More explicitly, it is defined by

$$
\left(H_{1} * H_{2}\right)(t, x)= \begin{cases}2 H_{1}(2 t, x) & 0 \leq t \leq 1 / 2 \\ 2 H_{2}(2 t-1, x) & 1 / 2 \leq t \leq 1\end{cases}
$$

(6) The action functional $\mathcal{A}_{H}: \widetilde{\mathcal{L}}_{0}(M) \rightarrow \mathbb{R}$ is defined by

$$
\mathcal{A}_{H}([\gamma, w])=-\int w^{*} \omega-\int_{0}^{1} H(t, \gamma(t)) d t .
$$

(7) We denote by $\operatorname{Per}(H)$ the set of periodic orbits of periodic Hamiltonian system associated to the Hamiltonian $H:[0,1] \times M \rightarrow \mathbb{R}$. $\operatorname{Crit}\left(\mathcal{A}_{H}\right)$ is the set of critical points of $\mathcal{A}_{H}$. Its element is denoted by $[\gamma, w]$ where 
$\gamma \in \operatorname{Per}(H)$ and $w$ is a disk which bounds $\gamma$. We identify $[\gamma, w]$ and $\left[\gamma, w^{\prime}\right]$ if $w$ is homotopic to $w^{\prime}$. We define $[\gamma, w] \sim\left[\gamma, w^{\prime}\right]$ if $\int w^{*} \omega=\int\left(w^{\prime}\right)^{*} \omega$ and denote by $\widehat{\operatorname{Per}}(H)$ the set of the equivalence classes. An element of $\widehat{\operatorname{Per}}(H)$ is denoted by $\llbracket \gamma, w \rrbracket$. There exists a map

$$
\operatorname{Crit}\left(\mathcal{A}_{H}\right) \stackrel{\pi}{\longrightarrow} \widehat{\operatorname{Per}}(H) \longrightarrow \operatorname{Per}(H)
$$

defined by $[\gamma, w] \mapsto \llbracket \gamma, w \rrbracket, \llbracket \gamma, w \rrbracket \mapsto \gamma$.

(8) $\mathcal{J}_{\omega}=$ the set of $\omega$-compatible almost complex structures. $j_{\omega}=\mathcal{L}\left(\mathcal{J}_{\omega}\right)=$ the set of $S^{1}$-family $J$ of compatible almost complex structures; $J=\left\{J_{t}\right\}_{t \in S^{1}}$.

(9) $\mathcal{P}\left(j_{\omega}\right)=\operatorname{Map}\left([0,1] \times S^{1}, \mathcal{J}_{\omega}\right) ; \quad(s, t) \in[0,1] \times S^{1} \mapsto J_{t}^{s} \in \mathcal{J}_{\omega}$.

(10) $\mathcal{K}=\{\chi: \mathbb{R} \rightarrow[0,1]\}$ where $\chi$ is a smooth function with $\chi^{\prime}(\tau) \geq 0, \chi(\tau) \equiv 0$ for $\tau \leq 0$ and $\chi(\tau) \equiv 1$ for $\tau \geq 1$. We define $\tilde{\chi}$ by $\tilde{\chi}=1-\chi$.

(11) For given $H \in C^{\infty}\left(S^{1} \times M, \mathbb{R}\right)$, we define the $\mathbb{R}$-family $H_{\chi}$ by

$$
H_{\chi}(\tau, t, x)=\chi(\tau) H(t, x) \text {. }
$$

(12) For $J \in \mathcal{P}\left(j_{\omega}\right)$ we take $J_{s}=\left\{J_{s, t} ; t \in S^{1}\right\}$ such that

$$
J_{1, t}=J_{t}, \quad J_{0, t}=J_{0}, \quad J_{s, 0}=J_{0},
$$

and put

$$
J_{\chi}(\tau, t)=J_{\chi(\tau), t} .
$$

(13) If $H \in C^{\infty}\left([0,1] \times S^{1} \times M, \mathbb{R}\right)$ and $J \in \mathcal{P}\left(j_{\omega}\right)$, we put

$$
H^{\chi}(\tau, t, x)=H(\chi(\tau), t, x), \quad J^{\chi}(\tau, t, x)=J(\chi(\tau), t, x) .
$$

(14) Let $\Omega_{*}(M) \widehat{\otimes} \Lambda^{\downarrow}$ be the completion of the algebraic tensor product $\Omega_{*}(M) \otimes$ $\Lambda^{\downarrow}$ with respect to the metric induced by the valuation $\mathfrak{v}_{q}$ on $\Lambda^{\downarrow}$ defined in item (18) below. The Piunikhin chain map

$$
\mathcal{P}_{\left(H_{\chi}, J_{\chi}\right)}^{\mathfrak{b}}: \Omega_{*}(M) \widehat{\otimes} \Lambda^{\downarrow} \rightarrow C F_{*}\left(M, H ; \Lambda^{\downarrow}\right)
$$

is associated to $\left(H_{\chi}, J_{\chi}\right)$ in (11),(12). (See Section 6). $\Lambda^{\downarrow}$ is defined in item (17). The map

$$
\mathcal{Q}_{\left(H_{\tilde{\chi}}, J_{\tilde{\chi}}\right)}^{\mathfrak{b}}: C F_{*}\left(M, H ; \Lambda^{\downarrow}\right) \rightarrow \Omega_{*}(M) \widehat{\otimes} \Lambda^{\downarrow}
$$

is associated to $\tilde{\chi}(\tau)=\chi(1-\tau)$. (See Section 26])

(15) We denote the set of shuffles of $\ell$ elements by

$$
\operatorname{Shuff}(\ell)=\left\{\left(\mathbb{L}_{1}, \mathbb{L}_{2}\right) \mid \mathbb{L}_{1} \cup \mathbb{L}_{2}=\{1, \ldots, \ell\}, \mathbb{L}_{1} \cap \mathbb{L}_{2}=\emptyset\right\} .
$$

For $\left(\mathbb{L}_{1}, \mathbb{L}_{2}\right) \in \operatorname{Shuff}(\ell)$ let $\# \mathbb{L}_{i}$ be the order of this subset. Then $\# \mathbb{L}_{1}+$ $\# \mathbb{L}_{2}=\ell$.

The set of triple shuffles is the set of $\left(\mathbb{L}_{1}, \mathbb{L}_{2}, \mathbb{L}_{3}\right)$ such that $\mathbb{L}_{1} \cup \mathbb{L}_{2} \cup \mathbb{L}_{3}=$ $\{1, \ldots, \ell\}$ and that $\mathbb{L}_{1}, \mathbb{L}_{2}, \mathbb{L}_{3}$ are mutually disjoint.

(16) The universal Novikov ring $\Lambda_{0}$ and its filed $\Lambda$ of fractions are defined by

$$
\begin{aligned}
\Lambda_{0} & =\left\{\sum_{i=1}^{\infty} a_{i} T^{\lambda_{i}} \mid a_{i} \in \mathbb{C}, \lambda_{i} \in \mathbb{R}_{\geq 0}, \lim _{i \rightarrow \infty} \lambda_{i}=+\infty\right\} \\
\Lambda & =\left\{\sum_{i=1}^{\infty} a_{i} T^{\lambda_{i}} \mid a_{i} \in \mathbb{C}, \lambda_{i} \in \mathbb{R}, \lim _{i \rightarrow \infty} \lambda_{i}=+\infty\right\} \cong \Lambda_{0}\left[T^{-1}\right] .
\end{aligned}
$$


The maximal ideal of $\Lambda_{0}$ is denoted by

$$
\Lambda_{+}=\left\{\sum_{i=1}^{\infty} a_{i} T^{\lambda_{i}} \mid a_{i} \in \mathbb{C}, \lambda_{i} \in \mathbb{R}_{>0}, \lim _{i \rightarrow \infty} \lambda_{i}=+\infty\right\} .
$$

We define the valuation $\mathfrak{v}_{T}$ on $\Lambda$ by

$$
\mathfrak{v}_{T}\left(\sum_{i=1}^{\infty} a_{i} T^{\lambda_{i}}\right)=\inf \left\{\lambda_{i} \mid a_{i} \neq 0\right\}, \quad \mathfrak{v}_{T}(0)=+\infty .
$$

(17) We also use the following (downward) Novikov ring $\Lambda_{0}^{\downarrow}$ and field $\Lambda^{\downarrow}$ :

$$
\begin{aligned}
& \Lambda_{0}^{\downarrow}=\left\{\sum_{i=1}^{\infty} a_{i} q^{\lambda_{i}} \mid a_{i} \in \mathbb{C}, \lambda_{i} \in \mathbb{R}_{\leq 0}, \lim _{i \rightarrow \infty} \lambda_{i}=-\infty\right\}, \\
& \Lambda^{\downarrow}=\left\{\sum_{i=1}^{\infty} a_{i} q^{\lambda_{i}} \mid a_{i} \in \mathbb{C}, \lambda_{i} \in \mathbb{R}, \lim _{i \rightarrow \infty} \lambda_{i}=-\infty\right\} \cong \Lambda_{0}^{\downarrow}[q] .
\end{aligned}
$$

The maximal ideal of $\Lambda_{0}^{\downarrow}$ is denoted by

$$
\Lambda_{-}^{\downarrow}=\left\{\sum_{i=1}^{\infty} a_{i} q^{\lambda_{i}} \in \Lambda^{\downarrow} \mid \lambda_{i}<0\right\} .
$$

(18) We define the valuation $\mathfrak{v}_{q}$ on $\Lambda^{\downarrow}$ by

$$
\mathfrak{v}_{q}\left(\sum_{i=1}^{\infty} a_{i} q^{\lambda_{i}}\right)=\sup \left\{\lambda_{i} \mid a_{i} \neq 0\right\}, \quad \mathfrak{v}_{q}(0)=-\infty .
$$

Of course, $\Lambda_{0}^{\downarrow}$ and $\Lambda^{\downarrow}$ are isomorphic to $\Lambda_{0}$ and $\Lambda$ respectively by the isomorphism $q \mapsto T^{-1}$. Under the isomorphism we have $\mathfrak{v}_{q}=-\mathfrak{v}_{T}$. The downward universal Novikov rings seem to be more commonly used in the study of spectral invariant (e.g., Oh4]), while the upward versions $\Lambda$ and $\Lambda_{0}$ are used in Lagrangian Floer theory (e.g., FOOO1, FOOO2]).

(19) Let $V$ be a $\mathbb{Z}$ graded vector space over $\mathbb{C}$. We put $B_{k} V=\underbrace{V \otimes \cdots \otimes V}_{k \text { times }}$ and $B V=\bigoplus_{k=0}^{\infty} B_{k} V$ where $B_{0} V=\mathbb{C}$. Then $B V$ has a structure of coassociative coalgebra with coproduct. We note that we have two kinds of coproduct structures on $B V$. One is the deconcatenation coproduct defined by

$\Delta_{\text {decon }}\left(x_{1} \otimes \cdots \otimes x_{k}\right)=\sum_{i=0}^{k}\left(x_{1} \otimes \cdots \otimes x_{i}\right) \otimes\left(x_{i+1} \otimes \cdots \otimes x_{k}\right)$.

The other is the shuffle coproduct defined by

$\Delta_{\text {shuff }}\left(x_{1} \otimes \cdots \otimes x_{k}\right)$

$=\sum_{\left(\mathbb{L}_{1}, \mathbb{L}_{2}\right) \in \operatorname{Shuff}(k)}(-1)^{*}\left(x_{\ell_{1}(1)} \otimes \cdots \otimes x_{\ell_{1}\left(k_{1}\right)}\right) \otimes\left(x_{\ell_{2}(1)} \otimes \cdots \otimes x_{\ell_{2}\left(k_{2}\right)}\right)$,

where $\mathbb{L}_{j}=\left\{\ell_{j}(1), \ldots, \ell_{j}\left(k_{j}\right)\right\}$ with $\ell_{j}(1)<\cdots<\ell_{j}\left(k_{j}\right)$ for $j=1,2$ and

$$
*=\sum_{\ell_{1}(i)>\ell_{2}(j)} \operatorname{deg} x_{\ell_{1}(i)} \operatorname{deg} x_{\ell_{2}(j)} .
$$


It is easy to see that

$$
\begin{aligned}
& \Delta_{\text {decon }}\left(\sum_{k=0}^{\infty} x^{\otimes k}\right)=\left(\sum_{k=0}^{\infty} x^{\otimes k}\right) \otimes\left(\sum_{k=0}^{\infty} x^{\otimes k}\right) \\
& \Delta_{\text {shuff }}\left(\sum_{k=0}^{\infty} \frac{x^{\otimes k}}{k !}\right)=\left(\sum_{k=0}^{\infty} \frac{x^{\otimes k}}{k !}\right) \otimes\left(\sum_{k=0}^{\infty} \frac{x^{\otimes k}}{k !}\right)
\end{aligned}
$$

if $\operatorname{deg} x$ is even. We write $e^{x}=\sum_{k=0}^{\infty} x^{\otimes k}$ or $e^{x}=\sum_{k=0}^{\infty} \frac{x^{\otimes k}}{k !}$ according as we use $\Delta_{\text {decon }}$ or $\Delta_{\text {shuff }}$ as coproduct structures.

Actually we only use $\Delta_{\text {decon }}$ for $B V$ in this article. However on $E V$, which we describe in item (20), we use $\Delta_{\text {shuff }}$, which is induced by $\Delta_{\text {shuff }}$ on $B V$.

(20) The symmetric group $\operatorname{Perm}(k)$ of order $k$ ! acts on $B_{k} V$ by

$$
\sigma \cdot\left(x_{1} \otimes \cdots \otimes x_{k}\right)=(-1)^{*} x_{\sigma(1)} \otimes \cdots \otimes x_{\sigma(k)},
$$

where $*=\sum_{i<j ; \sigma(i)>\sigma(j)} \operatorname{deg} x_{i} \operatorname{deg} x_{j}$. We denote by $E_{k} V$ the quotient of $B_{k} V$ by the submodule generated by $\sigma \cdot \mathbf{x}-\mathbf{x}$ for $\sigma \in \operatorname{Perm}(k), \mathbf{x} \in B_{k} V$. We denote by $[\mathbf{x}]$ an element of $E_{k} V$ and put $E V=\bigoplus_{k=0}^{\infty} E_{k} V$. The shuffle coproduct structure on $B V$ induces a coproduct structure on $E V$, which we also denote by $\Delta_{\text {shuff. }}$. It is given by

$$
\begin{aligned}
& \Delta_{\text {shuff }}\left(\left[x_{1} \otimes \cdots \otimes x_{k}\right]\right) \\
& =\sum_{\left(\mathbb{L}_{1}, \mathbb{L}_{2}\right) \in \operatorname{Shuff}(k)}(-1)^{*}\left(\left[x_{\ell_{1}(1)} \otimes \cdots \otimes x_{\ell_{1}\left(k_{1}\right)}\right]\right) \otimes\left(\left[x_{\ell_{2}(1)} \otimes \cdots \otimes x_{\ell_{2}\left(k_{2}\right)}\right]\right) .
\end{aligned}
$$

Here $*$ is the same as (1.24). Then $E V$ becomes a coassociative and graded cocommutative coalgebra.

In [FOOO1, FOOO3, FOOO4, we denote by $E_{k} V$ the Perm $(k)$-invariant subset of $B_{k} V$ and use the deconcatenation coproduct restricted to the subset. In [FOOO7, we use $E_{k} V$ as the quotient space and the shuffle coproduct on it as we do in this paper. This paper follows the conventions used in FOOO7.

(21) Let $L$ be a relatively spin closed Lagrangian submanifold of a symplectic manifold $(M, \omega)$.

(a) For the case $V=\Omega(L)[1]$, we always use the deconcatenation coproduct $\Delta_{\text {decon }}$ on $B(\Omega(L)[1])$

(b) For the case $V=\Omega(M)[2]$, we always use the shuffle coproduct $\Delta_{\text {shuff }}$ on $E(\Omega(M)[2])$.

Here $\Omega(L)[1]$ (resp. $\Omega(M)[2]$ ) is the degree shift by +1 of $\Omega(L)$, i.e., $(\Omega(L)[1])^{d}=\Omega^{d+1}(L)$ (resp. +2 of $\Omega(M)$, i.e., $(\Omega(M)[2])^{d}=\Omega^{d+2}(M)$.) Therefore, no confusion can occur even if we use the same notation $\Delta$ for the coproducts $\Delta_{\text {decon }}$ and $\Delta_{\text {shuff }}$.

(22) Sometimes we regard the de Rham complex $(\Omega(M), d)$ as a chain complex and consider its homology. In that case we put

$$
\Omega_{k}(M)=\Omega^{\operatorname{dim} M-k}(M), \quad \partial=(-1)^{\operatorname{deg}+1} d .
$$

See FOO01, Remark 3.5.8] for this sign convention. When a cohomology class $a \in H^{\operatorname{dim} M-k}(M)$ is represented by a differential form $\alpha$ and we regard $\alpha$ as an element of the chain complex $\left(\Omega_{*}(M), \partial\right)$, we denote the 
homology class by $a^{b} \in H_{k}(M)$. We identify the singular homology $H_{k}(M)$ with $H_{k}\left(\Omega_{*}(M), \delta\right)$ by identifying $a^{b} \in H_{k}\left(\Omega_{*}(M), \delta\right)$ with the singular homology class represented by a cycle $C$ satisfying $\int_{C} \beta=\int_{M} a^{b} \wedge \beta$ for all closed form $\beta$ on $M$.

More generally If $a \in H^{*}(M) \otimes \Lambda$ is expressed as

$$
a=\sum a_{i} T^{\lambda_{i}} \quad a_{i} \in H^{*}(M), \quad \lambda_{i} \rightarrow \infty
$$

then we define

$$
a^{b}=\sum a_{i}^{b} q^{-\lambda_{i}}, \quad-\lambda_{i} \rightarrow-\infty .
$$

(23) We always denote by $Q H^{*}(M)=Q H^{*}(M, \Lambda)$ the standard quantum cohomology ring with Novikov ring equipped with upward valuations. As a module, it is isomorphic to $Q H^{*}(M)=H^{*}(M) \otimes \Lambda \cong H^{*}(M) \otimes \Lambda^{\downarrow}=Q H_{*}(M)$.

(24) When we say that the boundary orientation of some moduli space is compatible with the orientation of strata corresponding to bubbling off disks (with boundary marked points), the compatibility means in the sense of [FOOO2, Proposition 8.3.3].

(25) In the present paper, we will exclusively use the quantum cohomology, the Hamiltonian Floer homology and Lagrangian Floer cohomology. Depending on the usage of homology or cohomology, we use different valuations $\mathfrak{v}_{T}$ or $\mathfrak{v}_{q}=-\mathfrak{v}_{T}$ on the Novikov ring $\Lambda$. When we emphasize we use parameter $q$ and valuation $\mathfrak{v}_{q}$ we write $\Lambda^{\downarrow}$.

1.3. Difference between Entov-Polterovich's convention and ours. In this subsection we explain the difference between our convention and the one of EntovPolterovich. The discussion of this subsection is not used anywhere else in this paper. Entov and Polterovich (see [EP3, equation (9)]) use the action functional

$$
\widetilde{\mathcal{A}}_{H}([\gamma, w])=-\int w^{*} \omega+\int_{0}^{1} H(t, \gamma(t)) d t
$$

instead of (1.2). We note the relationship

$$
\widetilde{\mathcal{A}}_{H}=\mathcal{A}_{-H} .
$$

They use it to define $c\left(a^{b} ; H\right)$ for a quantum homology class $a^{b} \in H_{*}\left(M ; \Lambda^{\downarrow}\right)$. (They do it in the same way as $\lambda_{H}$ given in (1.4) except the difference of convention mentioned above.) Put

$$
\rho^{E P}(H ; a)=-c\left(a^{b} ; H\right) .
$$

and define the function $\zeta_{e}^{E P}: C^{0}(M) \rightarrow \mathbb{R}:$

$$
\zeta_{e}^{E P}(H):=\lim _{n \rightarrow \infty} \frac{\rho^{E P}(n H ; e)}{n}
$$

for autonomous $C^{\infty}$ function $H$ and

$$
\mu_{e}^{E P}(\widetilde{\psi})=-\operatorname{vol}_{\omega}(M) \lim _{n \rightarrow \infty} \frac{\rho^{E P}\left(\widetilde{\psi}^{n} ; e\right)}{n},
$$

where $\rho^{E P}\left(\widetilde{\psi}^{n} ; e\right)$ is defined from $\rho^{E P}(H ; e)$ in the same way as in (1.7).

These functions exactly coincide with those defined by [EP1, EP2, EP3].

Now in the case $\mathfrak{b}=0$ (1.27) implies the equalities

$$
\zeta_{e}(H)=-\zeta_{e}^{E P}(-H), \quad \mu_{e}\left(\widetilde{\psi}_{H}\right)=-\mu_{e}^{E P}\left(\widetilde{\psi}_{-H}\right),
$$


which relate (1.8), (1.9) and (1.29), (1.30).

For example, when $e=1$ and $H$ is a $C^{2}$ small autonomous Morse function, we have

$$
\rho(H ; 1)=-\min H, \quad \rho^{E P}(H ; 1)=\max H,
$$

which also shows that the values of Entov-Polterovich's spectral invariant are different in general from ours (the latter is also the same as that of [Oh4).

Moreover we can find an example for which the identity $\zeta_{e}(H) \neq-\zeta_{e}^{E P}(H)$ holds, as follows. Consider an autonomous Hamiltonian $H$ on $T^{2}=S^{1} \times S^{1}$ of the form $\pi_{1}^{*} h$, where $\pi_{1}: S^{1} \times S^{1} \rightarrow S^{1}$ is the first projection. Note that there are no non-constant contractible periodic orbits of $X_{k H}$ for any real number $k$. We find that $\rho(k H ; 1)=-\min k H, \rho^{E P}(k H ; 1)=\max k H$. Therefore we have $\zeta_{1}(H)=-\min H$, while $\zeta_{1}^{E P}(H)=\max H$.

The identities (1.31) imply that, for the case $\zeta_{e}$ is actually homogeneous, not just semi-homogeneous, (namely if $\zeta_{e}(-H)=-\zeta_{e}(H)$ ), then the two (partial) quasistates $\zeta_{e}$ and $\zeta_{e}^{E P}$ coincide.

Remark 1.14. Note the direction of inequality we used in Definition 1.3 is opposite to the one in Entov-Polterovich [EP3. However, because of the minus sign in (1.31), Definition 1.3 is indeed consistent with that in EP3. We feel that the direction of the inequality above is easier to memorize and suits better for the general principle in symplectic topology in that getting a lower bound for the spectral invariants is much more nontrivial in general than getting an upper bound which is just a consequence of the general 'positivity' principle in symplectic topology.

We also remark that Entov-Polterovich use quantum homology and a Novikov ring similar to $\Lambda^{\downarrow}$. We use quantum cohomology and the Novikov ring $\Lambda$. 


\section{Part 1. Review of spectral invariants}

\section{Hamiltonian Floer-Novikov complex}

We first recall from Notations and Conventions (18) in Section 1 that we define a valuation $\mathfrak{v}_{q}$ on the (downward) universal Novikov field $\Lambda^{\downarrow}$ by

$$
\mathfrak{v}_{q}\left(\sum_{i=1}^{\infty} a_{i} q^{\lambda_{i}}\right)=\sup \left\{\lambda_{i} \mid a_{i} \neq 0\right\} .
$$

It satisfies the following properties:

(1) $\mathfrak{v}_{q}(x y)=\mathfrak{v}_{q}(x)+\mathfrak{v}_{q}(y)$,

(2) $\mathfrak{v}_{q}(x+y) \leq \max \left\{\mathfrak{v}_{q}(x), \mathfrak{v}_{q}(y)\right\}$

(3) $\mathfrak{v}_{q}(x)=-\infty$ if and only if $x=0$,

(4) $\mathfrak{v}_{q}(q)=1$,

(5) $\mathfrak{v}_{q}(a x)=\mathfrak{v}_{q}(x)$ if $a \in \mathbb{C} \backslash\{0\}$.

This field will be the coefficient field of the Floer chain module whose description is in order.

Let $\widetilde{\mathcal{L}}_{0}(M)$ be the set of all the pairs $[\gamma, w]$ where $\gamma$ is a loop $\gamma: S^{1} \rightarrow M$ and $w: D^{2} \rightarrow M$ a disc with $\left.w\right|_{\partial D^{2}}=\gamma$. We denote by $\pi_{2}(\gamma)$ the set of homotopy classes of such $w^{\prime}$ 's. We identify $[\gamma, w]$ and $\left[\gamma^{\prime}, w^{\prime}\right]$ if $\gamma=\gamma^{\prime}$ and $w$ is homotopic to $w^{\prime}$ relative to the boundary $\gamma$. When a one-periodic Hamiltonian $H:(\mathbb{R} / \mathbb{Z}) \times M \rightarrow \mathbb{R}$ is given, we consider the perturbed functional $\mathcal{A}_{H}: \widetilde{\mathcal{L}}_{0}(M) \rightarrow \mathbb{R}$ defined by

$$
\mathcal{A}_{H}([\gamma, w])=-\int w^{*} \omega-\int H(t, \gamma(t)) d t .
$$

For a Hamiltonian $H:[0,1] \times M \rightarrow \mathbb{R}$, we denote its flow, a Hamiltonian isotopy, by $\phi_{H}: t \mapsto \phi_{H}^{t} \in \operatorname{Ham}(M, \omega)$. This gives a one-to-one correspondence between equivalence classes of $H$ modulo the addition of a function on [0,1] and Hamiltonian isotopies. We denote the time-one map by $\psi_{H}:=\phi_{H}^{1}$. We put

$$
\operatorname{Fix} \psi_{H}=\left\{p \in M \mid \psi_{H}(p)=p\right\} \text {. }
$$

Each element $p \in$ Fix $\psi_{H}$ induces a map $z_{p}=z_{p}^{H}: S^{1} \rightarrow M$ by the correspondence

$$
z_{p}(t)=\phi_{H}^{t}(p)
$$

where $t \in \mathbb{R} / \mathbb{Z} \cong S^{1}$. The loop $z_{p}$ satisfies Hamilton's equation

$$
\dot{x}=X_{H}(t, x), \quad z_{p}(0)=p .
$$

Here $X_{H}$ is the (time-dependent) Hamiltonian vector field given by $X_{H}(t, x)=$ $X_{H_{t}}(x)$ where $X_{H_{t}}$ is the Hamiltonian vector field generated by the function $H_{t}$ : $C^{\infty}(M) \rightarrow \mathbb{R}$. We denote by $\operatorname{Per}(H)$ the set of one-periodic solutions of $\dot{x}=$ $X_{H}(t, x)$. Then (2.3) provides a one-to-one correspondence between Fix $\psi_{H}$ and $\operatorname{Per}(H)$. The next lemma is well-known.

Lemma 2.1. The set of critical points of $\mathcal{A}_{H}$ is given by

$$
\operatorname{Crit}\left(\mathcal{A}_{H}\right)=\left\{[\gamma, w]|\gamma \in \operatorname{Per}(H), w|_{\partial D^{2}}=\gamma\right\}
$$

Hereafter we assume that our Hamiltonian $H$ is normalized in the sense of (1.6) unless otherwise stated explicitly. 
We say that $H$ or its associated map $\psi_{H}$ is nondegenerate if at any $p \in \operatorname{Fix} \psi_{H}$, the differential $d_{p} \psi_{H}: T_{p} M \rightarrow T_{p} M$ does not have eigenvalue 1 . The cardinality of $\operatorname{Per}(H)$ is finite if $\psi_{H}$ is nondegenerate. For each nondegenerate Hamiltonian, we are given the canonical real and integer grading

$$
\mathcal{A}_{H}: \operatorname{Crit}\left(\mathcal{A}_{H}\right) \rightarrow \mathbb{R}, \quad \mu_{H}: \operatorname{Crit}\left(\mathcal{A}_{H}\right) \rightarrow \mathbb{Z}
$$

induced by the action functional and the Conley-Zehnder index [CZ respectively.

We define an equivalence relation $\sim$ on $\operatorname{Crit}\left(\mathcal{A}_{H}\right)$ such that $[\gamma, w] \sim\left[\gamma^{\prime}, w^{\prime}\right]$ if and only if $\gamma=\gamma^{\prime}$ and

$$
\int w^{*} \omega=\int\left(w^{\prime}\right)^{*} \omega
$$

We denote by $\widehat{\operatorname{Per}}(H)$ the set of such equivalence classes. The equivalence class of $[\gamma, w] \in \widehat{\operatorname{Per}}(H)$ is denoted by $\llbracket \gamma, w \rrbracket$. The map $\mathcal{A}_{H}$ in (2.4 induces a map $\widehat{\operatorname{Per}}(H) \rightarrow \mathbb{R}$, which we denote by the same symbol.

We define maps

$$
\operatorname{Crit}\left(\mathcal{A}_{H}\right) \stackrel{\pi}{\longrightarrow} \widehat{\operatorname{Per}}(H) \longrightarrow \operatorname{Per}(H)
$$

by $[\gamma, w] \mapsto \llbracket \gamma, w \rrbracket$ and $\llbracket \gamma, w \rrbracket \mapsto \gamma$. We denote by $\pi: \operatorname{Crit}\left(\mathcal{A}_{H}\right) \rightarrow \widehat{\operatorname{Per}}(H)$ the first map in (2.5).

Note that the obvious concatenation defines a map

$$
\#: \pi_{2}(M) \times \pi_{2}(\gamma) \rightarrow \pi_{2}(\gamma)
$$

We consider the map

$$
\pi_{2}(M) \rightarrow \mathbb{R}
$$

defined by $[v] \mapsto \int v^{*} \omega$ for $v: S^{2} \rightarrow M$. Let $K_{2}(M)$ be the kernel of this map.

Lemma 2.2. By the action (2.6) we can identify

$$
\widehat{\operatorname{Per}}(H)=\operatorname{Crit}\left(\mathcal{A}_{H}\right) / K_{2}(M) \text {. }
$$

The proof is easy and is omitted. See Lemma 4.5 for the relation between $\widehat{\operatorname{Per}}(H)$ and $\operatorname{Per}(H)$.

We consider the $\Lambda^{\downarrow}$ vector space $\widehat{C F}\left(M, H ; \Lambda^{\downarrow}\right)$ with a basis given by the elements of the set $\widehat{\operatorname{Per}}(H)$.

Definition 2.3. We define an equivalence relation $\sim$ on $\widehat{C F}\left(M, H ; \Lambda^{\downarrow}\right)$ so that $\llbracket \gamma, w \rrbracket \sim q^{c} \llbracket \gamma^{\prime}, w^{\prime} \rrbracket$ if and only if

$$
\gamma=\gamma^{\prime}, \quad \int_{D^{2}} w^{\prime *} \omega=\int_{D^{2}} w^{*} \omega+c .
$$

The quotient of $\widehat{C F}\left(M, H ; \Lambda^{\downarrow}\right)$ modded out by this equivalence relation $\sim$ is called the Floer complex of the periodic Hamiltonian $H$ and denoted by $C F\left(M, H ; \Lambda^{\downarrow}\right)$.

Here we ignore the $\mathbb{Z}$-grading, which is given by the Conley-Zehnder indices, in defining the equivalence relation unlike the case of standard literature of Hamiltonian Floer homology such as [HS], where additional requirement $c_{1}\left(\bar{w} \# w^{\prime}\right)=0$ is imposed in the definition of Floer complex, denoted by $C F(H)$. For the purpose of the current paper, the equivalence relation (2.8) is enough and more favorable in that it makes the associated Novikov ring becomes a field. To differentiate the current definition from $C F(H)$, we denote the complex used in the present paper by $C F\left(M, H ; \Lambda^{\downarrow}\right)$. 
K. FUKAYA, Y.-G. OH, H. OHTA, K. ONO

Lemma 2.4. As a $\Lambda^{\downarrow}$ vector space, $C F\left(M, H ; \Lambda^{\downarrow}\right)$ is isomorphic to the direct sum $\left(\Lambda^{\downarrow}\right) \# \operatorname{Per}(H)$.

Moreover the following holds: We fix a lifting $\llbracket \gamma, w_{\gamma} \rrbracket \in \widehat{\operatorname{Per}}(H)$ for each $\gamma \in$ $\operatorname{Per}(H)$. Then any element $x$ of $C F\left(M, H ; \Lambda^{\downarrow}\right)$ is uniquely written as a sum

$$
x=\sum_{\gamma \in \operatorname{Per}(H)} x_{\gamma} \llbracket \gamma, w_{\gamma} \rrbracket, \quad \text { with } x_{\gamma} \in \Lambda^{\downarrow} .
$$

The proof is easy and omitted.

Definition 2.5. (1) Let $x$ be as in (2.9). We define the level function

$$
\lambda_{H}(x)=\max \left\{\mathfrak{v}_{q}\left(x_{\gamma}\right)+\mathcal{A}_{H}\left(\llbracket \gamma, w_{\gamma} \rrbracket\right) \mid x_{\gamma} \neq 0\right\} .
$$

(2) We define a filtration $F^{\lambda} C F\left(M, H ; \Lambda^{\downarrow}\right)$ on $C F\left(M, H ; \Lambda^{\downarrow}\right)$ by

$$
F^{\lambda} C F\left(M, H ; \Lambda^{\downarrow}\right)=\left\{x \in C F\left(M, H ; \Lambda^{\downarrow}\right) \mid \lambda_{H}(x) \leq \lambda\right\} .
$$

We have

$$
F^{\lambda_{1}} C F\left(M, H ; \Lambda^{\downarrow}\right) \subset F^{\lambda_{2}} C F\left(M, H ; \Lambda^{\downarrow}\right)
$$

if $\lambda_{1}<\lambda_{2}$. We also have

$$
\bigcap_{\lambda} F^{\lambda} C F\left(M, H ; \Lambda^{\downarrow}\right)=\{0\}, \quad \bigcup_{\lambda} F^{\lambda} C F\left(M, H ; \Lambda^{\downarrow}\right)=C F(M, H) .
$$

(3) We define a metric $d_{q}$ on $C F\left(M, H ; \Lambda^{\downarrow}\right)$ by

$$
d_{q}\left(x, x^{\prime}\right)=e^{\lambda_{H}\left(x-x^{\prime}\right)} .
$$

Definition 2.5, together with (2.1) and (2.8), implies that

$$
\lambda_{H}(a \mathfrak{x})=\mathfrak{v}_{q}(a)+\lambda_{H}(\mathfrak{x})
$$

for $a \in \Lambda^{\downarrow}, \mathfrak{x} \in C F\left(M, H ; \Lambda^{\downarrow}\right)$. We also have

$$
q^{\lambda_{1}} F^{\lambda_{2}} C F\left(M, H ; \Lambda^{\downarrow}\right) \subseteq F^{\lambda_{1}+\lambda_{2}} C F\left(M, H ; \Lambda^{\downarrow}\right) .
$$

Lemma 2.6. (1) The definition of $\lambda_{H}$ given in Definition 2.5 is independent of the choice of the lifting $\gamma \mapsto\left[\gamma, w_{\gamma}\right]$ with $\gamma \in \operatorname{Per}(H)$.

(2) $C F\left(M, H ; \Lambda^{\downarrow}\right)$ is complete with respect to the metric $d_{q}$.

(3) The infinite sum

$$
\sum_{\llbracket \gamma, w \rrbracket \in \widehat{\operatorname{Per}}(H)} x_{\llbracket \gamma, w \rrbracket} \llbracket \gamma, w \rrbracket
$$

converges in $C F\left(M, H ; \Lambda^{\downarrow}\right)$ with respect to the metric $d_{q}$, provided

$$
\left\{\llbracket \gamma, w \rrbracket \in \operatorname{Per}(H) \mid \mathfrak{v}_{q}\left(x_{\llbracket \gamma, w \rrbracket}\right)+\mathcal{A}_{H}(\llbracket \gamma, w \rrbracket)>-C, x_{\llbracket \gamma, w \rrbracket} \neq 0\right\} .
$$

is finite for any $C \in \mathbb{R}$.

The proof is easy and omitted. 


\section{FLOER BOUNDARY MAP}

In this section we define the boundary operator $\partial_{(H, J)}$ on $C F\left(M, H ; \Lambda^{\downarrow}\right)$ so that it becomes a filtered complex. Let a nondegenerate one-periodic Hamiltonian function $H$ and a one-periodic family $J=\left\{J_{t}\right\}_{t \in S^{1}}$ of compatible almost complex structures be given. The study of the following perturbed Cauchy-Riemann equation

$$
\frac{\partial u}{\partial \tau}+J\left(\frac{\partial u}{\partial t}-X_{H_{t}}(u)\right)=0
$$

is the heart of the Hamiltonian Floer theory. Here and hereafter $J$ in (3.1) means $J_{t}$.

Remark 3.1. (1) In this paper, we never use perturbation of (a family of) compatible almost complex structures $J$ to achieve transversality of the moduli space of the Floer equations (3.1) but use abstract perturbations (multisections and CF-perturbations of the Kuranishi structure in the sense of [FO, FOOO2, FOOO11]) to achieve the necessary transversality. See Part 7 for a summary of Kuranishi structure and CF-perturbation.

(2) In Parts 1-3 (and somewhere in the appendix) we use a $t$-parameterized family of compatible almost complex structures $\left\{J_{t}\right\}_{t \in S^{1}}$. However we emphasize that we do not need to use an $S^{1}$-parameterized family of compatible almost complex structures but can use a fixed compatible almost complex structure $J$, to prove all of our main results of this paper. (We need to use a $t$-dependent $J$ for the construction in Sections 29 and 30.) The $t$-dependent $J$ is included only for the sake of consistency with the reference on spectral invariants. (Traditionally $t$-dependent $J$ had been used to achieve transversality. As we mentioned in (1), we do not need this extra freedom in this paper since we use abstract perturbations.)

The following definition is useful for the later discussions.

Definition 3.2. Let $\gamma, \gamma^{\prime} \in \operatorname{Per}(H)$. We denote by $\pi_{2}\left(\gamma, \gamma^{\prime}\right)$ the set of homotopy classes of smooth maps $u:[0,1] \times S^{1} \rightarrow M$ relative to the boundary $u(0, t)=\gamma(t)$, $u(1, t)=\gamma^{\prime}(t)$. We denote by $[u] \in \pi_{2}\left(\gamma, \gamma^{\prime}\right)$ its homotopy class.

As we defined at the beginning of Section $2 \pi_{2}(\gamma)$ denotes the set of relative homotopy classes of the maps $w: D^{2} \rightarrow M ;\left.w\right|_{\partial D^{2}}=\gamma$. For $C \in \pi_{2}\left(\gamma, \gamma^{\prime}\right)$, there is a natural map of $(\cdot) \# C: \pi_{2}(\gamma) \rightarrow \pi_{2}\left(\gamma^{\prime}\right)$ induced by the gluing map $w \mapsto w \# C$. There is also the natural gluing map

$$
\pi_{2}\left(\gamma_{0}, \gamma_{1}\right) \times \pi_{2}\left(\gamma_{1}, \gamma_{2}\right) \rightarrow \pi_{2}\left(\gamma_{0}, \gamma_{2}\right), \quad\left(u_{1}, u_{2}\right) \mapsto u_{1} \# u_{2} .
$$

For each $[\gamma, w],\left[\gamma^{\prime}, w^{\prime}\right] \in \operatorname{Crit}\left(\mathcal{A}_{H}\right)$, we will define a moduli space $\mathcal{M}\left(H, J ;[\gamma, w],\left[\gamma^{\prime}, w^{\prime}\right]\right)$. We begin with the definition of the energy.

Definition 3.3. (Energy) For a given smooth map $u: \mathbb{R} \times S^{1} \rightarrow M$, we define the energy of $u$ by

$$
E_{(H, J)}(u)=\frac{1}{2} \int\left(\left|\frac{\partial u}{\partial \tau}\right|_{J}^{2}+\left|\frac{\partial u}{\partial t}-X_{H_{t}}(u)\right|_{J}^{2}\right) d t d \tau .
$$

Here and hereafter we denote

$$
\left|\frac{\partial u}{\partial \tau}\right|_{J}^{2}=\omega\left(\frac{\partial u}{\partial \tau}, J_{t}\left(\frac{\partial u}{\partial \tau}\right)\right) .
$$


Definition 3.4. We denote by $\widehat{\stackrel{\mathcal{M}}{M}}\left(H, J ;[\gamma, w],\left[\gamma^{\prime}, w^{\prime}\right]\right)$ the set of all smooth maps $u: \mathbb{R} \times S^{1} \rightarrow M$ which satisfy the following conditions:

(1) The map $u$ satisfies the equation:

$$
\frac{\partial u}{\partial \tau}+J\left(\frac{\partial u}{\partial t}-X_{H_{t}}(u)\right)=0 .
$$

(2) The energy $E_{(H, J)}(u)$ is finite.

(3) The map $u$ satisfies the following asymptotic boundary condition.

$$
\lim _{\tau \rightarrow-\infty} u(\tau, t)=\gamma(t), \quad \lim _{\tau \rightarrow+\infty} u(\tau, t)=\gamma^{\prime}(t) .
$$

(4) The homotopy class of the concatenation $w \# u$ of $w$ and $u$ gives the same element as $\left[\gamma^{\prime}, w^{\prime}\right] \in \pi_{2}\left(\gamma^{\prime}\right)$. Namely $\left[\gamma^{\prime}, w \# u\right]=\left[\gamma^{\prime}, w^{\prime}\right]$.

It carries an $\mathbb{R}$-action induced by the domain translations in the $\tau$-direction. We denote the quotient space of this $\mathbb{R}$-action by $\stackrel{\circ}{\mathcal{M}}\left(H, J ;[\gamma, w],\left[\gamma^{\prime}, w^{\prime}\right]\right)$.

When $[\gamma, w]=\left[\gamma^{\prime}, w^{\prime}\right]$, we set the space $\stackrel{\circ}{\mathcal{M}}(H, J ;[\gamma, w],[\gamma, w])$ to be the empty set by definition.

Remark 3.5. The conditions (1) and (2) above make the convergence in (3) one of an exponential order, which in turn enables the statement (4) to make sense.

For any $\alpha \in \pi_{2}(M)$, we have a canonical homeomorphism

$$
\stackrel{\circ}{\mathcal{M}}(H, J ;[\gamma, w],[\gamma, w]) \cong \stackrel{\circ}{\mathcal{M}}(H, J ;[\gamma, \alpha \# w],[\gamma, \alpha \# w])
$$

This is because the condition $\left[\gamma^{\prime}, w \# u\right]=\left[\gamma^{\prime}, w^{\prime}\right]$ in item (4) is equivalent to $\left[\gamma^{\prime}, \alpha \# w \# u\right]=\left[\gamma^{\prime}, \alpha \# w^{\prime}\right]$.

Proposition 3.6. (1) The moduli space $\stackrel{\circ}{\mathcal{M}}\left(H, J ;[\gamma, w],\left[\gamma^{\prime}, w^{\prime}\right]\right)$ has a compactification $\mathcal{M}\left(H, J ;[\gamma, w],\left[\gamma^{\prime}, w^{\prime}\right]\right)$ that is Hausdorff.

(2) The space $\mathcal{M}\left(H, J ;[\gamma, w],\left[\gamma^{\prime}, w^{\prime}\right]\right)$ has an orientable Kuranishi structure with corners. (See Definitions 31.8 and 34.5 for the definition of Kuranishi structure with corners.)

(3) The normalized boundary of $\mathcal{M}\left(H, J ;[\gamma, w],\left[\gamma^{\prime}, w^{\prime}\right]\right)$ in the sense of $K u$ ranishi structure is described by

$$
\begin{aligned}
& \partial \mathcal{M}\left(H, J ;[\gamma, w],\left[\gamma^{\prime}, w^{\prime}\right]\right) \\
& =\bigcup \mathcal{M}\left(H, J ;[\gamma, w],\left[\gamma^{\prime \prime}, w^{\prime \prime}\right]\right) \times \mathcal{M}\left(H, J ;\left[\gamma^{\prime \prime}, w^{\prime \prime}\right],\left[\gamma^{\prime}, w^{\prime}\right]\right),
\end{aligned}
$$

where the union is taken over all $\left[\gamma^{\prime \prime}, w^{\prime \prime}\right] \in \operatorname{Crit}\left(\mathcal{A}_{H}\right)$.

(4) Then we have the following formula for the (virtual) dimension:

$$
\operatorname{dim} \mathcal{M}\left(H, J ;[\gamma, w],\left[\gamma^{\prime}, w^{\prime}\right]\right)=\mu_{H}\left(\left[\gamma^{\prime}, w^{\prime}\right]\right)-\mu_{H}([\gamma, w])-1 .
$$

(5) We can define orientations of $\mathcal{M}\left(H, J ;[\gamma, w],\left[\gamma^{\prime}, w^{\prime}\right]\right)$ so that (3) above is compatible with this orientation.

(6) The homeomorphism (3.3) extends to the compactifications and their $K u$ ranishi structures are identified by the homeomorphism.

\footnotetext{
${ }^{1}$ See Definition 34.2 for the definition of normalized boundary.
} 
This is proved in [FO, Chapter 4]. More precisely, the dimension formula (4) is proved in [Fl, SZ, and (1) is [FO, Theorem 19.12], (2), (3) are [FO, Theorem 19.14] and (5) is [FO, Lemma 21.4]. Thorough detail of the proof of this theorem is given also in FOOO10, Part 5]. In fact Proposition 3.6 is [FOOO10, Theorem 29.4] which is proved in [FOOO10, Section 31]. See [Fl, HS, On] etc. for the earlier works for the semi-positive cases.

We remark that $C F\left(M, H ; \Lambda^{\downarrow}\right)$ as a $\Lambda^{\downarrow}$-vector space carries a natural $\mathbb{Z}_{2}$-grading given by the homological degree $(\bmod 2)$ of $\llbracket \gamma, w \rrbracket$. This is because

$$
\mu_{H}\left(\left[\gamma, w^{\prime}\right]\right)-\mu_{H}([\gamma, w])=2 c_{1}(M) \cap\left[\bar{w} \# w^{\prime}\right]
$$

holds for any pair $[w],\left[w^{\prime}\right] \in \pi_{2}(\gamma)$. Here $\bar{w} \# w^{\prime}$ is a 2 -sphere obtained by gluing $\bar{w}$ and $w^{\prime}$ along $\gamma$ where $\bar{w}$ is the $w$ with opposite orientation. (See [Fl, page 557] and Oh6, Appendix] for its proof.) In particular, it implies that the parity of $\mu_{H}([\gamma, w])$ depends only on $\gamma \in \operatorname{Per}(H)$ but not on its lifting $[\gamma, w] \in \operatorname{Crit}\left(\mathcal{A}_{H}\right)$. This gives rise to a natural $\mathbb{Z}_{2}$-grading on $C F\left(M, H ; \Lambda^{\downarrow}\right)$, that is, the homological degree mod 2 of $\left[\gamma, w_{\gamma}\right]$, which is $n-\mu_{H}\left(\left[\gamma, w_{\gamma}\right]\right) \bmod 2$. So we put:

$$
\begin{aligned}
& C F_{1}\left(M, H ; \Lambda^{\downarrow}\right)=\bigoplus_{\gamma ; n-\mu_{H}\left(\left[\gamma, w_{\gamma}\right]\right) \text { is odd. }} \Lambda^{\downarrow} \llbracket \gamma, w_{\gamma} \rrbracket, \\
& C F_{0}\left(M, H ; \Lambda^{\downarrow}\right)=\bigoplus_{\gamma ; n-\mu_{H}\left(\left[\gamma, w_{\gamma}\right]\right) \text { is even. }} \Lambda^{\downarrow} \llbracket \gamma, w_{\gamma} \rrbracket .
\end{aligned}
$$

Remark 3.7. We remark that the degree of Floer chain defined above is shifted by $n$ from the Conley-Zehnder index $\mu_{H}$. By this shift, the degree will coincide with the degree of (quantum) cohomology group of $M$ by the isomorphism in Theorem 3.12. See also Remark 5.6 (2) and Oh6, Section 7.2].

We use Proposition 3.6 to define the Floer boundary map

$$
\partial_{(H, J)}: C F_{k+1}\left(M, H ; \Lambda^{\downarrow}\right) \rightarrow C F_{k}\left(M, H ; \Lambda^{\downarrow}\right)
$$

whose explanation is in order.

First for each given pair $[\gamma, w],\left[\gamma^{\prime}, w^{\prime}\right]$, it defines a homotopy class $B \in \pi_{2}\left(\gamma, \gamma^{\prime}\right)$ such that $[w \# B]=w^{\prime}$ in $\pi_{2}\left(\gamma^{\prime}\right)$. By an abuse of notation, we denote this class $B=\left[\bar{w} \# w^{\prime}\right]$. It follows from the Gromov-Floer compactness and the energy identity given (3.9) that for each given $[\gamma, w]$ the set of all $\left[\gamma^{\prime}, w^{\prime}\right] \in \operatorname{Crit} \mathcal{A}_{H}$ satisfying

$$
\mathcal{M}\left(H, J ;[\gamma, w],\left[\gamma^{\prime}, w^{\prime}\right]\right) \neq \emptyset, \quad\left[\bar{w} \# w^{\prime}\right] \cap[\omega]<A
$$

is finite for any fixed $A \in \mathbb{R}$. Using this finiteness, we construct a system of multisections $\mathfrak{s}$ on $\mathcal{M}\left(H, J ;[\gamma, w],\left[\gamma^{\prime}, w^{\prime}\right]\right)$ inductively over the symplectic area $\left(\bar{w} \# w^{\prime}\right) \cap \omega \in$ $\mathbb{R}_{\geq 0}$ so that they are transversal to 0 and compatible with the identification made in Proposition 3.6 (3). Such an inductive construction is proven to be possible for the relative version of the construction of Kuranishi structures in [FO, Theorem 6.12] (that is, FOOO2, Lemma A1.20]). Detail of the construction of such compatible system of Kuranishi structures is given in [FOOO10, Parts 4 and 5]. Construction of the multisection then is detailed in [FOOO11, Section 13]. Now we define the operator $\partial_{(H, J)}$ by the formula:

$$
\partial_{(H, J)}(\llbracket \gamma, w \rrbracket)=\sum_{\substack{\left[\gamma^{\prime}, w^{\prime}\right] \in \operatorname{rit}\left(\mathcal{A}_{H}\right) \\ \mu_{H}\left(\left[\gamma^{\prime}, w^{\prime}\right]\right)=\mu_{H}([\gamma, w])+1}} \mathfrak{n}_{H, J}\left([\gamma, w],\left[\gamma^{\prime}, w^{\prime}\right]\right) \llbracket \gamma^{\prime}, w^{\prime} \rrbracket .
$$


Here we put

$$
\mathfrak{n}_{H, J}\left([\gamma, w],\left[\gamma^{\prime}, w^{\prime}\right]\right)=\# \mathcal{M}\left(H, J ;[\gamma, w],\left[\gamma^{\prime}, w^{\prime}\right]\right)^{\mathfrak{s}} .
$$

The rational number $\# \mathcal{M}\left(H, J ;[\gamma, w],\left[\gamma^{\prime}, w^{\prime}\right]\right)^{\mathfrak{s}}$ is the virtual fundamental 0-chain of $\mathcal{M}\left(H, J ;[\gamma, w],\left[\gamma^{\prime}, w^{\prime}\right]\right)$ with respect to the multisection $\mathfrak{s}$. Namely it is the order of the zero set of $\mathfrak{s}$ counted with sign and multiplicity. (See [FO, Definition 4.6] or [FOOO2, Definition A1.28] for its precise definition. For the case of virtual dimension zero, which we use here, the detailed definition of virtual fundamental chain is given also in [FOOO11, Definitions 14.4 and 14.6].) Hereafter we omit $\mathfrak{s}$ and simply write $\mathcal{M}\left(H, J ;[\gamma, w],\left[\gamma^{\prime}, w^{\prime}\right]\right)$ for the perturbed moduli space.

By the above mentioned finiteness, Lemma 2.6 (3) implies that the right hand side of (3.7) converges in $d_{q}$-metric, i.e., with respect to the downward valuation.

Remark 3.8. To define the right hand side of (3.7) we assume $\llbracket \gamma, w \rrbracket \in \widehat{\operatorname{Per}}(H)$ is represented by $[\gamma, w] \in \operatorname{Crit}\left(\mathcal{A}_{H}\right)$, i.e., $\pi([\gamma, w])=\llbracket \gamma, w \rrbracket$ and the sum is taken over $\left[\gamma^{\prime}, w^{\prime}\right] \in \operatorname{Crit}\left(\mathcal{A}_{H}\right)$. 2 The right hand side of (3.7) is independent of the choice of such $[\gamma, w]$ as far as it represents the same element of $\widehat{\operatorname{Per}}(H)$. In fact we have

$$
\mathfrak{n}_{H, J}\left([\gamma, w],\left[\gamma^{\prime}, w^{\prime}\right]\right)=\mathfrak{n}_{H, J}\left([\gamma, \alpha \# w],\left[\gamma^{\prime}, \alpha \# w^{\prime}\right]\right)
$$

by Proposition 3.6 (5). (We take a multisection $\mathfrak{s}$ which is compatible with the isomorphism in Proposition 3.6 (5).) The independence of the right hand side of (3.7) then follows from Lemma 2.2 .

Lemma 2.2 also implies that this definition is consistent with the equivalence relation (2.8). (Hereafter in a similar situation we do not mention this kind of remarks.)

We can prove

$$
\partial_{(H, J)} \circ \partial_{(H, J)}=0
$$

by applying Proposition 3.6 (3) in the case when $\mu_{H}\left(\left[\gamma^{\prime}, w^{\prime}\right]\right)-\mu_{H}([\gamma, w])=2$. (See [FO, Lemma 20.2].) The boundary property (3.8) follows also from [FOOO11, Theorem 14.10].

Remark 3.9. Instead of multisections we can use CF-perturbations (See Definition 33.8.) to define the boundary operator $\partial_{(H, J)}$. If we use CF-perturbations instead of multisections, (3.8) follows from Stokes' formula, Theorem 34.8, and composition formula, Theorem 35.7 .

We use only CF-perturbations from now on in this paper, except in Part 4, where we use multisection sometimes. (See Remark 20.6 and other remarks quoted therein.) Actually we may regard a multisection itself as a CF-perturbation. (See Remark 28.17)

Lemma 3.10. For any $\lambda \in \mathbb{R}$,

$$
\partial_{(H, J)}\left(F^{\lambda} C F\left(M, H ; \Lambda^{\downarrow}\right)\right) \subset F^{\lambda} C F\left(M, H ; \Lambda^{\downarrow}\right) .
$$

Proof. We take $[\gamma, w] \in \operatorname{Crit}\left(\mathcal{A}_{H}\right)$ such that $\pi([\gamma, w])=\llbracket \gamma, w \rrbracket$ and suppose $\llbracket \gamma, w \rrbracket \in$ $\widehat{\operatorname{Per}}(H)$ satisfies $\mathcal{A}_{H}(\llbracket \gamma, w \rrbracket) \leq \lambda$.

\footnotetext{
${ }^{2}$ The map $\pi$ is defined as in 2.5.
} 


$$
\text { If } \begin{aligned}
u \in \widetilde{\mathcal{M}}\left(H, J ;[\gamma, w],\left[\gamma^{\prime}, w^{\prime}\right]\right) \text {, then } \\
\qquad \begin{aligned}
\int u^{*} \omega & =\int_{\tau \in \mathbb{R}} \int_{t \in S^{1}} \omega\left(\frac{\partial u}{\partial \tau}, \frac{\partial u}{\partial t}\right) d t d \tau \\
& =\int_{\tau \in \mathbb{R}} \int_{t \in S^{1}} \omega\left(\frac{\partial u}{\partial \tau}, J \frac{\partial u}{\partial \tau}+X_{H_{t}}(u)\right) d t d \tau \\
& =\int_{\tau \in \mathbb{R}} \int_{t \in S^{1}}\left|\frac{\partial u}{\partial \tau}\right|_{J}^{2} d t d \tau-\int_{\tau \in \mathbb{R}} \int_{t \in S^{1}}\left(d H_{t}(u(\tau, t))\right)\left(\frac{\partial u}{\partial \tau}\right) d t d \tau \\
& =\int_{\tau \in \mathbb{R}} \int_{t \in S^{1}}\left|\frac{\partial u}{\partial \tau}\right|_{J}^{2} d t d \tau-\int_{\tau \in \mathbb{R}}\left(\int_{t \in S^{1}} \frac{\partial}{\partial \tau}\left(H_{t}(u(\tau, t)) d t\right) d \tau\right. \\
& =E_{(H, J)}(u)-\int_{t \in S^{1}} H_{t}\left(\gamma^{\prime}(t)\right) d t+\int_{t \in S^{1}} H_{t}(\gamma(t)) d t .
\end{aligned}
\end{aligned}
$$

Therefore

$$
\int u^{*} \omega+\int_{t \in S^{1}} H_{t}\left(\gamma^{\prime}(t)\right) d t-\int_{t \in S^{1}} H_{t}(\gamma(t)) d t=E_{(H, J)}(u) \geq 0 .
$$

Combined with $w \# u \sim w^{\prime}$, this implies $\mathcal{A}_{H}([\gamma, w])-\mathcal{A}_{H}\left(\left[\gamma^{\prime}, w^{\prime}\right]\right)=E_{(H, J)}(u)$ and so

$$
\mathcal{A}_{H}\left(\left[\gamma^{\prime}, w^{\prime}\right]\right)=\mathcal{A}_{H}([\gamma, w])-E_{(H, J)}(u) \leq \mathcal{A}_{H}([\gamma, w])
$$

and hence Lemma 3.10 holds.

Definition 3.11. The Floer homology with $\Lambda^{\downarrow}$ coefficients is defined by

$$
H F_{*}\left(H, J ; \Lambda^{\downarrow}\right):=\frac{\operatorname{Ker} \partial_{(H, J)}}{\operatorname{Im} \partial_{(H, J)}} .
$$

Theorem 3.12. We may choose the orientation in Proposition 3.6 (5) so that $H F_{*}\left(H, J ; \Lambda^{\downarrow}\right)$ is isomorphic to the singular (co)homology $H\left(M ; \Lambda^{\downarrow}\right)$ with $\Lambda^{\downarrow}$ coefficients.

This is proved in FO, Theorem 22.1]. We will describe a construction of isomorphism (which is different from the one in [FO) below because we need to specify the isomorphism to encode each spectral invariant by the corresponding quantum cohomology class.

Definition 3.13. Consider a smooth function $\chi: \mathbb{R} \rightarrow[0,1]$ with the properties

$$
\begin{aligned}
& \chi(\tau)= \begin{cases}0 & \text { for } \tau \leq 0 \\
1 & \text { for } \tau \geq 1\end{cases} \\
& \chi^{\prime}(\tau) \geq 0 .
\end{aligned}
$$

We denote by $\mathcal{K}$ the set of such elongation functions.

We note that $\mathcal{K}$ is convex and so contractible. We will fix a choice of $\chi$ for our various constructions throughout the present paper but the Floer theoretic invariants coming out of such constructions are independent of the choice thanks to contractibility of $\mathcal{K}$.

For a given $S^{1}$-dependent family of almost complex structures $J$, we consider a two-parameter family $\left\{J_{s}\right\}_{s \in[0,1]}$ such that

$$
J_{0, t}=J_{0}, \quad J_{1, t}=J_{t}
$$


where $J_{0}$ is a time-independent almost complex structure. We also assume

$$
J_{s, t} \equiv J_{0}, \quad \text { for }(s, t) \in \partial[0,1]^{2} \backslash(\{1\} \times[0,1]) .
$$

For each nondegenerate $H: S^{1} \times M \rightarrow \mathbb{R}$, we define $\left(\mathbb{R} \times S^{1}\right)$-family to $\left(H_{\chi}, J_{\chi}\right)$ on $\mathbb{R} \times S^{1}$ by

$$
H_{\chi}(\tau, t)=\chi(\tau) H_{t}, \quad J_{\chi}(\tau, t)=J_{\chi(\tau), t} .
$$

Remark 3.14. It is very important that the family $J_{s}$ is $t$-independent for $s=0$. See the proof of Proposition 6.11

Definition 3.15. We denote by $\mathcal{\mathcal { M }}\left(H_{\chi}, J_{\chi} ; *,[\gamma ; w]\right)$ the set of all maps $u: \mathbb{R} \times$ $S^{1} \rightarrow M$ satisfying the following conditions:

(1) The map $u$ satisfies the equation:

$$
\frac{\partial u}{\partial \tau}+J_{\chi}\left(\frac{\partial u}{\partial t}-\chi(\tau) X_{H_{t}}(u)\right)=0 .
$$

Here and hereafter $J_{\chi}$ in (3.14) means $J_{\chi, t}$.

(2) The energy

$$
E_{\left(H_{\chi}, J_{\chi}\right)}(u):=\frac{1}{2} \int\left(\left|\frac{\partial u}{\partial \tau}\right|_{J_{\chi}}^{2}+\left|\frac{\partial u}{\partial t}-\chi(\tau) X_{H_{t}}(u)\right|_{J_{\chi}}^{2}\right) d t d \tau
$$

is finite.

(3) The map $u$ satisfies the following asymptotic boundary condition:

$$
\lim _{\tau \rightarrow+\infty} u(\tau, t)=\gamma(t) \text {. }
$$

In particular, $u$ defines a homotopy class $[u] \in \pi_{2}(\gamma)$.

(4) We have $[u]=[w]$ in $\pi_{2}(\gamma)$.

$$
\frac{\partial u}{\partial \tau}+J \frac{\partial u}{\partial t}=0 \quad u^{\frac{\partial u}{\partial \tau}+J_{t}\left(\frac{\partial u}{\partial t}-X_{H_{t}}\right)=0}
$$

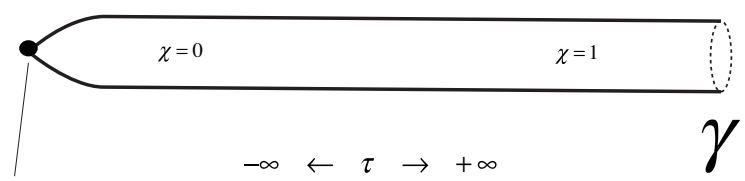

$\in M$

Figure 1. An element of $\stackrel{\circ}{\mathcal{M}}\left(H_{\chi}, J_{\chi} ; *,[\gamma ; w]\right)$

We note that since $\chi(\tau) X_{H_{t}} \equiv 0$ and $J_{\chi(\tau)} \equiv J_{0}$ for $\tau<-1$, which turns (3.14) into the genuine $J_{0}$-holomorphic curve equation, the removable singularity theorem (due to Sacks-Uhlenbeck and Gromov, see e.g., Sil, Theorem 4.5.1]) gives rise to a well-defined limit

$$
\lim _{\tau \rightarrow-\infty} u(\tau, t)
$$

which does not depend on $t$. Therefore the homotopy class condition required in (4) above makes sense.

We denote this assignment of the limit by

$$
\mathrm{ev}_{-\infty}: \stackrel{\circ}{\mathcal{M}}\left(H_{\chi}, J_{\chi} ; *,[\gamma, w]\right) \rightarrow M .
$$


Remark 3.16. We put $*$ on the left of $[\gamma ; w]$ in the notation of the moduli space to highlight the fact that $[\gamma, w]$ appears as positive asymptotic boundary condition of the cylinder $\mathbb{R} \times S^{1}$. We use similar notations using $*$ in various other Floer theoretic moduli spaces arising throughout the paper in a various different ways. Note we do not require the limit point (3.15) to be a particular base point of $M$ -we only require the convergence to some point of $M$.

Proposition 3.17. (1) The moduli space $\stackrel{\circ}{\mathcal{M}}\left(H_{\chi}, J_{\chi} ; *,[\gamma, w]\right)$ has a compactification $\mathcal{M}\left(H_{\chi}, J_{\chi} ; *,[\gamma, w]\right)$ that is Hausdorff.

(2) The space $\mathcal{M}\left(H_{\chi}, J_{\chi} ; *,[\gamma, w]\right)$ has an orientable Kuranishi structure with corners.

(3) The (normalized) boundary of $\mathcal{M}\left(H_{\chi}, J_{\chi} ; *,[\gamma, w]\right)$ is described by

$$
\begin{aligned}
& \partial \mathcal{M}\left(H_{\chi}, J_{\chi} ; *,[\gamma, w]\right) \\
& =\bigcup \mathcal{M}\left(H_{\chi}, J_{\chi} ; *,\left[\gamma^{\prime}, w^{\prime}\right]\right) \times \mathcal{M}\left(H, J ;\left[\gamma^{\prime}, w^{\prime}\right],[\gamma, w]\right),
\end{aligned}
$$

where the union is taken over all $\left[\gamma^{\prime}, w^{\prime}\right] \in \operatorname{Crit}\left(\mathcal{A}_{H}\right)$.

(4) The (virtual) dimension satisfies the following equality (3.18).

$$
\operatorname{dim} \mathcal{M}\left(H_{\chi}, J_{\chi} ; *,[\gamma, w]\right)=\mu_{H}([\gamma, w])+n .
$$

(5) We can define a system of orientations of $\mathcal{M}\left(H_{\chi}, J_{\chi} ; *,[\gamma, w]\right)$ so that (3) above is compatible with this orientation.

(6) The map ev $-\infty$ becomes a weakly submersive map in the sense of [FOOO2, Definition A1.13]. (See Definition 32.1.)

The proof is mostly the same as that of Proposition 3.6 and is omitted. We can prove the weak submersivity of $\mathrm{ev}_{-\infty}$ in item (6) in the same way as [FOOO2, Section 7]. It is proved more explicitly in [Fu3, Lemma 3.1]. (There the case of the evaluation map at the boundary marked point is treated. The case of $\mathrm{ev}_{-\infty}$, which is item (6) above, can be proved in the same way.) An element of the right hand side of (3.17) is drawn in Figure 2 below. We take a system of CF-perturbations $\left\{\widehat{\mathfrak{S}^{\epsilon}}\right\}$

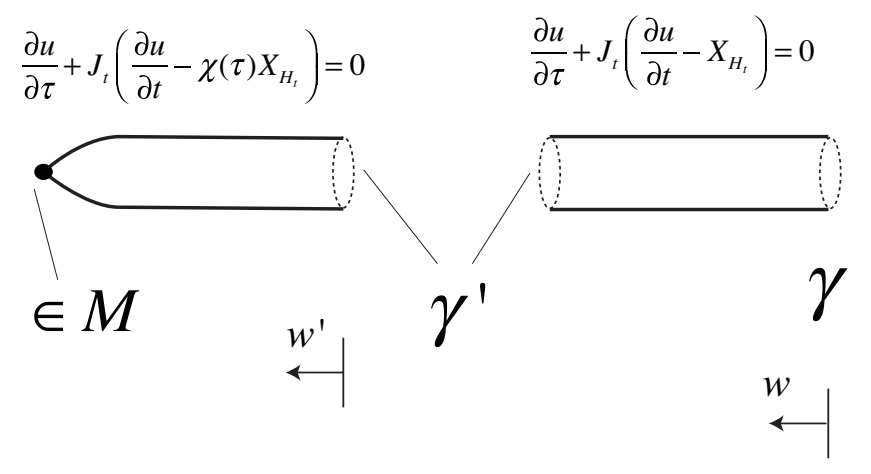

Figure 2. An element of (3.17)

on $\mathcal{M}\left(H_{\chi}, J_{\chi} ; *,[\gamma, w]\right)$ for various $[\gamma, w]$ so that it is compatible at the boundary described in (3.17) and $e_{-\infty}$ is strongly submersive with respect to $\left\{\widehat{\mathfrak{S}^{\epsilon}}\right\}$, in the sense of Definition 33.9 Existence of such CF-perturbations follows from Theorem 33.12 
Let $h$ be a differential $k$ form on $M$. We define

$$
\mathcal{P}_{\left(H_{\chi}, J_{\chi}\right)}(h)=\sum_{[\gamma, w] \in \operatorname{Crit}\left(\mathcal{A}_{H}\right)}\left(\int_{\left(\mathcal{M}\left(H_{\chi}, J_{\chi} ; *,[\gamma, w]\right), \widehat{\mathfrak{S}^{\epsilon}}\right)} \operatorname{ev}_{-\infty}^{*}(h)\right) \llbracket \gamma, w \rrbracket .
$$

(The symbol $\mathcal{P}$ stands for Piunikhin $[$ Piu .) Here the sum is taken over $[\gamma, w] \in$ $\operatorname{Crit}\left(\mathcal{A}_{H}\right)$ with $\mu_{H}([\gamma, w])=k-n=\operatorname{deg} h-n$ and $\widehat{\mathfrak{S}}$ is a CF-perturbation. The integration in (3.19) is defined as (34.3).

The definition (3.19) induces a map

$$
\mathcal{P}_{\left(H_{\chi}, J_{\chi}\right)}: \Omega(M) \widehat{\otimes} \Lambda^{\downarrow} \rightarrow C F\left(M, H ; \Lambda^{\downarrow}\right) .
$$

Here $\Omega(M) \widehat{\otimes} \Lambda^{\downarrow}$ is the completion with respect to the norm $\mathfrak{v}_{q}$ of the algebraic tensor product $\Omega(M) \otimes \Lambda^{\downarrow}$ (over $\mathbb{R}$ ).

Let $\left(\Omega^{*}(M), d\right)$ be the de Rham complex of $M$. We regard it as a chain complex $\left(\Omega_{*}(M), \partial\right)$, where

$$
\Omega_{k}(M)=\Omega^{\operatorname{dim} M-k}(M), \quad \partial=(-1)^{\operatorname{deg}+1} d .
$$

Lemma 3.18. $\mathcal{P}_{\left(H_{\chi}, J_{\chi}\right)}$ defines a chain map

$$
\mathcal{P}_{\left(H_{\chi}, J_{\chi}\right)}:(\Omega(M), \partial) \widehat{\otimes} \Lambda^{\downarrow} \rightarrow\left(C F\left(M, H ; \Lambda^{\downarrow}\right), \partial_{(H, J)}\right)
$$

from the de Rham complex to the Floer complex.

Proof. We can prove $\mathcal{P}_{\left(H_{\chi}, J_{\chi}\right)} \circ \partial=\partial_{(H, J)} \circ \mathcal{P}_{\left(H_{\chi}, J_{\chi}\right)}$ by applying Stokes' theorem (Theorem 34.8, [FOOO11, Proposition 9.26]), composition formula (Theorem 35.7 [FOOO11, Theorem 10.20]), Proposition 3.17(3) and the definition.

Lemma 3.19. $\mathcal{P}_{\left(H_{\chi}, J_{\chi}\right)}$ induces a chain homotopy equivalence.

The proof is similar to the argument established in various similar situations. (One of the closest descriptions we can find in the literature is [FOOO4, Section 8 Proposition 8.24], where a similar lemma is proved in the case of Lagrangian Floer theory.) We give a proof in the appendix for completeness' sake.

Remark 3.20. (1) Note we need to take care of the problem of running-out, which is explained in detail in [FOOO2, Section 7.2.3]. We explain it briefly below. In our situation we need to perturb infinitely many moduli spaces such as $\mathcal{M}\left(H, J ;[\gamma, w],\left[\gamma^{\prime}, w^{\prime}\right]\right)$ simultaneously. It is in general difficult to do so. We go around this problem as follows. For each $E>0$ we have only finitely many moduli spaces for which symplectic area of its element is less than $E$. We can perturb them appropriately for any fixed $E$. (The perturbation depends on $E$, however.) By doing so, we obtain a chain complex $C_{E}$ over $\Lambda_{0}^{\downarrow} / q^{-E} \Lambda_{0}^{\downarrow}$ for any $E$. We can also prove that, if $E^{\prime}>E$ then $C_{E^{\prime}} \bmod q^{-E} \Lambda_{0}^{\downarrow}$ is chain homotopy equivalent to $C_{E}$. We can use this fact and an elementary homological algebra to lift $C_{E}$ to a chain complex over $\Lambda_{0}^{\downarrow} / q^{-E^{\prime}} \Lambda_{0}^{\downarrow}$ so that it becomes chain homotopy equivalent to $C_{E^{\prime}}$. We repeat this process and by taking a projective limit we obtain a chain complex over $\Lambda_{0}^{\downarrow}$ whose reduction is chain homotopy equivalent to $C_{E}$ for any $E$.

The technical difficulty to perform this construction in our situation is much simpler than that of [FOOO2, Section 7.2], since we here need to take a projective limit of chain complex (or DGA) which is much simpler than 
that of $A_{\infty}$ algebra in general (which is discussed in [FOOO2, Section 7.2]). So we omit the detail.

(2) Here we work over the $\Lambda^{\downarrow}$ coefficients and so only with the $\mathbb{Z}_{2}$ grading given in (3.6). However if the minimal Chern number is $2 N$, we can define a $\mathbb{Z}_{2 N \text {-grading. }}$

(3) Here we use $\mathbb{C}$ as the ground field. Up until now, we can work with $\mathbb{Q}$ in the same way. We prefer to use $\mathbb{C}$ in this memoir since we will use de Rham theory later on to involve bulk deformations in our constructions. In addition, the de Rham theory is used for the Lagrangian Floer theory of toric manifolds in various calculations and applications developed in FOOO3 etc.

(4) We use de Rham cohomology of $M$ to define $\mathcal{P}_{\left(H_{\chi}, J_{\chi}\right)}$. There are several other ways of constructing this isomorphism. One uses singular (co)homology as in [FOOO2, Section 7.2] (especially [FOOO2, Proposition 7.2.21]) and references therein. This approach allows one to work with $\mathbb{Q}$ coefficients, which may have some additional applications. Other uses Morse homology as proposed in $\overline{\mathrm{RT}}$, $\overline{\mathrm{PSS}}$. The necessary analytic details of the latter approach has been established recently in $\mathrm{OZ}$.

\section{Spectral invariants}

The very motivating example of Floer-Novikov complex and its chain level theory was applied by the second named author in the Hamiltonian Floer theory Oh4, Oh7. Namely, a spectral number which we denote by $\rho(H ; a)$ is associated to $a \in Q H(M ; \Lambda)=H^{*}(M) \otimes \Lambda$ and a Hamiltonian $H$, and is proved to be independent of various choices, especially of $J$ in Oh4. Via the canonical identification $H^{*}(M) \otimes$ $\Lambda \cong H^{*}(M) \otimes \Lambda^{\downarrow}$ induced by the change of the formal variable $T \mapsto T^{-1}=: q$ which reverses the direction of valuation, we often abuse our notation for $a$ also to denote this formal change.

In this section we give a brief summary of this construction. Let $H: S^{1} \times M \rightarrow \mathbb{R}$ be a normalized time-dependent nondegenerate Hamiltonian.

Definition 4.1. We denote the set of (spherical) symplectic periods of $(M, \omega)$ by

$$
G(M, \omega)=\left\{\alpha \cap[\omega] \mid \alpha \in \pi_{2}(M)\right\} .
$$

The set $G(M, \omega)$ is a countable subset of $\mathbb{R}$ which is a subgroup of the additive group of $\mathbb{R}$ as a group. It may or may not be discrete.

For each given $H$, we define the action spectrum of $H$ by

$$
\operatorname{Spec}(H):=\left\{\mathcal{A}_{H}(\gamma, w) \in \mathbb{R} \mid[\gamma, w] \in \operatorname{Crit}\left(\mathcal{A}_{H}\right)\right\},
$$

i.e., the set of critical values of $\mathcal{A}_{H}: \widetilde{\mathcal{L}_{0}}(M) \rightarrow \mathbb{R}$.

Then for any given $H$ the subgroup $G(M, \omega)$ of $(\mathbb{R},+)$ acts on $\operatorname{Spec}(H)$ by an addition.

Definition 4.2. Let $G \subset \mathbb{R}$ be a submonoid. We say that a subset $G^{\prime} \subset \mathbb{R}$ is a $G$-set if $g \in G, g^{\prime} \in G^{\prime}$ implies $g+g^{\prime} \in G^{\prime}$.

With this definition, $\operatorname{Spec}(H)$ is $G(M, \omega)$-set

Lemma 4.3. If $\lambda \in \operatorname{Spec}(H)$ and $g \in G(M, \omega)$ then $\lambda \pm g \in \operatorname{Spec}(H)$. 
If $H$ is nondegenerate, then the quotient space $\operatorname{Spec}(H) / G(M, \omega)$ with the above action is a finite set and

$$
\#(\operatorname{Spec}(H) / G(M, \omega)) \leq \# \operatorname{Per}(H) .
$$

Proof. Let $[\gamma, w],\left[\gamma, w^{\prime}\right] \in \operatorname{Crit}\left(\mathcal{A}_{H}\right)$. We glue $w$ and $w^{\prime}$ along $\gamma$ and obtain $\bar{w} \# w^{\prime}$. Its homology class in $H_{2}(M ; \mathbb{Z})$ is well-defined. We have

$$
\mathcal{A}_{H}([\gamma, w])-\mathcal{A}_{H}\left(\left[\gamma, w^{\prime}\right]\right)=\int_{\bar{w} \# w^{\prime}} \omega .
$$

The lemma follows easily from this fact and the fact that $\operatorname{Per}(H)$ is a finite set.

Remark 4.4. One can actually make the equality \# $(\operatorname{Spec}(H) / G(M, \omega))=\# \operatorname{Per}(H)$ hold by further perturbing $H$, but we will not do that because it is not needed.

Lemma 4.5. $G(M, \omega)$ acts on $\widehat{\operatorname{Per}}(H)$ such that $\widehat{\operatorname{Per}}(H) / G(M, \omega)=\operatorname{Per}(H)$ and $\mathcal{A}_{H}(g \llbracket \gamma, w \rrbracket)=g+\mathcal{A}_{H}(\llbracket \gamma, w \rrbracket)$.

Proof. It is obvious from definition that $\pi_{2}(M)$ acts on $\operatorname{Crit}\left(\mathcal{A}_{H}\right) \operatorname{such}$ that $\operatorname{Crit}\left(\mathcal{A}_{H}\right) / \pi_{2}(M) \cong$ $\operatorname{Per}(H)$. The lemma then follows from the fact that $G(M, \omega)$ is the image of (2.7) and Lemma 2.2 in the same way as the proof of Lemma 4.3.

The following definition is standard.

Definition 4.6. We say that two one-periodic Hamiltonians $H$ and $H^{\prime}$ are homotopic if $\phi_{H}^{1}=\phi_{H^{\prime}}^{1}$ and if there exists $\left\{H^{s}\right\}_{s \in[0,1]}$ a one-parameter family of one periodic Hamiltonians such that $H^{0}=H, H^{1}=H^{\prime}$ and $\phi_{H}^{1}=\phi_{H^{s}}^{1}$ for all $s \in[0,1]$. In this case we denote $H \sim H^{\prime}$ and denote the set of equivalence classes by $\widehat{\operatorname{Ham}}(M, \omega)$.

The following lemma was proven in $\mathrm{Sc2}, \mathrm{P}$ in the aspherical case and in $\mathrm{Oh2}$ for the general case. We provide its proof in Section 10 for reader's convenience.

Proposition 4.7. Suppose that $H, H^{\prime}$ are normalized one periodic Hamiltonians. If $H \sim H^{\prime}$, we have $\operatorname{Spec}(H)=\operatorname{Spec}\left(H^{\prime}\right)$ as a subset of $\mathbb{R}$.

This enables one to make the following definition

Definition 4.8. We define the spectrum of $\widetilde{\psi} \in \widetilde{\operatorname{Ham}}(M, \omega)$ to be $\operatorname{Spec}(\widetilde{\psi}):=$ $\operatorname{Spec}(\underline{H})$ for a (and so any) Hamiltonian $H$ satisfying $\widetilde{\psi}=\left[\phi_{H}\right]$.

Here we denote the normalization of $H$ by

$$
\underline{H}(t, x)=H(t, x)-\frac{1}{\operatorname{vol}_{\omega}(M)} \int_{M} H_{t} \omega^{n} .
$$

Definition 4.9. Let $G$ be a subset of $\mathbb{R}$, which is a monoid. We denote by $\Lambda^{\downarrow}(G)$ the set of all elements

$$
\sum a_{i} q^{\lambda_{i}} \in \Lambda^{\downarrow}
$$

such that if $a_{i} \neq 0$ then $-\lambda_{i} \in G$. We note that $\Lambda^{\downarrow}(G)$ forms a subring of $\Lambda^{\downarrow}$ and $\Lambda^{\downarrow}(G)$ is a field if $G$ is a subgroup of the (additive) group $\mathbb{R}$. We write

$$
\Lambda^{\downarrow}(M)=\Lambda^{\downarrow}(G(M, \omega)) .
$$


Suppose that $H$ is nondegenerate. We denote by $C F(M, H)$ the subset of $C F\left(M, H ; \Lambda^{\downarrow}\right)$ consisting of the infinite sums

$$
\sum_{\llbracket \gamma, w \rrbracket \in \widehat{\operatorname{Per}}(H)} a_{\llbracket \gamma, w \rrbracket} \llbracket \gamma, w \rrbracket
$$

with $a_{\llbracket \gamma, w \rrbracket} \in \mathbb{C}$. We denote by $F^{\lambda} C F(M, H)$ the subset of $C F(M, H)$ consisting of elements (4.1) such that $\mathcal{A}_{H}(\llbracket \gamma, w \rrbracket) \leq \lambda$.

Lemma 4.10. (1) $C F(M, H)$ is a vector space over $\Lambda^{\downarrow}(M)$.

(2) $\left\{\llbracket \gamma, w_{\gamma} \rrbracket \mid \gamma \in \operatorname{Per}(H)\right\}$ is a basis of $C F(M, H)$ over $\Lambda^{\downarrow}(M)$.

(3) We have

$$
C F\left(M, H ; \Lambda^{\downarrow}\right) \cong C F(M, H) \otimes_{\Lambda \downarrow}(M) \Lambda^{\downarrow} .
$$

(4) The Floer boundary operator $\partial_{(H, J)}$ preserves the submodule $C F(M, H) \subset$ $C F\left(M, H ; \Lambda^{\downarrow}\right)$.

This is an easy consequence of Lemma 4.5 .

Lemma 4.11. The chain map $\mathcal{P}_{\left(H_{\chi}, J_{\chi}\right)}$ in (3.19) induces a $\Lambda^{\downarrow}(M)$-linear map, which we still denote

$$
\mathcal{P}_{\left(H_{\chi}, J_{\chi}\right)}: C\left(M ; \Lambda^{\downarrow}(M)\right) \rightarrow C F\left(M, H ; \Lambda^{\downarrow}(M)\right)
$$

which are chain-homotopic to one another for different choices of $\chi$.

This is immediate from the definition of $\mathcal{P}_{\left(H_{\chi}, J_{\chi}\right)}$. Therefore this together with Theorem 3.12 gives rise to an isomorphism

$$
\mathcal{P}_{\left(H_{\chi}, J_{\chi}\right), *}: H_{*}\left(M ; \Lambda^{\downarrow}(M)\right) \cong H F_{*}(M, H)
$$

where the right hand side is the homology of $(C F(M, H), \partial)$. This isomorphism does not depend on the choice of $\chi$ 's. We also use the isomorphism, which is the $\Lambda-\Lambda^{\downarrow}$ linear extension of the Poincaré duality isomorphism, denoted by

$$
\text { b : } Q H^{*}(M ; \Lambda)=H^{*}(M) \otimes \Lambda \rightarrow H_{*}(M) \otimes \Lambda^{\downarrow} \cong H_{*}\left(M, \Lambda^{\downarrow}\right)
$$

which is nothing but the map induced by first taking the Poincaré duality and then changing the formal variable $T \rightarrow q=T^{-1}$. For the simplicity of notation, we will just denote $Q H^{*}(M)=Q H^{*}(M ; \Lambda)$.

The filtration $F^{\lambda} C F\left(M, H ; \Lambda^{\downarrow}\right)$ induces a filtration $F^{\lambda} C F(M, H)$ on $C F(M, H)$ in an obvious way.

Definition 4.12. (1) Let $\mathfrak{x} \in H F(H, J)$ be any nonzero Floer homology class. We define its spectral invariant $\rho(\mathfrak{x})$ by

$$
\rho(\mathfrak{x})=\inf \left\{\lambda \mid x \in F^{\lambda} C F\left(M, H ; \Lambda^{\downarrow}\right), \partial_{(H, J)} x=0,[x]=\mathfrak{x}\right\} .
$$

(2) If $a \in H^{*}(M ; \Lambda(M))$ and $H$ is a nondegenerate time-dependent Hamiltonian, we define the spectral invariant $\rho(H ; a)$ by

$$
\rho(H, J ; a)=\rho\left(a_{H}^{b}\right), \quad a_{H}^{b}:=\mathcal{P}_{\left(H_{\chi}, J_{\chi}\right), *}\left(a^{b}\right)
$$

where the right hand side is defined in (1) and $a^{b}$ is the Poincaré dual of the cohomology class $a$. See Notations and Conventions (22). (Here $\Lambda(M) \subset \Lambda$ is a subring which is identified with $\Lambda^{\downarrow}(M) \subset \Lambda^{\downarrow}$ by the canonical isomorphism $\Lambda \cong \Lambda^{\downarrow}$.) 
It is proved in Oh4, Oh7 that $\rho(H, J ; a)$ is independent of $J$. The same can be proved in general under other choices involved in the definition such as the abstract perturbations in the framework of Kuranishi structure as long as the Hamiltonian $H$ is fixed. So we omit $J$ from notation and just denote it by $\rho(H ; a)$.

We introduce the following standard invariants associated to the Hamiltonian $H:[0,1] \times M \rightarrow \mathbb{R}$ called the positive and negative parts of Hofer norm $E^{ \pm}(H)$

$$
\begin{aligned}
E^{+}(H) & :=\int_{t \in S^{1}} \max _{x} H_{t} d t \\
E^{-}(H) & :=\int_{t \in S^{1}}-\min _{x} H_{t} d t
\end{aligned}
$$

for any Hamiltonian $H$. We have the Hofer norm $\|H\|=E^{+}(H)+E^{-}(H)$. We like to emphasize that $H$ is not necessarily one-periodic time-dependent family.

Lemma 4.13. We have

$$
-E^{+}\left(H^{\prime}-H\right) \leq \rho\left(H^{\prime} ; a\right)-\rho(H ; a) \leq E^{-}\left(H^{\prime}-H\right) .
$$

This lemma enables one to extend, by continuity, the definition of $\rho(H ; a)$ to any Hamiltonian $H: S^{1} \times M \rightarrow \mathbb{R}$ which is not necessarily nondegenerate. Lemma 4.13 is proved in a generalized form as Theorem 9.1 .

The following homotopy invariance is also proved in [Oh4, Oh7, Us1].

Theorem 4.14 (Homotopy invariance). Suppose $H, H^{\prime}$ are normalized. If $H \sim H^{\prime}$ then $\rho(H ; a)=\rho\left(H^{\prime} ; a\right)$.

We will prove it in Section 10 for completeness. This homotopy invariance enables one to extend the definition of $\rho(H ; a)$ to non-periodic $H:[0,1] \times M \rightarrow \mathbb{R}$.

Consider the set of smooth functions $\zeta:[0,1] \rightarrow[0,1]$ satisfying $\zeta(0)=0$, $\zeta(1)=1$ and $\zeta$ is constant in a neighborhood of 0 and 1 . Note that this set is convex and so contractible. Let $H:[0,1] \times M \rightarrow \mathbb{R}$ and $\zeta:[0,1] \rightarrow[0,1]$ be such a function. Denote

$$
H^{\zeta}(t, x)=\zeta^{\prime}(t) H(\zeta(t), x) .
$$

We note that $H^{\zeta}$ may be regarded as a map for $S^{1} \times M$ since $H=0$ in a neighborhood of $\{0,1\} \times M$. Moreover the above mentioned convexity implies that $H^{\zeta_{1}} \sim H^{\zeta_{2}}$. Therefore $\rho\left(H^{\zeta_{1}} ; a\right)=\rho\left(H^{\zeta_{2}} ; a\right)$ for any such $\zeta_{i}$. We define the common number to be $\rho(H ; a)$. This gives rise to the map

$$
\rho: C^{\infty}([0,1] \times M, \mathbb{R}) \times\left(Q H^{*}(M) \backslash\{0\}\right) \rightarrow \mathbb{R} .
$$

Its basic properties are summarized in the next theorem. For given $a \in Q H^{*}(M)$, we represent it by

$$
a=\sum_{g \in G(M, \omega)} a_{g} T^{-g}=\sum_{g \in G(M, \omega)} a_{g} q^{g}, \quad a_{g} \in H^{*}(M ; \mathbb{C}) .
$$

We define

$$
\lambda_{q}(a):=\max \left\{g \in \mathbb{R} \mid a_{g} \neq 0\right\} .
$$

Theorem 4.15. (Oh) Let $(M, \omega)$ be any closed symplectic manifold. Then the map $\rho$ in (4.5) satisfies the following properties: Let $H, H^{\prime} \in C^{\infty}([0,1] \times M, \mathbb{R})$ and $0 \neq a \in Q H^{*}(M)$. Then

(1) (Nondegenerate spectrality) $\rho(H ; a) \in \operatorname{Spec}(H)$, if $\widetilde{\psi}_{H}$ is nondegenerate.

(2) (Projective invariance) $\rho(H ; \lambda a)=\rho(H ; a)$ for any $0 \neq \lambda \in \mathbb{C}$. 
(3) (Normalization shift) For any function $c:[0,1] \rightarrow \mathbb{R}, \rho(H+c(t) ; a)=$ $\rho(H ; a)-\int_{0}^{1} c(t) d t$.

(4) (Normalization) $\rho(\underline{0} ; a)=\mathfrak{v}_{q}(a)=-\mathfrak{v}_{T}(a)$, where $\underline{0}$ is the identity in $\widetilde{\operatorname{Ham}}(M, \omega)$.

(5) (Symplectic invariance) $\rho\left(H \circ \eta ; \eta^{*} a\right)=\rho(\widetilde{\phi} ; a)$ for any symplectic diffeomorphism $\eta$. In particular, if $\eta \in \operatorname{Symp}_{0}(M, \omega)$, then we have $\rho(H \circ \eta ; a)=$ $\rho(H ; a)$.

(6) (Triangle inequality) $\rho\left(H \# H^{\prime} ; a \cup_{Q} b\right) \leq \rho(H ; a)+\rho\left(H^{\prime} ; b\right)$, where $a \cup_{Q} b$ is the quantum cup product.

(7) $\left(C^{0}\right.$-hamiltonian continuity) We have

$$
-E^{+}\left(H^{\prime}-H\right) \leq \rho\left(H^{\prime} ; a\right)-\rho(H ; a) \leq E^{-}\left(H^{\prime}-H\right) .
$$

(8) (Additive triangle inequality) $\rho(H ; a+b) \leq \max \{\rho(H ; a), \rho(H ; b)\}$.

Theorem 4.15 is stated by the second named author Oh4, Oh7 in the general context but without detailed account on the construction of virtual fundamental classes in the various moduli spaces entering in the proofs. In the present paper, we provide these details in the framework of Kuranishi structures [FO]. A purely algebraic treatment of the statement (1) is given by Usher [Us1.

By considering the normalization $\underline{H}(t, x)$ of $H(t, x)$ and the one-one correspondence between $\underline{H}$ and its Hamiltonian path $\phi_{H}$, we can interpret $\rho(H ; a)$ as the invariant of the associated Hamiltonian path $\phi_{H}$ by setting

$$
\rho\left(\phi_{H} ; a\right):=\rho(\underline{H} ; a) .
$$

The invariance of $H \mapsto \rho(H ; a)$ under the equivalence relation $H \sim H^{\prime}$ enables one to push this down to $\widetilde{\operatorname{Ham}}(M, \omega)$ which we denote $\rho\left(\phi_{H} ; a\right)$. We denote the resulting map by

$$
\rho: \widetilde{\operatorname{Ham}}(M, \omega) \times\left(Q H^{*}(M) \backslash\{0\}\right) \rightarrow \mathbb{R} .
$$

Its basic properties are summarized in the next theorem, which are immediate translation of those stated in Theorem 4.15 .

Theorem 4.16. Let $(M, \omega)$ be any closed symplectic manifold. Then the map $\rho$ in (4.7) has the following properties: Let $\widetilde{\psi}, \widetilde{\phi} \in \widetilde{\operatorname{Ham}}(M, \omega)$ and $0 \neq a \in$ $H^{*}(M ; \Lambda(M))$.

(1) (Nondegenerate spectrality) $\rho(\widetilde{\psi} ; a) \in \operatorname{Spec}(\widetilde{\psi})$, if $\widetilde{\psi}$ is nondegenerate.

(2) (Projective invariance) $\rho(\widetilde{\phi} ; \lambda a)=\rho(\widetilde{\phi} ; a)$ for any $0 \neq \lambda \in \mathbb{C}$.

(3) (Normalization) We have $\rho(\underline{0} ; a)=\mathfrak{v}_{q}(a)$.

(4) (Symplectic invariance) $\rho\left(\eta \circ \widetilde{\phi} \circ \eta^{-1} ; \eta^{*} a\right)=\rho(\widetilde{\phi} ; a)$ for any symplectic diffeomorphism $\eta$. In particular, if $\eta \in \operatorname{Symp}_{0}(M, \omega)$, then we have $\rho(\eta \circ$ $\left.\widetilde{\phi} \circ \eta^{-1} ; a\right)=\rho(\widetilde{\phi} ; a)$.

(5) (Triangle inequality) $\rho\left(\widetilde{\phi} \circ \widetilde{\psi} ; a \cup_{Q} b\right) \leq \rho(\widetilde{\phi} ; a)+\rho(\widetilde{\psi} ; b)$, where $a \cup_{Q} b$ is the quantum cup product.

(6) $\left(C^{0}\right.$-hamiltonian continuity) We have

$$
|\rho(\widetilde{\phi} ; a)-\rho(\widetilde{\psi} ; a)| \leq \max \left\{\left\|\widetilde{\phi} \circ \widetilde{\psi}^{-1}\right\|_{+},\left\|\widetilde{\phi} \circ \widetilde{\psi}^{-1}\right\|_{-}\right\}
$$

where $\|\cdot\|_{ \pm}$is the positive and negative parts of Hofer norm on $\widetilde{\operatorname{Ham}}(M, \omega)$.

(7) (Additive triangle inequality) $\rho(\widetilde{\phi} ; a+b) \leq \max \{\rho(\widetilde{\phi} ; a), \rho(\widetilde{\phi} ; b)\}$. 
Here we explain the meaning of the negative and positive parts of Hofer norm $\|\widetilde{\psi}\|_{ \pm}$. For $\widetilde{\psi} \in \widetilde{\operatorname{Ham}}(M, \omega)$, we define

$$
\|\widetilde{\psi}\|_{ \pm}=\inf _{H}\left\{E^{ \pm}(H) \mid\left[\phi_{H}\right]=\widetilde{\psi}\right\}
$$

respectively, and the (strong) Hofer norm $\|\widetilde{\psi}\|$ is defined by

$$
\|\widetilde{\psi}\|=\inf _{H}\left\{\|H\| \mid\left[\phi_{H}^{1}\right]=\widetilde{\psi}\right\} .
$$

There is another norm, sometimes called the medium Hofer norm, which is defined by

$$
\|\widetilde{\psi}\|_{\text {med }}=\|\widetilde{\psi}\|_{+}+\|\widetilde{\psi}\|_{-}
$$

Obviously we have

$$
|\rho(\widetilde{\psi} ; a)-\rho(\widetilde{i d} ; a)| \leq\|\widetilde{\psi}\|_{\operatorname{med}} \leq\|\widetilde{\psi}\|
$$

for all $a \in Q H^{*}(M ; \Lambda(M))$. Here $\widetilde{i d}$ stands for the constant Hamiltonian isotopy at the identity. If $a \in H^{*}(M ; \mathbb{C}) \subset Q H^{*}(M ; \Lambda(M))$, we find that

$$
|\rho(\widetilde{\psi} ; a)| \leq\|\widetilde{\psi}\|_{\text {med }} \leq\|\widetilde{\psi}\| .
$$

See the introduction of Oh5] for the related discussion.

Remark 4.17. There is another important property, that is compatibility with Poincaré duality observed by Entov-Polterovich [EP1] in the case $M$ is semi-positive and $M$ is rational. Those assumptions are removed by Usher Us3]. We will discuss some enhancement of this point later in Section 15.

We refer readers to the above references for the proof of Theorems 4.16. Later we will prove its enhancement including bulk deformations. Here are some remarks.

Remark 4.18. We like to note that the constructions of $\rho(H ; a)$ given in Oh4 can be carried over whether or not $H$ is normalized. We need the normalization only to descend the spectral function $H \mapsto \rho(H ; a)$ to the universal covering space $\widehat{\operatorname{Ham}}(M, \omega)$ as in Theorem 4.16.

Remark 4.19. In EP1, EP2, EP3], Entov-Polterovich used different sign conventions from the ones [Oh4] and the present paper. If we compare our convention with the one from EP3, the only difference lies in the definition of Hamiltonian vector field: our definition, which is the same as that of [0h4], is given by $d H=\omega\left(X_{H}, \cdot\right)$ while EP3 takes $d H=\omega\left(\cdot, X_{H}\right)$. Therefore by replacing $H$ by $-H$, one has the same set of closed loops as the periodic solutions of the corresponding Hamiltonian vector fields.

This also results in the difference in the definition of action functional: our definition, the same as the one in Oh4, is given by

$$
\mathcal{A}_{H}([\gamma, w])=-\int w^{*} \omega-\int_{0}^{1} H(t, \gamma(t)) d t
$$

while [EP1] and EP3] takes

$$
-\int w^{*} \omega+\int_{0}^{1} H(t, \gamma(t)) d t
$$

as its definition. We denote the definition (4.12) by $\widetilde{\mathcal{A}}_{H}([\gamma, w])$ for the purpose of comparison of the two below. 
Therefore under the change of $H$ by $-H$, one has the same set of $\operatorname{Crit}\left(\mathcal{A}_{H}\right)$ and $\operatorname{Crit}\left(\widetilde{\mathcal{A}}_{H}\right)$ with the same action integrals. Since both conventions use the same associated almost Kähler metric $\omega(\cdot, J \cdot)$, the associated perturbed Cauchy-Riemann equations are exactly the same.

In addition, Entov and Polterovich [EP1, EP2] use the notation $c(\mathfrak{a}, H)$ for the spectral numbers where $\mathfrak{a}$ is the quantum homology class. Our $\rho(H ; a)$ is nothing but

$$
\rho(H ; a)=c\left(a^{b} ; \widetilde{H}\right)=c\left(a^{b} ; \bar{H}\right)
$$

where $a^{b}$ is the homology class Poincaré dual to the cohomology class $a$ and $\widetilde{H}$ is the function defined by $\widetilde{H}(t, x)=-H(1-t, x)$ and $\bar{H}$ is the inverse Hamiltonian of $H$ given by

$$
\bar{H}(t, x)=-H\left(t, \phi_{H}^{t}(x)\right) .
$$

The second identity of (4.13) follows from the fact that $\widetilde{H} \sim \bar{H}$. More precisely, $\widetilde{H}$ generates the flow $\phi_{\widetilde{H}}: \phi_{H}^{1-t} \circ\left(\phi_{H}^{1}\right)^{-1}$ which can be deformed to $\phi_{\bar{H}}: t \mapsto\left(\phi_{H}^{t}\right)^{-1}$. In fact the following explicit formula provides such a deformation

$$
\phi_{s}^{t}= \begin{cases}\phi_{H}^{s-t} \circ\left(\phi_{H}^{s}\right)^{-1} & \text { for } 0 \leq t \leq s \\ \left(\phi_{H}^{t}\right)^{-1} & \text { for } s \leq t \leq 1\end{cases}
$$

for $0 \leq s \leq 1$. (See the proof of Oh5, Lemma 5.2] for this formula.)

With these understood, one can translate all the statements in [EP1, EP2] into the ones in terms of our notations. 


\section{Part 2. Bulk deformations of Hamiltonian Floer homology and spectral invariants}

In this part, we deform Hamiltonian Floer homology by the element $\mathfrak{b} \in H^{\text {even }}\left(M, \Lambda_{0}\right)$ in a way similar to the case of Lagrangian Floer theory in [FOOO1, Section 3.8]. We will denote the resulting $\mathfrak{b}$-deformation by $H F_{*}^{\mathfrak{b}}\left(H, J_{0} ; \Lambda\right)$. As a $\Lambda$-module, it is isomorphic to the singular homology $H_{*}(M ; \Lambda)$ for any $\mathfrak{b}$. Recall that we regard the de Rham complex as a chain complex (3.20).

Using the filtration we obtain a version of spectral invariants, the spectral invariants with bulk deformation, which contains various new information as we demonstrate later in Part 5.

\section{Big QUANTUm COHOMOlogy RING: REVIEW}

In this section, we exclusively denote by $J_{0}$ the time-independent almost complex structures.

The theory of spectral invariants explained in Part 1 is closely related to the (small) quantum cohomology. The spectral invariant with bulk we are going to construct is closely related to the big quantum cohomology, which we review in this section.

Let $(M, \omega)$ be a closed symplectic manifold and $J_{0}$ a compatible (time independent) almost complex structure. For $\alpha \in H_{2}(M ; \mathbb{Z})$ let $\mathcal{M}_{\ell}^{\mathrm{cl}}\left(\alpha ; J_{0}\right)$ be the moduli space of $J_{0}$-stable maps of genus zero, with $\ell$-marked points and of homology class $\alpha$. (See [FO, Definition 7.7] for example for the definition of this moduli space.) There exists an evaluation map

$$
\text { ev }: \mathcal{M}_{\ell}^{\mathrm{cl}}\left(\alpha ; J_{0}\right) \rightarrow M^{\ell} .
$$

The moduli space $\mathcal{M}_{\ell}^{\mathrm{cl}}\left(\alpha ; J_{0}\right)$ has a virtual fundamental cycle and hence defines a class

$$
\mathrm{ev}_{*}\left[\mathcal{M}_{\ell}^{\mathrm{cl}}\left(\alpha ; J_{0}\right)\right] \in H_{*}\left(M^{\ell} ; \mathbb{Q}\right)
$$

(See [FO, Theorem 1.3].) Here the sub-index $*$ in $H_{*}\left(M^{\ell} ; \mathbb{Q}\right)$ is given by $*=$ $2 n+2 c_{1}(M)(\alpha)+2 \ell-6$. Let $h_{1}, \ldots, h_{\ell}$ be closed differential forms on $M$ such that

$$
\sum \operatorname{deg} h_{i}=2 n+2 c_{1}(M)(\alpha)+2 \ell-6
$$

We define Gromov-Witten invariant by

$$
G W_{\ell}\left(\alpha: h_{1}, \ldots, h_{\ell}\right)=\int_{\mathcal{M}_{\ell}^{\mathrm{cl}}\left(\alpha ; J_{0}\right)} \operatorname{ev}^{*}\left(h_{1} \times \cdots \times h_{\ell}\right) \in \mathbb{R} .
$$

More precisely, we take a CF perturbation of the Kuranishi structure of $\mathcal{M}_{\ell}^{\mathrm{cl}}\left(\alpha ; J_{0}\right)$ and define the integration in (5.2) using it. (See (34.3) and FOOO11, Definition 10.22].) We can prove that (5.2) is independent of the almost complex structure $J_{0}$. We put $G W_{\ell}\left(\alpha: h_{1}, \ldots, h_{\ell}\right)=0$ unless (5.1) is satisfied. We now define

$$
G W_{\ell}\left(h_{1}, \ldots, h_{\ell}\right)=\sum_{\alpha} T^{\alpha \cap \omega} G W\left(\alpha: h_{1}, \ldots, h_{\ell}\right) \in \Lambda
$$

By Strokes' theorem (134.4), [FOOO11, Theorem 8.11]) we can prove that $G W_{\ell}\left(h_{1}, \ldots, h_{\ell}\right)$ depends only on the de Rham cohomology class of $h_{i}$ and is independent of the closed forms $h_{i}$ representing de Rham cohomology class. 
By extending the definition (5.3) linearly over to a $\Lambda$-module homomorphism, we obtain:

$$
G W_{\ell}: H(M ; \Lambda)^{\ell \otimes} \rightarrow \Lambda
$$

Definition 5.1. Let $\mathfrak{b} \in H^{\text {even }}\left(M ; \Lambda_{0}\right)$. For each given pair $\mathfrak{c}, \mathfrak{d} \in H^{*}(M ; \Lambda)$, we define a product $\mathfrak{c} \cup^{\mathfrak{b}} \mathfrak{d} \in H(M ; \Lambda)$ by the following formula

$$
\left\langle\mathfrak{c} \cup^{\mathfrak{b}} \mathfrak{d}, \mathfrak{e}\right\rangle_{\mathrm{PD}_{M}}=\sum_{\ell=0}^{\infty} \frac{1}{\ell !} G W_{\ell+3}(\mathfrak{c}, \mathfrak{d}, \mathfrak{e}, \mathfrak{b}, \ldots, \mathfrak{b}) .
$$

Here $\langle\cdot, \cdot\rangle_{\mathrm{PD}_{M}}$ denotes the Poincaré duality. We call $\cup^{\mathfrak{b}}$ the deformed quantum cup product.

Remark 5.2. We note that the right hand side of (5.4) is an infinite sum in general. If $\mathfrak{b} \in H^{\text {even }}\left(M ; \Lambda_{+}\right)$, it converges in $q$-adic topology so (5.4) makes sense. For a general element $\mathfrak{b} \in H^{\text {even }}\left(M ; \Lambda_{0}\right)$, we split

$$
\mathfrak{b}=\mathfrak{b}_{0}+\mathfrak{b}_{2}+\mathfrak{b}_{+}
$$

with $\mathfrak{b}_{0} \in H^{0}\left(M ; \Lambda_{0}\right), \mathfrak{b}_{2} \in H^{2}(M ; \mathbb{C})$, and $\mathfrak{b}_{+} \in H^{2}\left(M ; \Lambda_{+}\right) \oplus \bigoplus_{k \geq 2} H^{2 k}\left(M ; \Lambda_{0}\right)$ and define

$$
\left\langle\mathfrak{c} \cup^{\mathfrak{b}} \mathfrak{d}, \mathfrak{e}\right\rangle_{\mathrm{PD}_{M}}=\sum_{\ell=0}^{\infty} \sum_{\alpha} \frac{\exp \left(\mathfrak{b}_{2} \cap \alpha\right)}{\ell !} T^{\alpha \cap \omega} G W_{\ell+3}\left(\alpha: \mathfrak{c}, \mathfrak{d}, \mathfrak{e}, \mathfrak{b}_{+}, \ldots, \mathfrak{b}_{+}\right) .
$$

We can prove that (5.6) converges in $q$-adic topology. (This can be proved in the same way as in [FOOO4, Section 9]. See [FOOO7, Lemma 2.29].) We put the factor $\ell$ ! since our set of interior marked points is ordered.

Remark 5.3. Here the factor $T^{\alpha \cap \omega}$ is the usual weight we put in Gromov-Witten invariant and in Lagrangian Floer theory. The appearance of the exponential factor $\exp \left(\mathfrak{b}_{2} \cap \alpha\right)$ is related to the following formula

$$
G W_{\ell+3}\left(\alpha ; \mathfrak{c}, \mathfrak{d}, \mathfrak{e}, \mathfrak{b}_{2}, \ldots, \mathfrak{b}_{2}\right)=\frac{\left(\mathfrak{b}_{2} \cap \alpha\right)^{\ell}}{\ell !} G W_{3}(\alpha: \mathfrak{c}, \mathfrak{d}, \mathfrak{e})
$$

This formula is called the divisor axiom (See for example [FO, Theorem 23.1.4]). In fact if we formally 'expand' the formal sum

$$
\sum_{\ell=0}^{\infty} \sum_{\alpha} \frac{1}{\ell !} T^{\alpha \cap \omega} G W_{\ell+3}(\alpha: \mathfrak{c}, \mathfrak{o}, \mathfrak{e}, \mathfrak{b}, \ldots, \mathfrak{b})
$$

by substituting $\mathfrak{b}=\mathfrak{b}_{2}+\mathfrak{b}_{+}$, a formal calculation based on the divisor axiom gives rise to (5.6). However this sum as it is does not make sense since it does not converge in the $q$-adic topology.

Geometrically considering the element $\mathfrak{b} \in H^{2}\left(M ; \Lambda_{0}\right)$ corresponds to twisting the Hamiltonian Floer theory by a $B$-field and is the analog to Cho's trick of considering nonunitary line bundles [Fu1, Cho. (We remark that this $q$-adic convergence of Gromov-Witten invariant had been known for a long time.)

It is now well-established that the product $\cup^{\mathfrak{b}}$ is associative and graded commutative and is independent of $J_{0}$. We thus obtain a $\mathbb{Z}_{2}$-graded commutative ring

$$
Q H_{\mathfrak{b}}^{*}(M)=\left(H^{*}(M ; \Lambda), \cup^{\mathfrak{b}}\right) \cong\left(H^{*}(M) \otimes \Lambda, \cup^{\mathfrak{b}}\right)
$$


As we will see later, for the purpose of construction of spectral invariants and of partial symplectic quasi-states and quasi-morphisms, it is important to use a smaller Novikov ring than $\Lambda$. We discuss this point now.

Definition 5.4. Let $G$ be a discrete submonoid of $\mathbb{R}$. We say an element $\mathfrak{b} \in$ $H\left(M ; \Lambda_{0}\right)$ to be $G$-gapped if $\mathfrak{b}$ can be written as

$$
\mathfrak{b}=\sum_{g \in G} T^{g} b_{g}, \quad b_{g} \in H(M ; \mathbb{C}) .
$$

For each $\mathfrak{b} \in H\left(M ; \Lambda_{0}\right)$ there exists a smallest discrete submonoid $G$ such that $\mathfrak{b}$ is $G$-gapped. We write this monoid as $G_{0}(\mathfrak{b})$. Let $G_{0}(M, \omega)$ be the monoid generated by the set

$$
\left\{\alpha \cap \omega \mid \mathcal{M}_{\ell}^{\mathrm{cl}}\left(\alpha ; J_{0}\right) \neq \emptyset\right\} .
$$

Then $G_{0}(M, \omega)$ is discrete by the Gromov compactness. Let $G_{0}(M, \omega, \mathfrak{b})$ be the discrete monoid generated by $G_{0}(M, \omega) \cup G_{0}(\mathfrak{b})$. We define

$$
\Lambda_{0}(M, \omega, \mathfrak{b})=\left\{\sum a_{i} T^{\lambda_{i}} \in \Lambda_{0} \mid \lambda_{i} \in G_{0}(M, \omega, \mathfrak{b})\right\} .
$$

The following is easy to check.

Lemma 5.5. The bilinear map $\cup^{\mathfrak{b}}$ induces a ring structure on $H^{*}\left(M ; \Lambda_{0}(M, \omega, \mathfrak{b})\right)$.

We have thus obtained the associated quantum cohomology ring

$$
Q H_{\mathfrak{b}}^{*}\left(M ; \Lambda_{0}(M, \omega, \mathfrak{b})\right)=\left(H^{*}\left(M ; \Lambda_{0}(M, \omega, \mathfrak{b})\right), \cup^{\mathfrak{b}}\right) .
$$

Remark 5.6. (1) Via the identification $q=T^{-1}$, we will use

$$
\Lambda_{0}^{\downarrow}(M, \omega, \mathfrak{b})=\left\{\sum_{i} a_{i} q^{-\lambda_{i}} \in \Lambda \mid \lambda_{i} \in G_{0}(M, \omega ; \mathfrak{b}), \lambda_{i} \rightarrow \infty\right\}
$$

in place of $\Lambda_{0}(M, \omega, \mathfrak{b})$ in (5.8) in the construction of Floer homology as before.

(2) Entov-Polterovich [EP1, EP2, EP3] use quantum homology, where the degree is shifted by $2 n$ from the usual degree. The isomorphism in Theorem 3.12 then preserves the degree when we use the Conley-Zehnder index as the degree of Floer homology.

Here we use the usual degree convention of quantum cohomology but shift the degree of Floer homology by $n$ from the Conley-Zehnder index.

In this convention, the (quantum) cup product is $\left(\mathbb{Z}_{2}\right)$-degree preserving. In 'quantum homology', the product of degree $d_{1}$ and $d_{2}$ classes has degree $d_{1}+d_{2}-2 n$. We prefer to choose our convention so that the quantum product becomes degree-preserving as in the usual grading convention in the quantum cohomology ring.

\section{Hamiltonian Floer homology with BUlK DeFormations}

In this section we modify the construction of Section 3 and incorporate bulk deformations.

Let $[\gamma, w],\left[\gamma^{\prime}, w^{\prime}\right] \in \operatorname{Crit}\left(\mathcal{A}_{H}\right)$. Below we will need to consider the moduli space of marked Floer trajectories $\mathcal{M}_{\ell}\left(H, J ;[\gamma, w],\left[\gamma^{\prime}, w^{\prime}\right]\right)$ for each $\ell=0,1, \ldots$ The moduli space $\mathcal{M}_{0}\left(H, J ;[\gamma, w],\left[\gamma^{\prime}, w^{\prime}\right]\right)$ coincides with $\mathcal{M}\left(H, J ;[\gamma, w],\left[\gamma^{\prime}, w^{\prime}\right]\right)$ which is defined in Definition 3.4 and Proposition 3.6 
Definition 6.1. We denote by $\widehat{\mathcal{M}}_{\ell}\left(H, J ;[\gamma, w],\left[\gamma^{\prime}, w^{\prime}\right]\right)$ the set of all $\left(u ; z_{1}^{+}, \ldots, z_{\ell}^{+}\right)$, where $u$ is a map $u: \mathbb{R} \times S^{1} \rightarrow M$ which satisfies Conditions (1) (4) of Definition 3.4 and $z_{i}^{+}(i=1, \ldots, \ell)$ are mutually distinct points of $\mathbb{R} \times S^{1}$. It carries an $\mathbb{R}$-action by translations in $\tau$-direction. We denote its quotient space by $\stackrel{\circ}{\mathcal{M}}_{\ell}\left(H, J ;[\gamma, w],\left[\gamma^{\prime}, w^{\prime}\right]\right)$. We define the evaluation map

$$
\mathrm{ev}=\left(\mathrm{ev}_{1} \ldots, \mathrm{ev}_{\ell}\right): \stackrel{\circ}{\mathcal{M}}_{\ell}\left(H, J ;[\gamma, w],\left[\gamma^{\prime}, w^{\prime}\right]\right) \rightarrow M^{\ell}
$$

by

$$
\operatorname{ev}\left(u ; z_{1}^{+}, \ldots, z_{\ell}^{+}\right)=\left(u\left(z_{1}^{+}\right), \ldots, u\left(z_{\ell}^{+}\right)\right)
$$

We use the following notation in the next proposition. Denote the set of shuffles of $\ell$ elements by

$$
\operatorname{Shuff}(\ell)=\left\{\left(\mathbb{L}_{1}, \mathbb{L}_{2}\right) \mid \mathbb{L}_{1} \cup \mathbb{L}_{2}=\{1, \ldots, \ell\}, \mathbb{L}_{1} \cap \mathbb{L}_{2}=\emptyset\right\} .
$$

For $\left(\mathbb{L}_{1}, \mathbb{L}_{2}\right) \in \operatorname{Shuff}(\ell)$ let $\# \mathbb{L}_{i}$ be the order of this subset. Then $\# \mathbb{L}_{1}+\# \mathbb{L}_{2}=\ell$.

For any $\alpha \in \pi_{2}(M)$ there exists a canonical homeomorphism

$$
\stackrel{\circ}{\mathcal{M}}_{\ell}\left(H, J ;[\gamma, w],\left[\gamma^{\prime}, w^{\prime}\right]\right) \cong \stackrel{\circ}{\mathcal{M}}_{\ell}\left(H, J ;[\gamma, \alpha \# w],\left[\gamma^{\prime}, \alpha \# w^{\prime}\right]\right)
$$

Proposition 6.2. (1) The moduli space $\stackrel{\circ}{\mathcal{M}}_{\ell}\left(H, J ;[\gamma, w],\left[\gamma^{\prime}, w^{\prime}\right]\right)$ has a compactification $\mathcal{M}_{\ell}\left(H, J ;[\gamma, w],\left[\gamma^{\prime}, w^{\prime}\right]\right)$ that is Hausdorff.

(2) The space $\mathcal{M}_{\ell}\left(H, J ;[\gamma, w],\left[\gamma^{\prime}, w^{\prime}\right]\right)$ has an orientable Kuranishi structure with corners.

(3) The normalized boundary of $\mathcal{M}_{\ell}\left(H, J ;[\gamma, w],\left[\gamma^{\prime}, w^{\prime}\right]\right)$ is described by

$$
\begin{aligned}
& \partial \mathcal{M}_{\ell}\left(H, J ;[\gamma, w],\left[\gamma^{\prime}, w^{\prime}\right]\right)= \\
& \bigcup \mathcal{M}_{\# \mathbb{L}_{1}}\left(H, J ;[\gamma, w],\left[\gamma^{\prime \prime}, w^{\prime \prime}\right]\right) \times \mathcal{M}_{\# \mathbb{L}_{2}}\left(H, J ;\left[\gamma^{\prime \prime}, w^{\prime \prime}\right],\left[\gamma^{\prime}, w^{\prime}\right]\right),
\end{aligned}
$$

where the union is taken over all $\left[\gamma^{\prime \prime}, w^{\prime \prime}\right] \in \operatorname{Crit}\left(\mathcal{A}_{H}\right)$, and $\left(\mathbb{L}_{1}, \mathbb{L}_{2}\right) \in$ $\operatorname{Shuff}(\ell)$.

(4) Let $\mu_{H}: \operatorname{Crit}\left(\mathcal{A}_{H}\right) \rightarrow \mathbb{Z}$ be the Conley-Zehnder index. Then the (virtual) dimension satisfies the following equality (6.4).

$\operatorname{dim} \mathcal{M}_{\ell}\left(H, J ;[\gamma, w],\left[\gamma^{\prime}, w^{\prime}\right]\right)=\mu_{H}\left(\left[\gamma^{\prime}, w^{\prime}\right]\right)-\mu_{H}([\gamma, w])-1+2 \ell$.

(5) We can define orientations of $\mathcal{M}_{\ell}\left(H, J ;[\gamma, w],\left[\gamma^{\prime}, w^{\prime}\right]\right)$ so that (3) above is compatible with this orientation.

(6) The evaluation map ev extends to a map $\mathcal{M}_{\ell}\left(H, J ;[\gamma, w],\left[\gamma^{\prime}, w^{\prime}\right]\right) \rightarrow M^{\ell}$, which we denote also by ev. It is a strongly continuous and weakly submersive map in the sense of [FOOO11, Definition 4.6], [FOOO2, Definition A.1.13] (see also Definition 32.1) which is compatible with (3). Namely if we denote

$$
E_{1}=\left\{i_{1}, \ldots, i_{\# E_{1}}\right\}, E_{2}=\left\{j_{1}, \ldots, j_{\# E_{2}}\right\}
$$

with $i_{1}<\cdots<i_{\# E_{1}}, j_{1}<\cdots<j_{\# E_{2}}$, then $\mathrm{ev}_{k}$ of the first factor (resp. the second factor) of the right hand side of (6.3) coincides with ev $_{i_{k}}$ (resp. $\mathrm{ev}_{j_{k}}$ ) of the left hand side of (6.3).

(7) The homeomorphism (6.2) extends to the compactifications and their $K u$ ranishi structures are identified by the homeomorphism. 
The proof of Proposition 6.2 is the same as that of Propositions 3.6 and 3.17 The detail of the proof of [FOOO10, Theorem 29.4] given in [FOOO10, Section 31] proves Proposition 6.2 also. An element of (6.3) is drawn in Figure 3 below. We

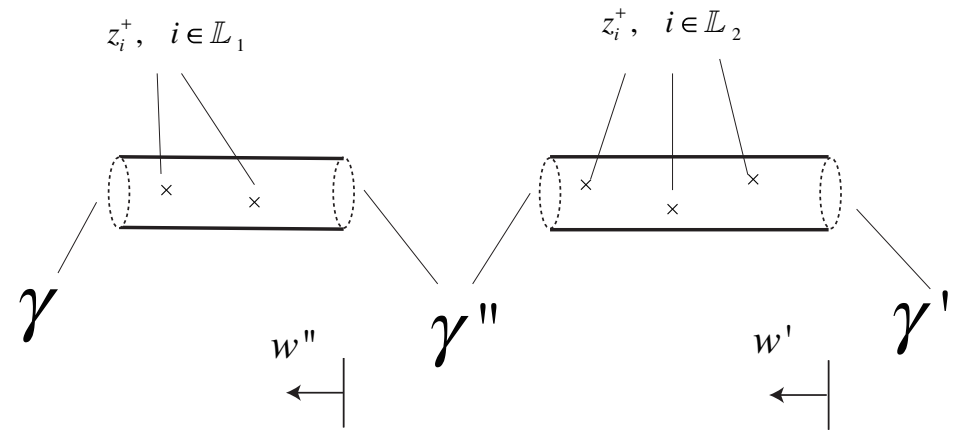

Figure 3. An element of (6.3)

are ready to define the deformed boundary map $\partial_{(H, J)}^{\mathfrak{b}}$. We start with defining the following operator:

Definition 6.3. Let $[\gamma, w],\left[\gamma^{\prime}, w^{\prime}\right] \in \operatorname{Crit}\left(\mathcal{A}_{H}\right)$ and $h_{i}(i=1, \ldots, \ell)$ be differential forms on $M$. We define $\mathfrak{n}_{(H, J) ; \ell}\left([\gamma, w],\left[\gamma^{\prime}, w^{\prime}\right]\right)\left(h_{1}, \ldots, h_{\ell}\right) \in \mathbb{C}$ by

$$
\mathfrak{n}_{(H, J) ; \ell}\left([\gamma, w],\left[\gamma^{\prime}, w^{\prime}\right]\right)\left(h_{1}, \ldots, h_{\ell}\right)=\int_{\mathcal{M}_{\ell}\left(H, J ;[\gamma, w],\left[\gamma^{\prime}, w^{\prime}\right]\right)} \operatorname{ev}_{1}^{*} h_{1} \wedge \cdots \wedge \mathrm{ev}_{\ell}^{*} h_{\ell} .
$$

By definition (6.5) is zero unless

$$
\sum_{i=1}^{\ell} \operatorname{deg} h_{i} \neq \operatorname{dim} \mathcal{M}_{\ell}\left(H, J ;[\gamma, w],\left[\gamma^{\prime}, w^{\prime}\right]\right),
$$

where the right hand side is as in (6.4).

Remark 6.4. In order to define the integration in (6.5) using Definition 33.20 we need to take a CF-perturbation of $\mathcal{M}_{\ell}\left(H, J ;[\gamma, w],\left[\gamma^{\prime}, w^{\prime}\right]\right)$ that is transversal to 0 . In our situation the integration (6.5) depends on the choice of a CF-perturbation as well as the small parameter $\epsilon>0$ since $\mathcal{M}_{\ell}\left(H, J ;[\gamma, w],\left[\gamma^{\prime}, w^{\prime}\right]\right)$ has codimension one boundary. We take a system of CF-perturbations of $\mathcal{M}_{\ell}\left(H, J ;[\gamma, w],\left[\gamma^{\prime}, w^{\prime}\right]\right)$ so that the system is compatible with the decomposition (6.3) of the normalized boundary $\partial \mathcal{M}_{\ell}\left(H, J ;[\gamma, w],\left[\gamma^{\prime}, w^{\prime}\right]\right)$. We take our CF-perturbations so that it is consistent with the isomorphism in Proposition 6.2 (7). 3 We also fix a sufficiently small constant $\epsilon>0$.

We linearly extend the definition of $\mathfrak{n}_{(H, J) ; \ell}\left([\gamma, w],\left[\gamma^{\prime}, w^{\prime}\right]\right)$ to a $\Lambda$-multilinear map and compose it with a canonical isomorphism $\Lambda \cong \Lambda^{\downarrow}$. We denote the composition $(\Omega(M) \widehat{\otimes} \Lambda)^{\ell} \rightarrow \Lambda^{\downarrow}$ by the same symbol $\mathfrak{n}_{(H, J) ; \ell}\left([\gamma, w],\left[\gamma^{\prime}, w^{\prime}\right]\right)$.

Let $\mathfrak{b} \in H^{\text {even }}\left(M ; \Lambda_{0}\right)$ and split $\mathfrak{b}=\mathfrak{b}_{0}+\mathfrak{b}_{2}+\mathfrak{b}_{+}$as in (5.5). We take closed forms that represent the cohomology classes $\mathfrak{b}_{0}, \mathfrak{b}_{2}, \mathfrak{b}_{+}$respectively and denote them by

${ }^{3}$ From now on we do not repeat this kind of remarks in the similar situations. 
the same symbols. We then define $\mathfrak{n}_{(H, J) ; \ell}^{\mathfrak{b}}\left([\gamma, w],\left[\gamma^{\prime}, w^{\prime}\right]\right) \in \Lambda^{\downarrow}$ for $[\gamma, w],\left[\gamma^{\prime}, w^{\prime}\right] \in$ $\operatorname{Crit}\left(\mathcal{A}_{H}\right)$ by

$$
\begin{aligned}
& \mathfrak{n}_{(H, J)}^{\mathfrak{b}}\left([\gamma, w],\left[\gamma^{\prime}, w^{\prime}\right]\right) \\
& =\sum_{\ell=0}^{\infty} \frac{\exp \left(\int\left(w^{\prime}\right)^{*} \mathfrak{b}_{2}-\int w^{*} \mathfrak{b}_{2}\right)}{\ell !} \mathfrak{n}_{(H, J) ; \ell}\left([\gamma, w],\left[\gamma^{\prime}, w^{\prime}\right]\right)(\underbrace{\mathfrak{b}_{+}, \ldots, \mathfrak{b}_{+}}_{\ell}) .
\end{aligned}
$$

Let $\llbracket \gamma, w \rrbracket, \llbracket \gamma^{\prime}, w^{\prime} \rrbracket \in \widehat{\operatorname{Per}}(H)$. Using (6.6) we define $\mathfrak{n}_{(H, J) ; \ell}^{\mathfrak{b}}\left(\llbracket \gamma, w \rrbracket, \llbracket \gamma^{\prime}, w^{\prime} \rrbracket\right) \in \Lambda^{\downarrow}$ as follows. Suppose $\llbracket \gamma, w \rrbracket$ is represented by $[\gamma, w] \in \operatorname{Crit}\left(\mathcal{A}_{H}\right)$, i.e., $\pi([\gamma, w])=\llbracket \gamma, w \rrbracket$. We then put:

$$
\mathfrak{n}_{(H, J)}^{\mathfrak{b}}\left(\llbracket \gamma, w \rrbracket, \llbracket \gamma^{\prime}, w^{\prime} \rrbracket\right)=\sum_{\substack{\left[\gamma^{\prime}, w^{\prime}\right] \in \operatorname{Crit}\left(\mathcal{A}_{H}\right) \\ \pi\left(\left[\gamma^{\prime}, w^{\prime}\right]\right)=\llbracket \gamma^{\prime}, w^{\prime} \rrbracket}} \mathfrak{n}_{(H, J)}^{\mathfrak{b}}\left([\gamma, w],\left[\gamma^{\prime}, w^{\prime}\right]\right) .
$$

Using Propositoin 6.2 (7) we can show that the right hand side of (6.7) is independent of $[\gamma, w]$ but depends only on $\llbracket \gamma, w \rrbracket$ in the same way as Remark 3.8

Lemma 6.5. When $\operatorname{deg} \mathfrak{b}_{+}>2$, the right hand sides of (6.6) and (6.7) are a finite sum. On the other hand, when $\operatorname{deg} \mathfrak{b}_{+}=2$, the right hand side of (6.6) and (6.7) converge in the q-adic topology.

Proof. Consider the case that $\operatorname{deg} \mathfrak{b}_{+}>2$. Suppose $\mathcal{M}_{\ell}\left(H, J ;[\gamma, w],\left[\gamma^{\prime}, w^{\prime}\right]\right) \neq$ $\emptyset$ and so $\mathcal{M}\left(H, J ;[\gamma, w],\left[\gamma^{\prime}, w^{\prime}\right]\right) \neq \emptyset$. The summand corresponding to $\ell$ in the right hand side of (6.6) vanishes unless $\ell \operatorname{deg} \mathfrak{b}_{+}=\operatorname{dim} \mathcal{M}_{\ell}\left(H, J ;[\gamma, w],\left[\gamma^{\prime}, w^{\prime}\right]\right)=$ $\mu_{H}\left(\left[\gamma^{\prime}, w^{\prime}\right]\right)-\mu_{H}([\gamma, w])+2 \ell-1$, i.e.,

$$
\ell\left(\operatorname{deg} \mathfrak{b}_{+}-2\right)=\mu_{H}\left(\left[\gamma^{\prime}, w^{\prime}\right]\right)-\mu_{H}([\gamma, w])-1 .
$$

Since the right hand side depends only on $[\gamma, w],\left[\gamma^{\prime}, w^{\prime}\right]$, it implies boundedness of the number of possible choices of $\ell$. This finishes the proof of the first statement.

For the second statement, we note that $\mathfrak{v}_{q}\left(\mathfrak{b}_{+}\right)=-\mathfrak{v}_{T}\left(\mathfrak{b}_{+}\right)<0$. Hence the value of $\mathfrak{v}_{q}$ of the $\ell$-th term in the right hand of (6.6) diverges to $-\infty$ as $\ell$ tends to $\infty$. Hence the proof.

Definition 6.6. We define a deformed Floer boundary map

$$
\partial_{(H, J)}^{\mathfrak{b}}: C F_{*}\left(M, H ; \Lambda^{\downarrow}\right) \rightarrow C F_{*}\left(M, H ; \Lambda^{\downarrow}\right)
$$

by

$$
\partial_{(H, J)}^{\mathfrak{b}}(\llbracket \gamma, w \rrbracket)=\sum_{\llbracket \gamma^{\prime}, w^{\prime} \rrbracket} \mathfrak{n}_{(H, J)}^{\mathfrak{b}}\left(\llbracket \gamma, w \rrbracket, \llbracket \gamma^{\prime}, w^{\prime} \rrbracket\right) \llbracket \gamma^{\prime}, w^{\prime} \rrbracket .
$$

We point out that the sum in (6.8) may not be a finite sum.

Lemma 6.7. The right hand side of (6.8) converges in $C F\left(M, H ; \Lambda^{\downarrow}\right)$ and $\partial_{(J, H)}^{\mathfrak{b}}$ is continuous in q-adic topology.

Proof. Let $E$ be any real number and $\left[\gamma^{\prime}, w^{\prime}\right] \in \operatorname{Crit} \mathcal{A}_{H}$. By Gromov-Floer compactness, the number of $\left[\gamma^{\prime}, w^{\prime}\right]$ such that $\mathcal{M}_{\ell}\left(H, J ;[\gamma, w],\left[\gamma^{\prime}, w^{\prime}\right]\right)$ is nonempty and

$$
\mathcal{A}_{H}([\gamma, w])-\mathcal{A}_{H}\left(\left[\gamma^{\prime}, w^{\prime}\right]\right)<E
$$

is finite. The lemma now follows from the definition of convergence in $C F\left(M, H ; \Lambda^{\downarrow}\right)$. 
Combining Proposition 6.2 (3) with Stokes' theorem [FOOO3, Lemma C.9] and composition formula [FOOO3, Lemma C.10], we can check

$$
\partial_{(H, J)}^{\mathfrak{b}} \circ \partial_{(H, J)}^{\mathfrak{b}}=0 .
$$

Definition 6.8. We define Floer homology of periodic Hamiltonian system with bulk deformation by

$$
H F_{*}^{\mathfrak{b}}\left(M, H, J ; \Lambda^{\downarrow}\right)=\frac{\operatorname{Ker} \partial_{(H, J)}^{\mathfrak{b}}}{\operatorname{Im} \partial_{(H, J)}^{\mathfrak{b}}} .
$$

Now we take two parameter family $\left\{\left(H_{\chi}, J_{\chi}\right)\right\}_{\tau \in \mathbb{R}}$ as in (3.13) in the proof of Theorem 3.12 .

Theorem 6.9. There exists a $\Lambda^{\downarrow}$-module isomorphism

$$
\mathcal{P}_{\left(H_{\chi}, J_{\chi}\right), *}^{\mathfrak{b}}: H_{*}\left(M ; \Lambda^{\downarrow}\right) \cong H F_{*}^{\mathfrak{b}}\left(M, H, J ; \Lambda^{\downarrow}\right)
$$

for all $\mathfrak{b}$. We call it the Piunikhin map with bulk.

Proof. The proof, which we discuss below, is similar to the proof of Theorem 3.12 We recall that we identify the de Rham complex with a chain complex

$$
\left(\Omega_{*}(M) \widehat{\otimes} \Lambda^{\downarrow}, \partial\right), \quad \Omega_{*}(M):=\Omega^{2 n-*}(M), \quad \partial=(-1)^{\operatorname{deg}+1} d .
$$

We denote by the map $b:\left(\Omega^{*}(M), \Lambda\right) \rightarrow\left(\Omega_{*}(M), \Lambda^{\downarrow}\right)$ this isomorphism between the two. In this section we only give the definition of the map $\mathcal{P}_{\left(H_{\chi}, J_{\chi}\right), *}^{\mathfrak{b}}$. In Section 26. we prove that it is indeed an isomorphism.

Definition 6.10. We denote by $\stackrel{\circ}{\mathcal{M}}_{\ell}\left(H_{\chi}, J_{\chi} ; *,[\gamma, w]\right)$ the set of all $\left(u ; z_{1}^{+}, \ldots, z_{\ell}^{+}\right)$ of maps $u: \mathbb{R} \times S^{1} \rightarrow M$ and $z_{i}^{+}, i=1, \ldots, \ell$ such that $u$ satisfies (1)-(4) of Definition 3.15 and $z_{i}^{+} \in \mathbb{R} \times S^{1}$ are mutually distinct.

The assignment $\left(u ; z_{1}^{+}, \ldots, z_{\ell}^{+}\right) \mapsto\left(u\left(z_{1}^{+}\right), \ldots, u\left(z_{\ell}^{+}\right)\right)$defines an evaluation map

$$
\mathrm{ev}=\left(\mathrm{ev}_{1} \ldots, \mathrm{ev}_{\ell}\right)=\stackrel{\circ}{\mathcal{M}}_{\ell}\left(H_{\chi}, J_{\chi} ; *,[\gamma, w]\right) \rightarrow M^{\ell} .
$$

Proposition 6.11. (1) $\stackrel{\circ}{\mathcal{M}}_{\ell}\left(H_{\chi}, J_{\chi} ; *,[\gamma, w]\right)$ has a compactification, denoted by $\mathcal{M}_{\ell}\left(H_{\chi}, J_{\chi} ; *,[\gamma, w]\right)$, that is Hausdorff.

(2) The space $\mathcal{M}_{\ell}\left(H_{\chi}, J_{\chi} ; *,[\gamma, w]\right)$ has an orientable Kuranishi structure with corners.

(3) The normalized boundary of $\mathcal{M}_{\ell}\left(H_{\chi}, J_{\chi} ; *,[\gamma, w]\right)$ is described by

$$
\begin{aligned}
& \partial \mathcal{M}_{\ell}\left(H_{\chi}, J_{\chi} ; *,[\gamma, w]\right) \\
& =\bigcup \mathcal{M}_{\# \mathbb{L}_{1}}\left(H_{\chi}, J_{\chi} ; *,\left[\gamma^{\prime}, w^{\prime}\right]\right) \times \mathcal{M}_{\# \mathbb{L}_{2}}\left(H, J ;\left[\gamma^{\prime}, w^{\prime}\right],[\gamma, w]\right)
\end{aligned}
$$

where the union is taken over all $\left[\gamma^{\prime}, w^{\prime}\right] \in \operatorname{Crit}\left(\mathcal{A}_{H}\right)$, and $\left(\mathbb{L}_{1}, \mathbb{L}_{2}\right) \in$ $\operatorname{Shuff}(\ell)$.

(4) Let $\mu_{H}: \operatorname{Crit}\left(\mathcal{A}_{H}\right) \rightarrow \mathbb{Z}$ be the Conley-Zehnder index. Then the (virtual) dimension satisfies the following equality:

$$
\operatorname{dim} \mathcal{M}_{\ell}\left(H_{\chi}, J_{\chi} ; *,[\gamma, w]\right)=\mu_{H}([\gamma, w])+n+2 \ell .
$$

(5) We can define orientations of $\mathcal{M}_{\ell}\left(H_{\chi}, J_{\chi} ; *,[\gamma, w]\right)$ so that (3) above is compatible with this orientation. 
(6) The map ev extends to a strongly smooth map $\mathcal{M}_{\ell}\left(H_{\chi}, J_{\chi} ; *,[\gamma, w]\right) \rightarrow M^{\ell}$, which we denote also by ev. It is compatible with (3) in the same sense as Proposition 6.2 (6).

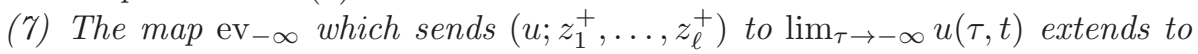
a strongly continuous smooth map $\mathcal{M}_{\ell}\left(H_{\chi}, J_{\chi} ; *,[\gamma, w]\right) \rightarrow M$, which we denote also by $\mathrm{ev}_{-\infty}$. It is compatible with (3).

Proof. The proof of Proposition 6.11 is mostly the same as that of Proposition 3.6 (See also [FOOO10, Parts 4 and 5].) We only need to see that in (6.9) the boundary component such as

$$
\mathcal{M}_{\# \mathrm{E}_{1}}\left(0, J_{0} ; *, * ; C\right) \times \mathcal{M}_{\# \mathrm{E}_{2}}\left(H_{\chi}, J_{\chi} ; *,[\gamma,(-C) \# w]\right)
$$

does not appear. (Here the first factor of (6.11) is a compactified moduli space of the $J_{0}$-holomorphic maps $\mathbb{R} \times S^{1} \rightarrow M$ with finite energy and of homotopy class $C \in \pi_{2}(M)$. The way of our usage of $*$ for the arguments used in the notation of the moduli spaces is the same as in Remark 3.16. In particular the second $*$ of the first factor of (6.11) highlights the fact that the limit at $+\infty$ of an element in the moduli space converges to an unspecified point of $M$.)

In fact, the moduli space $\mathcal{M}_{\# \mathrm{E}_{1}}\left(0, J_{0} ; *, * ; C\right)$ has an extra $S^{1}$ symmetry by the $S^{1}$ action of the domain $\mathbb{R} \times S^{1}$. (See Remark 3.14.) So after taking a quotient by this $S^{1}$ action, this component is of codimension 2. (See the proof of Lemma 26.9.)

Let $[\gamma, w] \in \operatorname{Crit}\left(\mathcal{A}_{H}\right)$ and $h_{i}(i=1, \ldots, \ell), h$ be differential forms on $M$. We take a system of CF-perturbations on $\mathcal{M}_{\ell}\left(H_{\chi}, J_{\chi} ; *,[\gamma, w]\right)$ such that it is compatible with (3). We use the system to define (6.12) below. See Remark 6.4. We define $\mathfrak{n}_{\left(H_{\chi}, J_{\chi}\right)}(h ;[\gamma, w])\left(h_{1}, \ldots, h_{\ell}\right) \in \mathbb{C}$ by

$$
\begin{aligned}
& \mathfrak{n}_{\left(H_{\chi}, J_{\chi}\right)}(h ;[\gamma, w])\left(h_{1}, \ldots, h_{\ell}\right) \\
& =\int_{\mathcal{M}_{\ell}\left(H_{\chi}, J_{\chi} ; *,[\gamma, w]\right)} \mathrm{ev}_{-\infty}^{*} h \wedge \mathrm{ev}_{1}^{*} h_{1} \wedge \cdots \wedge \mathrm{ev}_{\ell}^{*} h_{\ell} .
\end{aligned}
$$

We note that (6.12) is zero by definition unless the dimensional restriction

$$
\operatorname{deg} h+\sum_{i=1}^{\ell} \operatorname{deg} h_{i}=\operatorname{dim} \mathcal{M}_{\ell}\left(H_{\chi}, J_{\chi} ; *,[\gamma, w]\right)
$$

holds. We extend $\mathfrak{n}_{\left(H_{\chi}, J_{\chi}\right)}(h ;[\gamma, w])$ to a $\Lambda$-multilinear map $(\Omega(M) \widehat{\otimes} \Lambda) \rightarrow \Lambda$ and identify $\Lambda=\Lambda^{\downarrow}$ by $q=T^{-1}$. We denote it by the same symbol.

Let $\mathfrak{b} \in H^{\text {even }}\left(M ; \Lambda_{0}\right)$. We decompose $\mathfrak{b}=\mathfrak{b}_{0}+\mathfrak{b}_{2}+\mathfrak{b}_{+}$as in (5.5) and regard $\mathfrak{b}_{0}, \mathfrak{b}_{2}, \mathfrak{b}_{+}$as de Rham (co)homology classes by representing them by the closed differential forms. We define an element $\mathfrak{n}_{\left(H_{\chi}, J_{\chi}\right)}^{\mathfrak{b}}(h ;[\gamma, w]) \in \Lambda^{\downarrow}$ by :

$$
\mathfrak{n}_{\left(H_{\chi}, J_{\chi}\right)}^{\mathfrak{b}}(h ;[\gamma, w]):=\sum_{\ell=0}^{\infty} \frac{\exp \left(\int w^{*} \mathfrak{b}_{2}\right)}{\ell !} \mathfrak{n}_{\left(H_{\chi}, J_{\chi}\right), \ell}([\gamma, w])(h ; \underbrace{\mathfrak{b}_{+}, \ldots, \mathfrak{b}_{+}}_{\ell})
$$

for each given $[\gamma, w] \in \operatorname{Crit}\left(\mathcal{A}_{H}\right)$ and a differential form $h$ on $M$. We can prove that the sum in (6.13) converges in $q$-adic topology, in the same way as in Lemma 6.5. 
We now define

$$
\mathcal{P}_{\left(H_{\chi}, J_{\chi}\right)}^{\mathfrak{b}}(h):=\sum_{[\gamma, w] \in \operatorname{Crit}\left(\mathcal{A}_{H}\right)} \mathfrak{n}_{\left(H_{\chi}, J_{\chi}\right)}^{\mathfrak{b}}(h ;[\gamma, w]) \llbracket \gamma, w \rrbracket .
$$

We can prove that the right hand side is an element of $C F\left(H, J ; \Lambda^{\downarrow}\right)$ in the same way as in Lemma 6.7 Thus we have defined

$$
\mathcal{P}_{\left(H_{\chi}, J_{\chi}\right)}^{\mathfrak{b}}: \Omega_{*}(M) \widehat{\otimes} \Lambda^{\downarrow} \rightarrow C F_{*}\left(M, H ; \Lambda^{\downarrow}\right) .
$$

Then the identity

$$
\mathcal{P}_{\left(H_{\chi}, J_{\chi}\right)}^{\mathfrak{b}} \circ \partial=\partial_{(H, J)}^{\mathfrak{b}} \circ \mathcal{P}_{\left(H_{\chi}, J_{\chi}\right)}^{\mathfrak{b}}
$$

is a consequence of (6.9), Stokes' theorem (Theorem 34.8) and composition formula (Theorem 35.7). We can easily prove that $\mathcal{P}_{\left(H_{\chi}, J_{\chi}\right)}^{\mathfrak{b}}$ are chain homotopic to one another when $\chi$ is varied in $\mathcal{K}$. We denote by

$$
\mathcal{P}_{\left(H_{\chi}, J_{\chi}\right), *}^{\mathfrak{b}}: H_{*}\left(M ; \Lambda^{\downarrow}\right) \rightarrow H F_{*}^{\mathfrak{b}}\left(M, H, J ; \Lambda^{\downarrow}\right)
$$

the map induced on homology. We will prove in Section 26 that it is an isomorphism.

\section{Spectral inVariants With BULK DEFORMation}

We next modify the argument given in Section 4 and define spectral invariants with bulk. Let $\mathfrak{b} \in H^{\text {even }}\left(M ; \Lambda_{0}\right)$. We consider discrete submonoids $G_{0}(M, \omega)$ and $G_{0}(M, \omega, \mathfrak{b})$ of $\mathbb{R}$ in Definition 5.4.

Definition 7.1. We denote by $G(M, \omega)$ and $G(M, \omega, \mathfrak{b})$ the subgroup of $(\mathbb{R},+)$ generated by the monoids $G_{0}(M, \omega)$ and $G_{0}(M, \omega, \mathfrak{b})$, respectively.

We note that $G(M, \omega)$ and $G(M, \omega, \mathfrak{b})$ are not necessarily discrete. We also remark that $G(M, \omega, \mathfrak{b})$ may not even be finitely generated.

Let $H$ be a time-dependent Hamiltonian on $M$. We defined $\operatorname{Spec}(H)$ in Definition 4.1 .

Definition 7.2. We define

$$
\begin{aligned}
\operatorname{Spec}(H ; \mathfrak{b}) & =\operatorname{Spec}(H)+G(M, \omega, \mathfrak{b}) \\
& =\{\lambda+g \mid \lambda \in \operatorname{Spec}(H), g \in G(M, \omega, \mathfrak{b})\} .
\end{aligned}
$$

For a monoid $G \subset \mathbb{R}$, the ring $\Lambda^{\downarrow}(G)$ was defined in Definition 4.9 .

Definition 7.3. Suppose $H$ is nondegenerate. We put

$$
\Lambda_{\mathfrak{b}}^{\downarrow}(M)=\Lambda^{\downarrow}(G(M, \omega, \mathfrak{b}))
$$

and

$$
C F\left(M, H ; \Lambda_{\mathfrak{b}}^{\downarrow}(M)\right)=C F(M, H) \otimes_{\Lambda \downarrow(M)} \Lambda_{\mathfrak{b}}^{\downarrow}(M) .
$$

Here $C F(M, H)$ is defined in Definition 4.9 .

By definition, an element $x \in C F\left(M, H ; \Lambda_{\mathfrak{b}}^{\downarrow}(M)\right)$ can be written as

$$
x=\sum_{\gamma \in \operatorname{Per}(H)} x_{\gamma} \llbracket \gamma, w_{\gamma} \rrbracket, \quad x_{\gamma} \in \Lambda_{\mathfrak{b}}^{\downarrow}(M)
$$

similarly as in (2.9). We define the level function $\lambda_{H}: C F\left(M, H ; \Lambda_{\mathfrak{b}}^{\downarrow}(M)\right) \rightarrow \mathbb{R}$ by

$$
\lambda_{H}(x)=\max \left\{\mathfrak{v}_{q}\left(x_{\lambda}\right)+\mathcal{A}_{H}\left(\llbracket \gamma, w_{\gamma} \rrbracket\right) \mid x_{\gamma} \neq 0 \text { in the above sum }\right\} .
$$


Lemma 7.4. Suppose $H$ is nondegenerate.

(1) The set $\left\{\llbracket \gamma, w_{\gamma} \rrbracket \mid \gamma \in \operatorname{Per}(H)\right\}$ forms a basis of the vector space $C F\left(M, H ; \Lambda_{\mathfrak{b}}^{\downarrow}(M)\right)$ over the field $\Lambda_{\mathfrak{b}}^{\downarrow}(M)$.

(2) If $\mathfrak{x} \in C F(M, H ; \mathfrak{b}) \backslash\{0\}$ then $\lambda_{H}(\mathfrak{x}) \in \operatorname{Spec}(H ; \mathfrak{b})$.

Proof. Statement (1) follows from the fact that $\mathcal{A}_{H}([\gamma, w])-\mathcal{A}_{H}\left(\left[\gamma, w^{\prime}\right]\right) \in G(M, \omega, \mathfrak{b})$ for $\gamma \in \operatorname{Per}(H),[\gamma, w],\left[\gamma, w^{\prime}\right] \in \operatorname{Crit}\left(\mathcal{A}_{H}\right)$. Then statement (2) follows from statement (1).

We denote by $\operatorname{Ham}_{\text {nd }}(M, \omega)$ the set of nondegenerate Hamiltonian diffeomorphisms. By now, it is well-established that for any $H \in \operatorname{Ham}_{n d}(M, \omega)$ the map $\partial_{(H, J)}^{\mathfrak{b}}: C F\left(M, H ; \Lambda^{\downarrow}\right) \rightarrow C F\left(M, H ; \Lambda^{\downarrow}\right)$ is defined and preserves the subspace $C F\left(M, H ; \Lambda_{\mathfrak{b}}^{\downarrow}(M)\right)$ of $C F\left(M, H ; \Lambda^{\downarrow}\right)$. Moreover the filtration of $C F\left(M, H ; \Lambda^{\downarrow}(M)\right)$ induces one on $C F\left(M, H ; \Lambda_{\mathfrak{b}}^{\downarrow}(M)\right)$ given by

$$
F^{\lambda} C F\left(M, H ; \Lambda_{\mathfrak{b}}^{\downarrow}(M)\right)=F^{\lambda} C F\left(M, H ; \Lambda^{\downarrow}(M)\right) \cap C F\left(M, H ; \Lambda_{\mathfrak{b}}^{\downarrow}(M)\right) .
$$

We denote the homology of $\left(C F\left(M, H ; \Lambda_{\mathfrak{b}}^{\downarrow}(M)\right), \partial_{(H, J)}^{\mathfrak{b}}\right)$ by $H F^{\mathfrak{b}}\left(M, H, J ; \Lambda_{\mathfrak{b}}^{\downarrow}(M)\right)$. Then Lemma 7.4 implies

$$
H F_{*}^{\mathfrak{b}}\left(M, H, J ; \Lambda^{\downarrow}\right) \cong H F_{*}^{\mathfrak{b}}\left(M, H, J ; \Lambda_{\mathfrak{b}}^{\downarrow}(M)\right) \otimes_{\Lambda_{\mathfrak{b}}^{\downarrow}(M)} \Lambda^{\downarrow} .
$$

Therefore Theorem 6.9 implies:

Lemma 7.5. The map $\mathcal{P}_{\left(H_{\chi}, J_{\chi}\right), *}^{\mathfrak{b}}$ in (6.16) induces an isomorphism

$$
H\left(M ; \Lambda_{\mathfrak{b}}^{\downarrow}(M)\right) \cong H F^{\mathfrak{b}}\left(M, H, J ; \Lambda_{\mathfrak{b}}^{\downarrow}(M)\right) .
$$

Definition 7.6. (1) Let $\mathfrak{x} \in H F^{\mathfrak{b}}\left(M, H, J ; \Lambda^{\downarrow}\right)$. We define its spectral invariant $\rho^{\mathfrak{b}}(\mathfrak{x})$ by

$\rho^{\mathfrak{b}}(\mathfrak{x})=\inf \left\{\lambda \mid x \in F^{\lambda} C F\left(M, H, J ; \Lambda^{\downarrow}\right), \partial_{H, J}^{\mathfrak{b}}(x)=0,[x]=\mathfrak{x} \in H F^{\mathfrak{b}}\left(M, H, J ; \Lambda^{\downarrow}\right)\right\}$.

(2) If $a \in H^{*}\left(M ; \Lambda_{\mathfrak{b}}(M)\right)$ t 4 and $H$ is a nondegenerate time-dependent Hamiltonian, we define the spectral invariant with bulk $\rho^{\mathfrak{b}}(H ; a)$ by

$$
\rho^{\mathfrak{b}}(H ; a)=\rho^{\mathfrak{b}}\left(a_{H}^{\mathfrak{b} ; b}\right), \quad a_{H}^{\mathfrak{b} ; b}:=\mathcal{P}_{\left(H_{\chi}, J_{\chi}\right), *}^{\mathfrak{b}}\left(a^{\mathfrak{b}}\right) \in H F_{*}^{\mathfrak{b}}\left(M, H, J ; \Lambda^{\downarrow}\right)
$$

where the right hand side is as in (1), and we regard

$$
\mathcal{P}_{\left(H_{\chi}, J_{\chi}\right), *}^{\mathfrak{b}}\left(a^{b}\right) \in H F^{\mathfrak{b}}\left(M, H, J ; \Lambda_{\mathfrak{b}}^{\downarrow}(M)\right) \subset H F\left(M, H, J ; \Lambda^{\downarrow}\right) .
$$

By the same procedure exercised for the spectral invariant $\rho(H ; a)$, we can prove that $\rho^{\mathfrak{b}}\left(\mathcal{P}_{\left(H_{\chi}, J_{\chi}\right), *}^{\mathfrak{b}}(a)\right)$ do not depend on the choices of $J$ and $\chi$ or of other choices involved in the construction of virtual fundamental cycles, and hence $\rho^{\mathfrak{b}}(H ; a)$ is well-defined.

Theorem 7.7 (Homotopy invariance). $\quad$ (1) The spectral invariant $\rho^{\mathfrak{b}}(H ; a)$ is independent of the almost complex structure and other choices involved in the definition.

(2) The spectral invariant $\rho^{\mathfrak{b}}(H ; a)$ depends only on the homology class of $\mathfrak{b}$ and is independent of the choices of differential forms which represent it.

\footnotetext{
${ }^{4}$ Here $\Lambda_{\mathfrak{b}}(M)$ is a subalgebra of $\Lambda$ which is identified to the subalgebra $\Lambda_{\mathfrak{b}}^{\downarrow}(M)$ of $\Lambda^{\downarrow}$ by the canonical isomorphism $\Lambda \cong \Lambda^{\downarrow}$.
} 
(3) Suppose $\phi_{H}^{1}=\phi_{H^{\prime}}^{1}$ and the paths $\phi_{H}$ and $\phi_{H^{\prime}}$ are homotopic relative to the ends. Then

$$
\rho^{\mathfrak{b}}(H ; a)=\rho^{\mathfrak{b}}\left(H^{\prime} ; a\right) .
$$

Theorem 7.7(1) is proved in Section 9 Theorem 7.7 (3) is proved in Section 10. Theorem 7.7 (2) is proved in Section 27.

Theorem 7.7 implies that the function $H \mapsto \rho^{\mathfrak{b}}(\underline{H} ; a)$ descends to $\widetilde{\operatorname{Ham}}_{\text {nd }}(M, \omega)$, which is the inverse image of $\operatorname{Ham}_{\text {nd }}(M, \omega)$ in the universal covering $\widetilde{\operatorname{Ham}}(M, \omega) \rightarrow$ $\operatorname{Ham}(M, \omega)$. We denote by $\rho^{\mathfrak{b}}\left(\widetilde{\psi}_{H} ; a\right)=\rho^{\mathfrak{b}}(\underline{H} ; a)$ if $\widetilde{\psi}_{H}=\left[\phi_{H}\right] \in \widetilde{\operatorname{Ham}}_{\text {nd }}(M, \omega)$ associated to $H$ as before.

We have thus defined a map

$$
\rho^{\mathfrak{b}}: \widetilde{\operatorname{Ham}}_{\text {nd }}(M, \omega) \times\left(Q H_{\mathfrak{b}}^{*}(M) \backslash\{0\}\right) \rightarrow \mathbb{R} .
$$

It still satisfies the conclusions of Theorem 4.16. Namely we have:

Theorem 7.8. Let $(M, \omega)$ be any closed symplectic manifold. Then the map $\rho^{\mathfrak{b}}$ in (17.2) extends to

$$
\rho^{\mathfrak{b}}: \widetilde{\operatorname{Ham}}(M, \omega) \times\left(Q H_{\mathfrak{b}}^{*}(M) \backslash\{0\}\right) \rightarrow \mathbb{R} .
$$

It has the following properties. Let $\widetilde{\psi}, \widetilde{\phi} \in \widetilde{\operatorname{Ham}}(M, \omega)$ and $0 \neq a \in H^{*}\left(M ; \Lambda_{\mathfrak{b}}(M)\right)$.

(1) (Nondegenerate spectrality) If $\widetilde{\psi}$ is non-degenerate, then $\rho(\widetilde{\psi} ; a) \in \operatorname{Spec}(H ; \mathfrak{b})$.

(2) (Projective invariance) $\rho^{\mathfrak{b}}(\widetilde{\phi} ; \lambda a)=\rho^{\mathfrak{b}}(\widetilde{\phi} ; a)$ for any $0 \neq \lambda \in \mathbb{C}$.

(3) (Normalization) We have $\rho^{\mathfrak{b}}(\underline{0} ; a)=\lambda_{q}(a)$ where $\underline{0}$ is the identity in $\widetilde{\operatorname{Ham}}(M, \omega)$ and $\mathfrak{v}_{q}(a)$ is as in (4.6).

(4) (Symplectic invariance) $\rho^{\eta^{*} \mathfrak{b}}\left(\eta^{-1} \circ \widetilde{\phi} \circ \eta ; \eta^{*} a\right)=\rho^{\mathfrak{b}}(\widetilde{\phi} ; a)$ for any symplectic diffeomorphism $\eta$. In particular, if $\eta \in \operatorname{Symp}_{0}(M, \omega)$, then we have $\rho^{\mathfrak{b}}\left(\eta^{-1} \circ\right.$ $\widetilde{\phi} \circ \eta ; a)=\rho^{\mathfrak{b}}(\widetilde{\phi} ; a)$.

(5) (Triangle inequality) $\rho^{\mathfrak{b}}\left(\widetilde{\phi} \circ \widetilde{\psi} ; a \cup^{\mathfrak{b}} b\right) \leq \rho^{\mathfrak{b}}(\widetilde{\phi} ; a)+\rho^{\mathfrak{b}}(\widetilde{\psi} ; b)$, where $a \cup^{\mathfrak{b}} b$ is the $\mathfrak{b}$-deformed quantum cup product.

(6) ( $C^{0}$-Hamiltonian continuity) We have

$$
\left|\rho^{\mathfrak{b}}(\widetilde{\phi} ; a)-\rho^{\mathfrak{b}}(\widetilde{\psi} ; a)\right| \leq \max \left\{\left\|\widetilde{\phi} \circ \widetilde{\psi}^{-1}\right\|_{+},\left\|\widetilde{\phi} \circ \widetilde{\psi}^{-1}\right\|_{-}\right\}
$$

where $\|\cdot\|_{ \pm}$is the positive and negative parts of Hofer norm on $\widetilde{\operatorname{Ham}}(M, \omega)$. In particular, the function $\rho_{a}: \widetilde{\psi} \mapsto \rho^{\mathfrak{b}}(\widetilde{\psi} ; a)$ is continuous with respect to the quotient topology under the equivalence relation $\sim$ on the space of Hamiltonian paths $\left\{\widetilde{\psi}_{H} \mid H \in C^{\infty}\left(S^{1} \times M, \mathbb{R}\right)\right\}$.

(7) (Additive triangle inequality) $\rho^{\mathfrak{b}}(\widetilde{\psi} ; a+b) \leq \max \left\{\rho^{\mathfrak{b}}(\widetilde{\psi} ; a), \rho^{\mathfrak{b}}(\widetilde{\psi} ; b)\right\}$.

The proofs of Theorems 7.7 and 7.8 occupy the rest of this part. Most of the proofs are minor changes of the proofs of Theorem 4.16 in Oh4, Oh7] and of [Us1.

\section{Proof of the SPeCtrality axiom}

In this section we prove Theorem 7.8 (1). To include the case when $(M, \omega)$ is not rational we use some algebraic results exploited by Usher Us1. We reprove a similar result in Subsection 8.1 using the universal Novikov ring. 
8.1. Usher's spectrality lemma. Let $G$ be a subgroup of $\mathbb{R}$. (We do not assume that $G$ is discrete.) We define

$$
\begin{aligned}
& \Lambda^{\downarrow}(G)=\left\{\sum_{i=1}^{\infty} a_{i} q^{\lambda_{i}} \mid a_{i} \in \mathbb{C}, \lambda_{i} \in \mathbb{R}, \lambda_{i} \in G, \lim _{i \rightarrow \infty} \lambda_{i}=-\infty\right\}, \\
& \Lambda_{0}^{\downarrow}(G)=\left\{\sum_{i=1}^{\infty} a_{i} q^{\lambda_{i}} \mid a_{i} \in \mathbb{C}, \lambda_{i} \in \mathbb{R}_{\leq 0}, \lambda_{i} \in G, \lim _{i \rightarrow \infty} \lambda_{i}=-\infty\right\}, \\
& \Lambda_{+}^{\downarrow}(G)=\left\{\sum_{i=1}^{\infty} a_{i} q^{\lambda_{i}} \mid a_{i} \in \mathbb{C}, \lambda_{i} \in \mathbb{R}_{<0}, \lambda_{i} \in G, \lim _{i \rightarrow \infty} \lambda_{i}=-\infty\right\} .
\end{aligned}
$$

(Note the above definition of $\Lambda^{\downarrow}(G)$ coincides with Definition 4.9)

It is easy to see that $\Lambda^{\downarrow}(G)$ is a field of fraction of $\Lambda_{0}^{\downarrow}(G)$.

Let $\bar{C}$ be a finite dimensional $\mathbb{C}$ vector space. We put

$$
C=\bar{C} \otimes \Lambda^{\downarrow}, \quad C(G)=\bar{C} \otimes \Lambda^{\downarrow}(G) \subset C .
$$

Let $e_{i}(i=1, \ldots, N)$ be a $\mathbb{C}$-basis of $\bar{C}$ and $\lambda_{i}^{0}$ for $i=1, \ldots, N$ be real numbers. We define $\lambda_{q}: C \rightarrow \mathbb{R}$ by

$$
\lambda_{q}\left(\sum_{i=1}^{N} x_{i} e_{i}\right)=\sup \left\{\mathfrak{v}_{q}\left(x_{i}\right)+\lambda_{q}\left(e_{i}\right) \mid i=1, \ldots, N\right\},
$$

i.e., $\lambda_{q}\left(e_{i}\right)=\lambda_{i}^{0}$ for $i=1, \ldots, N$. It defines a norm with respect to which $C$ and $C(G)$ are complete. Then we define a $G$-set

$$
G^{\prime}=\bigcup_{i=1}^{N}\left\{\lambda_{i}^{0}+g \mid g \in G\right\} .
$$

It follows from the definition of $\lambda_{q}(x)$ that if $x \in C(G)$ then $\lambda_{q}(x) \in G^{\prime}$. We put

$$
F^{\lambda} C=\left\{x \in C \mid \lambda_{q}(x) \leq \lambda\right\}, \quad F^{\lambda} C(G)=F^{\lambda} C \cap C(G) .
$$

Suppose that $\bar{C}$ is $\mathbb{Z}_{2}$-graded, i.e., $\bar{C}=\bar{C}^{0} \oplus \bar{C}^{1}$ and each of the element of our basis $e_{i}$ lies in either $\bar{C}^{0}$ or $\bar{C}^{1}$. Let a $\mathbb{C}$-linear map

$$
\partial_{g}: \bar{C}^{i} \rightarrow \bar{C}^{i-1}
$$

be given for each $g \in G$. Assuming that $\left\{g \mid \partial_{g} \neq 0\right\} \cap \mathbb{R}_{>E}$ is a finite set for any $E \in \mathbb{R}$, we put

$$
\partial=\sum_{g \in G} q^{g} \partial_{g}: C \rightarrow C .
$$

It induces a linear map $C(G) \rightarrow C(G)$, which we also denote by $\partial$. If $\partial$ satisfies $\partial \partial=0,(C, \partial)$ and $(C(G), \partial)$ define chain complexes. Denote by $H(C), H(C(G))$ their homologies respectively, and denote by $H(C(G)) \rightarrow H(C)$ the natural homomorphism induced by $\Lambda^{\downarrow}(G) \hookrightarrow \Lambda^{\downarrow}$.

Definition 8.1. For $\mathfrak{x} \in H(C)$, we define the level

$$
\rho(\mathfrak{x})=\inf \left\{\lambda_{q}(x) \mid x \in C(G), \partial x=0,[x]=\mathfrak{x}\right\} .
$$

Now the following theorem is proved by Usher [Us1. Here we give its proof for completeness' sake exploiting the algebraic material developed in [FOO01, Subsection 6.3]. 
Proposition 8.2. (Usher) $\rho(\mathfrak{x}) \in G^{\prime}$ for any $\mathfrak{x} \in \operatorname{Im}(H(C(G)) \rightarrow H(C))$.

Proof. We first need to slightly modify the discussion in [FO001, Subsection 6.3] since the energy level of the basis $e_{i}$ is not zero but is $\lambda_{i}^{0}$ here.

We say

$$
e_{i} \sim e_{j} \quad \text { if and only if } \lambda_{i}^{0}-\lambda_{j}^{0} \in G \text {. }
$$

By re-choosing the basis $\left\{e_{i}\right\}_{1 \leq i \leq N}$ into the form $\left\{q^{\mu_{i}} e_{i}\right\}_{1 \leq i \leq N}$ with $\mu_{i} \in G$ if necessary, we may assume, without loss of generality, that $\lambda_{i}^{0}=\lambda_{j}^{0}$ if $e_{i} \sim e_{j}$. We assume this in the rest of this subsection.

For each $\lambda \in G^{\prime}$, define

$$
I(\lambda)=\left\{i \mid \lambda-\lambda_{i}^{0} \in G, 1 \leq i \leq N\right\} .
$$

We denote by $\mu(\lambda)$ the difference $\lambda-\lambda_{i}^{0}$ for $i \in I(\lambda)$. By the definition of $\sim$ and the hypothesis we put above, the value $\mu(\lambda)$ is independent of $i$. We take the direct sum

$$
\bar{C}(\lambda)=\bigoplus_{i \in I(\lambda)} \mathbb{C} e_{i}
$$

Let $x \in C(G)$ be a nonzero element and denote $\lambda=\lambda_{q}(x)$. Then there exists a unique $\sigma(x) \in \bar{C}(\lambda)$ such that

$$
\lambda_{q}\left(x-q^{\mu(\lambda)} \sigma(x)\right)<\lambda_{q}(x) .
$$

We call $\sigma(x)$ the symbol of $x$.

Definition 8.3. (Compare FOOO1, Section 6.3.1]) Let $V \subset C(G)$ be a $\Lambda^{\downarrow}(G)$ vector subspace. A basis $\left\{e_{i}^{\prime} \mid i=1, \ldots, N^{\prime}\right\}$ of $V$ is said to be a standard basis if the symbols $\left\{\sigma\left(e_{i}^{\prime}\right) \mid i=1, \ldots, N^{\prime}\right\}$ are linearly independent over $\mathbb{C}$.

If $\left\{e_{i}^{\prime} \mid i=1, \ldots, N^{\prime}\right\}$ is a standard basis, then we have

$$
\lambda_{q}\left(\sum_{i} a_{i} e_{i}^{\prime}\right)=\max \left\{\mathfrak{v}_{q}\left(a_{i}\right)+\lambda_{q}\left(e_{i}^{\prime}\right) \mid i=1, \ldots, N^{\prime}\right\} .
$$

Lemma 8.4. Any $V \subset C(G)$ has standard basis. Moreover if $V_{1} \subset V_{2} \subset C$ are $\Lambda^{\downarrow}(G)$ vector subspaces, then any standard basis of $V_{1}$ can be extended to one of $V_{2}$.

Proof. The proof is similar to the proof of FOOO1, Lemma 6.3.2 and Lemma 6.3.2bis]. We give the detail below since we considered $\Lambda$ in place of $\Lambda^{\downarrow}(G)$ in FOOO1.

Let $x_{1}, \ldots, x_{k}$ be a standard basis of $V_{1}$. We prove the following by induction on $\ell$.

Sublemma 8.5. For any given $\ell \leq \operatorname{dim} V_{2}-\operatorname{dim} V_{1}$, there exists $y_{1}, \ldots, y_{\ell}$ such that the set $\left\{\sigma\left(x_{1}\right), \ldots, \sigma\left(x_{k}\right), \sigma\left(y_{1}\right), \ldots, \sigma\left(y_{\ell}\right)\right\}$ is linearly independent over $\mathbb{C}$.

Proof. The proof is by induction on $\ell$. Suppose we have $y_{1}, \ldots, y_{\ell}$ as in the sublemma and $\operatorname{dim} V_{2}-\operatorname{dim} V_{1} \geq \ell+1$. We will find $y_{\ell+1}$.

Pick $z_{1}, \ldots, z_{m} \in \bar{C}$ such that $\left\{\sigma\left(x_{1}\right), \ldots, \sigma\left(x_{k}\right), \sigma\left(y_{1}\right), \ldots \sigma\left(y_{\ell}\right), \sigma\left(z_{1}\right), \ldots\right.$, $\left.\sigma\left(z_{m}\right)\right\}$ is a basis of $\bar{C}$ as a $\mathbb{C}$-vector space. In particular, $\left\{x_{1}, \ldots, x_{k}, y_{1}, \ldots, y_{\ell}\right.$, $\left.z_{1}, \ldots, z_{m}\right\}$ is a basis of $C$ as a $\Lambda(G)$-vector space. We define $A: C(G) \rightarrow C(G)$ a $\Lambda(G)$-linear isomorphism by

$$
A\left(x_{i}\right)=q^{\mu\left(\lambda_{q}\left(x_{i}\right)\right)} \sigma\left(x_{i}\right), \quad A\left(y_{j}\right)=q^{\mu\left(\lambda_{q}\left(y_{j}\right)\right)} \sigma\left(y_{j}\right), \quad A\left(z_{h}\right)=z_{h}
$$


for $i=1, \ldots, k, j=1, \ldots, \ell, h=1, \ldots, m$. Note that $A$ preserves filtration and $\sigma \circ A=\sigma$. We take $y^{\prime} \in V_{2}$ that is linearly independent of $\left\{x_{1}, \ldots, x_{k}, y_{1}, \ldots, y_{\ell}\right\}$ over $\Lambda(G)$. We write

$$
A\left(y^{\prime}\right)=\sum_{n=1}^{\infty} q^{\mu\left(\lambda_{n}\right)} \bar{y}_{n}^{\prime}
$$

where $\bar{y}_{n}^{\prime} \in \bar{C}\left(\lambda_{n}\right)$. Note $\lambda_{q}\left(q^{\mu\left(\lambda_{n}\right)} \bar{y}_{n}^{\prime}\right)=\lambda_{n}$. Moreover, we may assume that $\lambda_{n}>\lambda_{n+1}$ and $\lim _{n \rightarrow \infty} \lambda_{n}=-\infty$.

By the assumption that $\operatorname{dim} V_{2} \geq \operatorname{dim} V_{1}+\ell+1=k+\ell+1$ and that $\bar{y}^{\prime}$ is linearly independent of $\left\{x_{1}, \ldots, x_{k}, y_{1}, \ldots, y_{\ell}\right\}$, there exists $n$ such that

$$
\bar{y}_{n}^{\prime} \notin \bigoplus_{i=1}^{k} \mathbb{C} \sigma\left(x_{i}\right) \oplus \bigoplus_{j=1}^{\ell} \mathbb{C} \sigma\left(y_{j}\right)
$$

Let $n_{0}$ be the smallest number satisfying (8.3). Put

$$
y^{\prime \prime}=\sum_{n=n_{0}}^{\infty} q^{\mu\left(\lambda_{n}\right)} \bar{y}_{n}^{\prime}
$$

Clearly, $\sigma\left(y^{\prime \prime}\right)$ is linearly independent to $\sigma\left(x_{1}\right), \ldots, \sigma\left(x_{k}\right), \sigma\left(y_{1}\right), \ldots, \sigma\left(y_{\ell}\right)$. Hence $y_{\ell+1}=A^{-1}\left(y^{\prime \prime}\right)$ has the required property.

Lemma 8.4 easily follows from Sublemma 8.5

We now consider $\partial: C(G) \rightarrow C(G)$ and its matrix element with respect to a basis of $C(G)$. Choose a basis $\left\{e_{i}^{\prime} \mid i=1, \ldots, b\right\} \cup\left\{e_{i}^{\prime \prime} \mid i=1, \ldots, h\right\} \cup\left\{e_{i}^{\prime \prime \prime} \mid i=1, \ldots, b\right\}$ such that $\left\{e_{i}^{\prime} \mid i=1, \ldots, b\right\}$ is a standard basis of $\operatorname{Im} \partial,\left\{e_{i}^{\prime} \mid i=1, \ldots, b\right\} \cup\left\{e_{i}^{\prime \prime} \mid\right.$ $i=1, \ldots, h\}$ is a standard basis of $\operatorname{Ker} \partial$ and $\left\{e_{i}^{\prime} \mid i=1, \ldots, b\right\} \cup\left\{e_{i}^{\prime \prime} \mid i=\right.$ $1, \ldots, h\} \cup\left\{e_{i}^{\prime \prime \prime} \mid i=1, \ldots, b\right\}$ is a standard basis of $C$. (We may also assume that $e_{i}^{\prime}, e_{i}^{\prime \prime}, e_{i}^{\prime \prime \prime}$ are homogeneous so that they lie either in $C^{0}$ or in $C^{1}$.) Such a basis exists by Lemma 8.4.

Lemma 8.6. If $a \in H(C(G), \partial)$, there exists a unique $a_{i} \in \Lambda^{\downarrow}(G)$ such that $\sum_{i=1}^{h} a_{i} e_{i}^{\prime \prime}$ represents a. Moreover

$$
\inf \left\{\lambda_{q}(x) \mid x \in \operatorname{Ker} \partial, a=[x]\right\}=\lambda_{q}\left(\sum_{i=1}^{h} a_{i} e_{i}^{\prime \prime}\right)
$$

The proof is easy and so omitted.

We note that by the definition (8.1) of $G^{\prime}, \lambda_{q}\left(\sum_{i=1}^{h} a_{i} e_{i}^{\prime \prime}\right) \in G^{\prime}$. Proposition 8.2 is proved.

Remark 8.7. From the above discussion we have proved

$$
\inf \left\{\lambda_{q}(x) \mid x \in C(G), \partial x=0,[x]=\mathfrak{x}\right\}=\inf \left\{\lambda_{q}(x) \mid x \in C, \partial x=0,[x]=\mathfrak{x}\right\}
$$

for $\mathfrak{x} \in \operatorname{Im}(H(C(G)) \rightarrow H(C))$ at the same time. 
8.2. Proof of nondegenerate spectrality. In this subsection we apply Proposition 8.2 to prove the following theorem.

Theorem 8.8. If $H$ is nondegenerate, then $\rho^{\mathfrak{b}}(H ; a) \in \operatorname{Spec}(H ; \mathfrak{b})$.

Proof. We put $G=G(M, \omega, \mathfrak{b})$. Let $\bar{C}$ be the $\mathbb{C}$ vector space whose basis is given by $\{[\gamma] \mid \gamma \in \operatorname{Per}(H)\}$. Then we have

$$
C(G) \cong C F\left(M, H ; \Lambda_{\mathfrak{b}}^{\downarrow}(M)\right), \quad C \cong C F\left(M, H ; \Lambda^{\downarrow}\right) .
$$

In fact, an isomorphism $I: C(G) \cong C F\left(M, H ; \Lambda_{\mathfrak{b}}^{\downarrow}(M)\right)$ can be defined by

$$
I([\gamma])=\llbracket \gamma, w_{\gamma} \rrbracket
$$

where we take and fix a bounding disc $w_{\gamma}$ for each $\gamma$.

For each member $e_{i}=\left[\gamma_{i}\right]$ of the basis of $\bar{C}$, we put $\lambda_{i}^{0}=\mathcal{A}_{H}\left(\left[\gamma_{i}, w_{\gamma_{i}}\right]\right)$. Then $G^{\prime}=\operatorname{Spec}(H ; \mathfrak{b})$ and the map $I$ preserves filtration. Theorem 8.8 now follows from Proposition 8.2

\section{Proof of $C^{0}$-Hamiltonian continuity}

In this section we prove the following:

Theorem 9.1. Let $H, H^{\prime}: S^{1} \times M \rightarrow \mathbb{R}$ be smooth functions such that $\psi_{H}$ and $\psi_{H^{\prime}}$ are nondegenerate. Let $a \in Q H_{\mathfrak{b}}^{*}(M)$ and $\mathfrak{b} \in H^{\operatorname{even}}\left(M ; \Lambda_{0}\right)$. Then we have

$$
-E^{+}\left(H^{\prime}-H\right) \leq \rho^{\mathfrak{b}}\left(H^{\prime} ; a\right)-\rho^{\mathfrak{b}}(H ; a) \leq E^{-}\left(H^{\prime}-H\right) .
$$

Theorem 9.1 together with Theorem 7.7 implies Theorem 7.8 (6). (See the end of Section 10.) We will also prove the following theorem at the same time in this section.

Theorem 9.2. The value $\rho^{\mathfrak{b}}(H, J ; a)$ is independent of the choices of $J$ and the abstract perturbations of the moduli space we use during the construction of the number $\rho^{\mathfrak{b}}(H, J ; a)$.

Theorem 9.2 is Theorem 7.7 (1).

Proof. The proofs of Theorems 9.19 .2 are mostly the same as one presented in Oh4, Oh5, Oh7. Let $H, H^{\prime}$ be in Theorems 9.1 and $J, J^{\prime} \in j_{\omega}$. We interpolate them by the family in $\mathcal{P}\left(j_{\omega}\right)=\operatorname{Map}\left([0,1], j_{\omega}\right)$

$$
\left(F^{s}, J^{s}\right), \quad 0 \leq s \leq 1
$$

where $\left\{J^{s}\right\}_{0 \leq s \leq 1}$ with $J^{0}=J, J^{1}=J^{\prime}$ and

$$
F^{s}:=H+s\left(H^{\prime}-H\right): S^{1} \times M \rightarrow \mathbb{R} .
$$

(Note $J^{s} \neq J_{s}$ where $J_{s}$ is as in (3.12).) Let $\chi: \mathbb{R} \rightarrow[0,1]$ be as in Definition 3.13 and elongate the family to the $\left(\mathbb{R} \times S^{1}\right)$-family $\left(F^{\chi}, J^{\chi}\right)$ by

$$
F^{\chi}(\tau, t, x)=F^{\chi(\tau)}(t, x), \quad J_{t}^{\chi}=J_{t}^{\chi(\tau)} .
$$

We put $F_{t}^{\chi(\tau)}(x)=F^{\chi(\tau)}(t, x)$. Using this family, we construct a chain map

$$
\mathcal{P}_{(F \chi, J \chi), H, H^{\prime}}^{\mathfrak{b}}:\left(C F\left(M ; H ; \Lambda^{\downarrow}\right), \partial_{(H, J)}^{\mathfrak{b}}\right) \rightarrow\left(C F\left(M ; H^{\prime} ; \Lambda^{\downarrow}\right), \partial_{\left(H^{\prime}, J^{\prime}\right)}^{\mathfrak{b}}\right)
$$

by studying the equation

$$
\frac{\partial u}{\partial \tau}+J^{\chi}\left(\frac{\partial u}{\partial t}-X_{F_{t}^{\chi(\tau)}}(u)\right)=0
$$


with finite energy

$$
E_{(F \chi, J \chi)}(u)=\frac{1}{2} \int\left(\left|\frac{\partial u}{\partial \tau}\right|_{J \chi}^{2}+\left|\frac{\partial u}{\partial t}-X_{F_{t}^{\chi(\tau)}}(u)\right|_{J \chi}^{2}\right) d t d \tau
$$

To simplify the notation, we denote $\mathcal{P}_{(F \chi, J \chi), H, H^{\prime}}^{\mathfrak{b}}$ by $\mathcal{P}_{(F \chi, J \chi)}^{\mathfrak{b}}$ when no confusion can occur.

Let $[\gamma, w] \in \operatorname{Crit}\left(\mathcal{A}_{H}\right),\left[\gamma^{\prime}, w^{\prime}\right] \in \operatorname{Crit}\left(\mathcal{A}_{H^{\prime}}\right)$. First we prove the following bound for the action change.

Lemma 9.3. If the pair $[\gamma, w],\left[\gamma^{\prime}, w^{\prime}\right]$ carries a finite energy solution $u$ of (9.4) satisfying

$$
u(-\infty)=\gamma, u(\infty)=\gamma^{\prime}, \quad w \# u \sim w^{\prime}
$$

then

$$
\mathcal{A}_{H^{\prime}}\left(\left[\gamma^{\prime}, w^{\prime}\right]\right)-\mathcal{A}_{H}([\gamma, w]) \leq E^{-}\left(H^{\prime}-H\right) .
$$

Proof. Let $u$ be as above. Then by the same computation as in the proof of Lemma 3.10, we obtain

$$
\begin{aligned}
\mathcal{A}_{H^{\prime}}\left(\left[\gamma^{\prime}, w^{\prime}\right]\right)-\mathcal{A}_{H}([\gamma, w]) & =-E_{(H, J)}(u)-\int_{\mathbb{R}} \int_{S^{1}} \chi^{\prime}(\tau)\left(H^{\prime}-H\right) \circ u(\tau, t) d t d \tau \\
& \leq \int_{0}^{1}-\min _{x}\left(H_{t}^{\prime}(x)-H_{t}(x)\right) d t=E^{-}\left(H^{\prime}-H\right)
\end{aligned}
$$

where the inequality follows since $\chi^{\prime} \geq 0$ and $\int \chi^{\prime} d \tau=1$.

Definition 9.4. We denote by $\stackrel{\mathcal{M}}{\ell}_{\ell}\left(F^{\chi}, J^{\chi} ;[\gamma, w],\left[\gamma^{\prime}, w^{\prime}\right]\right)$ the set of all smooth maps $u: \mathbb{R} \times S^{1} \rightarrow M$ which satisfy the following conditions:

(1) The map $u$ satisfies the equation (9.4).

(2) The energy $E_{\left(F^{\chi}, J \chi\right)}(u)$ is finite.

(3) The map $u$ satisfies the following asymptotic boundary condition: 5

$$
\lim _{\tau \rightarrow-\infty} u(\tau, t)=\gamma(t), \quad \lim _{\tau \rightarrow+\infty} u(\tau, t)=\gamma^{\prime}(t) .
$$

(4) The homotopy class of $w \# u$ is $\left[w^{\prime}\right]$, where \# is the obvious concatenation.

(5) $z_{i}^{+}$are mutually distinct points in $\mathbb{R} \times S^{1}$.

The assignment $\left(u ; z_{1}^{+}, \ldots, z_{\ell}^{+}\right) \mapsto\left(u\left(z_{1}^{+}\right), \ldots, u\left(z_{\ell}^{+}\right)\right)$defines an evaluation map

$$
\mathrm{ev}=\left(\mathrm{ev}_{1} \ldots, \mathrm{ev}_{\ell}\right)=\stackrel{\circ}{\mathcal{M}}_{\ell}\left(F^{\chi}, J^{\chi} ;[\gamma, w],\left[\gamma^{\prime}, w^{\prime}\right]\right) \rightarrow M^{\ell} .
$$

We remark that for any $\alpha \in \pi_{2}(M)$ there exists a canonical homeomorphism

$$
\stackrel{\circ}{\mathcal{M}}_{\ell}\left(F^{\chi}, J^{\chi} ;[\gamma, w],\left[\gamma^{\prime}, w^{\prime}\right]\right) \cong \stackrel{\circ}{\mathcal{M}}_{\ell}\left(F^{\chi}, J^{\chi} ;[\gamma, \alpha \# w],\left[\gamma^{\prime}, \alpha \# w^{\prime}\right]\right) .
$$

Proposition 9.5. (1) The moduli space $\dot{\mathcal{M}}_{\ell}\left(F^{\chi}, J^{\chi} ;[\gamma, w],\left[\gamma^{\prime}, w^{\prime}\right]\right)$ has a compactification $\mathcal{M}_{\ell}\left(F^{\chi}, J^{\chi} ;[\gamma, w],\left[\gamma^{\prime}, w^{\prime}\right]\right)$ that is Hausdorff.

(2) The space $\mathcal{M}_{\ell}\left(F^{\chi}, J^{\chi} ;[\gamma, w],\left[\gamma^{\prime}, w^{\prime}\right]\right)$ has an orientable Kuranishi structure with corners.

\footnotetext{
${ }^{5}$ The moduli space $\mathcal{M}_{\ell}\left(F^{\chi}, J^{\chi} ;[\gamma, w],\left[\gamma^{\prime}, w^{\prime}\right]\right)$ is different from $\mathcal{M}_{\ell}\left(F_{\chi}, J_{\chi} ;[\gamma, w],\left[\gamma^{\prime}, w^{\prime}\right]\right)$, which is defined in Definition 3.15 Compare the definitions 9.2 and 3.12) of $F^{\chi}$ and $F_{\chi}$ respectively.
} 
(3) The normalized boundary of $\mathcal{M}_{\ell}\left(F^{\chi}, J^{\chi} ;[\gamma, w],\left[\gamma^{\prime}, w^{\prime}\right]\right)$ is described by

$$
\begin{aligned}
\partial \mathcal{M}_{\ell}\left(F^{\chi}, J^{\chi} ;[\gamma, w],\left[\gamma^{\prime}, w^{\prime}\right]\right) \\
=\bigcup \mathcal{M}_{\# \mathbb{L}_{1}}\left(H, J ;[\gamma, w],\left[\gamma^{\prime \prime} ; w^{\prime \prime}\right]\right) \times \mathcal{M}_{\# \mathbb{L}_{2}}\left(F^{\chi}, J^{\chi} ;\left[\gamma^{\prime \prime}, w^{\prime \prime}\right],\left[\gamma^{\prime}, w^{\prime}\right]\right) \\
\quad \cup \bigcup \mathcal{M}_{\# \mathbb{L}_{1}}\left(F^{\chi}, J^{\chi} ;[\gamma, w],\left[\gamma^{\prime \prime \prime} ; w^{\prime \prime \prime}\right]\right) \times \mathcal{M}_{\# \mathbb{L}_{2}}\left(H^{\prime} ; J^{\prime} ;\left[\gamma^{\prime \prime \prime} ; w^{\prime \prime \prime}\right],\left[\gamma^{\prime}, w^{\prime}\right]\right)
\end{aligned}
$$

where the first union is taken over all $\left(\gamma^{\prime \prime}, w^{\prime \prime}\right) \in \operatorname{Crit}\left(\mathcal{A}_{H}\right)$, and $\left(\mathbb{L}_{1}, \mathbb{L}_{2}\right) \in$ Shuff $(\ell)$ and the second union is taken over all $\left(\gamma^{\prime \prime \prime}, w^{\prime \prime \prime}\right) \in \operatorname{Crit}\left(\mathcal{A}_{H^{\prime}}\right)$, and $\left(\mathbb{L}_{1}, \mathbb{L}_{2}\right) \in \operatorname{Shuff}(\ell)$.

(4) Let $\mu_{H}: \operatorname{Crit}\left(\mathcal{A}_{H}\right) \rightarrow \mathbb{Z}, \mu_{H^{\prime}}: \operatorname{Crit}\left(\mathcal{A}_{H^{\prime}}\right) \rightarrow \mathbb{Z}$, be the Conley-Zehnder indices. Then the (virtual) dimension satisfies the following equality:

$$
\mathcal{M}_{\ell}\left(F^{\chi}, J^{\chi} ;[\gamma, w],\left[\gamma^{\prime}, w^{\prime}\right]\right)=\mu_{H^{\prime}}\left(\left[\gamma^{\prime}, w^{\prime}\right]\right)-\mu_{H}([\gamma, w])+2 \ell .
$$

(5) We can define orientations of $\mathcal{M}_{\ell}\left(F^{\chi}, J^{\chi} ;[\gamma, w],\left[\gamma^{\prime}, w^{\prime}\right]\right)$ so that (3) above is compatible with this orientation.

(6) ev extends to a weakly submersive map

$$
\mathcal{M}_{\ell}\left(F^{\chi}, J^{\chi} ;[\gamma, w],\left[\gamma^{\prime}, w^{\prime}\right]\right) \rightarrow M^{\ell}
$$

which we denote also by ev. It is compatible with (3).

(7) The homeomorphism (9.5) extends to the compactifications and their Kuranishi structures are identified by the homeomorphism.

The proof of Proposition 9.5 is the same as that of Proposition 3.6 (See [FOOO10, Section 31 and Part 4].) and so is omitted.

Definition 9.6. Let $[\gamma, w] \in \operatorname{Crit}\left(\mathcal{A}_{H}\right),\left[\gamma^{\prime}, w^{\prime}\right] \in \operatorname{Crit}\left(\mathcal{A}_{H^{\prime}}\right)$ and let $h_{i}(i=1, \ldots, \ell)$ be differential forms on $M$. We define $\mathfrak{n}_{(F \chi, J \chi) ;[\gamma, w],\left[\gamma^{\prime}, w^{\prime}\right]}\left(h_{1}, \ldots, h_{\ell}\right) \in \mathbb{C}$ by

$$
\mathfrak{n}_{(F \chi, J \chi) ;[\gamma, w],\left[\gamma^{\prime}, w^{\prime}\right]}\left(h_{1}, \ldots, h_{\ell}\right)=\int_{\mathcal{M}_{\ell}\left(F \chi, J \chi ;[\gamma, w],\left[\gamma^{\prime}, w^{\prime}\right]\right)} \mathrm{ev}_{1}^{*} h_{1} \wedge \cdots \wedge \mathrm{ev}_{\ell}^{*} h_{\ell} .
$$

(We take and use CF-perturbation to define the right hand side.)

By definition (9.8) is zero if

$$
\sum_{i=1}^{\ell} \operatorname{deg} h_{i} \neq \operatorname{dim} \mathcal{M}_{\ell}\left(F^{\chi}, J^{\chi} ;[\gamma, w],\left[\gamma^{\prime}, w^{\prime}\right]\right)
$$

where the right hand side is as in (9.7). We extend (9.8) to

$$
\mathfrak{n}_{(F \chi, J \chi) ;[\gamma, w],\left[\gamma^{\prime}, w^{\prime}\right]}: B_{\ell}(\Omega(M) \widehat{\otimes} \Lambda) \rightarrow \Lambda^{\downarrow}
$$

by $\left(\Lambda, \Lambda^{\downarrow}\right)$ linearity.

Note that we need to make an appropriate choice of a compatible system of CFperturbations in order to define the integral given in (9.8). See Remark 6.4. We sometimes omit this remark from now on.

Let $\mathfrak{b} \in H^{\text {even }}\left(M ; \Lambda_{0}\right)$. We split $\mathfrak{b}=\mathfrak{b}_{0}+\mathfrak{b}_{2}+\mathfrak{b}_{+}$as in (5.5). We take closed differential forms representing $\mathfrak{b}_{0}, \mathfrak{b}_{2}, \mathfrak{b}_{+}$which we still denote by the same letters respectively with an abuse of notations. Define $\mathfrak{n}_{(F \chi, J \chi)}^{\mathfrak{b}}\left([\gamma, w],\left[\gamma^{\prime}, w^{\prime}\right]\right) \in \Lambda^{\downarrow}$ by the sum

$$
\begin{aligned}
\mathfrak{n}_{(F \chi, J \chi)}^{\mathfrak{b}}\left([\gamma, w],\left[\gamma^{\prime}, w^{\prime}\right]\right)=\sum_{\ell=0}^{\infty} \frac{\exp \left(\int\left(w^{\prime}\right)^{*} \mathfrak{b}_{2}-\int w^{*} \mathfrak{b}_{2}\right)}{\ell !} \\
\mathfrak{n}_{(F \chi, J \chi) ;[\gamma, w],\left[\gamma^{\prime}, w^{\prime}\right]}(\underbrace{\mathfrak{b}_{+}, \ldots, \mathfrak{b}_{+}}_{\ell}) .
\end{aligned}
$$


We can prove that the sum in (9.9) converges in $q$-adic topology, in the same way as in Lemma 6.5. We now set

$$
\mathcal{P}_{(F \chi, J \chi)}^{\mathfrak{b}}(\llbracket \gamma, w \rrbracket)=\sum_{\left[\gamma^{\prime}, w^{\prime}\right] \in \operatorname{Crit}\left(\mathcal{A}_{H^{\prime}}\right)} \mathfrak{n}_{(F \chi, J \chi)}^{\mathfrak{b}}\left([\gamma, w],\left[\gamma^{\prime}, w^{\prime}\right]\right) \llbracket \gamma^{\prime}, w^{\prime} \rrbracket .
$$

Here we suppose $\llbracket \gamma, w \rrbracket$ is represented by $[\gamma, w]$, i.e., $\pi([\gamma, w])=\llbracket \gamma, w \rrbracket$. We then can show by using Proposition 9.5 (7) that the right hand side is independent of such a representative $[\gamma, w]$. (We take our CF-perturbation so that it is compatible with this isomorphism.)

We can prove that the right hand side defines an element of $C F\left(H^{\prime}, J^{\prime} ; \Lambda^{\downarrow}\right)$ in the same way as in Lemma 6.7. Thus we have defined (9.3). Then

$$
\mathcal{P}_{(F \chi, J \chi)}^{\mathfrak{b}} \circ \partial_{(H, J)}^{\mathfrak{b}}=\partial_{\left(H^{\prime}, J^{\prime}\right)}^{\mathfrak{b}} \circ \mathcal{P}_{(F \chi, J \chi)}^{\mathfrak{b}}
$$

is a consequence of (9.6), Stokes' theorem (Theorem 34.8) and the composition formula (Theorem 35.7).

Now we would like to study the relationship between the Piunikhin maps $\mathcal{P}_{\left(H_{\chi}, J_{\chi}\right)}^{\mathfrak{b}}$ as we vary the pair $(H, J)$ and the elongation function $\chi \in \mathcal{K}$ introduced in Definition 3.13. Let $\chi \in \mathcal{K}$ and consider the three maps $\mathcal{P}_{\left(H_{\chi}, J_{\chi}\right)}^{\mathfrak{b}}, \mathcal{P}_{\left(H_{\chi}^{\prime}, J_{\chi}^{\prime}\right)}^{\mathfrak{b}}$ and $\mathcal{P}_{(F \chi, J \chi)}^{\mathfrak{b}}$.

Proposition 9.7. $\mathcal{P}_{(F \chi, J \chi)}^{\mathfrak{b}} \circ \mathcal{P}_{\left(H_{\chi}, J_{\chi}\right)}^{\mathfrak{b}}$ is chain homotopic to $\mathcal{P}_{\left(H_{\chi}^{\prime}, J_{\chi}^{\prime}\right)}^{\mathfrak{b}}$.

Proof. Let $J_{s}, J_{s}^{\prime}$ be as in (3.12) and $\left(F^{s}, J^{s}\right)$ as in (9.2). For $S \in[1, \infty), \tau \in \mathbb{R}$, we define $G_{S}(\tau, t, x)$ by

$$
G_{S}(\tau, t, x)=\left\{\begin{array}{lll}
\chi(\tau+2 S) H_{t}(x) & \tau \leq 0, & S \geq 1 \\
F_{t}^{\chi(\tau-2 S)}(x) & \tau \geq 0, & S \geq 1 .
\end{array}\right.
$$

We also define $J_{S}(\tau, t, x)$ by

$$
J_{S}(\tau, t, x)=\left\{\begin{array}{lll}
J_{\chi(\tau+2 S), t} & \tau \leq 0, & S \geq 1 \\
J_{t}^{\chi(\tau-2 S)} & \tau \geq 0, & S \geq 1 .
\end{array}\right.
$$

We extend the definition of $G_{S}$ to those $S \in[0,1]$ by the formula,

$$
G_{S}(\tau, t, x)=(1-S) \chi(\tau) H^{\prime}(t, x)+S G_{1}(\tau, t, x) .
$$

Note that $G_{S}$ may not be smooth at $S=1$ on $S$ and on $\tau \in[-10,10]$. Here the specific number 10 does not assume any significance which can be replaced by any sufficiently large constant but fixed. It is chosen so that $G_{S}$ is smooth on $\tau$ at least outside $[-10,10]$. We modify $G_{S}$ on a small neighborhood of this set so that $G_{S}$ becomes a smooth family. We denote the resulting modification still by the same symbol $G_{S}$ by an abuse of notation.

We also extend the definition of $J_{S}$ to those $S \in[0,1]$ so that the following holds:

(1) At $S=0, J_{S}(\tau, t)$ coincides with $J_{\chi(\tau), t}^{\prime}$.

(2) $J_{S}$ is $t$ independent for $\tau<-10$. (It may be $S$-dependent there.)

We denote the family obtained above by

$$
(\mathcal{G}, \mathcal{J})=\left\{\left(G_{S}, J_{S}\right)\right\}_{S \in \mathbb{R}_{\geq 0}} .
$$

We put $G_{S ; \tau, t}(x)=G_{S}(\tau, t, x)$. Now for each $S \in \mathbb{R}_{\geq 0}$, we consider

$$
\frac{\partial u}{\partial \tau}+J_{S}\left(\frac{\partial u}{\partial t}-X_{G_{S ; \tau, t}}(u)\right)=0
$$


and define its moduli space $\stackrel{\circ}{\mathcal{M}}_{\ell}\left(G_{S}, J_{S} ; *,[\gamma, w]\right)$ defined in Definition 6.10, We put

$$
\stackrel{\circ}{\mathcal{M}}_{\ell}\left(\text { para } ; *,\left[\gamma^{\prime}, w^{\prime}\right]\right)=\bigcup_{S \in \mathbb{R}_{\geq 0}}\{S\} \times \stackrel{\circ}{\mathcal{M}}_{\ell}\left(G_{S}, J_{S} ; *,\left[\gamma^{\prime}, w^{\prime}\right]\right) .
$$

Here 'para' stands for the parameterized moduli space. We have the natural evaluation maps

$$
\text { ev }: \stackrel{\circ}{\mathcal{M}}_{\ell}\left(\text { para } ; *,\left[\gamma^{\prime}, w^{\prime}\right]\right) \rightarrow M^{\ell}, \quad \text { ev }_{-\infty}: \stackrel{\circ}{\mathcal{M}}_{\ell}\left(\text { para } ; *,\left[\gamma^{\prime}, w^{\prime}\right]\right) \rightarrow L .
$$

Lemma 9.8. (1) The moduli space $\stackrel{\mathcal{M}}{\ell}_{\ell}\left(\right.$ para; $\left.*,\left[\gamma^{\prime}, w^{\prime}\right]\right)$ has a compactification, denoted by $\mathcal{M}_{\ell}\left(\right.$ para $\left.; *,\left[\gamma^{\prime}, w^{\prime}\right]\right)$, that is Hausdorff.

(2) The space $\mathcal{M}_{\ell}\left(\right.$ para; $\left.*,\left[\gamma^{\prime}, w^{\prime}\right]\right)$ has an orientable Kuranishi structure with corners.

(3) The normalized boundary of $\mathcal{M}_{\ell}\left(\right.$ para; $\left.*,\left[\gamma^{\prime}, w^{\prime}\right]\right)$ is described by the following three types of components:

$\bigcup \mathcal{M}_{\# E_{1}}\left(\operatorname{para} ; *,\left[\gamma^{\prime \prime}, w^{\prime \prime}\right]\right) \times \mathcal{M}_{\# E_{2}}\left(H^{\prime}, J^{\prime} ;\left[\gamma^{\prime \prime}, w^{\prime \prime}\right],\left[\gamma^{\prime}, w^{\prime}\right]\right)$

where the union is taken over all $\left(L_{1}, t_{2}\right) \in \operatorname{Shuff}(\ell),\left[\gamma^{\prime \prime}, w^{\prime \prime}\right] \in \operatorname{Crit}\left(\mathcal{A}_{H^{\prime}}\right)$.

$$
\bigcup \mathcal{M}_{\# \mathbb{L}_{1}}\left(H_{\chi}, J_{\chi} ; *,[\gamma, w]\right) \times \mathcal{M}_{\# \mathbb{L}_{2}}\left(F^{\chi}, J^{\chi} ;[\gamma, w],\left[\gamma^{\prime}, w^{\prime}\right]\right)
$$

where the union is taken over all $\left(E_{1}, E_{2}\right) \in \operatorname{Shuff}(\ell),[\gamma, w] \in \operatorname{Crit}\left(\mathcal{A}_{H}\right)$.

$$
\mathcal{M}_{\ell}\left(H_{\chi}^{\prime}, J_{\chi}^{\prime} ; *,\left[\gamma^{\prime}, w^{\prime}\right]\right) \text {. }
$$

(4) Then the (virtual) dimension is given by

$$
\operatorname{dim} \mathcal{M}_{\ell}\left(\text { para } ; *,\left[\gamma^{\prime}, w^{\prime}\right]\right)=\mu_{H^{\prime}}\left(\left[\gamma^{\prime}, w^{\prime}\right]\right)+n+1+2 \ell .
$$

(5) We can define orientations of $\mathcal{M}_{\ell}\left(H_{\chi}, J_{\chi} ; *,\left[\gamma^{\prime}, w^{\prime}\right]\right)$ so that (3) above is compatible with this orientation.

(6) The evaluation map ev: $\stackrel{\mathcal{M}}{\ell}_{\ell}$ para; $\left.*,\left[\gamma^{\prime}, w^{\prime}\right]\right) \rightarrow M^{\ell}$ given in (9.14) extends to a weakly submersive map $\mathcal{M}_{\ell}\left(H_{\chi}, J_{\chi} ; *,\left[\gamma^{\prime}, w^{\prime}\right]\right) \rightarrow M^{\ell}$, which we denote also by ev. The family of evaluation maps are compatible with the boundary description (3).

Similarly the map ev $-\infty: \stackrel{\circ}{\mathcal{M}}_{\ell}\left(\right.$ para $\left.; *,\left[\gamma^{\prime}, w^{\prime}\right]\right) \rightarrow L$ can be also extended to $\mathcal{M}_{\ell}\left(H_{\chi}, J_{\chi} ; *,\left[\gamma^{\prime}, w^{\prime}\right]\right)$.

Proof. The proof is mostly the same as that of Proposition 3.6. We only mention how the boundary components are given as in (3).

The type (9.15) appears when there is a bubble to $\tau \rightarrow \infty$. The bubble to $\tau \rightarrow-\infty$ is of codimension 2 by the $S^{1}$-equivariance. (See the proof of Lemma 26.9 .)

The types (9.16) and (9.17) correspond to the cases of $S \rightarrow \infty$ and $S=0$ respectively.

We use this parameterized moduli space in the same way as we did in the definition of $\mathcal{P}_{\left(H_{\chi}, J_{\chi}\right)}^{\mathfrak{b}}$ and define a degree one map

$$
\mathcal{H}_{(\mathcal{G}, \mathcal{J})}^{\mathfrak{b}}: \Omega(M) \widehat{\otimes} \Lambda^{\downarrow} \rightarrow C F\left(M, H^{\prime} ; \Lambda^{\downarrow}\right) .
$$


We apply Lemma 9.8 together with Stokes' theorem (Theorem 34.8) and the composition formula (Theorem 35.7) to derive the equality

$$
\partial_{\left(H^{\prime}, J^{\prime}\right)}^{\mathfrak{b}} \circ \mathcal{H}_{(\mathcal{G}, \mathcal{J})}^{\mathfrak{b}}+\mathcal{H}_{(\mathcal{G}, \mathcal{J})}^{\mathfrak{b}} \circ \partial=\mathcal{P}_{\left(F^{\chi}, J^{\chi}\right)}^{\mathfrak{b}} \circ \mathcal{P}_{\left(H_{\chi}, J_{\chi}\right)}^{\mathfrak{b}}-P_{\left(H_{\chi}^{\prime}, J_{\chi}^{\prime}\right)}^{\mathfrak{b}} .
$$

Then Proposition 9.7 follows from (9.19).

Now we are ready to complete the proof of Theorem 9.1. By Lemma 9.3, we have

$$
\mathcal{P}_{(F \chi ; J \chi)}^{\mathfrak{b}}\left(F^{\lambda} C F\left(M, H ; \Lambda^{\downarrow}\right)\right) \subset F^{\lambda+E^{-}\left(H^{\prime}-H\right)} C F\left(M, H^{\prime} ; \Lambda^{\downarrow}\right) .
$$

Let $\rho=\rho^{\mathfrak{b}}(H ; a)$ and $\epsilon>0$. We take an element $x \in F^{\rho+\epsilon} C F\left(M, H ; \Lambda^{\downarrow}\right)$ representing the class $\mathcal{P}_{\left(H_{\chi}, J_{\chi}\right)}^{\mathfrak{b}}\left(a^{b}\right)$. Then the element $\mathcal{P}_{(F \chi, J \chi)}^{\mathfrak{b}}(x) \in F^{\rho+\epsilon-E^{-}\left(H^{\prime}-H\right)} C F\left(M, G ; \Lambda^{\downarrow}\right)$ represents the Floer homology class $\mathcal{P}_{(F \chi, J \chi)}^{\mathfrak{b}} \mathcal{P}_{\left(H_{\chi}, J_{\chi}\right)}^{\mathfrak{b}}\left(a^{b}\right)=\mathcal{P}_{\left(H_{\chi}^{\prime}, J_{\chi}^{\prime}\right)}^{\mathfrak{b}}\left(a^{b}\right)$. (Proposition 9.7). Therefore $\rho^{\mathfrak{b}}\left(H^{\prime} ; a\right) \leq \rho+\epsilon+E^{-}\left(H^{\prime}-H\right)$. Since $\epsilon$ is an arbitrary positive number, we have

$$
\rho^{\mathfrak{b}}\left(H^{\prime} ; a\right) \leq \rho^{\mathfrak{b}}(H ; a)+E^{-}\left(H^{\prime}-H\right) .
$$

By exchanging the role of $H^{\prime}$ and $H$ we have

$$
\rho^{\mathfrak{b}}(H ; a) \leq \rho^{\mathfrak{b}}\left(H^{\prime} ; a\right)+E^{+}\left(H^{\prime}-H\right) .
$$

The proof of Theorem 9.1 is complete.

We also note that Theorem 9.2 follows from the above argument applied to the case $H=H^{\prime}$ but $J \neq J^{\prime}$.

\section{Proof of HOMOTOPY INVARIANCE}

In this section we prove Theorem 7.7 (3) and Theorem 4.14 Let $H^{s}, s \in[0,1]$ be a one-parameter family of normalized periodic Hamiltonians $H^{s}: S^{1} \times M \rightarrow \mathbb{R}$ such that:

$$
\phi_{H^{s}}^{1} \equiv \psi, \phi_{H^{s}}^{0} \equiv i d \quad \text { for all } s \in[0,1] .
$$

We assume without loss of generality that $H^{s}(t, x) \equiv 0$ on a neighborhood of $\{[0]\} \times M \subset S^{1} \times M$.

We first define an isomorphism

$$
I_{s}: \operatorname{Crit}\left(\mathcal{A}_{H^{0}}\right) \rightarrow \operatorname{Crit}\left(\mathcal{A}_{H^{s}}\right) .
$$

Let $\gamma \in \operatorname{Per}\left(\mathcal{A}_{H^{0}}\right)$. Put $p=\gamma(0)$ and $\gamma_{s}=z_{p}^{H^{s}}$ defined by

$$
z_{p}^{H^{s}}(t)=\phi_{H^{s}}^{t}(p) .
$$

By (10.1), $z_{p}^{H^{s}}(1)=\gamma(1)=\psi(p)$ for all $s \in[0,1]$. Moreover, we have $z_{p}^{H^{s}} \in$ $\operatorname{Per}\left(\mathcal{A}_{H^{s}}\right)$. We note that $z_{p}^{H^{0}}=\gamma$.

Next let $[\gamma, w] \in \operatorname{Crit}\left(\mathcal{A}_{H^{0}}\right)$ be a lifting of $\gamma$. By concatenating $w$ with $\bigcup_{\sigma \leq s} \gamma_{\sigma}$ to obtain $w_{s}: D^{2} \rightarrow M$ such that $\left.w_{s}\right|_{\partial D^{2}}=\gamma_{s}$. We now define

$$
I_{s}([\gamma, w])=\left[\gamma_{s}, w_{s}\right] .
$$

The following is proved in [Sc2, Proposition 3.1] for the symplectically aspherical case and in Oh2] in general. The following proof is borrowed from Oh2]. 
Proposition 10.1. Suppose that each $H^{s}$ is normalized and satisfies (10.1). Then we have

$$
\mathcal{A}_{H^{s}}\left(I_{s}([\gamma, w])\right)=\mathcal{A}_{H^{0}}([\gamma, w])
$$

for all $s \in[0,1]$.

Proof. To prove the equality, it is enough to prove

$$
\frac{d}{d s} \mathcal{A}_{H^{s}}\left(I_{s}([\gamma, w])\right)=0
$$

for all $s \in[0,1]$.

Note that $\mathcal{A}_{H^{0}}\left(I_{0}([\gamma, w])\right)=\mathcal{A}_{H^{0}}([\gamma, w])$. Denote $H=H(s, t, x):=H^{s}(t, x)$ and denote by $K=K(s, t, x)$ the normalized Hamiltonian generating the vector field

$$
\frac{\partial \phi_{H^{s}}^{t}}{\partial s} \circ\left(\phi_{H^{s}}^{t}\right)^{-1}=: X_{K}
$$

in $s$-direction. We compute

$$
\frac{d}{d s} \mathcal{A}_{H^{s}}\left(I_{s}([\gamma, w])\right)=\left(d \mathcal{A}_{H^{s}}\left(I_{s}([\gamma, w])\right)\right)\left(\frac{d}{d s} I_{s}([\gamma, w])\right)-\int_{0}^{1} \frac{\partial H}{\partial s}\left(s, t, \gamma_{s}(t)\right) d t .
$$

Using that $I_{s}([\gamma, w]) \in \operatorname{Crit} \mathcal{A}_{H^{s}}$, this reduces to

$$
\frac{d}{d s} \mathcal{A}_{H^{s}}\left(I_{s}([\gamma, w])\right)=-\int_{0}^{1} \frac{\partial H}{\partial s}\left(s, t, \gamma_{s}(t)\right) d t
$$

By (10.1), we have

$$
X_{K}(s, 1, x)=0=X_{K}(s, 0, x)
$$

which implies $d K_{s, 1} \equiv 0$. Therefore $K_{s, 1} \equiv c(s)$ where $c:[0,1] \rightarrow \mathbb{R}$ is a function of $s$ alone. Then by the normalization condition, we obtain

$$
K_{s, 1} \equiv 0 \equiv K_{s, 0} .
$$

\section{Lemma 10.2.}

$$
\frac{\partial H}{\partial s}\left(s, t, \phi_{H^{s}}^{t}(p)\right)=\frac{\partial}{\partial t}\left(K\left(s, t, \phi_{H^{s}}^{t}(x)(p)\right)\right) .
$$

Proof. The following is proved

$$
\frac{\partial K}{\partial t}-\frac{\partial H}{\partial s}-\{H, K\}=0
$$

in [Ba1, Proposition I.1.1] for normalized family $H^{s}$. By rewriting this into

$$
\frac{\partial K}{\partial t}+\{K, H\}=\frac{\partial H}{\partial s}
$$

and recalling the definition

$$
\{K, H\}=\omega\left(X_{K}, X_{H}\right)=d K\left(X_{H}\right)
$$

of the Poisson bracket (in our convention), it is easy to check that this condition is equivalent to (10.7). Here the exterior differential and the Poisson bracket are taken over $M$ for each fixed $(s, t)$. 
Therefore we obtain

$$
\begin{aligned}
\int_{0}^{1} \frac{\partial H}{\partial s}\left(s, t, \gamma_{s}(t)\right) d t & =\int_{0}^{1} \frac{\partial H}{\partial s}\left(s, t, \phi_{H^{s}}^{t}(p)\right) d t \\
& =\int_{0}^{1} \frac{\partial}{\partial t}\left(K\left(s, t, \phi_{H^{s}}^{t}(p)\right)\right) d t \\
& =K\left(s, 1, \phi_{H^{s}}^{1}(p)\right)-K\left(s, 0, \phi_{H^{s}}^{0}(p)\right) \\
& =K(s, 1, \psi(p))-K(s, 0, p)=0
\end{aligned}
$$

where the last equality comes from (10.6). Substituting this into (10.5), we have finished the proof.

The following corollary is immediate.

Corollary 10.3. $\operatorname{Spec}\left(H^{0}\right)=\operatorname{Spec}\left(H^{s}\right)$. Moreover $\operatorname{Spec}\left(H^{0} ; \mathfrak{b}\right)=\operatorname{Spec}\left(H^{s} ; \mathfrak{b}\right)$.

The following lemma is proved for arbitrary $(M, \omega)$ by the second named author in $\mathrm{Oh} 3$. (The corresponding theorem in the aspherical case was proved in $\mathrm{Sc} 2$ generalizing a similar theorem in $\mathrm{HZ}$.)

Lemma 10.4. The set $\operatorname{Spec}(H)$ has measure zero for any periodic Hamiltonian $H$.

This, together with Lemma 10.4 and the fact that the $\operatorname{set} G(M, \omega, \mathfrak{b})$ is countable, implies

Corollary 10.5. $\operatorname{Spec}(H ; \mathfrak{b})$ has measure zero for any periodic Hamiltonian $H$ and $\mathfrak{b}$.

Proof of Theorem 7.7 (3). By Theorem 7.7 (1) which is proved in Section 9 the number $\rho^{\mathfrak{b}}\left(H^{s} ; a\right)$ is well-defined independent of the choices of $J$ and other choices such as abstract perturbations. By Theorem 9.1 the function $s \mapsto \rho^{\mathfrak{b}}\left(H^{s} ; a\right)$ is continuous. Moreover $\rho^{\mathfrak{b}}\left(H^{\rho} ; a\right)$ is contained in the set $\operatorname{Spec}\left(H^{s} ; \mathfrak{b}\right) \subset \mathbb{R}$ that is independent of $s$ and has Lebesgue measure 0. (This independence follows from Corollary 10.3) Therefore $s \mapsto \rho^{\mathfrak{b}}\left(H^{s} ; a\right)$ must be a constant function, as required.

Theorem 4.14 is a special case of Theorem 7.7 for $\mathfrak{b}=0$.

\section{Proof of the triangle inequality}

In this section we prove Theorem 7.8 (5). The proof is divided into several steps.

11.1. Pants products. In this subsection, we define a product structure of Floer homology of periodic Hamiltonian system. It is called the pants product. Let $J_{1}=\left\{J_{1, t}\right\}, J_{2}=\left\{J_{2, t}\right\}$ be two $S^{1}$-parameterized families of compatible almost complex structures on $M$. We assume that

$$
J_{1, t}=J_{2, t}=J_{0}, \quad \text { if } t \text { is in a neighborhood of }[1] \in S^{1} .
$$

Here $J_{0}$ is a certain compatible almost complex structure on $M$. We remark that we have already proved $J$-independence of the spectral invariant. So we may assume the above condition without loss of generality. (Actually we may also choose $J_{1, t}=$ $J_{2, t}=J_{0}$ without loss of generality. See Remark 3.1 (2).)

We next take time-dependent Hamiltonians $H_{1}, H_{2}$. After making the associated Hamiltonian isotopy constant neat $t=0,1$, we may assume

$$
H_{1, t}=H_{2, t}=0, \quad \text { if } t \text { is in a neighborhood of }[1] \in S^{1} .
$$


The pants product is defined by a chain map

$$
\begin{aligned}
\mathfrak{m}_{2}^{\mathrm{cl}}: C F\left(M, H_{1}, J_{1} ; \Lambda^{\downarrow}\right) & \otimes C F\left(M, H_{2}, J_{2} ; \Lambda^{\downarrow}\right) \\
& \rightarrow C F\left(M, H_{1} * H_{2}, J_{1} * J_{2} ; \Lambda^{\downarrow}\right)
\end{aligned}
$$

where

$$
\left(H_{1} * H_{2}\right)(t, x)= \begin{cases}2 H_{1}(2 t, x) & t \leq 1 / 2 \\ 2 H_{2}(2 t-1, x) & t \geq 1 / 2\end{cases}
$$

and

$$
\left(J_{1} * J_{2}\right)(t, x)= \begin{cases}J_{1}(2 t, x) & t \leq 1 / 2, \\ J_{2}(2 t-1, x) & t \geq 1 / 2 .\end{cases}
$$

Remark 11.1. Our definition of the product Hamiltonian $H_{1} * H_{2}$ is different from those used in $\mathrm{Sc2}, \mathrm{Oh} 4$. But the same definition is used in $\mathrm{ASc} 2$.

Remark 11.2. Note we include $J_{1}, J_{2}$ in the notation of $C F\left(M, H_{i}, J_{i} ; \Lambda^{\downarrow}\right)$, since the boundary operator depends on the almost complex structure $J_{i}$. (Its homology group $H F\left(M, H_{i}, J_{i} ; \Lambda^{\downarrow}\right)$ is independent of $J_{i}$.)

It is easy to see that $\psi_{H_{1} * H_{2}}=\psi_{H_{2}} \circ \psi_{H_{1}}$.

In the symplectically aspherical case, the detail of the construction (11.3) is written in [Sc1]. Its generalization to arbitrary symplectic manifold is rather immediate with the virtual fundamental chain technique in the framework of Kuranishi structure $[\mathrm{FO}$. We treat this construction for the general case here together with its generalization including bulk deformations.

Let $\Sigma=S^{2} \backslash\{3$ points $\}$. We choose a function $h: \Sigma \rightarrow \mathbb{R}$ with the following properties:

Condition 11.3. (1) It is proper.

(2) It is a Morse function with a unique critical point $z_{0}$ such that $h\left(z_{0}\right)=0$.

(3) For $\tau<0$, the preimage $h^{-1}(\tau)$ is a disjoint union of two $S^{1}$ 's, and for $\tau>0, h^{-1}(\tau)$ is one $S^{1}$.

See Figure 4. We fix a Riemannian metric on $\Sigma$ such that $\Sigma$ is isometric to the three copies of $S^{1} \times[0, \infty)$ outside a compact set. Let $\psi_{\nabla h}^{t}$ be the one parameter subgroup associated to the gradient vector field of $h$. We put

$$
\mathfrak{S}=\left\{z \in \Sigma \mid \lim _{t \rightarrow \infty} \psi_{\nabla h}^{t}(z)=z_{0}, \text { or } \lim _{t \rightarrow-\infty} \psi_{\nabla h}^{t}(z)=z_{0}\right\}
$$

i.e., the union of stable and unstable manifolds of $z_{0}$. Take a diffeomorphism

$$
\varphi: \mathbb{R} \times((0,1 / 2) \sqcup(1 / 2,1)) \rightarrow \Sigma \backslash \mathfrak{S}
$$

such that $h(\varphi(\tau, t))=\tau$ and put a complex structure $j_{\Sigma}$ on $\Sigma$ with respect to which $\varphi$ is conformal. Such a complex structure can be chosen by first pushing forward the standard one on $\mathbb{R} \times((0,1 / 2) \sqcup(1 / 2,1)) \subset \mathbb{C}$ and extending it to whole $\Sigma$. This choice of $\varphi$ and $j_{\Sigma}$ also provides the cylindrical ends near each puncture of $\Sigma$. See Figure 4.

We define a smooth function $H^{\varphi}: \Sigma \times M \rightarrow \mathbb{R}$ by:

$$
H^{\varphi}(\varphi(\tau, t), x)=\left(H_{1} * H_{2}\right)(t, x)
$$

on $\Sigma \backslash \mathfrak{S}$ and extending to $\mathfrak{S}$ by 0 . This is consistent with the assumption (11.2).

We define a $\Sigma$-parameterized family $J^{\varphi}$ of almost complex structures by

$$
J_{\varphi(\tau, t)}^{\varphi}=\left(J_{1} * J_{2}\right)_{t}
$$




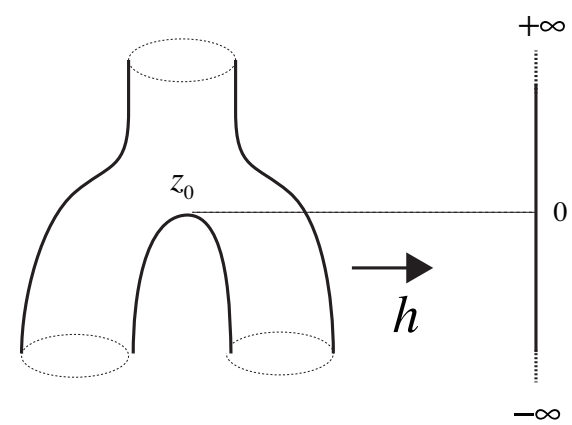

Figure 4. function $h$

first on $\Sigma \backslash \mathfrak{S}$. Note that the right hand side is $J_{0}$ in a neighborhood of $\mathfrak{S}$. Therefore we can trivially extend its definition across $\mathfrak{S}$ to whole $\Sigma$.

For $\tau<0$, we take the identification

$$
h^{-1}(\tau) \cong([0,1 / 2] / \sim) \sqcup([1 / 2,1] / \sim),
$$

where $0 \sim 1 / 2$ and $1 / 2 \sim 1$. Consider the natural diffeomorphisms

$$
\begin{aligned}
& \varphi_{1}: \quad([0,1 / 2] / \sim) \rightarrow([0,1] / \sim) ; t \mapsto 2 t \\
& \varphi_{2}:([1 / 2,1] / \sim) \rightarrow([0,1] / \sim) ; t \mapsto 2 t-1 .
\end{aligned}
$$

Then we have the identity

$$
\left(H_{1} * H_{2}\right) d t=\varphi_{i}^{*}\left(H_{i} d t\right), \quad i=1,2 .
$$

This can be easily seen from the definition of $H_{1} * H_{2}$.

Hereafter in this section, we assume that $H_{1}, H_{2}, H_{1} * H_{2}$ are all nondegenerate. Let $\left[\gamma_{1}, w_{1}\right] \in \operatorname{Crit}\left(\mathcal{A}_{H_{1}}\right),\left[\gamma_{2}, w_{2}\right] \in \operatorname{Crit}\left(\mathcal{A}_{H_{2}}\right)$ and $\left[\gamma_{3}, w_{3}\right] \in \operatorname{Crit}\left(\mathcal{A}_{H_{1} \# H_{2}}\right)$.

Definition 11.4. We denote by $\stackrel{\circ}{\mathcal{M}}_{\ell}\left(H^{\varphi}, J^{\varphi} ;\left[\gamma_{1} ; w_{1}\right],\left[\gamma_{2} ; w_{2}\right],\left[\gamma_{3}, w_{3}\right]\right)$ the set of all pairs $\left(u ; z_{1}^{+}, \ldots, z_{\ell}^{+}\right)$of maps $u: \Sigma \rightarrow M$ and marked points $z_{i}^{+} \in \Sigma$ that satisfy the following conditions:

(1) The map $\bar{u}=u \circ \varphi$ satisfies the equation:

$$
\frac{\partial \bar{u}}{\partial \tau}+J^{\varphi}\left(\frac{\partial \bar{u}}{\partial t}-X_{H^{\varphi}}(\bar{u})\right)=0
$$

(2) The energy

$$
E_{\left(H^{\varphi}, J^{\varphi}\right)}(u)=\frac{1}{2} \int\left(\left|\frac{\partial \bar{u}}{\partial \tau}\right|_{J^{\varphi}}^{2}+\left|\frac{\partial \bar{u}}{\partial t}-X_{H^{\varphi}}(\bar{u})\right|_{J^{\varphi}}^{2}\right) d t d \tau
$$

is finite.

(3) It satisfies the following three asymptotic boundary conditions:

$$
\begin{gathered}
\lim _{\tau \rightarrow+\infty} u(\varphi(\tau, t))=\gamma(t) . \\
\lim _{\tau \rightarrow-\infty} u(\varphi(\tau, t))= \begin{cases}\gamma_{1}(2 t) & t \leq 1 / 2, \\
\gamma_{2}(2 t-1) & t \geq 1 / 2 .\end{cases}
\end{gathered}
$$

(4) The homotopy class of $\left(w_{1} \sqcup w_{2}\right) \# u$ is $\left[w_{3}\right]$ in $\pi_{2}\left(\gamma_{3}\right)$. Here $\left(w_{1} \sqcup w_{2}\right) \# u$ is the obvious concatenation of $w_{1}, w_{2}$ and $u$.

(5) $z_{1}^{+}, \ldots, z_{\ell}^{+}$are mutually distinct. 
We denote by

$$
\mathrm{ev}=\left(\mathrm{ev}_{1}, \ldots, \mathrm{ev}_{\ell}\right): \stackrel{\circ}{\mathcal{M}}_{\ell}\left(H^{\varphi}, J^{\varphi} ;\left[\gamma_{1}, w_{1}\right],\left[\gamma_{2}, w_{2}\right],[\gamma, w]\right) \rightarrow M^{\ell}
$$

the evaluation map which associates the point $\left(u\left(z_{1}^{+}\right), \ldots, u\left(z_{\ell}^{+}\right)\right)$to $\left(u ; z_{1}^{+}, \ldots, z_{\ell}^{+}\right)$.

Remark 11.5. One can write the equation (11.8) in a more invariant fashion into the coordinate independent form

$$
\left(d u+P_{H \varphi}(u)\right)^{(0,1)}=0
$$

where $P_{H^{\varphi}}$ is a $u^{*}(T M)$-valued one form on $\Sigma$ and the $(0,1)$-part is taken with respect to $j_{\Sigma}(y)$ on $T_{y} \Sigma$ and $J^{\varphi}(u(y))$ on $T_{u(y)} M$ at each $y \in \Sigma$. In terms of $\varphi$, the pull-back $\varphi^{*}\left(P_{H^{\varphi}}\right)$ can be written as $\varphi^{*}\left(P_{H^{\varphi}}\right)=X_{H_{i}} d t, \quad i=1,2,3$ on the ends of $\Sigma$ near the punctures.

Now we have the following proposition that provides basic properties of the moduli space $\stackrel{\circ}{\mathcal{M}}_{\ell}\left(H^{\varphi}, J^{\varphi} ;\left[\gamma_{1}, w_{1}\right],\left[\gamma_{2}, w_{2}\right],\left[\gamma_{3}, w_{3}\right]\right)$.

For any $\alpha_{1}, \alpha_{2} \in \pi_{2}(M)$, there exists a canonical homeomorphism

$$
\begin{aligned}
& \stackrel{\circ}{\mathcal{M}}_{\ell}\left(H^{\varphi}, J^{\varphi} ;\left[\gamma_{1}, w_{1}\right],\left[\gamma_{2}, w_{2}\right],[\gamma, w]\right) \\
& \cong \stackrel{\circ}{\mathcal{M}}_{\ell}\left(H^{\varphi}, J^{\varphi} ;\left[\gamma_{1}, \alpha_{1} \# w_{1}\right],\left[\gamma_{2}, \alpha_{2} \# w_{2}\right],[\gamma, \alpha \# w]\right) .
\end{aligned}
$$

Here we put $\alpha=\alpha_{1}+\alpha_{2}$.

Proposition 11.6. (1) $\stackrel{\circ}{\mathcal{M}}_{\ell}\left(H^{\varphi}, J^{\varphi} ;\left[\gamma_{1}, w_{1}\right],\left[\gamma_{2}, w_{2}\right],\left[\gamma_{3}, w_{3}\right]\right)$ has a compactification $\mathcal{M}_{\ell}\left(H^{\varphi}, J^{\varphi} ;\left[\gamma_{1}, w_{1}\right],\left[\gamma_{2}, w_{2}\right],\left[\gamma_{3}, w_{3}\right]\right)$ that is Hausdorff.

(2) The space $\mathcal{M}_{\ell}\left(H^{\varphi}, J^{\varphi} ;\left[\gamma_{1}, w_{1}\right],\left[\gamma_{2}, w_{2}\right],\left[\gamma_{3}, w_{3}\right]\right)$ has an orientable Kuranishi structure with corners.

(3) The normalized boundary of $\mathcal{M}_{\ell}\left(H^{\varphi}, J^{\varphi} ;\left[\gamma_{1}, w_{1}\right],\left[\gamma_{2}, w_{2}\right],\left[\gamma_{3}, w_{3}\right]\right)$ is described by union of the following three types of direct products:

$\mathcal{M}_{\# \mathbb{L}_{1}}\left(H_{1}, J_{1} ;\left[\gamma_{1}, w_{1}\right],\left[\gamma_{1}^{\prime}, w_{1}^{\prime}\right]\right) \times \mathcal{M}_{\# \mathbb{L}_{2}}\left(H^{\varphi}, J^{\varphi} ;\left[\gamma_{1}^{\prime}, w_{1}^{\prime}\right],\left[\gamma_{2}, w_{2}\right],\left[\gamma_{3}, w_{3}\right]\right)$ where the union is taken over all $\left[\gamma_{1}^{\prime}, w_{1}^{\prime}\right] \in \operatorname{Crit}\left(H_{1}\right)$, and $\left(\mathbb{L}_{1}, \mathbb{L}_{2}\right) \in$ $\operatorname{Shuff}(\ell)$.

$\mathcal{M}_{\# \mathbb{L}_{1}}\left(H_{2}, J_{2} ;\left[\gamma_{2}, w_{2}\right],\left[\gamma_{2}^{\prime}, w_{2}^{\prime}\right]\right) \times \mathcal{M}_{\# \mathbb{L}_{2}}\left(H^{\varphi}, J^{\varphi} ;\left[\gamma_{1}, w_{1}\right],\left[\gamma_{2}^{\prime}, w_{2}^{\prime}\right],\left[\gamma_{3}, w_{3}\right]\right)$ where the union is taken over all $\left[\gamma_{2}^{\prime}, w_{2}^{\prime}\right] \in \operatorname{Crit}\left(H_{2}\right)$, and $\left(\mathbb{L}_{1}, \mathbb{L}_{2}\right) \in$ $\operatorname{Shuff}(\ell)$.

$$
\begin{aligned}
& \mathcal{M}_{\# \mathbb{L}_{1}}\left(H^{\varphi}, J^{\varphi} ;\left[\gamma_{1}, w_{1}\right],\left[\gamma_{2} ; w_{1}\right],\left[\gamma_{3}^{\prime}, w_{3}^{\prime}\right]\right) \\
& \times \mathcal{M}_{\# \mathbb{L}_{2}}\left(H_{1} * H_{2}, J_{1} * J_{2} ;\left[\gamma_{3}^{\prime}, w_{3}^{\prime}\right],\left[\gamma_{3}, w_{3}\right]\right)
\end{aligned}
$$

where the union is taken over all $\left[\gamma_{3}^{\prime}, w_{3}^{\prime}\right] \in \operatorname{Crit}\left(H_{1} * H_{2}\right)$, and $\left(\mathbb{L}_{1}, \mathbb{L}_{2}\right) \in$ $\operatorname{Shuff}(\ell)$.

(4) The (virtual) dimension is given by

$\operatorname{dim} \mathcal{M}_{\ell}\left(H^{\varphi}, J^{\varphi} ;\left[\gamma_{1}, w_{1}\right],\left[\gamma_{2}, w_{2}\right],\left[\gamma_{3}, w_{3}\right]\right)$

$=\mu_{H_{1} * H_{2}}\left(\left[\gamma_{3}, w_{3}\right]\right)-\mu_{H_{1}}\left(\left[\gamma_{1}, w_{1}\right]\right)-\mu_{H_{2}}\left(\left[\gamma_{2}, w_{2}\right]\right)+2 \ell-n$

where $\mu_{H}: \operatorname{Crit}\left(\mathcal{A}_{H}\right) \rightarrow \mathbb{Z}$ is the Conley-Zehnder index.

(5) There exists a system of orientations on the moduli spaces

$$
\mathcal{M}_{\ell}\left(H^{\varphi}, J^{\varphi} ;\left[\gamma_{1}, w_{1}\right],\left[\gamma_{2}, w_{2}\right],\left[\gamma_{3}, w_{3}\right]\right)
$$

such that the isomorphism (3) above is compatible with the orientations. 
(6) The map ev (11.9) extends to a strongly continuous smooth map

$$
\mathcal{M}_{\ell}\left(H^{\varphi}, J^{\varphi} ;\left[\gamma_{1}, w_{1}\right],\left[\gamma_{2}, w_{2}\right],\left[\gamma_{3}, w_{3}\right]\right) \rightarrow M^{\ell},
$$

which we denote also by $\mathrm{ev}$. The system of the evaluation maps is compatible with the boundary description (3) above.

(7) The homeomorphism (11.10) extends to the compactifications and their Kuranishi structures are identified by the homeomorphism.

The Figures 51617 below draw the elements corresponding to (11.11), (11.12), (11.13), respectively. The proof of Proposition 11.6 is the same as that of Propo-

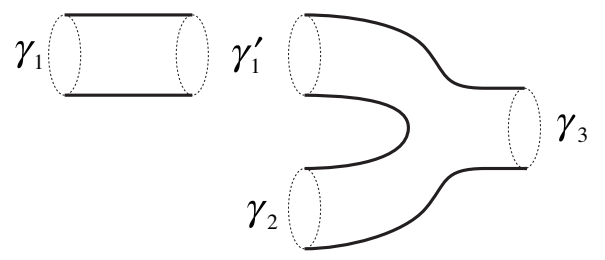

Figure 5. An element of (11.11)

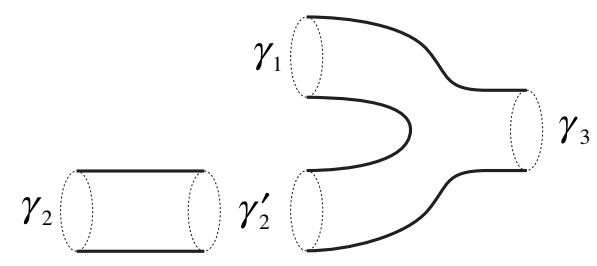

Figure 6. An element of (11.12)

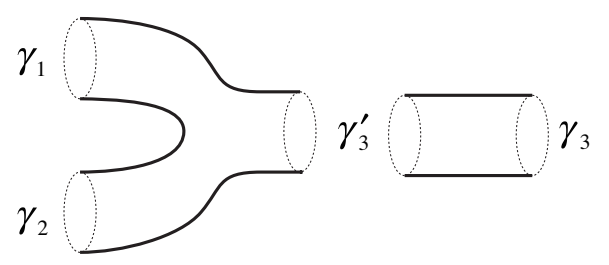

Figure 7. An element of (11.13)

sition 3.6 and so omitted. (Note that the domain of an element of the moduli space $\stackrel{\circ}{\mathcal{M}}_{\ell}\left(H^{\varphi}, J^{\varphi} ;\left[\gamma_{1}, w_{1}\right],\left[\gamma_{2}, w_{2}\right],\left[\gamma_{3}, w_{3}\right]\right)$ is a sphere with three punctures, which is stable. Therefore we do not need to add marked points thereto, in order to transport the element of the obstruction space to a nearby element. (See FOOO10, Definition 17.15].) The procedure of defining obstruction bundles of the factors such as $\mathcal{M}_{\# \mathbb{L}_{1}}\left(H_{1}, J_{1} ;\left[\gamma_{1}, w_{1}\right],\left[\gamma_{1}^{\prime}, w_{1}^{\prime}\right]\right)$ in (11.11) is determined in the course of the proof of Proposition 11.6, which is detailed in [FOOO10, Section 31]. Therefore the rest of the proof is the same as that of [FOOO10, Part 4].) 
Let $\mathfrak{b} \in H^{\text {even }}\left(M ; \Lambda_{0}\right)$ which we decompose into $\mathfrak{b}=\mathfrak{b}_{0}+\mathfrak{b}_{2}+\mathfrak{b}_{+}$as in (5.5). We choose their representative closed forms respectively as before. We then define the value $\mathfrak{n}_{H \varphi, J \varphi}^{\mathfrak{b}}\left(\left[\gamma_{1}, w_{1}\right],\left[\gamma_{2}, w_{2}\right],\left[\gamma_{3}, w_{3}\right]\right) \in \Lambda_{0}$ by

$$
\begin{aligned}
\mathfrak{n}_{H^{\varphi}, J \varphi}^{\mathfrak{b}} ; & \left(\left[\gamma_{1}, w_{1}\right],\left[\gamma_{2}, w_{2}\right],\left[\gamma_{3}, w_{3}\right]\right) \\
=\sum_{\ell=0}^{\infty} \frac{\exp \left(\int w_{3}^{*} \mathfrak{b}_{2}-\int w_{2}^{*} \mathfrak{b}_{2}-\int w_{1}^{*} \mathfrak{b}_{2}\right)}{\ell !} & \int_{\mathcal{M}_{\ell}\left(H^{\varphi}, J^{\varphi} ;\left[\gamma_{1}, w_{1}\right],\left[\gamma_{2}, w_{2}\right],\left[\gamma_{3}, w_{3}\right]\right)} \operatorname{ev}_{1}^{*} \mathfrak{b}_{+} \wedge \cdots \wedge \mathrm{ev}_{\ell}^{*} \mathfrak{b}_{+} \cdot
\end{aligned}
$$

We define a system of CF-perturbations on various $\mathcal{M}_{\ell}\left(H^{\varphi}, J^{\varphi} ;\left[\gamma_{1}, w_{1}\right],\left[\gamma_{2}, w_{2}\right],\left[\gamma_{3}, w_{3}\right]\right)$ that is compatible with the description of their boundaries given in Proposition 11.6 (3) and use the system to define the integration in the right hand side.

Definition 11.7. We put

$$
\begin{aligned}
& \mathfrak{m}_{2}^{\mathrm{cl}}\left(\llbracket \gamma_{1}, w_{1} \rrbracket \otimes \llbracket \gamma_{2}, w_{2} \rrbracket\right) \\
& =\sum_{\left[\gamma_{3}, w_{3}\right] \in \operatorname{Crit}\left(\mathcal{A}_{H_{1} * H_{2}}\right)} \mathfrak{n}_{H^{\varphi}, J \varphi}^{\mathfrak{b}}\left(\left[\gamma_{1}, w_{1}\right],\left[\gamma_{2}, w_{2}\right],\left[\gamma_{3}, w_{3}\right]\right) \llbracket \gamma_{3}, w_{3} \rrbracket .
\end{aligned}
$$

We can prove that the right hand side of (11.16) converges in $C F\left(\left(H_{1} * H_{2}, J_{2}\right) ; \Lambda^{\downarrow}\right)$ in the same way as the proof of Lemma 6.7. To define the right hand side of (11.16) we suppose that $\llbracket \gamma_{1}, w_{1} \rrbracket$ and $\llbracket \gamma_{2}, w_{2} \rrbracket$ are represented by $\left[\gamma_{1}, w_{1}\right] \in \operatorname{Crit}\left(\mathcal{A}_{H_{1}}\right)$ and $\left[\gamma_{2}, w_{2}\right] \in \operatorname{Crit}\left(\mathcal{A}_{H_{2}}\right)$, respectively, i.e., $\pi\left(\left[\gamma_{i}, w_{i}\right]\right)=\llbracket \gamma_{i}, w_{i} \rrbracket$. The independence of the right hand side of (11.16) of the the choice of the representatives follows from Proposition 11.6 (7). We have thus defined (11.3).

Lemma 11.8. The map $\mathfrak{m}_{2}^{\mathrm{cl}}$ satisfies

$$
\partial_{\left(H_{1} * H_{2}, J_{1} * J_{2}\right)}^{\mathfrak{b}} \circ \mathfrak{m}_{2}^{\mathrm{cl}}=\mathfrak{m}_{2}^{\mathrm{cl}} \circ\left(\partial_{\left(H_{1}, J_{1}\right)}^{\mathfrak{b}} \widehat{\otimes} 1+1 \widehat{\otimes} \partial_{\left(H_{2}, J_{2}\right)}^{\mathfrak{b}}\right) .
$$

Here $\widehat{\otimes}$ is the graded tensor product of the linear maps, which is given by

$$
(F \widehat{\otimes} G)(x \otimes y)=(-1)^{\operatorname{deg} G \operatorname{det}^{\prime} x} F(x) \otimes G(y) .
$$

Proof. This is a consequence of Proposition 11.6 (3), Stokes' theorem (Theorem 34.8) and composition formula (Theorem 35.7). In fact, the three boundary types, (11.11), (11.12), (11.13) correspond to the three operations, $\mathfrak{m}_{2}^{\mathrm{cl}} \circ \partial_{\left(H_{1}, J_{1}\right)}^{\mathfrak{b}} \widehat{\otimes} 1, \mathfrak{m}_{2}^{\mathrm{cl}} \circ$ $\partial_{\left(H_{2}, J_{2}\right)}^{\mathfrak{b}}$ and $\partial_{\left(H_{1} * H_{2}, J_{1} * J_{2}\right)}^{\mathfrak{b}} \circ \mathfrak{m}_{2}^{\mathrm{cl}}$, respectively.

Thus we have defined the pants product

$$
\begin{aligned}
\mathfrak{m}_{2}^{\mathrm{cl}}: H F\left(\left(H_{1}, J_{1}\right) ; \Lambda^{\downarrow}\right) & \otimes H F\left(M, H_{2}, J_{2} ; \Lambda^{\downarrow}\right) \\
& \rightarrow H F\left(M, H_{1} * H_{2}, J_{1} * J_{2} ; \Lambda^{\downarrow}\right) .
\end{aligned}
$$

The next proposition shows that it respects the filtration.

Proposition 11.9. For all $\lambda_{1}, \lambda_{2} \in \mathbb{R}$,

$$
\begin{aligned}
& \mathfrak{m}_{2}^{\mathrm{cl}}\left(F^{\lambda_{1}} C F\left(M, H_{1}, J_{1} ; \Lambda^{\downarrow}\right) \otimes F^{\lambda_{2}} C F\left(M, H_{2}, J_{2} ; \Lambda^{\downarrow}\right)\right) \\
\subseteq & F^{\lambda_{1}+\lambda_{2}} C F\left(M, H_{1} * H_{2}, J_{1} * J_{2} ; \Lambda^{\downarrow}\right) .
\end{aligned}
$$

Proof. We start with the following lemma 
Lemma 11.10. If $\mathcal{M}_{\ell}\left(H^{\varphi}, J^{\varphi} ;\left[\gamma_{1}, w_{1}\right],\left[\gamma_{2}, w_{2}\right],\left[\gamma_{3}, w_{3}\right]\right)$ is nonempty, then

$$
\mathcal{A}_{H_{1}}\left(\left[\gamma_{1}, w_{1}\right]\right)+\mathcal{A}_{H_{2}}\left(\left[\gamma_{2}, w_{2}\right]\right) \geq \mathcal{A}_{H_{1} * H_{2}}\left(\left[\gamma_{3}, w_{3}\right]\right) \text {. }
$$

Proof. Let $\left(u ; z_{1}^{+}, \ldots, z_{k}^{+}\right) \in \stackrel{\circ}{\mathcal{M}}_{\ell}\left(H^{\varphi}, J^{\varphi} ;\left[\gamma_{1}, w_{1}\right],\left[\gamma_{2}, w_{2}\right],\left[\gamma_{3}, w_{3}\right]\right)$ and $\tau_{0}<0$. We identify

$$
h^{-1}\left(\tau_{0}\right)=S_{1}^{1} \sqcup S_{2}^{1} .
$$

We denote the restriction of $u$ to $S_{1}^{1}$ by $\gamma_{1}^{\tau_{0}}$ and the restriction of $u$ to $S_{2}^{1}$ by $\gamma_{2}^{\tau_{0}}$.

We concatenate $w_{1}$ with $\cup_{\tau \leq \tau_{0}} \gamma_{1}^{\tau_{0}}$ to obtain $w_{1}^{\tau_{0}}$ which bounds $\gamma_{1}^{\tau_{0}}$. We define $w_{2}^{\tau_{0}}$ in the same way.

By the same way as in Lemma 3.10, we derive

$$
\mathcal{A}_{H_{1}}\left(\left[\gamma_{1}, w_{1}\right]\right) \geq \mathcal{A}_{H_{1}}\left(\left[\gamma_{1}^{\tau_{0}}, w_{1}^{\tau_{0}}\right]\right), \quad \mathcal{A}_{H_{2}}\left(\left[\gamma_{2}, w_{2}\right]\right) \geq \mathcal{A}_{H_{2}}\left(\left[\gamma_{2}^{\tau_{0}}, w_{2}^{\tau_{0}}\right]\right) .
$$

Next let $\tau_{0}>0$. We denote the restriction of $u$ to $h^{-1}\left(\tau_{0}\right)$ by $\gamma^{\tau_{0}}$. We concatenate $w_{1} \sqcup w_{2}$ and the restriction of $u$ to $\left\{z \in \Sigma \mid h(z) \leq \tau_{0}\right\}$ to obtain $w^{\tau_{0}}$. By the same way as in Lemma 3.10, we also derive

$$
\mathcal{A}_{H_{1} * H_{2}}\left(\left[\gamma^{\tau_{0}}, w^{\tau_{0}}\right]\right) \geq \mathcal{A}_{H_{1} * H_{2}}\left(\left[\gamma_{3}, w_{3}\right]\right) \text {. }
$$

It follows easily from definition that

$$
\lim _{\tau_{0} \rightarrow 0}\left(\mathcal{A}_{H_{1}}\left(\left[\gamma_{1}^{\tau_{0}}, w_{1}^{\tau_{0}}\right]\right)+\mathcal{A}_{H_{2}}\left(\left[\gamma_{2}^{\tau_{0}}, w_{2}^{\tau_{0}}\right]\right)\right)=\lim _{\tau_{0} \rightarrow 0} \mathcal{A}_{H_{1} * H_{2}}\left(\left[\gamma^{\tau_{0}}, w^{\tau_{0}}\right]\right) .
$$

Lemma 11.10 follows easily from (11.18), (11.19), (11.20).

Proposition 11.9 follows immediately from Lemma 11.10 .

11.2. Multiplicative property of Piunikhin isomorphism. In this subsection, we prove that the Piunikhin isomorphism interpolates the quantum product $\cup_{\mathfrak{b}}$ of $Q H$ and the $\mathfrak{b}$-deformed pants product of $H F$.

Let $\chi: \mathbb{R} \rightarrow[0,1]$ be as in Definition 3.13, For each $S \in \mathbb{R}$, we define

$$
H_{S}^{\varphi}(z, x)=\chi(h(z)+S) H^{\varphi}(z, x)
$$

where $H^{\varphi}$ is as in (11.6). Similarly we define a family $J_{S}^{\varphi}(z)$ so that

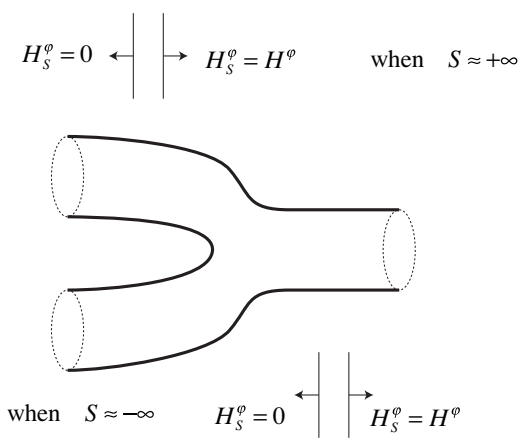

Figure 8. Function $H_{S}^{\varphi}$

$$
J_{S}^{\varphi}(\varphi(\tau, t))=J^{\varphi(\tau, t)}(\varphi(\tau+S, t)) .
$$

Due to the condition $J_{t} \equiv J_{0}$ near $t=0$, this definition smoothly extends to whole $\Sigma$.

With this preparation, we prove the following: 
Theorem 11.11. For $a_{1}, a_{2} \in H^{*}(M ; \Lambda) \backslash\{0\}$, we have

$$
\mathfrak{m}_{2}^{\mathrm{cl}}\left(\mathcal{P}_{\left(\left(H_{1}\right)_{\chi},\left(J_{1}\right)_{\chi}\right), *}\left(a_{1}^{b}\right), \mathcal{P}_{\left(\left(H_{2}\right)_{\chi},\left(J_{2}\right)_{\chi}\right), *}\left(a_{2}^{b}\right)\right)=\mathcal{P}_{\left(\left(H_{1} * H_{2}\right)_{\chi},\left(J_{1} * J_{2}\right)_{\chi}\right), *}\left(\left(a_{1} \cup^{\mathfrak{b}} a_{2}\right)^{b}\right) .
$$

Proof. Let $[\gamma, w] \in \operatorname{Crit}\left(\mathcal{A}_{H_{1} * H_{2}}\right)$.

Definition 11.12. We denote by $\mathcal{M}_{\ell}\left(H_{S}^{\varphi}, J_{S}^{\varphi} ; * *,[\gamma, w]\right)$ the set of all pairs $\left(u ; z_{1}^{+}, \ldots, z_{\ell}^{+}\right)$ of maps $u: \Sigma \rightarrow M$ and marked points $z_{i}^{+} \in \Sigma$, which satisfy the following conditions:

(1) The map $\bar{u}:=u \circ \varphi$ satisfies

$$
\frac{\partial \bar{u}}{\partial \tau}+J_{S}^{\varphi}\left(\frac{\partial \bar{u}}{\partial t}-X_{H_{S}^{\varphi}}(\bar{u})\right)=0
$$

(2) The energy

$$
E_{\left(H_{S}^{\varphi}, J_{S}^{\varphi}\right)}=\frac{1}{2} \int\left(\left|\frac{d \bar{u}}{d \tau}\right|_{J_{S}^{\varphi}}^{2}+\left|\frac{\partial \bar{u}}{\partial t}-X_{H_{S}^{\varphi}}(\bar{u})\right|_{J_{S}^{\varphi}}^{2}\right) d t d \tau
$$

is finite.

(3) It satisfies the following asymptotic boundary condition:

$$
\lim _{\tau \rightarrow+\infty} u(\varphi(\tau, t))=\gamma(t) .
$$

(4) Let $[u]$ be the homotopy class of $u$ in $\pi_{2}(\gamma)$. Then $[u]=[w]$.

(5) $z_{1}^{+}, \ldots, z_{\ell}^{+}$are mutually distinct.

Again here as in Remark 3.16 we put the $* *$ in the notation of the moduli space to highlight the fact that the domain curve carries two negative punctures and one positive puncture at which the asymptotic boundary condition $[\gamma, w]$ is assigned.

We note that (11.21) and the finiteness of energy imply that there exist $p_{1}, p_{2} \in$ $M$ such that

$$
\lim _{\tau \rightarrow-\infty} u(\varphi(\tau, t))= \begin{cases}p_{1} & t<1 / 2 \\ p_{2} & t>1 / 2\end{cases}
$$

Therefore the homotopy class of $u$ in $\pi_{2}(\gamma)$ is defined.

We define the evaluation map

$$
\mathrm{ev}_{-\infty}=\left(\mathrm{ev}_{-\infty, 1}, \mathrm{ev}_{-\infty, 2}\right): \stackrel{\circ}{\mathcal{M}}_{\ell}\left(H_{S}^{\varphi}, J_{S}^{\varphi} ; * *,[\gamma, w]\right) \rightarrow M^{2}
$$

by $\mathrm{ev}_{-\infty}(u)=\left(p_{1}, p_{2}\right)$ where $p_{1}, p_{2}$ are as in (11.22), and

$$
\mathrm{ev}=\left(\mathrm{ev}_{1}, \ldots, \mathrm{ev}_{\ell}\right): \stackrel{\circ}{\mathcal{M}}_{\ell}\left(H_{S}^{\varphi}, J_{S}^{\varphi} ; * *,[\gamma, w]\right) \rightarrow M^{\ell}
$$

by ev $\left(u ; z_{1}^{+}, \ldots, z_{\ell}^{+}\right)=\left(u\left(z_{1}^{+}\right), \ldots, u\left(z_{\ell}^{+}\right)\right)$.

We put

$$
\stackrel{\circ}{\mathcal{M}}_{\ell}\left(\operatorname{para} ; H^{\varphi}, J^{\varphi} ; * *,[\gamma, w]\right)=\bigcup_{S \in \mathbb{R}}\{S\} \times \stackrel{\circ}{\mathcal{M}}_{\ell}\left(H_{S}^{\varphi}, J_{S}^{\varphi} ; * *,[\gamma, w]\right) .
$$

The evaluation maps $\mathrm{ev}_{-\infty}$ and ev are defined on them in an obvious way.

Proposition 11.13. (1) The moduli space $\stackrel{\circ}{\mathcal{M}}_{\ell}\left(\right.$ para $\left.; H^{\varphi}, J^{\varphi} ; * *,[\gamma, w]\right)$ has a compactification $\mathcal{M}_{\ell}\left(\right.$ para $\left.; H^{\varphi}, J^{\varphi} ; * *,[\gamma, w]\right)$ that is Hausdorff.

(2) The space $\mathcal{M}_{\ell}\left(\right.$ para $\left.; H^{\varphi}, J^{\varphi} ; * *,[\gamma, w]\right)$ has an orientable Kuranishi structure with boundary and corners. 
(3) The normalized boundary of $\mathcal{M}_{\ell}\left(\right.$ para; $\left.H^{\varphi}, J^{\varphi} ; * *,[\gamma, w]\right)$ is described by the union of following three types of direct or fiber products: The first one is

$\mathcal{M}_{\# \mathbb{L}_{1}}\left(\right.$ para $\left.; H^{\varphi}, J^{\varphi} ; * *,\left[\gamma^{\prime}, w^{\prime}\right]\right) \times \mathcal{M}_{\# \mathbb{L}_{2}}\left(H_{1} * H_{2}, J_{1} * J_{2} ;\left[\gamma^{\prime}, w^{\prime}\right],[\gamma, w]\right)$

where the union is taken over all $\left[\gamma^{\prime}, w^{\prime}\right] \in \operatorname{Crit}\left(H_{1} * H_{2}\right)$ and $\left(\mathbb{L}_{1}, \mathbb{L}_{2}\right) \in$ $\operatorname{Shuff}(\ell)$, which corresponds to splitting-off a Floer trajectory at some finite parameters $S$. (Figure 9)

The second one corresponds to $S=-\infty$ which is

$\mathcal{M}_{3+\# \mathbb{L}_{1}}^{\mathrm{cl}}\left(\alpha ; J_{0}\right)_{\mathrm{ev}} \times_{\mathrm{ev}_{-\infty}} \mathcal{M}_{\# \mathbb{L}_{2}}\left(\left(H_{1} * H_{2}\right)_{\chi},\left(J_{1} * J_{2}\right)_{\chi} ; *,\left[\gamma^{\prime} ; w^{\prime}\right]\right)$.

Here $\mathcal{M}_{3+\# \mathbb{L}_{1}}^{\mathrm{cl}}\left(\alpha ; J_{0}\right)$ is as defined in Section 5 . The union is taken over all $\left(\mathbb{L}_{1}, \mathbb{L}_{2}\right) \in \operatorname{Shuff}(\ell)$ and $\alpha, w^{\prime}$ such that the obvious concatenation $\alpha \# w^{\prime}$ is homotopic to $w$ the fiber product is taken over M. (Figure 10) (See Definition 32.5 for the definition of fiber product of Kuranishi structure.)

The third type corresponds to $S=\infty$ which is

$$
\begin{array}{r}
\left(\mathcal{M}_{\# \mathbb{L}_{1}}\left(\left(H_{1}\right)_{\chi},\left(J_{1}\right)_{\chi} ; *,\left[\gamma_{1}, w_{1}\right]\right) \times \mathcal{M}_{\# \mathbb{L}_{2}}\left(\left(H_{2}\right)_{\chi},\left(J_{2}\right)_{\chi} ; *,\left[\gamma_{2}, w_{2}\right]\right)\right) \\
\times \mathcal{M}_{\mathbb{H}_{3}}\left(H^{\varphi}, J^{\varphi} ;\left[\gamma_{1}, w_{1}\right],\left[\gamma_{2}, w_{2}\right],[\gamma, w]\right) .
\end{array}
$$

where the union is taken over all $\left(\mathbb{L}_{1}, \mathbb{L}_{2}, \mathbb{L}_{3}\right)$ the triple shuffle of $\{1, \ldots, \ell\}$, and $\left[\gamma_{1}, w_{1}\right] \in \operatorname{Crit}\left(\mathcal{A}_{H_{1}}\right),\left[\gamma_{2}, w_{2}\right] \in \operatorname{Crit}\left(\mathcal{A}_{H_{2}}\right)$. (Figure 11)

(4) The (virtual) dimension is given by

$$
\operatorname{dim} \mathcal{M}_{\ell}\left(\text { para } ; H^{\varphi}, J^{\varphi} ; * *,[\gamma, w]\right)=\mu_{H_{1} * H_{2}}([\gamma, w])+2 \ell+1+n .
$$

(5) We can define a system of orientations on the collection of moduli spaces $\mathcal{M}_{\ell}\left(\right.$ para $\left.; H^{\varphi}, J^{\varphi} ; * *,[\gamma, w]\right)$ that is compatible with the isomorphism (3) above.

(6) The map ev on extends to a strongly continuous smooth map

$$
\mathcal{M}_{\ell}\left(\text { para } ; H^{\varphi}, J^{\varphi} ; * *,[\gamma, w]\right) \rightarrow M^{\ell},
$$

which we denote also by ev. The map is compatible with the boundary description (3) thereof.

(7) The map $\mathrm{ev}_{-\infty}$ defined above on $\stackrel{\mathcal{M}}{\ell}_{\ell}\left(\right.$ para $\left.; H^{\varphi}, J^{\varphi} ; * *,[\gamma, w]\right)$ extends also to a strongly continuous smooth map

$$
\mathcal{M}_{\ell}\left(\text { para } ; H^{\varphi}, J^{\varphi} ; * *,[\gamma, w]\right) \rightarrow M^{2},
$$

which we denote by $\mathrm{ev}_{-\infty}$. The map is compatible with the boundary description (3) thereof.

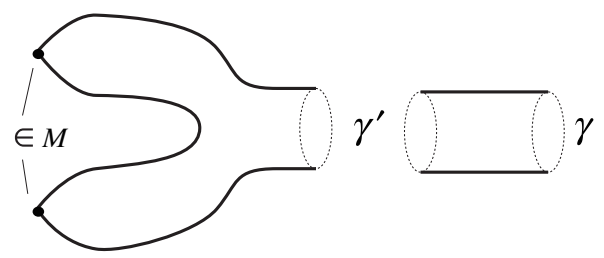

Figure 9. An element of (11.24) 


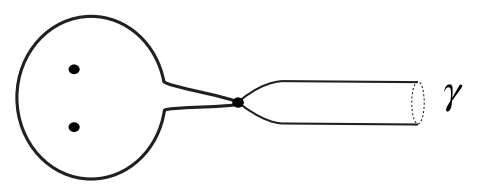

Figure 10. An element of (11.25)

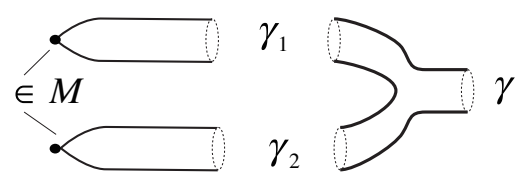

Figure 11. An element of (11.26)

Proof. The proof is the same as other similar statements appearing in this and several other previous papers, such as [FO, FOOO1, [FOOO2, [FOOO10. So it suffices to see how the boundary of our moduli space appears as in (3).

For each fixed $S$ the boundary of $\mathcal{M}_{\ell}\left(H_{S}^{\varphi}, J_{S}^{\varphi} ; * *,[\gamma, w]\right)$ is described by (11.24), with para being replaced by $S$. We note that there is a 'splitting end' where 'bubble' occurs at $\tau \rightarrow \infty$. See Figure 9 ,

The case $S \rightarrow-\infty$ is described by (11.25). We can prove the claim as follows. We recall that $\lim _{S \rightarrow-\infty}\left(H_{S}^{\varphi}, J_{S}^{\varphi}\right)=\left(0, J_{0}\right)$ where $J_{0}$ is time-independent. We also remark that the moduli space $\mathcal{M}_{3+\ell}^{\mathrm{cl}}\left(\alpha ; J_{0}\right)$ is identified with the moduli space of $\left(u ; z_{1}^{+}, \ldots, z_{\ell}^{+}\right)$such that the composition $\bar{u}=u \circ \varphi$ satisfies the equation:

$$
\frac{\partial \bar{u}}{\partial \tau}+J_{0}\left(\frac{\partial \bar{u}}{\partial t}\right)=0
$$

and $\int u^{*} \omega<\infty,[u]=\alpha$ in $H_{2}(M)$. Therefore the 'bubble' which slides to $\tau \rightarrow-\infty$ is described by $\mathcal{M}_{3+\ell}^{\mathrm{cl}}\left(\alpha ; J_{0}\right)$. (See Figure 10)

The other potential 'splitting end' where 'bubble' occurs at $\tau \rightarrow-\infty$ has codimension two and do not appear here. (This is because of the $S^{1}$ symmetry present on such a bubble.)

Finally the case $S \rightarrow+\infty$ is described by (11.26). (See Figure 11,)

To use $\mathcal{M}_{\ell}\left(\right.$ para $\left.; H^{\varphi}, J^{\varphi} ; * *,[\gamma, w]\right)$ to define an appropriate chain homotopy map we need to find a perturbation on it that is compatible with the description of its boundary given in Proposition 11.13 (3). Since (11.25) involves fiber product we need to find a perturbation so that $\mathrm{ev}_{3}$ is a submersion on the perturbed moduli space. We need to use a continuous family of perturbations (CF perturbation) for this purpose. The detail of the process of constructing such perturbations is given in [FOOO4, Section 12], [FOOO11, etc. In [FOOO4, Section 12] this notion is called continuous family of multisections. In [FOOO11, Definition 7.47] where thorough detail of this construction is given, we call it a CF-perturbation. In this paper we use the name of FOOO11. See Part 7 Definition 33.8 for the definition of CF-perturbation.

We regard $\mathcal{M}_{3+\ell}^{\mathrm{cl}}\left(\alpha ; J_{0}\right)$ as the compactified moduli space of the pair $\left(u ; z_{1}^{+}, \ldots, z_{\ell}^{+}\right)$ satisfying (11.28) etc. Then we have a CF-perturbation $\widehat{\mathcal{S}^{\epsilon}}$ on certain good coordinate system compatible with the Kuranishi structure in Proposition 11.13, (See 
Definition 31.9 for the definition of good coordinate system and Definition 33.8 for the definition of CF-perturbation. Existence of a good coordinate system is proved as Theorem 31.11, [FOOO11, Theorem 3.30] and existence of a CF-perturbation is proved as Theorem 33.11, [FOOO11, Theorem 7.49].)

Here we use the evaluation maps at the $1,2,4, \ldots,(\ell+3)$-th marked points to receive an 'input' and the evaluation map at the 3-rd marked point to give out an 'output'. This gives rise to a map $\operatorname{Corr}\left(\mathcal{M}_{3+\ell}^{\text {cl }}\left(\alpha ; J_{0}\right)\right): \Omega(M)^{\otimes(2+\ell)} \rightarrow \Omega(M)$ defined by

$$
\begin{aligned}
& \operatorname{Corr}\left(\mathcal{M}_{3+\ell}^{\mathrm{cl}}\left(\alpha ; J_{0}\right)\right)\left(h_{1}, h_{2}, h_{3}, \ldots, h_{\ell+2}\right) \\
& =\mathrm{ev}_{3} !\left(\left(\mathrm{ev}_{1} \times \mathrm{ev}_{2} \times \mathrm{ev}_{4} \times \cdots \times \mathrm{ev}_{\ell+3}\right)^{*}\left(h_{1}, h_{2}, h_{3}, \ldots, h_{\ell+2}\right) ; \widehat{\mathfrak{S}}^{\epsilon}\right),
\end{aligned}
$$

where $\left(\mathrm{ev}_{3}\right)$ ! is the integration along the fibers of $\mathcal{M}_{3+\ell}^{\mathrm{cl}}\left(\alpha ; J_{0}\right)$ under the evaluation map ev ev $_{3} \mathcal{M}_{3+\ell}^{\mathrm{cl}}\left(\alpha ; J_{0}\right) \rightarrow M$, which is defined via a choice of CF-perturbation $\widehat{\mathfrak{S}}^{\epsilon}$. See [FOOO11, Definition 7.78] and Definition 33.18, The map depends on the choice of a CF-perturbation and $\epsilon$ on the chain level but independent in the homology level. (See [FOOO11, Proposition 8.15].) We extend the above assignment $\operatorname{Corr}\left(\mathcal{M}_{3+\ell}^{\mathrm{cl}}\left(\alpha ; J_{0}\right)\right)$ to a $\Lambda$-multi-linear map $\operatorname{Corr}\left(\mathcal{M}_{3+\ell}^{\mathrm{cl}}\left(\alpha ; J_{0}\right)\right):(\Omega(M) \widehat{\otimes} \Lambda)^{\otimes(2+\ell)} \rightarrow$ $\Omega(M) \widehat{\otimes} \Lambda$ in the obvious way.

Let $\mathfrak{b} \in H^{\text {even }}\left(M ; \Lambda_{0}\right)$ and decompose $\mathfrak{b}=\mathfrak{b}_{0}+\mathfrak{b}_{2}+\mathfrak{b}_{+}$as in (5.5). We also regard them as differential forms by the same kind of abuse of notations. Let $a_{1}, a_{2} \in \Omega(M)$. We put

$$
\mathfrak{g}_{2 ; \alpha}^{\mathrm{cl}}\left(a_{1}, a_{2}\right)=\sum_{\ell} \frac{\exp \left(\mathfrak{b}_{2} \cap \alpha\right)}{\ell !} \operatorname{Corr}\left(\mathcal{M}_{3+\ell}^{\mathrm{cl}}\left(\alpha ; J_{0}\right)\right)\left(a_{1}, a_{2}, \mathfrak{b}_{+}, \ldots, \mathfrak{b}_{+}\right) .
$$

We then define

$$
\mathfrak{g w}_{2}\left(a_{1}, a_{2}\right)=\sum_{\alpha} T^{\alpha \cap \omega} \mathfrak{g w}_{2 ; \alpha}\left(a_{1}, a_{2}\right) .
$$

We can easily prove that the right hand side of (11.29) converges in $\Omega(M) \widehat{\otimes} \Lambda$. Using the fact that Gromov-Witten invariant is well-defined in the homology level (this follows from the fact that $\mathcal{M}_{3+\ell}^{\mathrm{cl}}\left(\alpha ; J_{0}\right)$ has a Kuranishi structure without boundary), we can easily show that $\mathfrak{g w}_{2}$ induces a product map $\cup^{\mathfrak{b}}$ in the cohomology level.

We now go back to the study of the moduli space $\mathcal{M}_{\ell}\left(\right.$ para $\left.; H^{\varphi}, J^{\varphi} ; * *,[\gamma, w]\right)$. We will need to construct a system of CF-perturbations thereon inductively over the energy the explanation of which is in order. We note that we have already defined CF-perturbations of the moduli spaces which appear in the right hand of Proposition 11.13 (3). The fiber product in (11.25) induces a fiber product of our CF-perturbations in the sense of [FOOO11, Definition 10.3], since $\mathrm{ev}_{3}$ is strongly submersive with respect to our CF-perturbation in the sense of Definition 33.9.

Other products appearing in (11.24) and (11.26) are direct products so the perturbation of each of the factors immediately induce one on the product. Thus we have defined a CF-perturbation on the boundary. It is compatible at the corners by the inductive construction of CF-perturbations. Therefore we can extend the given CF-perturbation on the boundary to the whole $\mathcal{M}_{\ell}\left(\right.$ para $\left.; H^{\varphi}, J^{\varphi} ; * *,[\gamma, w]\right)$ by the general theory of Kuranishi structure. We use this CF-perturbation to define the integration on these moduli spaces given below. 
We now use our CF-perturbations to define an integration along the fibers and put

$$
\begin{aligned}
& \mathfrak{n}_{a_{1}, a_{2}, \mathfrak{b}, \text { para } ; H^{\varphi}, J^{\varphi} ;[\gamma, w]} \\
& =\sum_{\ell} \frac{\exp \left(\mathfrak{b}_{2} \cap \alpha\right)}{\ell !} \int_{\mathcal{M}_{\ell}\left(\text { para } ; H^{\varphi}, J \varphi ;[\gamma, w]\right)} \operatorname{ev}_{-\infty}^{*}\left(a_{1}, a_{2}\right) \wedge \operatorname{ev}^{*}\left(\mathfrak{b}_{+} \wedge \cdots \wedge \mathfrak{b}_{+}\right) .
\end{aligned}
$$

\section{Definition 11.14.}

$$
\mathfrak{H}_{H^{\varphi}, J^{\varphi}}^{\mathfrak{b}}\left(a_{1}, a_{2}\right)=\sum_{[\gamma, w] \in \operatorname{Crit}\left(\mathcal{A}_{H_{1} * H_{2}}\right)} \mathfrak{n}_{a_{1}, a_{2}, \mathfrak{b}, \text { para } ; H^{\varphi}, J^{\varphi} ;[\gamma, w]} \llbracket \gamma, w \rrbracket .
$$

We can prove that the right hand side converges in $C F\left(M, H_{1} * H_{2}, J_{1} * J_{2} ; \Lambda\right)$ in the same way as in the proof of convergence of the right hand side of (3.7).

Lemma 11.15. We have

$$
\begin{aligned}
& \partial_{\left(\left(H_{1} * H_{2}\right)_{\chi},\left(J_{1} * J_{2}\right)_{\chi}\right)}^{\mathfrak{b}} \circ \mathfrak{H}_{H^{\varphi}, J^{\varphi}}^{\mathfrak{b}}+\mathfrak{H}_{H^{\varphi}, J^{\varphi}}^{\mathfrak{b}}(\partial \widehat{\otimes} 1+1 \widehat{\otimes} \partial) \\
& =\mathcal{P}_{\left(\left(H_{1} * H_{2}\right)_{\chi} ;\left(J_{1} * J_{2}\right)_{\chi}\right)}^{\mathfrak{b}} \circ \mathfrak{g w}_{2}-\mathfrak{m}_{2}^{\mathrm{cl}} \circ\left(\mathcal{P}_{\left(\left(H_{1}\right)_{\chi} ;\left(J_{1}\right)_{\chi}\right)}^{\mathfrak{b}} \otimes \mathcal{P}_{\left(\left(H_{2}\right)_{\chi} ;\left(J_{2}\right)_{\chi}\right)}^{\mathfrak{b}}\right) .
\end{aligned}
$$

Proof. Using the description of the moduli spaces given in Proposition 11.13 we derive the lemma from Stokes' formula (Theorem 34.8, [FOOO11, Theorem 8.10]) and composition formula (Theorem 35.7, [FOOO11, Theorem 10.20]) similarly as before.

Theorem 11.11 follows immediately from Lemma 11.15

11.3. Wrap-up of the proof of triangle inequality. Now we prove:

Theorem 11.16. Assume that $H_{1}, H_{2}, H_{1} * H_{2}$ are all nondegenerate. Then for any $a_{1}, a_{2} \in Q H_{\mathfrak{b}}^{*}(M ; \Lambda)$ we have:

$$
\rho^{\mathfrak{b}}\left(H_{1} ; a_{1}\right)+\rho^{\mathfrak{b}}\left(H_{2} ; a_{2}\right) \geq \rho^{\mathfrak{b}}\left(H_{1} * H_{2} ; a_{1} \cup^{\mathfrak{b}} a_{2}\right) .
$$

Proof. Let $\epsilon>0$ and $\rho_{i}=\rho\left(H_{i} ; a_{i} ; \mathfrak{b}\right)$. Let $x_{i} \in F^{\rho_{i}+\epsilon} C F\left(M, H_{i}, J_{i}\right)$ such that $\partial_{\left(H_{i}, J_{i}\right)}^{\mathfrak{b}}\left(x_{i}\right)=0$ and $\left[x_{i}\right]=\mathcal{P}_{\left(\left(H_{i}\right)_{\chi},\left(J_{i}\right)_{\chi}\right), *}^{\mathfrak{b}}\left(a_{i}^{\mathfrak{b}}\right) \in \operatorname{HF}\left(M, H_{i}, J_{i}\right)(i=1,2)$.

By Proposition 11.9 we have

$$
\mathfrak{m}_{2}^{\mathrm{cl}}\left(x_{1}, x_{2}\right) \in F^{\rho_{1}+\rho_{2}+2 \epsilon} C F\left(M, H_{1} * H_{2}, J_{1} * J_{2}\right) .
$$

By Theorem 11.11 we have

$$
\left[\mathfrak{m}_{2}^{\mathrm{cl}}\left(x_{1}, x_{2}\right)\right]=\mathcal{P}_{\left(\left(H_{1} * H_{2}\right)_{\chi},\left(J_{1} * J_{2}\right)_{\chi}\right), *}^{\mathfrak{b}}\left(\left(a_{1} \cup^{\mathfrak{b}} a_{2}\right)^{\mathfrak{b}}\right) .
$$

Therefore by definition

$$
\rho\left(H_{1} * H_{2} ; a_{1} \cup^{\mathfrak{b}} a_{2} ; \mathfrak{b}\right) \leq \rho_{1}+\rho_{2}+2 \epsilon .
$$

Since $\epsilon>0$ is arbitrary, Theorem 11.16 follows. 


\section{Proofs of Other AXIOMS}

We are now ready to complete the proof of Theorem 7.8 ,

Note that the proof of Theorem 7.7(1),(3) has been completed in Section10 and hence the invariant $\rho^{\mathfrak{b}}(\widetilde{\phi} ; a)$ is well-defined for $\widetilde{\phi} \in \widetilde{\operatorname{Ham}}_{\mathrm{nd}}(M ; \omega)$.

For general $\widetilde{\psi}_{H} \in \widetilde{\operatorname{Ham}}(M ; \omega)$, not necessarily nondegenerate, we take nondegenerate $H_{i}$ which converges to $H$ in $C^{0}$-sense and take the $\operatorname{limit}_{\lim } \lim _{i \rightarrow \infty} \rho^{\mathfrak{b}}\left(\widetilde{\psi}_{H_{i}} ; a\right)$. This limit exists and is independent of $H_{i}$ by Theorem 9.1. We define this limit to be $\rho^{\mathfrak{b}}(\widetilde{\phi} ; a)$ and have thus defined $\rho^{\mathfrak{b}}(\widetilde{\phi} ; a)$ in general. We prove that it satisfies (1) - (7) of Theorem 7.8

Statement (1) is Theorem 8.8

Statement (2) is immediate from definition.

Now let us prove (3). In a way similar to the proof of Lemma 9.3, we prove the following:

Lemma 12.1. If $\mathcal{M}_{\ell}\left(H_{\chi}, J_{\chi} ; *,[\gamma, w]\right)$ is nonempty, then $\mathcal{A}_{H}([\gamma, w]) \leq E^{-}(H)$.

Therefore if $\lambda_{H}(a)<\lambda$ then

$$
\mathcal{P}_{\left(H_{\chi}, J_{\chi}\right)}^{\mathfrak{b}}\left(a^{b}\right) \in F^{\lambda+\left\|H_{i}\right\|_{-}} C F(M, H, J) .
$$

It follows that

$$
\rho^{\mathfrak{b}}(H ; a) \leq \lambda+E^{-}(H)
$$

We apply this inequality to a sequence $H_{i}$ of Hamiltonians converging to 0 such that $\widetilde{\psi}_{H_{i}}$ are nondegenerate. By taking the limit, we have

$$
\rho^{\mathfrak{b}}(\underline{0} ; a) \leq \lambda \text {. }
$$

Since this holds for any $\lambda>\mathfrak{v}_{q}(a), \rho^{\mathfrak{b}}(\underline{0} ; a) \leq \mathfrak{v}_{q}(a)$. We refer to Proposition 26.10 in Section 26 for the proof of opposite inequality

$$
\rho^{\mathfrak{b}}(\underline{0} ; a) \geq \mathfrak{v}_{q}(a) .
$$

Statement (4) is immediate from construction.

Statement (5) is Theorem [11.16 in the nondegenerate case. The general case then follows by an obvious limit argument.

Statement (6) immediately follows from Theorem 9.1 .

Statement (7) is obvious from construction. We have thus completed the proof of Theorem 7.8 except the opposite inequality (12.1) which is deferred to Section 26. 


\section{Part 3. Quasi-states and quasi-morphisms via spectral invariants with bulk}

In this part, we show that Entov-Polterovich's theory can be enhanced by involving spectral invariants with bulk, which we have developed in Part 2. The generalization is rather straightforward requiring only a small amount of new ideas. So a large portion of this part is actually a review of the works by Entov-Polterovich and Usher [EP1, EP2, EP3, Os2, Us1, Us3. (It seems, however, that the proof of Theorem 15.1 below is not written in detail to the level of generality that we provide here.)

\section{Partial Symplectic QuASI-STATES}

We start this section by recalling the definition of Calabi homomorphism. Let $H:[0,1] \times M \rightarrow \mathbb{R}$ be a time dependent Hamiltonian and $\phi_{H}^{t}$ be the $t$ parameter family of Hamiltonian diffeomorphisms induced by it. We note that we do not assume that $H$ is normalized. For an open proper subset $U \subset M$ we define

$$
\operatorname{Ham}_{U}(M, \omega)=\left\{\psi_{H} \in \operatorname{Ham}(M, \omega) \mid \operatorname{supp} H_{t} \subset U \text { for any } t\right\} .
$$

We denote the universal covering space of $\operatorname{Ham}_{U}(M, \omega)$ by $\widetilde{\operatorname{Ham}}_{U}(M, \omega)$. Each time dependent Hamiltonian $H$ supported in $U$ determines an element $\psi_{H}=\phi_{H}^{1} \in$ $\operatorname{Ham}_{U}(M, \omega)$, together with its lifting $\widetilde{\psi}_{H}=\left[\phi_{H}\right]_{U} \in \widehat{\operatorname{Ham}}_{U}(M, \omega)$. Here $[\cdot]_{U}$ is the path homotopy class of $\phi_{H}$ in $\operatorname{Ham}_{U}(M, \omega)$. We recall the following lemma due to [Ca, whose proof we omit. (See for example Ba2, Theorem 4.2.7], MS, p.328-p.329].)

Lemma 13.1. If supp $H_{t} \subset U$ for all $t$, then the integral

$$
\int_{0}^{1} d t \int_{M} H_{t} \omega^{n}
$$

depends only on $\widetilde{\psi}_{H} \in \widetilde{\operatorname{Ham}}_{U}(M, \omega)$.

Definition 13.2. We define the homomorphism $\mathrm{Cal}_{U}: \widetilde{\operatorname{Ham}}_{U}(M, \omega) \rightarrow \mathbb{R}$ by

$$
\operatorname{Cal}_{U}\left(\widetilde{\psi}_{H}\right)=\int_{0}^{1} d t \int_{M} H_{t} \omega^{n}
$$

which is called a Calabi homomorphism. We also put $\mathrm{Cal}=\mathrm{Cal}_{M}: \widetilde{\operatorname{Ham}}(M, \omega) \rightarrow$ $\mathbb{R}$.

This is well-defined by Lemma 13.1

We next recall the notion of partial symplectic quasi-states introduced by EntovPolterovich [EP2]. We say that a subset $U$ of $M$ is displaceable (or Hamiltonian displaceable) if there exists $\phi \in \operatorname{Ham}(M, \omega)$ such that $\phi(U) \cap \bar{U}=\emptyset$.

Definition 13.3 (EP2, EP3]). A partial symplectic quasi-state is defined to be a function $\zeta: C^{0}(M) \rightarrow \mathbb{R}$ that satisfies the following properties:

(1) (Lipschitz continuity) $\left|\zeta\left(F_{1}\right)-\zeta\left(F_{2}\right)\right| \leq\left\|F_{1}-F_{2}\right\|_{C^{0}}$.

(2) (Semi-homogeneity) $\zeta(\lambda F)=\lambda \zeta(F)$ for any $F \in C^{0}(M)$ and $\lambda \in \mathbb{R}_{>0}$.

(3) (Monotonicity) $\zeta\left(F_{1}\right) \leq \zeta\left(F_{2}\right)$ for $F_{1} \leq F_{2}$.

(4) (Additivity over constants and Normalization) $\zeta(F+\alpha)=\zeta(F)+\alpha$ for any continuous function $F$ and any real number $\alpha$. In particular $\zeta(1)=1$. 
(5) (Partial additivity) If two $F_{1}, F_{2} \in C^{\infty}(M)$ satisfy $\left\{F_{1}, F_{2}\right\}=0$ and $\operatorname{supp} F_{2}$ is displaceable, then $\zeta\left(F_{1}+F_{2}\right)=\zeta\left(F_{1}\right)$.

(6) (Symplectic invariance) $\zeta(F)=\zeta(F \circ \psi)$ for any $\psi \in \operatorname{Symp}_{0}(M, \omega)$.

(7) (Vanishing) $\zeta(F)=0$, provided $\operatorname{supp} F$ is displaceable.

(8) (Triangle inequality) If $\left\{F_{1}, F_{2}\right\}=0, \zeta\left(F_{1}+F_{2}\right) \geq \zeta\left(F_{1}\right)+\zeta\left(F_{2}\right)$.

The triangle inequality property is required in the definition in EP3, though it is not in EP2. The triangle inequality (8) is different from the one in [EP3] and are adapted to our convention. Namely, for a partial symplectic quasi-state $\zeta^{E P}$ in the sense of Entov-Polterovich, $\zeta(H)=-\zeta^{E P}(-H)$ gives a partial symplectic quasi-state in the sense of Definition 13.3. We would like to point out that the above vanishing property (7) is actually an immediate consequence of the axiom, partial additivity (5).

The upshot of Entov-Polterovich's discovery is that the spectral invariant function $H \mapsto \rho(H ; 1)$ naturally gives rise to an example of partial symplectic quasistates, which we denote by $\zeta_{1}$. In fact, this spectral partial quasi-states is the only known example of such partial symplectic quasi-states so far. We call any such partial symplectic quasi-states constructed out of spectral invariants and its bulkdeformed ones as a whole spectral partial quasi-states. The main result of the next section is to generalize Entov-Polterovich's construction of spectral partial (symplectic) quasi-states by involving the spectral invariants with bulk.

Recall that the Lie algebra of $\widetilde{\operatorname{Ham}}(M, \omega)$ or $\operatorname{Ham}(M, \omega)$ can be identified with $C^{\infty}(M) / \mathbb{R} \cong C^{\infty}(M)_{0}$, the set of normalized autonomous Hamiltonian functions. The functional $\zeta_{1}^{\infty}=\left.\zeta_{1}\right|_{C^{\infty}(M)}$ is defined on the central extension $C^{\infty}(M)$ of this Lie algebra.

In fact, $\zeta_{1}$ can be regarded as a 'linearization' of another nonlinear functional $\mu: \widetilde{\operatorname{Ham}}(M, \omega) \rightarrow \mathbb{R}$ which has the properties established in EP2 section 7. This becomes a genuine quasi-morphism under a suitable algebraic condition such as semisimplicity of the quantum cohomology ring of the underlying symplectic manifold $(M, \omega)$.

We recall that the Hofer norm $\|\widetilde{\phi}\|$ for $\widetilde{\phi} \in \widetilde{\operatorname{Ham}}(M, \omega)$ is defined by

$$
\|\widetilde{\phi}\|=\inf \left\{\|H\| \mid\left[\phi_{H}\right]=\widetilde{\phi}\right\} .
$$

Following [EP2], we define another norm called the fragmentation norm.

Definition 13.4. Let $U$ be a given open subset of $M$. For any $\widetilde{\phi} \in \widetilde{\operatorname{Ham}}(M, \omega)$, we say $\|\widetilde{\phi}\|_{U} \leq m$ if and only if there exists $\widetilde{\psi}_{i} \in \widetilde{\operatorname{Ham}}(M, \omega), \widetilde{\phi}_{i} \in \widetilde{\operatorname{Ham}_{U}}(M, \omega)$ for $i=1, \ldots, m$ such that

$$
\widetilde{\phi}=\prod_{i=1}^{m}\left(\widetilde{\psi}_{i} \widetilde{\phi}_{i} \widetilde{\psi}_{i}^{-1}\right)
$$

The following fragmentation lemma of Banyaga Ba1] shows that the norm $\|\widetilde{\phi}\|_{U}$ is always finite.

Lemma 13.5 (Banyaga). Let $U_{i} \subset M$ be open sets for $i=1, \ldots, N, U=\bigcup_{i=1}^{N} U_{i}$, and $\widetilde{\phi} \in \widetilde{\operatorname{Ham}}_{U}(M, \omega)$. Then there exists $\widetilde{\phi}_{j}$ such that $\widetilde{\phi}_{j} \in \operatorname{Ham}_{U_{i(j)}}(M, \omega)$ for some $i(j) \in\{1, \ldots, N\}$ and

$$
\widetilde{\phi}=\widetilde{\phi}_{1} \ldots \widetilde{\phi}_{N}
$$


Proof. We give a self contained proof below for the sake of completeness and for readers' convenience. By an obvious induction argument it suffices to consider the case $N=2$, namely $U=U_{1} \cup U_{2}$. Let $\phi=\phi_{H}^{1} \in \operatorname{Ham}_{U}(M, \omega)$. We may assume without loss of generality that $\widetilde{\phi}=\widetilde{\psi}_{H}$ and $\|H\|_{C^{1}}<\epsilon$, where $\epsilon$ is a positive number depending only on $U_{1}, U_{2}$ and $U$ to be determined later. (This is because any element of $\widetilde{\operatorname{Ham}}_{U}(M, \omega)$ is a product of finitely many such $\widetilde{\phi}$ 's.)

We take a pair of open subsets $U_{1}^{\prime \prime} \subset U_{1}^{\prime}$ so that $\bar{U}_{1}^{\prime \prime} \subset U_{1}^{\prime} \subset \bar{U}_{1}^{\prime} \subset U_{1}$ and $U_{1}^{\prime \prime} \cup U_{2} \supset \operatorname{supp} H$.

Let $\eta: M \rightarrow[0,1]$ be a smooth cut-off function such that supp $\eta \subset U_{1}$ and that $\eta=1$ on $U_{1}^{\prime}$. and put $\phi_{1}=\psi_{\eta H}$. It is easy to see that if $\epsilon$ is sufficiently small then $\phi_{1}=\phi$ on $U_{1}^{\prime \prime}$, where $\phi \in \operatorname{Ham}_{U}(M, \omega)$ is the projection of $\widetilde{\phi}$. Moreover we may assume that $\phi_{1}(x)=x$ for $x \notin U_{1}^{\prime \prime} \cup U_{2}$.

Therefore the support of $\phi_{2}=\phi_{1}^{-1} \phi$ is on $U_{2}$ and the support of $\phi_{1}$ is on $U_{1}$. Using the fact that they are $C^{1}$-close to the identity, it follows that $\widetilde{\phi}_{1} \widetilde{\phi}_{2}=\widetilde{\phi}$.

Definition 13.6. A partial quasi-morphism on $\widetilde{\operatorname{Ham}}(M, \omega)$ is a function $\mu: \widetilde{\operatorname{Ham}}(M, \omega) \rightarrow$ $\mathbb{R}$ that satisfies following conditions: Let $\widetilde{\psi}, \widetilde{\phi} \in \widetilde{\operatorname{Ham}}(M, \omega)$.

(1) (Lipschitz continuity) $|\mu(\widetilde{\psi})-\mu(\widetilde{\phi})| \leq C\left\|\widetilde{\psi} \widetilde{\phi}^{-1}\right\|$, where \|\| is the Hofer norm and $C$ is a constant independent of $\widetilde{\psi}, \widetilde{\phi}$.

(2) (Semi-homogeneity) $\mu\left(\widetilde{\phi}^{n}\right)=n \mu(\widetilde{\phi})$ for any $n \in \mathbb{Z}_{\geq 0}$.

(3) (Controlled quasi-additivity) If $U \subset M$ is displaceable, then there exists a constant $K$ depending only on $U$ such that

$$
|\mu(\widetilde{\psi} \widetilde{\phi})-\mu(\widetilde{\psi})-\mu(\widetilde{\phi})|<K \min \left(\|\widetilde{\psi}\|_{U},\|\widetilde{\phi}\|_{U}\right) .
$$

(4) (Symplectic invariance) $\mu(\widetilde{\phi})=\mu\left(\psi \circ \widetilde{\phi} \circ \psi^{-1}\right)$ for all $\widetilde{\phi} \in \widetilde{\operatorname{Ham}}(M, \omega)$ and $\psi \in \operatorname{Symp}_{0}(M, \omega)$.

(5) (Calabi Property) If $U \subset M$ is displaceable, then the restriction of $\mu$ to $\widetilde{\operatorname{Ham}}_{U}(M, \omega)$ coincides with Calabi homomorphism $\mathrm{Cal}_{U}$.

Remark 13.7. (1) The notion given in Definition 13.6 was introduced by Entov and Polterovich EP2] in the course of their construction of spectral partial quasi-states. They did not name it. In the earlier versions of the present paper, which was originally posted in the arXiv in 2011 (arXiv:1105.5123), we proposed to use the name 'Entov-Polterovich pre-quasi-morphism' for this function $\mu$. This name has been used by other researchers. (See Ka, $\mathrm{WX}$, for example.) Because of the referee's request, who said that the 'same' notion was used in [MVZ, we change the name to the 'partial quasimorphism'.

(2) The name 'partial quasi-morphism' is used in the paper MVZ by A. Monzner, N. Vichery, F. Zapolsky, which was posted in the arXiv in 2011 (arXiv:1111.0287). The same name was also used in Monzner's thesis [Mo. The 'partial quasi-morphism' used in MVZ, Mo is a specialization of the notion given in Definition 13.6 to the cotangent bundle. In the definition of 'partial quasi-morphism' given in [Mo, Definition1.2] only the two conditions corresponding to (3),(2) of Definition 13.6 are required but not others put above in Definition 13.6. On the other hand, the 'partial quasi-morphism' constructed in [MVZ, Theorem 1.3] on the cotangent 
bundle, which is also duplicated as Theorem 3.5 in [Mo, satisfies the conditions corresponding to those put in Definition 13.6. More specifically, the conditions (1),(2),(3),(4),(5) of Definition 13.6 correspond to the ones (iii),(i),(v),(ii),(iv) in [MVZ, Theorem 1.3], respectively. On the cotangent bundle, MVZ, Mo shows that the partial quasi-morphism satisfies the additional properties like (vi),(vii),(ix) in [MVZ, Theorem 1.3]. It seems to be an interesting question to study how the properties (vi),(vii),(ix) in MVZ, Theorem 1.3], which are stated in terms of the presence of the zero section in cotangent bundle, can be generalized to the general symplectic manifold beyond the cotangent bundle.

\section{Construction By SPECTRAL invariant With BulK}

In this section we describe construction of an example of partial quasi-morphism out of spectral invariants with bulk. Let $\mathfrak{b} \in H^{*}(M) \otimes \Lambda_{0}$ and $0 \neq e \in Q H_{\mathfrak{b}}^{*}(M)$ satisfying

$$
e \cup^{\mathfrak{b}} e=e .
$$

An obvious example of such $e$ is $e=1 \in Q H^{*}(M)$. For given $\widetilde{\psi}_{H} \in \widetilde{\operatorname{Ham}}(M, \omega)$, we consider the limit

$$
\mu_{e}^{\mathfrak{b}}\left(\widetilde{\psi}_{H}\right)=\operatorname{vol}_{\omega}(M) \lim _{n \rightarrow+\infty} \frac{\rho^{\mathfrak{b}}\left(\left(\widetilde{\psi}_{H}\right)^{n} ; e\right)}{n} .
$$

We will later show that this limit always exists. In the mean time we recall the relationship

$$
\rho^{\mathfrak{b}}\left(\widetilde{\psi}_{H} ; e\right):=\rho^{\mathfrak{b}}(\underline{H} ; e)=\rho^{\mathfrak{b}}(H ; e)+\frac{1}{\operatorname{vol}_{\omega}(M)} \operatorname{Cal}(H)
$$

for any Hamiltonian $H$. In particular, the right hand side does not depend on $H$ as long as $\left[\tilde{\psi}_{H}\right]$ remains the same element of $\widetilde{\operatorname{Ham}}(M, \omega)$.

In particular, if $H$ is a autonomous Hamiltonian and so $\psi_{n H}=\left(\psi_{H}\right)^{n}, \widetilde{\psi}_{H}^{n}=$ $\left[\psi_{n H}\right]$, then $\mu_{e}^{\mathfrak{b}}\left(\widetilde{\psi}_{H}\right)$ can be also written as

$$
\mu_{e}^{\mathfrak{b}}\left(\widetilde{\psi}_{H}\right)=\operatorname{vol}_{\omega}(M) \lim _{n \rightarrow+\infty} \frac{\rho^{\mathfrak{b}}(n H ; e)}{n}+\operatorname{Cal}(H) .
$$

We define a (nonlinear) functional $\zeta_{e}^{\mathfrak{b}}: C^{0}(M) \rightarrow \mathbb{R}$ by

$$
\zeta_{e}^{\mathfrak{b}}(H)=-\lim _{n \rightarrow \infty} \frac{\rho^{\mathfrak{b}}(n H ; e)}{n}
$$

for $H \in C^{\infty}(M)$ and then extending to $C^{0}(M)$ by continuity. Then for any $\widetilde{\phi}$ generated by autonomous (smooth) Hamiltonian $H$, whether it is normalized or not, we obtain the relationship

$$
\frac{1}{\operatorname{vol}_{\omega}(M)} \mu_{e}^{\mathfrak{b}}\left(\widetilde{\psi}_{H}\right)=-\zeta_{e}^{\mathfrak{b}}(H)+\frac{1}{\operatorname{vol}_{\omega}(M)} \operatorname{Cal}(H) .
$$

If $H$ is normalized, $\operatorname{Cal}(H)=0$ hence $\mu_{e}^{\mathfrak{b}}\left(\widetilde{\psi}_{H}\right)=-\operatorname{vol}_{\omega}(M) \zeta_{e}^{\mathfrak{b}}(H)$.

Theorem 14.1. (1) The limit (14.2) exists and so does the limit (14.4).

(2) $\mu_{e}^{\mathfrak{b}}$ becomes a partial quasi-morphism on $\widetilde{\operatorname{Ham}}(M, \omega)$.

(3) $\zeta_{e}^{\mathfrak{b}}$ becomes a partial symplectic quasi-state on $M$. 
Remark 14.2. (1) In case $\mathfrak{b}=0$, Theorem 14.1 is proved by Entov-Polterovich EP2.

(2) Actually in [EP2 several additional assumptions are imposed on $(M, \omega)$. Those assumptions are now removed by Usher [Us1, Us3].

(3) See also Us4 for some works related to the themes of the present paper.

We mostly follow the arguments presented in [EP2, pp.86-88] for the proof. We give the proof of Theorem 14.1 in the following three subsections.

14.1. Existence of the limit. We begin with the following proposition.

Proposition 14.3. Let $U \subset M$ be an open set and $\phi: M \rightarrow M$ a Hamiltonian diffeomorphism such that $\phi(U) \cap \bar{U}=\emptyset$, and $\widetilde{\phi} \in \widetilde{\operatorname{Ham}}(M)$ its lift. Let $\widetilde{\psi} \in$ $\widetilde{\operatorname{Ham}}_{U}(M, \omega), \mathfrak{b} \in H^{\operatorname{even}}\left(M ; \Lambda_{0}^{\downarrow}\right)$ and $0 \neq a \in Q H_{\mathfrak{b}}^{*}(M)$. Then

$$
\rho^{\mathfrak{b}}(\widetilde{\phi} \widetilde{\psi} ; a)=\rho^{\mathfrak{b}}(\widetilde{\phi} ; a)+\frac{\operatorname{Cal}_{U}(\widetilde{\psi})}{\operatorname{vol}_{\omega}(M)} .
$$

Proof. The main idea of the proof of the proposition is due to Ostrover Os1. It was used by Entov-Polterovich for the proof of [EP2, Lemma 7.2], which we follow here.

Let $F:[0,1] \times M \rightarrow \mathbb{R}$ be a normalized Hamiltonian such that $\left[\phi_{F}\right]=\widetilde{\phi}$ and let $H:[0,1] \times M \rightarrow \mathbb{R}$ be a Hamiltonian such that $\operatorname{supp} H_{t}$ is compact and contained in $U$ for any $t$ and that $\widetilde{\psi}=\left[\phi_{H}\right]$.

By the assumption on $\phi$ and $\widetilde{\psi}$, we find that the fixed point set $\operatorname{Fix}\left(\phi \circ \phi_{H}^{t}\right)$ is independent of $t$. We note $\phi_{H^{s} * F}^{1}=\phi \circ \psi_{H^{s}}$, where $H^{s}$ is the Hamiltonian generating the rescaled flow $t \mapsto \phi_{H}^{s t}$ defined by

$$
H^{s}(t, x)=s H(s t, x)
$$

and $*$ is the concatenation defined as in (11.4). Using the condition $\phi(U) \cap \bar{U}=$, we find

$$
\operatorname{Crit}\left(\mathcal{A}_{H^{0} * F}\right)=\operatorname{Crit}\left(\mathcal{A}_{H^{s} * F}\right),
$$

which is the set consisting of $[\gamma, w]$ such that $\gamma(t)$ is the constant path at a fixed point of $\phi$ for $0 \leq t \leq 1 / 2$ and $\gamma(2 t-1)=\phi_{H}^{2 t-1}(\gamma(1 / 2))$. Note also that $\gamma(t)$ stays outside of $\operatorname{supp}\left(H_{t}\right)$ for $0 \leq t \leq 1 / 2$. This implies the following:

Lemma 14.4. For any $[\gamma, w] \in \operatorname{Crit}\left(\mathcal{A}_{H^{0} * F}\right)$, the number $\mathcal{A}_{H^{s} * F}([\gamma, w])$ is independent of $s$.

We now consider the normalization of $H^{s}$

$$
\underline{H}^{s}(t, x)=H^{s}(t, x)-\frac{s}{\operatorname{vol}_{\omega}(M)} \int_{M} H_{s t} \omega^{n} .
$$

Then Lemma 14.4 implies

$$
\begin{aligned}
\mathcal{A}_{\underline{H}^{s} * F}([\gamma, w]) & =\mathcal{A}_{H^{s} * F}([\gamma, w])+\frac{s}{\operatorname{vol}_{\omega}(M)} \int_{0}^{1} \int_{M} H_{s t} \omega^{n} d t \\
& =\mathcal{A}_{F}([\gamma, w])+\frac{s}{\operatorname{vol}_{\omega}(M)} \int_{0}^{1} \int_{M} H_{s t} \omega^{n} d t \\
& =\mathcal{A}_{F}([\gamma, w])+\frac{1}{\operatorname{vol}_{\omega}(M)} \int_{0}^{s} \int_{M} H_{r} \omega^{n} d r .
\end{aligned}
$$


Therefore we have

$$
\operatorname{Spec}\left(\widetilde{\phi} \circ\left[\phi_{H^{s}}\right] ; \mathfrak{b}\right)=\operatorname{Spec}(\widetilde{\phi} ; \mathfrak{b})+\mathcal{A}_{F}([\gamma, w])+\frac{1}{\operatorname{vol}_{\omega}(M)} \int_{0}^{s} \int_{M} H_{r} \omega^{n} d r
$$

and so the function

$$
s \mapsto \rho^{\mathfrak{b}}\left(\widetilde{\phi} \circ\left[\phi_{H^{s}}\right] ; a\right)-\mathcal{A}_{F}([\gamma, w])+\frac{1}{\operatorname{vol}_{\omega}(M)} \int_{0}^{s} \int_{M} H_{r} \omega^{n} d r
$$

is continuous and takes values in the $\operatorname{set} \operatorname{Spec}(\widetilde{\phi} ; \mathfrak{b})$, which is a set of measure 0 (see Corollary 10.5.) Therefore it must be constant. This finishes the proof of Proposition 14.3

Let $e$ and $\mathfrak{b}$ be as in (14.1).

Definition 14.5. Let $A$ be any displaceable closed subset of $M$. We define the $\rho_{e}^{\mathfrak{b}}$-spectral displacement energy $\mathfrak{e}(A ; e ; \mathfrak{b})$ by

$$
\mathfrak{e}(A ; e ; \mathfrak{b})=\inf \left\{\rho^{\mathfrak{b}}(\widetilde{\phi} ; e)+\rho^{\mathfrak{b}}\left(\widetilde{\phi}^{-1} ; e\right) \mid \widetilde{\phi} \in \widetilde{\operatorname{Ham}}(M, \omega), \widetilde{\phi}(A) \cap \bar{A}=\emptyset\right\} .
$$

Lemma 14.6. Let $U \subset M$ be an open set which is Hamiltonian displaceable and $\widetilde{\psi} \in \widetilde{\operatorname{Ham}}_{U}(M, \omega)$. Then

$$
\mathfrak{v}_{q}(e) \leq \rho^{\mathfrak{b}}(\widetilde{\psi} ; e)+\rho^{\mathfrak{b}}\left(\widetilde{\psi}^{-1} ; e\right) \leq 2 \mathfrak{e}(\bar{U} ; e ; \mathfrak{b}) .
$$

Proof. The following proof is the same as that of [EP2, Lemma 7.2]. First, Theorem $7.8(3),(5)$ and (14.1) imply

$$
\mathfrak{v}_{q}(e)=\rho(\underline{0} ; e ; \mathfrak{b}) \leq \rho^{\mathfrak{b}}\left(\widetilde{\psi}^{-1} ; e\right)+\rho^{\mathfrak{b}}(\widetilde{\psi} ; e)
$$

which proves the first inequality of (14.8).

Next, (14.6) implies

$$
\rho\left(\widetilde{\phi} \widetilde{\psi}^{-1} ; a ; \mathfrak{b}\right)=\rho^{\mathfrak{b}}(\widetilde{\phi} ; a)-\frac{\operatorname{Cal}_{U}(\widetilde{\psi})}{\operatorname{vol}_{\omega}(M)}
$$

for any $\phi$ displacing $U$.

By the triangle inequality, we also have

$$
\begin{aligned}
\rho^{\mathfrak{b}}(\widetilde{\psi} ; e) & \leq \rho^{\mathfrak{b}}(\widetilde{\phi} \widetilde{\psi} ; e)+\rho^{\mathfrak{b}}\left(\widetilde{\phi}^{-1} ; e\right) \\
\rho^{\mathfrak{b}}\left(\widetilde{\psi}^{-1} ; e\right) & \leq \rho^{\mathfrak{b}}\left(\widetilde{\phi} \widetilde{\psi}^{-1} ; e\right)+\rho^{\mathfrak{b}}\left(\widetilde{\phi}^{-1} ; e\right) .
\end{aligned}
$$

Combining (14.6), (14.9) and (14.10) we derive

$$
\begin{aligned}
\mathfrak{v}_{q}(e) & \leq \rho^{\mathfrak{b}}\left(\widetilde{\psi}^{-1} ; e\right)+\rho^{\mathfrak{b}}(\widetilde{\psi} ; e) \\
& \leq \rho^{\mathfrak{b}}\left(\widetilde{\phi} \widetilde{\psi}^{-1} ; e\right)+\rho^{\mathfrak{b}}(\widetilde{\phi} \widetilde{\psi} ; e)+2 \rho^{\mathfrak{b}}\left(\widetilde{\phi}^{-1} ; e\right) \\
& \leq 2 \rho^{\mathfrak{b}}(\widetilde{\phi} ; e)+2 \rho^{\mathfrak{b}}\left(\widetilde{\phi}^{-1} ; e\right) .
\end{aligned}
$$

Since this holds for all $\widetilde{\phi}$ displacing $U$, the second inequality of (14.8) follows.

Lemma 14.7. Suppose $U$ is displaceable and $\widetilde{\psi} \in \widetilde{\operatorname{Ham}}_{U}(M, \omega)$. Then for any $\widetilde{\phi} \in \widetilde{\operatorname{Ham}}(M, \omega)$,

$$
\rho^{\mathfrak{b}}(\widetilde{\phi} ; e)+\rho^{\mathfrak{b}}(\widetilde{\psi} ; e)-2 \mathfrak{e}(\bar{U} ; e ; \mathfrak{b}) \leq \rho^{\mathfrak{b}}(\widetilde{\phi} \widetilde{\psi} ; e) \leq \rho^{\mathfrak{b}}(\widetilde{\phi} ; e)+\rho^{\mathfrak{b}}(\widetilde{\psi} ; e) .
$$


Proof. The second inequality follows from Theorem 7.8 (5) and (14.1). The first inequality follows from

$$
\begin{aligned}
\rho^{\mathfrak{b}}(\widetilde{\phi} \widetilde{\psi} ; e) & \geq \rho^{\mathfrak{b}}(\widetilde{\phi} ; e)-\rho^{\mathfrak{b}}\left(\widetilde{\psi}^{-1} ; e\right) \\
& \geq \rho^{\mathfrak{b}}(\widetilde{\phi} ; e)+\rho^{\mathfrak{b}}(\widetilde{\psi} ; e)-2 \mathfrak{e}(A ; e ; \mathfrak{b}),
\end{aligned}
$$

where the first inequality follows from Theorem 7.8 (5) and the second follows from Lemma 14.6.

Corollary 14.8. Let $\widetilde{\psi}_{1}, \ldots, \widetilde{\psi}_{m} \in \widetilde{\operatorname{Ham}}(M, \omega)$ such that $\left\|\widetilde{\psi}_{i}\right\|_{U}=1$ for $i=$ $1, \ldots, m$. Then for any $\widetilde{\phi} \in \widetilde{\operatorname{Ham}}(M, \omega)$,

$$
\left|\rho^{\mathfrak{b}}\left(\widetilde{\psi}_{1} \cdots \widetilde{\psi}_{m} \widetilde{\phi} ; e\right)-\sum_{i=1}^{m} \rho^{\mathfrak{b}}\left(\widetilde{\psi}_{i} ; e\right)-\rho^{\mathfrak{b}}(\widetilde{\phi} ; e)\right|<2 m \mathfrak{e}(\bar{U} ; e ; \mathfrak{b}) .
$$

Proof. By the hypothesis $\left\|\widetilde{\psi}_{i}\right\|_{U}=1$, we can write $\widetilde{\psi}_{i}=\widetilde{\phi}_{i}^{-1} \widetilde{\psi}_{i}^{\prime} \widetilde{\phi}_{i}$ with $\widetilde{\psi}_{i}^{\prime} \in$ $\widetilde{\operatorname{Ham}}_{U}(M, \omega), \widetilde{\phi}_{i} \in \widetilde{\operatorname{Ham}}(M, \omega)$.

The case $m=1$ follows from Lemma 14.7 which we apply to $\psi_{1}(U)$ in place of $U$. (We note that $\mathfrak{e}(\bar{U} ; e ; \mathfrak{b})=\mathfrak{e}\left(\psi_{1}(\bar{U}) ; e ; \mathfrak{b}\right)$.)

Suppose the corollary is proved for $m-1$. Applying the induction hypothesis to the case $m=2$, we have

$$
\left|\rho^{\mathfrak{b}}\left(\widetilde{\psi}_{1} \cdots \widetilde{\psi}_{m} \widetilde{\phi} ; e\right)-\rho^{\mathfrak{b}}\left(\widetilde{\psi}_{1} ; e\right)-\rho^{\mathfrak{b}}\left(\widetilde{\psi}_{2} \cdots \widetilde{\psi}_{m} \widetilde{\phi} ; e\right)\right|<2 \mathfrak{e}(\bar{U} ; e ; \mathfrak{b})
$$

by Lemma 14.7. On the other hand, by the induction hypothesis we have

$$
\left|\rho^{\mathfrak{b}}\left(\widetilde{\psi}_{2} \cdots \widetilde{\psi}_{m} \widetilde{\phi} ; e\right)-\sum_{i=2}^{m} \rho^{\mathfrak{b}}\left(\widetilde{\psi}_{i} ; e\right)-\rho^{\mathfrak{b}}(\widetilde{\phi} ; e)\right|<2(m-1) \mathfrak{e}(\bar{U} ; e ; \mathfrak{b}) .
$$

The inequality (14.11) follows.

We now prove the convergence of the limit appearing in (14.2).

Let $\widetilde{\psi} \in \widetilde{\operatorname{Ham}}(M, \omega)$. We fix a displaceable open subset $U \subset M$ and cover $M=\cup_{i=1}^{m} \phi_{i}(U)$ by $\phi_{i} \in \operatorname{Ham}(M, \omega)$ for a sufficiently large $m$. Then by applying Lemma 13.5 to this decomposition of $M$, we can factorize $\widetilde{\psi}=\widetilde{\psi}_{1} \cdots \widetilde{\psi}_{m}$ so that $\operatorname{supp} \psi_{i} \subset \phi_{i}(U)$ for each $i=1, \cdots, m$. In particular, all $\widetilde{\psi}_{i}$ satisfy $\left\|\widetilde{\psi}_{i}\right\|_{U}=1$.

We apply Corollary 14.8 to these $\psi_{i}$ 's by putting $\widetilde{\phi}=\overline{0}$ and obtain

$$
\left|\rho^{\mathfrak{b}}\left(\widetilde{\psi}^{n} ; e\right)-n \sum_{i=1}^{m} \rho^{\mathfrak{b}}\left(\widetilde{\psi}_{i} ; e\right)\right| \leq 2 m n \mathfrak{e}(\bar{U} ; e ; \mathfrak{b}) .
$$

We put

$$
a_{n}=\rho^{\mathfrak{b}}\left(\widetilde{\psi}^{n} ; e\right)+2 m n \mathfrak{e}(\bar{U} ; e ; \mathfrak{b})+n m\left|\sup \left\{\rho^{\mathfrak{b}}\left(\widetilde{\psi}_{i} ; e\right) \mid i=1, \ldots, m\right\}\right| .
$$

(14.12) implies that $a_{n} \geq 0$. Theorem 7.8 (5) implies $a_{n}+a_{n^{\prime}} \geq a_{n+n^{\prime}}$. We recall the following:

Lemma 14.9 (M. Fekete [Fe]). If $a_{n} \geq 0$ and $a_{n}+a_{n^{\prime}} \geq a_{n+n^{\prime}}$, then $\lim _{n \rightarrow \infty} a_{n} / n$ converges.

Proof. The following proof is taken from Problem 98 of [PS, p 17]. Since $a_{2^{n}} / 2^{n}$ is non-increasing, $\alpha=\liminf _{n \rightarrow \infty} a_{n} / n$ is a finite number. Let $\epsilon>0$. We take 
any $n_{0}$ such that $\left|a_{n_{0}} / n_{0}\right| \leq \alpha+\epsilon$. If $n^{\prime}=n_{0} k+r$ with $r=1, \ldots, n_{0}-1$, then $a_{n^{\prime}}=a_{n_{0} k+r} \leq k a_{n_{0}}+a_{r}$. Therefore

$$
\frac{a_{n^{\prime}}}{n^{\prime}} \leq \frac{a_{n_{0}}}{n_{0}} \frac{k n_{0}}{k n_{0}+r}+\frac{a_{r}}{n^{\prime}} .
$$

Hence if $n^{\prime}$ is sufficiently large, we have $\alpha-\epsilon \leq a_{n^{\prime}} / n^{\prime}<\alpha+2 \epsilon$ as required.

We have thus proved that the limit

$$
\operatorname{vol}_{\omega}(M) \lim _{n \rightarrow+\infty} \frac{\rho^{\mathfrak{b}}\left(\widetilde{\phi}^{n} ; e\right)}{n}
$$

exists.

14.2. Partial quasi-morphism property of $\mu_{e}^{\mathfrak{b}}$. In this subsection, we prove Theorem 14.1 (2).

The limit $\mu_{e}^{\mathfrak{b}}(\widetilde{\phi})$ satisfies Definition 13.6 (2) by construction. Definition 13.6 (1) then follows from Theorem 7.8 (6). Definition 13.6 (4) follows from Theorem 7.8 (4).

We next prove the properties required in Definition 13.6 (3).

Lemma 14.10. We have

$$
\left|\mu_{e}^{\mathfrak{b}}(\widetilde{\psi} \tilde{\phi})-\mu_{e}^{\mathfrak{b}}(\widetilde{\psi})-\mu_{e}^{\mathfrak{b}}(\widetilde{\phi})\right| \leq 2 \mathfrak{e}(\bar{U} ; e ; \mathfrak{b}) \operatorname{vol}_{\omega}(M) \min \left(2\|\widetilde{\psi}\|_{U}-1,2\|\widetilde{\phi}\|_{U}-1\right) .
$$

Proof. Without loss of any generality, we may assume that $\|\widetilde{\psi}\|_{U} \leq\|\widetilde{\phi}\|_{U}$. The proof will be given by the induction over the fragmentation norm $\|\widetilde{\psi}\|_{U}$.

We first consider the case $\|\widetilde{\psi}\|_{U}=1$. Since $\left\|\widetilde{\phi^{j}} \widetilde{\psi}_{\phi^{-j}}\right\|_{U}=1$, Corollary 14.8, the identity

$$
(\widetilde{\psi} \widetilde{\phi})^{k}=\left(\prod_{j=0}^{k-1} \widetilde{\phi}^{j} \widetilde{\psi}^{-j}\right) \widetilde{\phi}^{k}
$$

and $\rho^{\mathfrak{b}}\left(\widetilde{\phi}^{j} \widetilde{\psi} \widetilde{\phi}^{-j} ; e\right)=\rho^{\mathfrak{b}}(\widetilde{\psi} ; e)$ (Theorem $\left.7.8(4)\right)$ imply

$$
\left|\rho^{\mathfrak{b}}\left((\widetilde{\psi} \widetilde{\phi})^{k} ; e\right)-k \rho^{\mathfrak{b}}(\widetilde{\psi} ; e)-\rho^{\mathfrak{b}}\left(\widetilde{\phi}^{k} ; e\right)\right| \leq 2 k \mathfrak{e}(\bar{U} ; e ; \mathfrak{b}) .
$$

We use Corollary 14.8 again to derive

$$
\left|\rho^{\mathfrak{b}}\left(\widetilde{\psi}^{k} ; e\right)-k \rho^{\mathfrak{b}}(\widetilde{\psi} ; e)\right| \leq 2 k \mathfrak{e}(\bar{U} ; e ; \mathfrak{b}) .
$$

Therefore

$$
\left|\rho^{\mathfrak{b}}\left((\widetilde{\psi} \widetilde{\phi})^{k} ; e\right)-\rho^{\mathfrak{b}}\left(\widetilde{\psi}^{k} ; e\right)-\rho^{\mathfrak{b}}\left(\widetilde{\phi}^{k} ; e\right)\right| \leq 4 k \mathfrak{e}(\bar{U} ; e ; \mathfrak{b}) .
$$

The case $m=1$ of the lemma follows.

Now suppose that the lemma is proved for all $\widetilde{\psi} \in \widetilde{\operatorname{Ham}}(M, \omega)$ that satisfy $\|\widetilde{\psi}\|_{U}=m-1$. Let $\widetilde{\psi}$ be any element of $\widetilde{\operatorname{Ham}}(M, \omega)$ with $\|\widetilde{\psi}\|_{U}=m$. We write $\widetilde{\psi}=\widetilde{\psi}_{1} \widetilde{\psi}_{2}$ with $\left\|\widetilde{\psi}_{1}\right\|_{U}=m-1$ and $\left\|\widetilde{\psi}_{2}\right\|_{U}=1$. Then by the induction hypothesis

$$
\left|\mu_{e}^{\mathfrak{b}}(\widetilde{\psi} \widetilde{\phi})-\mu_{e}^{\mathfrak{b}}\left(\widetilde{\psi}_{1}\right)-\mu_{e}^{\mathfrak{b}}\left(\widetilde{\psi}_{2} \widetilde{\phi}\right)\right| \leq 2 \mathfrak{e}(\bar{U} ; e ; \mathfrak{b}) \operatorname{vol}_{\omega}(M)(2(m-1)-1) .
$$

The case $m=1$ gives

$$
\left|\mu_{e}^{\mathfrak{b}}\left(\widetilde{\psi}_{2} \widetilde{\phi}\right)-\mu_{e}^{\mathfrak{b}}\left(\widetilde{\psi}_{2}\right)-\mu_{e}^{\mathfrak{b}}(\widetilde{\phi})\right| \leq 2 \mathfrak{e}(\bar{U} ; e ; \mathfrak{b}) \operatorname{vol}_{\omega}(M)
$$

and

$$
\left|\mu_{e}^{\mathfrak{b}}(\widetilde{\psi})-\mu_{e}^{\mathfrak{b}}\left(\widetilde{\psi}_{1}\right)-\mu_{e}^{\mathfrak{b}}\left(\widetilde{\psi}_{2}\right)\right| \leq 2 \mathfrak{e}(\bar{U} ; e ; \mathfrak{b}) \operatorname{vol}_{\omega}(M) .
$$

By combining these three inequalities, we have finished the proof of Lemma 14.10 
Clearly Lemma 14.10 implies

$$
\left|\mu_{e}^{\mathfrak{b}}(\widetilde{\psi} \widetilde{\phi})-\mu_{e}^{\mathfrak{b}}(\widetilde{\psi})-\mu_{e}^{\mathfrak{b}}(\widetilde{\phi})\right| \leq 4 \mathfrak{e}(\bar{U} ; e ; \mathfrak{b}) \operatorname{vol}_{\omega}(M) \min \left(\|\widetilde{\psi}\|_{U},\|\widetilde{\phi}\|_{U}\right) .
$$

Thus we have proved the property of Definition 13.6 (3).

Remark 14.11. We may take $K=4 \mathfrak{e}(\bar{U} ; e ; \mathfrak{b}) \operatorname{vol}_{\omega}(M)$ for the constant in Definition $13.6(3)$.

We next prove Definition13.6(5). Let $U \subset M$ be a displaceable open subset and $\widetilde{\psi} \in \widetilde{\operatorname{Ham}}_{U}(M, \omega)$. Let $\widetilde{\phi} \in \widetilde{\operatorname{Ham}}(M, \omega)$ such that $\phi(U) \cap \bar{U}=\emptyset$. By Proposition 14.3 applied to $\widetilde{\psi}^{n}$ we have

$$
\rho^{\mathfrak{b}}\left(\widetilde{\phi} \widetilde{\psi}^{n} ; e\right)=\rho^{\mathfrak{b}}(\widetilde{\phi} ; e)+\frac{n \operatorname{Cal}_{U}(\widetilde{\psi})}{\operatorname{vol}_{\omega}(M)} .
$$

Using this equality and Lemma 14.7 we obtain

$$
\begin{aligned}
\left|\rho^{\mathfrak{b}}\left(\widetilde{\psi}^{n} ; e\right)-\frac{n \operatorname{Cal}_{U}(\widetilde{\psi})}{\operatorname{vol}_{\omega}(M)}\right| & =\left|\rho^{\mathfrak{b}}\left(\widetilde{\psi}^{n} ; e\right)+\rho^{\mathfrak{b}}(\widetilde{\phi} ; e)-\rho^{\mathfrak{b}}\left(\widetilde{\phi} \widetilde{\psi}^{n} ; e\right)\right| \\
& \leq 2 \mathfrak{e}(\bar{U} ; e ; \mathfrak{b})<\infty .
\end{aligned}
$$

Then dividing this inequality by $\frac{n \operatorname{Cal}_{U}(\widetilde{\psi})}{\operatorname{vol}_{\omega}(M)}$ and letting $n \rightarrow \infty$, we obtain $\mu^{\mathfrak{b}}(\widetilde{\psi})=$ $\mathrm{Cal}_{U}(\widetilde{\psi})$. The proof of Theorem $14.1(2)$ is complete.

14.3. Partial symplectic quasi-state property of $\zeta_{e}^{\mathfrak{b}}$. In this subsection, we give the proof of Theorem 14.1 (3), i.e., the functional $\zeta_{e}^{\mathfrak{b}}: C^{0}(M) \rightarrow \mathbb{R}$ is a partial symplectic quasi-state. For this purpose, we have only to consider autonomous smooth Hamiltonian $F$ 's in the rest of the proof. Let $F$ be an autonomous Hamiltonian and take its normalization

$$
\underline{F}=F-\frac{1}{\operatorname{vol}_{\omega}(M)} \int_{M} F \omega^{n} .
$$

Then

$$
\rho^{\mathfrak{b}}(n F ; e)+\int_{M} n F \omega^{n}=\rho^{\mathfrak{b}}(n \underline{F} ; e)=\rho^{\mathfrak{b}}\left(\widetilde{\psi}^{n} ; e\right)
$$

for $\widetilde{\psi}=\left[\phi_{F}\right]$. Dividing this equation by $n$, we obtain

$$
\frac{\rho^{\mathfrak{b}}(n F ; e)}{n}+\frac{1}{\operatorname{vol}_{\omega}(M)} \int_{M} F \omega^{n}=\frac{\rho^{\mathfrak{b}}\left(\widetilde{\psi}^{n} ; e\right)}{n} .
$$

Therefore convergence of (14.4) follows from the convergence of (14.2). Thus $\zeta_{e}^{\mathfrak{b}}(F)$ is defined for $F \in C^{\infty}(M)$.

Definition 13.3 (1) is a consequence of Theorem 7.8 (6). We can extend $\zeta_{e}^{\mathfrak{b}}$ to $C^{0}(M)$ by the $F \in C^{\infty}(M)$ case of Definition 13.3 (1). The property of Definition 13.3 (1) in the case $F \in C^{0}(M)$ then follows for this extended $\zeta_{e}^{\mathfrak{b}}$.

Since $\widetilde{\psi}_{H / m}^{m}=\widetilde{\psi}_{H}$ holds for autonomous Hamiltonian $H$, we can prove the property of Definition 13.3 (2) in the case $\lambda \in \mathbb{Q}_{\geq 0}$ by using Definition 13.6 (2) and (14.16). Then the case $\lambda \in \mathbb{R}_{\geq 0}$ follows from Definition 13.3 (1).

Definition 13.3 (4) is immediate from (14.16).

The property of Definition 13.3 (6) is a consequence of Theorem 7.8 (4). 
Let us prove the property of Definition $13.3(7)$. Suppose $U$ is displaceable and the support of time independent Hamiltonian $F$ is in $U$. We define $U^{\prime}$ as in (14.15). We take $\widetilde{\phi} \in \widetilde{\operatorname{Ham}}(M, \omega)$ such that $\phi(U) \cap \bar{U}=\emptyset$. By Proposition 14.3, we have:

$$
\rho^{\mathfrak{b}}\left(\widetilde{\phi} \widetilde{\psi}_{F}^{n} ; e\right)=\rho^{\mathfrak{b}}(\widetilde{\phi} ; e)+\frac{n \operatorname{Cal}_{U}\left(\widetilde{\psi}_{F}\right)}{\operatorname{vol}_{\omega}(M)} .
$$

(Here we use the fact that $\mathrm{Cal}_{U}$ is a homomorphism.)

By (14.11) we also have

$$
\left|\rho^{\mathfrak{b}}\left(\widetilde{\phi} \widetilde{\psi}_{F}^{n} ; e\right)-\rho^{\mathfrak{b}}(\widetilde{\phi} ; e)-\rho^{\mathfrak{b}}\left(\widetilde{\psi}_{F}^{n} ; e\right)\right|<2 \mathfrak{e}(\bar{U} ; e ; \mathfrak{b}) .
$$

Substituting (14.17) into this inequality, and then dividing by $n$ and taking the limit, we obtain

$$
\lim _{n \rightarrow \infty} \frac{\rho^{\mathfrak{b}}\left(\widetilde{\psi}_{F}^{n} ; e\right)}{n}=\frac{\operatorname{Cal}_{U}\left(\widetilde{\psi}_{F}\right)}{\operatorname{vol}_{\omega}(M)}=\frac{1}{\operatorname{vol}_{\omega}(M)} \int_{M} F \omega^{n} .
$$

On the other hand, we have

$$
\lim _{n \rightarrow \infty} \frac{\rho^{\mathfrak{b}}\left(\widetilde{\psi}_{F}^{n} ; e\right)}{n}=\lim _{n \rightarrow \infty} \frac{\rho^{\mathfrak{b}}(n \underline{F} ; e)}{n}=-\zeta_{e}^{\mathfrak{b}}(\underline{F})
$$

and hence

$$
\zeta_{e}^{\mathfrak{b}}(F)=\zeta_{e}^{\mathfrak{b}}(\underline{F})+\frac{1}{\operatorname{vol}_{\omega}(M)} \int_{M} F \omega^{n}=0 .
$$

We next prove the property of Definition 13.3 (3). Let $F_{1} \leq F_{2}$. We put $H=F_{1}$ and $H^{\prime}=F_{2}$ and apply the argument of the proof of Theorem 9.2 and obtain a chain map

$$
\mathcal{P}_{(F \chi ; J \chi), \#}^{\mathfrak{b}}:\left(C F\left(M ; F_{1} ; \Lambda^{\downarrow}\right), \partial_{\left(F_{1}, J_{1}\right)}^{\mathfrak{b}}\right) \rightarrow\left(C F\left(M ; F_{2} ; \Lambda^{\downarrow}\right), \partial_{\left(F_{2}, J_{2}\right)}^{\mathfrak{b}}\right) .
$$

Using $F_{1}<F_{2}$ and Lemma 9.3 we have

$$
\left.\mathcal{P}_{(F \chi ; J \chi), \#}^{\mathfrak{b}}\left(F^{\lambda} C F\left(M ; F_{1} ; \Lambda^{\downarrow}\right)\right) \subset F^{\lambda} C F\left(M ; F_{2} ; \Lambda^{\downarrow}\right)\right) .
$$

Let $x \in F^{\lambda} C F\left(M ; F_{1} ; \Lambda^{\downarrow}\right)$ such that $[x]=\left[\mathcal{P}_{\left(\left(F_{1}\right)_{\chi},\left(J_{1}\right)_{\chi}\right), \#}^{\mathfrak{b}}\left(e^{b}\right)\right]$ and $\left|\lambda-\rho\left(F_{1} ; e\right)\right|<$ $\epsilon$. Then by Proposition 9.7 we have $\left[\mathcal{P}_{(F \chi, J \chi)}^{\mathfrak{b}}, \#(x)\right]=\left[\mathcal{P}_{\left(\left(F_{2}\right)_{\chi},\left(J_{2}\right)_{\chi}\right), \#}^{\mathfrak{b}}\left(e^{b}\right)\right]$ and $\left.\mathcal{P}_{(F \chi, J \chi)}^{\mathfrak{b}}(x) \in F^{\lambda} C F\left(M ; F_{2} ; \Lambda^{\downarrow}\right)\right)$. Therefore $\rho^{\mathfrak{b}}\left(F_{2} ; e\right) \leq \rho^{\mathfrak{b}}\left(F_{1} ; e\right)+\epsilon$. It implies $\zeta_{e}^{\mathfrak{b}}\left(F_{1}\right) \leq \zeta_{e}^{\mathfrak{b}}\left(F_{2}\right)$, as required.

Next we prove the property of Definition $13.3(5)$. By the assumption $\left\{F_{1}, F_{2}\right\}=$ 0 we have

$$
\widetilde{\psi}_{F_{1}} \widetilde{\phi}_{F_{2}}=\widetilde{\psi}_{F_{2}} \widetilde{\phi}_{F_{1}}=\widetilde{\psi}_{F_{1}+F_{2}} .
$$

Therefore by Definition 13.6 (3) we have

$$
\begin{aligned}
& \left|\rho^{\mathfrak{b}}\left(\left(\widetilde{\psi}_{F_{1}} \widetilde{\psi}_{F_{2}}\right)^{n} ; e\right)-\rho^{\mathfrak{b}}\left(\left(\widetilde{\psi}_{F_{1}}\right)^{n} ; e\right)-\rho^{\mathfrak{b}}\left(\left(\widetilde{\psi}_{F_{2}}\right)^{n} ; e\right)\right| \\
& =\left|\rho^{\mathfrak{b}}\left(\left(\widetilde{\psi}_{F_{1}}\right)^{n}\left(\widetilde{\psi}_{F_{2}}\right)^{n} ; e\right)-\rho^{\mathfrak{b}}\left(\left(\widetilde{\psi}_{F_{1}}\right)^{n} ; e\right)-\rho^{\mathfrak{b}}\left(\left(\widetilde{\psi}_{F_{2}}\right)^{n} ; e\right)\right| \leq K\left\|\left(\widetilde{\psi}_{F_{2}}\right)^{n}\right\|_{U}=K .
\end{aligned}
$$

Here $U$ is a displaceable open set containing the support of $F_{2}$. Therefore we have

$$
\mu_{e}^{\mathfrak{b}}\left(\widetilde{\psi}_{F_{1}} \widetilde{\psi}_{F_{2}}\right)=\mu_{e}^{\mathfrak{b}}\left(\widetilde{\psi}_{F_{1}}\right)+\mu_{e}^{\mathfrak{b}}\left(\widetilde{\psi}_{F_{2}}\right)=\mu_{e}^{\mathfrak{b}}\left(\widetilde{\psi}_{F_{1}}\right)+\operatorname{Cal}_{U}\left(F_{2}\right) .
$$

(We use Definition 13.6 (5) in the second equality.)

$$
\zeta_{e}^{\mathfrak{b}}\left(F_{1}+F_{2}\right)=\zeta_{e}^{\mathfrak{b}}\left(F_{1}\right)
$$


is now a consequence of (14.4). The triangle inequality for $\zeta_{e}^{\mathfrak{b}}$ follows the triangle inequality for the spectral invariant $\rho^{\mathfrak{b}}$, since $\zeta_{e}^{\mathfrak{b}}(F)=-\rho^{\mathfrak{b}}(F ; e)$. The proof of Theorem 14.1 is now complete.

\section{Poincaré DUALity AND SPECTRAL INVARIANT}

15.1. Statement of the result. Let $\pi: \Lambda^{\downarrow} \rightarrow \mathbb{C}$ be the projection to $\mathbb{C} \subset \Lambda^{\downarrow}$. Denote by $\langle\cdot, \cdot\rangle: \Omega(M) \otimes \Omega(M) \rightarrow \mathbb{C}$ the Poincaré duality pairing $\left\langle h_{1}, h_{2}\right\rangle=$ $\int_{M} h_{1} \wedge h_{2}$. We extend the pairing to

$$
\langle\cdot, \cdot\rangle:\left(\Omega(M) \widehat{\otimes} \Lambda^{\downarrow}\right) \otimes\left(\Omega(M) \widehat{\otimes} \Lambda^{\downarrow}\right) \rightarrow \Lambda^{\downarrow}
$$

so that it becomes $\Lambda^{\downarrow}$-bilinear. We put $\Pi(a, b)=\pi(\langle a, b\rangle)$ which induces a $\mathbb{C}$ bilinear pairing

$$
\Pi: H\left(M ; \Lambda^{\downarrow}\right) \otimes H\left(M ; \Lambda^{\downarrow}\right) \rightarrow \mathbb{C} .
$$

The main result of this section is:

Theorem 15.1. Let $\mathfrak{b} \in H\left(M ; \Lambda_{0}^{\downarrow}\right), a \in Q H_{\mathfrak{b}}^{*}(M)$, and $\widetilde{\phi} \in \widetilde{\operatorname{Ham}}(M, \omega)$. Then we have

$$
\rho^{\mathfrak{b}}(\widetilde{\phi} ; a)=-\inf _{b}\left\{\rho^{\mathfrak{b}}\left(\widetilde{\phi}^{-1} ; b\right) \mid \Pi(a, b) \neq 0\right\} .
$$

Remark 15.2. For the case $\mathfrak{b}=0$, this theorem is due to Entov-Polterovich under the monotonicity assumption. (See [EP1, Lemma 2.2].) The assumptions on $M$ which EP1 imposed are removed and Theorem 15.1 itself is proved by Usher in Us4.

15.2. Algebraic preliminary. In this section we prove some algebraic lemmas used in the proof of Theorem 15.1. A similar discussion was given by Usher in Us3.

We work in the situation of Subsections 8.1 We put $G=\mathbb{R}$ in this subsection. Namely $C(G)=C\left(G^{\prime}\right)=C$. Note in this case we may take the basis $e_{i}$ such that $\lambda_{q}\left(e_{i}\right)=0$. Let $\partial: C \rightarrow C$ be a boundary operator. We choose the standard basis $e_{i}^{\prime}, e_{i}^{\prime \prime}, e_{i}^{\prime \prime \prime}$ as in Subsection 8.1. Let $D$ be another finite dimensional $\Lambda^{\downarrow}$ vector space. We assume that there exists a $\Lambda^{\downarrow}$ bilinear pairing

$$
\langle\cdot, \cdot\rangle: C \times D \rightarrow \Lambda^{\downarrow}
$$

that is perfect. (Namely it induces an isomorphism $C \rightarrow D^{*}$ to the dual space $D^{*}$ of D.) Let $\left\{e_{i}^{*} \mid i=1, \ldots, N\right\}$ be the dual basis of $\left\{e_{i} \mid i=1, \ldots, N\right\}$. We use it to define the filtration $F^{\lambda} D$ in the same way as $F^{\lambda} C$. (We assume $\lambda_{q}\left(e_{i}^{*}\right)=0$.)

It is easy to see that if $x \in F^{\lambda_{1}} C, y \in F^{\lambda_{2}} D$ then

$$
\langle x, y\rangle \in F^{\lambda_{1}+\lambda_{2}} \Lambda^{\downarrow} \text {. }
$$

We define the adjoint $\partial^{*}: D \rightarrow D$ by

$$
\left\langle x, \partial^{*} y\right\rangle=\langle\partial x, y\rangle .
$$

It is easy to see that $\partial^{*} \circ \partial^{*}=0$ and $\partial^{*}\left(F^{\lambda} D\right) \subset F^{\lambda} D$.

Definition 15.3. We call $\left(D, \partial^{*}\right)$ the filtered dual complex of $(C, \partial)$.

We take a dual basis of $\left\{e_{i}^{\prime} \mid i=1, \ldots, b\right\} \cup\left\{e_{i}^{\prime \prime} \mid i=1, \ldots, h\right\} \cup\left\{e_{i}^{\prime \prime \prime} \mid i=1, \ldots, b\right\}$. Namely we take the basis so that

$$
\left\langle e_{i}^{\prime}, e_{* i}^{\prime \prime \prime}\right\rangle=1, \quad\left\langle e_{i}^{\prime \prime}, e_{* i}^{\prime \prime}\right\rangle=1, \quad\left\langle e_{i}^{\prime \prime \prime}, e_{* i}^{\prime}\right\rangle=1
$$


and all the other pairings among the basis are zero. It is easy to see that $\left\{e_{* i}^{\prime} \mid i=\right.$ $1, \ldots, b\}$ is a basis of $\operatorname{Im} \partial^{*}$ and $\left\{e_{* i}^{\prime} \mid i=1, \ldots, b\right\} \cup\left\{e_{* i}^{\prime \prime} \mid i=1, \ldots, h\right\}$ is a basis of Ker $\partial^{*}$.

In the same way as in (8.4) we have

$$
\inf \left\{\lambda_{q}(x) \mid x \in \operatorname{Ker} \partial^{*}, b=[x]\right\}=\lambda_{q}\left(\sum_{i=1}^{h} b_{i} e_{* i}^{\prime \prime}\right)
$$

for $b \in H\left(D ; \partial^{*}\right)$. We define $\lambda_{q}(b)$ for $b \in H\left(D ; \partial^{*}\right)$ by the left hand side.

The pairing $\langle\cdot, \cdot\rangle$ induces a perfect $\Lambda^{\downarrow}$ pairing between $H(C ; \partial)$ and $H\left(D ; \partial^{*}\right)$, which we also denote by $\langle\cdot, \cdot\rangle$. By applying (8.4) and (15.3) to $(C, \partial)$ instead of $\left(D, \partial^{*}\right)$, we obtain

Lemma 15.4.

$$
\lambda_{q}(a)=\sup \left\{\mathfrak{v}_{q}(\langle a, b\rangle) \mid b \in H\left(F^{0} D ; \partial^{*}\right)\right\}
$$

for $a \in H(C ; \partial)$.

15.3. Duality between Floer homologies. Let $H$ be a one-periodic time dependent Hamiltonian on $M$ such that $\psi_{H}$ is non-degenerate. We consider the chain complex $\left(C F\left(M, H ; \Lambda^{\downarrow}\right), \partial_{(H, J)}^{\mathfrak{b}}\right)$ which is defined in Section 6 .

Let $\left\{\gamma_{i} \mid i=1, \ldots, N\right\}=\operatorname{Per}(H)$ and fix a choice of their lifts $\llbracket \gamma_{i}, w_{i} \rrbracket \in \widehat{\operatorname{Per}}(H)$. Then we put

$$
e_{i}=q^{-\mathcal{A}_{H}\left(\llbracket \gamma_{i}, w_{i} \rrbracket\right)} \llbracket \gamma_{i}, w_{i} \rrbracket \in C F\left(M, H ; \Lambda^{\downarrow}\right) .
$$

We note that $\lambda_{H}\left(e_{i}\right)=0$ and $e_{i}$ is independent of $w_{i} .\left\{e_{i} \mid 1, \ldots, N\right\}$ is a $\Lambda^{\downarrow}$ basis of $C F\left(M, H ; \Lambda^{\downarrow}\right)$. It is easy to see that the filtration of $C F\left(M, H ; \Lambda^{\downarrow}\right)$ defined as in Subsection 8.1 coincides with the filtration defined in Definition 2.5

We define $\widetilde{H}$ by

$$
\widetilde{H}(t, x)=-H(1-t, x) .
$$

We have $\phi_{\widetilde{H}}^{t}=\phi_{H}^{1-t} \circ\left(\phi_{H}^{1}\right)^{-1}$. In particular, $\psi_{\widetilde{H}}=\left(\psi_{H}\right)^{-1}$. Hence $\psi_{\widetilde{H}}$ is also non-degenerate.

The main result of this subsection is as follows:

Proposition 15.5. We can choose the perturbation etc. that are used in the definition of $\left(C F\left(M, \widetilde{H} ; \Lambda^{\downarrow}\right), \partial_{(\widetilde{H}, J)}^{\mathfrak{b}}\right)$ such that there exists a perfect pairing

$$
\langle\cdot, \cdot\rangle: C F\left(M, H ; \Lambda^{\downarrow}\right) \times C F\left(M, \widetilde{H} ; \Lambda^{\downarrow}\right) \rightarrow \Lambda^{\downarrow}
$$

by which the filtered complex $\left(C F\left(M, \widetilde{H} ; \Lambda^{\downarrow}\right), \partial_{(\widetilde{H}, \widetilde{J})}^{\mathfrak{b}}\right)$ is identified with the dual filtered complex of $\left(C F\left(M, H ; \Lambda^{\downarrow}\right), \partial_{(H, J)}^{\mathfrak{b}}\right)$.

Proof. Let $\gamma \in \operatorname{Per}(H)$. It is then easy to see that

$$
\widetilde{\gamma}(t)=\gamma(1-t) \in \operatorname{Per}(\widetilde{H}) .
$$

If $w: D^{2} \rightarrow M$ satisfies $\left.w\right|_{\partial D}=\gamma$, then $\widetilde{w}(z)=w(\bar{z})$ satisfies $\left.\widetilde{w}\right|_{\partial D}=\widetilde{\gamma}$. We have thus defined

$$
\iota: \operatorname{Crit}\left(\mathcal{A}_{H}\right) \rightarrow \operatorname{Crit}\left(\mathcal{A}_{\widetilde{H}}\right)
$$

by $[\gamma, w] \mapsto[\widetilde{\gamma}, \widetilde{w}]$. It is easy to see

$$
\mathcal{A}_{H}([\gamma, w])+\mathcal{A}_{\widetilde{H}}([\widetilde{\gamma}, \widetilde{w}])=0
$$


and

$$
\int w^{*} \omega+\int \widetilde{w}^{*} \omega=0
$$

Let $\left(u ; z_{1}^{+}, \ldots, z_{\ell}^{+}\right) \in \stackrel{\circ}{\mathcal{M}}_{\ell}\left(H, J ;[\gamma, w],\left[\gamma^{\prime}, w^{\prime}\right]\right)$. We define $\iota: \mathbb{R} \times S^{1} \rightarrow \mathbb{R} \times S^{1}$ by $\iota(\tau, t)=(-\tau, 1-t)$ and put

$$
\widetilde{u}=u \circ \iota .
$$

It is easy to find that

$$
\left(\widetilde{u} ; \widetilde{z}_{1}^{+}, \ldots, \widetilde{z}_{\ell}^{+}\right) \in \stackrel{\circ}{\mathcal{M}}_{\ell}\left(\widetilde{H}, \widetilde{J} ;\left[\widetilde{\gamma}^{\prime}, \widetilde{w}^{\prime}\right],[\widetilde{\gamma}, \widetilde{w}]\right) .
$$

We have thus defined a homeomorphism

$$
\mathfrak{I}: \mathcal{M}_{\ell}\left(H, J ;[\gamma, w],\left[\gamma^{\prime}, w^{\prime}\right]\right) \rightarrow \mathcal{M}_{\ell}\left(\widetilde{H}, \widetilde{J} ;\left[\widetilde{\gamma}^{\prime}, \widetilde{w}^{\prime}\right],[\widetilde{\gamma}, \widetilde{w}]\right)
$$

by

$$
\Im\left(u ; z_{1}^{+}, \ldots, z_{\ell}^{+}\right)=\left(\widetilde{u} ; \widetilde{z}_{1}^{+}, \ldots, \widetilde{z}_{\ell}^{+}\right) .
$$

We can extend it to their compactifications and then it becomes an isomorphism between spaces with Kuranishi structure:

$$
\mathfrak{I}: \mathcal{M}_{\ell}\left(H, J ;[\gamma, w],\left[\gamma^{\prime}, w^{\prime}\right]\right) \rightarrow \mathcal{M}_{\ell}\left(\widetilde{H}, \widetilde{J} ;\left[\widetilde{\gamma}^{\prime}, \widetilde{w}^{\prime}\right],[\widetilde{\gamma}, \widetilde{w}]\right) .
$$

We take a CF-perturbation of $\mathcal{M}_{\ell}\left(\widetilde{H}, \widetilde{J} ;\left[\widetilde{\gamma}^{\prime}, \widetilde{w}^{\prime}\right],[\widetilde{\gamma}, \widetilde{w}]\right)$ so that it coincides with one for $\mathcal{M}_{\ell}\left(H, J ;[\gamma, w],\left[\gamma^{\prime}, w^{\prime}\right]\right)$ by the above isomorphism. Then we have

$$
\mathfrak{n}_{(H, J) ; \ell}\left([\gamma, w],\left[\gamma^{\prime}, w^{\prime}\right]\right)\left(h_{1}, \ldots, h_{\ell}\right)=\mathfrak{n}_{(\widetilde{H}, \widetilde{J}) ; \ell}\left(\left[\widetilde{\gamma}^{\prime}, \widetilde{w}^{\prime}\right],[\widetilde{\gamma}, \widetilde{w}]\right)\left(h_{1}, \ldots, h_{\ell}\right),
$$

where the left hand side is defined in (6.5). Therefore

$$
\mathfrak{n}_{(H, J)}^{\mathfrak{b}}\left(\llbracket \gamma, w \rrbracket, \llbracket \gamma^{\prime}, w^{\prime} \rrbracket\right)=\mathfrak{n}_{(\widetilde{H}, \widetilde{J})}^{\mathfrak{b}}\left(\llbracket \widetilde{\gamma}^{\prime}, \widetilde{w}^{\prime} \rrbracket, \llbracket \widetilde{\gamma}, \widetilde{w} \rrbracket\right) .
$$

Here the left hand side is defined in (6.7).

Definition 15.6. Let $\llbracket \gamma, w \rrbracket \in \widehat{\operatorname{Per}}(H), \llbracket \widetilde{\gamma}^{\prime}, \widetilde{w}^{\prime} \rrbracket \in \widehat{\operatorname{Per}}(\widetilde{H})$. We define

$$
\left\langle\llbracket \gamma, w \rrbracket, \llbracket \widetilde{\gamma}^{\prime}, \widetilde{w}^{\prime} \rrbracket\right\rangle= \begin{cases}0 & \text { if } \gamma \neq \gamma^{\prime}, \\ q^{-\left(w \cap \omega+\widetilde{w}^{\prime} \cap \omega\right)} & \text { if } \gamma=\gamma^{\prime} .\end{cases}
$$

We can extend (15.11) to a $\Lambda^{\downarrow}$ bilinear pairing

$$
\langle\cdot, \cdot\rangle: C F\left(M, H ; \Lambda^{\downarrow}\right) \times C F\left(M, \widetilde{H} ; \Lambda^{\downarrow}\right) \rightarrow \Lambda^{\downarrow},
$$

which becomes a perfect pairing.

By (15.8) we have

$$
\langle\llbracket \gamma, w \rrbracket, \llbracket \widetilde{\gamma}, \widetilde{w} \rrbracket\rangle=1
$$

\section{Lemma 15.7.}

$$
\left\langle\partial_{(H, J)}^{\mathfrak{b}}\left(\llbracket \gamma_{1}, w_{1} \rrbracket\right), \llbracket \widetilde{\gamma}_{2}, \widetilde{w}_{2} \rrbracket\right\rangle=\left\langle\llbracket \gamma_{1}, w_{1} \rrbracket, \partial_{(\widetilde{H}, \widetilde{J})}^{\mathfrak{b}}\left(\llbracket \widetilde{\gamma}_{2}, \widetilde{w}_{2} \rrbracket\right)\right\rangle .
$$

Proof. By definition the left hand side is

$$
\begin{aligned}
& \left.\sum_{w_{2}^{\prime} \in \pi_{2}\left(\gamma_{2}\right)} \mathfrak{n}_{(H, J)}^{\mathfrak{b}}\left(\left[\gamma_{1}, w_{1}\right]\right),\left[\gamma_{2}, w_{2}^{\prime}\right]\right) q^{-\left(w_{2}^{\prime} \cap \omega+\widetilde{w}_{2} \cap \omega\right)} \\
= & \left.\sum_{\alpha \in \pi_{2}(M)} \mathfrak{n}_{(H, J)}^{\mathfrak{b}}\left[\gamma_{1}, w_{1}\right],\left[\gamma_{2}, \alpha \# w_{2}\right]\right) q^{-\alpha \cap \omega} .
\end{aligned}
$$


On the other hand, the right hand side is

$$
\begin{aligned}
& \left.\sum_{\widetilde{w}_{1}^{\prime} \in \pi_{2}\left(\gamma_{1}^{\prime}\right)} \mathfrak{n}_{(\widetilde{H}, \widetilde{J})}^{\mathfrak{b}}\left(\left[\widetilde{\gamma}_{2}, \widetilde{w}_{2}\right]\right),\left[\widetilde{\gamma}_{1}, \widetilde{w}_{1}^{\prime}\right]\right) q^{-\left(w_{1} \cap \omega+\widetilde{w}_{1}^{\prime} \cap \omega\right)} \\
= & \left.\sum_{\alpha \in \pi_{2}(M)} \mathfrak{n}_{(\widetilde{H}, \widetilde{J})}^{\mathfrak{b}}\left(\left[\widetilde{\gamma}_{2}, \widetilde{w}_{2}\right]\right),\left[\widetilde{\gamma}_{1}, \overline{(-\alpha) \# w_{1}}\right]\right) q^{-\alpha \cap \omega} .
\end{aligned}
$$

By (15.10) this is equal to

$$
\mathfrak{n}_{(H, J)}^{\mathfrak{b}}\left(\left[\gamma_{1},(-\alpha) \# w_{1}\right],\left[\gamma_{2}, w_{2}\right]\right) q^{-\alpha \cap \omega} .
$$

Since

$$
\mathfrak{n}_{(H, J)}^{\mathfrak{b}}\left(\left[\gamma_{1},(-\alpha) \# w_{1}\right],\left[\gamma_{2}, w_{2}\right]\right)=\mathfrak{n}_{(H, J)}^{\mathfrak{b}}\left(\left[\gamma_{1}, w_{1}\right],\left[\gamma_{2}, \alpha \# w_{2}\right]\right),
$$

by Proposition 6.2 (7), the lemma follows.

Then (15.12) and (15.13) imply the proposition.

15.4. Duality and Piunikhin isomorphism. In this subsection we prove:

Theorem 15.8. For $a, a^{\prime} \in H^{*}(M ; \Lambda)$ we denote by $a^{b},\left(a^{\prime}\right)^{b}$ the homology classes Poincaré dual to a, $a^{\prime}$ respectively. (See Notations and Conventions (22).) Then we have

$$
\left\langle\mathcal{P}_{\left(H_{\chi}, J_{\chi}\right), *}^{\mathfrak{b}}\left(a^{b}\right), \mathcal{P}_{\left(\widetilde{H}_{\chi}, \widetilde{J}_{\chi}\right), *}^{\mathfrak{b}}\left(\left(a^{\prime}\right)^{b}\right)\right\rangle=\left\langle a, a^{\prime}\right\rangle .
$$

Proof. We consider two chain maps : $(\Omega(M) \widehat{\otimes} \Lambda) \otimes(\Omega(M) \widehat{\otimes} \Lambda) \rightarrow \Lambda \cong \Lambda^{\downarrow}$

$$
h \otimes h^{\prime} \mapsto \int_{M} h \wedge h^{\prime}
$$

and

$$
h \otimes h^{\prime} \mapsto\left\langle\mathcal{P}_{\left(H_{\chi}, J_{\chi}\right), \#}^{\mathfrak{b}}\left(h^{b}\right), \mathcal{P}_{\left(\widetilde{H}_{\chi}, \widetilde{J}_{\chi}\right), \#}^{\mathfrak{b}}\left(\left(h^{\prime}\right)^{b}\right)\right\rangle .
$$

Here we regard $\Lambda^{\downarrow}$ as a chain complex with trivial boundary operator. To prove Theorem 15.8 it suffices to show that 15.15) is chain homotopic to (15.16). For this purpose, we will use the following parameterized moduli space

$$
\mathcal{M}_{\ell}\left(\text { para } ; H_{\chi}, J_{\chi} ; *, * ; C\right)=\bigcup_{S \geq 0}\{S\} \times \mathcal{M}_{\ell}\left(H_{\chi}^{S}, J_{\chi}^{S} ; *, * ; C\right)
$$

equipped with Kuranishi structure and CF-perturbation that is compatible along the boundary. We refer readers to Definition 26.6 in Section 26 for the precise description of the moduli space $\mathcal{M}_{\ell}\left(\right.$ para $\left.; H_{\chi}, J_{\chi} ; *, * ; C\right)$ defined in (26.15). Here $C \in \pi_{2}(M)$.

We denote $\tilde{\chi}=\tilde{\chi}(\tau)=\chi(1-\tau)$. Some boundary component of $\mathcal{M}_{\ell}\left(\right.$ para $\left.; H_{\chi}, J_{\chi} ; *, * ; C\right)$ in (26.18) will contain a direct factor of the type $\mathcal{M}_{\# \mathbb{L}_{2}}\left(H_{\tilde{\chi}}, J_{\tilde{\chi}} ;[\gamma, w], *\right)$ whose definition is given in Definition 26.2. We consider the map

$$
\mathfrak{J}: \mathcal{M}_{\ell}\left(\widetilde{H}_{\chi}, \widetilde{J}_{\chi} ; *,[\widetilde{\gamma}, \widetilde{w}]\right) \rightarrow \mathcal{M}_{\ell}\left(H_{\tilde{\chi}}, J_{\tilde{\chi}} ;[\gamma, w], *\right)
$$

defined by

$$
\mathfrak{J}\left(\left(u ; z_{1}^{+}, \ldots, z_{\ell}^{+}\right)\right)=\left(\tilde{u} ; \tilde{z}_{1}^{+}, \ldots, \tilde{z}_{\ell}^{+}\right),
$$

where the right hand side is defined as in (15.9). The map (15.17) is extended to an isomorphism of spaces with Kuranishi structures. 
Recall that when we considered $\mathcal{P}_{\left(\widetilde{H}_{\chi}, \widetilde{J}_{\chi}\right)}^{\mathfrak{b}}$, we made a choice of a CF-perturbation on $\mathcal{M}_{\ell}\left(\widetilde{H}_{\chi}, \widetilde{J}_{\chi} ; *,[\widetilde{\gamma}, \widetilde{w}]\right)$. This CF-perturbation induces a CF-perturbation on $\mathcal{M}_{\ell}\left(H_{\tilde{\chi}}, J_{\tilde{\chi}} ;[\gamma, w], *\right)$ via the isomorphism (15.17).

We equip $\mathcal{M}_{\ell}\left(\right.$ para; $\left.H_{\chi}, J_{\chi} ; *, * ; C\right)$ with a system of CF-perturbations that is compatible along the boundary with respect to this choice of CF-perturbation on the direct factor $\mathcal{M}_{\ell}\left(H_{\tilde{\chi}}, J_{\tilde{\chi}} ;[\gamma, w], *\right)$ appearing in (26.18).

Remark 15.9. On the other hand, when we will define $\mathcal{Q}_{\left(H_{\tilde{\chi}}, J_{\tilde{\chi}}\right)}^{\mathfrak{b}}$ in Section 26, we take another family of CF-perturbations on $\mathcal{M}_{\ell}\left(H_{\tilde{\chi}}, J_{\tilde{\chi}} ;[\gamma, w], *\right)$. This is different from the CF-perturbation defined above.

Now let $h, h^{\prime}$ be differential forms on $M$. We define

$$
\begin{aligned}
\overline{\mathcal{H}}_{\left(H_{\chi}, J_{\chi}\right)}^{\mathfrak{b}}\left(h, h^{\prime}\right) \\
=\sum_{C} \sum_{\ell=0}^{\infty} \frac{\exp \left(\int_{C} \mathfrak{b}_{2}\right)}{\ell !} q^{-C \cap \omega} \\
\quad \int_{\mathcal{M}_{\ell}\left(\text { para } ; H_{\chi}, J_{\chi} ; *, * ; C\right)} e v_{+\infty}^{*} h \wedge e v_{-\infty}^{*} h^{\prime} \wedge \mathrm{ev}^{*}(\underbrace{\mathfrak{b}_{+}, \ldots, \mathfrak{b}_{+}}_{\ell}),
\end{aligned}
$$

where $\mathfrak{b}_{2}$ is the summand (more precisely its representative closed two-form) in the decomposition $\mathfrak{b}=\mathfrak{b}_{0}+\mathfrak{b}_{2}+\mathfrak{b}_{+}$as before and we use the above chosen CFperturbation on $\mathcal{M}_{\ell}\left(\right.$ para $\left.; H_{\chi}, J_{\chi} ; *, * ; C\right)$ to define an integration on it. The formula (15.18) defines a map

$$
\overline{\mathcal{H}}_{\left(H_{\chi}, J_{\chi}\right)}^{\mathfrak{b}}:(\Omega(M) \widehat{\otimes} \Lambda) \otimes(\Omega(M) \widehat{\otimes} \Lambda) \rightarrow \Lambda^{\downarrow} .
$$

It follows from Lemma $26.8(3)$ that $\overline{\mathcal{H}}_{\left(H_{\chi}, J_{\chi}\right)}^{\mathfrak{b}}$ is a chain homotopy between (15.15) and (15.16). The proof of Theorem 15.8 is complete.

15.5. Proof of Theorem 15.1. Now we prove Theorem 15.1 Let $\mathfrak{b}$ be given. Once Theorem 15.8 is established, the proof is the same as [EP2. It suffices to prove it in the case when $\widetilde{\phi}$ is nondegenerate. We take $H$ such that $\widetilde{\phi}=\widetilde{\psi}_{H}$. Let $a \in Q H_{\mathfrak{b}}^{*}(M)$ and $\epsilon>0$. By Lemma 15.4, we have $b^{\prime} \in Q H_{\mathfrak{b}}^{*}(M)$ such that

$$
\mathfrak{v}_{q}\left(\left\langle\mathcal{P}_{\left(H_{\chi}, J_{\chi}\right), *}^{\mathfrak{b}}\left(a^{b}\right), \mathcal{P}_{\left(\widetilde{H}_{\chi}, \widetilde{J}_{\chi}\right), *}^{\mathfrak{b}}\left(\left(b^{\prime}\right)^{b}\right)\right\rangle\right) \geq \rho^{\mathfrak{b}}(H ; a)-\epsilon
$$

and

$$
\lambda_{\widetilde{H}^{(}}\left(\mathcal{P}_{\left(\widetilde{H}_{\chi}, \widetilde{J}_{\chi}\right), *}\left(\left(b^{\prime}\right)^{b}\right)\right) \leq 0 .
$$

Let $\lambda$ be the left hand side of (15.19). Then

$$
0=\mathfrak{v}_{q}\left(\left\langle\mathcal{P}_{\left(H_{\chi}, J_{\chi}\right), *}^{\mathfrak{b}}\left(a^{b}\right), \mathcal{P}_{\left(\widetilde{H}_{\chi}, \widetilde{J}_{\chi}\right), *}^{\mathfrak{b}}\left(q^{-\lambda}\left(b^{\prime}\right)^{b}\right)\right\rangle\right)=\mathfrak{v}_{q}\left(\left\langle a, q^{-\lambda} b^{\prime}\right\rangle\right) .
$$

(We use Theorem 15.8 here.) We put $b=q^{-\lambda} b^{\prime}$. Then by definition

$$
\Pi(a, b) \neq 0 \text {. }
$$

Thus, since $\left.\left.\lambda_{\widetilde{H}} \mathcal{P}_{\left(\widetilde{H}_{\chi}, \widetilde{J}_{\chi}\right), *}^{\mathfrak{b}}\left(b^{b}\right)\right)=-\lambda+\lambda_{\widetilde{H}^{(}}{ }_{\left(\mathcal{P}_{\chi}, \widetilde{J}_{\chi}\right), *}^{\mathfrak{b}}\left(\left(b^{\prime}\right)^{b}\right)\right) \leq-\lambda$, we have

$$
\rho^{\mathfrak{b}}(H ; a)-\epsilon \leq \lambda \leq-\inf \left\{\rho^{\mathfrak{b}}\left(\widetilde{\psi}_{H}^{-1} ; b\right) \mid \Pi(a, b) \neq 0\right\} .
$$

Hence

$$
\rho^{\mathfrak{b}}\left(\widetilde{\psi}_{H} ; a\right) \leq-\inf \left\{\rho^{\mathfrak{b}}\left(\widetilde{\psi}_{H}^{-1} ; b\right) \mid \Pi(a, b) \neq 0\right\}
$$


On the other hand, if $\Pi(a, b) \neq 0$ then

$$
\mathfrak{v}_{q}(\langle a, b\rangle) \geq 0 .
$$

It implies

$$
\mathfrak{v}_{q}\left(\left\langle\mathcal{P}_{\left(H_{\chi}, J_{\chi}\right), *}^{\mathfrak{b}}\left(a^{b}\right), \mathcal{P}_{\left(\widetilde{H}_{\chi}, \widetilde{J}_{\chi}\right), *}^{\mathfrak{b}}\left(b^{b}\right)\right\rangle\right) \geq 0 .
$$

Hence

$$
\lambda_{H}\left(\mathcal{P}_{\left(H_{\chi}, J_{\chi}\right), *}^{\mathfrak{b}}\left(a^{b}\right)\right)+\lambda_{\widetilde{H}^{\prime}}\left(\mathcal{P}_{\left(\widetilde{H}_{\chi}, \widetilde{J}_{\chi}\right), *}^{\mathfrak{b}}\left(b^{b}\right)\right) \geq 0 .
$$

Therefore

$$
\rho^{\mathfrak{b}}\left(\widetilde{\psi}_{H} ; a\right) \geq-\inf \left\{\rho^{\mathfrak{b}}\left(\widetilde{\psi}_{H}^{-1} ; b\right) \mid \Pi(a, b) \neq 0\right\} .
$$

(15.20) and (15.21) imply Theorem 15.1.

\section{Construction of QuAsi-Morphisms Via SPECTRAL InVARIANT With BulK}

The next definition is due to Entov-Polterovich (see [EP1, Section 1.1]).

Definition 16.1. A function $\mu: \widetilde{\operatorname{Ham}}(M, \omega) \rightarrow \mathbb{R}$ is called a homogeneous Calabi quasi-morphism if the following three conditions are satisfied.

(1) It is a quasi-morphism. Namely there exists a constant $C$ such that for any $\widetilde{\phi}, \widetilde{\psi} \in \widetilde{\operatorname{Ham}}(M, \omega)$ we have

$$
|\mu(\widetilde{\phi} \widetilde{\psi})-\mu(\widetilde{\phi})-\mu(\widetilde{\psi})|<C,
$$

where $C$ is independent of $\widetilde{\phi}, \widetilde{\psi}$.

(2) It is homogeneous. Namely $\mu\left(\widetilde{\phi}^{n}\right)=n \mu(\widetilde{\phi})$ for $n \in \mathbb{Z}$.

(3) If $\widetilde{\phi} \in \widetilde{\operatorname{Ham}}_{U}(M, \omega)$ and $U$ is a displaceable open subset of $M$, then we have $\mu(\widetilde{\phi})=\operatorname{Cal}_{U}(\widetilde{\phi})$.

Remark 16.2. We note that we have the canonical homomorphism $\widetilde{\operatorname{Ham}}_{U}(M, \omega) \rightarrow \widetilde{\operatorname{Ham}}(M, \omega)$. We use this homomorphism to make sense out of the left hand side of the identity (3) above.

The following is the analog to [EP1, Theorem 3.1] whose proof is essentially the same once Theorem 15.1 is at our disposal.

Theorem 16.3. Let $\mathfrak{b} \in H^{\mathrm{even}}\left(M ; \Lambda_{0}\right)$. Suppose that there is a ring isomorphism $Q H_{\mathfrak{b}}^{*}(M) \cong \Lambda \times Q$ and let $e \in Q H_{\mathfrak{b}}^{*}(M)$ be the idempotent corresponding to the unit of the first factor of the right hand side. Then the function

$$
\mu_{e}^{\mathfrak{b}}: \widetilde{\operatorname{Ham}}(M, \omega) \rightarrow \mathbb{R}
$$

is a homogeneous Calabi quasi-morphism.

Remark 16.4. An observation by McDuff is that a sufficient condition for the existence of Calabi quasi-morphism is an existence of a direct product factor of a quantum cohomology that is a field. [EP1] used quantum homology over $\Lambda^{\downarrow}(\mathbb{Q})$, that is the set of $\sum a_{i} q^{\lambda_{i}}$ with $a_{i} \in \mathbb{Q}$. Here we use the (downward) universal Novikov ring $\Lambda^{\downarrow}$, where $a_{i} \in \mathbb{C}$. Since $\Lambda^{\downarrow}$ is an algebraically closed field (see [FOOO3, Appendix A]) and the quantum cohomology ring is finite dimensional thereover, the direct product factor of a quantum cohomology is isomorphic to $\Lambda$, if it is a field. So our assumption of Theorem 16.3 is equivalent to McDuff's in case $\mathfrak{b}=0$. 
Proof. Let $\mathfrak{b}$ and $e$ be as in Theorem 16.3. We first prove the property (1) of Definition 16.1. We begin with the following lemma.

\section{Lemma 16.5.}

$$
\rho^{\mathfrak{b}}(\widetilde{\phi} ; e) \leq 3 \mathfrak{v}_{q}(e)-\rho^{\mathfrak{b}}\left(\widetilde{\phi}^{-1} ; e\right) .
$$

Proof. Let $b \in H(M ; \Lambda)$ such that $\Pi(e, b) \neq 0$. Such a $b$ exists by the nondegeneracy of the Poincaré pairing. We write $b=\left(b_{1}, b_{2}\right)$ with respect to the decomposition $Q H_{\mathfrak{b}}^{*}(M) \cong \Lambda^{\downarrow} \times Q$. Using the Frobenius property of quantum cohomology (see, for example, Man]) we obtain

$$
\langle e, b\rangle=\left\langle e \cup^{\mathfrak{b}} e, b\right\rangle=\left\langle e, e \cup^{\mathfrak{b}} b\right\rangle=\left\langle e, b_{1}\right\rangle .
$$

Therefore $\Pi\left(e, b_{1}\right)=\Pi(e, b) \neq 0$.

Sublemma 16.6. $\mathfrak{v}_{q}\left(b_{1}\right) \geq 0$.

Proof. We have $b_{1}=x e$ for some $x \in \Lambda \backslash\{0\}$. We decompose $e=\sum_{d=0}^{2 n} e_{d}$ with $e_{d} \in H^{d}(M ; \mathbb{C}) \otimes \Lambda$. We denote by $\mathbf{1} \in H^{0}(M ; \mathbb{C})$ the unit of the cohomology ring. Then

$$
\begin{aligned}
\Pi\left(e, b_{1}\right) & =\pi\left(\left\langle e, b_{1}\right\rangle\right)=\pi(\langle e, x e\rangle)=\pi\left(\left\langle e \cup^{\mathfrak{b}} e, x \mathbf{1}\right\rangle\right) \\
& =\pi(\langle e, x \mathbf{1}\rangle)=\pi(\langle x e, \mathbf{1}\rangle)=\pi\left(\left\langle b_{1}, \mathbf{1}\right\rangle\right) .
\end{aligned}
$$

Therefore $\Pi\left(e, b_{1}\right) \neq 0$ implies $\mathfrak{v}_{q}\left(\left\langle b_{1}, \mathbf{1}\right\rangle\right)>0$. Since $\mathfrak{v}_{q}\left(b_{1}\right) \geq \mathfrak{v}_{q}\left(\left\langle b_{1}, \mathbf{1}\right\rangle\right)$, we obtain $\mathfrak{v}_{q}\left(b_{1}\right) \geq 0$ as required.

Let $x e=b_{1}$ and $x \in \Lambda \backslash\{0\}$ as above. Then

$$
\mathfrak{v}_{q}(x)+\mathfrak{v}_{q}(e)=\mathfrak{v}_{q}\left(b_{1}\right) \geq 0 .
$$

Since $b_{1}^{-1}=x^{-1} e$, we get

$$
\mathfrak{v}_{q}\left(b_{1}^{-1}\right)=-\mathfrak{v}_{q}(x)+\mathfrak{v}_{q}(e)=\left(\mathfrak{v}_{q}(e)-\mathfrak{v}_{q}\left(b_{1}\right)\right)+\mathfrak{v}_{q}(e) \leq 2 \mathfrak{v}_{q}(e) .
$$

Therefore

$$
\begin{aligned}
\rho^{\mathfrak{b}}\left(\widetilde{\phi}^{-1} ; b\right) & \geq \rho^{\mathfrak{b}}\left(\widetilde{\phi}^{-1} ; b_{1}\right)-\rho^{\mathfrak{b}}(\underline{0} ; e) \\
& \geq \rho^{\mathfrak{b}}\left(\widetilde{\phi}^{-1} ; e\right)-\rho^{\mathfrak{b}}\left(\underline{0} ; b_{1}^{-1}\right)-\rho^{\mathfrak{b}}(\underline{0} ; e)=\rho^{\mathfrak{b}}\left(\widetilde{\phi}^{-1} ; e\right)-\mathfrak{v}_{q}\left(b_{1}^{-1}\right)-\mathfrak{v}_{q}(e) \\
& \geq \rho^{\mathfrak{b}}\left(\widetilde{\phi}^{-1} ; e\right)-3 \mathfrak{v}_{q}(e) .
\end{aligned}
$$

Here we use the identity $b_{1} \cup^{\mathfrak{b}} b_{1}^{-1}=e$ and the triangle inequality for the second inequality. Lemma 16.5 now follows from Theorem 15.1. Here $\underline{0}$ is the identity element.

\section{Corollary 16.7.}

$$
\rho^{\mathfrak{b}}(\widetilde{\phi} ; e)+\rho^{\mathfrak{b}}(\widetilde{\psi} ; e) \geq \rho^{\mathfrak{b}}(\widetilde{\psi} \widetilde{\phi} ; e) \geq \rho^{\mathfrak{b}}(\widetilde{\phi} ; e)+\rho^{\mathfrak{b}}(\widetilde{\psi} ; e)-3 \mathfrak{v}_{q}(e) .
$$

Proof. The first inequality is a consequence of Theorem 7.8 (5). We have

$$
\rho^{\mathfrak{b}}(\widetilde{\psi} \tilde{\phi} ; e) \geq \rho^{\mathfrak{b}}(\widetilde{\psi} ; e)-\rho^{\mathfrak{b}}\left(\widetilde{\phi}^{-1} ; e\right) \geq \rho^{\mathfrak{b}}(\widetilde{\psi} ; e)+\rho^{\mathfrak{b}}(\widetilde{\phi} ; e)-3 \mathfrak{v}_{q}(e) .
$$

Here the first inequality follows from Theorem 7.8 (5) and the second inequality follows from Lemma 16.5 
We use Corollary 16.7 inductively to show

$$
\left|\rho^{\mathfrak{b}}\left(\widetilde{\phi}_{1} \cdots \widetilde{\phi}_{k} ; e\right)-\sum_{i=1}^{k} \rho^{\mathfrak{b}}\left(\widetilde{\phi}_{i} ; e\right)\right| \leq 3 k \mathfrak{v}_{q}(e) .
$$

Therefore

$$
\begin{gathered}
\left|\rho^{\mathfrak{b}}\left((\widetilde{\phi} \widetilde{\psi})^{n} ; e\right)-n \rho^{\mathfrak{b}}(\widetilde{\phi} ; e)-n \rho^{\mathfrak{b}}(\widetilde{\psi} ; e)\right| \leq 6 n \mathfrak{v}_{q}(e) . \\
\left|\rho^{\mathfrak{b}}\left(\widetilde{\phi}^{n} ; e\right)-n \rho^{\mathfrak{b}}(\widetilde{\phi} ; e)\right| \leq 3 n \mathfrak{v}_{q}(e) . \\
\left|\rho^{\mathfrak{b}}\left(\widetilde{\psi}^{n} ; e\right)-n \rho^{\mathfrak{b}}(\widetilde{\psi} ; e)\right| \leq 3 n \mathfrak{v}_{q}(e) .
\end{gathered}
$$

Hence

$$
\left|\rho^{\mathfrak{b}}\left((\widetilde{\phi} \widetilde{\psi})^{n} ; e\right)-\rho^{\mathfrak{b}}\left(\widetilde{\phi}^{n} ; e\right)-\rho^{\mathfrak{b}}\left(\widetilde{\psi}^{n} ; e\right)\right| \leq 12 n \mathfrak{v}_{q}(e) .
$$

By dividing the last inequality by $n$ and taking its limit as $n \rightarrow \infty$, we obtain

$$
\left|\mu_{e}^{\mathfrak{b}}(\widetilde{\phi} \widetilde{\psi})-\mu_{e}^{\mathfrak{b}}(\widetilde{\phi})-\mu_{e}^{\mathfrak{b}}(\psi)\right| \leq 12 \mathfrak{v}_{q}(e) .
$$

Thus, $\mu_{e}^{\mathfrak{b}}$ is a quasi-morphism.

Remark 16.8. (1) The constant $C$ in Definition 16.1 can be taken to be $12 \mathfrak{v}_{q}(e)$ for the quasi-morphism in Theorem 16.3.

(2) Our proof of Lemma16.5 is slightly simpler than [EP1, Lemma 3.2], since we may assume that the field which is a direct factor of quantum cohomology is $\Lambda$ and so we do not need a result from general non-Archimedean geometry which is quoted in EP1]. By the same reason we obtain an explicit bound.

Definition 16.1 (2) follows from Theorem 13.6 (5).

The homogeneity of $\mu_{e}^{\mathfrak{b}}$ follows from

$$
\rho^{\mathfrak{b}}(\underline{0} ; e) \leq \rho^{\mathfrak{b}}\left(\widetilde{\phi}^{n} ; e\right)+\rho^{\mathfrak{b}}\left(\widetilde{\phi}^{-n} ; e\right) \leq 3 \mathfrak{v}_{q}(e)
$$

and Definition 13.6 (2). The proof of Theorem 16.3 is complete.

Theorem 1.1 is immediate from Theorem 16.3 , 


\section{Part 4. Spectral invariants and Lagrangian Floer theory}

The purpose of this part is to prove Theorem 1.6. The proof is based on openclosed Gromov-Witten theory developed in [FOOO1, Section 3.8], which induces a map from the quantum cohomology of the ambient symplectic manifold to the Hochschild cohomology of $A_{\infty}$ algebra (or more generally that of Fukaya category of $(M, \omega))$. This map is defined in FOOO1 for arbitrary compact symplectic manifold and its (weakly) unobstructed Lagrangian submanifold. See also FOOO7, Section 4.7] for several properties of this map and various related works. For our purpose, we need only a small portion thereof, that is, the part constructed in FOOO1, Theorem 3.8.62] to which we restrict ourselves in this paper, except in Section 25.1.

The main new part of the proof is the construction of a map from Floer homology of periodic Hamiltonians to the Floer cohomology of Lagrangian submanifold, through which the map from quantum cohomology to Floer cohomology of Lagrangian submanifold factors (Definition 18.15 and Proposition [18.20). We also study its properties, especially those related to the filtration.

In Parts 4 and 5, we fix a compatible almost complex structure $J$ that is $t$ independent.

\section{Operator q; REVIEW}

In this section, we review a part of the results from [FOOO1, Section 3.8].

Let $(M, \omega)$ be a compact symplectic manifold and $L$ its relatively spin Lagrangian submanifold. We consider smooth differential forms on $M$. Note that in [FOOO1, FOOO2, FOOO4 we used smooth singular chains instead of differential forms to represent cohomology classes on $M$. In this paper we use differential forms because we use them in the discussion of Floer homology in Part 2. The construction of the operator $\mathfrak{q}$ in this section is a minor modification of the one given in FOOO1, Section 3.8] where smooth singular chains on $M$ are used.

We will introduce a family of operators denoted by

$$
\mathfrak{q}_{\ell, k ; \beta}: E_{\ell}(\Omega(M)[2]) \otimes B_{k}(\Omega(L)[1]) \rightarrow \Omega(L)[1] .
$$

Explanation of the various notations appearing in (17.1) is in order. $\beta$ is an element of the image of $\pi_{2}(M, L) \rightarrow H_{2}(M, L ; \mathbb{Z})$ and $C[i]$ is the degree shift of a $\mathbb{Z}$ graded $\mathbb{C}$-vector space $C$ by $i$ defined by $(C[i])^{d}=C^{d+i}$. We recall from Notations and Conventions (19)-(20) that $E_{\ell} C$ is the quotient of $B_{\ell} C=\underbrace{C \otimes \cdots \otimes C}_{\ell \text { times }}$ by the symmetric group action. The map (17.1) is a $\mathbb{C}$-linear map of degree $1-\mu_{L}(\beta)$ here $\mu_{L}$ is the Maslov index. (See [FOOO1, Definition 2.1.15].)

We next describe the main properties of $\mathfrak{q}_{\ell, k ; \beta}$. Recall from Notations and Conventions (19)-(20) again that both $B C=\bigoplus_{k=0}^{\infty} B_{k} C$ and $E C=\bigoplus_{\ell=0}^{\infty} E_{\ell} C$ have the structure of coassociative coalgebra with coproduct $\Delta$ respectively. We also consider a map $\Delta^{n-1}: B C \rightarrow(B C)^{\otimes n}$ or $E C \rightarrow(E C)^{\otimes n}$ defined by

$$
\Delta^{n-1}=(\Delta \otimes \underbrace{i d \otimes \cdots \otimes i d}_{n-2}) \circ(\Delta \otimes \underbrace{i d \otimes \cdots \otimes i d}_{n-3}) \circ \cdots \circ \Delta .
$$

Following Sweedler's notation $\underline{\mathrm{Sw}}$, we express an element $\mathbf{x} \in B C$ as

$$
\Delta^{n-1}(\mathbf{x})=\sum_{c} \mathbf{x}_{c}^{n ; 1} \otimes \cdots \otimes \mathbf{x}_{c}^{n ; n}
$$


where $c$ runs over some index set depending on $\mathbf{x}$. Here we note that by Notations and Conventions (20) we always use the coproducts $\Delta_{\text {decon }}$ on $B(\Omega(L)[1])$ and $\Delta_{\text {shuff }}$ on $E(\Omega(M)[2])$, respectively. Thus for $\mathbf{x} \in B(\Omega(L)[1])$ the equation (17.2) expresses the decomposition of $\Delta_{\text {decon }}^{n-1}(\mathbf{x})$, while for $\mathbf{y} \in E(\Omega(M)[2])$ the equation (17.2) expresses the decomposition of $\Delta_{\text {shuff }}^{n-1}(\mathbf{y})$. For an element $\mathbf{x}=x_{1} \otimes \cdots \otimes x_{k} \in$ $B_{k}(\Omega(L)[1])$ we put the shifted degree $\operatorname{deg}^{\prime} x_{i}=\operatorname{deg} x_{i}-1$ and $\operatorname{deg}^{\prime} \mathbf{x}=\sum \operatorname{deg}^{\prime} x_{i}=$ $\operatorname{deg} \mathbf{x}-k$. (Recall $\operatorname{deg} x_{i}$ is the cohomological degree of $x_{i}$ before shifted.) The next result is the de Rham version of [FOOO1, Theorem 3.8.32].

Theorem 17.1. The operators $\mathfrak{q}_{\beta ; \ell, k}$ satisfy the following properties:

(1) For each $\beta$ and $\mathbf{x} \in B_{k}(\Omega(L)[1]), \mathbf{y} \in E_{k}(\Omega(M)[2])$,

$$
0=\sum_{\beta_{1}+\beta_{2}=\beta} \sum_{c_{1}, c_{2}}(-1)^{*} \mathfrak{q}_{\beta_{1}}\left(\mathbf{y}_{c_{1}}^{2 ; 1} ; \mathbf{x}_{c_{2}}^{3 ; 1} \otimes \mathfrak{q}_{\beta_{2}}\left(\mathbf{y}_{c_{1}}^{2 ; 2} ; \mathbf{x}_{c_{2}}^{3 ; 2}\right) \otimes \mathbf{x}_{c_{2}}^{3 ; 3}\right)
$$

where $*=\operatorname{deg}^{\prime} \mathbf{x}_{c_{2}}^{3 ; 1}+\operatorname{deg}^{\prime} \mathbf{x}_{c_{2}}^{3 ; 1} \operatorname{deg} \mathbf{y}_{c_{1}}^{2 ; 2}+\operatorname{deg} \mathbf{y}_{c_{1}}^{2 ; 1}$. In (17.3) and hereafter, we write $\mathfrak{q}_{\beta}(\mathbf{y} ; \mathbf{x})$ in place of $\mathfrak{q}_{\ell, k ; \beta}(\mathbf{y} ; \mathbf{x})$ if $\mathbf{y} \in E_{\ell}(\Omega(M)[2]), \mathbf{x} \in B_{k}(\Omega(L)[1])$. We use notation (17.2) in (17.3).

(2) If $1 \in E_{0}(\Omega(M)[2])$ and $\mathbf{x} \in B_{k}(\Omega(L)[1])$ then

$$
\mathfrak{q}_{0, k ; \beta}(1 ; \mathbf{x})=\mathfrak{m}_{k ; \beta}(\mathbf{x}) .
$$

Here $\mathfrak{m}_{k ; \beta}$ is the filtered $A_{\infty}$ structure on $\Omega(L)$.

(3) Let $\mathbf{e}$ be the 0 form (function) on $L$ which is 1 everywhere. Let $\mathbf{x}_{i} \in$ $B(\Omega(L)[1])$ and we put $\mathbf{x}=\mathbf{x}_{1} \otimes \mathbf{e} \otimes \mathbf{x}_{2} \in B(\Omega(L)[1])$. Then

$$
\mathfrak{q}_{\beta}(\mathbf{y} ; \mathbf{x})=0
$$

except the following case :

$$
\mathfrak{q}_{\beta_{0}}(1 ; \mathbf{e} \otimes x)=(-1)^{\operatorname{deg} x} \mathfrak{q}_{\beta_{0}}(1 ; x \otimes \mathbf{e})=x,
$$

where $\beta_{0}=0 \in H_{2}(M, L ; \mathbb{Z})$ and $x \in \Omega(L)[1]=B_{1}(\Omega(L)[1])$. Note 1 in (17.6) is $1 \in E_{0}(\Omega(M)[2])$.

The singular homology version of Theorem 17.1 is proved in [FOOO1, FOOO2, Sections 3.8 and 7.4]. The version where we use de Rham cohomology for $L$ and cycles (of smooth submanifolds) on $M$ is given in [FOOO3, Section 6] for the case when $M$ is a toric manifold and $L$ is a Lagrangian torus fiber of $M$.

Since the details of this construction will be needed for the proof of Theorem 18.8 later in Section 18, we explain the construction of the relevant operators and the main ideas used in the proof of Theorem 17.1 here, although it is a straightforward modification of the constructions given in [FOOO1, FOOO2, FOOO3,

Definition 17.2. We denote by $\stackrel{\circ}{\mathcal{M}}_{k+1 ; \ell}(L ; \beta)$ the set of all $\sim$ equivalence classes of triples $\left(u ; z_{1}^{+}, \ldots, z_{\ell}^{+} ; z_{0}, \ldots, z_{k}\right)$ satisfying the following:

(1) $u:\left(D^{2}, \partial D^{2}\right) \rightarrow(M, L)$ is a pseudo-holomorphic map such that $u\left(\partial D^{2}\right) \subset$ $L$.

(2) $z_{1}^{+}, \ldots, z_{\ell}^{+}$are points in the interior of $D^{2}$ which are mutually distinct.

(3) $z_{0}, \ldots, z_{k}$ are points on the boundary $\partial D^{2}$ of $D^{2}$. They are mutually distinct. $z_{0}, \ldots, z_{k}$ respects the counterclockwise cyclic order on $\partial D^{2}$.

(4) The homology class $u_{*}\left(\left[D^{2}, \partial D^{2}\right]\right)$ is $\beta \in H_{2}(M, L ; \mathbb{Z})$. 
We say that $\left(u ; z_{1}^{+}, \ldots, z_{\ell}^{+} ; z_{0}, \ldots, z_{k}\right) \sim\left(u^{\prime} ; z_{1}^{\prime+}, \ldots, z_{\ell}^{\prime+} ; z_{0}^{\prime}, \ldots, z_{k}^{\prime}\right)$ if there exists a biholomorphic map $v: D^{2} \rightarrow D^{2}$ such that

$$
u^{\prime} \circ v=u, \quad v\left(z_{i}^{+}\right)=z_{i}^{\prime+}, \quad v\left(z_{i}\right)=z_{i}^{\prime} .
$$

We define an evaluation map

$$
\left(\mathrm{ev}, \mathrm{ev}^{\partial}\right)=\left(\mathrm{ev}_{1}, \ldots, \mathrm{ev}_{\ell} ; \mathrm{ev}_{0}^{\partial}, \ldots, \mathrm{ev}_{k}^{\partial}\right): \stackrel{\circ}{\mathcal{M}}_{k+1 ; \ell}(L ; \beta) \rightarrow M^{\ell} \times L^{k+1}
$$

by

$$
\mathrm{ev}_{i}\left(\left[u ; z_{1}^{+}, \ldots, z_{\ell}^{+} ; z_{0}, \ldots, z_{k}\right]\right)=u\left(z_{i}^{+}\right), \quad \operatorname{ev}_{i}^{\partial}\left(\left[u ; z_{1}^{+}, \ldots, z_{\ell}^{+} ; z_{0}, \ldots, z_{k}\right]\right)=u\left(z_{i}\right) .
$$

Proposition 17.3. (1) The moduli space $\stackrel{\circ}{\mathcal{M}}_{k+1 ; \ell}(L ; \beta)$ has a compactification $\mathcal{M}_{k+1 ; \ell}(L ; \beta)$ that is Hausdorff.

(2) The space $\mathcal{M}_{k+1 ; \ell}(L ; \beta)$ has an orientable Kuranishi structure with corners.

(3) The (normalized) boundary of $\mathcal{M}_{k+1 ; \ell}(L ; \beta)$ in the sense of Kuranishi structure is described by the following fiber product over $L$.

$$
\partial \mathcal{M}_{k+1 ; \ell}(L ; \beta)=\bigcup \mathcal{M}_{k_{1}+1 ; \# E_{1}}\left(L ; \beta_{1}\right)_{\mathrm{ev}_{0}^{\partial}} \times_{\mathrm{ev}_{i}^{\partial}} \mathcal{M}_{k_{2}+1 ; \# E_{2}}\left(L ; \beta_{2}\right),
$$

where the union is taken over all $\left(E_{1}, E_{2}\right) \in \operatorname{Shuff}(\ell), k_{1}, k_{2} \in \mathbb{Z}_{\geq 0}$ with $k_{1}+k_{2}=k$ and $\beta_{1}, \beta_{2} \in H_{2}(M, L ; \mathbb{Z})$ with $\beta_{1}+\beta_{2}=\beta$.

(4) There exists a map $\mu_{L}: H_{2}(M, L ; \mathbb{Z}) \rightarrow 2 \mathbb{Z}$, Maslov index, such that the (virtual) dimension satisfies the following equality (17.8).

$$
\operatorname{dim} \mathcal{M}_{k+1 ; \ell}(L ; \beta)=n+\mu_{L}(\beta)+k-2+2 \ell \text {. }
$$

(5) We can define orientations of $\mathcal{M}_{k+1 ; \ell}(L ; \beta)$ so that (3) above is compatible with this orientation in the sense of [FOOO2, Proposition 8.3.3].

(6) The evaluation map is extended to the compactification so that it is compatible with (17.7).

(7) $e v_{0}^{\partial}$ is weakly submersive.

(8) The Kuranishi structure is compatible with the forgetful map of the boundary marked points.

(9) The Kuranishi structure is invariant under the permutation of interior marked points.

(10) The Kuranishi structure is invariant under the cyclic permutation of the boundary marked points.

Remark 17.4. We remark that we require weak submersivity of $e v_{0}^{\partial}$, not for other evaluation maps. This is because we pull back differential forms by $\mathrm{ev}_{i}^{\partial}$ for $i \geq 1$ while we push forward them by $\mathrm{ev}_{0}^{\partial}$. As well-known, it is straightforward to pull back differential forms but one needs some submersivity of the map to push forward them. We would also remark that one cannot require the evaluation maps at all marked points simultaneously weakly submersive. (See [Fu3, Remark 3.2] for more explanation for this point.)

An element of the right hand side of (17.7) is drawn in Figure 12 below. Proposition 17.3 (1) - (7) is proved in FOOO2, Propositions 7.1.1,7.1.2] for the case of $\ell=0$. The same proof applies to case $\ell \neq 0$. The Kuranishi structure satisfying the additional properties (8), (9), (10) is constructed in [Fu3, Corollary 3.1]. We refer [Fu3, Definition 3.1] for the precise meaning of the statement (8).

Lemma 17.5. There exists a system of CF-perturbations on the moduli spaces $\mathcal{M}_{k+1 ; \ell}(L ; \beta)$ such that the following holds: 


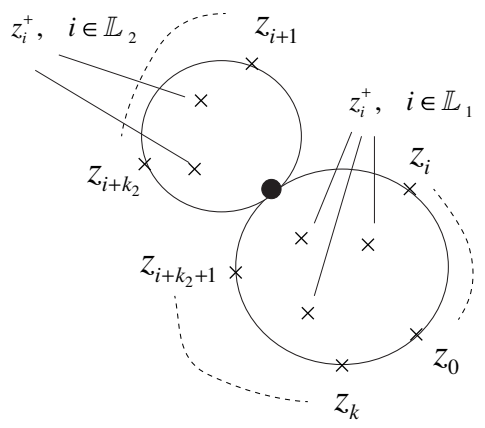

Figure 12. An element of the right hand side of (17.7)

(1) It is transversal to zero in the sense of Definition 33.9.

(2) It is compatible with the description of the boundary in Proposition 17.3 (3) above.

(3) It is compatible with the forgetful map of the boundary marked points.

(4) It is compatible with the permutation of interior marked points.

(5) It is compatible with cyclic permutation of the boundary marked points.

(6) $\mathrm{ev}_{0}^{\partial}$ restricted to the zero set of this system of CF-perturbations is a submersion. In other words, $\mathrm{ev}_{0}^{\partial}$ is strongly submersive with respect to our CF-perturbation in the sense of Definition 33.9, [FOOO11, Definition 7.48].

Proof. Existence of such a system of CF-perturbations is established in Fu3, Corollary 5.2] by an induction over $\beta \cap \omega$ and $\ell$. (See also [FOOO11, Theorem 7.49].)

Remark 17.6. Strictly speaking, we need to fix $E_{0}$ and $\ell_{0}$ and restrict ourselves to those moduli spaces $\mathcal{M}_{k+1 ; \ell}(L ; \beta)$ satisfying $\beta \cap \omega \leq E_{0}$ and $\ell \leq \ell_{0}$, in order to take care of the problem of 'running out' pointed out in [FOOO2, Subsection 7.2.3]. We can handle this in the same way as in FOOO2. With the de Rham version it is simpler to resolve this problem than with the singular homology version used in FOOO2. The detail of this de Rham version is provided in [Fu3, Section 14] for the case $\ell=0$. The case $\ell \neq 0$ can be handled by the same way with the homological algebra developed in [FOOO2, Section 7.4].

Let $g_{1}, \ldots, g_{\ell} \in \Omega(M)$ and $h_{1}, \ldots, h_{k} \in \Omega(L)$ and $\beta$ with $(\beta, \ell) \neq(0,0)$. We define

$$
\begin{aligned}
& \mathfrak{q}_{\ell, k ; \beta}\left(g_{1}, \ldots, g_{\ell}, h_{1}, \ldots, h_{k}\right) \\
& =\left(\mathrm{ev}_{0}^{\partial}\right) !\left(\mathrm{ev}_{1}^{*} g_{1} \wedge \cdots \wedge \mathrm{ev}_{\ell}^{*} g_{\ell} \wedge \mathrm{ev}_{1}^{\partial *} h_{1} \wedge \cdots \wedge \mathrm{ev}_{k}^{\partial *} h_{k}\right) .
\end{aligned}
$$

Here we use the evaluation map

$$
\left(\mathrm{ev}, \mathrm{ev}^{\partial}\right)=\left(\mathrm{ev}_{1}, \ldots, \mathrm{ev}_{\ell} ; \mathrm{ev}_{0}^{\partial}, \ldots, \mathrm{ev}_{k}^{\partial}\right): \mathcal{M}_{k+1 ; \ell}(L ; \beta) \rightarrow M^{\ell} \times L^{k+1}
$$

as the correspondence via the $\mathrm{CF}$-perturbation of the moduli space $\mathcal{M}_{k+1 ; \ell}(L ; \beta)$ given in Lemma 17.5. (See Definition 33.20] and [FOOO11, Definition 9.13] for the definition of $\left(\mathrm{ev}_{0}^{\partial}\right.$ !.) For $\beta=\beta_{0}=0, \ell=0$ we put

$$
\mathfrak{q}_{0, k ; \beta_{0}}\left(h_{1}, \ldots, h_{k}\right)= \begin{cases}0 & k \neq 1,2 \\ (-1)^{n+1+\operatorname{deg} h_{1}} d h_{1} & k=1 \\ (-1)^{\operatorname{deg} h_{1}\left(\operatorname{deg} h_{1}+1\right)} h_{1} \wedge h_{2} & k=2\end{cases}
$$


Theorem 17.1 (1) is a consequence of Proposition 17.3 (2)(3), Stokes' formula (Theorem 34.8 and [FOOO11, Theorem 9.26]) and the composition formula (Theorem 35.7, [FOOO11, Theorem 10.20]).

We may regard (17.4) as the definition of its right hand side. So Theorem 17.1 (2) is obvious.

Theorem 17.1 (3) is a consequence of Proposition 17.3 (8) and the compatibility of CF-perturbations to this forgetful map. See Fu3, Section 7] for a detailed explanation of this point. The proof of Theorem 17.1 is now complete.

Remark 17.7. (1) For $g_{1} \otimes \cdots \otimes g_{\ell} \in B_{\ell}(\Omega(M))$ and $h_{1} \otimes \cdots \otimes h_{k} \in B_{k}(\Omega(L))$ we defined $\mathfrak{q}_{\ell, k ; \beta}\left(g_{1}, \ldots, g_{\ell}, h_{1}, \ldots, h_{k}\right)$ by (17.9). Thanks to Proposition 17.3 (9) this is invariant under the permutation of $g_{1}, \ldots, g_{\ell}$. Thus the operator $\mathfrak{q}_{\ell, k ; \beta}$ descends to the operator

$$
\mathfrak{q}_{\ell, k ; \beta}: E_{\ell}(\Omega(M)[2]) \otimes B_{k}(\Omega(L)[1]) \rightarrow \Omega(L)[1] .
$$

(2) The right hand side of (17.9) is the same as that of [FOOO7, Definition 6.10] even with the same constant in front, but different from the one given in [FOOO1, (3.8.68)] and [FOOO4, (6.10)] in the coefficient. In FOOO1, FOOO3, FOOO4, as we noted in Notations and Conventions (20), we denoted by $E_{\ell} C$ the $\operatorname{Perm}(\ell)$-invariant subset of $B C$ and used the deconcatenation coproduct on it. Indeed, we have the equality $\mathfrak{q}_{\ell, k ; \beta}^{\text {book }}=\frac{1}{\ell !} \mathfrak{q}_{\ell, k ; \beta}$ where we denote by $\mathfrak{q}_{\ell, k ; \beta}^{\text {book }}$ the operator given in FOOO4, (6.10)] (or in [FOOO1, (3.8.68)]). However, we can see that this difference does not cause any trouble in the proof of Theorem 17.1 by just noticing the identity $\mathbf{y}_{c}^{2 ; 1} \otimes \mathbf{y}_{c}^{2 ; 2}=\frac{\ell_{1} ! \ell_{2} !}{\ell !} \mathbf{y}_{c}^{2 ; 1 \prime} \otimes \mathbf{y}_{c}^{2 ; 2 \prime}$ on $E_{\ell_{1}} C \otimes E_{\ell_{2}} C$, where the left (resp. right) hand side is the $\left(\ell_{1}, \ell_{2}\right)$-component in the decomposition of $\Delta_{\text {decon }} \mathbf{y}$ for the invariant set (resp. $\Delta_{\text {shuff }} \mathbf{y}$ for the quotient space). Here we identify the quotient set with the invariant subset by the map $\left[y_{1} \otimes \cdots \otimes y_{\ell}\right] \mapsto \frac{1}{\ell !} \sum_{\sigma \in \mathfrak{S}_{\ell}}(-1)^{*} y_{\sigma(1)} \otimes \cdots \otimes y_{\sigma(\ell)}$ with $*=\sum_{i<j ; \sigma(i)>\sigma(j)} \operatorname{deg} y_{i} \operatorname{deg} y_{j}$.

We next explain how we use the map q to deform the filtered $A_{\infty}$ structure m on $L$. In this section we use the universal Novikov ring $\Lambda_{0}$.

Definition 17.8. Put $\mathbf{b}=\left(\mathfrak{b}_{0}, \mathbf{b}_{2 ; 1}, \mathfrak{b}_{+}, b_{+}\right)$where

$$
\begin{array}{lr}
\mathfrak{b}_{0} \in H^{0}\left(M ; \Lambda_{0}\right), & \mathbf{b}_{2 ; 1} \in H^{2}(M, L ; \mathbb{C}), \\
\mathfrak{b}_{+} \in H^{2}\left(M ; \Lambda_{+}\right) \oplus \bigoplus_{k \geq 2} H^{2 k}\left(M ; \Lambda_{0}\right), & b_{+} \in \Omega^{1}(L) \widehat{\otimes} \Lambda_{+} \oplus \bigoplus_{k \geq 2} \Omega^{2 k-1}(L) \widehat{\otimes} \Lambda_{0} .
\end{array}
$$

Here $\Omega^{1}(L) \widehat{\otimes} \Lambda_{+}$is a completion of an algebraic tensor product, $\Omega^{1}(L) \otimes \Lambda_{+}$.

We represent $\mathfrak{b}_{0}, \mathfrak{b}_{+}$by closed differential forms which are denoted by the same letters. For each $k \neq 0$, we define $\mathfrak{m}_{k}^{\mathbf{b}}$ by

$$
\begin{aligned}
& \mathfrak{m}_{k}^{\mathbf{b}}\left(x_{1}, \ldots, x_{k}\right) \sum_{\beta \in H_{2}(M, L: \mathbb{Z})} \sum_{\ell=0}^{\infty} \sum_{m_{0}=0}^{\infty} \cdots \sum_{m_{k}=0}^{\infty} T^{\omega \cap \beta} \frac{\exp \left(\mathbf{b}_{2 ; 1} \cap \beta\right)}{\ell !} \\
& \mathfrak{q}_{\ell, k+\sum_{i=0}^{k} m_{i} ; \beta}\left(\mathfrak{b}_{+}^{\otimes \ell} ; b_{+}^{\otimes m_{0}}, x_{1}, b_{+}^{\otimes m_{1}}, \ldots, b_{+}^{\otimes m_{k-1}}, x_{k}, b_{+}^{\otimes m_{k}}\right),
\end{aligned}
$$

where $x_{i} \in \Omega(L)$. We extend it $\Lambda_{0}$-linearly to $\Omega(L) \widehat{\otimes} \Lambda_{0}$. 
For $k=0$, we define $\mathfrak{m}_{0}^{\mathbf{b}}$ by

$$
\mathfrak{m}_{0}^{\mathbf{b}}(1)=\mathfrak{b}_{0}+\sum_{\beta \in H_{2}(M, L: \mathbb{Z})} \sum_{\ell=0}^{\infty} \sum_{m=0}^{\infty} T^{\omega \cap \beta} \frac{\exp \left(\mathbf{b}_{2 ; 1} \cap \beta\right)}{\ell !} \mathfrak{q}_{\ell, k+m ; \beta}\left(\mathfrak{b}_{+}^{\otimes \ell} ; b_{+}^{\otimes m}\right) .
$$

Here we embed $H^{0}\left(M ; \Lambda_{0}\right)=\Lambda_{0} \subset \Omega^{0}(L) \widehat{\otimes} \Lambda_{0}$ as $\Lambda_{0}$-valued constant functions on $M$.

We can prove that the right hand sides in (17.11), (25.4) converge in $T$-adic topology in the same way as in Lemma 6.5.

Remark 17.9. The weight appearing in (17.11) is mostly the same as the one appearing in the definition of Gromov-Witten invariants. (See Remark 5.3, and [FOOO7, Section 4.1].)

Lemma 17.10. The family $\left\{\mathfrak{m}_{k}^{\mathrm{b}}\right\}_{k=0}^{\infty}$ defines a filtered $A_{\infty}$ structure on $\Omega(L) \widehat{\otimes} \Lambda_{0}$.

Proof. The proof is a straightforward calculation using Theorem 17.1, See [FOOO1, Lemma 3.8.39] for the detail of the proof of such a statement in the purely abstract context.

We regard the constant function 1 on $L$ as a differential zero-form which we denote by $\mathbf{e}_{L}$.

Definition 17.11. Denote by $\widehat{\mathcal{M}}_{\text {weak,def }}\left(L ; \Lambda_{0}\right)$ the set of all the elements $\mathbf{b}=$ $\left(\mathfrak{b}_{0}, \mathbf{b}_{2 ; 1}, \mathfrak{b}_{+}, b_{+}\right)$as in Definition 17.8 that satisfy the equation

$$
\mathfrak{m}_{0}^{\mathbf{b}}(1)=c \mathbf{e}_{L}
$$

for $c=c(\mathbf{b}) \in \Lambda_{+}$. We define $\mathfrak{P O}(\mathbf{b}) \in \Lambda_{+}$to be the coefficient $c(\mathbf{b})$, i.e., by the equation

$$
\mathfrak{m}_{0}^{\mathbf{b}}(1)=\mathfrak{P O}(\mathbf{b}) \mathbf{e}_{L} .
$$

We call the map $\mathfrak{P O}: \widehat{\mathcal{M}}_{\text {weak,def }}\left(L ; \Lambda_{0}\right) \rightarrow \Lambda_{+}$the potential function. We also define the projection

$\pi: \widehat{\mathcal{M}}_{\text {weak,def }}\left(L ; \Lambda_{0}\right) \rightarrow H^{0}\left(M ; \Lambda_{0}\right) \oplus H^{2}(M, L ; \mathbb{C}) \oplus H^{2}\left(M ; \Lambda_{+}\right) \oplus \bigoplus_{k \geq 2} H^{2 k}\left(M ; \Lambda_{0}\right)$ by $\pi\left(\mathfrak{b}_{0}, \mathbf{b}_{2 ; 1}, \mathfrak{b}_{+}, b_{+}\right)=\left(\mathfrak{b}_{0}, \mathbf{b}_{2 ; 1}, \mathfrak{b}_{+}\right)$.

Let $\mathbf{b}^{(i)}=\left(\mathfrak{b}_{0}^{(i)}, \mathbf{b}_{2 ; 1}^{(i)}, \mathfrak{b}_{+}^{(i)}, b_{+}^{(i)}\right) \in \widehat{\mathcal{M}}_{\text {weak,def }}\left(L ; \Lambda_{0, \text { nov }}^{+}\right)(i=1,2)$ such that $\pi\left(\mathbf{b}^{(1)}\right)=$ $\pi\left(\mathbf{b}^{(0)}\right)$. We define an operator $\delta^{\mathbf{b}^{(1)}, \mathbf{b}^{(0)}}: \Omega(L) \widehat{\otimes} \Lambda_{0} \rightarrow \Omega(L) \widehat{\otimes} \Lambda_{0}$ of degree +1 by

$$
\delta^{\mathbf{b}^{(1)}, \mathbf{b}^{(0)}}(x)=\sum_{k_{1}, k_{0}} \mathfrak{m}_{k_{1}+k_{0}+1}^{\overline{\mathbf{b}}}\left(\left(b_{+}^{(1)}\right)^{\otimes k_{1}} \otimes x \otimes\left(b_{+}^{(0)}\right)^{\otimes k_{0}}\right),
$$

where $\overline{\mathbf{b}}=\left(\mathfrak{b}_{0}^{(0)}, \mathbf{b}_{2 ; 1}^{(0)}, \mathfrak{b}_{+}^{(0)}, 0\right)=\left(\mathfrak{b}_{0}^{(1)}, \mathbf{b}_{2 ; 1}^{(1)}, \mathfrak{b}_{+}^{(1)}, 0\right)$. We remark that if $\mathbf{b}_{1}=\mathbf{b}_{0}=\mathbf{b}$ we have

$$
\delta^{\mathbf{b}^{(1)}, \mathbf{b}^{(0)}}=\mathfrak{m}_{1}^{\mathbf{b}}
$$

Lemma 17.12.

$$
\left(\delta^{\mathbf{b}^{(1)}, \mathbf{b}^{(0)}} \circ \delta^{\mathbf{b}^{(1)}, \mathbf{b}^{(0)}}\right)(x)=\left(-\mathfrak{P O}\left(\mathbf{b}^{(1)}\right)+\mathfrak{P O}\left(\mathbf{b}^{(0)}\right)\right) x .
$$

Proof. This is an easy consequence of Theorem 17.1, See [FOO01, Proposition 3.7.17] for its proof. 
This enables us to give the following definition

Definition 17.13. ([FOOO1, Definition 3.8.61].) For a given pair $\mathbf{b}^{(1)}, \mathbf{b}^{(0)} \in$ $\widehat{\mathcal{M}}_{\text {weak,def }}\left(L ; \Lambda_{0}\right)$ satisfying $\pi\left(\mathbf{b}^{(1)}\right)=\pi\left(\mathbf{b}^{(0)}\right), \quad \mathfrak{P O}\left(\mathbf{b}^{(1)}\right)=\mathfrak{P O}\left(\mathbf{b}^{(0)}\right)$, we define the Lagrangian Floer (cohomology by:

$$
H F\left(\left(L, \mathbf{b}^{(1)}\right),\left(L, \mathbf{b}^{(0)}\right) ; \Lambda_{0}\right)=\frac{\operatorname{Ker}\left(\delta^{\mathbf{b}^{(1)}, \mathbf{b}^{(0)}}\right)}{\operatorname{Im}\left(\delta^{\mathbf{b}^{(1)}, \mathbf{b}^{(0)}}\right)} .
$$

When $\mathbf{b}^{(1)}=\mathbf{b}^{(0)}=\mathbf{b}$, we just write $H F\left((L, \mathbf{b}) ; \Lambda_{0}\right)$ for simplicity.

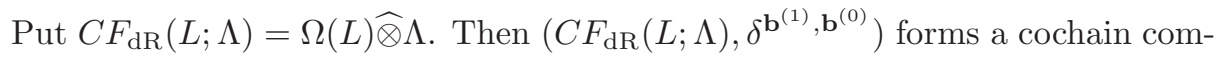
plex. The cochain complex $C F_{\mathrm{dR}}(L ; \Lambda)$ carries a natural filtration given by

$$
F^{\lambda} C F_{\mathrm{dR}}(L ; \Lambda)=T^{\lambda} \Omega(L) \widehat{\otimes} \Lambda_{0}
$$

Lemma 17.14. We have

$$
\delta^{\mathbf{b}^{(1)}, \mathbf{b}^{(0)}}\left(F^{\lambda} C F_{\mathrm{dR}}(L ; \Lambda)\right) \subset F^{\lambda} C F_{\mathrm{dR}}(L ; \Lambda) .
$$

Proof. Since the symplectic area of a pseudo-holomorphic map is nonnegative, $\beta \cap$ $\omega \geq 0$ if $\mathcal{M}_{k+1 ; \ell}(L ; \beta)$ is nonempty. Therefore if $\mathfrak{q}_{\ell, k ; \beta}$ is nonzero then $\beta \cap \omega$ is nonnegative. The lemma follows from this fact and the definition.

This enables us to define the following Lagrangian version of spectral numbers associated to $L$.

Definition 17.15. For $x \in H F\left(\left(L, \mathbf{b}^{(1)}\right),\left(L, \mathbf{b}^{(0)}\right) ; \Lambda\right)$ we put

$$
\begin{array}{r}
\rho_{L}^{\mathbf{b}^{(1)}, \mathbf{b}^{(0)}}(x)=-\sup \left\{\lambda \mid \exists \widehat{x} \in F^{\lambda} C F_{\mathrm{dR}}(L ; \Lambda)\right), \delta^{\mathbf{b}^{(1)}, \mathbf{b}^{(0)}}(\widehat{x})=0, \\
\left.[\widehat{x}]=x \in H F\left(\left(L, \mathbf{b}^{(1)}\right),\left(L, \mathbf{b}^{(0)}\right) ; \Lambda\right)\right\} .
\end{array}
$$

Remark 17.16. We put minus sign in (17.15) for the sake of consistency with Parts 2 and 3 . In fact, $\mathfrak{v}_{q}=-\mathfrak{v}_{T}$ via the isomorphism $\Lambda^{\downarrow} \cong \Lambda$.

We can show

$$
\rho_{L}^{\mathbf{b}^{(1)}, \mathbf{b}^{(0)}}(x)>-\infty
$$

if $x \neq 0$. (See Us1] or Lemma 18.16 of this paper for the detail.)

We next define a closed-open map from the cohomology of the ambient space to the Floer cohomology of $L$. Let $\mathbf{b}=\left(\mathfrak{b}_{0}, \mathbf{b}_{2 ; 1}, \mathfrak{b}_{+}, b_{+}\right) \in \widehat{\mathcal{M}}_{\text {weak,def }}\left(L ; \Lambda_{0}\right)$, take $g \in \Omega(M)$ and define a map $i_{\mathrm{qm}, \mathbf{b}}(g): \Omega(M) \otimes \Lambda_{0} \rightarrow C F_{\mathrm{dR}}\left(L ; \Lambda_{0}\right)$ by

$$
\begin{gathered}
i_{\mathrm{qm}, \mathbf{b}}(g)=(-1)^{\operatorname{deg} g} \sum_{\beta \in H_{2}(M, L: \mathbb{Z})} \sum_{\ell_{1}=0}^{\infty} \sum_{\ell_{2}=0}^{\infty} \sum_{k=0}^{\infty} T^{\omega \cap \beta} \frac{\exp \left(\mathbf{b}_{2 ; 1} \cap \beta\right)}{\left(\ell_{1}+\ell_{2}+1\right) !} \times \\
\mathfrak{q}_{\ell_{1}+\ell_{2}+1, k ; \beta}\left(\mathfrak{b}_{+}^{\otimes \ell_{1}} \otimes g \otimes \mathfrak{b}_{+}^{\otimes \ell_{2}} ; b_{+}^{\otimes k}\right) .
\end{gathered}
$$

Here 'qm' in the subindex of the map $i_{\mathrm{qm}, \mathrm{b}}$ stands for the 'quantum effect' or the effect of pseudoholomorphic discs. This effect also has some interaction with deformation parameter $\mathbf{b}$.

It follows in the same way as in Lemma 6.5 that the right hand side converges in $T$-adic topology.

Lemma 17.17. The map $i_{\mathrm{qm}, \mathbf{b}}$ is a chain map. Namely,

$$
\delta^{\mathbf{b}, \mathbf{b}} \circ i_{\mathrm{qm}, \mathbf{b}}= \pm i_{\mathrm{qm}, \mathbf{b}} \circ d .
$$


Proof. This is a consequence of Theorem 17.1 See [FOOO1, Theorem 3.8.62]. We recall from [FOO01, Remark 3.5.8] that $\mathfrak{m}_{1 ; \beta_{0}}$ in (17.4) satisfies $\mathfrak{m}_{1 ; \beta_{0}}(h)=$ $(-1)^{n+\operatorname{deg} h+1} d h$ for $h \in \Omega^{\operatorname{deg} h}(M)$.

We thus obtain a homomorphism

$$
i_{\mathrm{qm}, \mathbf{b}}^{*}: H^{*}\left(M ; \Lambda_{0}\right) \rightarrow H F^{*}\left((L, \mathbf{b}) ; \Lambda_{0}\right) .
$$

Remark 17.18. The homomorphism (17.18) is indeed a ring homomorphism. It is proved in [FOOO7, Section 2.6] for the toric case. See [FOOO7, Section 4.7] and AFOOO1 for the general case.

Composing the map $i_{\mathrm{qm}, \mathbf{b}}^{*}$ with $\rho_{L}^{\mathbf{b}^{(1)}, \mathbf{b}^{(0)}}$ in Definition 17.15, we introduce:

Definition 17.19. For each $0 \neq a \in H^{*}(M ; \Lambda)$, we define

$$
\rho_{L}^{\mathbf{b}}(a)=\rho_{L}^{\mathbf{b}, \mathbf{b}}\left(i_{\mathrm{qm}, \mathbf{b}}^{*}(a)\right)
$$

for $\mathbf{b} \in \widehat{\mathcal{M}}_{\text {weak,def }}\left(L ; \Lambda_{0}\right)$.

Therefore by the finiteness (17.16),$\rho_{L}^{\mathbf{b}}(a)>-\infty$ for any $a \neq 0$, provided there exists some element $\mathbf{b} \in \widehat{\mathcal{M}}_{\text {weak,def }}\left(L ; \Lambda_{0}\right)$ such that $i_{\mathrm{qm}, \mathbf{b}}^{*}(a) \neq 0$.

\section{CRiterion For heAviness of Lagrangian submanifolds}

In this section, we incorporate the Lagrangian Floer theory into the theory of spectral invariants and Calabi quasi-morphisms of Hamiltonian flows and symplectic quasi-states.

18.1. Statement of the results. We review the notions of heavy and superheavy subsets of a symplectic manifold $(M, \omega)$ introduced by Entov and Polterovich EP3, Definition 1.3]. (See also $[\mathrm{Al}, \mathrm{BC}$ for some related results.)

Definition 18.1. Let $\zeta$ be a partial symplectic quasi-state on $(M, \omega)$. A closed subset $Y \subset M$ is called $\zeta$-heavy if

$$
\zeta(H) \leq \sup \{H(p) \mid p \in Y\}
$$

for any $H \in C^{0}(M)$.

A closed subset $Y \subset M$ is called $\zeta$-superheavy if

$$
\zeta(H) \geq \inf \{H(p) \mid p \in Y\}
$$

for any $H \in C^{0}(M)$.

Remark 18.2. (1) Due to the different sign conventions from EP3 as mentioned in Subsection [1.3. Remark 4.19 and also because we use quantum cohomology class $a$ in the definition of the spectral invariants $\rho(H ; a)$, the above definition looks opposite to that of EP3. However after taking these different convention and usage, this definition of heaviness or of superheaviness of a given subset $S \subset(M, \omega)$ indeed is equivalent to that of [EP3].

(2) Following the proof of [EP3, Proposition 4.1], we can obtain a characterization of a $\zeta$-heavy set or a $\zeta$-superheavy set as follows: A closed subset $Y \subset M$ is $\zeta$-heavy if and only if for every $H \in C^{\infty}(M)$ with $\left.H\right|_{Y}=0$, $H \geq 0$ one has $\zeta(H)=0$. A closed subset $Y \subset M$ is $\zeta$-superheavy if and only if for every $H \in C^{\infty}(M)$ with $\left.H\right|_{Y}=0, H \leq 0$ one has $\zeta(H)=0$. Due to the different sign convention again, this statement is in a slightly 
different form [EP3, Proposition 4.1]. Using this characterization and our triangle inequality Definition 13.3 (8) and the monotonicity (3), we can show that every $\zeta$-superheavy subset is $\zeta$-heavy. This is nothing but EP3, Proposition 4.2].

(3) Furthermore, we can show [EP3, Proposition 4.3] as it is. Namely for any $\zeta$-superheavy set $Y$, and any $\alpha \in \mathbb{R}$ and $H \in C^{\infty}(M)$ with $\left.H\right|_{Y}=\alpha$ we have $\zeta(H)=\alpha$.

(4) [EP3, Entov-Polterovich Theorem 1.4 (iii)] proved that for any partial symplectic quasi-state $\zeta$, every $\zeta$-superheavy set intersects every $\zeta$-heavy subset. See Theorem 18.7.

The definitions of heaviness and super-heaviness EP3 involve only autonomous Hamiltonian. We first enhance the definition by involving time-dependent Hamiltonian. For this purpose, the following definition is useful.

Definition 18.3. Let $H:[0,1] \times M \rightarrow \mathbb{R}$ be a Hamiltonian and $Y \subset M$ be a closed subset. We put $H_{t}(x)=H(t, x)$. For such a pair $(H, Y)$ we associate two constants $E^{ \pm}(H ; Y)$ by

$$
\begin{aligned}
E^{-}(H ; Y) & =\int_{0}^{1}-\min \left(\left.H_{t}\right|_{Y}\right) d t=\int_{0}^{1} \max \left(-\left.H_{t}\right|_{Y}\right) d t \\
E^{+}(H, Y) & =\int_{0}^{1} \max \left(\left.H_{t}\right|_{Y}\right) \\
E(H ; Y) & =E^{-}(H ; Y)+E^{+}(H ; Y) .
\end{aligned}
$$

We remark that when $Y=M, E^{ \pm}(H ; M)$ often appear in relation to the energy estimate and Hofer geometry (see Oh5, Theorem 3.1] for example), and $E(H ; M)$ is the Hofer norm, $\|H\|$.

We note

$$
E^{ \pm}(\underline{H} ; Y)=E^{ \pm}(H ; Y) \mathfrak{p} \frac{1}{\operatorname{vol}_{\omega}(M)} \operatorname{Cal}(H)
$$

and so

$$
E^{-}(H ; Y)+E^{+}(H ; Y)=E^{-}(\underline{H} ; Y)+E^{+}(\underline{H} ; Y)
$$

depend only on the Hamiltonian path $\phi_{H}$, but not on the normalization constant.

Definition 18.4. For $\widetilde{\psi} \in \widetilde{\operatorname{Ham}}(M, \omega)$, we define

$$
\begin{aligned}
e^{-}(\widetilde{\psi} ; Y) & =\inf _{H}\left\{E^{-}(\underline{H} ; Y) \mid \widetilde{\psi}=\left[\phi_{H}\right]\right\} \\
e^{+}(\widetilde{\psi} ; Y) & =\inf _{H}\left\{E^{+}(\underline{H} ; Y) \mid \widetilde{\psi}=\left[\phi_{H}\right]\right\} \\
e(\widetilde{\psi} ; Y) & =\inf _{H}\left\{E(H ; Y) \mid \widetilde{\psi}=\left[\phi_{H}\right]\right\} .
\end{aligned}
$$

Note thanks to (18.4) we do not need to normalize $H$ in the definition of $e(\widetilde{\psi} ; Y)$.

We note $e(\widetilde{\psi} ; Y) \geq e^{+}(\widetilde{\psi} ; Y)+e^{-}(\widetilde{\psi} ; Y)$.

Definition 18.5. Let $\mu: \widetilde{\operatorname{Ham}}(M, \omega) \rightarrow \mathbb{R}$ be an partial quasi-morphism. A closed subset $Y \subset M$ is called $\mu$-heavy if we have

$$
-\mu(\widetilde{\psi}) \leq \operatorname{vol}_{\omega}(M) e^{+}(\widetilde{\psi} ; Y)
$$


for any $\widetilde{\psi}$. A closed subset $Y \subset M$ is called $\mu$-superheavy if we have

$$
-\mu(\widetilde{\psi}) \geq-\operatorname{vol}_{\omega}(M) e^{-}(\widetilde{\psi} ; Y)
$$

for any $\widetilde{\psi}$.

Remark 18.6. We note that our definition of $\mu$-heaviness is given in terms of the universal covering space of $\operatorname{Ham}(M, \omega)$ while the $\zeta$-heaviness is in terms of the autonomous functions. Now consider $\widetilde{\psi}=\left[\phi_{H}\right]$ for an autonomous $H$. Then by (14.5), we derive

$$
-\mu(\widetilde{\psi})=-\mu\left(\phi_{H}\right)=\operatorname{vol}_{\omega}(M) \zeta(\underline{H})=\left(\operatorname{vol}_{\omega}(M) \zeta(H)-\operatorname{Cal}(H)\right)
$$

for autonomous $H$. On the other hand, we also have

$$
-e^{+}(\widetilde{\psi} ; Y) \geq-E^{+}(\underline{H} ; Y)=-E^{+}(H ; Y)+\frac{1}{\operatorname{vol}_{\omega}(M)} \operatorname{Cal}(H)
$$

for arbitrary time-dependent Hamiltonian $H$. Therefore $\mu$-heaviness of $L$ implies $\zeta$-heaviness of $L$. Similarly, we can also see that $\mu$-superheaviness implies $\zeta$-superheaviness. However, since not every element $\widetilde{\psi}$ can be realized by an autonomous Hamiltonian, a priori the $\mu$-heaviness (resp. $\mu$-super-heaviness) is a stronger notion than the $\zeta$-heaviness (resp. $\zeta$-super-heaviness). In fact, the definition of $\mu$-heaviness can be given by replacing the right hand side of (18.6) by some invariant defined in terms of the loop space of the Lagrangian submanifolds. (See Section 25.4 for the related remark.) It is an interesting problem to further investigate their relationship.

The following result is due to Entov-Polterovich [EP3] which will be used later in Section 23. We give a proof for reader's convenience.

Theorem 18.7. ([EP3, Theorem 1.4]) Let $\zeta$ be a partial symplectic quasi-state. If $Y \subset M$ is $\zeta$-superheavy and $Z \subset M$ is $\zeta$-heavy, then for any $\psi \in \operatorname{Symp}_{0}(M, \omega)$ we have

$$
\psi(Y) \cap Z \neq \emptyset .
$$

Proof. Since superheaviness is invariant under symplectic diffeomorphisms contained in $\operatorname{Symp}_{0}(M, \omega)$, we may assume that $\psi$ is the identity map. Suppose $Y \cap Z=\emptyset$. We define $H: M \rightarrow \mathbb{R}$ such that $H=1$ on $Y$ and $H=-1$ on $Z$. Then since $Y$ is $\zeta$-superheavy, $\zeta(H) \geq \inf \{H(y) \mid y \in Y\}=1$. On the other hand, since $Z$ is $\zeta$-heavy, we have $\zeta(H) \leq \sup \{H(z) \mid z \in Z\}=-1$. This is a contradiction.

Now the following is the main theorem of this paper whose proof is completed in Subsection 18.5

Theorem 18.8. Let $L \subset M$ be a relatively spin compact Lagrangian submanifold, and $\mathbf{b}=\left(\mathfrak{b}_{0}, \mathbf{b}_{2 ; 1}, \mathfrak{b}_{+}, b_{+}\right) \in \widehat{\mathcal{M}}_{\text {weak, def }}\left(L ; \Lambda_{0}\right)$ as in Definition 17.11, We put

$$
\mathfrak{b}=i^{*}\left(\mathbf{b}_{2 ; 1}\right)+\mathfrak{b}_{+} \in H^{\text {even }}\left(M ; \Lambda_{0}\right),
$$

where $i^{*}: H^{2}\left(M, L ; \Lambda_{0}\right) \rightarrow H^{2}\left(M ; \Lambda_{0}\right)$ is the natural homomorphism. Let $e \in$ $H^{*}(M ; \Lambda)$. 
(1) If $e \cup^{\mathfrak{b}} e=e$ and

$$
0 \neq i_{\mathrm{qm}, \mathbf{b}}^{*}(e) \in H F^{*}((L, \mathbf{b}) ; \Lambda),
$$

then $L$ is $\zeta_{e}^{\mathfrak{b}}$-heavy and is $\mu_{e}^{\mathfrak{b}}$-heavy.

(2) If there is a direct factor decomposition $Q H_{\mathfrak{b}}^{*}(M ; \Lambda) \cong \Lambda \times Q^{\prime}$ as a ring and $e$ comes from the unit of the direct factor $\Lambda$ which satisfies (18.8), then $L$ is $\zeta_{e}^{\mathfrak{b}}$-superheavy and is $\mu_{e}^{\mathfrak{b}}$-superheavy.

18.2. Floer homologies of periodic Hamiltonians and of Lagrangian submanifolds. The main part of the proof of Theorem 18.8 is the proof of the following proposition.

Proposition 18.9. Let $L, \mathbf{b}$, and $\mathfrak{b}$ be as in Theorem 18.8 and $a \in Q H_{\mathfrak{b}}^{*}(M ; \Lambda)$. Then

$$
\rho^{\mathfrak{b}}(H ; a) \geq-E^{+}(H ; L)+\rho_{L}^{\mathbf{b}}(a)
$$

for any Hamiltonian $H$. Here $\rho_{L}^{\mathbf{b}}(a)$ is as in (17.19). Equivalently, we have

$$
\rho^{\mathfrak{b}}(\widetilde{\psi} ; a) \geq-e^{+}(\widetilde{\psi} ; L)+\rho_{L}^{\mathbf{b}}(a) .
$$

For the proof of Proposition 18.9 we will use a map $\mathfrak{I}_{(H, J)}^{\mathbf{b}, \mathfrak{b}}: C F\left(M, H ; \Lambda^{\downarrow}\right) \rightarrow$ $C F_{\mathrm{dR}}\left(L ; \Lambda^{\downarrow}\right)$ to be introduced in Definition 18.15 the main properties of which we state in Propositions 18.18 and 18.20. The proof of Proposition 18.9 will be completed in Subsection 18.5.

To give the definition of the map $\mathfrak{I}_{(H, J)}^{\mathbf{b}, \mathfrak{b}}$, we start with introducing a moduli spaces relating the Hamiltonian periodic orbits of $H$ and Lagrangian submanifold $L$ as Albers did in [Al]. We refer readers to Remark 18.12 for detailed remark related to this moduli space and its usage. See also [FOOO7, Subsection 4.7]. We recall that we fix a $t$-independent $J$ throughout in Part 4.

Definition 18.10. Let $[\gamma, w] \in \operatorname{Crit}\left(\mathcal{A}_{H}\right)$ and $\beta \in H_{2}(M, L ; \mathbb{Z})$. We denote by $\stackrel{\circ}{\mathcal{M}}_{k+1 ; \ell}(H, J ;[\gamma, w], L ; \beta)$ the set of all triples $\left(u ; z_{1}^{+}, \ldots, z_{\ell}^{+} ; z_{0}, \ldots, z_{k}\right)$ satisfying the following:

(1) $u:(-\infty, 0] \times S^{1} \rightarrow M$ is a smooth map such that $u(0, t) \in L$.

(2) The map $u$ satisfies the equation

$$
\frac{\partial u}{\partial \tau}+J\left(\frac{\partial u}{\partial t}-X_{H_{t}}(u)\right)=0 .
$$

Here $H_{t}(x)=H(t, x)$

(3) The energy

$$
E_{(H, J) ; L}=\frac{1}{2} \int\left(\left|\frac{\partial u}{\partial \tau}\right|_{J}^{2}+\left|\frac{\partial u}{\partial t}-X_{H_{t}}(u)\right|_{J}^{2}\right) d t d \tau
$$

is finite.

(4) The map $u$ satisfies the following asymptotic boundary condition

$$
\lim _{\tau \rightarrow-\infty} u(\tau, t)=\gamma(t) .
$$

(5) $z_{1}^{+}, \ldots, z_{\ell}^{+}$are points in $(-\infty, 0) \times S^{1}$ which are mutually distinct.

(6) $z_{0}, \ldots, z_{k}$ are mutually distinct points on the boundary which are ordered counterclockwise on $S^{1}$ with respect to the boundary orientation coming from $(-\infty, 0] \times S^{1}$. We always set $z_{0}=(0,0)$.

(7) The homology class of the concatenation of $w$ and $u$ is $\beta$. 


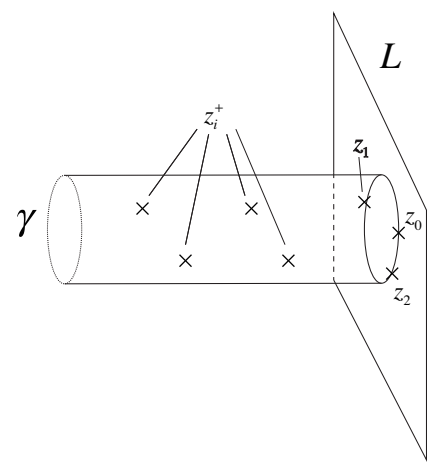

Figure 13. An element of $\stackrel{\circ}{\mathcal{M}}_{k+1 ; \ell}(H, J ;[\gamma, w], L ; \beta)$

For any $\alpha \in \pi_{2}(\gamma)$ we have a canonical homeomorphism

$$
\stackrel{\circ}{\mathcal{M}}_{k+1 ; \ell}(H, J ;[\gamma, \alpha \# w], L ; \beta) \cong \stackrel{\circ}{\mathcal{M}}_{k+1 ; \ell}(H, J ;[\gamma, w], L ; \beta+\alpha) .
$$

In particular if $w$ is homologous to $w^{\prime}$ we have

$$
\stackrel{\circ}{\mathcal{M}}_{k+1 ; \ell}(H, J ;[\gamma, w], L ; \beta) \cong \stackrel{\circ}{\mathcal{M}}_{k+1 ; \ell}\left(H, J ;\left[\gamma, w^{\prime}\right], L ; \beta\right) .
$$

We define an evaluation map

$$
\left(\mathrm{ev} \mathrm{ev}^{\partial}\right)=\left(\mathrm{ev}_{1}, \ldots, \mathrm{ev}_{\ell} ; \mathrm{ev}_{0}^{\partial}, \ldots, \mathrm{ev}_{k}^{\partial}\right): \stackrel{\circ}{\mathcal{M}}_{k+1 ; \ell}(H, J ;[\gamma, w], L ; \beta) \rightarrow M^{\ell} \times L^{k+1}
$$

where

$$
\operatorname{ev}_{i}\left(\left[u ; z_{1}^{+}, \ldots, z_{\ell}^{+} ; z_{0}, \ldots, z_{k}\right]\right)=u\left(z_{i}^{+}\right), \quad \operatorname{ev}_{i}^{\partial}\left(\left[u ; z_{1}^{+}, \ldots, z_{\ell}^{+} ; z_{0}, \ldots, z_{k}\right]\right)=u\left(z_{i}\right) .
$$

Lemma 18.11. (1) The moduli space $\stackrel{\mathcal{M}}{k+1 ; \ell}(H, J ;[\gamma, w], L ; \beta)$ has a compactification $\mathcal{M}_{k+1 ; \ell}(H, J ;[\gamma, w], L ; \beta)$ that is Hausdorff.

(2) The space $\mathcal{M}_{k+1 ; \ell}(H, J ;[\gamma, w], L ; \beta)$ has an orientable Kuranishi structure with corners.

(3) The normalized boundary of $\mathcal{M}_{k+1 ; \ell}(H, J ;[\gamma, w], L ; \beta)$ is described by the union of the following two types of fiber or direct products.

$\bigcup \mathcal{M}_{\# \mathbb{L}_{1}}\left(H, J ;[\gamma, w],\left[\gamma^{\prime}, w^{\prime}\right]\right) \times \mathcal{M}_{k+1 ; \# \mathbb{L}_{2}}\left(H, J ;\left[\gamma^{\prime}, w^{\prime}\right], L ; \beta\right)$,

where the union is taken over all $\left(\gamma^{\prime}, w^{\prime}\right) \in \operatorname{Crit}\left(\mathcal{A}_{H}\right)$, and $\left(\mathbb{L}_{1}, \mathbb{L}_{2}\right) \in$ $\operatorname{Shuff}(\ell)$.

$\bigcup \mathcal{M}_{k_{1}+1 ; \# E_{1}}\left(L ; \beta_{1}\right)_{\mathrm{ev}_{0}^{\partial}} \times_{\mathrm{ev}_{i}^{\partial}} \mathcal{M}_{k_{2}+1 ; \# E_{2}}\left(H, J ;[\gamma, w], L ; \beta_{2}\right)$,

where the union is taken over all $\left(\mathbb{L}_{1}, \mathbb{L}_{2}\right) \in \operatorname{Shuff}(\ell), k_{1}, k_{2}$ with $k_{1}+k_{2}=k$, $i \leq k_{2}$, and $\beta_{1}, \beta_{2}$ with $\beta_{1}+\beta_{2}=\beta$. (See (6.1) for the notation $\operatorname{Shuff}(\ell)$.)

(4) Let $\mu_{H}: \operatorname{Crit}\left(\mathcal{A}_{H}\right) \rightarrow \mathbb{Z}$ be the Conley-Zehnder index and $\mu_{L}: H_{2}(M, L ; \mathbb{Z}) \rightarrow$ $2 \mathbb{Z}$ the Maslov index. Then the (virtual) dimension is given by

$\operatorname{dim} \mathcal{M}_{k+1 ; \ell}(H, J ;[\gamma, w], L ; \beta)=\mu_{L}(\beta)-\mu_{H}([\gamma, w])+2 \ell+k-2+n$.

(5) We can define a system of orientations on the moduli spaces $\mathcal{M}_{k+1 ; \ell}(H, J ;[\gamma, w], L ; \beta)$ that is compatible with the isomorphism (3) above. The compatibility for the boundary of type (18.16) is in the sense of [FOOO2, Proposition 8.3.3]. 
(6) The evaluation map $\left(\mathrm{ev}, \mathrm{ev}^{\partial}\right)$ given above extends to a strongly continuous smooth map

$$
\mathcal{M}_{k+1 ; \ell}(H, J ;[\gamma, w], L ; \beta) \rightarrow M^{\ell} \times L^{k+1},
$$

which we denote also by the same symbol. It is compatible with (3).

(7) $e v_{0}^{\partial}$ is weakly submersive.

(8) The Kuranishi structure is compatible with the forgetful map of the boundary marked points.

(9) The Kuranishi structure is invariant under the permutation of the interior marked points.

(10) The Kuranishi structure is invariant under the cyclic permutation of the boundary marked points.

(11) The homeomorphisms (18.13), 18.14) extend to the compactifications and their Kuranishi structures are identified by the homeomorphisms.

Elements of (18.15) and (18.16) are drawn in Figure 14 and 15 below, respectively.

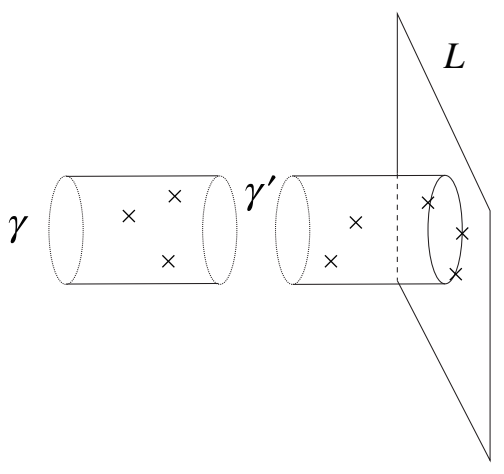

Figure 14. An element of 18.15

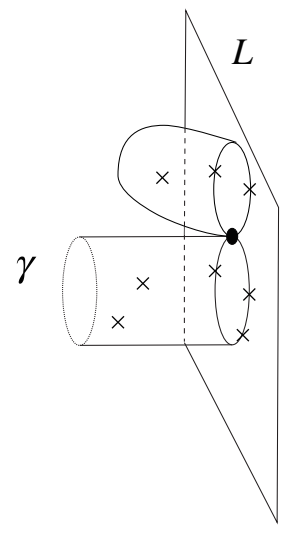

FiguRe 15. An element of (18.16)

The proof of Lemma 18.11 is the same as those of Propositions 3.6, 17.3, which are detailed in [FOOO10, Parts 4 and 5], and so omitted.

Remark 18.12. (1) The same moduli space was used by Albers Al in the monotone case. According to Entov-Polterovich [EP3, p.779], their motivation to define the heaviness comes from $\mathrm{Al}$. See also [FOOO12 for the relevant analytic details needed for the construction of the moduli space. Note that $\mathrm{FOOO12}$ treats the case when $H=0$. However, as is explained in FOOO10, Section 30] (see especially [FOOO10, Lemma 30.24]) the analytic detail given in [FOOO12 can be applied to Floer's equation 18.11) without change.

(2) We refer to [FOOO7, Section 4.7] for a usage of the map $i_{\mathrm{qm}, \mathbf{b}}$ in the study of mirror symmetry. In the monotone case for $\mathbf{b}=0$, the map $i_{\mathrm{qm}, \mathbf{b}}$ coincides with the map considered by Albers [A] and also by Biran-Cornea [BC]. We refer to [FOOO7, Remark 4.7.8] for further details on this relationship. 
Lemma 18.13. There exists a system of CF-perturbations on our moduli space $\mathcal{M}_{k+1 ; \ell}(H, J ;[\gamma, w], L ; \beta)$ with the following properties.

(1) It is transversal to 0.

(2) It is compatible with the description of the boundary in Proposition 18.11 (3).

(3) The evaluation map $\mathrm{ev}_{0}^{\partial}$ is strongly submersive with respect to our $\mathrm{CF}$ perturbation in the sense of Definition [33.9] or [FOOO11, Definition 7.48].

(4) It is compatible with forgetful map of the boundary marked points.

(5) It is invariant under the permutation of the interior marked points.

(6) It is invariant under the cyclic permutation of the boundary marked points.

The compatibility in item (2) above is described as follows. The description of the boundary in Proposition 18.11 (3) identifies the normalized boundary of $\mathcal{M}_{k+1 ; \ell}(H, J ;[\gamma, w], L ; \beta)$ with the fiber product of similar moduli spaces. Using the fact that $\mathrm{ev}_{0}^{\partial}$ is strongly submersive etc. we can define the fiber product CFperburbation. (See Subsection 35.2) The compatibility here means that the restriction of the CF-perturbation of $\mathcal{M}_{k+1 ; \ell}(H, J ;[\gamma, w], L ; \beta)$ to its boundary coincides with the fiber product CF-perturbation.

The proof of Lemma 18.13 is similar to the proof of Lemma 17.5 (and also that of [Fu3, Corollary 5.2] and [FOOO11, Theorem 7.49]) and so omitted.

Let $C F(M, H ; \mathbb{C})$ be the $\mathbb{C}$ vector space over the basis $\operatorname{Crit}\left(\mathcal{A}_{H}\right)$. We use this moduli space to define an operator for $[\gamma, w] \in \operatorname{Crit}\left(\mathcal{A}_{H}\right)$ and $\beta \in H_{2}(M, L ; \mathbb{Z})$.

$$
\mathfrak{q}_{\ell, k ; \beta}^{H ;[\gamma, w]}: E_{\ell}(\Omega(M)[2]) \otimes B_{k}(\Omega(L)[1]) \rightarrow \Omega(L)[1]
$$

as follows. Let $g_{1}, \ldots, g_{\ell} \in \Omega(M)$ and $h_{1}, \ldots, h_{k} \in \Omega(L)$. We define

$$
\begin{aligned}
& \quad \mathfrak{q}_{\ell, k ; \beta}^{H ;[\gamma, w]}\left(g_{1}, \ldots, g_{\ell} ; h_{1}, \ldots, h_{k}\right) \\
& \quad=\left(\operatorname{ev}_{0}^{\partial}\right) !\left(\mathrm{ev}_{1}^{*} g_{1} \wedge \cdots \wedge \mathrm{ev}_{\ell}^{*} g_{\ell} \wedge \mathrm{ev}_{1}^{\partial *} h_{1} \wedge \cdots \wedge \operatorname{ev}_{k}^{\partial *} h_{k}\right) .
\end{aligned}
$$

Here we use the evaluation map

$$
\left(\mathrm{ev}, \mathrm{ev}^{\partial}\right)=\left(\mathrm{ev}_{1}, \ldots, \mathrm{ev}_{\ell} ; \mathrm{ev}_{0}^{\partial}, \ldots, \mathrm{ev}_{k}^{\partial}\right): \mathcal{M}_{k+1 ; \ell}(H, J ;[\gamma, w], L ; \beta) \rightarrow M^{\ell} \times L^{k+1}
$$

and the correspondence given by this moduli space via our CF-perturbation. (See Part 7 and [FOOO11, Definition 7.78].) The next proposition states the main property of this operator.

Proposition 18.14. The operators $\mathfrak{q}_{\ell, k ; \beta}^{H ;[\gamma, w]}$ have the following properties:

(1) $\mathfrak{q}_{\ell, k ; \beta}^{H ;[\gamma, w]}$ satisfies

$$
\begin{aligned}
& 0= \sum_{\beta_{1}+\beta_{2}=\beta} \sum_{c_{1}, c_{2}}(-1)^{*} \mathfrak{q}_{\beta_{1}}\left(\mathbf{y}_{c_{1}}^{2 ; 1} ; \mathbf{x}_{c_{2}}^{3 ; 1} \otimes \mathfrak{q}_{\beta_{2}}^{H ;[\gamma, w]}\left(\mathbf{y}_{c_{1}}^{2 ; 2} ; \mathbf{x}_{c_{2}}^{3 ; 2}\right) \otimes \mathbf{x}_{c_{2}}^{3 ; 3}\right) \\
&+\sum_{c_{1}, c_{2}} \sum_{\left[\gamma^{\prime} w^{\prime}\right] \in \operatorname{Crit}\left(\mathcal{A}_{H}\right)}(-1)^{* *} \mathfrak{n}_{(H, J) ;\left|\mathbf{y}_{c_{2}}^{2 ; 2}\right|}\left([\gamma, w],\left[\gamma^{\prime}, w^{\prime}\right]\right)\left(\mathbf{y}_{c_{2}}^{2 ; 2}\right) \\
& \mathfrak{q}_{\beta}^{H ;\left[\gamma^{\prime}, w^{\prime}\right]}\left(\mathbf{y}_{c_{1}}^{2 ; 1} ; \mathbf{x}\right)
\end{aligned}
$$

where $*=\operatorname{deg}^{\prime} \mathbf{x}_{c_{2}}^{3 ; 1}+\operatorname{deg}^{\prime} \mathbf{x}_{c_{2}}^{3 ; 1} \operatorname{deg} \mathbf{y}_{c_{1}}^{2 ; 2}+\operatorname{deg} \mathbf{y}_{c_{1}}^{2 ; 1}, * *=\operatorname{deg} \mathbf{y}_{c_{1}}^{2 ; 1}$.

The number $\mathfrak{n}_{(H, J) ;\left|\mathbf{y}_{c_{2}}^{2 ; 2}\right|}\left([\gamma, w],\left[\gamma^{\prime}, w^{\prime}\right]\right)\left(\mathbf{y}_{c_{2}}^{2 ; 2}\right) \in \mathbb{C}$ is defined in (6.5). (Here $\left|\mathbf{y}_{c_{2}}^{2 ; 2}\right|$ is defined by $\mathbf{y}_{c_{2}}^{2 ; 2} \in E_{\left|\mathbf{y}_{c_{2}}^{2 ; 2}\right|}(\Omega(M)[2])$.) 
In (18.20) and hereafter, we simplify our notation by writing $\mathfrak{q}_{\beta}^{H ;[\gamma, w]}(\mathbf{y} ; \mathbf{x})$, $\mathfrak{q}_{\beta}(\mathbf{y} ; \mathbf{x})$ in place of $\mathfrak{q}_{\ell, k ; \beta}^{H ;[\gamma, w]}(\mathbf{y} ; \mathbf{x}), \mathfrak{q}_{\ell, k ; \beta}(\mathbf{y} ; \mathbf{x})$ if $\mathbf{y} \in E_{\ell}(\Omega(M)[2]), \mathbf{x} \in$ $B_{k}(\Omega(L)[1])$. We use the notation (17.2) here.

(2) Let $\mathbf{e}_{L}$ be the constant function 1 which we regard degree 0 differential form on L. Let $\mathbf{x}_{i} \in B(H(L ; R)[1])$ and we put $\mathbf{x}=\mathbf{x}_{1} \otimes \mathbf{e}_{L} \otimes \mathbf{x}_{2} \in B(H(L ; R)[1])$. Then

$$
\mathfrak{q}_{\beta}^{H ;[\gamma, w]}(\mathbf{y} ; \mathbf{x})=0 .
$$

Proof. Using Stokes' formula (Theorem 34.8, [FOOO11, Theorem 8.11]) and composition formula (Theorem 35.7 [FOOO11, Theorem 10.20]), Statement (1) follows from Lemma 18.13 (2) and Proposition18.11(3). Statement (2) follows from 18.13 (4) and Proposition 18.11 (8).

Let $\mathbf{b}=\left(\mathfrak{b}_{0}, \mathbf{b}_{2 ; 1}, \mathfrak{b}_{+}, b_{+}\right)$as in Definition 17.8. We put $\mathfrak{b}=i^{*}\left(\mathbf{b}_{2 ; 1}\right)+\mathfrak{b}_{+}$. We extend $\mathfrak{q}_{\ell, k ; \beta}^{H ;[\gamma, w]}$ by $\Lambda$ linearity in the formula (18.22) below.

Definition 18.15. We define $\mathfrak{I}_{(H, J)}^{\mathrm{b}, \mathfrak{b}}: C F\left(M, H ; \Lambda^{\downarrow}\right) \rightarrow C F_{\mathrm{dR}}\left(L ; \Lambda^{\downarrow}\right)$ by

$$
\begin{gathered}
\mathfrak{I}_{(H, J)}^{\mathbf{b}, \mathfrak{b}}(\llbracket \gamma, w \rrbracket)=\sum_{\beta} \sum_{\ell=0}^{\infty} \sum_{k=0}^{\infty} q^{-(\beta \cap \omega-w \cap \omega)} \frac{\exp \left(\mathbf{b}_{2 ; 1} \cap \beta-i^{*}\left(\mathbf{b}_{2 ; 1}\right) \cap w\right)}{\ell !} \\
\mathfrak{q}_{\ell, k ; \beta}^{H,[\gamma, w]}\left(\mathfrak{b}_{+}^{\otimes \ell} ; b_{+}^{\otimes k}\right) .
\end{gathered}
$$

Here we suppose $\llbracket \gamma, w \rrbracket \in \widehat{\operatorname{Per}}(H)$ is represented by $[\gamma, w] \in \operatorname{Crit}\left(\mathcal{A}_{H}\right)$, i.e., $\pi([\gamma, w])=$ $\llbracket \gamma, w \rrbracket$, to define the right hand side. We can show the independence of the representative $[\gamma, w]$ as follows. Suppose we take another choice $\left[\gamma, w^{\prime}\right]$. Then $w^{\prime}=\alpha \# w^{\prime}$ for $\alpha \in K_{2}(\gamma)$. (Lemma 2.2.) We find

$$
\mathfrak{q}_{\ell, k ; \beta}^{H,\left[\gamma, w^{\prime}\right]}\left(\mathfrak{b}_{+}^{\otimes \ell} ; b_{+}^{\otimes k}\right)=\mathfrak{q}_{\ell, k ; \beta+\alpha}^{H,[\gamma, w]}\left(\mathfrak{b}_{+}^{\otimes \ell} ; b_{+}^{\otimes k}\right)
$$

by Proposition 18.11(11). Therefore the sum in the right hand side is independent of the choice of $[\gamma, w]$.

We can prove the convergence of the right hand side of (18.22) in $q$-adic topology in the same way as in Lemma 6.5.

Lemma 18.16. We have

$$
\delta^{\mathbf{b}} \circ \mathfrak{I}_{(H, J)}^{\mathbf{b}, \mathfrak{b}}=\mathfrak{I}_{(H, J)}^{\mathbf{b}, \mathfrak{b}} \circ \partial_{(H, J)}^{\mathfrak{b}}, \quad \delta^{\mathbf{b}}(x)=(-1)^{\operatorname{deg} x} \mathfrak{m}_{1}^{\mathbf{b}}(x) .
$$

The proof is a straightforward calculation using Proposition 18.14 and (6.8) so omitted. This gives rise to a map

$$
\mathfrak{I}_{(H, J)}^{\mathbf{b}, \mathfrak{b}, *}: H F_{*}\left(M, H ; \Lambda^{\downarrow}\right) \rightarrow H F^{*}\left((L, \mathbf{b}) ; \Lambda^{\downarrow}\right) .
$$

Remark 18.17. We can show that the map (18.23) is a ring homomorphism with respect to the pants product in the left hand side and $\pm \mathfrak{m}_{2}$ in the right hand side up to sign. We do not prove it here since we do not use it. 
18.3. Filtration and the map $\mathfrak{I}_{(H, J)}^{\mathbf{b}, \mathfrak{b}}$. We define a filtration on $C F_{\mathrm{dR}}\left(L ; \Lambda^{\downarrow}\right)$

$$
F^{-\lambda} C F_{\mathrm{dR}}\left(L ; \Lambda^{\downarrow}\right):=F^{\lambda} C F_{\mathrm{dR}}(L ; \Lambda)=T^{\lambda}\left(\Omega(L) \widehat{\otimes} \Lambda_{0}\right)
$$

by identifying $C F_{\mathrm{dR}}\left(L ; \Lambda^{\downarrow}\right)$ with $C F_{\mathrm{dR}}(L ; \Lambda)$ via the change $T=q^{-1}$ of formal parameters. Similarly we put

$$
F^{\lambda}\left(\Omega(M) \widehat{\otimes} \Lambda^{\downarrow}\right)=q^{-\lambda}\left(\Omega(M) \widehat{\otimes} \Lambda_{0}^{\downarrow}\right) .
$$

This is consistent with Definitions 2.3, 2.5. See Notations and Conventions (18) in Section 1 and also Remark 17.16 In this subsection we prove the following:

Proposition 18.18. For all $\lambda \in \mathbb{R}$,

$$
\mathfrak{I}_{(H, J)}^{\mathbf{b}, \mathfrak{b}}\left(F^{\lambda}\left(\Omega(M) \widehat{\otimes} \Lambda^{\downarrow}\right)\right) \subseteq F^{\lambda+E^{+}(H ; Y)} C F_{d R}\left(L ; \Lambda^{\downarrow}\right) .
$$

Proof. The proposition immediately follows from Lemma 18.19 below.

Lemma 18.19. If $\mathcal{M}_{k+1 ; \ell}(H, J ;[\gamma, w], L ; \beta)$ is nonempty, we have

$$
\mathcal{A}_{H}([\gamma, w]) \geq-E^{+}(H ; Y)-\beta \cap \omega .
$$

Proof. Let $\left(u ; z_{1}^{+} \ldots, z_{\ell}^{+}, z_{0}, \ldots, z_{k}\right) \in \stackrel{\circ}{\mathcal{M}}_{k+1 ; \ell}(H, J ;[\gamma, w], L ; \beta)$. We calculate

$$
\begin{aligned}
\int u^{*} \omega & =E_{H, J}(u)-\int_{(-\infty, 0] \times S^{1}} \frac{\partial}{\partial \tau}(H \circ u) d \tau d t \\
& \geq \lim _{\tau \rightarrow-\infty} \int_{S^{1}} H(t, u(\tau, t)) d t-\int_{S^{1}} H(0, u(0, t)) d t \\
& =\int_{S^{1}} H(t, \gamma(t)) d t-\int_{0}^{1} H(0, u(0, t)) d t \\
& \geq \int_{S^{1}} H_{t}(\gamma(t)) d t-E^{+}(H ; Y) .
\end{aligned}
$$

Recalling $\beta \cap \omega-\int w^{*} \omega=\int u^{*} \omega$ from $\beta=[w \# u]$, we obtain

$$
-\int w^{*} \omega-\int_{S^{1}} H_{t}(\gamma(t)) d t \geq-E^{+}(H ; Y)-\beta \cap \omega .
$$

The lemma follows.

18.4. Identity $\mathfrak{I}_{(H, J)}^{\mathbf{b}, \mathfrak{b}, *} \circ \mathcal{P}_{\left(H_{\chi}, J\right), *}^{\mathfrak{b}}=i_{\mathrm{qm}, \mathbf{b}}^{*}$. In this subsection we prove:

Proposition 18.20. For any $a \in H^{*}(M) \otimes \Lambda$ the identity

$$
\mathfrak{I}_{(H, J)}^{\mathbf{b}, \mathfrak{b}, *} \circ \mathcal{P}_{\left(H_{\chi}, J\right), *}^{\mathfrak{b}}\left(a^{b}\right)=i_{\mathrm{qm}, \mathbf{b}}^{*}(a)
$$

holds in cohomology. Here $a^{b} \in H_{*}\left(M ; \Lambda^{\downarrow}\right)$ is the homology class Poincarè dual to $a \in H^{*}(M) \otimes \Lambda$ as in Notations and Conventions (22).

Proof. For $S \geq 0$ we put

$$
H^{S}(\tau, t, x)=\chi(\tau+S+20) H(t, x)
$$

where $\chi$ is as in Definition 3.13. We also put $H_{\tau, t}^{S}(x)=H^{S}(\tau, t, x)$.

Definition 18.21. Denote by

$$
\stackrel{\circ}{\mathcal{M}}_{k+1 ; \ell}\left(H^{S}, J ; *, L ; \beta\right)
$$

the set of all triples $\left(u ; z_{1}^{+}, \ldots, z_{\ell}^{+} ; z_{0}, \ldots, z_{k}\right)$ satisfying the following: 
(1) $u:(-\infty, 0] \times S^{1} \rightarrow M$ is a smooth map such that $u(0, t) \subset L$.

(2) $u$ satisfies the equation

$$
\frac{\partial u}{\partial \tau}+J\left(\frac{\partial u}{\partial t}-X_{H_{\tau, t}^{S}}(u)\right)=0 .
$$

(3) The energy

$$
\frac{1}{2} \int\left(\left|\frac{\partial u}{\partial \tau}\right|_{J}^{2}+\left|\frac{\partial u}{\partial t}-X_{H_{\tau, t}^{S}}(u)\right|_{J}^{2}\right) d t d \tau
$$

is finite.

(4) $z_{1}^{+}, \ldots, z_{\ell}^{+}$are points in $(-\infty, 0) \times S^{1}$ which are mutually distinct.

(5) $z_{0}, \ldots, z_{k}$ are points on the boundary $\{0\} \times S^{1}$. They are mutually distinct. $z_{0}, \ldots, z_{k}$ respects the counterclockwise cyclic order on $S^{1}$. We always set $z_{0}=(0,0)$.

(6) The homology class of $u$ is $\beta$.

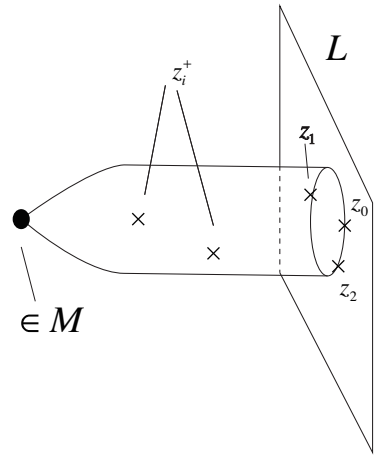

Figure 16. An element of $\stackrel{\circ}{\mathcal{M}}_{k+1 ; \ell}\left(H^{S}, J ; *, L ; \beta\right)$

We define an evaluation map

$$
\left(\mathrm{ev}, \mathrm{ev}^{\partial}\right)=\left(\mathrm{ev}_{1}, \ldots, \mathrm{ev}_{\ell} ; \mathrm{ev}_{0}^{\partial}, \ldots, \mathrm{ev}_{k}^{\partial}\right): \stackrel{\circ}{\mathcal{M}}_{k+1 ; \ell}\left(H^{S}, J ; *, L ; \beta\right) \rightarrow M^{\ell} \times L^{k+1}
$$

by

$$
\mathrm{ev}_{i}\left(u ; z_{1}^{+}, \ldots, z_{\ell}^{+} ; z_{0}, \ldots, z_{k}\right)=u\left(z_{i}^{+}\right), \quad \operatorname{ev}_{i}^{\partial}\left(\left[u ; z_{1}^{+}, \ldots, z_{\ell}^{+} ; z_{0}, \ldots, z_{k}\right]\right)=u\left(z_{i}\right) .
$$

We also define $\mathrm{ev}_{-\infty}: \stackrel{\circ}{\mathcal{M}}_{k+1 ; \ell}\left(H^{S}, J ; *, L ; \beta\right) \rightarrow M$ by

$$
\mathrm{ev}_{-\infty}\left(u ; z_{1}^{+}, \ldots, z_{\ell}^{+} ; z_{0}, \ldots, z_{k}\right)=\lim _{\tau \rightarrow-\infty} u(\tau, t) .
$$

By (2), (3) and the removable singularity theorem, the limit of the right hand side exists and is independent of $t$. We put

$$
\stackrel{\circ}{\mathcal{M}}_{k+1 ; \ell}(\operatorname{para} ; H, J ; *, L ; \beta)=\bigcup_{S \in[0, \infty)}\{S\} \times \stackrel{\circ}{\mathcal{M}}_{k+1 ; \ell}\left(H^{S}, J ; *, L ; \beta\right),
$$

where $\left(e v, \mathrm{ev}^{\partial}\right)$ and $\mathrm{ev}_{-\infty}$ are defined on it in an obvious way.

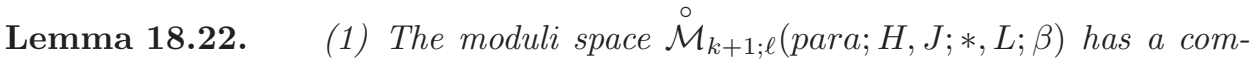
pactification $\mathcal{M}_{k+1 ; \ell}($ para $; H, J ; *, L ; \beta)$ that is Hausdorff. 
(2) The space $\mathcal{M}_{k+1 ; \ell}($ para $; H, J ; *, L ; \beta)$ has an orientable Kuranishi structure with corners.

(3) The normalized boundary of $\mathcal{M}_{k+1 ; \ell}($ para; $H, J ; *, L ; \beta)$ is described by the union of the three types of direct or fiber products:

$$
\bigcup \mathcal{M}_{\# E_{1}}\left(H_{\chi}, J ; *,[\gamma, w]\right) \times \mathcal{M}_{k+1 ; \# E_{2}}(H, J ;[\gamma, w], L ; \beta),
$$

where the union is taken over all $[\gamma, w] \in \operatorname{Crit}\left(\mathcal{A}_{H}\right),\left(\mathbb{L}_{1}, \mathbb{L}_{2}\right) \in \operatorname{Shuff}(\ell)$. (Figure 17) (Here $\mathcal{M}_{\# E_{1}}\left(H_{\chi}, J ; *,[\gamma, w]\right)$ is the moduli space defined in Definition 6.10 and Proposition 6.11. We write $J$ in place of $J_{\chi}$ since in Part 4 we use a fixed $J$ which is independent of $t$ and $\tau$.)

$$
\bigcup \mathcal{M}_{k_{1}+1 ; \# E_{1}}\left(L ; \beta_{1}\right)_{\mathrm{ev}_{0}^{\partial}} \times_{\mathrm{ev}_{i}^{\partial}} \mathcal{M}_{k_{2}+1 ; \# L_{2}}\left(\operatorname{para} ; H, J ; *, L ; \beta_{2}\right),
$$

(Figure 18) where the union is taken over all $\left(\mathbb{L}_{1}, \mathbb{L}_{2}\right) \in \operatorname{Shuff}(\ell), k_{1}, k_{2}$ with $k_{1}+k_{2}=k, i \leq k_{2}$, and $\beta_{1}, \beta_{2}$ with $\beta_{1}+\beta_{2}=\beta$.

$$
\mathcal{M}_{k+1 ; \ell}\left(H^{0}, J ; *, L ; \beta\right),
$$

that is a compactification of the $S=0$ case of $\dot{\mathcal{M}}_{k+1 ; \ell}\left(H^{S}, J ; *, L ; \beta\right)$.

(4) Let $\mu_{L}: H_{2}(M, L ; \mathbb{Z}) \rightarrow 2 \mathbb{Z}$ be the Maslov index. Then the (virtual) dimension satisfies the following equality:

$$
\left.\operatorname{dim} \mathcal{M}_{k+1 ; \ell} \text { (para; } H, J ; *, L ; \beta\right)=\mu_{L}(\beta)+2 \ell+k-1+n .
$$

(5) We can define a system of orientations on the collection of moduli spaces $\mathcal{M}_{k+1 ; \ell}($ para $; H, J ; *, L ; \beta)$ that is compatible with the isomorphism (3) above. For the boundary of type (18.27) the compatibility means the same as in Lemma 18.11 (5).

(6) $\left(\mathrm{ev}, \mathrm{ev}^{2}, \mathrm{ev}_{-\infty}\right)$ extends to a strongly continuous smooth map

$$
\mathcal{M}_{k+1 ; \ell}(\text { para } ; H, J ; *, L ; \beta) \rightarrow M^{\ell+1} \times L^{k+1},
$$

which we denote by the same symbol. It is compatible with (3).

(7) $e v_{0}^{\partial}$ is weakly submersive.

(8) The Kuranishi structure is compatible with forgetful map of the boundary marked points.

(9) The Kuranishi structure is invariant under the permutation of the interior marked points.

(10) The Kuranishi structure is invariant under the cyclic permutation of the boundary marked points.

The proof of Lemma 18.22 is the same as that of Propositions 3.6, which is detailed in FOOO10 Parts 4 and 5. It suffices to observe that (18.26) appears at the limit $S \rightarrow \infty$.

Lemma 18.23. There exists a system of CF-perturbations on our moduli spaces $\mathcal{M}_{k+1 ; \ell}($ para $; H, J ; *, L ; \beta)$ with the following properties:

(1) It is transversal to 0.

(2) It is compatible with the description of the boundary in Proposition 18.22 (3). 


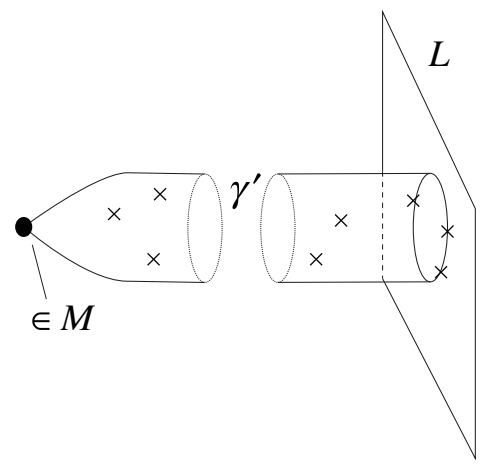

Figure 17. An element of 18.26)

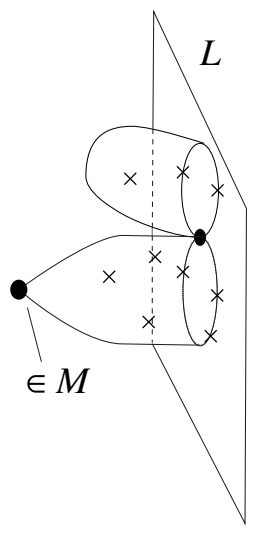

Figure 18. An element of 18.27)

(3) The map $\mathrm{ev}_{0}^{\partial}$ is strongly submersive with respect to our CF-perturbation in the sense of [FOOO11, Definition 7.48].

(4) It is compatible with forgetful map of the boundary marked points.

(5) It is invariant under the permutation of the interior marked points.

(6) It is invariant under the cyclic permutation of the boundary marked points.

The proof is the same as that of Lemma[18.13 (and Fu3, Corollary 5.2], FOOO11, Theorem 7.49]) and is omitted. We now define

$$
\mathfrak{q}_{\ell, k ; \beta}^{H, S \geq 0}: E_{\ell}(\Omega(M)[2]) \otimes \Omega(M)[1] \otimes B_{k}(\Omega(L)[1]) \rightarrow \Omega(L)[1]
$$

by sending $\left(g_{1}, \ldots, g_{\ell} ; h ; h_{1}, \ldots, h_{k}\right)$ to

$$
\left(\operatorname{ev}_{0}^{\partial}\right) !\left(\mathrm{ev}_{1}^{*} g_{1} \wedge \cdots \wedge \mathrm{ev}_{\ell}^{*} g_{\ell} \wedge \mathrm{ev}_{-\infty}^{*} h \wedge \mathrm{ev}_{1}^{\partial *} h_{1} \wedge \cdots \wedge \mathrm{ev}_{k}^{\partial *} h_{k}\right) .
$$

Here

$$
\begin{aligned}
\left(\mathrm{ev} \mathrm{ev}^{\partial}, \mathrm{ev}_{-\infty}\right) & =\left(\mathrm{ev}_{1}, \ldots, \mathrm{ev}_{\ell} ; \mathrm{ev}^{\partial} ; \mathrm{ev}_{0}^{\partial}, \ldots, \mathrm{ev}_{k}^{\partial}\right) \\
& : \mathcal{M}_{k+1 ; \ell}(\text { para } ; H, J ; *, L ; \beta) \rightarrow M^{\ell+1} \times L^{k+1}
\end{aligned}
$$

is the natural evaluation map and $\left(\mathrm{ev}_{0}^{\partial}\right)$ ! is the integration along the fibers of $\mathrm{ev}_{0}^{\partial}$. (The precise definition of this integration associated to the CF-perturbation is given in [FOO011, Definition 7.78]. See also Definition 33.20) We define

$$
\mathfrak{q}_{\ell, k ; \beta}^{H, S=0}: E_{\ell}(\Omega(M)[2]) \otimes \Omega(M)[1] \otimes B_{k}(\Omega(L)[1]) \rightarrow \Omega(L)[1]
$$

by using $\mathcal{M}_{k+1 ; \ell}\left(H^{0}, J ; *, L ; \beta\right)$ in (18.28) in the same way.

Definition 18.24. Define a map $\mathfrak{J}_{\left(H^{0}, J\right)}^{\mathrm{b}, \mathfrak{b}}: \Omega(M) \widehat{\otimes} \Lambda^{\downarrow} \rightarrow C F_{\mathrm{dR}}\left(L ; \Lambda^{\downarrow}\right)$ by

$$
\mathfrak{J}_{\left(H^{0}, J\right)}^{\mathbf{b}, \mathfrak{b}}(h)=\sum_{\beta} \sum_{\ell=0}^{\infty} \sum_{k=0}^{\infty} q^{-\beta \cap \omega} \frac{\exp \left(\mathbf{b}_{2 ; 1} \cap \beta\right)}{\ell !} \mathfrak{q}_{\ell, k ; \beta}^{H, S=0}\left(\mathfrak{b}_{+}^{\otimes \ell}, h, b_{+}^{\otimes k}\right) .
$$

We also define $\mathfrak{H}_{(H, J)}^{\mathbf{b}, \mathfrak{b}}: \Omega(M) \widehat{\otimes} \Lambda^{\downarrow} \rightarrow C F_{\mathrm{dR}}\left(L ; \Lambda^{\downarrow}\right)$ by

$$
\mathfrak{H}_{(H, J)}^{\mathbf{b}, \mathfrak{b}}(h)=\sum_{\beta} \sum_{\ell=0}^{\infty} \sum_{k=0}^{\infty} q^{-\beta \cap \omega} \frac{\exp \left(\mathbf{b}_{2 ; 1} \cap \beta\right)}{\ell !} \mathfrak{q}_{\ell, k ; \beta}^{H, S \geq 0}\left(\mathfrak{b}_{+}^{\otimes \ell}, h, b_{+}^{\otimes k}\right) .
$$


Lemma 18.25. We have

$$
\delta^{\mathbf{b}} \circ \mathfrak{H}_{(H, J)}^{\mathbf{b}, \mathfrak{b}} \pm \mathfrak{H}_{(H, J)}^{\mathbf{b}, \mathfrak{b}} \circ d=\mathfrak{I}_{(H, J)}^{\mathbf{b}, \mathfrak{b}} \circ \mathcal{P}_{\left(H_{\chi}, J\right)}^{\mathfrak{b}} \circ b-\mathfrak{J}_{\left(H^{0}, J\right)}^{\mathbf{b}, \mathfrak{b}} .
$$

Here $b: \Omega^{*}(M) \widehat{\otimes} \Lambda^{\downarrow} \cong \Omega_{\operatorname{dim} M-*}(M) \widehat{\otimes} \Lambda^{\downarrow}$ as in (3.20).

Proof. This follows from Lemma 18.22 after considering the correspondence by using the moduli space in Lemma 18.22 (1) and using Stokes' formula (Theorem 34.8, [FO0011, Theorem 8.11]) and composition formula (Theorem 35.7, FO0011, Theorem 10.20]). Indeed, the first term of left hand side corresponds to (18.27). The first and second terms of the right hand side correspond to (18.26) and (18.28), respectively.

We next construct a chain homotopy between $\mathfrak{J}_{\left(H^{0}, J\right)}^{\mathbf{b}, \mathfrak{b}}$ and $i_{\mathrm{qm}, \mathbf{b}}$. Let $\sigma \in[0,1]$. We replace (18.24) by

$$
\frac{\partial u}{\partial \tau}+J\left(\frac{\partial u}{\partial t}-\sigma X_{H_{t}^{0}}(u)\right)=0
$$

in Definition 18.21 to define $\stackrel{\circ}{\mathcal{M}}_{k+1 ; \ell}\left(\sigma, H^{0}, J ; *, L ; \beta\right)$. We put

$$
\stackrel{\mathcal{M}}{k+1 ; \ell}_{k}\left([0,1], H^{0}, J ; *, L ; \beta\right)=\bigcup_{\sigma \in[0,1]}\{\sigma\} \times \stackrel{\circ}{\mathcal{M}}_{k+1 ; \ell}\left(\sigma, H^{0}, J ; *, L ; \beta\right) .
$$

We can prove a lemma similar to Lemmas $18.22[18.23$ using the compactification $\mathcal{M}_{k+1 ; \ell}\left([0,1], H^{0}, J ; *, L ; \beta\right)$ in place of $\mathcal{M}_{k+1 ; \ell}($ para, $H, J ; *, L ; \beta)$ in (18.31) and (18.33), and define

$$
\overline{\mathfrak{H}}_{\left(H^{0}, J\right)}^{\mathbf{b}, \mathfrak{b}}: \Omega(M) \widehat{\otimes} \Lambda^{\downarrow} \rightarrow C F_{\mathrm{dR}}\left(L ; \Lambda^{\downarrow}\right) .
$$

Then in a similar way we can show

$$
\delta^{\mathbf{b}} \circ \overline{\mathfrak{H}}_{\left(H^{0}, J\right)}^{\mathbf{b}, \mathfrak{b}} \pm \overline{\mathfrak{H}}_{\left(H^{0}, J\right)}^{\mathbf{b}, \mathfrak{b}} \circ d=\mathfrak{J}_{\left(H^{0}, J\right)}^{\mathbf{b}, \mathfrak{b}}-i_{\mathrm{qm}, \mathbf{b}}
$$

Combining this with Lemma 18.4, we finish the proof of Proposition 18.20.

18.5. Heaviness of $L$. We are now ready to complete the proofs of Proposition 18.9 and Theorem 18.8 .

Proof of Proposition 18.9. For $\epsilon>0$ we take $x \in F^{\lambda} C F\left(M, H ; \Lambda^{\downarrow}\right) \cong F^{-\lambda} C F(M, H ; \Lambda)$ such that $[x]=\mathcal{P}_{\left(H_{\chi}, J\right), *}^{\mathfrak{b}}\left(a^{\mathfrak{b}}\right)$ and $\lambda \leq \rho^{\mathfrak{b}}(H ; a)+\epsilon$. By Proposition 18.18 we have

$$
\mathfrak{I}_{(H, J)}^{\mathbf{b}, \mathfrak{b}}(x) \in H^{\lambda+E^{+}(H ; Y)+\epsilon} C F\left(L ; \Lambda^{\downarrow}\right) .
$$

On the other hand, Proposition 18.20 shows that

$$
\left[\mathfrak{I}_{(H, J)}^{\mathbf{b}, \mathfrak{b}}(x)\right]=\mathfrak{I}_{(H, J)}^{\mathbf{b}, \mathfrak{b}, *} \circ \mathcal{P}_{\left(H_{\chi}, J\right), *}^{\mathfrak{b}}\left(a^{\mathfrak{b}}\right)=i_{\mathrm{qm}, \mathbf{b}}^{*}(a) .
$$

Therefore $\lambda+E^{+}(H ; Y) \geq \rho_{L}^{\mathbf{b}}(a)$ by definition. It implies $\rho^{\mathfrak{b}}(H ; a)+\epsilon \geq \rho_{L}^{\mathbf{b}}(a)-$ $E^{+}(H ; Y)$. The proof of Proposition 18.9 is complete.

Proof of Theorem 18.8, We first prove (1). By Remark 18.6 it suffices to prove $\mu_{e}^{\mathfrak{b}}$-heaviness. Let $H: M \times S^{1} \rightarrow \mathbb{R}$ be a normalized periodic Hamiltonian. We put

$$
H_{(n)}(t, x)=n H(n t-[n t], x),
$$


where $[c]$ is the largest integer such that $c \geq[c]$. It is easy to see that $\widetilde{\psi}_{H_{(n)}}=\left(\widetilde{\psi}_{H}\right)^{n}$. We apply Proposition 18.9 to $H_{(n)}$ and obtain

$$
\rho^{\mathfrak{b}}\left(\left(\widetilde{\psi}_{H}\right)^{n} ; e\right) \geq-n \inf E^{+}(H ; L)+\rho_{L}^{\mathbf{b}}(e) .
$$

Therefore by definition we have

$$
-\mu_{e}^{\mathfrak{b}}\left(\widetilde{\psi}_{H}\right) \leq \operatorname{vol}_{\omega}(M) E^{+}(H ; L) .
$$

Thus Theorem 18.8 (1) is proved.

We turn to the proof of (2). Again it suffices to prove $\mu_{e}^{\mathfrak{b}}$-superheaviness.

We use our assumption to apply Lemma 16.5 and obtain

$$
\rho^{\mathfrak{b}}\left(\left(\widetilde{\psi}_{H}\right)^{n} ; e\right) \leq 3 \mathfrak{v}_{q}(e)-\rho^{\mathfrak{b}}\left(\left(\widetilde{\psi}_{H}\right)^{-n} ; e\right) .
$$

We put $\widetilde{H}(t, x)=-H(1-t, x)$ and then obtain $\widetilde{H}_{(n)}$ as in (18.36). We then apply Proposition 18.9 to $\widetilde{H}_{(n)}$ and obtain

$$
\rho^{\mathfrak{b}}\left(\left(\widetilde{\psi}_{H}\right)^{-n} ; e\right) \geq-n E^{-}(H ; L)+\rho_{L}^{\mathbf{b}}(e) .
$$

By (18.37) and (18.38) we have

$$
\rho^{\mathfrak{b}}\left(\left(\widetilde{\psi}_{H}\right)^{n} ; e\right) \leq n E^{-}(H ; L)+3 \mathfrak{v}_{q}(e)-\rho_{L}^{\mathbf{b}}(e)
$$

Therefore

$$
-\mu_{e}^{\mathfrak{b}}\left(\widetilde{\psi}_{H}\right) \geq-\operatorname{vol}_{\omega}(M) E^{-}(H ; L)
$$

as required. The proof of Theorem 18.8 is now complete.

\section{LINEAR INDEPENDENCE OF QUASI-MORPHISMS.}

In this section we prove Corollary [1.9. We use the same notations as those in this corollary. Let $U_{i} \subset M, i=-N, \ldots, N$ be open sets such that $\bar{U}_{i} \cap \bar{U}_{j}=\emptyset$ for $i \neq j$ and $L_{i} \subset U_{i}$ for $i=1, \ldots, N$. For $i=-N, \ldots, N$, let $\rho_{i}$ be nonnegative smooth functions on $M$ such that $\operatorname{supp} \rho_{i} \subset U_{i}(i=-N, \ldots, N), \rho_{i} \equiv 1$ on $L_{i}$ $(i=1, \ldots, N)$, and $\int_{M} \rho_{i} \omega^{n}=c$ where $c>0$ is independent of $i(i=1, \ldots, N)$. We then put

$$
H_{i}=\operatorname{vol}_{\omega}(M)^{-1}\left(\rho_{i}-\rho_{-i}\right)
$$

and regard them as time independent normalized Hamiltonian functions. We put $\widetilde{\psi}_{i}=\widetilde{\psi}_{H_{i}}$. Since the support of $H_{i}$ is disjoint from that of $H_{j}$ it follows that $\widetilde{\psi}_{i}$ for $i \neq j$ commutes with $\widetilde{\psi}_{j}$. Namely they generate a subgroup isomorphic to $\mathbb{Z}^{N}$.

For $\left(k_{1}, \ldots, k_{N}\right) \in \mathbb{Z}^{N}$ we consider

$$
\widetilde{\phi}=\prod_{i=1}^{N} \widetilde{\psi}_{i}^{k_{i}} .
$$

Note that $\widetilde{\phi}=\widetilde{\psi}_{H}$ where $H=\sum_{i=1}^{N} k_{i} H_{i}$. Since $L_{i}$ is $\mu_{e_{i}}^{\mathfrak{b}_{i}}$-superheavy and $\mu_{e_{i}}^{\mathfrak{b}_{i}}$-heavy, we have

$$
\operatorname{vol}_{\omega}(M) \inf \left\{-H(x) \mid x \in L_{i}\right\} \leq \mu_{e_{i}}^{\mathfrak{b}_{i}}(\widetilde{\phi}) \leq \operatorname{vol}_{\omega}(M) \sup \left\{-H(x) \mid x \in L_{i}\right\} .
$$

Therefore $\mu_{e_{i}}^{\mathfrak{b}_{i}}(\widetilde{\phi})=-k_{i}$. The proof of Corollary 1.9 is complete. 


\section{Part 5. Applications}

In this part, we provide applications of the results obtained in the previous parts. Especially combining them with the calculations we carried out in a series of papers FOOO3, FOOO4, FOOO7, in the case of toric manifolds, we prove Corollary 1.2. and Theorem 1.10 for the case of $k(\geq 2)$ points blow up of $\mathbb{C} P^{2}$. The latter example has been studied in FOOO4. We also examine a continuum of Lagrangian tori in $S^{2} \times S^{2}$ discovered by the present authors in [FOOO6] and prove Theorem 1.10,

\section{LAGRANGian Floer theORY OF TORIC FIBERS: REVIEW}

20.1. Toric manifolds: review. In this subsection we review a very small portion of the theory of toric variety. See for example Ful for a detailed account of toric varieties.

Let $(M, \omega, J)$ be a Kähler manifold, where $J$ is its complex structure and $\omega$ its Kähler form. Let $n$ be the complex dimension of $M$. We assume $n$ dimensional real torus $T^{n}=\left(S^{1}\right)^{n}$ acts effectively on $M$ such that $J$ and $\omega$ are preserved by the action. We call such $(M, \omega, J)$ a Kähler toric manifold if the $T^{n}$ action has a moment map in the sense we describe below. Hereafter we simply say $(M, \omega, J)$ (or $M)$ is a toric manifold.

Let $(M, \omega, J)$ be as above. We say a map $\pi=\left(\pi_{1}, \ldots, \pi_{n}\right): M \rightarrow \mathbb{R}^{n}$ is a moment map if the following holds. We consider the $i$-th factor $S_{i}^{1}$ of $T^{n}$. (Here $i=1, \ldots, n$.) Then $\pi_{i}: M \rightarrow \mathbb{R}$ is the moment map of the action of $S_{i}^{1}$. In other words, we have the following identity of $\pi_{i}$

$$
2 \pi d \pi_{i}=\omega\left(\widetilde{\mathfrak{t}}_{i}, \cdot\right)
$$

where $\widetilde{\mathfrak{t}}_{i}$ is the Killing vector field associated to the action of the circle $S_{i}^{1}$ on $X$.

Remark 20.1. We put $2 \pi$ in Formula (20.1) in order to eliminate this factor from (20.5). See Remark 20.3.

Let $\mathbf{u} \in \operatorname{Int} P$. Then the inverse image $\pi^{-1}(\mathbf{u})$ is a Lagrangian submanifold which is an orbit of the $T^{n}$ action. We put

$$
L(\mathbf{u})=\pi^{-1}(\mathbf{u})
$$

This is a Lagrangian torus.

It is well-known that $P=\pi(M)$ is a convex polytope. We can find a finitely many affine functions $\ell_{j}: \mathbb{R}^{n} \rightarrow \mathbb{R}(j=1, \ldots, m)$ such that

$$
P=\left\{\mathbf{u} \in \mathbb{R}^{n} \mid \ell_{j}(\mathbf{u}) \geq 0, \quad \forall j=1, \ldots, m\right\} .
$$

We put $\partial_{j} P=\left\{\mathbf{u} \in P \mid \ell_{j}(\mathbf{u})=0\right\}$ and $D_{j}=\pi^{-1}\left(\partial_{j} P\right) .\left(\operatorname{dim}_{\mathbb{R}} \partial_{j} P=n-1\right.$. $)$ $D_{1} \cup \cdots \cup D_{m}$ is called the toric divisor.

Moreover we may choose $\ell_{j}$ so that the following holds.

Condition 20.2. (1) We put

$$
d \ell_{j}=\vec{v}_{j}=\left(v_{j, 1}, \ldots, v_{j, n}\right) \in \mathbb{R}^{n} .
$$

Then $v_{j, i} \in \mathbb{Z}$.

(2) Let $p$ be a vertex of $P$. Then the number of faces $\partial_{j} P$ which contain $p$ is $n$. Let $\partial_{j_{1}} P, \ldots, \partial_{j_{n}} P$ be those faces. Then $\vec{v}_{j_{1}}, \ldots, \vec{v}_{j_{n}}$ (which is contained in $\mathbb{Z}^{n}$ by item (1)) is a basis of $\mathbb{Z}^{n}$. 
The existence of such $\ell_{j}$ and the property above is proved in [D]. The affine functions $\ell_{j}$ have the following geometric interpretation. Let $\mathbf{u} \in \operatorname{Int} P$. There exists $m$ elements $\beta_{j} \in H_{2}(M, L(\mathbf{u}) ; \mathbb{Z})$ such that

$$
\beta_{j} \cap D_{j^{\prime}}= \begin{cases}1 & j=j^{\prime} \\ 0 & j \neq j^{\prime}\end{cases}
$$

Then the following area formula

$$
\int_{\beta_{j}} \omega=\ell_{j}(\mathbf{u})
$$

is proved in [CO, Theorem 8.1]. (See [FOOO3, Section 2] also.)

Remark 20.3. Note in [CO, Theorem 8.1], FOOO3, Section 2] there is a factor $2 \pi$ in the right hand side of (20.5). We eliminate it by slightly changing the notation of moment map (See Remark 20.1, ) Note in FOOO3 the contribution of the pseudoholomorphic disc of homology class $\beta$ in $\mathfrak{m}_{k}$ has weight $T^{\beta \cap \omega / 2 \pi}$. In this paper and in FOOO1, FOOO2 the weight is $T^{\beta \cap \omega}$.

20.2. Review of Floer cohomology of toric fiber. Let $\widehat{\mathcal{H}}^{2 k}$ be the $\mathbb{C}$ vector space whose basis is given by the cohomology classes which are the Poincaré-dual to the fundamental homology classes of complex codimension $k$ submanifolds of $M$ which arise as transversal intersections of $k$ irreducible components $D_{j_{1}}, \ldots, D_{j_{k}}$ of the toric divisor. For $k=0$ we let $\widehat{\mathcal{H}}^{0}=\mathbb{C}$ and its basis is regarded as a codimension 0 submanifold $M$ itself. For $k \neq 0$ the inclusion map induces an isomorphism

$$
\widehat{\mathcal{H}}^{2 k} \cong H_{2 n-2 k}(M \backslash L(\mathbf{u}) ; \mathbb{C}) \cong H^{2 k}(M, L(\mathbf{u}) ; \mathbb{C}) .
$$

There exists a short exact sequence

$$
0 \rightarrow H_{2 n-2 k}(M ; \mathbb{Z}) \rightarrow H_{2 n-2 k}(M, L(\mathbf{u}) ; \mathbb{Z}) \rightarrow H_{2 n-2 k-1}(L(\mathbf{u}) ; \mathbb{Z}) \rightarrow 0 .
$$

Note that $L(\mathbf{u})$ is a torus and so $H(L(\mathbf{u}) ; \mathbb{Z})$ is a free abelian group. We fix a splitting of (20.7) and identify

$$
H_{2}(M, L(\mathbf{u}) ; \mathbb{Z}) \cong H_{2}(M ; \mathbb{Z}) \oplus H_{1}(L(\mathbf{u}) ; \mathbb{Z}) .
$$

For $k \neq 0$, we also fix a $\mathbb{C}$-linear subspace $\mathcal{H}^{2 k} \subset \widehat{\mathcal{H}}^{2 k}$ such that the homomorphism induced by the inclusion $H_{2 n-2 k}(M \backslash L(\mathbf{u}) ; \mathbb{Z}) \rightarrow H_{2 n-2 k}(M ; \mathbb{Z})$ restricts to an isomorphism from $\mathcal{H}^{2 k}$ to $H_{2 n-2 k}(M ; \mathbb{C})$. For $k=0$, we have

$$
\mathcal{H}^{0} \cong \mathbb{C} \cong H^{0}(M ; \mathbb{C})
$$

where the first isomorphism is given by definition and the second is induced by the canonical unit of $H^{0}(M ; \mathbb{C})$. We recall that the odd degree cohomology of toric manifolds are all trivial.

We take the direct sum and denote $\mathcal{H}=\bigoplus_{k=0}^{n} \mathcal{H}^{2 k}$. We take its basis $\left\{P D\left(\left[D_{a}\right]\right) \mid\right.$ $a=0, \ldots, B\}$ so that $D_{0}=[M]$ (whose Poincaré dual is the unit), each of $D_{1}, \ldots, D_{B_{2}}$ is an irreducible component of the toric divisor $\left(B_{2}=\operatorname{rank} H_{2}(M ; \mathbb{Q})\right)$ and $D_{B_{2}+1} \ldots, D_{B}$ are transversal intersection of irreducible components of the toric divisors. $\left(B+1=\operatorname{rank} H(M ; \mathbb{Q})\right.$.) We put $\mathbf{e}_{a}^{M}=P D\left(\left[D_{a}\right]\right)$.

We put $\underline{B}=\{1, \ldots, B\}$ and denote the set of all maps $\mathbf{p}:\{1, \ldots, \ell\} \rightarrow \underline{B}$ by $\operatorname{Map}(\ell, \underline{B})$. We write $|\mathbf{p}|=\ell$ if $\mathbf{p} \in \operatorname{Map}(\ell, \underline{B})$. 
For $k, \ell \in \mathbb{Z}_{\geq 0}, \mathbf{p} \in \operatorname{Map}(\ell, \underline{B})$ and $\beta \in H_{2}(M, L(\mathbf{u}) ; \mathbb{Z})$ we define a fiber product

$$
\mathcal{M}_{k+1 ; \ell}(L(\mathbf{u}) ; \beta ; \mathbf{p})=\mathcal{M}_{k+1 ; \ell}(L(\mathbf{u}) ; \beta)_{\left(e v_{1}, \ldots, e v_{\ell}\right)} \times_{M^{\ell}} \prod_{i=1}^{\ell} D_{\mathbf{p}(i)},
$$

where $\mathcal{M}_{k+1 ; \ell}(L(\mathbf{u}) ; \beta)$ is the moduli space defined in Definition 17.2 and Proposition 17.3 .

Let $\operatorname{Perm}(\ell)$ be the symmetric group of order $\ell$ !. It acts on $\mathcal{M}_{k+1 ; \ell}(L(\mathbf{u}) ; \beta)$ as the permutation of the interior marked points. We define $\sigma \cdot \mathbf{p}=\mathbf{p} \circ \sigma^{-1}$. They induce a map $\sigma_{*}: \mathcal{M}_{k+1 ; \ell}(L(\mathbf{u}) ; \beta ; \mathbf{p}) \rightarrow \mathcal{M}_{k+1 ; \ell}(L(\mathbf{u}) ; \beta ; \sigma \cdot \mathbf{p})$.

Since $L(\mathbf{u})$ is a $T^{n}$ orbit, $\mathcal{M}_{k+1 ; \ell}(L(\mathbf{u}) ; \beta ; \mathbf{p})$ has a $T^{n}$ action induced by the action on $M$. To describe the boundary of $\mathcal{M}_{k+1 ; \ell}(L(\mathbf{u}) ; \beta ; \mathbf{p})$ we need to prepare some notations. We will define a map

$$
\text { Split : } \operatorname{Shuff}(\ell) \times \operatorname{Map}(\ell, \underline{B}) \longrightarrow \bigcup_{\ell_{1}+\ell_{2}=\ell} \operatorname{Map}\left(\ell_{1}, \underline{B}\right) \times \operatorname{Map}\left(\ell_{2}, \underline{B}\right),
$$

as follows: Let $\mathbf{p} \in \operatorname{Map}(\ell, \underline{B})$ and $\left(\mathbb{L}_{1}, \mathbb{L}_{2}\right) \in \operatorname{Shuff}(\ell)$. We put $\ell_{j}=\#\left(\mathbb{L}_{j}\right)$ and let $\mathfrak{i}_{j}:\left\{1, \ldots, \ell_{j}\right\} \rightarrow \mathbb{L}_{j}$ be the order preserving bijection. We consider the map $\mathbf{p}_{j}:\left\{1, \ldots, \ell_{j}\right\} \rightarrow \underline{B}$ defined by $\mathbf{p}_{j}(i)=\mathbf{p}\left(\mathfrak{i}_{j}(i)\right)$, and set

$$
\operatorname{Split}\left(\left(\mathbb{L}_{1}, \mathbb{L}_{2}\right), \mathbf{p}\right):=\left(\mathbf{p}_{1}, \mathbf{p}_{2}\right) .
$$

Lemma 20.4. (1) $\mathcal{M}_{k+1 ; \ell}(L(\mathbf{u}) ; \beta ; \mathbf{p})$ has a Kuranishi structure with corners.

(2) The Kuranishi structure is invariant under the $T^{n}$ action.

(3) Its normalized boundary is described by the union of fiber products:

$$
\mathcal{M}_{k_{1}+1 ; \# E_{1}}\left(L(\mathbf{u}) ; \beta_{1} ; \mathbf{p}_{1}\right)_{\mathrm{ev}_{0}^{\partial}} \times_{\mathrm{ev}_{i}^{\partial}} \mathcal{M}_{k_{2}+1 ; \# E_{2}}\left(L(\mathbf{u}) ; \beta_{2} ; \mathbf{p}_{2}\right)
$$

where the union is taken over all $\left(E_{1}, E_{2}\right) \in \operatorname{Shuff}(\ell), k_{1}, k_{2}$ with $k_{1}+k_{2}=k$ and $\beta_{1}, \beta_{2} \in H_{2}(M, L(\mathbf{u}) ; \mathbb{Z})$ with $\beta=\beta_{1}+\beta_{2}$. We put $\operatorname{Split}\left(\left(\mathbb{L}_{1}, \mathbb{L}_{2}\right), \mathbf{p}\right)=$ $\left(\mathbf{p}_{1}, \mathbf{p}_{2}\right)$.

(4) The virtual dimension is given by

$\operatorname{dim} \mathcal{M}_{k+1 ; \ell}(L(\mathbf{u}) ; \beta ; \mathbf{p})=n+\mu_{L(\mathbf{u})}(\beta)+k-2+2 \ell-\sum_{i=1}^{\ell} 2 \operatorname{deg} D_{\mathbf{p}(i)}$.

(5) The evaluation maps $\mathrm{ev}_{i}^{\partial}$ at the boundary marked points of $\mathcal{M}_{k+1 ; \ell}(L(\mathbf{u}) ; \beta)$ define strongly continuous smooth maps on $\mathcal{M}_{k+1 ; \ell}(L(\mathbf{u}) ; \beta ; \mathbf{p})$, which we also denote by $\mathrm{ev}_{i}^{\partial}$. They are compatible with the description (3) of the boundary.

(6) We can define an orientation of the Kuranishi structure so that it is compatible with (3).

(7) The evaluation map $\mathrm{ev}_{0}^{\partial}$ is weakly submersive.

(8) The Kuranishi structure is compatible with the permutation of the interior marked points.

(9) The Kuranishi structure is compatible with the forgetful map of the $i$-th boundary marked point for $i=1, \ldots, k$. (We do not require the compatibility with the forgetful map of the 0 -th marked point.)

Lemma 20.4 is proved in [FOOO4, Section 6] and [FOOO7, Section 4.3]. See Figure 12

Lemma 20.5. There exists a system of multisections on $\mathcal{M}_{k+1 ; \ell}(L(\mathbf{u}) ; \beta ; \mathbf{p})$ with the following properties: 
(1) They are transversal to 0.

(2) They are invariant under the $T^{n}$ action.

(3) They are compatible with the description of the boundary in Lemma 20.4 (3).

(4) The restriction of $\mathrm{ev}_{0}^{\partial}$ to the zero set of this multisection is a submersion.

(5) They are invariant under permutation of the interior marked points.

(6) The multisection is compatible with the forgetful map of the $i$-th boundary marked point for $i=1, \ldots, k$.

This is also proved in [FOOO4, Section 6] and [FOOO7, Section 4.4]. We note that (4) is a consequence of (2).

Let $h_{1}, \ldots, h_{k} \in \Omega(L(\mathbf{u}))$. We then define a differential form on $L(\mathbf{u})$ by

$$
\mathfrak{q}_{\ell, k ; \beta}^{T}\left(\mathbf{p} ; h_{1}, \ldots, h_{k}\right)=\left(\operatorname{ev}_{0}^{\partial}\right) !\left(\operatorname{ev}_{1}^{\partial}, \ldots, \operatorname{ev}_{k}^{\partial}\right)^{*}\left(h_{1} \wedge \cdots \wedge h_{k}\right) .
$$

Here the superscript $T$ stands for $T^{n}$ equivariance. By Lemma 20.5 (4) the integration along the fibers $\left(\mathrm{ev}_{0}^{\partial}\right)$ ! is well-defined. Then Lemma 20.5 (5) implies that the operation (20.13) is invariant under the permutation of the factors of $\mathbf{p}$. Therefore we can $\mathbb{C}$-linearly extend the definition (20.13) to define a map

$$
\mathfrak{q}_{\ell, k ; \beta}^{T}: E_{\ell}(\mathcal{H}[2]) \otimes B_{k}(\Omega(L(\mathbf{u}))[1]) \rightarrow \Omega(L(\mathbf{u}))[1] .
$$

Identification of the de Rham cohomology group $H(L(\mathbf{u}) ; \mathbb{C})$ of $L(\mathbf{u})$ with the subspace of $T^{n}$-invariant differential forms on the torus $L(\mathbf{u})$ and the property stated in Lemma 20.5 (2) imply that the operations $\mathfrak{q}_{\ell, k ; \beta}^{T}$ induce

$$
\mathfrak{q}_{\ell, k ; \beta}^{T}: E_{\ell}(\mathcal{H}[2]) \otimes B_{k}(H(L(\mathbf{u}) ; \mathbb{C})[1]) \rightarrow H(L(\mathbf{u}) ; \mathbb{C})[1] .
$$

For $\beta=\beta_{0}=0$, we define $\mathfrak{q}_{0, k ; \beta_{0}}^{T}$ to be the map given in (18.19). Then the operators $\mathfrak{q}_{\ell, k ; \beta}^{T}$ satisfy all the conclusions of Theorem 17.1. We use Theorem 17.1 to define $\mathfrak{m}_{k}^{T, \mathbf{b}}$ for $\mathbf{b}=\left(\mathfrak{b}_{0}, \mathbf{b}_{2 ; 1}, \mathfrak{b}_{+}, b_{+}\right)$in the same way as we did in Definition 17.8. We have thus obtained a filtered $A_{\infty}$ algebra $\left(C F_{\mathrm{dR}}\left(L(\mathbf{u}) ; \Lambda_{0}\right),\left\{\mathfrak{m}_{k}^{T, \mathbf{b}}\right\}_{k=0}^{\infty}\right)$ with

$$
C F_{\mathrm{dR}}\left(L(\mathbf{u}) ; \Lambda_{0}\right)=\Omega(L(\mathbf{u})) \widehat{\otimes} \Lambda_{0} .
$$

This is the filtered $A_{\infty}$ algebra used in FOOO4. (In FOOO4 $\mathfrak{q}^{T}$ is denoted by $\mathfrak{q}^{d R}$.) In particular, if $\mathfrak{m}_{0}^{T, \mathbf{b}}(1) \equiv 0 \bmod \mathbf{e}_{L} \Lambda_{+}, \mathfrak{m}_{1}^{T, \mathbf{b}} \circ \mathfrak{m}_{1}^{T, \mathbf{b}}=0$. We put $\delta^{T, \mathbf{b}}=\mathfrak{m}_{1}^{T, \mathbf{b}}$ and define its associated Floer cohomology by

$$
H F_{T}\left((L, \mathbf{b}) ; \Lambda_{0}\right)=\frac{\operatorname{Ker} \delta^{T, \mathbf{b}}}{\operatorname{Im} \delta^{T, \mathbf{b}}} .
$$

We put subscript $T$ in the notation to indicate that we are using a $T^{n}$-equivariant perturbation. In a series of papers [FOOO3, FOOO4, FOOO7, we studied Floer cohomology (20.16) and described its nonvanishing property in terms of the critical point theory of certain non-Archimedean analytic function, called the potential function. An explanation of this potential function is given in Subsection 20.4.

Remark 20.6. Here we note that we use a system of multisections and integrations on their zero sets in Lemma 20.5. They are described in [FOOO3, appendix C]. In other part of this paper, we use a system of $\mathrm{CF}$-perturbations and define integration along the fiber using CF-perturbation. See Remarks 28.6 and 28.17. 
20.3. Relationship with the Floer cohomology in Section 17. To apply the Floer cohomology (20.16) for the purpose of studying spectral invariants, we need to show that (20.16) is isomorphic to the Floer cohomology we used in Part 3. We use the next proposition for this purpose. We denote $\mathbf{b}^{(0)}=\left(\mathfrak{b}_{0}, \mathbf{b}_{2 ; 1}, \mathfrak{b}_{+}, 0\right)$ as before.

Proposition 20.7. Let $\mathbf{u} \in \operatorname{Int} P$. The filtered $A_{\infty}$ algebra $\left(C F_{\mathrm{dR}}\left(L(\mathbf{u}) ; \Lambda_{0}\right),\left\{\mathfrak{m}_{k}^{\mathbf{b}^{(0)}}\right\}_{k=0}^{\infty}\right)$ is homotopy equivalent to $\left(C F_{\mathrm{dR}}\left(L(\mathbf{u}) ; \Lambda_{0}\right),\left\{\mathfrak{m}_{k}^{T, \mathbf{b}^{(0)}}\right\}_{k=0}^{\infty}\right)$ as a unital filtered $A_{\infty}$ algebra.

Here the first filtered $A_{\infty}$ algebra is defined in Lemma 17.10 and the second one is defined at the end of Subsection 20.2. They are de Rham versions of the filtered $A_{\infty}$ algebra associated to a Lagrangian submanifold, which was established in [FOOO1, Theorem A]. Since the two constructions are slightly different from each other in technical points, we give a proof of Proposition 20.7 in Section 28 for completeness' sake.

Let $\mathbf{e}_{L}=1$ be the differential 0-form on $L$ which is the unit of our filtered $A_{\infty}$ algebra. We put:

$$
\begin{aligned}
& \widehat{\mathcal{M}}_{\text {weak,def }}\left(L(\mathbf{u}) ; \Lambda_{+} ; \mathbf{b}^{(0)}\right) \\
& =\left\{b_{+} \in H^{\text {odd }}\left(L(\mathbf{u}) ; \Lambda_{+}\right) \mid \sum_{k=0}^{\infty} \mathfrak{m}_{k}^{\mathbf{b}^{(0)}}\left(b_{+}^{k}\right) \equiv 0 \quad \bmod \mathbf{e}_{L} \Lambda_{+}\right\}
\end{aligned}
$$

and

$$
\begin{aligned}
& \widehat{\mathcal{M}}_{\text {weak,def }}^{T}\left(L(\mathbf{u}) ; \Lambda_{+} ; \mathbf{b}^{(0)}\right) \\
& =\left\{b_{+} \in H^{o d d}\left(L(\mathbf{u}) ; \Lambda_{+}\right) \mid \sum_{k=0}^{\infty} \mathfrak{m}_{k}^{T, \mathbf{b}^{(0)}}\left(b_{+}^{k}\right) \equiv 0 \quad \bmod \mathbf{e}_{L} \Lambda_{+}\right\} .
\end{aligned}
$$

We write $\mathbf{b}\left(b_{+}\right)=\left(\mathfrak{b}_{0}, \mathbf{b}_{2 ; 1}, \mathfrak{b}_{+}, b_{+}\right)$. Then

$$
\mathbf{b}\left(b_{+}\right) \in \widehat{\mathcal{M}}_{\text {weak }, \mathrm{def}}\left(L(\mathbf{u}) ; \Lambda_{0}\right)
$$

for $b_{+} \in \widehat{\mathcal{M}}_{\text {weak }, \text { def }}\left(L(\mathbf{u}) ; \Lambda_{+} ; \mathbf{b}^{(0)}\right)$.

Similar fact holds for $b_{+} \in \widehat{\mathcal{M}}_{\text {weak,def }}^{T}\left(L(\mathbf{u}) ; \Lambda_{+} ; \mathbf{b}^{(0)}\right)$.

Proposition 20.7 and the homotopy theory of filtered $A_{\infty}$ algebras as given in [FOO01, Chapter 4] immediately imply the following:

Corollary 20.8. There exists a map

$$
\mathfrak{J}_{*}: \widehat{\mathcal{M}}_{\text {weak,def }}^{T}\left(L(\mathbf{u}) ; \Lambda_{+} ; \mathbf{b}^{(0)}\right) \rightarrow \widehat{\mathcal{M}}_{\text {weak,def }}\left(L(\mathbf{u}) ; \Lambda_{+} ; \mathbf{b}^{(0)}\right)
$$

such that for each $b_{+} \in \widehat{\mathcal{M}}_{\text {weak,def }}^{T}\left(L(\mathbf{u}) ; \Lambda_{+} ; \mathbf{b}^{(0)}\right)$ there exists a chain homotopy equivalence

$$
\left.\left.\mathfrak{J}_{*}^{b_{+}}:(C F(L(\mathbf{u})) ; \Lambda), \delta^{T, \mathbf{b}\left(b_{+}\right)}\right) \rightarrow(C F(L(\mathbf{u})) ; \Lambda), \delta^{\mathbf{b}\left(\mathfrak{J}_{*}\left(b_{+}\right)\right)}\right)
$$

that preserves the filtration.

(We note that $\mathfrak{J}_{*}$ induces an isomorphism after taking a gauge equivalence. We do not use this fact in this paper.)

We next use $\mathfrak{q}_{\ell, k ; \beta}^{T}$ in place of $\mathfrak{q}_{\ell, k ; \beta}$ in (17.17) to define a chain map

$$
i_{\mathrm{qm}, \mathbf{b}}^{T}:(\Omega(M) \widehat{\otimes} \Lambda, d) \rightarrow\left(C F(L(\mathbf{u}) ; \Lambda), \delta^{T, \mathbf{b}}\right) .
$$


Lemma 20.9. $\mathfrak{J}_{*}^{b_{+}} \circ i_{\mathrm{qm}, \mathbf{b}}$ is chain homotopic to $i_{\mathrm{qm}, \mathbf{b}}^{T}$.

The proof is parallel to Proposition 20.7 and is given in Section 28 .

Now the following is an immediate consequence.

Corollary 20.10. If we replace the condition (18.8) in Theorem 18.8 by $H F((L, \mathbf{b}) ; \Lambda)$ and $i_{\mathrm{qm}}^{*}$ by $H_{T}((L, \mathbf{b}) ; \Lambda)$ and $i_{\mathrm{qm}}^{T, *}$ respectively, then the conclusion of Theorem 18.8 still holds.

20.4. Properties of Floer cohomology $H F_{T}((L, \mathbf{b}) ; \Lambda)$ : review. We now go back to the study of Floer cohomology $H F_{T}((L, \mathbf{b}) ; \Lambda)$ introduced in Subsection 20.2. The main properties of $H F_{T}((L, \mathbf{b}) ; \Lambda)$ were established in [FOOO3, FOOO4.

Proposition 20.11. If $b_{+} \in H^{1}\left(L(\mathbf{u}) ; \Lambda_{+}\right)$, then

$$
\mathfrak{m}_{0}^{T, \mathbf{b}\left(b_{+}\right)}(1) \equiv 0 \quad \bmod \mathbf{e}_{L} \Lambda_{+} .
$$

In particular, we have an embedding $H^{1}\left(L(\mathbf{u}) ; \Lambda_{+}\right) \hookrightarrow \widehat{\mathcal{M}}_{\text {weak,def }}^{T}\left(L(\mathbf{u}) ; \Lambda_{+} ; \mathbf{b}^{(0)}\right)$.

This is nothing but [FOOO3, Proposition 4.3] and [FOOO4, Proposition 3.1]. We omit its proof and refer readers to the above references for the details. The proof is based on a dimension counting argument. We remark that the proof of Proposition 20.11 does not work if we replace $\mathfrak{m}_{0}^{T, \mathbf{b}\left(b_{+}\right)}$by $\mathfrak{m}_{0}^{\mathbf{b}\left(b_{+}\right)}$. This is because we used a continuous family of multisections (or CF-perturbations) for the definition of $\mathfrak{m}_{k}^{\mathbf{b}\left(b_{+}\right)}$in Section 17, which obstructs the above mentioned dimension counting argument. (See FOOO7, Remark 3.2.2].) Actually this is the reason why we use $T^{n}$-invariant cycles $D_{a}$ instead of differential forms to represent cohomology classes of $M$ in [FOOO3, FOOO4] and in this section.

Now we consider $\mathbf{b}^{(0)}=\left(\mathfrak{b}_{0}, \mathbf{b}_{2 ; 1}, \mathfrak{b}_{+}, 0\right)$ and $b_{+} \in H^{1}\left(L(\mathbf{u}) ; \Lambda_{+}\right)$. By Proposition 20.11 we have

$$
\mathbf{b}\left(b_{+}\right) \in \widehat{\mathcal{M}}_{\text {weak,def }}^{T}\left(L(\mathbf{u}) ; \Lambda_{0}\right) .
$$

We use the splitting (20.8) to regard $\mathbf{b}\left(b_{+}\right)=\left(\mathfrak{b}_{0}, \mathbf{b}_{2 ; 1}, \mathfrak{b}_{+}, b_{+}\right)$as an element of $H^{\text {even }}\left(M ; \Lambda_{0}\right) \oplus H^{1}\left(L(\mathbf{u}) ; \Lambda_{0}\right)$. So hereafter we define $H F_{T}\left((L(\mathbf{u}),(\mathfrak{b}, b)) ; \Lambda_{0}\right)$ for $\mathfrak{b} \in H^{\text {even }}\left(M ; \Lambda_{0}\right)$ and $b \in H^{1}\left(L(\mathbf{u}) ; \Lambda_{0}\right)$. This is the Floer cohomology we studied in $\mathrm{FOOO} 4$.

We define the potential function

$$
\mathfrak{P O}^{\mathbf{u}}: H^{\text {even }}\left(M ; \Lambda_{0}\right) \times H^{1}\left(L(\mathbf{u}) ; \Lambda_{0}\right) \rightarrow \Lambda_{+}
$$

by the equation

$$
\mathfrak{m}_{0}^{T,(\mathfrak{b}, b)}(1)=\mathfrak{P O}^{\mathbf{u}}(\mathfrak{b}, b) \mathbf{e}_{L} .
$$

We now review the results of FOOO3, FOOO4 on the potential function $\mathfrak{P O}^{\mathbf{u}}$ and its role in the study of Floer cohomology.

We let $\mathbf{u} \in \operatorname{Int} P$ and fix a basis $\left\{\mathbf{e}_{i}\right\}_{i=1}^{n}$ of $H^{1}(L(\mathbf{u}) ; \mathbb{Z})$. Identifying $L(\mathbf{u})$ with $T^{n}$ by the action, we can find a basis $\left\{\mathbf{e}_{i}\right\}_{i=1}^{n}$ for all $\mathbf{u} \in \operatorname{Int} P$ simultaneously in a canonical way. Let $b \in H^{1}\left(L(\mathbf{u}) ; \Lambda_{0}\right)$ express it as a linear combination

$$
b=\sum x_{i}^{\mathbf{u}} \mathbf{e}_{i}
$$

where $x_{i}^{\mathbf{u}} \in \Lambda_{0}$. Thus $\left(x_{1}^{\mathbf{u}}, \ldots, x_{n}^{\mathbf{u}}\right)$ are coordinates on $H^{1}\left(L(\mathbf{u}) ; \Lambda_{0}\right)$. (To specify that the coordinate system is defined on the torus $L(\mathbf{u})$ associated to $\mathbf{u}$ we put $\mathbf{u}$ in the expression $x_{i}^{\mathbf{u}}$ above.) Let $x_{i}^{\mathbf{u}}=x_{i, 0}^{\mathbf{u}}+x_{i,+}^{\mathbf{u}}$ where $x_{i, 0}^{\mathbf{u}} \in \mathbb{C}$ and $x_{i,+}^{\mathbf{u}} \in \Lambda_{+}$. We put

$$
y_{i}^{\mathbf{u}}=\exp \left(x_{i, 0}^{\mathbf{u}}\right) \exp \left(x_{i,+}^{\mathbf{u}}\right) \in \Lambda_{0} \backslash \Lambda_{+} .
$$


We note that $\exp \left(x_{i, 0}^{\mathbf{u}}\right) \in \mathbb{C} \backslash\{0\}$ makes sense in the usual Archimedean sense, and

$$
\exp \left(x_{i,+}^{\mathbf{u}}\right):=\sum_{k=0}^{\infty}\left(x_{i,+}^{\mathbf{u}}\right)^{k} / k !
$$

converges in $T$-adic topology since $\mathfrak{v}_{T}\left(x_{i,+}^{\mathbf{u}}\right)>0$.

Let $S_{i}^{1}$ be the $i$-th factor of $T^{n}$ which represents the basis element $\mathbf{e}_{i}$. We choose our moment map $\pi: M \rightarrow \mathbb{R}^{n}$ so that its $i$-th component is the moment map of the $S_{i}^{1}$ action. In this way we fix the coordinates on the affine space $\mathbb{R}^{n}$ which contains $P$. Note that there is still a freedom to choose the origin $\mathbf{0} \in \mathbb{R}^{n}$. We do not specify this choice since it does not affect the story.

Let $\mathbf{u}=\left(u_{1}, \ldots, u_{n}\right) \in \operatorname{Int} P$. We put

$$
y_{i}=T^{u_{i}} y_{i}^{\mathbf{u}} .
$$

We do not put $\mathbf{u}$ in the notation $y_{i}$ above. This is justified by Theorem 20.15]

Remark 20.12. For the notational convenience we assume $\mathbf{0} \in P$. Then we will have $y_{i}=y_{i}^{0}$.

With respect to the above coordinates, we may regard $\mathfrak{P O}^{\mathbf{u}}$ as a function of $\left(x_{1}^{\mathbf{u}}, \cdots, x_{n}^{\mathbf{u}}\right)$

$$
\mathfrak{P O}^{\mathbf{u}}(\mathfrak{b} ; b)=\mathfrak{P O}_{\mathfrak{b}}^{\mathbf{u}}\left(x_{1}^{\mathbf{u}}, \ldots, x_{n}^{\mathbf{u}}\right)
$$

where $x_{k}^{\mathbf{u}}(k=1, \ldots, n)$ are the variables defined in (20.21).

As we will see in Theorem 20.15, $\mathfrak{P O}_{\mathfrak{b}}^{\mathbf{u}}$ as a function of $y_{1}, \ldots, y_{n}$ will be independent of $\mathbf{u}$. The resulting function is contained in an appropriate completion of the Laurent polynomial ring $\Lambda\left[y_{1}, \ldots, y_{n}, y_{1}^{-1}, \ldots, y_{n}^{-1}\right]$. Description of this completion is in order now. By the change of variables (20.23), there exists an isomorphism

$$
\Lambda\left[y_{1}, \ldots, y_{n}, y_{1}^{-1}, \ldots, y_{n}^{-1}\right] \cong \Lambda\left[y_{1}^{\mathbf{u}}, \ldots, y_{n}^{\mathbf{u}},\left(y_{1}^{\mathbf{u}}\right)^{-1}, \ldots,\left(y_{n}^{\mathbf{u}}\right)^{-1}\right]
$$

for any $\mathbf{u} \in \operatorname{Int} P$. In other words any element of $\mathfrak{P} \in \Lambda\left[y_{1}, \ldots, y_{n}, y_{1}^{-1}, \ldots, y_{n}^{-1}\right]$ can be written as a finite sum

$$
\mathfrak{P}=\sum a_{k_{1}, \ldots, k_{n}}\left(y_{1}^{\mathbf{u}}\right)^{k_{1}} \ldots\left(y_{n}^{\mathbf{u}}\right)^{k_{n}} .
$$

In other words $a_{k_{1}, \ldots, k_{n}} \in \Lambda$ are zero except for a finite number of them. We define a valuation

$$
\mathfrak{v}_{\mathbf{u}}(\mathfrak{P})=\min \left\{\mathfrak{v}_{T}\left(a_{k_{1}, \ldots, k_{n}}\right) \mid a_{k_{1}, \ldots, k_{n}} \neq 0\right\}
$$

for each $\mathbf{u} \in \operatorname{Int} P$. In particular $\mathfrak{v}_{\mathbf{u}}\left(y_{i}^{\mathbf{u}}\right)=0$. This defines a family of nonArchimedean valuations on the ring $\Lambda\left[y_{1}, \ldots, y_{n}, y_{1}^{-1}, \ldots, y_{n}^{-1}\right]$. This valuation is characterized by the equation

$$
\mathfrak{v}_{\mathbf{u}}\left(y_{i}\right)=u_{i}
$$

for $\mathbf{u} \in \operatorname{Int} P$. From this expression, the family can be extended to the full closed moment polytope $P$ in the obvious way.

We now put

$$
\mathfrak{v}_{P}(\mathfrak{P})=\inf \left\{\mathfrak{v}_{\mathbf{u}}(\mathfrak{P}) \mid \mathbf{u} \in P\right\} .
$$

This defines a norm (but not a valuation) on $\Lambda\left[y_{1}, \ldots, y_{n}, y_{1}^{-1}, \ldots, y_{n}^{-1}\right]$ and the function $d_{P}$ defined by

$$
d_{P}(\mathfrak{P}, \mathfrak{Q})=\exp ^{-\mathfrak{v}_{P}(\mathfrak{P}-\mathfrak{Q})}
$$

gives rise to a metric thereon.

For $\epsilon>0$, denote

$$
P_{\epsilon}=\left\{\mathbf{u} \in P \mid \forall i \ell_{i}(\mathbf{u}) \geq \epsilon\right\} .
$$


We define another metric on $\Lambda\left[y_{1}, \ldots, y_{n}, y_{1}^{-1}, \ldots, y_{n}^{-1}\right]$ by

$$
d_{P}(\mathfrak{P}, \mathfrak{Q})=\sum_{n=n_{0}}^{\infty} 2^{-n} \exp ^{-\mathfrak{v}_{P_{1 / n}}(\mathfrak{P}-\mathfrak{Q})} .
$$

(Here we take $n_{0}$ sufficiently large so that $P_{1 / n_{0}}$ is nonempty.) This series obviously converges because $\mathfrak{v}_{P_{\epsilon^{\prime}}} \leq \mathfrak{v}_{P_{\epsilon}}$ if $\epsilon^{\prime}<\epsilon$.

Definition 20.13. We denote the completion of $\Lambda\left[y_{1}, \ldots, y_{n}, y_{1}^{-1}, \ldots, y_{n}^{-1}\right]$ with respect to the metric $d_{P}$ by $\Lambda\left\langle\left\langle y, y^{-1}\right\rangle\right\rangle^{P}$.

We denote by $\Lambda\left\langle\left\langle y, y^{-1}\right\rangle\right\rangle^{\stackrel{\circ}{ }}$ the completion of $\Lambda\left[y_{1}, \ldots, y_{n}, y_{1}^{-1}, \ldots, y_{n}^{-1}\right]$ with respect to the metric $d_{P}$.

In other words, $\Lambda\left\langle\left\langle y, y^{-1}\right\rangle\right\rangle^{P}$ (resp. $\Lambda\left\langle\left\langle y, y^{-1}\right\rangle\right\rangle^{\stackrel{\circ}{ }}$ ) is the set of all $\mathfrak{P}$ 's such that for any $\mathbf{u} \in P$ (resp. $\mathbf{u} \in \operatorname{Int} P$ ) we may write $\mathfrak{P}$ as a possibly infinite sum of the form (20.24) such that $\lim _{\left|k_{1}\right|+\cdots+\left|k_{n}\right| \rightarrow \infty} \mathfrak{v}_{T}\left(a_{k_{1}, \ldots, k_{n}}\right)=+\infty$.

Remark 20.14. In FOOO4, we used a slightly different notation $\Lambda^{P}\left\langle\left\langle y, y^{-1}\right\rangle\right\rangle$ instead of $\Lambda\left\langle\left\langle y, y^{-1}\right\rangle\right\rangle^{P}$.

Now we have:

Theorem 20.15. If $\mathfrak{b} \in H^{\text {even }}\left(M ; \Lambda_{0}\right)$, then

$$
\mathfrak{P O}_{\mathfrak{b}}^{\mathbf{u}} \in \Lambda\left\langle\left\langle y, y^{-1}\right\rangle\right\rangle^{P} .
$$

If $\mathfrak{b} \in H^{\text {even }}\left(M ; \Lambda_{+}\right)$, then

$$
\mathfrak{P O} \mathfrak{O}_{\mathfrak{b}}^{\mathbf{u}} \in \Lambda\left\langle\left\langle y, y^{-1}\right\rangle\right\rangle^{P} .
$$

We explain the meaning of (20.28). Let $\mathfrak{P} \in \Lambda\left\langle\left\langle y, y^{-1}\right\rangle\right\rangle^{P}$ and $\mathbf{u} \in \operatorname{Int} P$. As we mention above, $\mathfrak{P}$ is written as a series of the form (20.24) with

$$
\lim _{\left|k_{1}\right|+\cdots+\left|k_{n}\right| \rightarrow \infty} \mathfrak{v}_{T}\left(a_{k_{1}, \ldots, k_{n}}\right)=+\infty .
$$

Let $b=\sum x_{i}^{\mathbf{u}} \mathbf{e}_{i}$. Then by putting (20.22) and plugging it in (20.24) the series converges in $T$-adic topology and we obtain an element of $\Lambda$. Thus we obtain a function

$$
\mathfrak{P}^{\mathbf{u}}: H^{1}(L(\mathbf{u}) ; \Lambda) \rightarrow \Lambda .
$$

The statement (20.28) means that there exists $\mathfrak{P} \in \Lambda\left\langle\left\langle y, y^{-1}\right\rangle\right\rangle^{P}$ such that the above $\mathfrak{P}^{\mathbf{u}}$ coincides with $\mathfrak{P O}_{\mathfrak{b}}^{\mathbf{u}}$ for any $\mathbf{u} \in \operatorname{Int} P$. (We note that we require $\mathfrak{P}$ to be independent of $\mathbf{u}$.) The meaning of (20.29) is similar.

Actually we can show the following:

Lemma 20.16. Let $\mathfrak{P} \in \Lambda\left\langle\left\langle y, y^{-1}\right\rangle\right\rangle^{P}$. Then $\mathfrak{P}$ is written as a series

$$
\mathfrak{P}=\sum a_{k_{1}, \ldots, k_{n}} y_{1}^{k_{1}} \ldots y_{n}^{k_{n}}
$$

which converges in $d_{P}$ topology. For any $\left(\mathfrak{y}_{1}, \ldots, \mathfrak{y}_{n}\right) \in \Lambda^{n}$ with

$$
\left(\mathfrak{v}_{T}\left(\mathfrak{y}_{1}\right), \ldots, \mathfrak{v}_{T}\left(\mathfrak{y}_{n}\right)\right) \in \operatorname{Int} P
$$

the series

$$
\sum a_{k_{1}, \ldots, k_{n}} \mathfrak{y}_{1}^{k_{1}} \ldots \mathfrak{y}_{n}^{k_{n}}
$$


converges in T-adic topology.

Let $\mathfrak{P} \in \Lambda\left\langle\left\langle y, y^{-1}\right\rangle\right\rangle^{P}$. Then $\mathfrak{P}$ is written as a series (20.30) which converges in $d_{P}$ topology. For any $\left(\mathfrak{y}_{1}, \ldots, \mathfrak{y}_{n}\right) \in \Lambda^{n}$ with

$$
\left(\mathfrak{v}_{T}\left(\mathfrak{y}_{1}\right), \ldots, \mathfrak{v}_{T}\left(\mathfrak{y}_{n}\right)\right) \in P
$$

the series (20.31) converges in T-adic topology.

The proof is elementary and so omitted.

Theorem 20.15 is [FOOO4, Theorem 3.14]. We do not discuss its proof in this paper but refer to [FOOO4 for the details.

We next discuss the relationship between the potential function and the nonvanishing of Floer cohomology. We first note that one can define the logarithmic derivative

$$
y_{i} \frac{\partial \mathfrak{P}}{\partial y_{i}}
$$

for an element $\mathfrak{P}$ of $\Lambda\left\langle\left\langle y, y^{-1}\right\rangle\right\rangle^{\stackrel{\circ}{P}}$. In fact, regarding the expression (20.30) of $\mathfrak{P}$ as a power series, we define

$$
y_{i} \frac{\partial \mathfrak{P}}{\partial y_{i}}=\sum a_{k_{1}, \ldots, k_{n}} k_{i} y_{1}^{k_{1}} \ldots y_{n}^{k_{n}} .
$$

It is easy to see that this series converges with respect to $d_{P}$-topology and so defines an element of $\Lambda\left\langle\left\langle y, y^{-1}\right\rangle \stackrel{\circ}{P}^{\text {. }}\right.$.

Definition 20.17. Let $\mathfrak{P} \in \Lambda\left\langle\left\langle y, y^{-1}\right\rangle\right\rangle^{P}$ and $\mathfrak{y}=\left(\mathfrak{y}_{1}, \ldots, \mathfrak{y}_{n}\right) \in \Lambda^{n}$ with

$$
\left(\mathfrak{v}_{T}\left(\mathfrak{y}_{1}\right), \ldots, \mathfrak{v}_{T}\left(\mathfrak{y}_{n}\right)\right) \in \operatorname{Int} P .
$$

We say that $\mathfrak{y}$ is a critical point of $\mathfrak{P}$ if it satisfies

$$
\left(y_{i} \frac{\partial \mathfrak{P}}{\partial y_{i}}\right)(\mathfrak{y})=0
$$

for all $i=1, \ldots, n$.

For each critical point $\mathfrak{y}$, we define a point $\mathbf{u}(\mathfrak{y}) \in \operatorname{Int} P$ by

$$
\mathbf{u}(\mathfrak{y})=\left(\mathfrak{v}_{T}\left(\mathfrak{y}_{1}\right), \ldots, \mathfrak{v}_{T}\left(\mathfrak{y}_{n}\right)\right),
$$

and an element $b=b(\mathfrak{y}) \in H^{1}\left(L(\mathbf{u}(\mathfrak{y})), \Lambda_{0}\right)$ by

$$
b(\mathfrak{y})=\sum x_{i}^{\mathfrak{y}} e_{i}, \quad x_{i}^{\mathfrak{y}}=\log \left(T^{-\mathfrak{v}_{T}\left(\mathfrak{y}_{i}\right)} y_{i}\right) .
$$

Here the meaning of log in (20.34) is as follows. Note that $\mathfrak{v}_{T}\left(T^{-\mathfrak{v}_{T}\left(\mathfrak{y}_{i}\right)} y_{i}\right)=0$. Therefore we can write

$$
T^{-\mathfrak{v}_{T}\left(\mathfrak{y}_{i}\right)} y_{i}=c_{1}\left(1+c_{2}\right)
$$

for some $c_{1} \in \mathbb{C} \backslash\{0\}, c_{2} \in \Lambda_{+}$. Then we define

$$
\log \left(T^{-\mathfrak{v}_{T}\left(\mathfrak{y}_{i}\right)} y_{i}\right)=\log c_{1}+\sum_{n=1}^{\infty}(-1)^{n} \frac{c_{2}^{n+1}}{n+1} .
$$

(Here we choose a branch of $\log c_{1}$ so that its imaginary part lies in $[0,2 \pi$ ), for example.) 


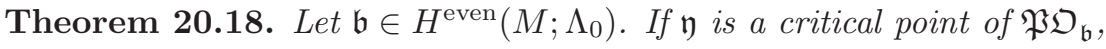

$$
H F\left((L(\mathbf{u}(\mathfrak{y})),(\mathfrak{b}, b(\mathfrak{y})) ; \Lambda) \cong H\left(T^{n} ; \Lambda\right) .\right.
$$

Conversely if

$$
H F((L(\mathbf{u}),(\mathfrak{b}, b)) ; \Lambda) \neq 0,
$$

there exists a critical point $\mathfrak{y}$ of $\mathfrak{P O}_{\mathfrak{b}}$ such that

$$
\mathbf{u}=\mathbf{u}(\mathfrak{y}), \quad b=b(\mathfrak{y}) .
$$

Theorem 20.18 is nothing but [FOOO4, Theorem 5.5]. We refer readers to FOOO4 for its proof.

We next describe the relation of $\mathfrak{P \mathfrak { O } _ { \mathfrak { b } }}$ to the quantum cohomology. Consider the closed ideal of the Fréchet ring $\Lambda\left\langle\left\langle y, y^{-1}\right\rangle\right\rangle^{P}$ generated by $\left\{y_{i} \frac{\partial \mathfrak{P O}_{\mathfrak{b}}}{\partial y_{i}} \mid i=1, \ldots, n\right\}$. We denote the quotient ring by

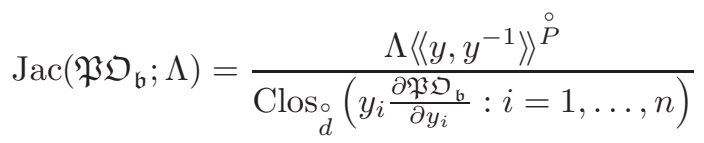

which we call the Jacobian ring of $\mathfrak{P O}_{\mathfrak{b}}$. We define a map

$$
\mathfrak{k}_{\mathfrak{b}}: H(M ; \Lambda) \rightarrow \operatorname{Jac}\left(\mathfrak{P O}_{\mathfrak{b}} ; \Lambda\right)
$$

called Kodaira-Spencer map as follows. Let $\left\{\mathbf{e}_{i}^{M}\right\}$ be a basis of $H(M ; \mathbb{Q})$. We write an element of $H(M ; \Lambda)$ as $\sum w_{i} \mathbf{e}_{i}^{M}, w_{i} \in \Lambda$. We may express

$$
\mathfrak{P O}_{\mathfrak{b}}=\sum a_{k_{1}, \ldots, k_{n}}(\mathfrak{b}) y_{1}^{k_{1}} \ldots y_{n}^{k_{n}}
$$

where $a_{k_{1}, \ldots, k_{n}}(\mathfrak{b})$ is a function of $w_{i}$ (where $\mathfrak{b}=\sum w_{i} \mathbf{e}_{i}^{M}$ ). (See 20.43) for the precise expression of $\mathfrak{P O}_{\mathfrak{b}}$.) Then $a_{k_{1}, \ldots, k_{n}}(\mathfrak{b})$ is a formal power series of $w_{i}$ with coefficients in $\Lambda$ which converges in $T$-adic topology.

Therefore the partial derivatives $\frac{\partial a_{k_{1}, \ldots, k_{n}}}{\partial w_{i}}$ are well-defined. Then we have

$$
\frac{\partial \mathfrak{P O}}{\partial w_{i}}(\mathfrak{b})=\sum \frac{\partial a_{k_{1}, \ldots, k_{n}}}{\partial w_{i}}(\mathfrak{b}) y_{1}^{k_{1}} \ldots y_{n}^{k_{n}} .
$$

For each $\mathfrak{b} \in H\left(M ; \Lambda_{0}\right)$, the right hand side converges and defines an element of

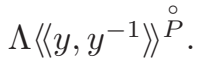

Now we define the map $\mathfrak{k}_{\mathfrak{b}}$ by setting its value to be

$$
\mathfrak{k}_{\mathfrak{b}}\left(\mathbf{e}_{i}^{M}\right)=\left[\frac{\partial \mathfrak{P O}}{\partial w_{i}}(\mathfrak{b})\right] \in \operatorname{Jac}\left(\mathfrak{P O}_{\mathfrak{b}} ; \Lambda\right) .
$$

Theorem 20.19. The map $\mathfrak{k}_{\mathfrak{S}_{\mathfrak{b}}}$ defines a ring isomorphism

$$
\left(Q H(M ; \Lambda), \cup^{\mathfrak{b}}\right) \cong \operatorname{Jac}\left(\mathfrak{P O}_{\mathfrak{b}} ; \Lambda\right) .
$$

This is [FOOO7, Theorem 1.1.1] for whose proof we refer readers thereto.

Remark 20.20. FOOO7, Theorem 1.1.1] is stated as a result over $\Lambda_{0}$-coefficients which is stronger than Theorem 20.19. We do not use this stronger isomorphism over $\Lambda_{0}$-coefficients in the present paper.

We also need a result on the structure of the Jacobian $\operatorname{ring} \operatorname{Jac}(\mathfrak{P O} \mathfrak{b} ; \Lambda)$. 
Definition 20.21. We say a critical point $\mathfrak{y}$ of $\mathfrak{P O}_{\mathfrak{b}}$ is nondegenerate if

$$
\operatorname{det}\left[y_{i} y_{j} \frac{\partial^{2} \mathfrak{P} \mathfrak{O}_{\mathfrak{b}}}{\partial y_{i} \partial y_{j}}\right]_{i, j=1}^{i, j=n}(\mathfrak{y}) \neq 0 \text {. }
$$

We say $\mathfrak{P O}_{\mathfrak{b}}$ is a Morse function if all of its critical points are nondegenerate.

Let $\operatorname{Crit}\left(\mathfrak{P O}_{\mathfrak{b}}\right)$ be the set of all critical points of $\mathfrak{P O}_{\mathfrak{b}}$.

Definition 20.22. For $\mathfrak{y}=\left(\mathfrak{y}_{1}, \ldots, \mathfrak{y}_{n}\right) \in \operatorname{Crit}\left(\mathfrak{P O}_{\mathfrak{b}}\right)$, we define the subset $\operatorname{Jac}\left(\mathfrak{P O} \mathfrak{O}_{\mathfrak{b}} ; \mathfrak{y}\right) \subset$ $\operatorname{Jac}\left(\mathfrak{P O}_{\mathfrak{b}} ; \Lambda\right)$ as follows: If we regard $y_{i} \in \Lambda\left\langle\left\langle y, y^{-1}\right\rangle\right\rangle^{P}$, the multiplication by $y_{i}$ induces an action on $\operatorname{Jac}\left(\mathfrak{P O}_{\mathfrak{b}} ; \Lambda\right)$. We denote the corresponding endomorphism by $\widehat{y}_{i}$. Then we put

$$
\begin{aligned}
\operatorname{Jac}\left(\mathfrak{P O}_{\mathfrak{b}} ; \mathfrak{y}\right)=\left\{x \in \operatorname{Jac}\left(\mathfrak{P O}_{\mathfrak{b}} ; \Lambda\right) \mid\right. & \left(\widehat{y}_{i}-\mathfrak{y}_{i}\right)^{N} x=0, \\
& \text { for all } i \text { and sufficiently large } N\} .
\end{aligned}
$$

Proposition 20.23. (1) There is a factorization of the Jacobian ring

$$
\operatorname{Jac}\left(\mathfrak{P O}_{\mathfrak{b}} ; \Lambda\right) \cong \prod_{\mathfrak{y} \in \operatorname{Crit}\left(\mathfrak{P O} \mathfrak{O}_{\mathfrak{b}}\right)} \operatorname{Jac}\left(\mathfrak{P \mathfrak { O } _ { \mathfrak { b } }} ; \mathfrak{y}\right)
$$

as a direct product of rings.

(2) For each critical point $\mathfrak{y} \in \operatorname{Crit}\left(\mathfrak{P O}_{\mathfrak{b}}\right), \operatorname{Jac}\left(\mathfrak{P O} \mathfrak{O}_{\mathfrak{b}} ; \mathfrak{y}\right)$ is a local ring.

(3) $\mathfrak{y}$ is a nondegenerate critical point of $\mathfrak{P \mathcal { O } _ { \mathfrak { b } }}$ if and only if $\operatorname{Jac}(\mathfrak{P O} \mathfrak{b} ; \mathfrak{y}) \cong \Lambda$.

Proposition 20.23 is [FOOO7, Proposition 1.2.16], to which we refer readers for its proof.

It follows from Proposition 20.23 that the set of indecomposable idempotents of $\operatorname{Jac}\left(\mathfrak{P O}_{\mathfrak{b}} ; \Lambda\right)$ one-one corresponds to $\operatorname{Crit}\left(\mathfrak{P O}_{\mathfrak{b}}\right)$. We denote by $1_{\mathfrak{y}} \in \operatorname{Jac}(\mathfrak{P O} \mathfrak{\mathfrak { b }} ; \mathfrak{y})$ the unit of the ring $\operatorname{Jac}\left(\mathfrak{P O}_{\mathfrak{b}} ; \mathfrak{y}\right)$ which corresponds to an idempotent of $\operatorname{Jac}\left(\mathfrak{P O}_{\mathfrak{b}} ; \Lambda\right)$. Denote by $e_{\mathfrak{n}}$ the idempotent of $\left(Q H(M ; \Lambda), \cup^{\mathfrak{b}}\right)$ corresponding to $1_{\mathfrak{y}}$ under the isomorphism $\mathfrak{k}_{\mathfrak{s}_{\mathfrak{b}}}$ in Theorem 20.19

We are finally ready to describe the map

$$
i_{\mathrm{qm},(\mathfrak{b}, b)}^{T, *}: Q H_{\mathfrak{b}}^{*}(M ; \Lambda) \rightarrow H F^{*}((L(\mathbf{u}),(\mathfrak{b}, b)) ; \Lambda)
$$

in our situation.

Theorem 20.24. Let $\mathfrak{b} \in H^{\operatorname{even}}\left(M ; \Lambda_{0}\right), \mathfrak{y}$ a critical point of $\mathfrak{P O}_{\mathfrak{b}}, a \in H(M ; \Lambda)$ and let $\mathfrak{P} \in \Lambda\left\langle\left\langle y, y^{-1}\right\rangle\right\rangle^{P}$ satisfy

$$
\mathfrak{k}_{\mathfrak{b} \mathfrak{b}}(a)=[\mathfrak{P}] \bmod \operatorname{Clos}_{d}\left(y_{i} \frac{\partial \mathfrak{P \mathfrak { O } _ { \mathfrak { b } }}}{\partial y_{i}}: i=1, \ldots, n\right) .
$$

Then we have

$$
i_{\mathrm{qm},(\mathfrak{b}(\mathfrak{y}), b(\mathfrak{y}))}^{T, *}(a)=\mathfrak{P}(\mathfrak{y}) \mathbf{e}_{L(\mathbf{u}(\mathfrak{y}))} .
$$

Proof. This is [FOOO7, Lemma 3.3.1]. Since its proof was omitted in [FOOO7, we provide its proof here.

We note that the right hand side of (20.39) is independent of the choices of $\mathfrak{P}$ satisfying (20.38) because $y_{i} \frac{\partial \mathfrak{P \mathfrak { O } _ { \mathfrak { b } }}}{\partial y_{i}}$ is zero at $\mathfrak{y}$. 
Let $\mathfrak{b}=\mathfrak{b}_{0}+\mathfrak{b}_{2}+\mathfrak{b}_{+}$be as in (5.5). We express $\mathfrak{b}=\sum w_{i}(\mathfrak{b}) \mathbf{e}_{i}^{M}$ and $b=$ $\sum y_{i}(b) \mathbf{e}_{i}=b_{0}+b_{+}$where $b_{0} \in H^{1}(L(\mathbf{u}) ; \mathbb{C})$ and $b_{+} \in H^{1}\left(L(\mathbf{u}) ; \Lambda_{+}\right)$. By definition, we have

$$
\mathfrak{P O}_{\mathfrak{b}}(b)=\mathfrak{b}_{0}+\sum_{\beta, k, \ell} T^{\omega \cap \beta} \frac{\exp \left(\mathfrak{b}_{2} \cap \beta+b_{0} \cap \partial \beta\right)}{\ell !} \mathfrak{q}_{k, \ell, \beta}^{T}\left(\mathfrak{b}_{+}^{\otimes \ell}, b_{+}^{\otimes k}\right),
$$

where we identify $H^{0}(L(\mathbf{u}) ; \Lambda)=\Lambda$.

We now consider the splitting $\mathfrak{b}=\mathfrak{b}_{0}+\widehat{\mathfrak{b}}_{2}+\mathfrak{b}_{\text {high }}$ such that

$$
\widehat{\mathfrak{b}}_{2} \in H^{2}\left(M ; \Lambda_{0}\right), \quad \mathfrak{b}_{\text {high }} \in \bigoplus_{k>1} H^{2 k}\left(M ; \Lambda_{0}\right) .
$$

Using a relative version of the divisor equation ([FOOO4, Lemmas 7.1 and 9.2]), we can rewrite (20.40) as

$$
\mathfrak{P O}_{\mathfrak{b}}(b)=\mathfrak{b}_{0}+\sum_{\beta, \ell} T^{\omega \cap \beta} \frac{\exp \left(\widehat{\mathfrak{b}}_{2} \cap \beta+b \cap \partial \beta\right)}{\ell !} \mathfrak{q}_{0, \ell, \beta}^{T}\left(\mathfrak{b}_{\text {high }}^{\otimes \ell}, 1\right) .
$$

We re-enumerate $D_{1}, D_{2}, \ldots$ so that $\left\{D_{1}, \ldots, D_{B_{1}}\right\}$ becomes a $\mathbb{Q}$-basis of $H^{2}(M ; \mathbb{Q})$ and let $w_{1}, \ldots, w_{B_{1}}$ are the corresponding coordinates of $H^{2}(M ; \mathbb{Q})$. Then we write $\mathfrak{b}_{\text {high }}=\sum_{i=B_{1}+1}^{B} w_{i} \mathbf{e}_{i}^{M}$ for $\mathfrak{b}_{\text {high }}$ where we use the coordinate $w^{\prime}=\left(w_{B_{1}+1}, \ldots, w_{B}\right)$ for $\mathfrak{b}_{\text {high }}$. Then we define a series $P_{\beta}$ of $w^{\prime}$ by the equation

$$
P_{\beta}\left(w^{\prime}\right)=\sum_{\ell=0}^{\infty} \frac{1}{\ell !} \mathfrak{q}_{0, \ell, \beta}^{T}\left(\mathfrak{b}_{\text {high }}^{\otimes \ell}, 1\right) .
$$

Lemma 20.25. The series $P_{\beta}$ is indeed a polynomial of $w_{B_{1}+1}, \ldots, w_{B}$, i.e.,

$$
P_{\beta}\left(w^{\prime}\right) \in \Lambda\left[w_{B_{1}+1}, \ldots, w_{B}\right] .
$$

Proof. Since each of the component of $\mathfrak{b}_{\text {high }}$ has degree 4 or higher, we can show by a dimension counting argument that $\mathfrak{q}_{0, \ell, \beta}^{T}\left(\mathfrak{b}_{\text {high }}^{\otimes \ell}, 1\right)$ is nonzero only for a finite number of $\ell$. Since each $\mathfrak{q}_{0, \ell, \beta}^{T}\left(\mathfrak{b}_{\text {high }}^{\otimes \ell}, 1\right)$ is a polynomial of $w^{\prime}, P_{\beta}\left(w^{\prime}\right)$ is also a polynomial as asserted.

We put

$$
\mathfrak{w}_{i}=e^{w_{i}}=\sum_{k=0}^{\infty} \frac{w_{i}^{k}}{k !}
$$

for $i=1, \ldots, B_{1}$. It follows from (20.41) and (20.42) that we can write

$$
\mathfrak{P O}_{\mathfrak{b}}=w_{0}+\sum_{\beta} T^{\omega \cap \beta} \mathfrak{w}_{1}^{\beta \cap D_{1}} \cdots \mathfrak{w}_{B_{1}}^{\beta \cap D_{B_{1}}} y_{1}^{\partial \beta \cap e_{1}} \cdots y_{n}^{\partial \beta \cap e_{n}} P_{\beta}\left(w^{\prime}\right) .
$$

Here we regard $\beta$ as an element of $H_{2}(M ; L(\mathbf{0}) ; \mathbb{Z})$ to define the cap product $\omega \cap \beta$ in (20.43).

We will compare the value $i_{\mathrm{qm},(\mathfrak{b}(\mathfrak{y}), b(\mathfrak{y}))}^{T, *}\left(\mathbf{e}_{i}^{M}\right)$ with the $w_{i}$-derivative of (20.43). By definition, we have

$$
\begin{aligned}
& i_{\mathrm{qm},(\mathfrak{b}(\mathfrak{y}), b(\mathfrak{y}))}^{T, *}\left(\mathbf{e}_{i}^{M}\right) \\
& =\sum_{\beta, k, \ell_{1}, \ell_{2}} T^{\omega \cap \beta} \frac{\exp \left(\mathfrak{b}_{2} \cap \beta+b_{0} \cap \partial \beta\right)}{\left(\ell_{1}+\ell_{2}+1\right) !} \mathfrak{q}_{k, \ell_{1}+\ell_{2}+1, \beta}^{T}\left(\mathfrak{b}_{+}^{\otimes \ell_{1}} \otimes \mathbf{e}_{i}^{M} \otimes \mathfrak{b}_{+}^{\otimes \ell_{2}}, b_{+}^{\otimes k}\right) .
\end{aligned}
$$


For a further analysis of the power series, we consider the following three cases separately:

(Case $1 ; i=0)$ : It is easy to see that

$$
\mathfrak{q}_{k, \ell, \beta}^{T}\left(\mathfrak{b}_{+}^{\otimes \ell_{1}} \otimes \mathbf{e}_{0}^{M} \otimes \mathfrak{b}_{+}^{\otimes \ell_{2}} ; b_{+}^{\otimes k}\right)=0
$$

unless $\beta=0$ and $k=\ell=0$. Therefore we have $i_{\mathrm{qm},(\mathfrak{b}(\mathfrak{y}), b(\mathfrak{y}))}^{T, *}\left(\mathbf{e}_{0}^{M}\right)=\mathfrak{q}_{0,0,0}^{T}\left(\mathbf{e}_{0}^{M}\right)=$ $\mathbf{e}_{L}$. Since $\frac{\partial \mathfrak{P \mathcal { O } _ { \mathfrak { b } } ( w )}}{\partial w_{0}}=1$ by (20.43), we have (20.39) for $a=\mathbf{e}_{0}^{M}$.

(Case 2; $i>B_{1}$ ): By [FOOO4, Lemmas 7.1 and 9.2] we can rewrite (20.44) to

$$
\begin{aligned}
& i_{\mathrm{qm},(\mathfrak{b}(\mathfrak{y}), b(\mathfrak{y}))}^{T, *}\left(\mathbf{e}_{i}^{M}\right) \\
= & \sum_{\ell_{1}, \ell_{2}, \beta} \frac{\mathfrak{w}_{1}^{\beta \cap D_{1}} \cdots \mathfrak{w}_{B_{1}}^{\beta \cap D_{B_{1}}} \mathfrak{y}_{1}^{\partial \beta \cap e_{1}} \cdots \mathfrak{y}_{n}^{\partial \beta \cap e_{n}}}{\left(\ell_{1}+\ell_{2}+1\right) !} \mathfrak{q}_{0, \ell_{1}+\ell_{2}+1, \beta}^{T}\left(\mathfrak{b}_{\text {high }}^{\otimes \ell_{1}} \otimes \mathbf{e}_{i}^{M} \otimes \mathfrak{b}_{\text {high }}^{\otimes \ell_{2}}, 1\right) .
\end{aligned}
$$

By differentiating (20.42) in $w_{i}$ and recalling $\mathfrak{b}_{\text {high }}=\sum_{i=B_{1}+1}^{B} w_{i} \mathbf{e}_{i}^{M}$, we compute

$$
\frac{\partial P_{\beta}}{\partial w_{i}}=\sum_{\ell_{1}, \ell_{2}} \frac{1}{\left(\ell_{1}+\ell_{2}+1\right) !} \mathfrak{q}_{0, \ell_{1}+\ell_{2}+1, \beta}^{T}\left(\mathfrak{b}_{\text {high }}^{\otimes \ell_{1}} \otimes \mathbf{e}_{i}^{M} \otimes \mathfrak{b}_{\text {high }}^{\otimes \ell_{2}}, 1\right) .
$$

Therefore comparing this with (20.45), we conclude

$$
i_{\mathrm{qm},(\mathfrak{b}(\mathfrak{y}), b(\mathfrak{y}))}^{T, *}\left(\mathbf{e}_{i}^{M}\right)=\frac{\partial \mathfrak{P} \mathfrak{O}_{\mathfrak{b}}}{\partial w_{i}}(\mathfrak{y}),
$$

as required.

(Case $\left.3 ; i=1, \ldots, B_{1}\right)$ : The equality (20.45) also holds in this case. Then, by [FOOO4, Lemma 7.1 and 9.2] ("(relative) divisor equation"), we obtain

$$
\begin{aligned}
& i_{\mathrm{qm},(\mathfrak{b}(\mathfrak{y}), b(\mathfrak{y}))}^{T, *}\left(\mathbf{e}_{i}^{M}\right) \\
& =\sum_{\ell, \beta}\left(\beta \cap D_{i}\right) \frac{\mathfrak{w}_{1}^{\beta \cap D_{1}} \cdots \mathfrak{w}_{B_{1}}^{\beta \cap D_{B_{1}}} \mathfrak{y}_{1}^{\partial \beta \cap e_{1}} \cdots \mathfrak{y}_{n}^{\partial \beta \cap e_{n}}}{\left(\ell_{1}+\ell_{2}+1\right) !} \mathfrak{q}_{0, \ell_{1}+\ell_{2}+1, \beta}^{T}\left(\mathfrak{b}_{\text {high }}^{\otimes \ell}, 1\right) .
\end{aligned}
$$

Substitution of

$$
\frac{\partial \mathfrak{w}_{i}^{\beta \cap D_{i}}}{\partial w_{i}}=\left(\beta \cap D_{i}\right) \mathfrak{w}_{i}^{\beta \cap D_{i}}
$$

into the right hand side of (20.47) turns it into the derivative $\frac{\partial \mathfrak{P O} \mathfrak{O}_{\mathfrak{b}}}{\partial w_{i}}(\mathfrak{y})$ and hence gives rise to 20.46 also in this case. The proof of Theorem 20.24 is now complete.

\section{SPECTRAL INVARIANTS AND QUASI-MORPHISMS FOR TORIC MANIFOLDS}

21.1. $\mu_{e}^{\mathfrak{b}}$-heaviness of the Lagrangian fibers in toric manifolds. Let $(M, \omega)$ be a compact toric manifold, $P$ its moment polytope. Let $\mathfrak{b} \in H^{\text {even }}\left(M ; \Lambda_{0}\right)$. We consider the factorization

$$
Q H_{\mathfrak{b}}^{*}(M ; \Lambda) \cong \prod_{\mathfrak{y} \in \operatorname{Crit}\left(\mathfrak{R \mathcal { O } _ { \mathfrak { b } }}\right)} Q H_{\mathfrak{b}}(M ; \mathfrak{y})
$$

corresponding to the one given in Proposition 20.23 via Theorem 20.19 so that $Q H_{\mathfrak{b}}(M ; \mathfrak{y})$ is the factor corresponding to $\operatorname{Jac}\left(\mathfrak{P O}_{\mathfrak{b}} ; \mathfrak{y}\right)$. 
Theorem 21.1. Let $\mathfrak{y}=\left(\mathfrak{y}_{1}, \ldots, \mathfrak{y}_{n}\right) \in \operatorname{Crit}\left(\mathfrak{P O}_{\mathfrak{b}}\right)$ and $e_{\mathfrak{y}} \in Q H_{\mathfrak{b}}(M ; \mathfrak{y})$ be the corresponding idempotent. We put

$$
\mathbf{u}(\mathfrak{y})=\left(\mathfrak{v}_{T}\left(\mathfrak{y}_{1}\right), \ldots, \mathfrak{v}_{T}\left(\mathfrak{y}_{n}\right)\right) \in \operatorname{Int} P .
$$

Then the following holds:

(1) $L(\mathbf{u}(\mathfrak{y}))$ is $\mu_{e_{\mathfrak{y}}}^{\mathfrak{b}}$-heavy.

(2) If $\mathfrak{y}$ is a nondegenerate critical point, then $L(\mathbf{u}(\mathfrak{y}))$ is $\mu_{e_{\mathfrak{y}}}^{\mathfrak{b}}$-superheavy.

Proof. Theorem 21.1 follows from Theorems 18.8, 20.24, Proposition 20.23 combined with the following lemma below.

Lemma 21.2. Let $\mathfrak{k}_{\mathfrak{b}}\left(e_{\mathfrak{y}}\right)=1_{\mathfrak{y}}=[\mathfrak{P}]$ for an element $\mathfrak{P} \in \Lambda\left\langle\left\langle y, y^{-1}\right\rangle\right\rangle^{P}$. Then $\mathfrak{P}$ satisfies

$$
\mathfrak{P}(\mathfrak{y})=1 .
$$

Proof. The ring homomorphism $\mathfrak{P} \mapsto \mathfrak{P}(\mathfrak{y}): \Lambda\left\langle\left\langle y, y^{-1}\right\rangle\right\rangle^{P} \rightarrow \Lambda$ induces a ring homomorphism eval $\mathfrak{y}_{\mathfrak{y}} \operatorname{Jac}\left(\mathfrak{P O}_{\mathfrak{b}} ; \Lambda\right) \rightarrow \Lambda$. The ring homomorphism eval $\mathfrak{y}_{\mathfrak{y}}$ is unital and so is surjective.

Let $\mathfrak{y}^{\prime} \in \operatorname{Crit}\left(\mathfrak{P \mathcal { O } _ { \mathfrak { b } }}\right), \mathfrak{y}^{\prime} \neq \mathfrak{y}$ and let $\mathfrak{P} \in \Lambda\left\langle\left\langle y, y^{-1}\right\rangle\right\rangle^{\stackrel{\circ}{ }}$ be an element such that $[\mathfrak{P}] \in \operatorname{Jac}\left(\mathfrak{P O}_{\mathfrak{b}} ; \mathfrak{y}^{\prime}\right)$. By definition $\left(\widehat{y}_{i}-\mathfrak{y}_{i}^{\prime}\right)^{N}[\mathfrak{P}]=0$ in $\operatorname{Jac}\left(\mathfrak{P O}_{\mathfrak{b}} ; \Lambda\right)$ for some large integer $N \in \mathbb{N}$. Therefore applying eval $\mathfrak{y}_{\mathfrak{y}}$ we obtain $\left(\mathfrak{y}_{i}-\mathfrak{y}_{i}^{\prime}\right)^{N}$ eval $_{\mathfrak{y}}([\mathfrak{P}])=0$. Since $\mathfrak{y}_{i}-\mathfrak{y}_{i}^{\prime} \neq 0$ for some $i$ by the hypothesis, we conclude eval $\mathfrak{y}_{\mathfrak{y}}([\mathfrak{P}])=0$.

Therefore by Proposition 20.23, the homomorphism eval $\mathfrak{l}_{\mathfrak{y}}$ is nonzero on the factor $\operatorname{Jac}(\mathfrak{P O} \mathfrak{b} ; \mathfrak{y})$. Since $1_{\mathfrak{y}}=[\mathfrak{P}]$ is the unit of this factor, we conclude $\mathfrak{P}(\mathfrak{y})=1$, as required.

21.2. Calculation of the leading order term of the potential function in the toric case: review. We put

$$
z_{i}=T^{\ell_{i}(\mathbf{0})} y_{1}^{\partial \beta_{i} \cap \mathbf{e}_{1}} \cdots y_{n}^{\partial \beta_{i} \cap \mathbf{e}_{n}} \in \Lambda\left\langle\left\langle y, y^{-1}\right\rangle\right\rangle^{\stackrel{\circ}{\circ}}
$$

for $i=1, \ldots, m$. We assume

$$
\mathfrak{b}-\sum_{i=1}^{B_{1}} \overline{\mathfrak{b}}_{i} \mathbf{e}_{i}^{M} \in H^{2}\left(M ; \Lambda_{+}\right) \oplus \bigoplus_{k \neq 1} H^{2 k}\left(M ; \Lambda_{0}\right),
$$

where $\overline{\mathfrak{b}}_{i} \in \mathbb{C}$.

Theorem 21.3. We have

$$
\mathfrak{P O}_{\mathfrak{b}}=\mathfrak{b}_{0}+\sum_{i=1}^{m} e^{\overline{\mathfrak{b}}_{i}} z_{i}+\sum_{j} T^{\lambda_{j}} P_{j}\left(z_{1}, \ldots, z_{m}\right)
$$

where $P_{j} \in \Lambda\left[z_{1}, \ldots, z_{m}\right], \lambda_{j} \in \mathbb{R}_{>0}, \lim _{j \rightarrow \infty} \lambda_{j}=\infty$.

In case $(M, \omega)$ is Fano and $\mathfrak{b} \in H^{2}\left(M ; \Lambda_{0}\right)$, we have

$$
\mathfrak{P O}_{\mathfrak{b}}=\sum_{i=1}^{m} e^{w_{i}} z_{i}
$$

where $\mathfrak{b}=\sum w_{i} \mathbf{e}_{i}^{M}$

Proof. Theorem 21.3 is [FOOO4, Theorem 3.5]. (See also [FOOO8, Theorem 8.2].) We sketch its proof below. The proof uses the result of Cho-Oh $\mathrm{CO}$ which was summarized in [FOOO3, Theorem 11.1] as follows: 
(1) If $\stackrel{\circ}{\mathcal{M}}_{1 ; 0}(\beta) \neq \emptyset, \mu_{L(\mathbf{u})}(\beta)=2$ then $\beta=\beta_{j}$ for $j=1, \ldots, m$, where $\beta_{j}$ is as in (20.4). In this case $\stackrel{\circ}{\mathcal{M}}_{1 ; 0}\left(\beta_{j}\right)=\mathcal{M}_{1 ; 0}\left(\beta_{j}\right)=T^{n}$ and the evaluation map $\mathrm{ev}_{0}^{\partial}: \mathcal{M}_{1 ; 0}\left(\beta_{j}\right) \rightarrow L(\mathbf{u})$ has degree 1 .

(2) If $\mathcal{M}_{1 ; 0}(\beta) \neq \emptyset, \beta \neq \beta_{j}(j=1, \ldots, m)$ then

$$
\beta=\sum_{j=1}^{m} k_{j} \beta_{j}+\alpha
$$

where $\sum k_{j}>0, k_{j} \geq 0$ and $\alpha \in \pi_{2}(M)$ with $\alpha \cap \omega>0$.

Using this description of the moduli space $\mathcal{M}_{1,0}(\beta)$, we calculate the summands of the right hand side of (20.43) now.

For $\beta=\beta_{j}$ we have

$$
\begin{aligned}
& T^{\omega \cap \beta_{j}} \mathfrak{w}_{1}^{\beta_{j} \cap D_{1}} \cdots \mathfrak{w}_{B_{1}}^{\beta_{j} \cap D_{B_{1}}} y_{1}^{\partial \beta_{j} \cap \mathbf{e}_{1}} \cdots y_{n}^{\partial \beta_{j} \cap \mathbf{e}_{n}} P_{\beta_{j}}\left(w^{\prime}\right) \\
& =e^{w_{j}} z_{j}=\left(e^{\overline{\mathfrak{b}}_{j}}+(\text { higher order })\right) z_{j} .
\end{aligned}
$$

For $\beta \neq \beta_{j}(j=1, \ldots, m)$ we have

$$
T^{\omega \cap \beta} \mathfrak{w}_{1}^{\beta \cap D_{1}} \cdots \mathfrak{w}_{B_{1}}^{\beta \cap D_{B_{1}}} y_{1}^{\partial \beta \cap \mathbf{e}_{1}} \cdots y_{n}^{\partial \beta \cap \mathbf{e}_{n}} P_{\beta}\left(w^{\prime}\right)=T^{\alpha \cap \omega} \prod_{j=1}^{m}\left(e^{k_{j} w_{j}} z_{j}^{k_{j}}\right) .
$$

Therefore combining the above discussion, we have proved Theorem 21.3.

21.3. Existence of Calabi quasi-morphism on toric manifolds. In this subsection we complete the proof of Corollary 1.2. We begin with the following lemma

Lemma 21.4. The set of vectors $\left(c_{1}, \ldots, c_{m}\right) \in(\mathbb{C} \backslash\{0\})^{m}$ with the following properties is dense in $(\mathbb{C} \backslash\{0\})^{m}$ :

The function $f$ defined by

$$
f\left(y_{1}, \ldots, y_{n}\right)=\sum_{i=1}^{m} c_{i} y_{1}^{\partial \beta \cap \mathbf{e}_{1}} \cdots y_{n}^{\partial \beta \cap \mathbf{e}_{n}}
$$

restricts to a Morse function on $(\mathbb{C} \backslash\{0\})^{n}$.

This lemma is proved in [Ku (see [Iri1, Corollary 5.12], [FOOO3, Proposition 8.8] for the discussion in this context).

Corollary 21.5. Write $\mathfrak{b}=\sum_{i=1}^{m} \log c_{i}\left[D_{i}\right] \in H(M ; \mathbb{C})$ and consider the sum

$$
\mathfrak{P O}_{\overline{\mathfrak{b}}, 0}=\sum_{i=1}^{m} c_{i} z_{i} \in \Lambda\left[y_{1}, \ldots, y_{n}, y_{1}^{-1}, \ldots, y_{n}^{-1}\right] .
$$

Then the set of $\left(c_{1}, \ldots, c_{m}\right) \in(\mathbb{C} \backslash\{0\})^{m}$ for which $\mathfrak{P O}_{\overline{\mathfrak{b}}, 0}$ becomes a Morse function is dense in $(\mathbb{C} \backslash\{0\})^{m}$.

Proof. Suppose that $\mathfrak{P \mathfrak { O } _ { \overline { b } , 0 }}$ is not a Morse function. Consider a degenerate critical point $\mathfrak{y}=\left(\mathfrak{y}_{1}, \ldots, \mathfrak{y}_{n}\right)$ each of whose coordinates is a 'formal Laurent power series' of $T$. (We put 'formal Laurent power series' in the quote since the exponents of $T$ are real numbers which are not necessarily integers.) By [FOOO3, Lemma 8.5], those series are convergent when we put $T=\epsilon$ for sufficiently small $\epsilon>0$. Then for $c_{i}^{\prime}=c_{i} \epsilon^{\ell_{i}(\mathbf{0})}$ the function (21.5) will not be a Morse function. Corollary 21.5 follows from this observation and Lemma 21.4 
Corollary 21.6. For any compact toric manifold $M$ there exists an element $\mathfrak{b} \in$ $H^{\text {even }}(M ; \Lambda)$ such that $\mathfrak{P O}_{\mathfrak{b}}$ is a Morse function.

Proof. By [FOOO3, Theorem 10.4] we can prove that if $\mathfrak{P O}_{\overline{\mathfrak{b}}, 0}$ is a Morse function then $\mathfrak{P O}_{\mathfrak{b}}$ is also a Morse function. (Actually the case $\overline{\mathfrak{b}}=0$ is stated there. However the general case can be proved in the same way.) Therefore Corollary 21.6 follows from Corollary 21.5,

Corollary 1.2 follows immediately from Corollary 21.6. Proposition 20.23, Theorem 20.19 and Theorem 16.3 .

21.4. Defect estimate of a quasi-morphism $\mu_{e}^{\mathfrak{b}}$. Using the calculations we have performed, we can obtain some explicit estimates of the norm of the defect Def $\mu_{e}$ of spectral quasi-morphism $\mu_{e}$. We define

$$
|\operatorname{Def}|\left(\mu_{e}\right)=\sup _{\widetilde{\psi}, \widetilde{\phi}}\left|\mu_{e}(\widetilde{\psi} \widetilde{\phi})-\mu_{e}(\widetilde{\psi})-\mu_{e}(\widetilde{\phi})\right| .
$$

We illustrate this estimate by an example.

We consider $(M, \omega)=\mathbb{C} P^{n}$ with moment polytope

$$
\left\{\left(u_{1}, \ldots, u_{n}\right) \mid u_{i} \geq 0, \sum u_{i} \leq 1\right\} .
$$

Set $\mathfrak{b}=\mathbf{0}$. It is well known that the small quantum cohomology $Q H\left(\mathbb{C} P^{n} ; \Lambda\right)$ is isomorphic to $\Lambda[x] /\left(x^{n+1}-T\right)$, where $x \in H^{2}\left(\mathbb{C} P^{n} ; \mathbb{C}\right)$ is the standard generator. This is isomorphic to the direct product of $n+1$ copies of $\Lambda$. Therefore we have $n+1$ quasi-morphisms $\mu_{e_{k}}$. $(k=0, \ldots, n$. $)$ It is actually defined on the Hamiltonian diffeomorphism group $\operatorname{Ham}\left(\mathbb{C} P^{n}, \omega\right)$ itself. (EP1, Section 4.3].) (It is unknown whether they are different from one another.)

Proposition 21.7. Let $e_{k}$ and $\mu_{e_{k}}$ for $k=0, \ldots, n$ be as above. Then

$$
|\operatorname{Def}|\left(\mu_{e_{k}}\right) \leq \frac{12 n}{n+1}
$$

Proof. We have

$$
\mathfrak{P O}_{0}=y_{1}+\cdots+y_{n}+T\left(y_{1} \ldots y_{n}\right)^{-1} .
$$

See for example FOOO3, Example 5.2].

Let $\chi_{k}=\exp (2 \pi k \sqrt{-1} /(n+1))$. The critical points of $\mathfrak{P O _ { 0 }}$ are

$$
\mathfrak{y}_{k}=T^{1 /(n+1)}\left(\chi_{k}, \ldots, \chi_{k}\right), \quad k=0,1, \ldots, n .
$$

We put

Since

$$
P_{k}=\frac{\prod_{i \neq k}\left(y_{1}-T^{1 /(n+1)} \chi_{i}\right)}{\prod_{i \neq k}\left(T^{1 /(n+1)} \chi_{k}-T^{1 /(n+1)} \chi_{i}\right)} .
$$

$$
P_{k}\left(\mathfrak{y}_{\ell}\right)= \begin{cases}0 & k \neq \ell \\ 1 & k=\ell\end{cases}
$$

it follows that $\left[P_{k}\right]=\mathfrak{k}_{\mathfrak{s}_{0}}\left(e_{\mathfrak{y}_{k}}\right)$ in the Jacobian ring. Therefore, using $\mathfrak{k}_{\mathfrak{s}_{0}}(x)=\left[y_{1}\right]$ also, we have

$$
\mathfrak{v}_{q}\left(e_{\mathfrak{y}_{k}}\right)=-\mathfrak{v}_{T}\left(e_{\mathfrak{y}_{k}}\right)=\frac{n}{n+1} .
$$

Proposition 21.7 now follows from Remark 16.8 (1). 20.3.)

Note we chose our symplectic form $\omega$ so that $\int_{\mathbb{C} P^{1}} \omega=1$. (See 20.5) and Remark 


\section{LAGRANGIAN TORI IN $k$-POINTS BLOW UP OF $\mathbb{C} P^{2}(k \geq 2)$.}

In this section, we prove Theorem 1.10 (3) in the case of $k$-points blow up of $\mathbb{C} P^{2}(k \geq 2)$. We use the example of [FOOO4, Section 5], which we review now.

We first consider 2-points blow up $M$ of $\mathbb{C} P^{2}$. We put a toric Kähler form on it $\omega_{\alpha, \beta}$ such that the moment polytope is given by

$$
P_{\alpha, \beta}=\left\{\left(u_{1}, u_{2}\right) \mid 0 \leq u_{1} \leq 1,0 \leq u_{2} \leq 1-\alpha, \beta \leq u_{1}+u_{2} \leq 1\right\} .
$$

Here

$$
(\alpha, \beta) \in\{(\alpha, \beta) \mid 0 \leq \alpha, \beta, \alpha+\beta \leq 1\} .
$$

We are interested in the case $\beta=(1-\alpha) / 2$ and write $M_{\alpha}=\left(M, \omega_{\alpha,(1-\alpha) / 2}\right)$ where $\alpha>1 / 3$. We denote

$$
D_{1}=\pi^{-1}\left(\partial P_{\alpha,(1-\alpha) / 2} \cap\left\{\left(u_{1}, u_{2}\right) \mid u_{2}=0\right\}\right)
$$

and put

$$
\mathfrak{b}_{\kappa}=T^{\kappa} P D\left(\left[D_{1}\right]\right) \in H^{2}\left(M_{\alpha} ; \Lambda_{+}\right), \quad \kappa>0 .
$$

Then by (21.4), we have obtained

$$
\mathfrak{P O}_{\mathfrak{b}_{\kappa}}=y_{1}+e^{T^{\kappa}} y_{2}+T^{1-\alpha} y_{2}^{-1}+T y_{1}^{-1} y_{2}^{-1}+T^{-(1-\alpha) / 2} y_{1} y_{2} .
$$

Now consider a family of Lagrangian torus fibers

$$
L(u)=L(u,(1-\alpha) / 2),
$$

for $(1-\alpha) / 2<u<(1+\alpha) / 4$. Note that $\alpha>1 / 3$ implies $(1-\alpha) / 2<1 / 3<(1+\alpha) / 4$. Then for any such $u$ we can show the following.

Theorem 22.1. If $1 / 3 \leq u<(1+\alpha) / 4$, we take $\kappa(u)=(1+\alpha) / 2-2 u>0$. If $(1-\alpha) / 2<u<1 / 3$, we take $\kappa(u)=u-(1-\alpha) / 2>0$. Then $L(u) \subset M_{\alpha}$ is $\mu_{e}^{\mathfrak{b}_{\kappa(u)}}$-superheavy with respect to an appropriate idempotent e of $Q H_{\mathfrak{b}_{\kappa(u)}^{*}}^{*}\left(M_{\alpha} ; \Lambda\right)$.

Proof. Let $\mathbf{u}=(u,(1-\alpha) / 2)$. We put

$$
y_{1}^{\mathbf{u}}=T^{-u_{1}} y_{1}=T^{-u} y_{1}, y_{2}^{\mathbf{u}}=T^{-u_{2}} y_{2}=T^{-(1-\alpha) / 2} y_{2}
$$

in (22.4) to obtain

$$
\begin{aligned}
\mathfrak{P O}_{\mathfrak{b}_{\kappa(u)}}=T^{u} y_{1}^{\mathbf{u}} & +e^{T^{\kappa(u)}} T^{(1-\alpha) / 2} y_{2}^{\mathbf{u}}+T^{(1-\alpha) / 2}\left(y_{2}^{\mathbf{u}}\right)^{-1} \\
& +T^{(1+\alpha) / 2-u}\left(y_{1}^{\mathbf{u}}\right)^{-1}\left(y_{2}^{\mathbf{u}}\right)^{-1}+T^{u} y_{1}^{\mathbf{u}} y_{2}^{\mathbf{u}} .
\end{aligned}
$$

See [FOOO4, (5.10)]. We first consider the case that $1 / 3<u<(1+\alpha) / 4$ and $\kappa(u)=(1+\alpha) / 2-2 u$. Then the calculation in Case 1 of [FOOO4, Section 5] shows that the potential function $\mathfrak{P O}_{\mathfrak{b}_{\kappa(u)}}$ has nondegenerate critical points $\mathfrak{y}(u)=$ $\left(\mathfrak{y}_{1}(u), \mathfrak{y}_{2}(u)\right)$ such that

$$
\left(T^{-u} \mathfrak{y}_{1}(u), T^{-(1-\alpha) / 2} \mathfrak{y}_{2}(u)\right) \equiv( \pm \sqrt{-2},-1) \quad \bmod \Lambda_{+} .
$$

Each of them corresponds to an idempotent $e_{\mathfrak{y}(u)}$ of $Q H_{\mathfrak{b}_{\kappa(u)}}\left(M_{\alpha} ; \Lambda\right)$. Theorem 21.1 implies that $L(u)$ is $\mu_{e_{\mathfrak{y}(u)}}^{\mathfrak{b}_{\kappa(u)}}$-superheavy. When $u=1 / 3$ and $\kappa(u)=(1+\alpha) / 2-2 u$, Case 4 of [FOOO4, Section 5] shows that there are nondegenerate critical points. (Note that we are using $\mathfrak{b}_{\kappa}$ as (22.3) so $w=1$ in [FOOO4, (5.14)].) If $(1-\alpha) / 2<$ $u<1 / 3$ and $\kappa(u)=u-(1-\alpha) / 2$, Case 3 of [FOOO4, Section 5] shows that there is a nondegenerate critical point as well. Thus Theorem 22.1 follows from Theorem 21.1 . 
Proof of Theorem 1.10 (3). Since $\mathfrak{y}(u)$ is a nondegenerate critical point, Theorem 20.19 and Proposition 20.23 imply that $e=e_{\mathfrak{y}(u)}$ is the unit of the direct factor

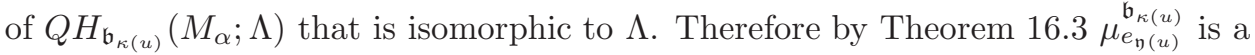

Calabi quasi-morphism. By Corollary [1.9], the set $\left\{\mu_{e_{\mathfrak{n}(u)}}^{\mathfrak{b}_{\kappa(u)}}\right\}_{u \in((1-\alpha) / 2,(1+\alpha) / 4)}$ is linearly independent. Thus we have constructed a continuum of linearly independent Calabi quasi-morphisms parametrized by $u \in((1-\alpha) / 2,(1+\alpha) / 4)$. The proof of Theorem 1.10 for the case of two-points blow-up of $\mathbb{C} P^{2}$ is complete.

To prove the existence of a continuum of linearly independent Calabi quasimorphisms for the case of three-points blow-up of $\mathbb{C} P^{2}$, we consider the Kähler toric surface $(M, \omega)$ whose moment polytope is given by

$$
P_{\alpha,(1-\alpha) / 2} \backslash\left\{\left(u_{1}, u_{2}\right) \mid 1-\epsilon<u_{2}\right\}
$$

for a sufficiently small $\epsilon$. Then $(M, \omega)$ is a three-points blow-up of $\mathbb{C} P^{2}$. Its potential function is given by

$$
\text { (22.6) }+T^{1-\epsilon} y_{1}^{-1} \text {. }
$$

It is easy to see that the extra term $T^{1-\epsilon} y_{1}^{-1}$ is of higher order, when $\left(\mathfrak{v}_{T}\left(y_{1}\right), \mathfrak{v}_{T}\left(y_{2}\right)\right)$ $=(u,(1-\alpha) / 2), u \in(1 / 3,(1+\alpha) / 4)$. So by the same argument as in the case of two-points blow-up, we can prove Theorem 1.10. For the general $k$-points blow-up with $k \geq 3$, we can repeat the same argument. (See [FOOO3, page 111] for a relevant study of $k$-points blow-up of $\mathbb{C} P^{2}$.)

\section{LAGRANGIAN TORI IN $S^{2} \times S^{2}$}

In this section we prove Theorem 1.10 for the case of $S^{2} \times S^{2}$, which is equipped with the symplectic structure $\omega \oplus \omega$. We also prove Theorem 1.12. We first recall the description of the family of Lagrangian tori constructed in [FOOO6].

23.1. Review of the construction from FOOO6]. We consider the toric Hirzebruch surface $F_{2}(\alpha)(\alpha>0)$ whose moment polytope is

$$
P(\alpha)=\left\{\left(u_{1}, u_{2}\right) \in \mathbb{R}^{2} \mid u_{i} \geq 0, u_{2} \leq 1-\alpha, u_{1}+2 u_{2} \leq 2\right\} .
$$

Note that $F_{2}(\alpha)$ is not Fano but nef, i.e. every holomorphic sphere has non-negative Chern number. In fact, the divisor $D_{1} \cong \mathbb{C} P^{1}$ associated to the facet $\partial_{1} P(\alpha)=$ $\left\{u \in \partial P(\alpha) \mid u_{2}=1-\alpha\right\}$ has $c_{1}\left(D_{1}\right)=0$.

Theorem 23.1. ([FOOO6, Theorem 3.1]) We put $\mathfrak{b}=\mathbf{0}$. The potential function $\mathfrak{P O}_{0}$ of $F_{2}(\alpha)$ has the form

$$
\mathfrak{P O _ { 0 }}=y_{1}+y_{2}+T^{2} y_{1}^{-1} y_{2}^{-2}+T^{1-\alpha}\left(1+T^{2 \alpha}\right) y_{2}^{-1} .
$$

We consider the limit $\alpha \rightarrow 0$ of the Hirzebruch surface $F_{2}(\alpha)$. At $\alpha=0$, the limit polytope is the triangle

$$
P(0)=\left\{\left(u_{1}, u_{2}\right) \in \mathbb{R}^{2} \mid u_{i} \geq 0, u_{2} \leq 1, u_{1}+2 u_{2} \leq 2\right\}
$$

and the limit $F_{2}(0)$ is an orbifold with a singularity of the form $\mathbb{C}^{2} /\{ \pm 1\}$. We cut out a neighborhood of the singularity of $F_{2}(0)$ and paste the Milnor fiber back into the neighborhood to obtain the desired manifold. We denote it by $\widehat{F}_{2}(0)$.

Consider the preimage $Y(\varepsilon)$ of $P(\varepsilon) \subset P(0), 0<\varepsilon<1$, under the moment map $\pi: F_{2}(0) \backslash\{O\} \rightarrow P(0) \backslash\{(0,1)\}$, where $O$ is the singularity of $F_{2}(0)$. We can put a natural glued symplectic form on $\widehat{F}_{2}(0)=Y(\varepsilon) \cup D_{r}\left(T^{*} S^{2}\right)$ in a way that the given toric symplectic form on $Y(\varepsilon)$ is unchanged on $Y(\varepsilon) \backslash N(\varepsilon) \subset Y(\varepsilon) \backslash \partial Y(\varepsilon)$, where 
$N(\varepsilon)$ is a collar neighborhood of $\partial Y(\varepsilon)$. Since $H^{2}\left(S^{3} /\{ \pm 1\} ; \mathbb{Q}\right)=0$, the glued symplectic form does not depend on the choices of $\varepsilon>0$ or the gluing data up to the symplectic diffeomorphism. This symplectic manifold is symplectomorphic to $\left(S^{2}, \omega_{\text {std }}\right) \times\left(S^{2}, \omega_{\text {std }}\right)$ ([FOO06, Proposition 5.1].) In other words, we have symplectomorphisms

$$
\phi_{\varepsilon}:\left(\widehat{F}_{2}(0), \omega_{\varepsilon}\right) \rightarrow\left(S^{2} \times S^{2}, \omega_{\text {std }} \oplus \omega_{\text {std }}\right) .
$$

We denote

$$
T(\rho)=\phi_{\varepsilon}(L(1 / 2-\rho, 1 / 2+\rho)), \quad 0 \leq \rho<\frac{1}{2}
$$

where $L(1 / 2-\rho, 1 / 2+\rho)=\pi^{-1}(1 / 2-\rho, 1 / 2+\rho)$ regarded as a Lagrangian submanifold of $\left(\widehat{F}_{2}(0), \omega_{\varepsilon}\right)$. We refer to [FOOO6, Sections 3 and 4] for the detailed explanation of this construction.

23.2. Superheaviness of $T(\rho)$. Recall from [FOOO6, Section 5] that we have a family $\bigcup_{a \in \mathbb{C}} X_{a}$ where $X_{a}$ is biholomorphic to $\mathbb{C} P^{1} \times \mathbb{C} P^{1}$ for $a \neq 0$ and $X_{0}$ is biholomorphic to $F_{2}$. (See [FOOO6, Lemma 5.1].) The smooth trivialization of the simultaneous resolution $\bigcup_{a \in \mathbb{C}} X_{a}$ of $F_{2}(0)$ constructed in [FOOO6, Section 6] identifies the homology class $\left[D_{1}\right]$ in $X_{0}$ and $\left[S_{\text {van }}^{2}\right]$ in $X_{a}$. Beside this, the relative homology class $\beta_{1}$ in $X_{0}$ which satisfies $\beta_{1} \cap\left[D_{1}\right]=1$ and does not intersect with other toric divisors can be also regarded as a homology class in $X_{a}$. The homology classes $\beta_{1}$ and $\beta_{1}+\left[S_{\mathrm{van}}^{2}\right]$ satisfy the relations

$$
\beta_{1} \cap\left[S_{\text {van }}^{2}\right]=1, \quad\left(\beta_{1}+\left[S_{\text {van }}^{2}\right]\right) \cap\left[S_{\text {van }}^{2}\right]=-1 .
$$

We consider the cohomology class

$$
\mathfrak{b}(\rho)=T^{\rho} P D\left[S_{\text {van }}^{2}\right] \in H^{2}\left(\widehat{F}_{2}(0), \Lambda_{+}\right) .
$$

Using the 4-dimensionality and special properties of $\widehat{F}_{2}(0)$, we proved the following in [FOOO6, Lemma 4.2].

\section{Lemma 23.2.}

$$
H^{1}\left(T(u) ; \Lambda_{0}\right) \subset\left\{b \in H^{\text {odd }}\left(T(u) ; \Lambda_{0}\right) \mid(\mathfrak{b}(\rho), b) \in \widehat{\mathcal{M}}_{\text {def,weak }}\left(T(u) ; \Lambda_{0}\right)\right\} .
$$

In FOOO6], we showed that the potential function for $T(0)$, i.e., $\mathbf{u}_{0}=(1 / 2,1 / 2)$ is

$$
\mathfrak{P O}=T^{1 / 2}\left(y_{1}^{\mathbf{u}_{\mathbf{0}}}+y_{2}^{\mathbf{u}_{\mathbf{o}}}+\left(y_{1}^{\mathbf{u}_{\mathbf{0}}}\right)^{-1}\left(y_{2}^{\mathbf{u}_{\mathbf{0}}}\right)^{-2}+2\left(y_{2}^{\mathbf{u}_{\mathbf{o}}}\right)^{-1}\right) .
$$

We find that there are two critical points $\pm(1 / 2,2)$, see [FOOO6, Digression 4.1]. Hence there exist two $b \in H^{1}\left(T(0) ; \Lambda_{0}\right)$ modulo $H^{1}(T(0) ; 2 \pi \sqrt{-1} \mathbb{Z})$ such that $H F((T(0), b) ; \Lambda) \neq 0$.

When we consider the bulk deformation by $\mathfrak{b}(\rho)$, 23.6) and Theorem 23.1 imply that the potential function of $T(\mathbf{u})$ with bulk, $\mathfrak{P} \mathfrak{O}_{\mathfrak{b}(\rho)}$, becomes

$$
\begin{aligned}
& \mathfrak{P O}_{\mathfrak{b}(\rho)}=T^{u_{1}} y_{1}^{\mathbf{u}}+T^{u_{2}} y_{2}^{\mathbf{u}}+T^{2-u_{1}-2 u_{2}}\left(y_{1}^{\mathbf{u}}\right)^{-1}\left(y_{2}^{\mathbf{u}}\right)^{-2} \\
& +\left(e^{T^{\rho}}+e^{-T^{\rho}}\right) T^{1-u_{2}}\left(y_{2}^{\mathbf{u}}\right)^{-1} .
\end{aligned}
$$

(See [FOOO4, Theorem 3.5] and [FOOO6, Formula (47)].) Now we put

$$
2 \rho=u_{2}-u_{1}=u_{2}-\left(1-u_{2}\right)=2 u_{2}-1
$$

and consider (23.7) at $\mathbf{u}=\left(u_{1}, u_{2}\right)$ for some $\rho$. Namely, $\mathbf{u}=(1 / 2-\rho, 1 / 2+\rho)$. Then the potential function with bulk $\mathfrak{b}(\rho)$ of $T(0)$ is written as

$$
T^{1 / 2}\left(y_{1}^{\mathbf{u}_{\mathbf{o}}}+y_{2}^{\mathbf{u}_{\mathbf{o}}}+\left(y_{1}^{\mathbf{u}_{\mathbf{o}}}\right)^{-1}\left(y_{2}^{\mathbf{u}_{\mathbf{o}}}\right)^{-2}+\left(e^{T^{\rho}}+e^{-T^{\rho}}\right)\left(y_{2}^{\mathbf{u}_{\mathbf{o}}}\right)^{-1}\right) .
$$


See [FOO06, Formula (47)] with $u_{1}=u_{2}=1 / 2$. There are two critical points, which are $\left(\mathfrak{y}_{1}^{0}(\rho), \mathfrak{y}_{2}^{0}(\rho)\right)=\left(\epsilon\left(e^{T^{\rho / 2}}+e^{-T^{\rho / 2}}\right)^{-1}, \epsilon\left(e^{T^{\rho / 2}}+e^{-T^{\rho / 2}}\right)\right)$ with $\epsilon= \pm 1$. Hence $b^{0}(\rho)=b\left(\mathfrak{y}^{0}(\rho)\right)=\left(\log \mathfrak{y}_{1}^{0}(\rho), \log \mathfrak{y}_{2}^{0}(\rho)\right) \in H^{1}\left(T(0) ; \Lambda_{0}\right)$,

$$
H F\left(\left(T(0),\left(\mathfrak{b}(\rho), b^{0}(\rho)\right)\right) ; \Lambda\right) \neq 0 .
$$

For $T(\rho)$, the potential function with bulk $\mathfrak{b}_{\rho}$ is written as

$$
T^{1 / 2-\rho}\left(y_{1}^{\mathbf{u}}+T^{2 \rho} y_{2}^{\mathbf{u}}+\left(y_{1}^{\mathbf{u}}\right)^{-1}\left(y_{2}^{\mathbf{u}}\right)^{-2}+\left(e^{T^{\rho}}+e^{-T^{\rho}}\right)\left(y_{2}^{\mathbf{u}}\right)^{-1}\right) .
$$

See [FOO06, Formula (47)] with $u_{1}=1 / 2-\rho, u_{2}=1 / 2+\rho$. There are two critical points, which are $\left(\mathfrak{y}_{1}(\rho), \mathfrak{y}_{2}(\rho)\right)=\left(\epsilon T^{\rho}\left(e^{T^{\rho / 2}}-e^{-T^{\rho / 2}}\right)^{-1},-\epsilon T^{-\rho}\left(e^{T^{\rho / 2}}-e^{-T^{\rho / 2}}\right)\right)$. It follows that for $b(\rho)=b(\mathfrak{y}(\rho))=\left(\log \mathfrak{y}_{1}(\rho), \log \mathfrak{y}_{2}(\rho)\right) \in H^{1}\left(T(\rho) ; \Lambda_{0}\right)$,

$$
H F((T(\rho),(\mathfrak{b}(\rho), b(\rho))) ; \Lambda) \neq 0 .
$$

In summary, we have:

Lemma 23.3. (1) There exist two $b \in H^{1}\left(T(0) ; \Lambda_{0}\right) / H^{1}(T(0) ; 2 \pi \sqrt{-1} \mathbb{Z})$ such that

$$
H F((T(0), b) ; \Lambda) \neq 0 .
$$

(2) There exist two $b^{0}(\rho) \in H^{1}\left(T(0) ; \Lambda_{0}\right) / H^{1}(T(0) ; 2 \pi \sqrt{-1} \mathbb{Z})$ such that

$$
H F\left(\left(T(0),\left(\mathfrak{b}(\rho), b^{0}(\rho)\right) ; \Lambda\right) \neq 0 .\right.
$$

(3) There exist two $b(\rho) \in H^{1}\left(T(\rho) ; \Lambda_{0}\right) / H^{1}(T(\rho) ; 2 \pi \sqrt{-1} \mathbb{Z})$ such that

$$
H F((T(\rho),(\mathfrak{b}(\rho), b(\rho)) ; \Lambda) \neq 0 .
$$

Using this lemma, we obtain the following results.

Theorem 23.4. (1) There exists an idempotent e of a field factor of $Q H\left(S^{2} \times\right.$ $\left.S^{2} ; \Lambda\right)$ such that $T(0)$ is $\mu_{e}$-superheavy.

(2) For any $0 \leq \rho<\frac{1}{2}$, there exist idempotents $e_{\rho}$ and $e_{\rho}^{0}$, each of which is an idempotent of a field factor of $Q H_{\mathfrak{b}(\rho)}\left(S^{2} \times S^{2} ; \Lambda\right)$ such that $T(\rho)$ is $\mu_{e_{\rho}}^{\mathfrak{b}(\rho)}$-superheavy and $T(0)$ is $\mu_{e_{\rho}^{0}}^{\mathfrak{b}(\rho)}$-superheavy.

Proof. We first show that $Q H\left(S^{2} \times S^{2} ; \Lambda\right)$ and $Q H_{\mathfrak{b}(\rho)}\left(S^{2} \times S^{2} ; \Lambda\right)$ are semi-simple. For this purpose, we consider the toric structure as the monotone product of $S^{2}$. Let $\mathfrak{b}=a\left[S_{\text {van }}^{2}\right], a \in \Lambda_{+} \cdot\left(\mathfrak{b}=\mathfrak{b}(\rho)\right.$ if $a=T^{\rho}$, while $\mathfrak{b}=0$ if $a=0$.) We pick points $p t_{1}$ (resp. pt $t_{2}$ ) on the first (resp. the second) factor of $S^{2} \times S^{2}$ in the hemisphere in the classes $\beta_{1}, \beta_{2}$ that contributes to the coefficients of $y_{1}, y_{2}$ respectively in the potential function. We represent the homology class $\left[S_{\text {van }}^{2}\right]$ by the anti-diagonal whose homology class is given by $\left[S^{2} \times p t_{2}\right]-\left[p t_{1} \times S^{2}\right]$ in $H_{2}\left(S^{2} \times S^{2}\right)$. The potential function of $S_{\text {eq }}^{1} \times S_{\text {eq }}^{1}$ with bulk $\mathfrak{b}$ is written as

$$
\mathfrak{P O}_{\mathfrak{b}}=T^{1 / 2}\left(e^{a} y_{1}+y_{1}^{-1}+e^{-a} y_{2}+y_{2}^{-1}\right) .
$$

It has four nondegenerate critical points $\left(\epsilon_{1} e^{-a / 2}, \epsilon_{2} e^{a / 2}\right)$ with $\epsilon_{1}, \epsilon_{2}= \pm 1$. The critical values are $2\left(\epsilon_{1} e^{a / 2}+\epsilon_{2} e^{a / 2}\right) T^{1 / 2}$. By FOOO3, Theorem 6.1] (Fano toric case) and [FOOO7, Theorem 1.1], we find that the quantum cohomology with bulk deformation by $\mathfrak{b}$ is factorized into four copies of $\Lambda$ :

$$
Q H_{\mathfrak{b}}\left(S^{2} \times S^{2} ; \Lambda\right) \cong \bigoplus_{i=1}^{4} \Lambda \mathbf{e}_{i}^{\mathfrak{b}} .
$$


Here $\mathbf{e}_{1}^{\mathfrak{b}}, \ldots, \mathbf{e}_{4}^{\mathfrak{b}}$ are the idempotents corresponding to the critical points of $\mathfrak{P O}_{\mathfrak{b}}$ with $\left(\epsilon_{1}, \epsilon_{2}\right)=(1,1),(1,-1),(-1,1),(-1,-1)$, respectively. (When $\mathfrak{b}=0$, we simply write them as $\mathbf{e}_{i}$.) In particular, $Q H_{\mathfrak{b}}\left(S^{2} \times S^{2} ; \Lambda\right)$ is semi-simple.

By Lemma223.3 (1) and (3), there exists $b($ resp. $b(\rho))$ such that $H F((T(0), b) ; \Lambda) \neq$ 0 (resp. $\operatorname{HF}(T(\rho),(\mathfrak{b}(\rho), b(\rho)) ; \Lambda) \neq 0)$. Hence [FOOO1, Theorem 3.8.62], with [FOOO1, (3.8.36.2)] taken into account, implies that the maps

$$
\begin{gathered}
i_{\mathrm{qm}, T(0), b}^{*}: Q H\left(S^{2} \times S^{2} ; \Lambda\right) \rightarrow H F((T(0), b) ; \Lambda), \\
i_{\mathrm{qm}, T(\rho),(\mathfrak{b}(\rho), b(\rho))}^{*}: Q H_{\mathfrak{b}(\rho)}\left(S^{2} \times S^{2} ; \Lambda\right) \rightarrow H F((T(\rho)(\mathfrak{b}(\rho), b(\rho)) ; \Lambda)
\end{gathered}
$$

are nontrivial. In particular, there is at least one idempotent $e_{0} \in Q H\left(S^{2} \times S^{2} ; \Lambda\right)$ (resp. $\left.e_{\rho} \in Q H_{\mathfrak{b}(\rho)}\left(S^{2} \times S^{2} ; \Lambda\right)\right)$ such that $i_{\mathrm{qm}, T(0), b_{i}}^{*}\left(e_{0}\right) \neq 0\left(\operatorname{resp} . i_{\mathrm{qm}, T(\rho),(\mathfrak{b}(\rho), b(\rho))}^{*}\left(e_{\rho}\right) \neq\right.$ $0)$. Hence $T(0)$ is $\mu_{e^{-}}$-superheavy and $T(\rho)$ is $\mu_{e_{\rho}}^{\mathfrak{b}(\rho)}$-superheavy.

The sphere $S_{\text {van }}^{2}$ is a Lagrangian submanifold, which is disjoint from $T(\rho)$. We have the following

Lemma 23.5. The Lagrangian sphere $S_{\mathrm{van}}^{2}$, which is the anti-diagonal in $S^{2} \times S^{2}$, is unobstructed and

$$
H F\left(S_{\text {van }}^{2} ; \Lambda\right) \cong H\left(S_{\text {van }}^{2} ; \Lambda\right) \neq 0 .
$$

Proof. Note that the anti-diagonal in $S^{2} \times S^{2}$ can be seen as a fixed point set of an anti-symplectic involution. Then Theorem 1.3 with $k=0, \ell=0$ in [FOOO5] implies that $\mathfrak{m}_{0}(1)=0$, since the Maslov index of any holomorphic disc in $\left(S^{2} \times S^{2}, S_{\text {van }}^{2}\right)$ is divisible by 4 . See also [FOOO5, Corollary 1.6]. The second assertion follows from [FOOO1, Theorem D (D.3)].

Since $H^{1}\left(S_{\text {van }}^{2} ; \Lambda\right)=0$, there is at most one bounding cochain up to gauge equivalence. Lemma 23.5 implies existence of bounding cochain that satisfies $\mathfrak{m}^{b}(1)=$ 0 .(Recall that in general a weak bounding cochain satisfies $\mathfrak{m}^{b}(1)=\mathfrak{P O}(b) \mathbf{e}_{L}$.) and so the value of the potential function at $b$ is zero. By the same argument as in the case of $T(0)$, we find an idempotent $e^{\prime}$ of a field factor of $Q H\left(S^{2} \times S^{2} ; \Lambda\right)$ and such that $i_{\mathrm{qm}, \mathrm{S}_{\mathrm{van}}^{2}}^{*}\left(e^{\prime}\right) \neq 0$.

Since each of $e_{0}, e^{\prime}$ is an idempotent of a field factor of $Q H\left(S^{2} \times S^{2} ; \Lambda\right)$ and $e_{\rho}$ is an idempotent of a field factor of $Q H_{\mathfrak{b}(\rho)}\left(S^{2} \times S^{2} ; \Lambda\right)$, there exist corresponding Calabi quasi-morphisms $\mu_{e_{0}}^{0}, \mu_{e^{\prime}}, \mu_{e_{\rho}}^{\mathfrak{b}(\rho)}$ from $\widetilde{\operatorname{Ham}}\left(S^{2} \times S^{2}\right)$ to $\mathbb{R}$. Since $T(\rho)$, $\rho \in[0,1 / 2)$ and $S_{\text {van }}^{2}$ are mutually disjoint, Corollary 1.9 implies Theorem 1.10 (1). This completes the proof of Theorem 1.10 (1).

Furthermore, since homogenous quasi-morphisms are homomorphisms on abelian subgroups and $\pi_{1}\left(\operatorname{Ham}\left(S^{2} \times S^{2}\right)\right) \cong \mathbb{Z} / 2 \mathbb{Z} \times \mathbb{Z} / 2 \mathbb{Z}$ (see [Gr]), they descend to quasimorphisms on $\operatorname{Ham}\left(S^{2} \times S^{2}\right)$. We denote them by $\bar{\mu}_{e_{0}}^{0}, \bar{\mu}_{e^{\prime}}$ and $\bar{\mu}_{e_{\rho}}^{\mathfrak{b}(\rho)}$. Thus we also obtain the following.

Corollary 23.6. The Calabi quasi-morphisms $\bar{\mu}_{e_{0}}^{0}, \bar{\mu}_{e^{\prime}}$ and $\bar{\mu}_{e_{\rho}}^{\mathfrak{b}(\rho)}$ on $\operatorname{Ham}\left(S^{2} \times S^{2}\right)$ are linearly independent from one another.

Generally, let $(M, \omega)$ be a closed symplectic manifold. Suppose that $\widetilde{\operatorname{Ham}}(M, \omega)$ has infinitely many linearly independent homogeneous quasimoprhisms $\mu_{i}$. Now, under this hypothesis, we state a sufficient condition for the existence of infinitely many linearly independent homogeneous quasi-morphisms on $\operatorname{Ham}(M, \omega)$. 
Proposition 23.7. Suppose that $\pi_{1}(\operatorname{Ham}(M, \omega))$ is finitely generated. If there are infinitely many linearly independent homogeneous quasi-morphisms $\mu_{i}$ on $\widetilde{\operatorname{Ham}}(M, \omega)$, then there are infinitely many linearly independent homogeneous quasi-morphisms on $\operatorname{Ham}(M, \omega)$. The same statement holds for Calabi quasi-morphisms.

Proof. Let $\mu_{i}$ for $i \in \mathbb{N}$ be the given infinite family of linearly independent homogeneous quasi-morphisms. Pick generators $g_{1}, \ldots, g_{A} \in \pi_{1}(\operatorname{Ham}(M, \omega))$. Denote by $K$ the maximal integer such that, for some $i_{1}, \ldots, i_{K}$, the vectors

$$
\left(\mu_{i_{j}}\left(g_{1}\right), \ldots, \mu_{i_{j}}\left(g_{A}\right)\right) \in \mathbb{R}^{A}, \quad j=1, \ldots, K
$$

are linearly independent. We re-arrange the ordering of $g_{1}, \ldots, g_{A}$ so that $i_{1}=$ $1, \ldots, i_{K}=K$. For $k>K$, we can find $a_{\ell}(k) \in \mathbb{R}$ such that the quasi-morphism

$$
\mu_{k}^{\prime}:=\mu_{k}-\sum_{\ell=1}^{K} a_{\ell}(k) \mu_{\ell}
$$

vanish at $g_{i}$ for all $i=1, \ldots, A$. Since the restriction of a homogeneous quasimorphism on an abelian subgroup is a homomorphism, $\mu_{k}^{\prime}$ vanishes on $\pi_{1}(\operatorname{Ham}(M, \omega))$, which we regard as a subgroup of $\widetilde{\operatorname{Ham}}(M, \omega)$. (Recall that $\pi_{1}(\operatorname{Ham}(M, \omega))$ is abelian since $\operatorname{Ham}(M, \omega))$ is a topological group.) Therefore all $\mu_{k}^{\prime}$, for $k>K$, descend to homogeneous quasi-morphisms on $\operatorname{Ham}(M, \omega)$. Linear independence of $\mu_{k}^{\prime}, k>K$, follows from the standing hypothesis of linear indepedence of the set $\left\{\mu_{i} \mid i \in \mathbb{N}\right\}$.

For the statement concerning Calabi quasi-morphisms, we take one more $\mu_{K+1}$. Then, for $k>K+1$, choose $a_{i}(k), i=1, \ldots, K+1$ such that $\mu_{k}^{\prime}\left(g_{i}\right)=\mu_{k}\left(g_{i}\right)-$ $\sum_{i=1}^{K+1} a_{i}(k) \mu_{i}\left(g_{i}\right)$ are zero for $i=1, \ldots, A$ and $\sum_{i=1}^{K+1} a_{i}(k) \neq 1$. Then after a suitable rescaling, $\mu_{k}^{\prime}$ becomes a Calabi quasi-morphism. $\left\{\mu_{k}^{\prime} \mid k>K+1\right\}$ is the set of linearly independent Calabi quasi-morphisms.

Remark 23.8. In case $M$ is either a $k(\geq 3)$ points blow up of $\mathbb{C} P^{2}$ or cubic surface, we can descend our family of Calabi quasi-morphisms on $\widetilde{\operatorname{Ham}}(M, \omega)$ to one on $\operatorname{Ham}(M, \omega)$ in the same way as above if we can show that $\pi_{1}(\operatorname{Ham}(M, \omega))$ is a finitely generated group.

Next we give the proof of Theorem 1.12 using Theorem 23.4 together with Theorem 18.7 .

Proof of Theorem 1.12, Note that $T(u)$ in Theorem 1.12 is $T(\rho)$. Since $S_{\text {eq }}^{1} \times S_{\text {eq }}^{1}$ is the unique Lagrangian torus fiber with respect to the monotone toric structure on $S^{2} \times S^{2}, S_{\text {eq }}^{1} \times S_{\text {eq }}^{1}$ is superheavy with respect to the quasi-morphism $\mu_{e}^{\mathfrak{b}}$ associated with any idempotent $e$ of the field factor of $Q H_{\mathfrak{b}_{\rho}}\left(S^{2} \times S^{2} ; \Lambda\right)$. (Here we consider $\mathfrak{b}=\mathfrak{b}_{\rho}=T^{\rho}\left[S_{\text {van }}^{2}\right]$.)

From Theorem 23.4 we know that $T(\rho)$ is superheavy with respect to the quasi-

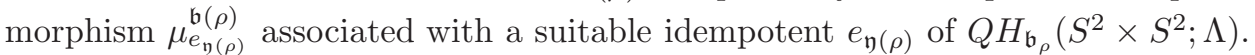
Since the superheaviness is invariant under symplectomorphisms, $\varphi(T(\rho))$ is also $\mu_{e_{\mathfrak{y}(\rho)}}^{\mathfrak{b}(\rho)}$-superheavy for any $\varphi \in \operatorname{Ham}\left(S^{2}(1) \times S^{2}(1)\right)$. Since superheavy sets with respect to the same quasi-morphism must intersect by Theorem 18.7, we have

$$
\varphi(T(\rho)) \cap\left(S_{\text {eq }}^{1} \times S_{\text {eq }}^{1}\right) \neq \emptyset .
$$

By [Gr, Theorem 0.3.C], $\operatorname{Symp}\left(S^{2}(1) \times S^{2}(1)\right)$ deformation retracts to the trivial $\mathbb{Z} / 2 \mathbb{Z}$ extension of $S O(3) \times S O(3)$ where $\mathbb{Z} / 2 \mathbb{Z}$ is generated by switching the factors. 
Thus the identity component of $\operatorname{Symp}\left(S^{2}(1) \times S^{2}(1)\right)$ is the same as $\operatorname{Ham}\left(S^{2}(1) \times\right.$ $\left.S^{2}(1)\right)$ and switching the factors fixes $S_{\text {eq }}^{1} \times S_{\text {eq }}^{1}$. Hence (23.8) holds for any $\varphi \in$ $\operatorname{Symp}\left(S^{2}(1) \times S^{2}(1)\right)$.

Remark 23.9. We can also give a different proof of Theorem 23.4 which follows the way similar to the toric case as follows. (A similar argument is used in Section 24) It follows from [FOOO7, Theorem 9.1] that for $\alpha>0$ the map

$$
\mathfrak{k}_{\mathfrak{b}(\rho)}: Q H_{\mathfrak{b}(\rho)}\left(F_{2}(\alpha) ; \Lambda\right) \rightarrow \operatorname{Jac}\left(\mathfrak{P O}_{\mathfrak{b}(\rho)} ; \Lambda\right)
$$

is a ring homomorphism. (Here $\mathfrak{P O}_{\mathfrak{b}(\rho)}$ is the potential function (23.7) of $F_{2}(\alpha)$ computed with respect to its canonical toric structure.) Since we can take limit $\alpha \rightarrow 0$ of $\mathfrak{P O}_{\mathfrak{b}(\rho)}$ and the potential function of $\widehat{F}_{2}(0)$ with respect to its toric structure is nothing but this limit by the result from [FOOO6, we find that

$$
\mathfrak{k}_{\mathfrak{b}(\rho)}: Q H_{\mathfrak{b}(\rho)}\left(\widehat{F}_{2}(0) ; \Lambda\right) \rightarrow \operatorname{Jac}\left(\mathfrak{P O} \mathfrak{O}_{\mathfrak{b}(\rho)} ; \Lambda\right)
$$

is also a ring homomorphism. (See AFOOO1] for the proof of this multiplicative property.)

Lemma 23.10. The map $\mathfrak{k}_{\mathfrak{b}(\rho)}$ is surjective.

Proof. We can check that $\mathfrak{P O}_{\mathfrak{b}(\rho)}$ has exactly 4 critical points if $\rho \in(0,1)$ and has exactly 2 critical points in case $\rho=0$. We can also check that those critical points are nondegenerate. Therefore $\operatorname{Jac}\left(\mathfrak{P O}_{\mathfrak{b}(\rho)} ; \Lambda\right) \cong \Lambda^{4}$ if $\rho \in(0,1)$ and $\operatorname{Jac}\left(\mathfrak{P O}_{\mathfrak{b}(\rho)} ; \Lambda\right) \cong \Lambda^{2}$ if $\rho=0$. We put

$$
z_{1}=T^{1-u_{2}}\left(y_{2}^{\mathbf{u}}\right)^{-1}, \quad z_{2}=T^{u_{1}} y_{1}^{\mathbf{u}}, \quad z_{3}=T^{u_{2}} y_{2}^{\mathbf{u}}, \quad z_{4}=T^{2-u_{1}-2 u_{2}}\left(y_{1}^{\mathbf{u}}\right)^{-1}\left(y_{2}^{\mathbf{u}}\right)^{-2} .
$$

By the same way as in [FOOO7, Lemma 1.2.4], we can show that $z_{1}, \ldots, z_{4}$ generate a $\Lambda$-subalgebra that is dense in $\Lambda\left\langle\left\langle y, y^{-1}\right\rangle\right\rangle^{\stackrel{\circ}{2}}$. (See Definition 20.13 for the notation.) Therefore, since $\operatorname{Jac}\left(\mathfrak{P O}_{\mathfrak{b}(\rho)} ; \Lambda\right)$ is finite dimensional, they generate $\operatorname{Jac}\left(\mathfrak{P O} \mathfrak{b}_{\mathfrak{b}(\rho)} ; \Lambda\right)$ as a $\Lambda$-algebra.

Let $D_{i}(i=1, \ldots, 4)$ be the divisors of $X$ associated to the facets of the moment polytope $u_{2}=1-\alpha, u_{1}=0, u_{2}=0, u_{1}+2 u_{2}=2$ respectively. It is easy to see that $\mathfrak{k}_{\mathfrak{b}(\rho)}\left(P D\left[D_{i}\right]\right)=z_{i}$ for $i=2,3,4$ and $\mathfrak{k}_{\mathfrak{b}(\rho)}\left(P D\left[D_{1}\right]\right)=\left(e^{T^{\rho}}-e^{-T^{\rho}}\right) z_{1}$. The lemma follows.

Let $\rho \neq 0$. Then $\operatorname{Jac}\left(\mathfrak{P O}_{\mathfrak{b}(\rho)} ; \Lambda\right) \cong \Lambda^{4}$. Since the Betti number of $\widehat{F}_{0}(0)$ is 4 , Lemma 23.10 implies that $\mathfrak{k}_{\mathfrak{b}(\rho)}$ is an isomorphism.

Let $\rho=0$. Using the fact that $Q H_{\mathfrak{b}(0)}(X ; \Lambda)$ is semisimple, and $\operatorname{Jac}\left(\mathfrak{P O}_{\mathfrak{b}(0)} ; \Lambda\right)$ $\cong \Lambda^{2}$, we derive from Lemma 23.10 that there exists an idempotent $e_{\mathfrak{y}(0)}$ that is a unit of the direct factor $\cong \Lambda$ of $Q H_{\mathfrak{b}(0)}(X ; \Lambda)$, such that $\mathfrak{k}_{\mathfrak{b}(0)}\left(e_{\mathfrak{y}(0)}\right) \neq 0$. (In the case $\rho \neq 0$, existence of such an idempotent $e_{\mathfrak{y}(\rho)}$ is immediate from the fact that $\mathfrak{k}_{\mathfrak{b}_{\mathfrak{b}}(\rho)}$ is an isomorphism.)

Thus in the way similar to the proofs of Theorem 20.24 and Lemma 21.2 we find that $i_{\mathrm{qm},\left(\mathfrak{b}(\rho), b\left(\mathfrak{y}^{u}\right)\right)}^{*}\left(e_{\mathfrak{y}(\rho)}\right) \neq 0$. In fact, we can use a de Rham representative of the Poincaré dual to $\left[S_{\text {van }}^{2}\right]$ that is supported in a small neighborhood of $S_{\text {van }}^{2}$ and in particular can be taken to be disjoint from $T(\rho)$. Therefore the above calculation of $\mathfrak{P O}_{\mathfrak{b}(\rho)}$ makes sense in the homology level. 
23.3. Critical values and eigenvalues of $c_{1}(M)$. In the course of the proof of Theorem 1.10 (1) given in Subsection 23.2 we proved existence of an idempotent associated to a field factor of quantum cohomology which is not in the kernel of the maps $i_{\mathrm{qm}, T(0), b_{i}}^{*}, i_{\mathrm{qm}, T(\rho),(\mathfrak{b}(\rho), b(\rho))}^{*}$. At the end of this subsection, we specify those idempotents.

We give a digression on the critical values of the potential function and the eigenvalues of the quantum multiplication by the first Chern $\operatorname{class} c_{1}(M)$ on a general closed symplectic manifold $(M, \omega)$. We start with an easy observation.

Lemma 23.11. For an oriented Lagrangian submanifold $L \subset M$, there is a cycle $D$ of codimension 2 in $M \backslash L$ such that the Maslov index is equal to twice of the intersection number with $D$, i.e., $\mu(\beta)=2 \beta \cdot D$ for any $\beta \in H_{2}(M, L ; \mathbb{Z})$.

Proof. Since $L$ is an oriented Lagrangian submanifold, the top exterior power $\bigwedge_{\mathbb{C}}^{n} T M$ is a trivial complex line bundle on $L$, where $2 n=\operatorname{dim} M$. Moreover, the volume form of $L$ gives a non-vanishing section $s_{L}$ of $\left.\bigwedge_{\mathbb{C}}^{n} T M\right|_{L}$. We extend $s_{L}$ to a section $s$ of $\bigwedge_{\mathbb{C}}^{n} T M$, which is transversal to the zero section. Then the zero locus $D$ of $s$ represents the Poincaré dual of the first Chern class $c_{1}(M)$ and the Maslov index $\mu_{L}: H_{2}(M, L ; \mathbb{Z}) \rightarrow \mathbb{Z}$ is given by the twice of the intersection number with $D$.

For our present purpose, we restrict ourselves to the case of the triple $(M, \omega, J)$ and an oriented Lagrangian submanifold $L \subset X$ satisfy the property that $\mu(\beta) \geq 2$ whenever the moduli space $\mathcal{M}(L ; J ; \beta)$ of ( $J$-holomorphic) bordered stable maps in class $\beta$ is nonempty. 6 of bordered stable maps in the class $\beta \neq 0$. See [FOOO6, Appendix 1] for some related results under this condition. The following theorem was proved (in the Fano toric case) by Auroux [Au, Theorem 6.1].

Theorem 23.12. Let $\mathfrak{b}$ be a cycle of codimension 2 in $M$ with coefficients in $\Lambda_{+}$ and $b \in \mathcal{M}_{\text {weak,def }}(L ; \mathfrak{b})$. Then, for any cycle $A$ in $M$,

$$
i_{\mathrm{qm},(\mathfrak{b}, b)}^{*}\left(c_{1}(M) \cup_{\mathfrak{b}} P D(A)\right)=\mathfrak{P O}_{\mathfrak{b}}(b) i_{\mathrm{qm},(\mathfrak{b}, b)}^{*}(P D(A))
$$

in $H F((L, \mathfrak{b}, b) ; \Lambda)$.

Proof. Let $D$ be the cycle in $M \backslash L$ obtained in Lemma 23.11 Since $c_{1}(M)$ is the Poincaré dual of $D$ as a cycle in $M$, we use $D$ to prove the formula (23.9).

The strategy of the proof is the same as in the proof of [FOOO7, Theorem 2.6.1]. Let $\mathcal{M}_{k+1 ; \ell+2}$ be the moduli space of genus zero bordered stable curves with $k+1$ boundary marked points $z_{0}, \ldots, z_{k}$ and $\ell+2$ interior marked points $z_{1}^{+}, \ldots, z_{\ell+2}^{+}$with connected boundary. Let $\mathcal{M}_{k+1 ; \ell+2}(L ; \beta)$ be the moduli space of bordered stable maps in $M$ attached to $L$ in class $\beta \in H_{2}(M, L ; \mathbb{Z})$ whose domain is a genus zero bordered semi-stable curves with $k+1$ boundary marked points and $\ell+2$ interior marked points with connected boundary. Here the boundary marked points are ordered counter-clockwise. We denote by $\mathrm{ev}_{j}^{+}$the evaluation map at the $j$-th interior marked point and by $\mathrm{ev}_{i}$ the evaluation map at the $i$-th boundary marked point $z_{i}$. We set $\mathrm{ev}^{+}=\left(\mathrm{ev}_{1}^{+}, \ldots, \mathrm{ev}_{\ell+2}^{+}\right)$and $\mathrm{ev}=\left(\mathrm{ev}_{1}, \ldots, \mathrm{ev}_{k}\right)$.

For cycles $Q_{1}, \ldots, Q_{\ell+2}$ in $M$ and chains $P_{1}, \ldots, P_{k}$ in $L$, we define

$$
\begin{gathered}
\mathcal{M}_{k+1, \ell+2}\left(\beta ; Q_{1}, \ldots, Q_{\ell+2} ; P_{1}, \ldots, P_{k}\right) \\
:=\mathcal{M}_{k+1, \ell+2}(\beta)_{\mathrm{ev}+\times \mathrm{ev}} \times_{\left(M^{\ell+2} \times L^{k}\right)}\left(Q_{1} \times \cdots \times Q_{\ell+2} \times P_{1} \times \cdots \times P_{k}\right) .
\end{gathered}
$$

${ }^{6}$ See Section 2.1.2 [FOOO1] for the definition of this moduli space. 
By stabilizing the domain of the stable map and forgetting the boundary marked points $z_{1}, \ldots, z_{k}$ and the interior marked points $z_{3}^{+}, \ldots, z_{\ell+2}^{+}$, we obtain the forgetful map

$$
\text { forget : } \mathcal{M}_{k+1, \ell+2}\left(\beta ; A \otimes D \otimes \mathfrak{b}^{\otimes \ell} ; b^{\otimes k}\right) \rightarrow \mathcal{M}_{1 ; 2} .
$$

The moduli space $\mathcal{M}_{1 ; 2}$ of bordered stable curves of genus 0 , connected boundary with two interior marked points and one boundary marked point is a complex manifold with boundary of complex dimension 1 . It can be easily shown to be homeomorphic to the unit disc (see e.g., FOOO7, Section 2.6]). We pick two distinguished points $\left[\Sigma_{0}\right],\left[\Sigma_{1}\right]$ in $\mathcal{M}_{1 ; 2}$ given as follows.

The bordered stable curve $\Sigma_{0}$ is the union of the unit disc with $z_{0}=1$ on its boundary and the Riemann sphere with two marked points $z_{1}^{+}, z_{2}^{+}$, away from the nodal point of $\Sigma_{0}$. On the other hand, the bordered stable curve $\Sigma_{1}$ consists of the union of two copies $D_{0}, D_{1}$ of the unit disc with a boundary node so that we can put $z_{0}=1, z_{1}^{+}=0$ in $D_{0}, z_{2}^{+}=0$ in $D_{1}$ and the boundary node corresponds to $-1 \in \partial D_{0}, 1 \in \partial D_{1}$. (See Figures 19, 20.)

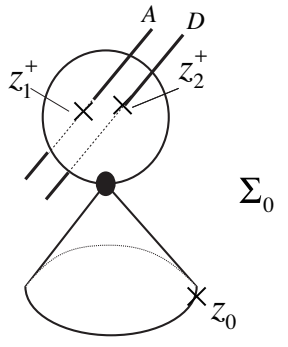

FiguRE 19. $\Sigma_{0}$

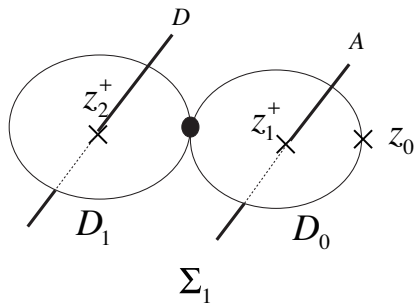

FiguRE 20. $\Sigma_{1}$

In our case, since the Maslov index $\mu(\beta)$ is at least 2 if $\mathcal{M}(L ; J ; \beta) \neq \emptyset$, and $\mathfrak{b}$ is represented by a codimension 2 cycle, it is enough to study holomorphic discs of Maslov index 2 for the computation of $\mathfrak{m}_{0}^{\mathfrak{b}, b}(1)$. We also recall that the Maslov index of a holomorphic discs attached to $L \subset M$ is equal to $2(\beta \cap D)$ where $\beta \cap D$ is the intersection number with $D$. In particular, if $\mu(\beta)=2$, we have $\beta \cap D=1$. Combining the above discussions, we compute

$$
\begin{aligned}
\mathfrak{q}\left(\left[D \otimes e^{\mathfrak{b}}\right] ; e^{b}\right) & :=\mathfrak{q}\left(\sum_{\ell_{1}, \ell_{2}} \frac{1}{\left(\ell_{1}+\ell_{2}+1\right) !} \mathfrak{b}^{\otimes \ell_{1}} \otimes D \otimes \mathfrak{b}^{\otimes \ell_{2}} ; e^{b}\right) \\
& =\mathfrak{q}\left(e^{\mathfrak{b}} ; e^{b}\right)=\mathfrak{P \mathcal { O } _ { \mathfrak { b } }}(b) \cdot \mathbf{e}
\end{aligned}
$$

for $b \in \mathcal{M}_{\text {def,weak }}(L, \mathfrak{b})$. Here $\mathbf{e}$ is the unit.

Once we obtain this identity, we can derive that the sum of contributions from $\mathrm{ev}_{0}: \mathfrak{f o r g e t}^{-1}\left(\left[\Sigma_{1}\right]\right) \rightarrow L$ gives rise to the right hand side of (23.9), which is $\mathfrak{P O}_{\mathfrak{b}}(b) i_{\mathrm{qm},(\mathfrak{b}, b)}^{*}(P D(A))$. We use the evaluation map at the marked point corresponding to the boundary node of $D_{1}$ to obtain a differential form on $L$ using the moduli space of $D_{1}$ in Figure 23.2 as a correspondence. Then it gives $\mathfrak{m}_{0}^{\mathfrak{b}}(1)=\mathfrak{P O}_{\mathfrak{b}}(b) \cdot 1$, which is proportional to the unit (the fundamental class). We remark that we fix the conformal structure of $D_{0}$. So there is no freedom of 
moving the node on $D_{0}$. Therefore when we use the evaluation map at $z_{0}$ to obtain a differential form of $L$ from the moduli space of Figure 23.2, it gives rise to $\mathfrak{P O}_{\mathfrak{b}}(b) i_{\mathrm{qm},(\mathfrak{b}, b)}^{*}(P D(A))$ as claimed.

On the other hand, it is straightforward to derive from construction that the contribution of $\mathrm{ev}_{0}: \mathfrak{f o r g e t}^{-1}\left(\left[\Sigma_{0}\right]\right) \rightarrow L$ is equal to $i_{\mathrm{qm},(\mathfrak{b}, b)}^{*}\left(c_{1}(M) \cup_{\mathfrak{b}} P D(A)\right)$ which is the left hand side of (23.9). Finally the proof of the equality of the two contributions from the two correspondences

$$
\mathrm{ev}_{0}: \mathfrak{f o r g e t}^{-1}\left(\left[\Sigma_{0}\right]\right) \rightarrow L, \quad \mathrm{ev}_{0}: \mathfrak{f o r g e t}^{-1}\left(\left[\Sigma_{1}\right]\right) \rightarrow L
$$

follows the way similar to that of [FOOO7 Theorem 2.6.1, especially using Lemma 2.6.3 therein. This then completes the proof of Theorem 23.12.

Corollary 23.13. If $A$ is an eigenvector of $c_{1}(M) \cup^{\mathfrak{b}}$ on $Q H_{\mathfrak{b}}(M ; \Lambda)$ with eigenvalue $\lambda$ and $i_{\mathrm{qm},(\mathfrak{b}, b)}^{*}(P D(A)) \neq 0$, then $\lambda=\mathfrak{P O}_{\mathfrak{b}}(b)$.

We return to the discussion on $T(\rho)$. For $T(0)=T(\rho=0)$, we can find that the potential function (without bulk deformations) of $T(0)$ has two critical points with critical values $\pm 4 T^{1 / 2}$, by the result of the calculation in FOOO6, Digression 4.1], where $T(0)$ is denoted by $T\left(\mathbf{u}_{0}\right)$. We have two bounding cochains $b_{1}, b_{2}$ with critical values $4 T^{1 / 2},-4 T^{1 / 2}$ up to gauge equivalence.

[FOOO1, Theorem 3.8.62] with [FOOO1, (3.8.36.2)] and Lemma23.5] and Lemma 23.3 (1) taking into account implies that

$$
i_{\mathrm{qm}, S_{\mathrm{van}}^{2}}^{*}: H\left(S^{2} \times S^{2} ; \Lambda\right) \rightarrow H F\left(S_{\mathrm{van}}^{2} ; \Lambda\right),
$$

resp.

$$
i_{\mathrm{qm}, T(0), b_{i}}^{*}: H\left(S^{2} \times S^{2} ; \Lambda\right) \rightarrow H F\left(\left(T(0), b_{i}\right) ; \Lambda\right)
$$

sends $\sum_{j=1}^{4} \mathbf{e}_{j}$ to the unit $P D\left[S_{\text {van }}^{2}\right] \neq 0$ of $H F\left(S_{\text {van }}^{2} ; \Lambda\right)$, resp. the unit $P D[T(0)] \neq$ 0 of $H F\left(\left(T(0), b_{i}\right) ; \Lambda\right), i=1,2$.

Recall that $Q H\left(S^{2} \times S^{2} ; \Lambda\right)$ is semi-simple and decomposes into $\bigoplus_{i=1}^{4} \Lambda \mathbf{e}_{i}$. We may assume that $\mathbf{e}_{1}, \mathbf{e}_{4}$ are eigenvectors of the quantum multiplication by $c_{1}\left(S^{2} \times S^{2}\right)$ with eigenvalues $\pm 4 T^{1 / 2}$ and $\mathbf{e}_{2}, \mathbf{e}_{3}$ are those with eigenvalue 0 . Comparing the critical values of the potential function and eigenvalues of the quantum multiplication by $c_{1}\left(S^{2} \times S^{2}\right)$, Theorem 23.12 implies that

$$
\begin{aligned}
i_{\mathrm{qm}, S_{\mathrm{van}}^{2}}^{*}\left(\mathbf{e}_{2}+\mathbf{e}_{3}\right) & =P D\left[S_{\mathrm{van}}^{2}\right], \\
i_{\mathrm{qm}, T(0), b_{1}}^{*}\left(\mathbf{e}_{1}\right) & =P D[T(0)], \\
i_{\mathrm{qm}, T(0), b_{2}}^{*}\left(\mathbf{e}_{4}\right) & =P D[T(0)] .
\end{aligned}
$$

We may assume that $i_{\mathrm{qm}, S_{\mathrm{van}}^{2}}^{*}\left(\mathbf{e}_{2}\right) \neq 0$. By Theorem $18.8(2)$, we find that $S_{\text {van }}^{2}$ is $\mu_{\mathbf{e}_{2}}$-superheavy and while $T(0)$ is $\mu_{\mathbf{e}_{1}}$-superheavy and $\mu_{\mathbf{e}_{4}}$-superheavy. On the other hand, since $S_{\text {van }}^{2}$ and $T(0)$ are disjoint, two quasi-morphisms corresponding to $\mu_{\mathbf{e}_{1}}$ and $\mu_{\mathbf{e}_{2}}$ are distinct by Theorem 18.7 (and Remark 18.6). This statement is mentioned without proof in [FOOO6, Remark 7.1].

As we showed in Subsection 23.2 the potential function of $T(0)$ with bulk deformation by $\mathfrak{b}_{\rho}$ has two critical points $\left(\epsilon\left(e^{T^{\rho / 2}}+e^{-T^{\rho / 2}}\right)^{-1}, \epsilon\left(e^{T^{\rho / 2}}+e^{-T^{\rho / 2}}\right)\right)$ with $\epsilon= \pm 1$. The associated critical values are $\pm 2\left(e^{T^{\rho} / 2}+e^{-T^{\rho} / 2}\right) T^{1 / 2}$.

For $T(\rho)$, the potential function with bulk $\mathfrak{b}_{\rho}$ has critical points $\left(\epsilon T^{\rho}\left(e^{T^{\rho / 2}}-\right.\right.$ $\left.e^{-T^{\rho / 2}}\right)^{-1},-\epsilon T^{-\rho}\left(e^{T^{\rho / 2}}-e^{-T^{\rho / 2}}\right)$. The critical values are $\pm 2\left(e^{T^{\rho} / 2}-e^{-T^{\rho} / 2}\right) T^{1 / 2}$. 
We now consider the maps

$$
\begin{gathered}
i_{\mathrm{qm}, T(0),\left(\mathfrak{b}(\rho), b_{i}\right)}^{*}: Q H_{\mathfrak{b}(\rho)}\left(S^{2} \times S^{2} ; \Lambda\right) \rightarrow H F\left(\left(T(0)\left(\mathfrak{b}(\rho), b_{i}\right) ; \Lambda\right),\right. \\
i_{\mathrm{qm}, T(\rho),\left(\mathfrak{b}(\rho), b(\rho)_{i}\right)}^{*}: Q H_{\mathfrak{b}(\rho)}\left(S^{2} \times S^{2} ; \Lambda\right) \rightarrow H F\left(\left(T(\rho)\left(\mathfrak{b}(\rho), b(\rho)_{i}\right) ; \Lambda\right) .\right.
\end{gathered}
$$

These maps have non-zero values at $b_{i} \in H^{1}\left(T(\rho), \Lambda_{0}\right)$ and $b(\rho)_{i} \in H^{1}\left(T(\rho), \Lambda_{0}\right), i=$ 1,2 respectively. (See the proof of Theorem 1.10 (1) given in Subsection 23.2) The eigenvalues of the $\mathfrak{b}(\rho)$-deformed quantum multiplication by $c_{1}\left(S^{2} \times S^{2}\right)$ are computed as follows. It follows from [FOOO3, Remark 5.3 and Theorem 1.9](Fano

toric case), [FOOO7, Theorem 1.1.4] (general toric case) that $\mathbf{e}_{1}^{\mathfrak{b}(\rho)}, \ldots, \mathbf{e}_{4}^{\mathfrak{b}(\rho)}$ are eigenvectors of the quantum multiplication with eigenvalues given by

$$
\begin{array}{cc}
2\left(e^{T^{\rho} / 2}+e^{-T^{\rho} / 2}\right) T^{1 / 2}, & 2\left(e^{T^{\rho} / 2}-e^{-T^{\rho} / 2}\right) T^{1 / 2}, \\
2\left(-e^{T^{\rho} / 2}+e^{-T^{\rho} / 2}\right) T^{1 / 2}, & -2\left(e^{T^{\rho} / 2}+e^{-T^{\rho} / 2}\right) T^{1 / 2},
\end{array}
$$

respectively.

Hence, by Corollary 23.13, $b_{i}, b(\rho)_{i}$ can be arranged so that

$$
i_{\mathrm{qm}, T(0),\left(\mathfrak{b}(\rho), b_{1}\right)}^{*}\left(\mathbf{e}_{1}^{\mathfrak{b}(\rho)}\right)=i_{\mathrm{qm}, T(0),\left(\mathfrak{b}(\rho), b_{2}\right)}^{*}\left(\mathbf{e}_{4}^{\mathfrak{b}(\rho)}\right)=P D[T(0)]
$$

and

$$
i_{\mathrm{qm}, T(\rho),\left(\mathfrak{b}(\rho), b(\rho)_{1}\right)}^{*}\left(\mathbf{e}_{2}^{\mathfrak{b}(\rho)}\right)=i_{\mathrm{qm}, T(\rho),\left(\mathfrak{b}(\rho), b(\rho)_{2}\right)}^{*}\left(\mathbf{e}_{3}^{\mathfrak{b}(\rho)}\right)=P D[T(\rho)] .
$$

Remark 23.14. The Lagrangian sphere $S_{\text {van }}^{2}$ is unobstructed without bulk deformation as we saw in Lemma 23.5. Since the self-intersection number of $S_{\text {van }}^{2}$ is $-2, \mathfrak{m}_{0}^{\mathfrak{b}_{\rho}}(1)=-2 T^{\rho} P D[p t]$, it gets obstructed after the bulk deformation by $\mathfrak{b}_{\rho}$ for $\rho \neq 0$.

\section{LAGRANGIAN TORI IN THE CUBIC SURFACE}

This section owes much to the paper NNU2 of Nishinou-Nohara-Ueda, especially its Subsection 4.1 of the version 1 (arXiv:0812.0066v1). That section contained an error which seems to be a reason why the subsection was removed from the second version (arXiv:0812.0066v2). However, using a result by Chan-Lau CL, (actually, in [NNU2, Section 5] of the second version they independently obtained the relevant result for the cubic surface by a different argument), we can correct this error. This provides an interesting example which we discuss in this section. We would like to emphasize that the idea of exploiting a toric degeneration in the calculation of the potential function of a non-toric manifold used in this section and in FOOO6 is due to Nishinou-Nohara-Ueda NNU1 who successfully applied the idea to various examples.

Following [NNU2, Subsection 4.1] of its version one, we consider a family of cubic surfaces given by

$$
M_{t}=\left\{[x: y: z: w] \in \mathbb{C} P^{3} \mid x y z-w^{3}=t\left(x^{3}+y^{3}+z^{3}+w^{3}\right)\right\}
$$

parametrized by $t \in \mathbb{C}$. For $t \neq 0$ this gives a smooth surface. For $t=0, M_{0}$ becomes a toric variety with the $\left(\mathbb{C}^{*}\right)^{2}$-action

$$
(\alpha, \beta)[x: y: z: w]=\left[\alpha x: \beta y: \alpha^{-1} \beta^{-1} z: w\right] .
$$


The Fubini-Study form on $\mathbb{C} P^{3}$ induces a symplectic structure on $M_{t}$. This symplectic structure on $M_{0}$ is invariant under the action of real torus $T^{2} \subset\left(\mathbb{C}^{*}\right)^{2}$. The moment polytope of this action is given by

$$
P=\left\{\left(u_{1}, u_{2}\right) \in \mathbb{R}^{2} \mid \ell_{i}\left(u_{1}, u_{2}\right) \geq 0, i=1,2,3\right\}
$$

where

$$
\begin{aligned}
& \ell_{1}\left(u_{1}, u_{2}\right)=-u_{1}+2 u_{2}+1, \\
& \ell_{2}\left(u_{1}, u_{2}\right)=2 u_{1}-u_{2}+1, \\
& \ell_{3}\left(u_{1}, u_{2}\right)=-u_{1}-u_{2}+1 .
\end{aligned}
$$

The moment polytope $P$ is an isosceles triangle, whose center of gravity is origin.

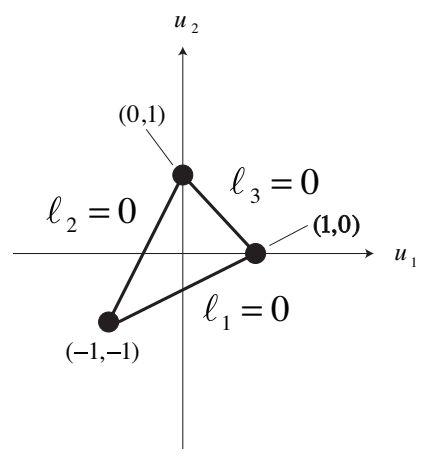

Figure 21. Polytope $P$

The three vertices of $P$ correspond to the three singular points of $M_{0}$. The variety $M_{0}$ is a toric orbifold with three singular points of $A_{2}$-type.

We can deform those three singular points by gluing the Milnor fiber of the $A_{2}$ singularity by the same way as in Section 23 to obtain a symplectic manifold $M$. It is easy to see that $M$ is symplectomorphic to $M_{t}$ for $t \neq 0$. (Note $M_{t}$ is symplectomorphic to $M_{t^{\prime}}$ if $t, t^{\prime} \neq 0$.)

We consider

$$
\mathfrak{Z}=\left(\mathbb{R}_{\geq 0}(1,0)\right) \sqcup\left(\mathbb{R}_{\geq 0}(0,1)\right) \sqcup\left(\mathbb{R}_{\geq 0}(-1,-1)\right) \cap \operatorname{Int} P .
$$

For $\mathbf{u} \in \mathfrak{Z}$ we consider $\pi^{-1}(\mathbf{u}) \subset M_{0}$. In the same way as in Section 23 we may regard it as a Lagrangian torus in $M$. We denote it by $T(\mathbf{u})$.

Theorem 24.1. For each $\mathbf{u} \in \mathfrak{Z}$, there exist $\mathfrak{b}(\mathbf{u}) \in H^{2}\left(M ; \Lambda_{+}\right)$and $b(\mathbf{u}) \in$ $H^{1}\left(T(\mathbf{u}) ; \Lambda_{0}\right)$ such that

$$
H F((T(\mathbf{u}),(\mathfrak{b}(\mathbf{u}), b(\mathbf{u}))) ; \Lambda) \neq 0 .
$$

Moreover there exists $e_{\mathbf{u}}$ that is a unit of a direct product factor $e_{\mathbf{u}} \Lambda=\Lambda$ of $Q H_{\mathfrak{b}(\mathbf{u})}(M ; \Lambda)$ such that

$$
i_{\mathrm{qm},(\mathfrak{b}(\mathbf{u}), b(\mathbf{u}))}^{*}\left(e_{\mathbf{u}}\right) \neq 0 \in H F((T(\mathbf{u}),(\mathfrak{b}(\mathbf{u}), b(\mathbf{u}))) ; \Lambda) .
$$

We can use this theorem in the same way as in Section 23 to show the following.

Corollary 24.2. (1) Each of $T(\mathbf{u})$ is non-displaceable.

(2) $T(\mathbf{u})$ is not Hamiltonian isotopic to $T\left(\mathbf{u}^{\prime}\right)$ if $\mathbf{u} \neq \mathbf{u}^{\prime}$. 


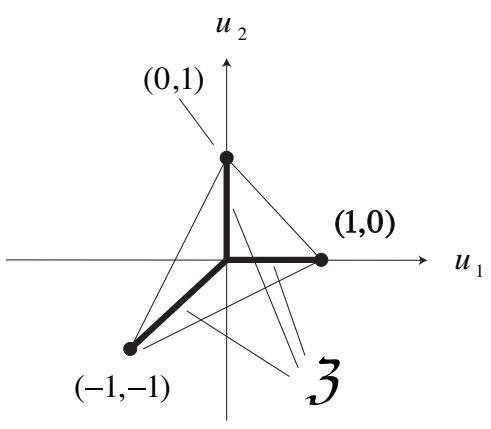

Figure 22. Set $\mathfrak{Z}$

(3) There exist uncountably many homogeneous Calabi quasi-morphisms

$$
\mu_{e_{\mathbf{u}}}^{\mathfrak{b}(\mathbf{u})}: \widetilde{\operatorname{Ham}}(M ; \omega) \rightarrow \mathbb{R}
$$

which are linearly independent.

Proof of Theorem 24.1. We consider a toric resolution of our orbifold $M_{0}$, which we denote by $M(\epsilon)$. We may take it so that its moment polytope is

$$
P_{\epsilon}=\left\{\left(u_{1}, u_{2}\right) \in P \mid \ell_{i}^{\epsilon}\left(u_{1}, u_{2}\right) \geq 0, \quad i=4, \ldots, 9\right\},
$$

where

$$
\begin{aligned}
& \ell_{4}^{\epsilon}\left(u_{1}, u_{2}\right)=u_{1}+1-\epsilon=\frac{1}{3}\left(2 \ell_{1}+\ell_{2}\right)-\epsilon, \\
& \ell_{5}^{\epsilon}\left(u_{1}, u_{2}\right)=u_{2}+1-\epsilon=\frac{1}{3}\left(\ell_{1}+2 \ell_{2}\right)-\epsilon, \\
& \ell_{6}^{\epsilon}\left(u_{1}, u_{2}\right)=u_{1}-u_{2}+1-\epsilon=\frac{1}{3}\left(2 \ell_{2}+\ell_{3}\right)-\epsilon, \\
& \ell_{7}^{\epsilon}\left(u_{1}, u_{2}\right)=-u_{2}+1-\epsilon=\frac{1}{3}\left(\ell_{2}+2 \ell_{3}\right)-\epsilon, \\
& \ell_{8}^{\epsilon}\left(u_{1}, u_{2}\right)=-u_{1}+1-\epsilon=\frac{1}{3}\left(2 \ell_{3}+\ell_{1}\right)-\epsilon, \\
& \ell_{9}^{\epsilon}\left(u_{1}, u_{2}\right)=-u_{1}+u_{2}+1-\epsilon=\frac{1}{3}\left(\ell_{3}+2 \ell_{1}\right)-\epsilon .
\end{aligned}
$$

We put

$$
D_{i}=\pi^{-1}\left(\partial_{i} P_{\epsilon}\right), \quad \partial_{i} P_{\epsilon}=\left\{\left(u_{1}, u_{2}\right) \in P_{\epsilon} \mid \ell_{i}^{\epsilon}\left(u_{1}, u_{2}\right)=0\right\},
$$

for $i=4, \ldots, 9$. $\left(D_{i}, i=1,2,3\right.$ are defined in the same way.)

We note that $M(\epsilon)$ is nef but is not Fano. In fact, $c_{1}(M(\epsilon)) \cap D_{i}=0$ for $i=4, \ldots, 9$. The potential function of $M(\epsilon)$ is calculated by Chan and Lau. In fact, $M(\epsilon)$ is $X_{11}$ in the table given in [CL, p.19].

Using the fact that $M$ is monotone, we can apply the argument of [FOOO6, Section 6] to show that we can take the limit $\epsilon \rightarrow 0$ to calculate the potential function of $T(\mathbf{u})$ in $M$. The result is the following.

Let $e_{1}, e_{2}$ be a basis of $H^{1}(T(\mathbf{u}) ; \mathbb{Z})$ and put $b=x_{1} e_{1}+x_{2} e_{2} \in H^{1}\left(T(\mathbf{u}) ; \Lambda_{0}\right)$. We put $\bar{y}_{i}=e^{x_{i}}$ and $y_{i}=T^{u_{i}} \bar{y}_{i}$, where $\mathbf{u}=\left(u_{1}, u_{2}\right)$. 


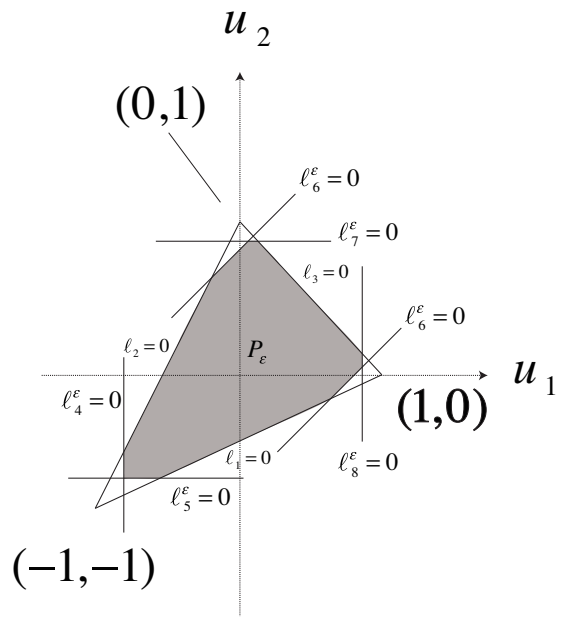

Figure 23. Polytope $P_{\epsilon}$

Theorem 24.3. The potential function of $T(\mathbf{u}) \subset M$ is given by

$$
\begin{aligned}
\mathfrak{P O}=T & \left(y_{1}^{-1} y_{2}^{-1}\left(y_{1}+y_{2}\right)^{3}+y_{1}^{-1} y_{2}^{2}\left(y_{1} y_{2}^{-1}+y_{2}^{-1}\right)^{3}\right. \\
& \left.+y_{1}^{2} y_{2}^{-1}\left(y_{1}^{-1}+y_{1}^{-1} y_{2}\right)^{3}-y_{1}^{-1} y_{2}^{2}-y_{1}^{2} y_{2}^{-1}-y_{1}^{-1} y_{2}^{-1}\right) .
\end{aligned}
$$

We postpone the proof of Theorem 24.3 till later when we prove Theorem 24.6 using (24.14).

Corollary 24.4. For each $\mathbf{u} \in \mathfrak{Z}$, there exists $b \in H^{1}\left(T(\mathbf{u}), \Lambda_{0}\right)$ such that

$$
H F\left((T(\mathbf{u}), b),(T(\mathbf{u}), b) ; \Lambda_{0}\right) \cong H\left(T^{2} ; \Lambda_{0}\right) .
$$

Proof. We define $Y_{1}, Y_{2}$ by the formula

$$
\begin{aligned}
Y_{1}^{3} & =y_{1}^{2} y_{2}^{-1}, \\
Y_{1}^{2} Y_{2} & =y_{1} .
\end{aligned}
$$

Note for each $y_{1}, y_{2}$ there are 3 choices of $Y_{1}$ satisfying (24.7). Then (24.8) uniquely determines $Y_{2}$. Thus $\left(Y_{1}, Y_{2}\right) \mapsto\left(y_{1}, y_{2}\right)$ is a three to one correspondence.

Now we can rewrite (24.6) as follows:

$$
\begin{aligned}
\mathfrak{P O} & \left.=T\left\{\left(Y_{1}+Y_{2}\right)^{3}+\left(Y_{1}+Y_{1}^{-1} Y_{2}^{-1}\right)^{3}+\left(Y_{2}+Y_{1}^{-1} Y_{2}^{-1}\right)^{3}-Y_{1}^{3}-Y_{2}^{3}-Y_{1}^{-3} Y_{2}^{-3}\right)\right\} \\
& =T\left(\left(Y_{1}+Y_{2}+Y_{1}^{-1} Y_{2}^{-1}\right)^{3}-6\right) .
\end{aligned}
$$

Therefore

$$
\begin{aligned}
& \frac{1}{T} \frac{\partial \mathfrak{P O}}{\partial Y_{1}}=3\left(1-Y_{1}^{-2} Y_{2}^{-1}\right)\left(Y_{1}+Y_{2}+Y_{1}^{-1} Y_{2}^{-1}\right)^{2} \\
& \frac{1}{T} \frac{\partial \mathfrak{P O}}{\partial Y_{2}}=3\left(1-Y_{1}^{-1} Y_{2}^{-2}\right)\left(Y_{1}+Y_{2}+Y_{1}^{-1} Y_{2}^{-1}\right)^{2} .
\end{aligned}
$$

Therefore the critical point is either

$$
Y_{1}=Y_{2}, \quad Y_{1}^{3}=1
$$

or

$$
Y_{1}+Y_{2}+Y_{1}^{-1} Y_{2}^{-1}=0
$$


The equation (24.9) gives a single solution $y_{1}=y_{2}=1$.

By (24.7), (24.8), the equation (24.10) is equivalent to $y_{1}+y_{2}+1=0$ in old coordinates $\left(y_{1}, y_{2}\right)$. Therefore the point $\left(y_{1}, y_{2}\right)$ given by the coordinates

$$
\begin{aligned}
& y_{1}=Y_{1}^{2} Y_{2}=-c^{3} T^{3 v}\left(1-c^{-3} T^{-3 v}+\cdots\right), \\
& y_{2}=Y_{1} Y_{2}^{2}=c^{3} T^{3 v}\left(1-2 c^{-3} T^{-3 v}+\cdots\right),
\end{aligned}
$$

is a critical point, when $\mathfrak{v}_{T}(c)=0$. Therefore the expression shows that it is a critical point with its valuation vector given by $\mathbf{u}=(3 v, 3 v) \in \mathbb{R}_{>0}(-1,-1)$, for any $v<0$. The corollary now follows from the obvious $\mathbb{Z}_{3}$-symmetry.

Remark 24.5. Corollary 24.4 implies that the Jacobian ring

$$
\operatorname{Jac}(\mathfrak{P O} ; \Lambda)=\frac{\Lambda\left\langle\left\langle y, y^{-1}\right\rangle\right\rangle^{P}}{\operatorname{Clos}_{d_{\odot}}\left(y_{i} \frac{\partial \mathfrak{P O}}{\partial y_{i}} ; i=1,2\right)}
$$

is infinite dimensional over $\Lambda$. Recall that in the toric case the Jacobian ring is always finite dimensional since the Kodaira-Spencer map $\mathfrak{k}_{\mathfrak{s}_{0}}: Q H(X ; \Lambda) \rightarrow$ $\operatorname{Jac}(\mathfrak{P O} ; \Lambda)$ is an isomorphism. (See Theorem 20.19.)

Corollary 24.4 implies the existence of a continuum of mutually disjoint nondisplaceable Lagrangian tori in a cubic surface. To show the existence of infinitely many Calabi quasi-morphisms and prove Theorem 1.10 (2), we need to study bulk deformations. Let

$$
\vec{w}=\left(w_{1}, \ldots, w_{9}\right) \in \Lambda_{0}^{9} .
$$

We put

$$
\mathfrak{b}(\vec{w})=\sum_{i=1}^{9} w_{i} P D\left(D_{i}\right)
$$

Theorem 24.6. We have

$$
\begin{aligned}
& \frac{1}{T}\left(\mathfrak{P O}_{\mathfrak{b}(\vec{w})}-\mathfrak{P O}\right) \\
= & \left(e^{w_{1}}-1\right) y_{1}^{-1} y_{2}^{2}+\left(e^{w_{2}}-1\right) y_{1}^{2} y_{2}^{-1}+\left(e^{w_{3}}-1\right) y_{1}^{-1} y_{2}^{-1} \\
& +\left(e^{w_{4}}+e^{w_{5}-w_{4}}+e^{-w_{5}}-3\right) y_{1}+\left(e^{w_{5}}+e^{w_{4}-w_{5}}+e^{-w_{4}}-3\right) y_{2} \\
& +\left(e^{w_{6}}+e^{w_{7}-w_{6}}+e^{-w_{7}}-3\right) y_{1} y_{2}^{-1}+\left(e^{w_{7}}+e^{w_{6}-w_{7}}+e^{-w_{6}}-3\right) y_{2}^{-1} \\
& +\left(e^{w_{8}}+e^{w_{9}-w_{8}}+e^{-w_{9}}-3\right) y_{1}^{-1}+\left(e^{w_{9}}+e^{w_{8}-w_{9}}+e^{-w_{8}}-3\right) y_{1}^{-1} y_{2} .
\end{aligned}
$$

Proof. We consider the term $3 y_{1}^{-1} y_{2}^{-1} y_{1}^{2} y_{2}=3 y_{1}$ in (24.6). This term comes from the moduli space $\mathcal{M}(\beta)$ where $\beta=\beta_{4}+\alpha$ with

$$
\beta_{4} \cap D_{j}= \begin{cases}1 & j=4 \\ 0 & j \neq 4\end{cases}
$$

and $\alpha \in H_{2}(M ; \mathbb{Z})$ with

$$
\alpha=k_{1}\left[D_{4}\right]+k_{2}\left[D_{5}\right]
$$

We define

$$
d\left(k_{1}, k_{2}\right)=\operatorname{deg}\left(\operatorname{ev}_{0}: \mathcal{M}_{1}\left(\beta_{4}+k_{1}\left[D_{4}\right]+k_{2}\left[D_{5}\right]\right) \rightarrow L(\mathbf{u})\right)
$$


By the result of Chan-Lau, CL, Theorem 1.1], (and the fact that the potential functions are continuous with respect to the limit $\epsilon \rightarrow 0$ ), we derive

$$
d\left(k_{1}, k_{2}\right)= \begin{cases}1 & \left(k_{1}, k_{2}\right)=(0,0),(1,0),(1,1) \\ 0 & \text { otherwise }\end{cases}
$$

This result is also obtained independently in Section 5 of the second version of NNU2 based on a different argument. Therefore, by the proof of FOOO4, Proposition 9.4], the coefficient of $y_{1}$ in $\mathfrak{P O}_{\mathfrak{b}_{a, b}(u)}$ is given by

$$
\begin{aligned}
\sum_{k_{1}, k_{2}} d\left(k_{1}, k_{2}\right) \exp \left(w_{4}\left[D_{4}\right] \cap\left[\beta_{4}+k_{1}\left[D_{4}\right]+k_{2}\left[D_{5}\right]\right]\right) \\
\exp \left(w_{5}\left[D_{5}\right] \cap\left[\beta_{4}+k_{1}\left[D_{4}\right]+k_{2}\left[D_{5}\right]\right]\right) \\
=e^{w_{4}}+e^{w_{5}-w_{4}}+e^{-w_{5}} .
\end{aligned}
$$

(Here we use (24.13) and $\left[D_{4}\right] \cdot\left[D_{4}\right]=\left[D_{5}\right] \cdot\left[D_{5}\right]=-2,\left[D_{4}\right] \cdot\left[D_{5}\right]=1$.)

In the same way the coefficient of $y_{2}$ in $\mathfrak{P O}_{\mathfrak{b}(\vec{w})}$ is given by $e^{w_{5}}+e^{w_{4}-w_{5}}+e^{-w_{4}}$. This proves the second line of the right hand side formula. The third and fourth lines of the formula can be proved in the same way. The proof of the first line is easier and so omitted.

We put

$$
\vec{w}_{0}=\left(0,0,0, w_{0}, w_{0}, w_{0}, w_{0}, w_{0}, w_{0}, w_{0}\right), \quad e^{2 w_{0}}+e^{w_{0}}+1=0 .
$$

Theorem 24.6 implies

$$
\mathfrak{P O}_{\mathfrak{b}\left(\vec{w}_{0}\right)}=T\left(y_{1}^{-1} y_{2}^{2}+y_{1}^{2} y_{2}^{-1}+y_{1}^{-1} y_{2}^{-1}\right) .
$$

Remark 24.7. According to [ri2, Proposition 3.10], the function (24.15) is the Landau-Ginzburg superpotential of the mirror of the toric orbifold $M_{0}$.

Lemma 24.8. $\mathfrak{P O}_{\mathfrak{b}\left(\vec{w}_{0}\right)}$ has 9 critical points. All of them have valuation 0 and are nondegenerate.

Proof. We can easily check that the critical points are given by $y_{1}^{3}=y_{2}^{3}=1$.

Lemma 24.9. For a generic $\vec{w}$ the set of critical points of $\mathfrak{P O}_{\mathfrak{b}(\vec{w})}$ consists of 9 elements all of which are nondegenerate.

Proof. The Newton polytope of the Laurent polynomial $\mathfrak{P O}_{\mathfrak{b}(\vec{w})}$ of $y$ has volume $9 / 2$. Therefore by the result of Kushnirenko $\mathrm{Ku}$ the number of critical points are at most 9 . Since it is exactly 9 for $\vec{w}=\vec{w}_{0}$, it is so for a generic $\vec{w}$. Since the number is maximal it must be nondegenerate.

Lemma 24.10. Suppose that the set of critical points of $\mathfrak{P O}_{\mathfrak{b}(\vec{w})}$ consists of 9 elements all of which are nondegenerate. We also assume that the valuation vectors of the critical points are in the interior of the moment polytope P. We also assume that none of the following happens.

(1) $e^{3 w_{4}}=1, e^{2 w_{4}}=e^{w_{5}}$.

(2) $e^{3 w_{6}}=1, e^{2 w_{6}}=e^{w_{7}}$.

(3) $e^{3 w_{8}}=1, e^{2 w_{8}}=e^{w_{9}}$. 
Then the homomorphism

$$
\mathfrak{k}_{\mathfrak{b}(\vec{w})}: Q H_{\mathfrak{b}(\vec{w})}(X ; \Lambda) \rightarrow \operatorname{Jac}\left(\mathfrak{P O}_{\mathfrak{b}(\vec{w})} ; \Lambda\right)
$$

is an isomorphism. Moreover $Q H_{\mathfrak{b}(\vec{w})}(X ; \Lambda)$ is semi-simple.

Proof. We can prove that $\mathfrak{k}_{\mathfrak{S}_{\mathfrak{b}}(\vec{w})}$ is a ring homomorphism in a similar way as in [FOOO7, Theorem 2.6.1]. (See [AFOOO1] for the detail.) We put

$$
z_{i}=y_{1}^{\frac{\partial \ell_{i}}{\partial y_{1}}} y_{2}^{\frac{\partial \ell_{i}}{\partial y_{2}}}
$$

In a way similar to [FOOO7, Lemma 1.2.4], we can prove that the set $\left\{z_{i} \mid i=\right.$ $1, \ldots, 9\}$ generates a dense $\Lambda$-subalgebra of $\Lambda\left\langle\left\langle y, y^{-1}\right\rangle\right\rangle^{P}$. Since $\operatorname{Jac}\left(\mathfrak{P O}_{\mathfrak{b}(\vec{w})} ; \Lambda\right)$ is finite dimensional, it is generated by the images of $z_{i}, i=1, \ldots, 9$ as a $\Lambda$-algebra.

By differentiating the formula given in Theorem 24.6, we find that the cohomology class $P D\left[D_{i}\right]$ is mapped to $e^{w_{i}} z_{i}$ by $\mathfrak{k}_{\mathfrak{b}(\vec{w})}$ for all $i=1,2,3$. We calculate

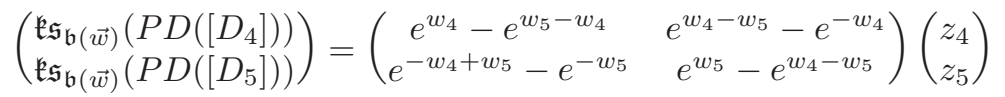

By assumption the matrix in the right hand side is nonzero. Therefore the image of $\mathfrak{k}_{\mathfrak{G}(\vec{w})}$ contains either $z_{4}$ or $z_{5}$. Since $z_{4} z_{5}=z_{1} z_{2}$, it contains both of $z_{4}$ and $z_{5}$. In a similar way we find that the image of $\mathfrak{k}_{\mathfrak{b}}(\vec{w})$ contains $z_{6}, \ldots, z_{9}$. Therefore $\mathfrak{k}_{\mathfrak{b}}(\vec{w})$ is surjective.

The rank of $\operatorname{Jac}\left(\mathfrak{P O}_{\mathfrak{b}(\vec{w})} ; \Lambda\right)$ is 9 that is equal to the Betti number of $X$. Therefore $\mathfrak{k}_{\mathfrak{b}(\vec{w})}$ is an isomorphism and $Q H_{\mathfrak{b}(\vec{w})}(X ; \Lambda) \cong \Lambda^{9}$. This in particular proves that the ring $Q H_{\mathfrak{b}(\vec{w})}(X ; \Lambda)$ is semi-simple.

We next put

$$
\vec{w}_{u ; c}=(0,0,0, w(u ; c), w(u ; c), 0,0,0,0), \quad e^{w(u ; c)}+1+e^{-w(u ; c)}=3+c T^{u}
$$

with $c \in \mathbb{C} \backslash\{0\}, u \geq 0$. (We observe that $\mathfrak{v}_{T}(w(u ; c))=u / 2$.)

Lemma 24.11. Let $\vec{w}_{u ; c}$ be the vector given as above, and assume $c$ is chosen generically for $u=0$. Then

(1) $\mathfrak{P O}_{\mathfrak{b}\left(\vec{w}_{u ; c}\right)}$ has exactly 3 nonzero critical points.

(2) The valuation of the critical points are $(0,0)$.

(3) All the three critical points are nondegenerate.

Proof. We change the variable from $\left(y_{1}, y_{2}\right)$ to $\left(Y_{1}, Y_{2}\right)$ determined by the relation (24.7), (24.8). Then by substituting the vector $w=\vec{w}_{u ; c}$ above into the formula given in Theorem 24.6 we obtain

$$
\mathfrak{P O}_{\mathfrak{b}\left(\vec{w}_{u ; c}\right)}=T\left(\left(Y_{1}+Y_{2}+Y_{1}^{-1} Y_{2}^{-1}\right)^{3}-6\right)+c T^{1+u}\left(Y_{1}+Y_{2}\right) Y_{1} Y_{2} .
$$

Therefore the critical point equation $\nabla \mathfrak{P O} \mathfrak{b}_{\mathfrak{b}\left(\vec{w}_{u ; c}\right)}=0$ is equivalent to

$$
\begin{aligned}
& 3\left(1-Y_{1}^{-2} Y_{2}^{-1}\right)\left(Y_{1}+Y_{2}+Y_{1}^{-1} Y_{2}^{-1}\right)^{2}+c T^{u} Y_{2}\left(2 Y_{1}+Y_{2}\right)=0, \\
& 3\left(1-Y_{1}^{-1} Y_{2}^{-2}\right)\left(Y_{1}+Y_{2}+Y_{1}^{-1} Y_{2}^{-1}\right)^{2}+c T^{u} Y_{1}\left(2 Y_{2}+Y_{1}\right)=0 .
\end{aligned}
$$

In particular we obtain the equation

$$
\frac{2 Y_{1}+Y_{2}}{Y_{1}^{2} Y_{2}-1}=\frac{2 Y_{2}+Y_{1}}{Y_{2}^{2} Y_{1}-1}
$$

Since $Y_{1} \neq 0, Y_{2} \neq 0$ this equation implies of the following two alternatives:

$$
\text { either } Y_{1}=Y_{2} \quad \text { or } Y_{1} Y_{2}\left(Y_{1}+Y_{2}\right)=-1 \text {. }
$$


In case $Y_{1} Y_{2}\left(Y_{1}+Y_{2}\right)=-1$, (24.16) and (24.17) imply $2 Y_{1}+Y_{2}=0$ and $2 Y_{2}+Y_{1}=$ 0 respectively. But this equation also has trivial solution which is ruled out since $Y_{1} \neq 0 \neq Y_{2}$. Therefore $Y_{1}=Y_{2}$ must hold. If we put $x=Y_{1}^{3}=Y_{2}^{3}$, then the system of equations (24.16), (24.17) is equivalent to

$$
(x-1)(2 x+1)^{2}+c T^{u} x^{3}=0 .
$$

This equation has three simple roots. (We use genericity of $c$ in case $u=0$.) This proves $(1)$. (Note $y_{1}=y_{2}=x$.)

Now we prove (2). If $u=0 x \in \mathbb{C} \backslash\{0\}$. Therefore the valuations of $y_{1}, y_{2}$ are 0 . If $u>0$, then $x \equiv 1$ or $-1 / 2$ modulo $\Lambda_{+}$. Therefore $\mathfrak{v}_{T}(x)=0$ also. This proves (2).

We next prove (3). We calculate

$$
\left(y_{i} y_{j} \frac{\partial^{2} \mathfrak{P} \mathfrak{O}_{\mathfrak{b}(\rho)}}{\partial y_{i} \partial y_{j}}\right)_{i, j=1}^{2}=\left(\begin{array}{cc}
A & B \\
B & A
\end{array}\right)
$$

where

$$
A=\frac{T}{x^{2}}\left(4 x^{3}+6 x^{2}+6 x+2\right), \quad B=\frac{T}{x^{2}}\left(-4 x^{3}-6 x^{2}+1\right) .
$$

Here we use (24.18) during the calculation. Therefore we get

$$
\operatorname{det}\left(y_{i} y_{j} \frac{\partial^{2} \mathfrak{P \mathfrak { O } _ { \mathfrak { b } ( \rho ) }}}{\partial y_{i} \partial y_{j}}\right)_{i, j=1}^{2}=3 T^{2} \frac{(2 x+1)^{4}}{x^{4}}
$$

and so (3) follows.

We now consider an affine line $C \cong \mathbb{C}$ contained in $\mathbb{C}^{9}$ so that it contains $\vec{w}_{0}$ and $\vec{w}_{0 ; c}$ where $c$ is generic. We denote by $\mathfrak{b}(a)$ the bulk ambient cycle associated to $a$ and consider the set

$$
\mathfrak{X}_{0}=\left\{\left(a ; y_{1}, y_{2}\right) \mid a \in C, \nabla\left(\mathfrak{P O}_{\mathfrak{b}(a)}\right)=0 \text { at }\left(y_{1}, y_{2}\right) \in \mathbb{C}^{2}\right\} .
$$

We take the Zariski closure of $\mathfrak{X}_{0}$ in $C \times \mathbb{C} P^{1} \times \mathbb{C} P^{1}$ and denotes it by $\mathfrak{X}$. We have a natural projection $\pi: \mathfrak{X} \rightarrow C$. At a generic point $a \in C$ the fiber of $\pi$ consists of 9 points. At $a=\vec{w}_{0 ; c}$ the fiber of $\pi$ intersects with $\mathfrak{X}_{0}$ at 3 points and those three points are all simple. Therefore $\pi^{-1}\left(\vec{w}_{0 ; c}\right) \backslash \mathfrak{X}_{0} \neq \emptyset$. Then there exist Laurent power series

$$
y_{1}(w)=\sum_{k \geq k_{0,1}} y_{1, k} w^{k}, \quad y_{2}(w)=\sum_{k \geq k_{0,2}} y_{2, k} w^{k}
$$

and

$$
a(w)=\sum_{k \geq 0} a_{k} w^{k}
$$

such that the following holds:

(1) $y_{1}(w), y_{2}(w)$ converge for $|w| \in(0, \epsilon)$.

(2) $a(w)$ converges for $|w| \in[0, \epsilon)$.

(3) $\left(a(w) ; y_{1}(w), y_{2}(w)\right) \in \mathfrak{X}_{0}$ for $|w| \in(0, \epsilon)$.

(4) $\left(y_{1}(0), y_{2}(0)\right) \in\left(\mathbb{C} P^{1}\right)^{2} \backslash(\mathbb{C} \backslash\{0\})^{2}$.

(5) $a(0)=\vec{w}_{0 ; c}$.

Now we consider $\left(y_{1}\left(T^{\rho}\right), y_{2}\left(T^{\rho}\right)\right) \in \Lambda^{2}$ and $a\left(T^{\rho}\right) \in \Lambda_{0}^{9}$. Then (3) implies that $\left(y_{1}\left(T^{\rho}\right), y_{2}\left(T^{\rho}\right)\right)$ is a critical point of $\mathfrak{P O}_{\mathfrak{b}\left(a\left(T^{\rho}\right)\right)}$. 
Lemma 24.12. If $\left(\mathfrak{v}_{T}\left(y_{1}\left(T^{\rho}\right)\right), \mathfrak{v}_{T}\left(y_{2}\left(T^{\rho}\right)\right)\right) \in P$, then

$$
\left(\mathfrak{v}_{T}\left(y_{1}\left(T^{\rho}\right)\right), \mathfrak{v}_{T}\left(y_{2}\left(T^{\rho}\right)\right)\right) \in \mathfrak{Z} .
$$

Proof. If $\left(u_{1}, u_{2}\right)=\left(\mathfrak{v}_{T}\left(y_{1}\right), \mathfrak{v}_{T}\left(y_{2}\right)\right) \in P \backslash \mathfrak{Z}$, then there exist $i=1,2,3$ such that $\ell_{i}\left(u_{1}, u_{2}\right)<\ell_{j}\left(u_{1}, u_{2}\right)$ for each $j \in\{1, \ldots, 9\}, j \neq i$. It easily follows that $\left(y_{1}, y_{2}\right)$ is not a critical point of $\mathfrak{P O}_{\mathfrak{b}\left(a\left(T^{\rho}\right)\right)}$.

It is also easy to see that $\rho \mapsto\left(\mathfrak{v}_{T}\left(y_{1}\left(T^{\rho}\right)\right), \mathfrak{v}_{T}\left(y_{2}\left(T^{\rho}\right)\right)\right)$ is continuous and

$$
\lim _{\rho \rightarrow 0}\left(\mathfrak{v}_{T}\left(y_{1}\left(T^{\rho}\right)\right), \mathfrak{v}_{T}\left(y_{2}\left(T^{\rho}\right)\right)\right)=(0,0) .
$$

Moreover we find that $\mathfrak{v}_{T}\left(y_{1}\left(T^{\rho}\right)\right)$ and $\mathfrak{v}_{T}\left(y_{2}\left(T^{\rho}\right)\right)$ are either increasing or decreasing, and $\left(\mathfrak{v}_{T}\left(y_{1}\left(T^{\rho}\right)\right), \mathfrak{v}_{T}\left(y_{2}\left(T^{\rho}\right)\right)\right)$ diverges as $\rho \rightarrow \infty$. (This is a consequence of (4) above.) Therefore there exists $\rho_{1}>0$ such that

$$
\rho \mapsto\left(\mathfrak{v}_{T}\left(y_{1}\left(T^{\rho}\right)\right), \mathfrak{v}_{T}\left(y_{2}\left(T^{\rho}\right)\right)\right)
$$

defines a homeomorphism between $\left(0, \rho_{1}\right)$ and one of the sets

$$
\mathfrak{Z}_{1}=\{(-u,-u) \mid u \in(0,1)\}, \mathfrak{Z}_{2}=\{(u, 0) \mid u \in(0,1)\}, \mathfrak{Z}_{3}=\{(0, u) \mid u \in(0,1)\} .
$$

Note that there exist 6 choices of such $\left(y_{1}(q), y_{2}(q)\right)$ for given $a(w)$, after replacing $C$ by an appropriate branched cover branching at $w=\vec{w}_{0 ; c}$. This is because the order of the set $\pi^{-1}(a(w)) \subset \mathfrak{X}$ is 9 for generic $w$ and the set $\pi^{-1}(a(0)) \subset \mathfrak{X}$ consists of 3 points all of which are simple. Each of such 6 choices determines $\rho_{1}$ above. We take its minimum and denote it by $\rho_{0}$. Thus we have proved the following:

Lemma 24.13. (1) For each $\rho \in\left(0, \rho_{0}\right)$, there exist exactly 9 critical points of $\mathfrak{P O}_{\mathfrak{b}\left(a\left(T^{\rho}\right)\right)}$. They are simple and their valuation vectors are always contained in the interior of $P$.

(2) We may take a choice of $\left(y_{1}(q), y_{2}(q)\right)$ as above so that

$$
\rho \mapsto\left(\mathfrak{v}_{T}\left(y_{1}\left(T^{\rho}\right)\right), \mathfrak{v}_{T}\left(y_{2}\left(T^{\rho}\right)\right)\right)
$$

defines a homeomorphism between $\left(0, \rho_{0}\right)$ and one of $\mathfrak{Z}_{1}, \mathfrak{Z}_{2}, \mathfrak{Z}_{3}$.

Lemmas 24.10 24.13 and Theorem 18.8 imply that the following holds for one of $i=1,2,3$. We also note that we can prove Theorem 20.24 (20.39) in our situation, where we replace $i_{\mathrm{qm},(\mathfrak{b}(\mathfrak{y}), b(\mathfrak{y}))}^{T, *}$ by $i_{\mathrm{qm},\left(\mathfrak{b}\left(a\left(T^{\rho}\right)\right), b\left(a\left(T^{\rho}\right)\right)\right)}^{*}$.

Lemma 24.14. For each $\mathbf{u} \in \mathfrak{Z}_{i}$, there exist $\mathfrak{b}(\mathbf{u})$ and $e(\mathbf{u}) \in Q H_{\mathfrak{b}(\mathbf{u})}(X ; \Lambda)$ such that:

(1) $e(\mathbf{u}) \Lambda \subset Q H_{\mathfrak{b}(\mathbf{u})}(X ; \Lambda)$ is a direct factor.

(2) $T(\mathbf{u})$ is $\zeta_{e(\mathbf{u})}^{\mathfrak{b}(\mathbf{u})}$-superheavy.

Once we have Lemma 24.14 for some $i$, then by symmetry we obtain the same conclusion for the other two of $i=1,2,3$. The proof of Theorem 24.1 is now completed.

\section{Detecting spectral invariant via Hochschild COHOMOlOgy}

In this section we prove the following theorem. For a critical point $\mathfrak{y}$ of the potential function $\mathfrak{P O}_{\mathfrak{b}}$ we recall the subset $\operatorname{Jac}\left(\mathfrak{P O}_{\mathfrak{b}} ; \mathfrak{y}\right) \subset \operatorname{Jac}\left(\mathfrak{P O}_{\mathfrak{b}} ; \Lambda\right)$ from Definition 20.22. Corresponding to this subspace, we put

$$
Q H_{\mathfrak{b}}(M ; \Lambda ; \mathfrak{y}):=\left\{s \in Q H_{\mathfrak{b}}(M ; \Lambda) \mid \mathfrak{k}_{\mathfrak{b}}(s) \in \operatorname{Jac}\left(\mathfrak{P O} \mathfrak{O}_{\mathfrak{b}} ; \mathfrak{y}\right)\right\} .
$$


Theorem 25.1. Let $(M, \omega)$ be a compact toric manifold and $\mathfrak{b} \in H^{\text {even }}\left(M ; \Lambda_{0}\right)$. Suppose that $\mathfrak{y}$ is a critical point of the potential function $\mathfrak{P O}_{\mathfrak{b}}$. Let $\mathbf{u}=\mathbf{u}(\mathfrak{y})$ and let $b=b(\mathfrak{y})$ be those defined as in Theorem 20.18. Denote by $e_{\mathfrak{y}}$ the corresponding idempotent of $Q H_{\mathfrak{b}}(M, \omega ; \mathfrak{y})$. Then $L(\mathbf{u})$ is $\mu_{e_{\mathfrak{y}}}^{\mathfrak{b}}$-superheavy.

This theorem improves Theorem 21.1 in that superheaviness holds without assuming nondegeneracy of $\mathfrak{y}$.

Problem 25.2. Let $\mathfrak{y}$ be a degenerate critical point of $\mathfrak{P O}_{\mathfrak{b}}$. When does $\mu_{e_{\mathfrak{y}}}^{\mathfrak{b}}$ become a quasi-morphism?

25.1. Hochschild cohomology of filtered $A_{\infty}$ algebra: review. We will use Hochschild cohomology in the proof of Theorem 25.1, Let $\left(C,\left\{\mathfrak{m}_{k}\right\}_{k=0}^{\infty}\right)$ be a unital and gapped filtered $A_{\infty}$ algebra. (See [FOOO1, Section 3] for the definition of filtered $A_{\infty}$ algebra etc.) In the situation of Theorem 25.1 we have $\mathfrak{m}_{0}(1)=$ $\mathfrak{P O}(b) \cdot \mathbf{e}$ that is not zero in general. (Here e is the unit of $\left(C,\left\{\mathfrak{m}_{k}\right\}_{k=0}^{\infty}\right)$.) We redefine the $A_{\infty}$ structure by setting $\mathfrak{m}_{0}(1)=0$ but not changing other operators. By the unitality of the given $A_{\infty}$ algebra, we find that the $A_{\infty}$ relations still hold. After this modification we will assume

$$
\mathfrak{m}_{0}=0
$$

in this section.

We consider the Hochschild cochain modules

$$
\begin{aligned}
C H^{k}(C, C) & =H_{0 m}\left(B_{k} C[1], C[1]\right), \\
C H(C, C) & =\prod_{k=0}^{\infty} C H^{k}(C, C), \\
\mathcal{N}^{k} C H(C, C) & =C H(C, C) / \prod_{k^{\prime}>k} C H^{k^{\prime}}(C, C) .
\end{aligned}
$$

We define Hochschild differential $\delta_{H}: C H(C, C) \rightarrow C H(C, C)$ by

$$
\begin{aligned}
\delta_{H}(\varphi)\left(x_{1}, \ldots, x_{k}\right)= & \sum_{i, \ell}(-1)^{*_{1}} \varphi\left(x_{1}, \ldots, \mathfrak{m}_{\ell}\left(x_{i}, \ldots\right), \ldots\right) \\
& +\sum_{i, \ell}(-1)^{*_{2}} \mathfrak{m}_{\ell}\left(x_{1}, \ldots, \varphi\left(x_{i}, \ldots\right), \ldots\right),
\end{aligned}
$$

where $*_{1}=\operatorname{deg} \varphi\left(\operatorname{deg} x_{1}+\cdots+\operatorname{deg} x_{i-1}+i-1\right), *_{2}=\operatorname{deg} x_{1}+\cdots+\operatorname{deg} x_{i-1}+i$. It is easy to check $\delta_{H} \circ \delta_{H}=0$. So $\left(C H(C, C), \delta_{H}\right)$ is a (co)chain complex, which we call Hochschild cochain complex.

By our assumption $\mathfrak{m}_{0}=0$, we have

$$
\delta_{H}\left(\prod_{k^{\prime}>k} C H^{k^{\prime}}(C, C)\right) \subset \prod_{k^{\prime}>k} C H^{k^{\prime}}(C, C) .
$$

Therefore the map $\delta_{H}$ descends to a map $\delta_{H}: \mathcal{N}^{k} C H(C, C) \rightarrow \mathcal{N}^{k} C H(C, C)$ in the quotient. We call this filtration the number filtration. Note we have

$$
\left(C H(C, C), \delta_{H}\right)=\underset{k \rightarrow \infty}{\operatorname{proj}} \lim \left(\mathcal{N}^{k} C H(C, C), \delta_{H}\right) .
$$

We assume that $\left(C,\left\{\mathfrak{m}_{k}\right\}_{k=0}^{\infty}\right)$ has a strict unit e. We define its submodule

$$
C H^{\mathrm{red}, k}(C, C)=\left\{\varphi \in C H^{k}(C, C) \mid \varphi(\cdots, \mathbf{e}, \cdots)=0\right\}
$$


and define $C H^{\text {red }}(C, C), \mathcal{N}^{k} C H^{\text {red }}(C, C)$ in a similar way. We can easily show $\delta_{H}\left(C H^{\text {red }}(C, C)\right) \subset C H^{\text {red }}(C, C)$ which enables us to define reduced Hochschild cochain complex $\left(C H^{\text {red }}(C, C), \delta_{H}\right)$. The latter complex carries a number filtration. The cohomology of the reduced Hochschild cochain complex is written as $H H^{\text {red }}(C, C)$ and is called reduced Hochschild cohomology.

We note that

$$
C H^{k}(C, C) \cong \operatorname{Hom}_{\mathbb{C}}\left(B_{k} \bar{C}[1], \bar{C}[1]\right) \otimes \Lambda,
$$

where $\bar{C} \otimes_{\mathbb{C}} \Lambda=C$. We can then use the filtration $F^{\lambda} C=\bar{C} \otimes_{\mathbb{C}} T^{\lambda} \Lambda_{0}$ to define another filtration $F^{\lambda} C H^{k}(C, C)$ on $C H^{k}(C, C)$. We call this filtration the energy filtration. Using the condition $\mathfrak{m}_{k}\left(F^{\lambda_{1}} C \otimes \cdots \otimes F^{\lambda_{k}} C\right) \subset F^{\lambda_{1}+\cdots+\lambda_{k}} C$ ([FOOO1, (3.2.12.6)]), $\delta_{H}$ preserves the energy filtration.

25.2. From quantum cohomology to Hochschild cohomology. Let $L$ be a relative spin Lagrangian submanifold of a compact symplectic manifold $(M, \omega)$ and $\mathfrak{b} \in H^{\text {even }}\left(M ; \Lambda_{0}\right)$. Let $b=b_{0}+b_{+} \in \Omega^{\text {odd }} \otimes \Lambda_{0}$ be an element satisfying the Maurer-Cartan equation

$$
\sum_{\beta} \sum_{k=0}^{\infty} T^{\omega \cap \beta} \exp \left(b_{0} \cap \partial \beta\right) \mathfrak{m}_{k, \beta}\left(b_{+}, \ldots, b_{+}\right)=0 .
$$

For each such pair $(\mathfrak{b}, b)$, we obtain a unital gapped filtered $A_{\infty}$ algebra $\left(\Omega(L) \widehat{\otimes} \Lambda,\left\{\mathfrak{m}_{k}^{\mathfrak{b}, b}\right\}\right)$. We define

$$
\mathfrak{q}_{*}^{\mathbf{b}}=H(M ; \Lambda) \rightarrow C H^{\mathrm{red}}(\Omega(L) \widehat{\otimes} \Lambda, \Omega(L) \widehat{\otimes} \Lambda)
$$

as follows. We put $\mathbf{b}=\left(\mathfrak{b}_{0}, \mathbf{b}_{2 ; 1}, \mathfrak{b}_{+}, b_{+}\right)$and define for $k \neq 0$ :

$$
\begin{aligned}
& \mathfrak{q}_{*}^{\mathbf{b}}(a)\left(x_{1}, \ldots, x_{k}\right) \\
& =\sum_{\beta \in H_{2}(M, L: \mathbb{Z})} \sum_{\ell_{1}=0}^{\infty} \sum_{\ell_{2}=0}^{\infty} \sum_{m_{0}=0}^{\infty} \cdots \sum_{m_{k}=0}^{\infty} T^{\omega \cap \beta} \frac{\exp \left(\mathbf{b}_{2 ; 1} \cap \beta\right)}{\left(\ell_{1}+\ell_{2}+1\right) !} \\
& \mathfrak{q}_{\ell_{1}+\ell_{2}+1, k+\sum_{i=0}^{k} m_{i} ; \beta}\left(\mathfrak{b}_{+}^{\otimes \ell_{1}} \otimes a \otimes \mathfrak{b}_{+}^{\otimes \ell_{2}} ; b_{+}^{\otimes m_{0}}, x_{1}, b_{+}^{\otimes m_{1}}, \ldots, b_{+}^{\otimes m_{k-1}}, x_{k}, b_{+}^{\otimes m_{k}}\right),
\end{aligned}
$$

where $x_{i} \in \Omega(L)$. Here the notations are the same as in (17.11). Recall that we assume $\mathfrak{m}_{0}=0$ in this section.

Using Theorem 17.1 (1), we can show that $\delta_{H}\left(\mathfrak{q}_{*}^{\mathbf{b}}(a)\right)=0$. Therefore we have a map

$$
\mathfrak{q}_{*}^{\mathbf{b}}: H(M ; \Lambda) \rightarrow H H_{\mathbf{b}}^{\mathrm{red}}(\Omega(L) \widehat{\otimes} \Lambda, \Omega(L) \widehat{\otimes} \Lambda)
$$

to the reduced Hochschild cohomology. Here $H H_{\mathbf{b}}^{\text {red }}(\Omega(L) \widehat{\otimes} \Lambda, \Omega(L) \widehat{\otimes} \Lambda)$ is the reduced Hochschild cohomology with respect to the filtered $A_{\infty}$ structure $\mathfrak{m}^{\mathrm{b}}$.

Remark 25.3. (1) By composing $\mathfrak{q}_{*}^{\mathbf{b}}$ with the projection

$$
H H_{\mathbf{b}}^{\mathrm{red}}(\Omega(L) \widehat{\otimes} \Lambda, \Omega(L) \widehat{\otimes} \Lambda) \rightarrow \mathcal{N}_{0} H H_{\mathbf{b}}^{\mathrm{red}}(\Omega(L) \widehat{\otimes} \Lambda, \Omega(L) \widehat{\otimes} \Lambda)=H F^{*}((L, \mathbf{b}) ; \Lambda),
$$

we obtain a map $H(M ; \Lambda) \rightarrow H F^{*}((L, \mathbf{b}) ; \Lambda)$. This coincides with the map $i_{\mathrm{qm}, H F((L, \mathbf{b}) ; \Lambda)}^{*}$ given in (17.18).

(2) $H H_{\mathbf{b}}^{\text {red }}(\Omega(L) \widehat{\otimes} \Lambda, \Omega(L) \widehat{\otimes} \Lambda)$ has a filtered $A_{\infty}$ structure. (See FOOO7, 4.7.4 and Remark 4.7.1 (1)].) Moreover $\mathfrak{q}_{*}^{\mathbf{b}}$ is a ring homomorphism. (See AFOOO1 for the proof in general). 
25.3. Proof of Theorem 25.1. To prove Theorem 25.1 we need to explore some estimates of the spectral invariant which are analogs of the ones developed in Parts 1-4. In the previous parts we use $\Lambda^{\downarrow}$ coefficients and the valuation $\mathfrak{v}_{q}$, while we use $\Lambda$ coefficients and the valuation $\mathfrak{v}_{T}$ in Subsections 25.1, 25.2. To translate the valuation $\mathfrak{v}_{T}$ for any element $x$ defined over $\Lambda$ into $\mathfrak{v}_{q}$, we just define

$$
\mathfrak{v}_{q}(x)=-\mathfrak{v}_{T}(x),
$$

because $T=q^{-1}$. See Notations and Conventions (18). We use this notation throughout this subsection.

The following is an analog of Proposition 18.9.

Proposition 25.4. Let $L, \mathbf{b}$ be as above and $a \in H(M ; \Lambda)$. Then

$$
\rho^{\mathfrak{b}}(H ; a) \geq-E^{+}(H ; L)+\mathfrak{v}_{q}\left(\mathfrak{q}_{*}^{\mathbf{b}}(a)\right) .
$$

Here for $x \in H H^{\text {red }}(\Omega(L) \widehat{\otimes} \Lambda, \Omega(L) \widehat{\otimes} \Lambda)$ we define

$$
\mathfrak{v}_{q}(x)=-\mathfrak{v}_{T}(x)=-\sup \left\{\lambda \mid \exists \widetilde{x} \in F^{\lambda} C H^{\mathrm{red}}(\Omega(L) \widehat{\otimes} \Lambda, \Omega(L) \widehat{\otimes} \Lambda), x=[\widetilde{x}]\right\} .
$$

Proof. Using the operator $\mathfrak{q}_{\beta ; \ell ; k}^{H ;[\gamma, w]}$ in 18.18), we define

$$
\mathfrak{q}_{*}^{H, \mathbf{b}}: C F(M ; H ; \Lambda) \rightarrow C H_{\mathbf{b}}^{\mathrm{red}}(\Omega(L) \widehat{\otimes} \Lambda, \Omega(L) \widehat{\otimes} \Lambda)
$$

by

$$
\begin{aligned}
& \mathfrak{q}_{*}^{H, \mathbf{b}}(\llbracket \gamma, w \rrbracket)\left(x_{1}, \ldots, x_{k}\right) \\
& =\sum_{\beta \in H_{2}(M, L: \mathbb{Z})} \sum_{\ell=0}^{\infty} \sum_{m_{0}=0}^{\infty} \cdots \sum_{m_{k}=0}^{\infty} T^{\omega \cap \beta} \frac{\exp \left(\mathbf{b}_{2 ; 1} \cap \beta\right)}{\ell !} \\
& \mathfrak{q}_{\ell, k+\sum_{i=0}^{k} m_{i} ;[\gamma}\left(\mathfrak{b}_{+}^{\otimes \ell} ; b_{+}^{\otimes m_{0}}, x_{1}, b_{+}^{\otimes m_{1}}, \ldots, b_{+}^{\otimes m_{k-1}}, x_{k}, b_{+}^{\otimes m_{k}}\right) .
\end{aligned}
$$

Here we take a lift $[\gamma, w] \in \operatorname{Crit}\left(\mathcal{A}_{H}\right)$ of $\llbracket \gamma, w \rrbracket \in \widehat{\operatorname{Per}}(H)$ to define the right hand side and can show that it is independent of the lift $[\gamma, w]$ by Proposition 18.11(11).

Using Proposition 18.14, we can find that it induces a map

$$
\mathfrak{q}_{*}^{H, \mathbf{b}}: H F(M, H ; \Lambda) \rightarrow H H_{\mathbf{b}}^{\text {red }}(\Omega(L) \widehat{\otimes} \Lambda, \Omega(L) \widehat{\otimes} \Lambda) .
$$

Lemma 25.5. $\mathfrak{q}_{*}^{H, \mathbf{b}} \circ \mathcal{P}_{\left(H_{\chi}, J\right)}^{\mathfrak{b}}$ is chain homotopic $\mathfrak{q}_{*}^{\mathbf{b}}$.

The proof is the same as that of Proposition 18.20 and is omitted.

We can use Lemma 25.5 to prove Proposition 25.4 in the same way as we used Proposition 18.20 to prove Proposition 18.9. Thus Proposition 25.4 follows.

Now we restrict ourselves to the case of toric manifold $(M, \omega)$. For the toric case we can use the $T^{n}$-equivariant operator $\mathfrak{q}^{T}$ in place of $\mathfrak{q}$ in Proposition 25.4 and the Hochschild complex $C H(H(L(\mathbf{u}) ; \Lambda), H(L(\mathbf{u}) ; \Lambda))$ defined on de Rham cohomology group (instead on the space of differential forms). In fact the $A_{\infty}$ structure $\mathfrak{m}_{*}^{T}$ itself was defined thereon.

Let $\mathfrak{b} \in H^{\text {even }}(M ; \Lambda)$ and let $\mathfrak{y}$ be a critical point of $\mathfrak{P \mathfrak { O } _ { \mathfrak { b } }}, \mathbf{u}=\mathbf{u}(\mathfrak{y})$ and $b=b(\mathfrak{y})$ as in Theorem 20.18 We put $\mathbf{b}=\mathbf{b}(\mathfrak{y})=(\mathfrak{b}, b(\mathfrak{y}))$.

Lemma 25.6. The restriction of the map

$$
\mathfrak{q}_{*}^{T, \mathbf{b}}: Q H_{\mathfrak{b}}(M ; \Lambda) \rightarrow H H_{\mathbf{b}}^{\mathrm{red}}(H(L(\mathbf{u}) ; \Lambda), H(L(\mathbf{u}) ; \Lambda))
$$

to $Q H_{\mathfrak{b}}(M ; \Lambda ; \mathfrak{y}) \subset Q H_{\mathfrak{b}}(M ; \Lambda)$, is injective. 
Proof. We recall from [FOOO7, Lemma 4.7.5] that we have the map

$$
H H(H(L(\mathbf{u}) ; \Lambda), H(L(\mathbf{u}) ; \Lambda)) \rightarrow \operatorname{Jac}(\mathfrak{P O} ; \mathfrak{y}) .
$$

(More precisely, we constructed a map from $H H(H(L(\mathbf{u}) ; \Lambda), H(L(\mathbf{u}) ; \Lambda))$ to a formal power series version of the Jacobian ring in [FOOO7, Lemma 4.7.5]. As we mentioned at the end of [FOOO7, page 329], this formal power series version is isomorphic to the Jacobian ring $\operatorname{Jac}(\mathfrak{P O} ; \mathfrak{y})$, which is its adic convergent version. The detail of the proof of this isomorphism will be written in AFOOO2. It seems likely that this is known to the expert of rigid analytic geometry.) Its bulk deformation gives rise to a map

$$
H H_{\mathbf{b}}(H(L(\mathbf{u}) ; \Lambda), H(L(\mathbf{u}) ; \Lambda)) \rightarrow \operatorname{Jac}\left(\mathfrak{P O}_{\mathbf{b}} ; \mathfrak{y}\right) .
$$

Then the composition of the restriction of $\mathfrak{q}_{*}^{T, \mathbf{b}}$ to $Q H_{\mathfrak{b}}(M ; \Lambda ; \mathfrak{y})$ with the canonical injective map $H H_{\mathbf{b}}^{\text {red }}(H(L(\mathbf{u}) ; \Lambda), H(L(\mathbf{u}) ; \Lambda)) \rightarrow H H_{\mathbf{b}}(H(L(\mathbf{u}) ; \Lambda), H(L(\mathbf{u}) ; \Lambda))$ and (25.8) induces an isomorphism $Q H_{\mathfrak{b}}(M ; \Lambda ; \mathfrak{y}) \cong \operatorname{Jac}(\mathfrak{P O} \mathfrak{b} ; \mathfrak{y})$. Hence the lemma.

Since the image of the map $\mathfrak{q}_{*}^{T, \mathbf{b}}$ is a finite dimensional vector space over $\Lambda$, we can apply the argument of Subsection 8.1 to find a standard basis $\mathfrak{q}_{*}^{T, \mathbf{b}}\left(e_{1}\right), \ldots, \mathfrak{q}_{*}^{T, \mathbf{b}}\left(e_{k}\right)$ of the image of $\mathfrak{q}_{*}^{T, \mathbf{b}}$. Then we have

$$
\begin{aligned}
\mathfrak{v}_{q}\left(\mathfrak{q}_{*}^{T, \mathbf{b}}\left(\sum_{i=1}^{k} x_{i} e_{i}\right)\right) & =\max \left\{\mathfrak{v}_{q}\left(x_{i} \mathfrak{q}_{*}^{T, \mathbf{b}}\left(e_{i}\right)\right) \mid i=1, \ldots, k\right\} \\
& \geq \max \left\{\mathfrak{v}_{q}\left(x_{i}\right) \mid i=1, \ldots, k\right\}-C_{1}
\end{aligned}
$$

where $C_{1}$ is a constant independent of $x_{i}$.

Now we are ready to complete the proof of Theorem 25.1. Let $\widetilde{\psi}_{H} \in \widetilde{\operatorname{Ham}}(M, \omega)$. By Theorem 15.1 we have

$$
\rho^{\mathfrak{b}}\left(\widetilde{\psi}_{H}^{n} ; e_{\mathfrak{y}}\right)=-\inf \left\{\rho^{\mathfrak{b}}\left(\widetilde{\psi}_{H}^{-n} ; b\right) \mid \Pi\left(e_{\mathfrak{y}}, b\right) \neq 0\right\} .
$$

Let us estimate the right hand side of (25.10). Suppose $\Pi\left(e_{\mathfrak{y}}, b\right) \neq 0$. We put

$$
e_{\mathfrak{y}} \cup^{\mathfrak{b}} b=\sum_{i=1}^{k} x_{i} e_{i}, \quad x_{i} \in \Lambda .
$$

Since $\Pi\left(e_{\mathfrak{y}}, b\right) \neq 0$, Sublemma 16.6 implies $\mathfrak{v}_{q}\left(e_{\mathfrak{y}} \cup^{\mathfrak{b}} b\right) \geq 0$. Therefore

$$
\max \left\{\mathfrak{v}_{q}\left(x_{i}\right) \mid i=1, \ldots, k\right\} \geq C_{2},
$$

where $C_{2}=-\max \left\{\mathfrak{v}_{q}\left(e_{i}\right) \mid i=1, \ldots, k\right\}$.

By the triangle inequality,

$$
\rho^{\mathfrak{b}}\left(\widetilde{\psi}_{H}^{-n} ; b\right) \geq \rho^{\mathfrak{b}}\left(\widetilde{\psi}_{H}^{-n} ; \sum_{i=1}^{k} x_{i} e_{i}\right)-\rho^{\mathfrak{b}}\left(\underline{0} ; e_{\mathfrak{y}}\right) .
$$

Here $\underline{0}$ is the identity map $=\widetilde{\psi}_{0}$. It follows from Proposition 25.4 that the right hand side is not smaller than

$$
n \int_{0}^{1} \min \left(-\left.\widetilde{H}_{t}\right|_{L(\mathbf{u})}\right) d t+\mathfrak{v}_{q}\left(\mathfrak{q}_{*}^{T, \mathfrak{b}}\left(\sum_{i=1}^{k} x_{i} e_{i}\right)\right)-\rho^{\mathfrak{b}}\left(\underline{0} ; e_{\mathfrak{y}}\right) .
$$


Here $\tilde{H}$ is as in (15.5). By (25.9), this is not smaller than

$$
n \int_{0}^{1} \min \left(\left.H_{t}\right|_{L(\mathbf{u})}\right) d t+\max \left\{\mathfrak{v}_{q}\left(x_{i}\right) \mid i=1, \ldots, k\right\}-C_{1}-\rho^{\mathfrak{b}}\left(\underline{0} ; e_{\mathfrak{y}}\right) .
$$

Combining the above, we obtain

$$
\begin{aligned}
\rho^{\mathfrak{b}}\left(\widetilde{\psi}_{H}^{-n} ; b\right) & \geq \int_{0}^{1} \min \left(\left.H_{t}\right|_{L(\mathbf{u})}\right) d t+C_{2}-C_{1}-\rho^{\mathfrak{b}}(\underline{0} ; e) \\
& =-n \int_{0}^{1} \max \left(-\left.H_{t}\right|_{L(\mathbf{u})}\right) d t+C_{2}-C_{1}-\rho^{\mathfrak{b}}(\underline{0} ; e) \\
& =-n E^{-}(H ; L(\mathbf{u}))+C_{2}-C_{1}-\rho^{\mathfrak{b}}(\underline{0} ; e) .
\end{aligned}
$$

Therefore using (25.10), we obtain

$$
-\frac{\rho^{\mathfrak{b}}\left(\widetilde{\psi}_{H}^{n} ; e_{\mathfrak{y}}\right)}{n} \geq-E^{-}(H ; L(\mathbf{u}))-\frac{C_{3}}{n},
$$

where $C_{3}$ is independent of $n$. By letting $n \rightarrow \infty$, we have finished the proof of Theorem 25.1.

25.4. A remark. In Theorems 21.1 and 25.1, we use Lagrangian Floer theory to estimate the spectral invariant in terms of the values of the Hamiltonian on the Lagrangian submanifolds. One can use a variant of this technique to obtain an estimate of spectral invariant using various other invariant appearing in symplectic topology.

We introduce the invariant $E^{+}(H ; \mathcal{L}(Y))$ defined by

$$
E^{+}(H ; \mathcal{L}(Y)):=\sup \left\{\int_{0}^{1} H(t, \gamma(t)) d t \mid \gamma \in \mathcal{L}(Y)\right\} .
$$

This is an invariant stronger than $E^{+}(H ; Y)$ in that $E^{+}(H ; \mathcal{L}(Y)) \leq E^{+}(H ; Y)$ and more directly related to the loop space $\mathcal{L}(Y)$ of $Y$. Using this invariant, we can improve the statement of Proposition 18.9 to the following

$$
\rho^{\mathfrak{b}}(H ; a) \geq-E^{+}(H ; \mathcal{L}(Y))+\rho_{L}^{\mathbf{b}}(a) .
$$

This formula suggests that we may use symplectic homology $S H(V)([\overrightarrow{\mathrm{FH}}])$ of a subset $V \subset M$ and the Viterbo functoriality (Viterbo [Vi2, Abouzaid-Seidel [ASe] in place of Lagrangian Floer theory in certain cases, for example, in the case where $V$ is a Darboux-Weinstein neighborhood of a Lagrangian submanifold $L$. For the case where the Floer homology $H F(L)$ is isomorphic to $H(L)$ (such as the case $L$ is exact), the symplectic homology $S H(V)$ is related to the homology of the loop space of $L$ (Salamon-Weber [SW], Abbondandolo-Schwartz ASc1, which is in turn closely related to the Hochschild cohomology of $H(L)$. (See also [Fu2]).) Thus in that case the method using symplectic homology becomes equivalent to those using Hochschild cohomology that we have established in this section.

Eliashberg-Polterovich EIP use symplectic homology to estimate the spectral invariant in the case of Lagrangian tori in $S^{2} \times S^{2}$. Through the above mentioned equivalence, their argument is related to ours given in Section 23. 


\section{Part 6. Appendix}

\section{6. $\mathcal{P}_{\left(H_{\chi}, J_{\chi}\right), *}^{\mathfrak{b}}$ IS AN ISOMORPHISM}

In Section 6 we introduced the Piunikhin map $\mathcal{P}_{\left(H_{\chi}, J_{\chi}\right), *}^{\mathfrak{b}}$ with bulk deformation. In this section we complete the proof of Theorem 6.9.

Theorem 26.1. The Piunikhin map with bulk deformation

$$
\mathcal{P}_{\left(H_{\chi}, J_{\chi}\right), *}^{\mathfrak{b}}: H_{*}\left(M ; \Lambda^{\downarrow}\right) \rightarrow H F_{*}^{\mathfrak{b}}\left(M, H, J ; \Lambda^{\downarrow}\right)
$$

is a $\Lambda^{\downarrow}$-module isomorphism.

Proof. We first construct another map

$$
\mathcal{Q}_{\left(H_{\tilde{\chi}}, J_{\tilde{\chi}}\right), *}^{\mathfrak{b}}: H F_{*}^{\mathfrak{b}}\left(M, H ; \Lambda^{\downarrow}\right) \rightarrow H_{*}\left(M ; \Lambda^{\downarrow}\right)
$$

in the direction opposite to $\mathcal{P}_{\left(H_{\chi}, J_{\chi}\right), *}^{\mathfrak{b}}$. (Here and hereafter we sometimes omit $J$ in the notation of $H F_{*}^{\mathfrak{b}}\left(M, H, J ; \Lambda^{\downarrow}\right)$.) This will be carried out by constructing the associated chain map

$$
C F\left(M, H ; \Lambda^{\downarrow}\right) \rightarrow \Omega(M) \widehat{\otimes} \Lambda^{\downarrow} .
$$

Let $\chi \in \mathcal{K}$ be as in Definition 3.13 and $[\gamma, w] \in \operatorname{Crit}\left(\mathcal{A}_{H}\right)$. For the construction of this chain map, we need to consider the dual version of $\chi$. To distinguish the two different types of elongation functions, we recall that we denote

$$
\tilde{\chi}(\tau)=-\chi(1-\tau)
$$

for $\chi \in \mathcal{K}$. We also use $\left(H_{\chi}, J_{\chi}\right)$ defined in (3.13). (In this section $J=\left\{J_{t}\right\}$ is a $t \in S^{1}$ parametrized family of compatible almost complex structures.)

We consider the elongated family $\left(H_{\tilde{\chi}}, J_{\tilde{\chi}}\right)$ defined by:

$$
H_{\tilde{\chi}}(\tau, t, x)=\tilde{\chi}(\tau) H_{t}(x), \quad J_{\tilde{\chi}}(\tau, t)=J_{-\tilde{\chi}(\tau), t},
$$

where $J_{s, t}$ is as in (3.12).

Definition 26.2. We denote by $\stackrel{\circ}{\mathcal{M}}_{\ell}\left(H_{\tilde{\chi}}, J_{\tilde{\chi}} ;[\gamma, w], *\right)$ the set of all pairs $\left(u ; z_{1}^{+}, \ldots, z_{\ell}^{+}\right)$ of maps $u: \mathbb{R} \times S^{1} \rightarrow M$ and $z_{i}^{+} \in \mathbb{R} \times S^{1}$ which satisfy the following conditions:

(1) The map $u$ satisfies the equation:

$$
\frac{\partial u}{\partial \tau}+J_{\tilde{\chi}}\left(\frac{\partial u}{\partial t}-X_{H_{\tilde{\chi}}}(u)\right)=0 .
$$

(2) The energy

$$
E_{\left(H_{\tilde{\chi}}, J_{\tilde{\chi}}\right)}(u)=\frac{1}{2} \int\left(\left|\frac{\partial u}{\partial \tau}\right|_{J_{\tilde{\chi}}}^{2}+\left|\frac{\partial u}{\partial t}-X_{H_{\tilde{\chi}}}(u)\right|_{J_{\tilde{\chi}}}^{2}\right) d t d \tau
$$

is finite.

(3) The map $u$ satisfies the following asymptotic boundary condition.

$$
\lim _{\tau \rightarrow-\infty} u(\tau, t)=\gamma(t)
$$

(4) The homology class of the concatenation of $u$ and $w$ is homotopic to 0.

(5) $z_{i}^{+}$are all different from one another.

$$
\begin{aligned}
\left(u ; z_{1}^{+}, \ldots, z_{\ell}^{+}\right) & \mapsto\left(u\left(z_{1}^{+}\right), \ldots, u\left(z_{\ell}^{+}\right)\right) \text {defines an evaluation map } \\
\mathrm{ev} & =\left(\operatorname{ev}_{1} \ldots, \mathrm{ev}_{\ell}\right)=\stackrel{\circ}{\mathcal{M}}_{\ell}\left(H_{\tilde{\chi}}, J_{\tilde{\chi}} ;[\gamma, w], *\right) \rightarrow M^{\ell} .
\end{aligned}
$$


Lemma 26.3. (1) The moduli space $\stackrel{\circ}{\mathcal{M}}_{\ell}\left(H_{\tilde{\chi}}, J_{\tilde{\chi}} ;[\gamma, w], *\right)$ has a compactification $\left.\mathcal{M}_{\ell}\left(H_{\tilde{\chi}}, J_{\tilde{\chi}} ; \gamma, w\right], *\right)$ that is Hausdorff.

(2) The space $\mathcal{M}_{\ell}\left(H_{\tilde{\chi}}, J_{\tilde{\chi}} ; \gamma, w\right]$,*) has an orientable Kuranishi structure with corners.

(3) The normalized boundary of $\mathcal{M}_{\ell}\left(H_{\tilde{\chi}}, J_{\tilde{\chi}} ;[\gamma, w]\right.$,*) is described by

$$
\begin{aligned}
& \partial \mathcal{M}_{\ell}\left(H_{\tilde{\chi}}, J_{\tilde{\chi}} ;[\gamma, w], *\right) \\
& =\bigcup \mathcal{M}\left(H, J ;[\gamma, w],\left[\gamma^{\prime}, w^{\prime}\right]\right) \times \mathcal{M}\left(H_{\tilde{\chi}}, J_{\tilde{\chi}} ;\left[\gamma^{\prime}, w^{\prime}\right], *\right),
\end{aligned}
$$

where the union is taken over all $\left[\gamma^{\prime}, w^{\prime}\right] \in \operatorname{Crit}\left(\mathcal{A}_{H}\right)$ and $\left(\mathbb{L}_{1}, \mathbb{L}_{2}\right) \in \operatorname{Shuff}(\ell)$.

(4) Let $\mu_{H}: \operatorname{Crit}\left(\mathcal{A}_{H}\right) \rightarrow \mathbb{Z}$, be the Conley-Zehnder index. Then the (virtual) dimension satisfies the following equality:

$$
\operatorname{dim} \mathcal{M}_{\ell}\left(H_{\tilde{\chi}}, J_{\tilde{\chi}} ;[\gamma, w], *\right)=n-\mu_{H}([\gamma, w])+2 \ell .
$$

(5) We can define a system of orientations on $\mathcal{M}_{\ell}\left(H_{\tilde{\chi}}, J_{\tilde{\chi}} ;[\gamma, w]\right.$,*) so that the isomorphism (3) above is compatible with this orientation.

(6) ev extends to a strongly continuous smooth map $\mathcal{M}_{\ell}\left(H_{\tilde{\chi}}, J_{\tilde{\chi}} ;[\gamma, w], *\right) \rightarrow$ $M^{\ell}$, which we denote also by ev. It is compatible with (3).

(7) The map ev $+\infty$ which sends $\left(u ; z_{1}^{+}, \ldots, z_{\ell}^{+}\right)$to $\lim _{\tau \rightarrow+\infty} u(\tau, t)$ extends to a weakly submersive map $\mathcal{M}_{\ell}\left(H_{\tilde{\chi}}, J_{\tilde{\chi}} ;[\gamma, w], *\right) \rightarrow M$, which we also denote by $\mathrm{ev}_{+\infty}$. It is compatible with (3).

The proof of Lemma 26.3 is the same as that of Proposition 3.6, which is detailed in FOOO10, Parts 4 and 5], and so omitted.

We take a CF-perturbation $\widehat{\mathfrak{S}}$ on the moduli space $\mathcal{M}_{\ell}\left(H_{\tilde{\chi}}, J_{\tilde{\chi}} ;[\gamma, w], *\right)$ which is compatible with $(3)$ and such that $\mathrm{ev}_{+\infty}$ is strongly submersive with respect to $\widehat{\mathfrak{S}}$.

Let $h_{1}, \ldots, h_{\ell} \in \Omega(M)$. We define $\mathfrak{n}_{(H, J), *}([\gamma, w])\left(h_{1}, \ldots, h_{\ell}\right) \in \Omega(M)$ by

$$
\mathfrak{n}_{(H, J), *}([\gamma, w])\left(h_{1}, \ldots, h_{\ell}\right)=\left(\mathrm{ev}_{+\infty}\right) !\left(\mathrm{ev}_{1}^{*} h_{1} \wedge \cdots \wedge \mathrm{ev}_{\ell}^{*} h_{\ell} ; \widehat{\mathfrak{S}^{\epsilon}}\right) .
$$

Here $\left(\mathrm{ev}_{+\infty}\right)$ ! is the integration along the fibers of the map $\mathrm{ev}_{+\infty}$ which is defined by using a CF-perturbation $\widehat{\mathfrak{S}}$ of the space $\mathcal{M}_{\ell}\left(H_{\tilde{\chi}}, J_{\tilde{\chi}} ;[\gamma, w], *\right)$. (See Definition 33.20, [FOOO11, Definition 7.78].)

Let $\mathfrak{b} \in H^{\text {even }}\left(M ; \Lambda_{0}^{\downarrow}\right)$. We split $\mathfrak{b}=\mathfrak{b}_{0}+\mathfrak{b}_{2}+\mathfrak{b}_{+}$as in (5.5). We take closed forms which represent $\mathfrak{b}_{0}, \mathfrak{b}_{2}, \mathfrak{b}_{+}$and write them by the same symbols.

Definition 26.4. Let $\llbracket \gamma, w \rrbracket \in \widehat{\operatorname{Per}}(H)$. We define

$$
\begin{aligned}
\mathcal{Q}_{\left(H_{\tilde{\chi}}, J_{\tilde{\chi}}\right)}^{\mathfrak{b}}(\llbracket \gamma, w \rrbracket)= & \sum_{\alpha \in \pi_{2}(M)} \sum_{\ell=0}^{\infty} \frac{\exp \left(\int(\alpha \# w)^{*} \mathfrak{b}_{2}\right)}{\ell !} \\
& q^{-\int w^{*} \omega+\int(\alpha \# w)^{*} \omega_{\mathfrak{n}}(H, J), *}([\gamma, \alpha \# w])(\underbrace{\mathfrak{b}_{+}, \ldots, \mathfrak{b}_{+}}_{\ell}) .
\end{aligned}
$$

Note $\left\{[\alpha \# w] \mid \alpha \in \pi_{2}(M)\right\}=\pi_{2}(\gamma)$. We can use it to show that the right hand side is independent of the choice of $[\gamma, w] \in \operatorname{Crit}\left(\mathcal{A}_{H}\right)$.

We can prove that the sum in (26.7) converges in $q$-adic topology in the same way as in Lemma 6.5. We have thus defined (26.1). Then

$$
\partial \circ \mathcal{Q}_{\left(H_{\tilde{\chi}}, J_{\tilde{\chi}}\right)}^{\mathfrak{b}}=\mathcal{Q}_{\left(H_{\tilde{\chi}}, J_{\tilde{\chi}}\right)}^{\mathfrak{b}} \circ \partial_{(H, J)}^{\mathfrak{b}}
$$


is a consequence of Lemma 26.3 (3), Stokes' theorem (Theorem 34.8, FOOO11, Theorem 8.11]) and composition formula (Theorem 35.7 [FOOO11, Theorem 10.20]). (Here $\partial$ is defined by (3.20).)

Proposition 26.5. $\mathcal{Q}_{\left(H_{\tilde{\chi}}, J_{\tilde{\chi}}\right)}^{\mathfrak{b}} \circ \mathcal{P}_{\left(H_{\chi}, J_{\chi}\right)}^{\mathfrak{b}}$ is chain homotopic to the identity.

Proof. For $S \in[1, \infty)$ define $H_{\chi}^{S}$ as follows:

$$
H_{\chi}^{S}(\tau, t, x)= \begin{cases}\chi(\tau+S+1) H_{t}(x) & S \geq 1, \tau \leq 0 \\ \tilde{\chi}(\tau-S-1) H_{t}(x) & S \geq 1, \tau \geq 0 .\end{cases}
$$

We extend it to $S \in[0,1]$ by

$$
H_{\chi}^{S}(\tau, t, x)=S H_{\chi}^{1}(\tau, t, x) .
$$

The function $H_{\chi}^{S}$ may not be smooth on $S$ at $S=1, \tau \in[-10,10]$. We modify it on a neighborhood of $S=1, \tau \in[-10,10]$ so that it becomes smooth and denote it by the same symbol. We define $(S, \tau, t) \in[0, \infty) \times \mathbb{R} \times[0,1]$ parametrized family of compatible almost complex structures $J_{\chi}^{S}$ as follows. For $S \in[1, \infty)$ we put

$$
J_{\chi}^{S}(\tau, t)= \begin{cases}J_{\chi(\tau+S+1), t} & S \geq 1, \tau \leq 0 \\ J_{\tilde{\chi}(\tau-S-1), t} & S \geq 1, \tau \geq 0\end{cases}
$$

We extend it to $S \in[0,1]$ so that the following is satisfied.

$$
J_{\chi}^{S}(\tau, t)= \begin{cases}J_{0} & \tau \leq-10 \\ J_{0} & \tau \geq+10 \\ J_{0} & S=0, \\ J_{0} & t \text { is in a neighborhood of }[1] .\end{cases}
$$

Definition 26.6. Let $C \in \pi_{2}(M)$. For each $0 \leq S<\infty$, we denote by $\stackrel{\circ}{\mathcal{M}}_{\ell}\left(H_{\chi}^{S}, J_{\chi}^{S} ; *, * ; C\right)$ the set of all pairs $\left(u ; z_{1}^{+}, \ldots, z_{\ell}^{+}\right)$of maps $u: \mathbb{R} \times S^{1} \rightarrow M, z_{i}^{+} \in \mathbb{R} \times S^{1}$ which satisfy the following conditions:

(1) The map $u$ satisfies the equation:

$$
\frac{\partial u}{\partial \tau}+J_{\chi}^{S}\left(\frac{\partial u}{\partial t}-X_{H_{\chi}^{S}}(u)\right)=0
$$

(2) The energy

$$
\frac{1}{2} \int\left(\left|\frac{\partial u}{\partial \tau}\right|_{J \underset{\chi}{S}}^{2}+\left|\frac{\partial u}{\partial t}-X_{H_{\chi}^{S}}(u)\right|_{J_{\chi}^{S}}^{2}\right) d t d \tau
$$

is finite.

(3) The homotopy class of $u$ is $C$.

(4) $z_{i}^{+}$are all distinct each other.

We note that (26.13) and the finiteness of energy imply that there exist $p_{1}, p_{2} \in$ $M$ such that

$$
\lim _{\tau \rightarrow-\infty} u(\tau, t)=p_{1}, \quad \lim _{\tau \rightarrow+\infty} u(\tau, t)=p_{2} .
$$

Therefore the homology class of $u$ is well-defined. We define the evaluation map

$$
\left(\mathrm{ev}_{-\infty}, \mathrm{ev}_{+\infty}\right): \stackrel{\circ}{\mathcal{M}}_{\ell}\left(H_{\chi}^{S}, J_{\chi}^{S} ; *, * ; C\right) \rightarrow M^{2}
$$

by $\left(\mathrm{ev}_{-\infty}, \mathrm{ev}_{+\infty}\right)(u)=\left(p_{1}, p_{2}\right)$, where $p_{1}, p_{2}$ are as in (26.14). 
We put

$$
\stackrel{\circ}{\mathcal{M}}_{\ell}\left(\text { para } ; H_{\chi}, J_{\chi} ; *, * ; C\right)=\bigcup_{S \geq 0}\{S\} \times \stackrel{\circ}{\mathcal{M}}_{\ell}\left(H_{\chi}^{S}, J_{\chi}^{S} ; *, * ; C\right),
$$

where $\mathrm{ev}, \mathrm{ev}_{-\infty}$ and $\mathrm{ev}_{+\infty}$ are defined on it.

To describe the boundary of the compactification of $\stackrel{\circ}{\mathcal{M}}_{\ell}\left(\right.$ para; $\left.H_{\chi}, J_{\chi} ; *, * ; C\right)$ we define another moduli space.

Definition 26.7. We denote by $\widehat{\dot{\mathcal{M}}_{\ell}}\left(H=0, J_{0} ; *, * ; C\right)$ the set of all $\left(u ; z_{1}^{+}, \ldots, z_{\ell}^{+}\right)$ that satisfy (1),..,(4) of Definition 26.6 with $S=0$.

Note that $H$ actually does not appear in (1),..,(4) of Definition 26.6 in case $S=0$. There exists an $\mathbb{R} \times S^{1}$ action on $\widehat{\mathcal{M}_{\ell}}\left(H=0, J_{0} ; *, * ; C\right)$ that is induced by the $\mathbb{R} \times S^{1}$ action on $\mathbb{R} \times S^{1}$, the source of the map $u$. In fact, the equation 26.13) is preserved by $\mathbb{R} \times S^{1}$ action in case $S=0$. See [FOOO10, Definition 28.4] for the definition of $S^{1}$ equivariant Kuranishi structure and [FOOO10, Section 30] for its construction.

We define the evaluation maps

$$
\mathrm{ev}=\left(\mathrm{ev}_{1}, \ldots, \mathrm{ev}_{\ell}\right): \widehat{\stackrel{\circ}{M}}_{\ell}\left(H=0, J_{0} ; *, * ; C\right) \rightarrow M^{\ell}
$$

and

$$
\left(\mathrm{ev}_{+\infty}, \mathrm{ev}_{-\infty}\right): \widehat{\dot{\mathcal{M}}}_{\ell}\left(H=0, J_{0} ; *, * ; C\right) \rightarrow M^{2}
$$

in an obvious way. We put

$$
\begin{aligned}
& \stackrel{\circ}{\mathcal{M}}_{\ell}\left(H=0, J_{0} ; *, * ; C\right)=\widehat{\stackrel{\mathcal{M}}{\ell}}_{\ell}\left(H=0, J_{0} ; *, * ; C\right) / \mathbb{R}, \\
& \widehat{\stackrel{\circ}{\mathcal{M}}}_{\ell}\left(H=0, J_{0} ; *, * ; C\right)=\widehat{\circ}_{\mathcal{M}_{\ell}}\left(H=0, J_{0} ; *, * ; C\right) / S^{1} \text {, } \\
& \stackrel{\circ}{\mathcal{M}}_{\ell}\left(H=0, J_{0} ; *, * ; C\right)=\widehat{\grave{\mathcal{M}}}_{\ell}\left(H=0, J_{0} ; *, * ; C\right) /\left(\mathbb{R} \times S^{1}\right) \text {. }
\end{aligned}
$$

Then $\widehat{\grave{M}}_{\ell}\left(H=0, J_{0} ; *, * ; C\right), \stackrel{\circ}{\mathcal{M}}_{\ell}\left(H=0, J_{0} ; *, * ; C\right), \widehat{\stackrel{\circ}{\mathcal{M}}}_{\ell}\left(H=0, J_{0} ; *, * ; C\right)$ and $\stackrel{\circ}{\mathcal{M}}_{\ell}\left(H=0, J_{0} ; *, * ; C\right)$ can be compactified. We denote the corresponding compactifications by $\widehat{\mathcal{M}}_{\ell}\left(H=0, J_{0} ; *, * ; C\right), \mathcal{M}_{\ell}\left(H=0, J_{0} ; *, * ; C\right), \widehat{\bar{M}}_{\ell}\left(H=0, J_{0} ; *, * ; C\right)$ and $\overline{\mathcal{M}}_{\ell}\left(H=0, J_{0} ; *, * ; C\right)$, respectively. The compactifications are obtained as follows. Fix an identification of $\mathbb{R} \times S^{1}$ with $\mathbb{C} P^{1} \backslash\{N, S\}$, where $N, S$ are the limits as $\tau \rightarrow \pm \infty$, respectively. For each $\left(u ; z_{1}^{+}, \ldots, z_{\ell}^{+}\right) \in \widehat{\dot{\mathcal{M}}_{\ell}}\left(H=0, J_{0} ; *, * ; C\right)$, we regard $u$ as a map from $\mathbb{C} P^{1}$ and consider its graph in $\mathbb{C} P^{1} \times M$. Then we identify the space $\widehat{\stackrel{\circ}{\mathcal{M}}}_{\ell}\left(H=0, J_{0} ; *, * ; C\right)$ with the space $\stackrel{\circ}{\mathcal{N}}_{\ell}\left(H=0, J_{0} ; *, * ; C\right)$ of their graphs. Take its stable map compactification $\mathcal{N}_{\ell}\left(H=0, J_{0} ; *, * ; C\right)$, which is identified with $\widehat{\mathcal{M}}_{\ell}\left(H=0, J_{0} ; *, * ; C\right)$. (The component, which has degree 1 to $\mathbb{C} P^{1}$-factor is the component with a parametrized solution of (26.3).) The group $\mathbb{R} \times S^{1}$ acts on the first factor of $\mathbb{C} P^{1} \times M$ and induces an action on $\mathcal{N}_{\ell}\left(H=0, J_{0} ; *, * ; C\right)$. By taking the quotient of $\mathcal{N}_{\ell}\left(H=0, J_{0} ; *, * ; C\right)$ by $\mathbb{R}, S^{1}, \mathbb{R} \times S^{1}$, we obtain the compactification $\mathcal{M}_{\ell}\left(H=0, J_{0} ; *, * ; C\right), \widehat{\overline{\mathcal{M}}}_{\ell}\left(H=0, J_{0} ; *, * ; C\right)$ and $\overline{\mathcal{M}}_{\ell}\left(H=0, J_{0} ; *, * ; C\right)$, 
respectively. Each of them carries a Kuranishi structure and evaluation maps that extend to its compactification. We note that $\overline{\mathcal{M}}_{\ell}\left(H=0, J_{0} ; *, * ; C\right)$ is identified with $\mathcal{M}_{\ell+2}^{\mathrm{cl}}(C)$ which is introduced in Section 5 .

Lemma 26.8. (1) The moduli space $\mathcal{M}_{\ell}\left(\right.$ para $\left.; H_{\chi}, J_{\chi} ; *, * ; C\right)$ has a compactification $\mathcal{M}_{\ell}\left(\right.$ para $\left.; H_{\chi}, J_{\chi} ; *, * ; C\right)$ that is Hausdorff.

(2) The space $\mathcal{M}_{\ell}\left(\right.$ para; $\left.H_{\chi}, J_{\chi} ; *, * ; C\right)$ has an orientable Kuranishi structure with corners.

(3) The normalized boundary of $\mathcal{M}_{\ell}\left(\right.$ para; $\left.H_{\chi}, J_{\chi} ; *, * ; C\right)$ is described by the union of following four types of direct or fiber products:

(i)

$$
\mathcal{M}_{\# \mathbb{L}_{1}}\left(H_{\chi}, J_{\chi} ; *,[\gamma, w]\right) \times \mathcal{M}_{\# \mathbb{L}_{2}}\left(H_{\widetilde{\chi}}, J_{\widetilde{\chi}} ;\left[\gamma, w^{\prime}\right], *\right)
$$

where the union is taken over all $[\gamma, w] \in \operatorname{Crit}(H)$, and $\left(\mathbb{L}_{1}, \mathbb{L}_{2}\right) \in$ $\operatorname{Shuff}(\ell)$. Here the bounding disc $w^{\prime}$ is defined by $[C \# w]=\left[w^{\prime}\right]$. (Figure 24)

(ii)

$$
\mathcal{M}_{\# \mathbb{L}_{1}}\left(H=0, J_{0} ; *, * ; C_{1}\right)_{\mathrm{ev}_{+\infty}} \times_{\mathrm{ev}_{-\infty}} \mathcal{M}_{\# \mathbb{L}_{2}}\left(\text { para } ; H_{\chi}, J_{\chi} ; *, * ; C_{2}\right)
$$

where the union is taken over all $C_{1}, C_{2} \in \pi_{2}(M)$ and $\left(\mathbb{L}_{1}, \mathbb{L}_{2}\right) \in$ $\operatorname{Shuff}(\ell)$ such that $C_{1}+C_{2}=C$. The fiber product is taken over $M$. (iii) (Figure 25) (See Definition 32.5 for the definition of fiber product.)

$\mathcal{M}_{\# \mathbb{L}_{1}}\left(\text { para } ; H_{\chi}, J_{\chi} ; *, * ; C_{1}\right)_{\mathrm{ev}_{+\infty}} \times_{\mathrm{ev}_{-\infty}} \mathcal{M}_{\# \mathbb{L}_{2}}\left(H=0, J_{0} ; *, * ; C_{2}\right)$

where the union is taken over all $C_{1}, C_{2} \in \pi_{2}(M)$ and $\left(\mathbb{L}_{1}, \mathbb{L}_{2}\right) \in$ $\operatorname{Shuff}(\ell)$ such that $C_{1}+C_{2}=C$. The fiber product is taken over $M$. (Figure 26)

(iv) And

$$
\widehat{\mathcal{M}}_{\# \mathbb{L}}\left(H=0, J_{0} ; *, * ; C\right) .
$$

(4) The (virtual) dimension is given by

$\operatorname{dim} \mathcal{M}_{\ell}\left(\right.$ para $\left.; H^{\chi}, J^{\chi} ; *, * ; C\right)=2 c_{1}(M) \cap C+2 n+2 \ell-1$.

(5) We can define a system of orientations on $\mathcal{M}_{\ell}\left(\right.$ para; $\left.H_{\chi}, J_{\chi} ; *, * ; C\right)$ that is compatible with the isomorphism (3) above.

(6) The map ev (26.16) extends to a weakly submersive map $\mathcal{M}_{\ell}\left(\right.$ para $\left.; H_{\chi}, J_{\chi} ; *, * ; C\right) \rightarrow$ $M^{\ell}$, which we denote also by ev. It is compatible with (3).

(7) The map $\left(\mathrm{ev}_{-\infty}, \mathrm{ev}_{+\infty}\right)$ (26.17) also extends to a weakly submersive map

$$
\mathcal{M}_{\ell}\left(\text { para } ; H_{\chi}, J_{\chi} ; *, * ; C\right) \rightarrow M^{2},
$$

which we denote by $\left(\mathrm{ev}_{-\infty}, \mathrm{ev}_{+\infty}\right)$. It is compatible with (3).

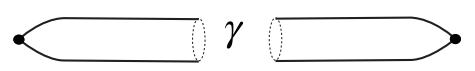

Figure 24. An element of (26.18) 


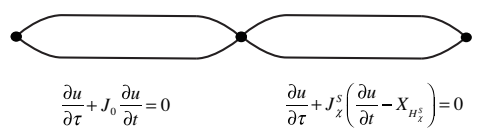

Figure 25. An element of (26.19)

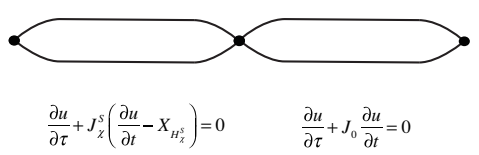

Figure 26. An element of (26.20)

Proof. The proof is similar to the proofs of Propositions 6.11, 3.6 (See [FOOO10, Parts 4 and 5]). So we only mention the way how the four different types of boundary components appear. In fact, (26.18) appears when $S \rightarrow \infty$, 26.21) appears when $S=0$. (26.19), 26.20) appear when $S$ is bounded and is away from 0 . (26.19) is the case there is some bubble which slides to $\tau \rightarrow-\infty$ and (26.20) is the case there is some bubble which slides to $\tau \rightarrow+\infty$.

We now use a system of CF-perturbations $\widehat{\mathfrak{S}}=\left\{\widehat{\mathfrak{S}^{\epsilon}}\right\}$ on $\mathcal{M}_{\ell}\left(\right.$ para $\left.; H_{\chi}, J_{\chi} ; *, * ; C\right)$ such that it is compatible with the description of its boundary given in Lemma 26.8 (3) and that $\mathrm{ev}_{+\infty}$ is strongly submersive with respect to $\widehat{\mathfrak{S}}$, in the sense of Definition 33.9. We need some particular choice thereof at some of the factors of the boundary component.

We observe that there exist maps

$$
\widehat{\mathcal{M}}_{\ell}\left(H=0, J_{0} ; *, * ; C\right) \rightarrow \overline{\mathcal{M}}_{\ell}\left(H=0, J_{0} ; *, * ; C\right)
$$

and

$$
\mathcal{M}_{\ell}\left(H=0, J_{0} ; *, * ; C\right) \rightarrow \overline{\mathcal{M}}_{\ell}\left(H=0, J_{0} ; *, * ; C\right) .
$$

Various evaluation maps factor through them. We take our CF-perturbation obtained by the pull-back with respect to the maps (26.24), (26.23) on the first factor of (26.19), the second factor of (26.20) and on (26.21).

Using the system of CF-perturbations as above, we define the map

$$
\mathfrak{H}_{H, J ; C}^{\mathfrak{b}}: \Omega(M) \widehat{\otimes} \Lambda^{\downarrow} \rightarrow \Omega(M) \widehat{\otimes} \Lambda^{\downarrow}
$$

by putting

$$
\mathfrak{H}_{H, J}^{\mathfrak{b}}(h)=\sum_{\ell=0}^{\infty} \sum_{C} \frac{\exp \left(C \cap \mathfrak{b}_{2}\right)}{\ell !} q^{-C \cap \omega} \mathrm{ev}_{+\infty} !\left(\mathrm{ev}_{-\infty}^{*} h \wedge \mathrm{ev}^{*}(\underbrace{\mathfrak{b}_{+}, \ldots, \mathfrak{b}_{+}}_{\ell}) ; \widehat{\mathfrak{S}^{\epsilon}}\right) .
$$

Here each summand of the right hand side is given by the correspondence by the moduli space $\mathcal{M}_{\ell}\left(\right.$ para $\left.; H_{\chi}, J_{\chi} ; *, * ; C\right)$. (See Definition 35.4, [FOOO11, Definition 7.85].)

\section{Lemma 26.9.}

$$
\partial \circ \mathfrak{H}_{H, J}^{\mathfrak{b}}+\mathfrak{H}_{H, J}^{\mathfrak{b}} \circ \partial=\mathcal{Q}_{\left(H_{\tilde{\chi}}, J_{\tilde{\chi}}\right)}^{\mathfrak{b}} \circ \mathcal{P}_{\left(H_{\chi}, J_{\chi}\right)}^{\mathfrak{b}}-i d .
$$


Proof. The proof is based on Lemma 26.8 (3) and Stokes' formula (Theorem 34.8 [FOOO4, Lemma 12.13], FOOO11, Corollary 8.13]) and the composition formula (Theorem 35.7 [FOOO4, Lemma 12.15], FOOO11, Theorem 10.20]). We note that (26.18) corresponds to the composition $\mathcal{Q}_{\left(H_{\tilde{\chi}}, J_{\tilde{\chi}}\right)}^{\mathfrak{b}} \circ \mathcal{P}_{\left(H_{\chi}, J_{\chi}\right)}^{\mathfrak{b}}$. Using the compatibility of the CF-perturbation and evaluation map to (26.24) it is easy to see that the contribution of (26.19) and (26.20) vanishes.

By the same reason, the contribution of (26.21) vanishes by the the compatibility with (26.23) $)$, except the case $\ell=0$ and $C=0$. In the latter cases the moduli space is identified with $M$ and $\mathrm{ev}_{ \pm \infty}$ with the identity map. Therefore the contribution induces the identity map : $\Omega(M) \rightarrow \Omega(M)$. This finishes the proof of Lemma 26.9 .

Therefore the proof of Proposition 26.5 is now complete.

Similarly as in Proposition 26.5 we can prove that $\mathcal{P}_{\left(H_{\chi}, J_{\chi}\right)}^{\mathfrak{b}} \circ \mathcal{Q}_{\left(H_{\tilde{\chi}}, J_{\tilde{\chi}}\right)}^{\mathfrak{b}}$ is chain homotopic to the identity. Hence the proof of Theorem 26.1 is now complete.

We now complete the proof of Theorem 7.8 (3). It remains to prove the following:

Proposition 26.10. For each $a \in Q H^{\mathfrak{b}}(M)$,

$$
\rho^{\mathfrak{b}}(\underline{0} ; a) \geq \mathfrak{v}_{q}(a)\left(=-\mathfrak{v}_{T}(a)\right) .
$$

Proof.

Lemma 26.11. If $\mathcal{M}_{\ell}\left(H_{\tilde{\chi}}, J_{\tilde{\chi}} ;[\gamma, w], *\right)$ is nonempty, then

$$
\mathcal{A}_{H}([\gamma, w]) \geq-E^{+}(H) \text {. }
$$

The proof is similar to the proof of Lemma 9.3 and so omitted.

\section{Corollary 26.12.}

$$
\mathcal{Q}_{\left(H_{\tilde{\chi}}, J_{\tilde{\chi}}\right)}^{\mathfrak{b}}\left(F^{\lambda} C F\left(M, H, J ; \Lambda^{\downarrow}\right)\right) \subseteq q^{\lambda+E^{+}(H)} \Omega(M) \widehat{\otimes} \Lambda^{\downarrow} .
$$

Proof. Let $x \in F^{\lambda} C F\left(M, H, J ; \Lambda^{\downarrow}\right)$. We choose $\left[\gamma, w_{\gamma}\right] \in \operatorname{Crit}\left(\mathcal{A}_{H}\right)$ for each of $\gamma \in \operatorname{Per}(H)$ and put

$$
x=\sum_{\gamma \in \operatorname{Per}(H)} x_{\gamma} \llbracket \gamma, w_{\gamma} \rrbracket,
$$

with

$$
\mathfrak{v}_{q}\left(x_{\gamma}\right)+\mathcal{A}_{H}\left(\llbracket \gamma, w_{\gamma} \rrbracket\right) \leq \lambda .
$$

By (26.7) we have

$$
\lambda_{q}\left(\mathcal{Q}_{\left(H_{\tilde{\chi}}, J_{\tilde{\chi}}\right)}^{\mathfrak{b}}(x)\right) \leq \max _{\left[\gamma, w^{\prime}\right]}\left(-w_{\gamma} \cap \omega+w^{\prime} \cap \omega+\mathfrak{v}_{q}\left(x_{\gamma}\right)\right),
$$

where the maximum in the right hand side is taken over all $\left[\gamma, w^{\prime}\right] \in \operatorname{Crit}\left(\mathcal{A}_{H}\right)$ such that $\mathcal{M}_{\ell}\left(H, J ;\left[\gamma, w^{\prime}\right], *\right)$ is nonempty.

We note that

$$
-w_{\gamma} \cap \omega+w^{\prime} \cap \omega=-\mathcal{A}_{H}\left(\llbracket \gamma, w^{\prime} \rrbracket\right)+\mathcal{A}_{H}\left(\llbracket \gamma, w_{\gamma} \rrbracket\right) .
$$

By (26.25), 26.26), (26.27) we obtain

$$
\lambda_{q}\left(\mathcal{Q}_{\left(H_{\tilde{\chi}}, J_{\tilde{\chi}}\right)}^{\mathfrak{b}}(x)\right) \leq \lambda+E^{+}(H)
$$

as required. 
We take a sequence of normalized Hamiltonians $H_{i}$ such that $\lim _{i \rightarrow \infty}\left\|H_{i}\right\|=0$ and $\widetilde{\psi}_{H_{i}}$ is nondegenerate. Let $x \in C F\left(M, H_{i}, J ; \Lambda^{\downarrow}\right)$ such that $\partial_{\left(H_{i}, J\right)}^{\mathfrak{b}} x=0$, $[x]=\mathcal{P}_{\left(\left(H_{i}\right)_{\chi}, J_{\chi}\right)}^{\mathfrak{b}}\left(a^{b}\right)$, and

$$
\left|\lambda_{H_{i}}(x)-\rho^{\mathfrak{b}}\left(H_{i} ; a\right)\right|<\epsilon .
$$

Then $\left[\mathcal{Q}_{\left(\left(H_{i}\right)_{\tilde{\chi}}, J_{\tilde{\chi})}\right.}^{\mathfrak{b}}(x)\right]=a^{b}$ and

$$
\lambda_{q}\left(\mathcal{Q}_{\left(\left(H_{i}\right)_{\tilde{\chi}}, J_{\tilde{\chi}}\right)}^{\mathfrak{b}}(x)\right) \leq \rho^{\mathfrak{b}}\left(H_{i} ; a\right)+\epsilon+E^{+}\left(H_{i}\right) .
$$

Since $\epsilon$ is arbitrary small and $\lim _{i \rightarrow \infty} E^{+}\left(H_{i}\right)=0$, we obtain the proposition.

Remark 26.13. Actually there is a problem of 'running-out of the given Kuranishi neighborhood' in the above proof when the energy level increases as mentioned in FOOO2, Section 7.2.3]. In order to handle the problem, we first work over the $\Lambda_{0}^{\downarrow}$ coefficients and perform the construction up to a pre-given finite energy level. Then we take an inductive limit as we let energy level go to infinity. The technical difficulty performing this construction is much simpler than that of [FOOO2, Section 7.2], since here we need to take an inductive limit of chain complex (or DGA). This is much simpler than the case of $A_{\infty}$ algebra in general discussed in FOOO2, Section 7.2]. So we omit the detail.

\section{Independence on the De Rham Representative of $\mathfrak{b}$.}

In this section we prove Theorem 7.7 (2). Let $H$ be a one periodic Hamiltonian on $M$ such that $\psi_{H}$ is nondegenerate. Let $\mathfrak{b}(0), \mathfrak{b}(1) \in \Omega(M) \widehat{\otimes} \Lambda^{\downarrow}$ such that $d \mathfrak{b}(0)=$ $d \mathfrak{b}(1)=0$. We assume that there exists $\mathfrak{c} \in \Omega(M) \widehat{\otimes} \Lambda^{\downarrow}$ such that

$$
\mathfrak{b}(1)-\mathfrak{b}(0)=d \mathfrak{c} .
$$

Then we prove that $\rho^{\mathfrak{b}(0)}\left(\widetilde{\phi}_{H} ; a\right)=\rho^{\mathfrak{b}(1)}\left(\widetilde{\phi}_{H} ; a\right)$. Firstly we consider the case that $\mathfrak{b}_{2}(0)=\mathfrak{b}_{2}(1)$. Here $\mathfrak{b}_{2}(0), \mathfrak{b}_{2}(1) \in H^{2}(M ; \mathbb{C})$ as in (5.5). After establishing Theorem 7.7 (2) under the condition that $\mathfrak{b}_{2}(0)=\mathfrak{b}_{2}(1)$, we show that the invariant $\rho^{\mathfrak{b}}\left(\widetilde{\phi}_{H} ; a\right)$ does not depend on the choice of representative of the cohomology class $\left[\mathfrak{b}_{2}\right]$.

We consider the ring of strictly convergent power series

$$
\Lambda^{\downarrow}\langle\langle s\rangle\rangle=\left\{\sum_{k=0}^{\infty} x_{k} s^{k} \mid x_{k} \in \Lambda^{\downarrow}, \lim _{k \rightarrow \infty} \mathfrak{v}_{q}\left(x_{k}\right)=-\infty\right\} .
$$

Here $s$ is a formal parameter. We denote by $\operatorname{Poly}\left(\mathbb{R} ; C F\left(M ; H ; \Lambda^{\downarrow}\right)\right)$ the set of formal expressions of the form

$$
x(s)+d s \wedge y(s)
$$

where

$$
x(s), y(s) \in C F\left(M ; H ; \Lambda^{\downarrow}\right) \otimes_{\Lambda^{\downarrow}} \Lambda^{\downarrow}\langle\langle s\rangle\rangle .
$$

For $s_{0} \in \mathbb{R}$ we define

$$
\operatorname{Eval}_{s=s_{0}}: \operatorname{Poly}\left(\mathbb{R} ; C F\left(M ; H ; \Lambda^{\downarrow}\right)\right) \rightarrow C F\left(M ; H ; \Lambda^{\downarrow}\right)
$$

by

$$
\operatorname{Eval}_{s=s_{0}}(x(s)+d s \wedge y(s))=x\left(s_{0}\right) .
$$


We note that, for $x(s)=\sum_{k=0}^{\infty} x_{k} s^{k} \in C F\left(M ; H ; \Lambda^{\downarrow}\right) \otimes_{\Lambda^{\downarrow}} \Lambda^{\downarrow}\langle\langle s\rangle\rangle$ with $x_{k} \in$ $C F\left(M ; H ; \Lambda^{\downarrow}\right)$, the series $x\left(s_{0}\right)=\sum_{k=0}^{\infty} x_{k} s_{0}^{k}$ converges in $q$-adic topology for $s_{0} \in \mathbb{R}$.

We put

$$
\mathfrak{b}(s)=s \mathfrak{b}(1)+(1-s) \mathfrak{b}(0)
$$

For each $s_{0} \in \mathbb{R}$ we define

$$
\partial_{(H, J)}^{\mathfrak{b}\left(s_{0}\right)}: C F\left(M ; H ; \Lambda^{\downarrow}\right) \rightarrow C F\left(M ; H ; \Lambda^{\downarrow}\right)
$$

by (6.8).

Lemma 27.1. There exists a $\Lambda^{\downarrow}\langle\langle s\rangle$-module homomorphism

$$
\partial_{(H, J)}^{\mathfrak{b}(\cdot)}: C F\left(M ; H ; \Lambda^{\downarrow}\right) \otimes_{\Lambda \downarrow} \Lambda^{\downarrow}\langle\langle s\rangle\rangle \rightarrow C F\left(M ; H ; \Lambda^{\downarrow}\right) \otimes_{\Lambda \downarrow} \Lambda^{\downarrow}\langle\langle s\rangle\rangle
$$

such that

$$
\operatorname{Eval}_{s=s_{0}} \circ \partial_{(H, J)}^{\mathfrak{b}(\cdot)}=\partial_{(H, J)}^{\mathfrak{b}\left(s_{0}\right)} \circ \mathrm{Eval}_{s=s_{0}}, \quad \partial_{(H, J)}^{\mathfrak{b}(\cdot)} \circ \partial_{(H, J)}^{\mathfrak{b}(\cdot)}=0 .
$$

Proof. We split $\mathfrak{b}(s)=\mathfrak{b}_{0}(s)+\mathfrak{b}_{2}(s)+\mathfrak{b}_{+}(s)$ as in (5.5). Then we have $\mathfrak{b}_{2}(s)=$ $s \mathfrak{b}_{2}(1)+(1-s) \mathfrak{b}_{2}(0)$ etc. We use it to see that

$$
\mathfrak{n}_{(H, J) ; \ell}\left([\gamma, w],\left[\gamma^{\prime}, w^{\prime}\right]\right)(\underbrace{\mathfrak{b}_{+}(s), \ldots, \mathfrak{b}_{+}(s)}_{\ell})
$$

is a polynomial of order $\leq \ell$ in $s$ with coefficients in $\mathbb{C}$. (See (6.5), (6.6).)

Therefore the series (6.6) of the current context converges for each $s$ and so the totality thereof define an element

$$
\mathfrak{n}_{(H, J)}^{\mathfrak{b}(s)}\left([\gamma, w],\left[\gamma^{\prime}, w^{\prime}\right]\right) \in \Lambda^{\downarrow}\langle\langle s\rangle\rangle .
$$

Hence we can define the map $\partial_{(H, J)}^{\mathfrak{b}(\cdot)}$ by replacing $\mathfrak{b}$ by $\mathfrak{b}(s)$ in $(6.8)$. The first formula in (27.5) is easy to show. The second formula follows from the first one.

We next put

$$
\begin{aligned}
& \mathfrak{n}_{(H, J)}^{\mathfrak{c}, 1}\left([\gamma, w],\left[\gamma^{\prime}, w^{\prime}\right]\right) \\
& =\sum_{\ell_{1}=0}^{\infty} \sum_{\ell_{2}=0}^{\infty} \frac{\exp \left(w^{\prime} \cap \mathfrak{b}_{2}(s)-w \cap \mathfrak{b}_{2}(s)\right)}{\left(\ell_{1}+\ell_{2}+1\right) !} \\
& \quad \mathfrak{n}_{(H, J) ; \ell_{1}+\ell_{2}+1}\left([\gamma, w],\left[\gamma^{\prime}, w^{\prime}\right]\right)(\underbrace{\mathfrak{b}_{+}(s), \ldots, \mathfrak{b}_{+}(s)}_{\ell_{1}}, \mathfrak{c}, \underbrace{\mathfrak{b}_{+}(s), \ldots, \mathfrak{b}_{+}(s)}_{\ell_{2}}) \\
& \in \Lambda^{\downarrow}\langle\langle s\rangle\rangle
\end{aligned}
$$

and define a map $\partial_{(H, J)}^{\mathfrak{c}}: C F\left(M ; H ; \Lambda^{\downarrow}\right) \rightarrow C F\left(M ; H ; \Lambda^{\downarrow}\right)$ by

$$
\partial_{(H, J)}^{\mathfrak{c}}(\llbracket \gamma, w \rrbracket)=\sum_{\left[\gamma^{\prime}, w^{\prime}\right] \in \operatorname{Crit}\left(\mathcal{A}_{H}\right)} \mathfrak{n}_{(H, J)}^{\mathfrak{c}, 1}\left([\gamma, w],\left[\gamma^{\prime}, w^{\prime}\right]\right) \llbracket \gamma^{\prime}, w^{\prime} \rrbracket .
$$

Lemma 27.2.

$$
\frac{\partial}{\partial s} \circ \partial_{(H, J)}^{\mathfrak{b}(\cdot)}-\partial_{(H, J)}^{\mathfrak{b}(\cdot)} \circ \frac{\partial}{\partial s}=\partial_{(H, J)}^{\mathfrak{c}} \circ \partial_{(H, J)}^{\mathfrak{b}(\cdot)}-\partial_{(H, J)}^{\mathfrak{b}(\cdot)} \circ \partial_{(H, J)}^{\mathfrak{c}} \cdot
$$


Proof. Using Proposition 6.2 (3), Stokes' formula (Theorem 34.8) and the composition formula (Theorem 35.7), we obtain

$$
\begin{aligned}
& \sum_{i=1}^{\ell}(-1)^{*} \mathfrak{n}_{(H, J) ; \ell}\left([\gamma, w],\left[\gamma^{\prime}, w^{\prime}\right]\right)\left(h_{1}, \ldots, d h_{i}, \ldots, h_{\ell}\right) \\
& =\sum_{\left(\mathrm{E}_{1}, \mathrm{E}_{2}\right) \in \operatorname{Shuff}(\ell)} \sum_{\left[\gamma^{\prime \prime}, w^{\prime \prime}\right]}(-1)^{* *} \mathfrak{n}_{(H, J) ; \# \mathrm{E}_{1}}\left([\gamma, w],\left[\gamma^{\prime \prime}, w^{\prime \prime}\right]\right)\left(h_{i_{1}}, \ldots, h_{i_{\# \mathrm{E}_{1}}}\right) \\
& \mathfrak{n}_{(H, J) ; \# \mathrm{E}_{2}}\left(\left[\gamma^{\prime \prime}, w^{\prime \prime}\right],\left[\gamma^{\prime}, w^{\prime}\right]\right)\left(h_{j_{1}}, \ldots, h_{j_{\# \mathrm{E}_{2}}}\right),
\end{aligned}
$$

where $\mathrm{E}_{1}=\left\{i_{1}, \ldots, i_{\# \mathrm{E}_{1}}\right\}, \mathrm{E}_{2}=\left\{j_{1}, \ldots, j_{\# \mathrm{E}_{2}}\right\}$,

$$
*=\operatorname{deg} h_{1}+\cdots+\operatorname{deg} h_{i-1}, \quad * *=\sum_{i \in \mathrm{E}_{1}, j \in \mathrm{E}_{2} ; j<i} \operatorname{deg} h_{i} \operatorname{deg} h_{j} .
$$

Using (27.4) and (27.8) we can prove Lemma 27.2 easily.

We define the map

$$
\partial_{(H, J)}^{(\mathfrak{b}(\cdot), \mathfrak{c})}: \operatorname{Poly}\left(\mathbb{R} ; C F\left(M ; H ; \Lambda^{\downarrow}\right)\right) \rightarrow \operatorname{Poly}\left(\mathbb{R} ; C F\left(M ; H ; \Lambda^{\downarrow}\right)\right)
$$

by

$$
\begin{aligned}
& \partial_{(H, J)}^{(\mathfrak{b}(\cdot), \mathfrak{c})}(x(s)+d s \wedge y(s)) \\
& =\partial_{(H, J)}^{\mathfrak{b}(\cdot)}(x(s))-d s \wedge \frac{\partial}{\partial s}(x(s))+d s \wedge \partial_{(H, J)}^{\mathfrak{c}}(x(s))-d s \wedge \partial_{(H, J)}^{\mathfrak{b}(\cdot)}(y(s)) .
\end{aligned}
$$

Then the second formula of (27.5) and Lemma 27.2 imply

$$
\partial_{(H, J)}^{(\mathfrak{b}(\cdot), \mathfrak{c})} \circ \partial_{(H, J)}^{(\mathfrak{b}(\cdot), \mathfrak{c})}=0 .
$$

Thus $\left(\operatorname{Poly}\left(\mathbb{R} ; C F\left(M ; H ; \Lambda^{\downarrow}\right)\right), \partial_{(H, J)}^{(\mathfrak{b}(\cdot), \mathfrak{c})}\right)$ is a chain complex. The first formula of (27.5) implies that

$$
\operatorname{Eval}_{s=s_{0}}:\left(\operatorname{Poly}\left(\mathbb{R} ; C F\left(M ; H ; \Lambda^{\downarrow}\right)\right), \partial_{(H, J)}^{(\mathfrak{b}(\cdot), \mathfrak{c})}\right) \rightarrow\left(C F\left(M ; H ; \Lambda^{\downarrow}\right), \partial_{(H, J)}^{\mathfrak{b}\left(s_{0}\right)}\right)
$$

is a chain map.

We define a filtration $F^{\lambda} \operatorname{Poly}\left(\mathbb{R} ; C F\left(M ; H ; \Lambda^{\downarrow}\right)\right)$ on $\operatorname{Poly}\left(\mathbb{R} ; C F\left(M ; H ; \Lambda^{\downarrow}\right)\right)$ by

$$
\begin{aligned}
& F^{\lambda} \operatorname{Poly}\left(\mathbb{R} ; C F\left(M ; H ; \Lambda^{\downarrow}\right)\right) \\
& =\left\{x(s)+d s \wedge y(s) \mid x(s)=\sum x_{k} s^{k}, y(s)=\sum y_{k} s^{k}, \lambda_{H}\left(x_{k}\right), \lambda_{H}\left(y_{k}\right) \leq \lambda\right\} .
\end{aligned}
$$

Lemma 27.3. $\partial_{(H, J)}^{(\mathfrak{b}(\cdot), \mathfrak{c})}$ and $\mathrm{Eval}_{s=s_{0}}$ preserves the filtration $F^{\lambda}$.

The proof is easy and is omitted.

Lemma 27.4. The map (27.10) is a chain homotopy equivalence.

Proof. If $x(s)+d s \wedge y(s) \in F^{\lambda} \operatorname{Poly}\left(\mathbb{R} ; C F\left(M ; H ; \Lambda^{\downarrow}\right)\right)$, then we have

$$
\partial_{(H, J)}^{(\mathfrak{b}(\cdot), \mathfrak{c})}(x(s)+d s \wedge y(s))-d s \wedge \frac{\partial x}{\partial s}(s) \in F^{\lambda-\epsilon} \operatorname{Poly}\left(\mathbb{R} ; C F\left(M ; H ; \Lambda^{\downarrow}\right)\right)
$$

for some positive $\epsilon$. We use this fact to prove Lemma 27.4 by the same way as in the proof given in [FOOO1, Proposition 4.3.18]. 
We next define the map

$$
\mathcal{P}_{\left(H_{\chi}, J_{\chi}\right)}^{(\mathfrak{b}(\cdot), \mathfrak{c})}: \Omega(M) \otimes \Lambda^{\downarrow} \rightarrow \operatorname{Poly}\left(\mathbb{R} ; C F\left(M ; H ; \Lambda^{\downarrow}\right)\right) .
$$

First, for each fixed $s_{0}$, we define a map

$$
\mathcal{P}_{\left(H_{\chi}, J_{\chi}\right)}^{\mathfrak{b}\left(s_{0}\right)}: \Omega(M) \otimes \Lambda^{\downarrow} \rightarrow C F\left(M ; H ; \Lambda^{\downarrow}\right)
$$

by the formula (6.14). We then obtain a map

$$
\mathcal{P}_{\left(H_{\chi}, J_{\chi}\right)}^{\mathfrak{b}(\cdot)}: \Omega(M) \otimes \Lambda^{\downarrow} \rightarrow C F\left(M ; H ; \Lambda^{\downarrow}\right) \otimes_{\Lambda^{\downarrow}} \Lambda^{\downarrow}\langle\langle s\rangle\rangle
$$

that satisfies

$$
\operatorname{Eval}_{s=s_{0}} \circ \mathcal{P}_{\left(H_{\chi}, J_{\chi}\right)}^{\mathfrak{b}(\cdot)}=\mathcal{P}_{\left(H_{\chi}, J_{\chi}\right)}^{\mathfrak{b}\left(s_{0}\right)} .
$$

Similarly to (6.13), we define the term

$$
\begin{aligned}
& \mathfrak{n}_{\left(H_{\chi}, J_{\chi}\right)}^{\mathfrak{c}, 1}(h ;[\gamma, w]) \\
& =\sum_{\substack{\ell_{1}=0 \\
\ell_{\ell_{2}=0}}}^{\infty} \frac{\exp \left(\int w^{*} \mathfrak{b}_{2}(s)\right)}{\left(\ell_{1}+\ell_{2}+1\right) !} \\
& \quad \mathfrak{n}_{(H, J) ; \ell_{1}+\ell_{2}+1}(h ;[\gamma, w])(\underbrace{\mathfrak{b}_{+}(s), \ldots, \mathfrak{b}_{+}(s)}_{\ell_{1}}, \mathfrak{c}, \underbrace{\mathfrak{b}_{+}(s), \ldots, \mathfrak{b}_{+}(s)}_{\ell_{2}}) \\
& \in \Lambda^{\downarrow}\langle\langle s\rangle\rangle .
\end{aligned}
$$

Using this, we then define a map $\mathcal{P}_{\left(H_{\chi}, J_{\chi}\right)}^{\mathfrak{c}}: \Omega(M) \otimes \Lambda^{\downarrow} \rightarrow C F\left(M ; H ; \Lambda^{\downarrow}\right)$ by

$$
\mathcal{P}_{\left(H_{\chi}, J_{\chi}\right)}^{\mathfrak{c}}(h)=\sum_{[\gamma, w] \in \operatorname{Crit}\left(\mathcal{A}_{H}\right)} \mathfrak{n}_{\left(H_{\chi}, J_{\chi}\right)}^{\mathfrak{c}, 1}(h ;[\gamma, w]) \llbracket \gamma, w \rrbracket .
$$

Finally we define the map (27.11) by putting

$$
\mathcal{P}_{\left(H_{\chi}, J_{\chi}\right)}^{(\mathfrak{b}(\cdot), \mathfrak{c})}(h)=\mathcal{P}_{\left(H_{\chi}, J_{\chi}\right)}^{\mathfrak{b}(\cdot)}(h)+d s \wedge \mathcal{P}_{\left(H_{\chi}, J_{\chi}\right)}^{\mathfrak{c}}(h) .
$$

Lemma 27.5. We have

$$
\partial_{(H, J)}^{(\mathfrak{b}(\cdot), \mathfrak{c})} \circ \mathcal{P}_{\left(H_{\chi}, J_{\chi}\right)}^{(\mathfrak{b}(\cdot), \mathfrak{c})}=\mathcal{P}_{\left(H_{\chi}, J_{\chi}\right)}^{(\mathfrak{b}(\cdot), \mathfrak{c})} \circ \partial
$$

and

$$
\operatorname{Eval}_{s=s_{0}} \circ \partial_{(H, J)}^{(\mathfrak{b}(\cdot), \mathfrak{c})}=\mathcal{P}_{\left(H_{\chi}, J_{\chi}\right)}^{\mathfrak{b}\left(s_{0}\right)}
$$

The proof is straightforward calculation and is omitted.

Combining Lemmas 27.3, 27.4, 27.5, we easily prove $\rho^{\mathfrak{b}(0)}\left(\widetilde{\psi}_{H}, a\right)=\rho^{\mathfrak{b}(1)}\left(\widetilde{\psi}_{H}, a\right)$. The proof of Theorem 7.7 (2) is complete under the condition that $\mathfrak{b}_{2}(0)=\mathfrak{b}_{2}(1)$.

Next, for $\mathfrak{b}(0), \mathfrak{b}(1)$ such that $\mathfrak{b}(1)-\mathfrak{b}(0)=d \mathfrak{c}$ for some $\mathfrak{c}$, we consider $\mathfrak{b}^{\prime}=$ $\mathfrak{b}(0)+d\left(\mathfrak{c}-\mathfrak{c}_{1}\right)$. Here $\mathfrak{c}_{1}$ is the $\Omega^{1}(M ; \mathbb{C})$-component of $\mathfrak{c}$ in the decomposition $\Omega^{1}(M ; \mathbb{C}) \oplus \Omega^{1}\left(M ; \Lambda_{-}^{\downarrow}\right) \oplus \Omega^{\geq 3}\left(M ; \Lambda^{\downarrow}\right)$. We showed that $\rho^{\mathfrak{b}(0)}\left(\widetilde{\phi}_{H}, a\right)=\rho^{\mathfrak{b}^{\prime}}\left(\widetilde{\phi}_{H}, a\right)$. The remaining task is to show that $\rho^{\mathfrak{b}^{\prime}}\left(\widetilde{\phi}_{H}, a\right)=\rho^{\mathfrak{b}(1)}\left(\widetilde{\phi}_{H}, a\right)$. Namely we prove Theorem 7.7 (2) in the case that $\mathfrak{b}(1)-\mathfrak{b}(0)=d \mathfrak{c}_{1}$ with $\mathfrak{c}_{1} \in \Omega^{1}(M ; \mathbb{C})$.

We define $I: C F\left(M ; H ; \Lambda^{\downarrow}\right) \rightarrow C F\left(M ; H ; \Lambda^{\downarrow}\right)$ by

$$
I(\llbracket \gamma, w \rrbracket)=\exp \left(\int_{S^{1}} \gamma^{*} \mathfrak{c}_{1}\right) \llbracket \gamma, w \rrbracket .
$$


Then we find that $I$ gives an isomorphism of Floer chain complexes

$$
I:\left(C F\left(M ; H ; \Lambda^{\downarrow}\right), \partial_{(J, H)}^{\mathfrak{b}^{\prime}}\right) \rightarrow\left(C F\left(M ; H ; \Lambda^{\downarrow}\right), \partial_{(J, H)}^{\mathfrak{b}(1)}\right)
$$

and

$$
I \circ \mathcal{P}_{\left(H_{\chi}, J_{\chi}\right)}^{\mathfrak{b}^{\prime}}=\mathcal{P}_{\left(H_{\chi}, J_{\chi}\right)}^{\mathfrak{b}(1)} .
$$

Hence the proof of Theorem 7.7 (2).

Remark 27.6. A cocycle $\mathfrak{b} \in \Omega^{2}(M ; \mathbb{C})$ induces a representation

$$
\mathfrak{r e p}_{\mathfrak{b}}: a \in \pi_{1}\left(\mathcal{L}(M) ; \ell_{0}\right) \mapsto \exp \left(\int_{C_{a}} \mathfrak{b}\right) \in \mathbb{C}^{*},
$$

where $C_{a}: S^{1} \times S^{1} \rightarrow M$ is the mapping corresponding to the loop $a$ in $\mathcal{L}(M)$. Then we can consider Floer complex of the Hamiltonian system with coefficients in the local system corresponding to $\mathfrak{r e p}_{\mathfrak{b}}$. If $\mathfrak{b}(0)$ and $\mathfrak{b}(1)$ are cohomologous, the corresponding local systems are isomorphic, hence Floer cohomology groups with coefficients in these local systems are isomorphic. Here we gave the isomorphism $I$ directly without dealing with the isomorphism of the local systems.

\section{Proof of Proposition 20.7.}

The purpose of this section is to prove Proposition 20.7 and Lemma 20.9. In this section we fix a $t$-independent $J$.

28.1. Pseudo-isotopy of filtered $A_{\infty}$ algebra. In this subsection, we review the notion of pseudo-isotopy of filtered $A_{\infty}$ algebra, which was introduced in [Fu3] Definition 8.5. We consider $L \times[0,1]$ and use $s$ for the coordinate of the interval $[0,1]$. We put $\bar{C}=\Omega(L)$ and

$$
C^{\infty}([0,1] \times \bar{C})=\Omega([0,1] \times L) .
$$

An element of $C^{\infty}([0,1] \times \bar{C})$ is written uniquely as

$$
x(s)+d s \wedge y(s)
$$

where $x(s), y(s)$ are smooth differential forms on $[0,1] \times L$ that do not contain $d s$, i.e., that satisfies $\left.\left.\frac{\partial}{\partial s}\right\rfloor x(s)=0=\frac{\partial}{\partial s}\right\rfloor y(s)=0$. It follows that $x\left(s_{0}\right), y\left(s_{0}\right) \in \bar{C}$ for each fixed $s_{0}$.

Suppose that, for each $s \in[0,1], k, \ell, \beta \in \pi_{2}(M ; L)$ we have operators

$$
\mathfrak{m}_{k, \beta}^{s}: B_{k}(\bar{C}[1]) \rightarrow \bar{C}[1]
$$

of degree $-\mu(\beta)+1$ and

$$
\mathfrak{c}_{k, \beta}^{s}: B_{k}(\bar{C}[1]) \rightarrow \bar{C}[1]
$$

of degree $-\mu(\beta)$.

Definition 28.1. We say $\mathfrak{m}_{k, \beta}^{s}$ is smooth if for each $x_{1}, \ldots, x_{k} \in \bar{C}$ we may regard $\mathfrak{m}_{k, \beta}^{s}\left(x_{1}, \ldots, x_{k}\right)$ as an element of $C^{\infty}([0,1], \bar{C})$ with vanishing $d s$ component. The smoothness of $\mathfrak{c}_{k, \beta}^{s}$ is defined in the same way.

Suppose that there exists a subset $\widehat{G}$ of $H_{2}(M, L ; \mathbb{Z})$ such that $\{\omega \cap \beta \mid \beta \in \widehat{G}\}$ is a discrete subset of $\mathbb{R}_{\geq 0}$. Let $G$ be the monoid generated by this set. Here we assume that the operators $\mathfrak{m}_{k, \beta}^{s}, \mathfrak{c}_{k, \beta}^{s}$ are given for $\beta \in \widehat{G}$. 
Definition 28.2. We say $\left(C,\left\{\mathfrak{m}_{k, \beta}^{s}\right\},\left\{\mathfrak{c}_{k, \beta}^{s}\right\}\right)$ is a pseudo-isotopy of $G$-gapped filtered $A_{\infty}$ algebra: 7 if the following holds:

(1) $\mathfrak{m}_{k, \beta}^{s}$ and $\mathfrak{c}_{k, \beta}^{s}$ are smooth.

(2) For each (but fixed) $s,\left(C,\left\{\mathfrak{m}_{k, \beta}^{s}\right\}\right)$ defines a filtered $A_{\infty}$ algebra.

(3) For each given $x_{i} \in \bar{C}[1], i=1, \ldots, k$, the operators satisfy

$$
\begin{aligned}
& \frac{d}{d s} \mathfrak{m}_{k, \beta}^{s}\left(x_{1}, \ldots, x_{k}\right) \\
& +\sum_{k_{1}+k_{2}=k} \sum_{\beta_{1}+\beta_{2}=\beta} \sum_{i=1}^{k-k_{2}+1}(-1)^{*} \mathfrak{c}_{k_{1}, \beta_{1}}^{s}\left(x_{1}, \ldots, \mathfrak{m}_{k_{2}, \beta_{2}}^{s}\left(x_{i}, \ldots\right), \ldots, x_{k}\right) \\
& -\sum_{k_{1}+k_{2}=k} \sum_{\beta_{1}+\beta_{2}=\beta} \sum_{i=1}^{k-k_{2}+1} \mathfrak{m}_{k_{1}, \beta_{1}}^{s}\left(x_{1}, \ldots, \mathfrak{c}_{k_{2}, \beta_{2}}^{s}\left(x_{i}, \ldots\right), \ldots, x_{k}\right) \\
& =0 .
\end{aligned}
$$

Here $*=\operatorname{deg}^{\prime} x_{1}+\ldots+\operatorname{deg}^{\prime} x_{i-1}$.

(4) $\mathfrak{m}_{k, \beta_{0}}^{s}$ is independent of $s$, and $\mathfrak{c}_{k, \beta_{0}}^{s}=0$. Here $\beta_{0}=0 \in H_{2}(M ; L ; \mathbb{Z})$.

We consider $x_{i}(s)+d s \wedge y_{i}(s)=\mathbf{x}_{i} \in C^{\infty}([0,1], \bar{C})$. We define the operators $\widehat{\mathfrak{m}}_{k, \beta}: C^{\infty}([0,1], \bar{C})^{\otimes k} \rightarrow C^{\infty}([0,1], \bar{C})$ by

$$
\widehat{\mathfrak{m}}_{k, \beta}\left(\mathbf{x}_{1}, \ldots, \mathbf{x}_{k}\right)=x(s)+d s \wedge y(s),
$$

where

$$
\begin{gathered}
x(s)=\mathfrak{m}_{k, \beta}^{s}\left(x_{1}(s), \ldots, x_{k}(s)\right) \\
y(s)=\mathfrak{c}_{k, \beta}^{s}\left(x_{1}(s), \ldots, x_{k}(s)\right) \\
-\sum_{i=1}^{k}(-1)^{*_{i}} \mathfrak{m}_{k, \beta}^{t}\left(x_{1}(s), \ldots, x_{i-1}(s), y_{i}(s), x_{i+1}(s), \ldots, x_{k}(s)\right)
\end{gathered}
$$

if $(k, \beta) \neq\left(1, \beta_{0}\right)$ and

$$
y(s)=\frac{d}{d s} x_{1}(s)+\mathfrak{m}_{1,0}^{s}\left(y_{1}(s)\right)
$$

if $(k, \beta)=\left(1, \beta_{0}\right)$. Here $*_{i}$ in (28.5b) is $*_{i}=\operatorname{deg}^{\prime} x_{1}+\ldots+\operatorname{deg}^{\prime} x_{i-1}$.

Lemma 28.3. The equation (28.3) is equivalent to the filtered $A_{\infty}$ relation of $\left\{\widehat{\mathfrak{m}}_{k, \beta}\right\}$ defined by (28.5).

The proof is a straightforward calculation.

Definition 28.4. A pseudo-isotopy $\left(C,\left\{\mathfrak{m}_{k, \beta}^{s}\right\},\left\{\mathfrak{c}_{k, \beta}^{s}\right\}\right)$ is said to be unital if there exists $\mathbf{e} \in \bar{C}^{0}$ such that $\mathbf{e}$ is a unit of $\left(C,\left\{\mathfrak{m}_{k, \beta}^{s}\right\}\right)$ for each $s$ and if $\mathfrak{c}_{k, \beta}^{s}(\ldots, \mathbf{e}, \ldots)=0$ for each $k, \beta$ and $s$.

In our situation the unit $\mathbf{e}$ is always $\mathbf{e}^{L}$, the constant function 1 on $L$.

Theorem 28.5. If $\left(C,\left\{\mathfrak{m}_{k, \beta}^{s}\right\},\left\{\mathfrak{c}_{k, \beta}^{s}\right\}\right)$ is a unital pseudo-isotopy, then there exists a unital filtered $A_{\infty}$ homomorphism from $\left(C,\left\{\mathfrak{m}_{k, \beta}^{0}\right\}\right)$ to $\left(C,\left\{\mathfrak{m}_{k, \beta}^{1}\right\}\right)$ that has a homotopy inverse.

\footnotetext{
${ }^{7}$ See [FOOO1, Definition 3.2.26] for the definition of $G$-gapped-ness of a filtered $A_{\infty}$ algebra.
} 
Proof. The cyclic version of this theorem is [Fu3, Theorem 8.2]. Since we do not require cyclic symmetry here, the proof of Theorem 28.5 is easier. In fact, it follows from [FOOO1, Theorem 4.2.45] as follows. We have a filtered $A_{\infty}$ homomorphism

$$
\operatorname{Eval}_{s=s_{0}}:\left(C^{\infty}([0,1] \times \bar{C}),\left\{\widehat{\mathfrak{m}}_{k, \beta}^{s}\right\}\right) \rightarrow\left(\bar{C},\left\{\mathfrak{m}_{k, \beta}^{s_{0}}\right\}\right)
$$

defined by $\left(\operatorname{Eval}_{s=s_{0}}\right)_{1}(a(s)+d s \wedge b(s))=a\left(s_{0}\right)$ and $\left(\operatorname{Eval}_{s=s_{0}}\right)_{k}=0$ for $k \neq 1$. Then using the $A_{\infty}$ Whitehead theorem ([FOOO1, Lemma 4.2.45]) we can show that it is a homotopy equivalence. Theorem 28.5 follows.

28.2. Difference between $\mathfrak{m}^{T}$ and $\mathfrak{m}$. We will construct a pseudo-isotopy between two filtered $A_{\infty}$ structures $\left\{\mathfrak{m}_{k, \beta}^{T, \mathfrak{b}}\right\}$ and $\left\{\mathfrak{m}_{k, \beta}^{\mathfrak{b}}\right\}$ on $\bar{C}=\Omega(L)$. Here the first one is defined in Section 20 and the second one is defined in Section 17. We note that the difference of these two constructions are roughly as follows:

(1) We represent $\mathfrak{b}$ by a $T^{n}$-invariant cycle $D_{a}$ that is a submanifold to define $\left\{\mathfrak{m}_{k, \beta}^{T, \mathfrak{b}}\right\}$. In other words, we use a current that may not be smooth when we define $\left\{\mathfrak{m}_{k, \beta}^{T, \mathfrak{b}}\right\}$. On the other hand, we represent $\mathfrak{b}$ by a smooth differential form when we define $\left\{\mathfrak{m}_{k, \beta}^{\mathfrak{b}}\right\}$.

(2) In the definition of $\left\{\mathfrak{m}_{k, \beta}^{T, \mathfrak{b}}\right\}$ we first take the fiber product 20.9) and then use a multisection to achieve transversality. On the other hand, in the definition of $\left\{\mathfrak{m}_{k, \beta}^{\mathfrak{b}}\right\}$, we first perturb (by CF-perturbations) the moduli space $\mathcal{M}_{k+1: \ell}(\beta)$ then pull back the differential form representing $\mathfrak{b}$ to the zero set of the multisection. In other words the perturbation to define $\left\{\mathfrak{m}_{k, \beta}^{\mathfrak{b}}\right\}$ is independent of the ambient cohomology class $\mathfrak{b}$.

Remark 28.6. We note that there are various reasons why we need to take cycles and multisections (rather than taking CF-perturbation), when we construct $\left\{\mathfrak{m}_{k, \beta}^{T, \mathfrak{b}}\right\}$ in the toric case. The most important reason lies in Proposition 20.11. This is related to point (1) above. The reason why we first need to take the fiber product (20.9) is explained in [FOOO3, Remark 11.4].

On the other hand to develop the theory of spectral invariant with bulk deformation in the general setting, it seems simplest to always use de Rham representative and CF-perturbations.

In the next subsection, we will construct a pseudo-isotopy of filtered $A_{\infty}$ structures interpolating $\left\{\mathfrak{m}_{k, \beta}^{T, \mathfrak{b}}\right\}$ and $\left\{\mathfrak{m}_{k, \beta}^{\mathfrak{b}}\right\}$. Below we handle the above (1) and (2) separately. We construct the pseudo-isotopy resolving (1) in Subsection 28.3 and construct the pseudo-isotopy resolving (2) in Subsection 28.5

28.3. Smoothing $T^{n}$-invariant chains. Let $D_{a}=D_{i_{1}} \cap \cdots \cap D_{i_{k}}$ be a transversal intersection of $k$ irreducible components of the toric divisor, $N D_{a}$ its normal bundle, and $\exp : N D_{a} \rightarrow M$ the exponential map with respect to a $T^{n}$-invariant Riemannian metric. Let $\mathcal{U}_{a} \subset \Gamma\left(N D_{a}\right)$ be a finite dimensional submanifold of the space of smooth sections of $N D_{a}$ such that if $\mathfrak{u} \in \mathcal{U}_{a}$ and $\rho \in[0,1]$ then $\rho \mathfrak{u} \in \mathcal{U}_{a}$. We assume that it has the following properties.

Properties 28.7. (1) The exponential map Exp : $D_{a} \times \mathcal{U}_{a} \rightarrow M$ defined by

$$
\operatorname{Exp}(\mathfrak{u}, x)=\exp (\mathfrak{u}(x))
$$

is a submersion.

(2) $\|\mathfrak{u}(x)\|<\epsilon$, where $\epsilon$ is a sufficiently small positive number determined later. 
We put $d_{a}=\operatorname{dim} \mathcal{U}_{a}$.

Let $\mathbf{p}:\{1, \ldots, \ell\} \rightarrow \underline{B}$ be as in the beginning of Subsection 20.2. We put

$$
\mathcal{U}(\mathbf{p})=\prod_{i=1}^{\ell} \mathcal{U}_{\mathbf{p}(i)}, \quad \mathbf{p}(\mathcal{U})=\prod_{i=1}^{\ell}\left(D_{\mathbf{p}(i)} \times \mathcal{U}_{\mathbf{p}(i)}\right) .
$$

The map (28.6) induces

$$
\operatorname{Exp}: \mathbf{p}(\mathcal{U}) \rightarrow M^{\ell}
$$

For $k, \ell \in \mathbb{Z}_{\geq 0}$ and $\beta \in H_{2}(M, L(\mathbf{u}) ; \mathbb{Z})$ we define a fiber product

$$
\mathcal{M}_{k+1 ; \ell}(L(\mathbf{u}) ; \beta ; \mathbf{p}(\mathcal{U}))=\mathcal{M}_{k+1 ; \ell}(L(\mathbf{u}) ; \beta)_{\left(e v_{1}, \ldots, e v_{\ell}\right)} \times_{\operatorname{Exp}} \mathbf{p}(\mathcal{U}),
$$

where $\mathcal{M}_{k+1 ; \ell}(L(\mathbf{u}) ; \beta)$ is a moduli space defined in Definition 17.2 and Proposition 17.3. (Compare (20.9).) We can define the evaluation map at the boundary marked points

$$
\mathrm{ev}^{\partial}=\left(\mathrm{ev}_{1}^{\partial}, \ldots, \mathrm{ev}_{k}^{\partial}\right): \mathcal{M}_{k+1 ; \ell}(L(\mathbf{u}) ; \beta ; \mathbf{p}(\mathcal{U})) \rightarrow L(\mathbf{u})^{k+1}
$$

in an obvious way. We also have a projection

$$
\pi_{\mathcal{U}}: \mathcal{M}_{k+1 ; \ell}(L(\mathbf{u}) ; \beta ; \mathbf{p}(\mathcal{U})) \rightarrow \mathcal{U}(\mathbf{p})
$$

to the $\mathcal{U}_{a}$-factors. By definition we have

$$
\pi_{\mathcal{U}}^{-1}(\mathbf{0})=\mathcal{M}_{k+1 ; \ell}(L(\mathbf{u}) ; \beta ; \mathbf{p}) .
$$

Lemma 28.8. (1) $\mathcal{M}_{k+1 ; \ell}(L(\mathbf{u}) ; \beta ; \mathbf{p}(\mathcal{U}))$ has a Kuranishi structure with corners.

(2) It induces the Kuranishi structure on $\pi_{\mathcal{U}}^{-1}(\mathbf{0})$ as described in Lemma 20.4 .

(3) Its normalized boundary is described by the union of the following fiber products:

$$
\mathcal{M}_{k_{1}+1 ; \# E_{1}}\left(L(\mathbf{u}) ; \beta_{1} ; \mathbf{p}_{1}(\mathcal{U})\right)_{\mathrm{ev}_{0}^{\partial}} \times_{\operatorname{ev}_{i}^{\partial}} \mathcal{M}_{k_{2}+1 ; \# E_{2}}\left(L(\mathbf{u}) ; \beta_{2} ; \mathbf{p}_{2}(\mathcal{U})\right)
$$

where the union is taken over all $\left(E_{1}, E_{2}\right) \in \operatorname{Shuff}(\ell), k_{1}, k_{2}$ with $k_{1}+k_{2}=k$ and $\beta_{1}, \beta_{2} \in H_{2}(M, L(\mathbf{u}) ; \mathbb{Z})$ with $\beta=\beta_{1}+\beta_{2}$. We put $\operatorname{Split}\left(\left(\mathbb{L}_{1}, \mathbb{L}_{2}\right), \mathbf{p}\right)=$ $\left(\mathbf{p}_{1}, \mathbf{p}_{2}\right)$.

(4) The dimension is

$$
\begin{aligned}
& \operatorname{dim} \mathcal{M}_{k+1 ; \ell}(L(\mathbf{u}) ; \beta ; \mathbf{p}(\mathcal{U})) \\
& =n+\mu_{L(\mathbf{u})}(\beta)+k-2+2 \ell-\sum_{i=1}^{\ell} 2 \operatorname{deg} D_{\mathbf{p}(i)}+\sum_{i=1}^{\ell} d_{\mathbf{p}(i)} .
\end{aligned}
$$

(5) The evaluation maps $\mathrm{ev}_{i}^{\partial}$ at the boundary marked points of $\mathcal{M}_{k+1 ; \ell}(L(\mathbf{u}) ; \beta)$ define maps on $\mathcal{M}_{k+1 ; \ell}(L(\mathbf{u}) ; \beta ; \mathbf{p}(\mathcal{U}))$, which we denote by $\mathrm{ev}_{i}^{\partial}$ also. They are compatible with (3).

(6) We can define an orientation of the Kuranishi structure so that it is compatible with (3).

(7) $\mathrm{ev}_{0}^{\partial} \times \pi_{\mathcal{U}}$ is weakly submersive.

(8) The Kuranishi structure is invariant under the permutation of interior marked points.

(9) The Kuranishi structure is compatible with the forgetful map of the 1st, 2 nd, $\ldots, k$-th boundary marked points. (We do not require that it is compatible with the forgetful map of the 0-th marked point.)

The proof is the same as that of Lemma 20.4. We note that (7) is a consequence of (2) if we take $\epsilon$ in Properties 28.7 (2) to be small enough. 
Lemma 28.9. There exists a system of multisections on $\mathcal{M}_{k+1 ; \ell}(L(\mathbf{u}) ; \beta ; \mathbf{p}(\mathcal{U}))$ with the following properties.

(1) They are transversal to $\mathbf{0 .}$

(2) They coincide with the multisection in Lemma20.5 on the induced Kuranishi charts of $\pi_{\mathcal{U}}^{-1}(\mathbf{0})$.

(3) They are compatible with the description of the boundary in Lemma 20.4 (3).

(4) The restriction of $\operatorname{ev}_{0}^{\partial} \times \pi_{\mathcal{U}}$ to the zero set of this multisection is a submersion.

(5) They are invariant under the permutation of interior marked points.

(6) They are compatible with the forgetful map of the 1 st, $2 n d, \ldots, k$-th boundary marked points.

The proof is mostly the same as the proof of Lemma 20.5. We only observe that (4) is a consequence of (2) if $\epsilon$ is sufficiently small.

For each $a=1, \ldots, B$, we choose a compactly supported smooth differential form $\chi_{a}$ of top degree on $\mathcal{U}_{a}$ such that $\int_{\mathcal{U}_{a}} \chi_{a}=1$. For $\mathbf{p}:\{1, \ldots, \ell\} \rightarrow \underline{B}$, we put

$$
\chi_{\mathbf{p}}=\prod_{i=1}^{\ell} \chi_{\mathbf{p}(i)} \in \Omega(\mathcal{U}(\mathbf{p})) .
$$

Let $h_{1}, \ldots, h_{k} \in \Omega(L(\mathbf{u}))$. We then define a differential form on $L(\mathbf{u})$ by

$$
\mathfrak{q}_{\ell, k ; \beta}^{S}\left(\mathbf{p} ; h_{1}, \ldots, h_{k}\right)=\left(\mathrm{ev}_{0}^{\partial}\right) !\left(\operatorname{ev}_{1}^{\partial}, \ldots, \mathrm{ev}_{k}^{\partial}, \pi_{\mathcal{U}}\right)^{*}\left(h_{1} \wedge \cdots \wedge h_{k} \wedge \chi_{\mathbf{p}}\right),
$$

where we use the evaluation map

$$
\left(\mathrm{ev}_{0}^{\partial}, \ldots, \mathrm{ev}_{k}^{\partial}, \pi_{\mathcal{U}}\right): \mathcal{M}_{k+1 ; \ell}(L(\mathbf{u}) ; \beta ; \mathbf{p}(\mathcal{U})) \rightarrow L(\mathbf{u})^{k+1} \times \mathcal{U}(\mathbf{p})
$$

and $\left(\mathrm{ev}_{0}\right)$ ! is the integration along the fibers. (See [FOOO3, Definition C.1].) Here the superscript $S$ stands for smoothing. By Lemma 28.9 (4), the integration along the fibers is well-defined. By Lemma 28.9 (5) the operators $\mathfrak{q}_{\ell, k ; \beta}^{S}$ is invariant under the permutation of the components of $\mathbf{p}$. Therefore by the $\mathbb{C}$-linearity we define

$$
\mathfrak{q}_{\ell, k ; \beta}^{S}: E_{\ell}(\mathcal{H}[2]) \otimes B_{k}(\Omega(L(\mathbf{u}))[1]) \rightarrow \Omega(L(\mathbf{u}))[1] .
$$

Using these operators in the same way as in Definition 17.8 to define $\left\{\mathfrak{m}_{k}^{S, \mathbf{b}}\right\}$ for $\mathbf{b}=$ $\left(\mathfrak{b}_{0}, \mathbf{b}_{2 ; 1}, \mathfrak{b}_{+}, b_{+}\right)$. Thus we have obtained a filtered $A_{\infty}$ algebra $\left(C F_{\mathrm{dR}}\left(L(\mathbf{u}) ; \Lambda_{0}\right),\left\{\mathfrak{m}_{k}^{S, \mathbf{b}}\right\}_{k=0}^{\infty}\right)$. Here we recall

$$
C F_{\mathrm{dR}}\left(L(\mathbf{u}) ; \Lambda_{0}\right)=\Omega(L(\mathbf{u})) \widehat{\otimes} \Lambda_{0} .
$$

Lemma 28.10. The filtered $A_{\infty}$ algebra $\left(C F_{\mathrm{dR}}\left(L(\mathbf{u}) ; \Lambda_{0}\right),\left\{\mathfrak{m}_{k}^{S, \mathbf{b}}\right\}_{k=0}^{\infty}\right)$ is pseudoisotopic to $\left(C F_{\mathrm{dR}}\left(L(\mathbf{u}) ; \Lambda_{0}\right),\left\{\mathfrak{m}_{k}^{T, \mathbf{b}}\right\}_{k=0}^{\infty}\right)$ as a unital filtered $A_{\infty}$ algebra.

Proof. Let $\delta_{0}^{a}$ be the distributional $d_{a}$-form on $\mathcal{U}_{a}$ supported at the origin $\mathbf{0}$ and satisfy $\int \delta_{\mathbf{0}}^{a}=1$. (Namely it is the delta function times the volume form.) We also take a distributional $\left(d_{a}-1\right)$-form $\kappa_{a}$ on $\mathcal{U}_{a}$ with the following properties.

Properties 28.11. (1) $d \kappa_{a}=\chi_{a}-\delta_{0}^{a}$.

(2) $\kappa_{a}$ is smooth outside the origin.

We put

$$
\chi_{a}^{s}=s \chi_{a}+(1-s) \delta_{0}^{a}
$$


and

$$
\chi_{\mathbf{p}}^{s}=\prod_{i=1}^{\ell} \chi_{\mathbf{p}(i)}^{s}
$$

which defines a distributional $\sum d_{\mathbf{p}(i)}$ form on $\mathcal{U}(\mathbf{p})$. We then define

$$
\mathfrak{q}_{\ell, k ; \beta}^{S, s}\left(\mathbf{p} ; h_{1}, \ldots, h_{k}\right)=\left(\operatorname{ev}_{0}^{\partial}\right) !\left(\operatorname{ev}_{1}^{\partial}, \ldots, \mathrm{ev}_{k}^{\partial}\right)^{*}\left(h_{1} \wedge \cdots \wedge h_{k} \wedge \chi_{\mathbf{p}}^{s}\right) .
$$

Note that because $\chi_{\mathbf{p}}^{s}$ is only a distributional form, existence of the pull-back thereof is not automatic. However using Lemma 28.9 (4) we can show that the pull-back exists and the right hand side of (28.16) gives rise to a smooth differential form.

By extending the definition (28.16) multi-linearly, we obtain the homomorphism

$$
\mathfrak{q}_{\ell, k ; \beta}^{S, s}: E_{\ell}(\mathcal{H}[2]) \otimes B_{k}(\Omega(L(\mathbf{u}))[1]) \rightarrow \Omega(L(\mathbf{u}))[1] .
$$

We use them to define $\left\{\mathfrak{m}_{k}^{S, s, \mathbf{b}}\right\}$ in the same way as in Definition 17.8, Then it is smooth (with respect to $s$ coordinate) in the sense of Definition 28.1.

Sublemma 28.12. $\left(C F_{\mathrm{dR}}\left(L(\mathbf{u}) ; \Lambda_{0}\right),\left\{\mathfrak{m}_{k}^{S, s, \mathbf{b}}\right\}_{k=0}^{\infty}\right)$ is a unital filtered $A_{\infty}$ algebra. Moreover we have:

$$
\mathfrak{m}_{k}^{S, 0, \mathbf{b}}=\mathfrak{m}_{k}^{T, \mathbf{b}}, \quad \mathfrak{m}_{k}^{S, 1, \mathbf{b}}=\mathfrak{m}_{k}^{S, \mathbf{b}} .
$$

The proof is easy and omitted.

We next denote

$$
\kappa_{i, \mathbf{p}}^{s}=\chi_{\mathbf{p}(1)}^{s} \wedge \cdots \wedge \chi_{\mathbf{p}(i-1)}^{s} \wedge \kappa_{\mathbf{p}(i)} \wedge \chi_{\mathbf{p}(i+1)}^{s} \wedge \cdots \wedge \chi_{\mathbf{p}(\ell)}^{s}
$$

and define

$$
\begin{aligned}
& \mathfrak{q c}_{\beta ; \ell, k}^{S, s}\left(\mathbf{p} ; h_{1}, \ldots, h_{k}\right) \\
& =\sum_{i=1}^{\ell}(-1)^{*(i)}\left(\operatorname{ev}_{0}^{\partial}\right) !\left(\operatorname{ev}_{1}^{\partial}, \ldots, \operatorname{ev}_{k}^{\partial}\right)^{*}\left(h_{1} \wedge \cdots \wedge h_{k} \wedge \kappa_{i, \mathbf{p}}^{s}\right) .
\end{aligned}
$$

See Remark 28.16 for the sign. In the same way as the operator $\mathfrak{q}_{\ell, k ; \beta}^{S, s}$ defines $\mathfrak{m}_{k}^{S, s, \mathbf{b}}$, the operator $\mathfrak{q c}_{\beta ; \ell, k}^{S, s}$ induces an operator, which we write $\mathfrak{c}_{k}^{S, s, \mathbf{b}}$. Using Stokes' formula ([FOOO3, Lemma C.9]) and Composition formula ([FOOO3, Lemma C.10]), together with Lemmas 28.8, 28.9, we easily derive that

$$
\left(C F_{\mathrm{dR}}\left(L(\mathbf{u}) ; \Lambda_{0}\right),\left\{\mathfrak{m}_{k}^{S, s, \mathbf{b}}\right\}_{k=0}^{\infty},\left\{\mathfrak{c}_{k}^{S, s, \mathbf{b}}\right\}_{k=0}^{\infty}\right)
$$

is the required pseudo-isotopy. The proof of Lemma 28.10 is complete.

28.4. Wrap-up of the proof of Proposition 20.7. In this subsection, we construct a pseudo-isotopy between $\left(C F_{\mathrm{dR}}\left(L(\mathbf{u}) ; \Lambda_{0}\right),\left\{\mathfrak{m}_{k}^{S, \mathbf{b}}\right\}_{k=0}^{\infty}\right)$ (which is defined in Subsection 28.3) and $\left(C F_{\mathrm{dR}}\left(L(\mathbf{u}) ; \Lambda_{0}\right),\left\{\mathfrak{m}_{k}^{\mathbf{b}}\right\}_{k=0}^{\infty}\right)$ (which is defined in Definition 17.8.)

Together with Theorem 28.5 and Lemma 28.10 this will complete the proof of Proposition 20.7

In Section 27 we already proved that the homotopy equivalence class of

$$
\left(C F_{\mathrm{dR}}\left(L(\mathbf{u}) ; \Lambda_{0}\right),\left\{\mathfrak{m}_{k}^{\mathbf{b}}\right\}_{k=0}^{\infty}\right)
$$

is independent of the choice of de Rham representative $\mathbf{b}$. We make this choice more specifically below.

Let $D_{a}$ be as in the beginning of Subsection 28.3 We put

$$
\mathfrak{b}_{a}=\operatorname{Exp} !\left(\pi_{\mathcal{U}}^{*} \chi_{a}\right),
$$


where we use the map $\left(\operatorname{Exp}, \pi_{\mathcal{U}}\right): D_{a} \times \mathcal{U}_{a} \rightarrow M \times \mathcal{U}_{a}$ as a correspondence. Clearly $\mathfrak{b}_{a}$ is a de Rham representative of the Poincaré dual to $\left[D_{a}\right]$. The de Rham cohomology classes $\left\{\left[\mathfrak{b}_{a}\right]\right\}_{a=1}^{B}$ form a basis of $\bigoplus_{k \neq 0} H^{k}(M ; \mathbb{C})$. We use them to specify the de Rham representatives of the elements of $\bigoplus_{k \neq 0} H^{k}(M ; \Lambda)$. (We represent the 0 -th cohomology class by the constant function.)

We next review two Kuranishi structures on $\mathcal{M}_{k+1 ; \ell}(L(\mathbf{u}) ; \beta ; \mathbf{p}(\mathcal{U}))$ and their perturbations that will enter in the construction of a pseudo-isotopy used in the proof of Proposition 20.7

(Kuranishi structure and perturbations 1) Consider the natural projection

$$
\pi: \mathcal{M}_{k+1 ; \ell}(L(\mathbf{u}) ; \beta ; \mathbf{p}(\mathcal{U})) \rightarrow \mathcal{M}_{k+1 ; \ell}(L(\mathbf{u}) ; \beta) .
$$

We have chosen and fixed a Kuranishi structure on $\mathcal{M}_{k+1 ; \ell}(L(\mathbf{u}) ; \beta)$ in Proposition 17.3. We pull it back by the map (28.20). It defines a Kuranishi structure $\mathcal{K}_{1}$.

In Lemma 17.5 we took and fixed a CF-perturbation on $\mathcal{M}_{k+1 ; \ell}(L(\mathbf{u}) ; \beta)$. We pull it back by the map (28.20) and obtain a CF-perturbation of the Kuranishi structure $\mathcal{K}_{1}$. We denote it by $\widehat{\mathfrak{S}_{1}}=\left\{\widehat{\mathfrak{S}_{1}^{\epsilon}}\right\}$. We use them to define $\mathfrak{q}_{\ell, k ; \beta}^{\widehat{\mathfrak{S}_{\epsilon}^{\epsilon}}}$ by

$$
\mathfrak{q}_{\ell, k ; \beta}^{\widehat{\mathfrak{S}_{1}^{\epsilon}}}\left(\mathbf{p} ; h_{1}, \ldots, h_{k}\right)=\left(\operatorname{ev}_{0}^{\partial}\right) !\left(\left(\mathrm{ev}_{1}^{\partial}, \ldots, \mathrm{ev}_{k}^{\partial}, \pi_{\mathcal{U}}\right)^{*}\left(h_{1} \wedge \cdots \wedge h_{k} \wedge \chi_{\mathbf{p}}\right) ; \widehat{\mathfrak{S}_{1}^{\epsilon}}\right),
$$

where we use the evaluation map

$$
\left(\operatorname{ev}_{0}^{\partial}, \ldots, \mathrm{ev}_{k}^{\partial}, \pi_{\mathcal{U}}\right): \mathcal{M}_{k+1 ; \ell}(L(\mathbf{u}) ; \beta ; \mathbf{p}(\mathcal{U})) \rightarrow L(\mathbf{u})^{k+1} \times \mathcal{U}(\mathbf{p}),
$$

and the $\mathrm{CF}$-perturbation $\widehat{\mathfrak{S}_{1}}$ to define integration along the fiber in the right hand side. See [FOOO11, Definitions 7.78 and 9.13].

Lemma 28.13. Let $\mathfrak{q}_{\ell, k ; \beta}$ be the map defined in (17.9). Then we have $\mathfrak{q}_{\ell, k ; \beta}^{\widehat{\mathfrak{S}_{\epsilon}^{\epsilon}}}=$ $\mathfrak{q}_{\ell, k ; \beta}$.

This lemma is obvious from the definition and (28.19).

(Kuranishi structure and perturbations 2) In Lemma 28.8 we equipped $\mathcal{M}_{k+1 ; \ell}(L(\mathbf{u}) ; \beta ; \mathbf{p}(\mathcal{U}))$ with a Kuranishi structure. We name the Kuranishi structure $\mathcal{K}_{2}$. In Lemma 28.9 we took a multisection of $\mathcal{K}_{2}$. We name the multisection $\mathfrak{s}_{2}$. They determine the operators $\mathfrak{q}_{\ell, k ; \beta}^{S}$ by (28.13).

Thus we have described two systems of Kuranishi structures and perturbations. We next define a system of Kuranishi structures and CF-perturbations on $[0,1] \times$ $\mathcal{M}_{k+1 ; \ell}(L(\mathbf{u}) ; \beta ; \mathbf{p}(\mathcal{U}))$ which interpolate the two systems.

We define

$$
\widehat{\mathrm{ev}}_{i}^{\partial}:[0,1] \times \mathcal{M}_{k+1 ; \ell}(L(\mathbf{u}) ; \beta ; \mathbf{p}(\mathcal{U})) \rightarrow[0,1] \times L(\mathbf{u})
$$

by $\widehat{\operatorname{ev}}_{i}^{\partial}=\left(\pi_{s}, \mathrm{ev}_{i}^{\partial}\right)$ where $\pi_{s}$ is the projection to the $[0,1]$ factor. (We use $s$ as the coordinate of this factor.)

Lemma 28.14. (1) $[0,1] \times \mathcal{M}_{k+1 ; \ell}(L(\mathbf{u}) ; \beta ; \mathbf{p}(\mathcal{U}))$ has a Kuranishi structure with corners.

(2) Its restriction to $\{0\} \times \mathcal{M}_{k+1 ; \ell}(L(\mathbf{u}) ; \beta ; \mathbf{p}(\mathcal{U}))$ coincides with $\mathcal{K}_{1}$ and its restriction to $\{1\} \times \mathcal{M}_{k+1 ; \ell}(L(\mathbf{u}) ; \beta ; \mathbf{p}(\mathcal{U}))$ coincides with $\mathcal{K}_{2}$.

(3) Its normalized boundary is described by the union of

$$
\partial([0,1]) \times \mathcal{M}_{k_{1}+1 ; \# E_{1}}\left(L(\mathbf{u}) ; \beta_{1} ; \mathbf{p}_{1}(\mathcal{U})\right) .
$$


and the union of fiber products

$$
\begin{aligned}
& \left([0,1] \times \mathcal{M}_{k_{1}+1 ; \# E_{1}}\left(L(\mathbf{u}) ; \beta_{1} ; \mathbf{p}_{1}(\mathcal{U})\right)\right) \\
& {\widehat{\mathrm{ev}_{0}}}_{\partial} \times{\widehat{\mathrm{ev}_{i}}}^{\partial}\left([0,1] \times \mathcal{M}_{k_{2}+1 ; \# L_{2}}\left(L(\mathbf{u}) ; \beta_{2} ; \mathbf{p}_{2}(\mathcal{U})\right)\right)
\end{aligned}
$$

where the union is taken over all $\left(E_{1}, E_{2}\right) \in \operatorname{Shuff}(\ell), k_{1}, k_{2}$ with $k_{1}+k_{2}=k$ and $\beta_{1}, \beta_{2} \in H_{2}(M, L(\mathbf{u}) ; \mathbb{Z})$ with $\beta=\beta_{1}+\beta_{2}$. We put $\operatorname{Split}\left(\left(\mathbb{L}_{1}, \mathbb{L}_{2}\right), \mathbf{p}\right)=$ $\left(\mathbf{p}_{1}, \mathbf{p}_{2}\right)$.

(4) The (virtual) dimension is given by

$$
\begin{aligned}
& \operatorname{dim} \mathcal{M}_{k+1 ; \ell}(L(\mathbf{u}) ; \beta ; \mathbf{p}(\mathcal{U})) \\
& =n+\mu_{L(\mathbf{u})}(\beta)+k-1+2 \ell-\sum_{i=1}^{\ell} 2 \operatorname{deg} D_{\mathbf{p}(i)}+\sum_{i=1}^{\ell} d_{\mathbf{p}(i)} .
\end{aligned}
$$

(5) The evaluation map $\widehat{\mathrm{ev}}_{i}^{\partial}$ at any boundary marked point of $\mathcal{M}_{k+1 ; \ell}(L(\mathbf{u}) ; \beta)$ defines a map on $\mathcal{M}_{k+1 ; \ell}(L(\mathbf{u}) ; \beta ; \mathbf{p}(\mathcal{U}))$, which we also denote by $\widehat{\operatorname{ev}}_{i}^{\partial}$. It is compatible with (3).

(6) We can define a coherent system of orientations on the Kuranishi structures so that it is compatible with (3).

(7) $\widehat{\mathrm{ev}}_{0}^{\partial} \times \pi_{\mathcal{U}}$ is weakly submersive.

(8) The Kuranishi structure is invariant under the permutation of interior marked points.

(9) The Kuranishi structure is compatible with the forgetful map of the 1st, 2nd, ..., $k$-th boundary marked points. (We do not require that it is compatible with the forgetful map of the 0-th marked point.)

The proof is the same as that of Lemma 20.4 and is omitted.

Lemma 28.15. There exists a system of CF-perturbations $\widehat{\mathfrak{S}}=\left\{\widehat{\mathfrak{S}}^{\epsilon}\right\}$ of the $K u$ ranishi structure on $[0,1] \times \mathcal{M}_{k+1 ; \ell}(L(\mathbf{u}) ; \beta ; \mathbf{p}(\mathcal{U}))$ given in Lemma 28.14 with the following properties:

(1) They are transversal to 0.

(2) Its restriction to $\{0\} \times \mathcal{M}_{k+1 ; \ell}(L(\mathbf{u}) ; \beta ; \mathbf{p}(\mathcal{U}))$ coincides with $\widehat{\mathfrak{S}_{1}}$ and its restriction to $\{1\} \times \mathcal{M}_{k+1 ; \ell}(L(\mathbf{u}) ; \beta ; \mathbf{p}(\mathcal{U}))$ coincides with $\mathfrak{s}_{2}$.

(3) They are compatible with the description of the boundary in Lemma 28.14 (3).

(4) The map $\widehat{\operatorname{ev}}_{0}^{\partial} \times \pi_{\mathcal{U}}$ is strongly submersive with respece to $\widehat{\mathfrak{S}}$.

(5) They are invariant under the permutation of interior marked points.

(6) They are compatible with the forgetful map of the 1 st, $2 n d, \ldots, k$-th boundary marked points.

As we show in Remark 28.17 we can identify a multisection as a special case of the CF-perturbation. Taken this fact and the existence theorem of CF-perturbation ([FOOO11, Theorem 7.49]) into account, the proof is the same as that of Lemma 20.5 and so omitted.

We now define operators

$$
\mathfrak{q}_{\ell, k ; \beta}^{\text {para }}: E_{\ell}(\mathcal{H}[2]) \otimes B_{k}(\Omega(L(\mathbf{u}))[1]) \rightarrow \Omega([0,1] \times L(\mathbf{u}))[1]
$$

by

$$
\mathfrak{q}_{\ell, k ; \beta}^{\text {para }}\left(\mathbf{p} ; h_{1}, \ldots, h_{k}\right)=\left(\widehat{\mathrm{ev}}_{0}^{\partial}\right) !\left(\left(\widehat{\mathrm{ev}}_{1}^{\partial}, \ldots, \widehat{\mathrm{ev}}_{k}^{\partial}, \pi_{\mathcal{U}}\right)^{*}\left(h_{1} \wedge \cdots \wedge h_{k} \wedge \chi_{\mathbf{p}}\right) ; \widehat{\mathfrak{S}}^{\epsilon}\right)
$$


where we use the evaluation map

$$
\left(\widehat{\mathrm{ev}}_{0}^{\partial}, \ldots, \widehat{\mathrm{ev}}_{k}^{\partial}, \pi_{\mathcal{U}}\right): \mathcal{M}_{k+1 ; \ell}(L(\mathbf{u}) ; \beta ; \mathbf{p}(\mathcal{U})) \rightarrow([0,1] \times L(\mathbf{u}))^{k+1} \times \mathcal{U}(\mathbf{p})
$$

and $\left(\widehat{\mathrm{ev}}_{0}\right)$ ! is the integration along the fibers defined via the CF-perturbation $\widehat{\mathfrak{S}^{\epsilon}}$. (Definition 33.20, FOOO11, Definition 7.78].)

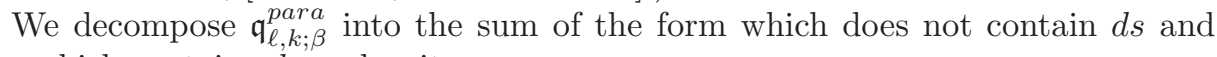
one which contains $d s$ and write:

$$
\mathfrak{q}_{\ell, k ; \beta}^{\text {para }}=\mathfrak{q}_{\ell, k ; \beta}^{\text {para }, 1}+d s \wedge \mathfrak{q}_{\ell, k ; \beta}^{\text {para }, 2}:
$$

More specifically, the two summands are given by

$$
\left.\mathfrak{q}_{\ell, k ; \beta}^{\text {para }, 2}=\frac{\partial}{\partial s}\right\rfloor \mathfrak{q}_{\ell, k ; \beta}^{\text {para }}, \quad \mathfrak{q}_{\ell, k ; \beta}^{\text {para }, 1}=\mathfrak{q}_{\ell, k ; \beta}^{\text {para }}-d s \wedge \mathfrak{q}_{\ell, k ; \beta}^{\text {para }, 2}
$$

Now we put

$$
\begin{aligned}
& \mathfrak{m}_{k}^{\mathbf{b}}\left(x_{1}, \ldots, x_{k}\right) \\
& =\sum_{\beta \in H_{2}(M, L: \mathbb{Z})} \sum_{\ell=0}^{\infty} \sum_{m_{0}=0}^{\infty} \cdots \sum_{m_{k}=0}^{\infty} T^{\omega \cap \beta} \frac{\exp \left(\mathbf{b}_{2 ; 1} \cap \beta\right)}{\ell !} \\
& \mathfrak{q}_{\ell, k+\sum_{i=0}^{k} m_{i} ; \beta}^{\text {para }, 1}\left(\mathfrak{b}_{+}^{\otimes \ell} ; b_{+}^{\otimes m_{0}}, x_{1}, b_{+}^{\otimes m_{1}}, \ldots, b_{+}^{\otimes m_{k-1}}, x_{k}, b_{+}^{\otimes m_{k}}\right), \\
& \mathfrak{c}_{k}^{\mathbf{b}}\left(x_{1}, \ldots, x_{k}\right) \\
& =\sum_{\beta \in H_{2}(M, L: \mathbb{Z})} \sum_{\ell=0}^{\infty} \sum_{m_{0}=0}^{\infty} \cdots \sum_{m_{k}=0}^{\infty} T^{\omega \cap \beta} \frac{\exp \left(\mathbf{b}_{2 ; 1} \cap \beta\right)}{\ell !}
\end{aligned}
$$

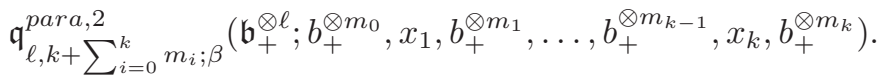

They define maps from $B_{k}(\Omega(L(\mathbf{u})) \widehat{\otimes} \Lambda)$ to $(\Omega([0,1] \times L(\mathbf{u}))) \widehat{\otimes} \Lambda$. By Lemmas $28.1428 .15, \mathfrak{m}_{k}^{\mathbf{b}}$ and $\mathfrak{c}_{k}^{\mathbf{b}}$ define a unital pseudo-isotopy between $\left(C F_{\mathrm{dR}}\left(L(\mathbf{u}) ; \Lambda_{0}\right),\left\{\mathfrak{m}_{k}^{S, \mathbf{b}}\right\}_{k=0}^{\infty}\right)$ and $\left(C F_{\mathrm{dR}}\left(L(\mathbf{u}) ; \Lambda_{0}\right),\left\{\mathfrak{m}_{k}^{\mathbf{b}}\right\}_{k=0}^{\infty}\right)$. The proof of Proposition 20.7 is now complete.

Remark 28.16. We can handle the sign needed in the argument of this section by the same way as in [FOOO3. (See the end of [FOOO3, Appendix C] for the relevant explanation.)

Remark 28.17. In this remark we explain how we can regard a multisection as a CF-perturbation. Let $X$ be a space with Kuranishi structure and $\widehat{\mathcal{U}}=\left\{\mathcal{U}_{\mathfrak{p}} \mid \mathfrak{p} \in \mathfrak{P}\right\}$ be a good coordinate system of $X$. Here $\mathcal{U}_{\mathfrak{p}}=\left(U_{\mathfrak{p}}, \mathcal{E}_{\mathfrak{p}}, \psi_{\mathfrak{p}}, s_{\mathfrak{p}}\right)$ is a Kuranishi chart. (Namely $U_{\mathfrak{p}}$ is an orbifold, $\mathcal{E}_{\mathfrak{p}}$ a vector bundle on it, $s_{\mathfrak{p}}$ its section, and $\psi_{\mathfrak{p}}$ is a homeomorphism from $s_{\mathfrak{p}}^{-1}(0)$ to an open set of $X$.) We suppress the coordinate change from the notation of Kuranishi chart for simplicity. Let $f:(X, \overrightarrow{\mathcal{U}}) \rightarrow N$ be a weakly submersive map to a manifold $N$.

We recall that a CF-perturbation is a continuous family parametrized by $\epsilon>0$, while a multisection does not involve such a parameter. We just call a multisection trasversal to $\{0\}$ a transveral multisection. Based on the consturction of a transveral multisection described in [FOOO11, Section 13], we can construct an $\epsilon$-parametrized family of multisections. We may regard it as a multisection defined on $(X \times[0,1), \widehat{\mathcal{U}} \times[0,1))$ that restricts to the Kuranishi map at $X \times\{0\}$. Moreover we may assume that it is transversal to $\{0\}$ on $X \times(0,1)$. Then for each generic 
$\epsilon>0$ the restriction $\mathfrak{s}_{\epsilon}$ of $\mathfrak{s}$ to $X \times\{\epsilon\}$ defines a transversal multisection. In the situation where we can take a multisection so that the restriction of $f$ to the zero set of the multisection on $(X, \hat{\mathcal{U}})$ is a submersion, we may require in addition that the restriction of $f$ to the zero set of $\mathfrak{s}_{\epsilon}$ becomes a submersion for generic $\epsilon$. This is the case such as that of Lemma 28.15, Lemma 20.5, or the case of $\operatorname{dim} N=0$ (for example (3.7)).

In the latter situation, we will associate to the above constructed multisection a CF-perturbation $\widehat{\mathfrak{S}}$ of $\widehat{\mathcal{U}}$ with the following properties:

(1) $\widehat{\mathfrak{S}}$ is transversal to 0 .

(2) $f$ is strongly submersive with respect to $\hat{\mathcal{U}}$.

(3) Let $h$ be a differential form on $(X, \widehat{\mathcal{U}})$ in the sense of Definition 33.1. Then for a generic choice of sufficiently small $\epsilon$ we have

$$
f !\left(h ; \widehat{\mathfrak{S}}^{\epsilon}\right)=f !\left(h ; \mathfrak{s}_{\epsilon}\right) .
$$

Here the left hand side is the integration along the fibers defined by using the CF-perturbation $\widehat{\mathfrak{S}}$ (See Definition 33.20, [FOOO11, Definition 7.78]) and the right hand side the integration along the fibers on the zero set of $\mathfrak{s}_{\epsilon}$, which is defined in FOOO3, Definition C.1].

Now construction of the CF-perturbation $\widehat{\mathfrak{S}}$ is in order.

First, we note that the given multisection $\mathfrak{s}$ by definition gives a multisection $\mathfrak{s}_{\mathfrak{p}}$ of $\mathcal{U}_{\mathfrak{p}} \times[0,1)$ for each $\mathfrak{p}$. We take an open coverging $U_{\mathfrak{p}}=\bigcup_{a} U_{\mathfrak{p}, a}$ so that each $a$ is associated to orbifold charts $\left(V_{\mathfrak{p}, a}, \Gamma_{\mathfrak{p}, a}, \phi_{\mathfrak{p}, a}\right)$ inducing a homeomorphism $V_{\mathfrak{p}, a} / \Gamma_{\mathfrak{p}, a} \cong U_{\mathfrak{p}, a}$. We also take a trivialization $\left(V_{\mathfrak{p}, a} \times E_{\mathfrak{p}, a}\right) /\left.\Gamma_{\mathfrak{p}, a} \cong \mathcal{E}_{\mathfrak{p}}\right|_{U_{\mathfrak{p}, a}}$. In this coordinate, the multisection $\mathfrak{s}_{\mathfrak{p}}$ induces a map

$$
\mathfrak{s}_{\mathfrak{p}, a}=\left(\mathfrak{s}_{\mathfrak{p}, a, i}\right)_{i=1}^{\ell_{\mathfrak{p}, a}}: V_{\mathfrak{p}, a} \times[0,1) \rightarrow E_{\mathfrak{p}, a}^{\ell_{\mathfrak{p}, a}}
$$

together with a group homomorphism $\sigma_{\mathfrak{p}, a}: \Gamma_{\mathfrak{p}, a} \rightarrow \operatorname{Perm}\left(\ell_{\mathfrak{p}, a}\right)$ to the permutation $\operatorname{group} \operatorname{Perm}\left(\ell_{\mathfrak{p}, a}\right)$ of order $\ell_{\mathfrak{p}, a}$ ! such that for each $i$ it satisfies

$$
\mathfrak{s}_{\mathfrak{p}, a, i}(\gamma x, \epsilon)=\mathfrak{s}_{\mathfrak{p}, a, \sigma_{\mathfrak{p}, a}(\gamma)(i)}(x, \epsilon)
$$

for $x \in V_{\mathfrak{p}, a}, \epsilon \in[0,1)$ and $\gamma \in \Gamma_{\mathfrak{p}, a}$.

We use this map to define a CF-perturbation $\mathcal{S}_{\mathfrak{p}, a}=\left(W_{\mathfrak{p}, a}^{\prime}, \omega_{\mathfrak{p}, a}, \mathfrak{s}_{\mathfrak{p}, a}\right)$ in terms of the given chart so that it satisfies the conditions given in [FOOO11, Definition $7.8]$ as follows.

We take an $\ell_{\mathfrak{p}, a}$ dimensional vector space $W_{\mathfrak{p}, a}=\mathbb{R}^{\ell_{\mathfrak{p}, a}}$ and $\Gamma_{\mathfrak{p}, a}$ acts on $W_{\mathfrak{p}, a}$ by $\sigma_{\mathfrak{p}, a}$. Let $w_{i}=(0, \ldots, 0,1,0, \ldots, 0) \in W_{\mathfrak{p}, a}$ with the $i$-th component 1 . We take a small neighborhood $W_{i}$ of $w_{i}$ in $W_{\mathfrak{p}, a}$ and let $W_{\mathfrak{p}, a}^{\prime}$ be the union of $W_{i}, i=1, \ldots, \ell_{\mathfrak{p}, a}$, which is $\Gamma_{\mathfrak{p}, a}$ invariant. We define a map $\mathfrak{s}_{\mathfrak{p}, a}: V_{\mathfrak{p}, a} \times[0,1) \times W_{\mathfrak{p}, a}^{\prime} \rightarrow E_{\mathfrak{p}, a}$ by setting

$$
\mathfrak{s}_{\mathfrak{p}, a}(x, \epsilon, w)=\mathfrak{s}_{\mathfrak{p}, a, i}(x, \epsilon)
$$

if $w \in W_{i}$. This map is $\Gamma_{\mathfrak{p}, a}$-equivariant. We take a compactly supported $\Gamma_{\mathfrak{p}, a^{-}}$ invariant smooth $\ell_{\mathfrak{p}, a}$-form $\omega_{\mathfrak{p}, a}$ on $W_{\mathfrak{p}, a}^{\prime}$ satisfying $\int_{W_{i}} \omega_{\mathfrak{p}, a}=\frac{1}{\ell_{\mathfrak{p}, a}}$ and put

$$
\mathcal{S}_{\mathfrak{p}, a}=\left(W_{\mathfrak{p}, a}^{\prime}, \omega_{\mathfrak{p}, a}, \mathfrak{s}_{\mathfrak{p}, a}\right) .
$$

Then $\mathcal{S}_{\mathfrak{p}, a}$ is a CF-perturbation and, by construction, the equality (28.27) holds if $h$ is supported on $U_{\mathfrak{p}, a}$.

Again by construction we may take them so that the restriction of $\mathcal{S}_{\mathfrak{p}, a}$ to $U_{\mathfrak{p}, a} \cap$ $U_{\mathfrak{p}, a^{\prime}}$ is equivalent to the restriction of $\mathcal{S}_{\mathfrak{p}^{\prime}, a}$ to $U_{\mathfrak{p}, a} \cap U_{\mathfrak{p}, a^{\prime}}$ in the sense of [FOOO11, 
Definition 7.16]. (See also Definition 33.4) We thus obtain a CF-perturbation $\mathfrak{S}_{\mathfrak{p}}$ of $\mathcal{U}_{\mathfrak{p}}$ for each $\mathfrak{p}$. By construction, $\left\{\mathfrak{S}_{\mathfrak{p}}\right\}$ is compatible with coordinate change in the sense of [FOOO11, Definition 7.47] (that is, Definition 33.7 (1),(2) hold) and so define a CF-perturbation $\widehat{\mathfrak{S}}$ of $(X, \widehat{\mathcal{U}})$. The CF-perturbation $\widehat{\mathfrak{S}}$ of our Kuranishi structure is induced from $\widehat{\mathfrak{S}}$ by [FO0011, Lemma 9.11]. Then (28.27) follows from the fact that $\mathcal{S}_{\mathfrak{p}, a}$ satisfies the equality in case $h$ is in one chart.

28.5. Proof of Lemma 20.9, In this subsection we prove Lemma 20.9, Let

$$
\mathfrak{m}_{k}^{1, \mathbf{b}}: B_{k}((\Omega([0,1] \times L(\mathbf{u})) \widehat{\otimes} \Lambda)[1]) \rightarrow(\Omega([0,1] \times L(\mathbf{u})) \widehat{\otimes} \Lambda)[1]
$$

be the filtered $A_{\infty}$ structure induced from the pseudo-isotopy defined in the proof of Lemma 28.10, Let

$$
\mathfrak{m}_{k}^{2, \mathbf{b}}: B_{k}((\Omega([0,1] \times L(\mathbf{u}) \widehat{\otimes} \Lambda)[1]) \rightarrow(\Omega([0,1] \times L(\mathbf{u})) \widehat{\otimes} \Lambda)[1]
$$

be the filtered $A_{\infty}$ structure induced from the pseudo-isotopy constructed in Subsection 28.4

They induce chain complexes

$$
\left((\Omega([0,1] \times L(\mathbf{u})) \widehat{\otimes} \Lambda), \mathfrak{m}_{1}^{1, \mathbf{b}}\right), \quad\left((\Omega([0,1] \times L(\mathbf{u})) \widehat{\otimes} \Lambda), \mathfrak{m}_{2}^{1, \mathbf{b}}\right) .
$$

We have chain homotopy equivalences

$$
\begin{aligned}
& \operatorname{Eval}_{s=0}:\left((\Omega([0,1] \times L(\mathbf{u})) \widehat{\otimes} \Lambda), \mathfrak{m}_{1}^{1, \mathbf{b}}\right) \rightarrow\left(\Omega(L(\mathbf{u}) \widehat{\otimes} \Lambda), \mathfrak{m}_{1}^{T, \mathbf{b}}\right), \\
& \operatorname{Eval}_{s=1}:\left((\Omega([0,1] \times L(\mathbf{u})) \widehat{\otimes} \Lambda), \mathfrak{m}_{1}^{1, \mathbf{b}}\right) \rightarrow\left(\Omega(L(\mathbf{u}) \widehat{\otimes} \Lambda), \mathfrak{m}_{1}^{S, \mathbf{b}}\right),
\end{aligned}
$$

and

$$
\begin{aligned}
& \operatorname{Eval}_{s=0}:\left((\Omega([0,1] \times L(\mathbf{u})) \widehat{\otimes} \Lambda), \mathfrak{m}_{1}^{2, \mathbf{b}}\right) \rightarrow\left(\Omega(L(\mathbf{u}) \widehat{\otimes} \Lambda), \mathfrak{m}_{1}^{S, \mathbf{b}}\right), \\
& \operatorname{Eval}_{s=1}:\left(\left(\Omega([0,1] \times L(\mathbf{u}) \widehat{\otimes} \Lambda), \mathfrak{m}_{1}^{2, \mathbf{b}}\right) \rightarrow\left(\Omega(L(\mathbf{u}) \widehat{\otimes} \Lambda), \mathfrak{m}_{1}^{\mathbf{b}}\right),\right.
\end{aligned}
$$

that are defined by (27.3).

Therefore to prove Lemma 20.9 it suffices to construct chain maps:

$$
\begin{aligned}
& i_{\mathrm{qm}, \mathbf{b}}^{S}: \Omega(M) \widehat{\otimes} \Lambda \rightarrow \Omega\left(L(\mathbf{u}) \widehat{\otimes} \Lambda ; \mathfrak{m}_{1}^{T, \mathbf{b}}\right), \\
& i_{\mathrm{qm}, \mathbf{b}}^{1}: \Omega(M) \widehat{\otimes} \Lambda \rightarrow\left((\Omega([0,1] \times L(\mathbf{u})) \widehat{\otimes} \Lambda) ; \mathfrak{m}_{1}^{1, \mathbf{b}}\right), \\
& i_{\mathrm{qm}, \mathbf{b}}^{2}: \Omega(M) \widehat{\otimes} \Lambda \rightarrow\left((\Omega([0,1] \times L(\mathbf{u})) \widehat{\otimes} \Lambda) ; \mathfrak{m}_{1}^{2, \mathbf{b}}\right),
\end{aligned}
$$

that satisfy

$$
\begin{array}{ll}
\mathrm{Eval}_{s=0} \circ i_{\mathrm{qm}, \mathbf{b}}^{1}=i_{\mathrm{qm}, \mathbf{b}}^{T}, & \mathrm{Eval}_{s=1} \circ i_{\mathrm{qm}, \mathbf{b}}^{1}=i_{\mathrm{qm}, \mathbf{b}}^{S}, \\
\mathrm{Eval}_{s=0} \circ i_{\mathrm{qm}, \mathbf{b}}^{2}=i_{\mathrm{qm}, \mathbf{b}}^{S}, & \mathrm{Eval}_{s=1} \circ i_{\mathrm{qm}, \mathbf{b}}^{2}=i_{\mathrm{qm}, \mathbf{b}} .
\end{array}
$$

We can construct such $i_{\mathrm{qm}, \mathbf{b}}^{S}, i_{\mathrm{qm}, \mathbf{b}}^{1}, i_{\mathrm{qm}, \mathbf{b}}^{2}$ by modifying the definition of $i_{\mathrm{qm}, \mathbf{b}}$ (17.17) in an obvious way. The proof of Lemma 20.9 is complete.

\section{Seidel homomorphism With BUlK}

In this section we generalize the Seidel homomorphism [Se to a version with bulk deformation. We then generalize, in the next section, the results by EntovPolterovich [EP1, Section 4] and by McDuff-Tolman [MT] on the relationship between the Seidel homomorphism and the Calabi quasi-morphism. These generalizations are rather straightforward and do not require novel ideas. 
29.1. Seidel homomorphism with bulk. In this subsection, we present a version of Seidel's construction Se] that incorporates bulk deformations.

Let $H$ be a one-periodic Hamiltonian such that $\phi_{H}:[0,1] \rightarrow \operatorname{Ham}(M, \omega)$ defines a loop, i.e. satisfies $\psi_{H}=\phi_{H}^{1}=i d$. Such a loop is called a Hamiltonian loop. For such $H$, there is a diffeomorphism $M \rightarrow \operatorname{Per}(H)$ : For each point $p \in M$, we assign a periodic orbit defined by

$$
z_{p}^{H}(t)=\phi_{H}^{t}(p)
$$

Then the map $p \mapsto z_{p}^{H}$ is a one-to-one correspondence $M \rightarrow \operatorname{Per}(H)$.

Let $v: \mathbb{R} \times S^{1} \rightarrow M$ be any continuous map. We define $u: \mathbb{R} \times S^{1} \rightarrow M$ by

$$
u(\tau, t)=\phi_{H}^{t}(v(\tau, t)) .
$$

Lemma 29.1. Let $p_{-}, p_{+} \in M$. Then

$$
\lim _{\tau \rightarrow-\infty} u(\tau, t)=z_{p_{-}}^{H}(t), \quad \lim _{\tau \rightarrow+\infty} u(\tau, t)=z_{p_{+}}^{H}(t),
$$

if and only if

$$
\lim _{\tau \rightarrow-\infty} v(\tau, t)=p_{-}, \quad \lim _{\tau \rightarrow+\infty} v(\tau, t)=p_{+} .
$$

The proof is a straightforward calculation. For a map $u$ satisfying the above conditions, we define a homology class $[u] \in H_{2}(M ; \mathbb{Z})$ by setting $[u]:=[v]$. (Note by the asymptotic conditions given $v$ extends to a map from $S^{2}$ so $[v] \in H_{2}(M ; \mathbb{Z})$ is defined.)

We define a symplectic fibration

$$
\pi: E_{\phi_{H}} \rightarrow \mathbb{C} P^{1}
$$

with its fiber isomorphic to $(M, \omega)$ as follows. Let $D_{ \pm}$be two copies of the unit disc in $\mathbb{C}$. Set $U_{1}=D_{-} \times M, U_{2}=\left(\mathbb{R} \times S^{1}\right) \times M$ and $U_{3}=D_{+} \times M$. We glue them by the gluing maps

$$
I_{-}:(-\infty, 0) \times S^{1} \times M \rightarrow D_{-} \backslash\{0\} \times M, \quad I_{-}((\tau, t), x)=\left(e^{2 \pi(\tau+\sqrt{-1} t)}, x\right)
$$

(where we regard $S^{1}=\mathbb{R} / \mathbb{Z}$, ) and

$I_{+}:(1, \infty) \times S^{1} \times M \rightarrow D_{+} \backslash\{\infty\} \times M, \quad I_{+}((\tau, t), x)=\left(e^{-2 \pi(\tau-1+\sqrt{-1} t)},\left(\phi_{H}^{t}\right)^{-1}(x)\right)$.

We thus obtain

$$
E_{\phi_{H}}=U_{1} \cup U_{2} \cup U_{3} .
$$

The projections to the second factor induce a map

$$
\pi: E_{\phi_{H}} \rightarrow D_{-} \cup\left(\mathbb{R} \times S^{1}\right) \cup D_{+} \cong \mathbb{C} P^{1} .
$$

This defines a locally trivial fiber bundle and the fiber of $\pi$ is diffeomorphic to $M$.

In fact, $E_{\phi_{H}} \rightarrow \mathbb{C} P^{1}$ becomes a Hamiltonian fiber bundle. See GLS for the precise definition of Hamiltonian fiber bundle and its associated coupling form $\Omega$ that we use below. We also refer to $[\mathrm{Sc} 2, \mathrm{E}, \mathrm{Oh} 4$ ] for their applications to the Floer theory and spectral invariants.

Lemma 29.2. The fibration $E_{\phi_{H}} \rightarrow \mathbb{C} P^{1}$ is a Hamiltonian fiber bundle, i.e., it carries a coupling form $\Omega$ on $E_{\phi_{H}}$ such that

(1) $\Omega$ is closed and $\left.\Omega\right|_{E_{\phi_{H}, \gamma}}=\omega$,

(2) $\pi ! \Omega^{n+1}=0$ where $\pi !$ is the integration over fiber and $2 n=\operatorname{dim} M$. 
Proof. On each of $U_{i}, i=1,2,3$, we pull back $\omega$ by the projection to $M$ and denote it by $\omega_{i}$. We put $\omega_{2}^{\prime}=\omega_{2}+d(\chi H d t)$. Then we find that $\omega_{1}$ on $U_{1}, \omega_{2}^{\prime}$ on $U_{2}$ and $\omega_{3}$ on $U_{3}$ are glued to a closed 2 -form $\Omega$ on $E_{\phi_{H}}$. The normalization condition on $H$ then gives rise to the condition $\pi ! \Omega^{n+1}=0$.

Let $u: \mathbb{R} \times S^{1} \rightarrow M$ be a continuous map. We denote the associated section $\widehat{u}: \mathbb{R} \times S^{1} \rightarrow E_{\phi_{H}}$ by the formula

$$
\widehat{u}(\tau, t)=((\tau, t), u(\tau, t))
$$

on $U_{2}$.

Lemma 29.3. Let $u: \mathbb{R} \times S^{1} \rightarrow M$ be a continuous map. The following is equivalent:

(1) There exists some $p_{-}, p_{+} \in M$ such that

$$
\lim _{\tau \rightarrow-\infty} u(\tau, t)=z_{p_{-}}^{H}(t), \quad \lim _{\tau \rightarrow+\infty} u(\tau, t)=z_{p_{+}}^{H}(t) .
$$

(2) The map $\widehat{u}$ extends to a section $s_{u}: \mathbb{C} P^{1} \rightarrow E_{\phi_{H}}$.

The proof is obvious by definition of $E_{\phi_{H}}$. Let $u_{1}, u_{2}$ satisfy the condition (1) above. We say that $u_{1}$ is homologous to $u_{2}$ if

$$
\left[\widehat{u}_{1}\right]=\left[\widehat{u}_{2}\right] \in H_{2}\left(E_{\phi_{H}} ; \mathbb{Z}\right) .
$$

Let $\Pi_{2}(M ; H)$ be the set of the homology classes of such $u$. We note that

$$
\left[\widehat{u}_{1}\right]-\left[\widehat{u}_{2}\right] \in \operatorname{Ker}\left(H_{2}\left(E_{\phi_{H}} ; \mathbb{Z}\right) \rightarrow H_{2}\left(\mathbb{C} P^{1} ; \mathbb{Z}\right)\right) .
$$

Therefore $\Pi_{2}(M ; H)$ is a principal homogeneous space of the group $\operatorname{Ker}\left(H_{2}\left(E_{\phi_{H}} ; \mathbb{Z}\right) \rightarrow\right.$ $\left.\mathrm{H}_{2}\left(\mathbb{C} P^{1} ; \mathbb{Z}\right)\right)$.

We also have a natural marking $M \cong E_{\{0\}}$ of the fibration $E_{\phi_{H}} \rightarrow \mathbb{C} P^{1}$ via the inclusion map

$$
M \times\{0\} \subset M \times \mathbb{C} \subset E_{\phi_{H}}
$$

which we will fix once and for all. Then the natural inclusion induces a map $H_{2}(M ; \mathbb{Z}) \rightarrow \operatorname{Ker}\left(H_{2}\left(E_{\phi_{H}} ; \mathbb{Z}\right) \rightarrow H_{2}\left(\mathbb{C} P^{1} ; \mathbb{Z}\right)\right)$. Therefore there exists an action

$$
H_{2}(M ; \mathbb{Z}) \times \Pi_{2}(M ; H) \rightarrow \Pi_{2}(M ; H)
$$

of the group $H_{2}(M ; \mathbb{Z})$ to $\Pi_{2}(M ; H)$.

Remark 29.4. Theorem 29.9 which we will prove later implies that

$$
H_{2}(M ; \mathbb{Q}) \cong \operatorname{Ker}\left(H_{2}\left(E_{\phi_{H}} ; \mathbb{Q}\right) \rightarrow H_{2}\left(\mathbb{C} P^{1} ; \mathbb{Q}\right)\right) \text {. }
$$

We however do not use this fact.

Let $J_{0}$ be a compatible almost complex structure on $M$. For $t \in S^{1}$, we define

$$
J_{t}^{H}=\left(\phi_{H}^{t}\right)_{*} J_{0} .
$$

Since $\phi_{H}^{t}$ is a symplectic diffeomorphism, $J_{t}^{H}$ is compatible with $\omega$. We denote by $J^{H}=\left\{J_{t}^{H}\right\}_{t \in S^{1}}$ the above $S^{1}$-parametrized family of compatible almost complex structures.

We take $\chi \in \mathcal{K}$ and consider $H_{\chi}$ as in (3.13). We also take an $\left(\mathbb{R} \times S^{1}\right)$ parametrized family of almost complex structures $J_{\chi}^{H}$ such that

$$
J_{\chi}^{H}(\tau, t)= \begin{cases}J_{0} & \tau \leq 0, \\ J_{t}^{H} & \tau \geq 1, \\ J_{0} & t \text { is in a neighborhood of }[0] \in S^{1} .\end{cases}
$$


Definition 29.5. For $\alpha \in \Pi_{2}(M ; H)$ we denote by $\stackrel{\circ}{\mathcal{M}}_{\ell}\left(H_{\chi}, J_{\chi}^{H} ; *, z_{*}^{H} ; \alpha\right)$ the set of all pairs $\left(u ; z_{1}^{+}, \ldots, z_{\ell}^{+}\right)$of maps $u: \mathbb{R} \times S^{1} \rightarrow M$ and $z_{1}^{+}, \ldots, z_{\ell}^{+} \in \mathbb{R} \times S^{1}$, which satisfy the following conditions:

(1) The map $u$ satisfies the equation:

$$
\frac{\partial u}{\partial \tau}+J_{\chi}^{H}\left(\frac{\partial u}{\partial t}-\chi(\tau) X_{H_{t}}(u)\right)=0 .
$$

(2) The energy

$$
\frac{1}{2} \int\left(\left|\frac{\partial u}{\partial \tau}\right|_{J_{\chi}^{H}}^{2}+\left|\frac{\partial u}{\partial t}-\chi(\tau) X_{H_{t}}(u)\right|_{J_{\chi}^{H}}^{2}\right) d t d \tau
$$

is finite.

(3) The map $u$ satisfies the condition that there exists $p_{+} \in M$ such that

$$
\lim _{\tau \rightarrow+\infty} u(\tau, t)=z_{p_{+}}^{H}(t) .
$$

(4) The homology class of $u$ in $\Pi_{2}(M ; H)$ is $\alpha$.

(5) $z_{i}^{+}$are all distinct.

By our construction, the map

$$
\bar{u}: \mathbb{R} \times S^{1} \rightarrow M, \quad \bar{u}(\tau, t)=\left(\phi_{H}^{t}\right)^{-1} u(\tau, t)
$$

is $J_{0}$-holomorphic on $[1, \infty) \times S^{1}$ on $M$. Therefore we can apply removable singularity theorem to $\bar{u}$ which gives rise to a section $\widehat{u}$ mentioned in Lemma 29.3.

We denote by

$$
\mathrm{ev}_{ \pm \infty}: \stackrel{\circ}{\mathcal{M}}_{\ell}\left(H_{\chi}, J_{\chi}^{H} ; *, z_{*}^{H} ; \alpha\right) \rightarrow M
$$

the map which associates to $u$ the $\operatorname{limit}_{\tau \rightarrow \pm \infty} \bar{u}(\tau, 0)$. We define the evaluation maps at $z_{i}$ :

$$
\mathrm{ev}=\left(\mathrm{ev}_{1}, \ldots, \mathrm{ev}_{\ell}\right): \stackrel{\circ}{\mathcal{M}}_{\ell}\left(H_{\chi}, J_{\chi}^{H} ; *, z_{*}^{H} ; \alpha\right) \rightarrow\left(E_{\phi_{H}}\right)^{\ell}
$$

by

$$
\mathrm{ev}_{i}\left(u ; z_{1}, \ldots, z_{\ell}\right)=\left(z_{i}, u\left(z_{i}\right)\right) \in U_{2} \subset E_{\phi_{H}} .
$$

Definition 29.6. For $\alpha \in H_{2}(M ; \mathbb{Z})$ we define $\widehat{\dot{M}_{\ell}}\left(H, J^{H} ; z_{*}^{H}, z_{*}^{H} ; \alpha\right)$ to be the set of all pairs $\left(u ; z_{1}^{+}, \ldots, z_{\ell}^{+}\right)$of maps $u: \mathbb{R} \times S^{1} \rightarrow M$ and marked points $z_{1}^{+}, \ldots, z_{\ell}^{+} \in$ $\mathbb{R} \times S^{1}$, which satisfy the following conditions:

(1) The map $u$ satisfies the equation:

$$
\frac{\partial u}{\partial \tau}+J^{H_{t}}\left(\frac{\partial u}{\partial t}-X_{H_{t}}(u)\right)=0
$$

(2) The energy

$$
\frac{1}{2} \int\left(\left|\frac{\partial u}{\partial \tau}\right|_{J^{H_{t}}}^{2}+\left|\frac{\partial u}{\partial t}-X_{H_{t}}(u)\right|_{J^{H_{t}}}^{2}\right) d t d \tau
$$

is finite.

(3) There exist points $p_{ \pm} \in M$ such that

$$
\lim _{\tau \rightarrow-\infty} u(\tau, t)=z_{p_{-}}^{H}, \quad \lim _{\tau \rightarrow+\infty} u(\tau, t)=z_{p_{+}}^{H} .
$$

(4) The homology class of $u$ is $\alpha$.

(5) $z_{i}^{+}$are all distinct. 


\section{$\widehat{o}$}

There is an $\mathbb{R}$-action on $\stackrel{\circ}{\mathcal{M}}_{\ell}\left(H, J^{H} ; z_{*}^{H}, z_{*}^{H} ; \alpha\right)$ that is induced by the translations of the $\mathbb{R}$ direction (namely $\tau \mapsto \tau+c$ ). The action is free if $\alpha \neq 0$ or $\ell \neq 0$. We denote its quotient space by $\stackrel{\circ}{\mathcal{M}}\left(H, J^{H} ; z_{*}^{H}, z_{*}^{H} ; \alpha\right)$. If $\alpha=0=\ell$, we define $\stackrel{\circ}{\mathcal{M}}_{\ell}\left(H, J^{H} ; z_{*}^{H}, z_{*}^{H} ; \alpha\right)$ to be the empty set.

We define evaluation maps $\operatorname{ev}_{ \pm \infty}: \widehat{\grave{M}}_{\ell}\left(H, J^{H} ; z_{*}^{H}, z_{*}^{H} ; \alpha\right) \rightarrow M$ by

$$
\mathrm{ev}_{ \pm \infty}(u)=\lim _{\tau \rightarrow \pm \infty}\left(\phi_{H}^{t}\right)^{-1}(u(\tau, t)) \text {. }
$$

Here we would like to point out that for any $u \in \widehat{\mathcal{M}_{\ell}}\left(H, J^{H} ; z_{*}^{H}, z_{*}^{H} ; \alpha\right)$ the right hand side of (29.9) converges to $p_{ \pm} \in M$ that is independent of $t$. Therefore the evaluation map is well-defined. The maps ev $\mathrm{e}_{ \pm}$factor through $\stackrel{\circ}{\mathcal{M}}_{\ell}\left(H, J^{H} ; z_{*}^{H}, z_{*}^{H} ; \alpha\right)$.

We define ev $=\left(\mathrm{ev}_{1}, \ldots, \mathrm{ev}_{\ell}\right): \widehat{\stackrel{\mathcal{M}}{\ell}}_{\ell}\left(H, J^{H} ; z_{*}^{H}, z_{*}^{H} ; \alpha\right) \rightarrow M^{\ell}$ as follows.

$$
\mathrm{ev}_{i}\left(u ; z_{1}^{+}, \ldots, z_{\ell}^{+}\right)=\phi_{H}^{-t}\left(u\left(z_{i}^{+}\right)\right)
$$

where $z_{i}^{+}=(\tau, t)$. It factors through $\stackrel{\mathcal{M}}{\ell}_{\ell}\left(H, J^{H} ; z_{*}^{H}, z_{*}^{H} ; \alpha\right)$ also.

We consider the case $H=0$ in $\widehat{\dot{M}}_{\ell}\left(H, J^{H} ; z_{*}^{H}, z_{*}^{H} ; \alpha\right)$ and write it $\widehat{\dot{M}}_{\ell}(H=$ $\left.0, J_{0} ; *, * ; \alpha\right)$. (Note that $J_{t}^{H}=J_{0}$ if $H=0$.)

Lemma 29.7. $\widehat{\mathcal{M}}_{\ell}\left(H, J^{H} ; z_{*}^{H}, z_{*}^{H} ; \alpha\right)$ is isomorphic to $\widehat{\mathcal{M}}_{\ell}\left(H=0, J_{0} ; *, * ; \alpha\right)$. The isomorphism is compatible with evaluation maps and $\mathbb{R}$ actions.

Proof. Let $\left(u ; z_{1}^{+}, \ldots, z_{\ell}^{+}\right) \in \dot{\mathcal{M}}_{\ell}\left(H, J^{H} ; z_{*}^{H}, z_{*}^{H} ; \alpha\right)$ we put

$$
v(\tau, t)=\left(\phi_{H}^{t}\right)^{-1}(u(\tau, t)) .
$$

Then $\left(v ; z_{1}^{+\prime}, \ldots, z_{\ell}^{+\prime}\right) \in \stackrel{\circ}{\mathcal{M}}_{\ell}\left(H=0, J_{0} ; *, * ; \alpha\right)$. The assignment $\left(u ; z_{1}^{+}, \ldots, z_{\ell}^{+}\right) \mapsto$ $\left(v ; z_{1}^{+\prime}, \ldots, z_{\ell}^{+\prime}\right)$ gives the required isomorphism.

We can prove that $\stackrel{\circ}{\mathcal{M}}_{\ell}\left(H, J^{H} ; z_{*}^{H}, z_{*}^{H} ; \alpha\right)$ and $\stackrel{\mathcal{M}}{\ell}_{\ell}\left(H=0, J_{0} ; *, * ; \alpha\right)$ have compactifications $\mathcal{M}_{\ell}\left(H, J^{H} ; z_{*}^{H}, z_{*}^{H} ; \alpha\right)$ and $\mathcal{M}_{\ell}\left(H=0, J_{0} ; *, * ; \alpha\right)$, respectively. They have Kuranishi structures which are isomorphic. We can define an $S^{1}$ action on $\mathcal{M}_{\ell}\left(H=0, J_{0} ; *, * ; \alpha\right)$ by using the $S^{1}$ action on $\mathbb{R} \times S^{1}$. We then use the isomorphism to define an $S^{1}$ action on the domain $\mathcal{M}_{\ell}\left(H, J^{H} ; z_{*}^{H}, z_{*}^{H} ; \alpha\right)$. The evaluation maps are compatible with this action. The isotropy group of this $S^{1}$ action is always finite. (We note that we have $\alpha \neq 0$ or $\ell \neq 0$ by definition.)

Lemma 29.8. (1) The moduli space $\stackrel{\mathcal{M}}{\ell}_{\ell}\left(H_{\chi}, J_{\chi}^{H} ; *, z_{*}^{H} ; \alpha\right)$ has a compactification $\mathcal{M}_{\ell}\left(H_{\chi}, J_{\chi}^{H} ; *, z_{*}^{H} ; \alpha\right)$ that is Hausdorff.

(2) The space $\mathcal{M}_{\ell}\left(H_{\chi}, J_{\chi}^{H} ; *, z_{*}^{H} ; \alpha\right)$ has an orientable Kuranishi structure with corners.

(3) The normalized boundary of $\mathcal{M}_{\ell}\left(H_{\chi}, J_{\chi}^{H} ; *, z_{*}^{H} ; \alpha\right)$ is described as the union of the following two types of fiber products.

$\bigcup \mathcal{M}_{\# \mathbb{L}_{1}}\left(H_{\chi}, J_{\chi}^{H} ; *, z_{*}^{H} ; \alpha_{1}\right)_{e v_{+\infty}} \times_{e v_{-\infty}} \mathcal{M}_{\# \mathbb{L}_{2}}\left(H, J^{H} ; z_{*}^{H}, z_{*}^{H} ; \alpha_{2}\right)$ 
where the union is taken over all $\alpha_{1}, \alpha_{2} \in \Pi_{2}(M ; H)$ with $\alpha_{1}+\alpha_{2}=\alpha$ and $\left(\mathbb{L}_{1}, \mathbb{L}_{2}\right) \in \operatorname{Shuff}(\ell)$. Here $\alpha_{1}+\alpha_{2}$ is as in (29.4). The fiber product is taken over $M$.

$\bigcup \mathcal{M}_{\# \mathbb{L}_{1}}\left(H=0, J_{0} ; *, * ; \alpha_{1}\right)_{e v_{+\infty}} \times_{e v_{-\infty}} \mathcal{M}_{\# \mathbb{L}_{2}}\left(H_{\chi}, J_{\chi}^{H} ; *, z_{*}^{H} ; \alpha_{2}\right)$

where the union is taken over all $\alpha_{1}, \alpha_{2} \in \Pi_{2}(M ; H)$ with $\alpha_{1}+\alpha_{2}=\alpha$ and $\left(\mathbb{L}_{1}, \mathbb{L}_{2}\right) \in \operatorname{Shuff}(\ell)$. The fiber product is taken over $M$.

(4) We may choose $\alpha_{0} \in \Pi_{2}(M ; H)$ such that the (virtual) dimension satisfies the following equality (29.13).

$$
\operatorname{dim} \mathcal{M}_{\ell}\left(H_{\chi}, J_{\chi}^{H} ; *, z_{*}^{H} ; \alpha_{0}+\alpha\right)=2 c_{1}(M) \cap \alpha+2 n+2 \ell .
$$

(5) We can define a system of orientations on $\mathcal{M}_{\ell}\left(H_{\chi}, J_{\chi}^{H} ; *, z_{*}^{H} ; \alpha\right)$ so that the isomorphisms (3) above are compatible with this orientations.

(6) The valuation maps (29.10) extend to $\mathcal{M}_{\ell}\left(H_{\chi}, J_{\chi}^{H} ; *, z_{*}^{H} ; \alpha\right)$ in a way compatible with (3) above.

(7) The map ev $+\infty$ becomes a weakly submersive map in the sense of [FOOO2, Definition A1.13] (Definition 32.1). Here $\mathrm{ev}_{+\infty}$ is defined in the same way as in (29.9).

(8) The Kuranishi structure is invariant under the permutation of interior marked points.

Here the compatibility with the evaluation maps claimed in (6) above is described as follows. Let us consider the boundary in (29.11). Let $i \in \mathrm{E}_{2}$ be the $j$-th element of $\mathrm{E}_{2}$. We have

$$
\mathrm{ev}_{i}: \mathcal{M}_{\ell}\left(H_{\chi}, J_{\chi}^{H} ; *, z_{*}^{H} ; \alpha\right) \rightarrow E_{\phi_{H}}
$$

and

$$
\mathrm{ev}_{j}: \mathcal{M}_{\# \mathrm{E}_{2}}\left(H, J^{H} ; z_{*}^{H}, z_{*}^{H} ; \alpha\right) \rightarrow M .
$$

Denote by $t$ the second coordinate of the marked point in $\mathbb{R} \times S^{1}$. Then $\left(\phi_{H}^{t}\right)^{-1} \circ \mathrm{ev}_{j}$ is equal to second factor of the $\mathrm{ev}_{i}$ with respect to $U_{3} \cong D_{+} \times M$.

The proof of Lemma 29.8 is the same as the proof of Proposition 3.6 and is omitted. Note the end of $\mathcal{M}_{\ell}\left(H_{\chi}, J_{\chi}^{H} ; *, z_{*}^{H} ; \alpha\right)$ at which an element of $\overline{\mathcal{M}}\left(0, J_{0} ; *, * ; \alpha\right)$ bubbles off as $\tau \rightarrow-\infty$ may be regarded as codimension 2 because of $S^{1}$ symmetry, where the latter moduli space corresponds to the case of $H=0$ for the moduli space $\overline{\mathcal{M}}\left(H_{\chi}, J_{\chi}^{H} ; *, z_{*}^{H} ; \alpha\right)$.

To define the operators involving bulk deformations, we need the following result due to Lalonde-McDuff-Polterovich [LMP.

Theorem 29.9 (Lalonde-McDuff-Polterovich). There exists a section

$$
H^{*}(M ; \mathbb{C}) \rightarrow H^{*}\left(E_{\phi_{H}} ; \mathbb{C}\right)
$$

of the $\mathbb{C}$-linear map $H^{*}\left(E_{\phi_{H}} ; \mathbb{C}\right) \rightarrow H^{*}(M ; \mathbb{C})$ which is induced by the inclusion $M \times\{0\} \rightarrow E_{\phi_{H}}$.

Remark 29.10. (1) Theorem 29.9 is [LMP, Theorem 3B]. We give a proof of Theorem 29.9 in Subsection 29.3 for completeness' sake. The proof we give in Subsection 29.3 is basically the same as the one given in [LMP.

(2) The proof by [LMP as well as our proof in Subsection 29.3] uses the construction which is closely related to the definition of Seidel homomorphism. We use Theorem 29.9 to define the Seidel homomorphism with bulk. However the argument is not circular by the following reason. We do not use 
Theorem 29.9 to define Seidel homomorphism in the case when the bulk deformation $\mathfrak{b}$ is zero. The proof of Theorem 29.9 uses the construction of Seidel homomorphism without bulk only, that is the case $\mathfrak{b}=0$.

Consider a system of CF-perturbations of $\mathcal{M}_{\ell}\left(H, J_{H} ; z_{*}^{H}, z_{*}^{H} ; \alpha\right)$ and of $\mathcal{M}_{\ell}(H=$ $\left.0, J_{0} ; *, * ; \alpha\right)$ which is transversal to $0, S^{1}$-equivariant and compatible with the isomorphism in Lemma 29.7. Moreover we may assume that the system is compatible with the identification

$$
\begin{aligned}
& \partial \mathcal{M}_{\ell}\left(H, J^{H} ; z_{*}^{H}, z_{*}^{H} ; \alpha\right) \\
& =\bigcup \mathcal{M}_{\# \mathbb{L}_{1}}\left(H, J^{H} ; z_{*}^{H}, z_{*}^{H} ; \alpha_{1}\right)_{\mathrm{ev}+\infty} \times_{\mathrm{ev}_{-\infty}} \mathcal{M}_{\# \mathbb{L}_{2}}\left(H, J^{H} ; z_{*}^{H}, z_{*}^{H} ; \alpha_{2}\right)
\end{aligned}
$$

of the boundary. Furthermore we may assume that the evaluation map $\mathrm{ev}_{+\infty}$ is strongly submersive with respect to this CF-perturbations.

Then, there exists a system of CF-perturbations of the moduli space $\mathcal{M}_{\ell}\left(H_{\chi}, J_{\chi}^{H} ; *, z_{*}^{H} ; \alpha\right)$ such that they are transversal to 0, compatible with the description of the boundary in Lemma 29.8 (3) and that $\mathrm{ev}_{+\infty}$ is strongly submersive with respect thereto.

Let $\mathfrak{b}=\mathfrak{b}_{0}+\mathfrak{b}_{2}+\mathfrak{b}_{+}$be as in (5.5). Using Theorem 29.9, we regard them as de Rham cohomology classes of $E_{\phi_{H}}$ and denote them as $\widehat{\mathfrak{b}}_{2}, \widehat{\mathfrak{b}}_{+}$.

Now we define a map

$$
\mathcal{S}_{\left(H_{\chi}, J_{\chi}\right)}^{\mathfrak{b}}: \Omega(M) \widehat{\otimes} \Lambda \rightarrow \Omega(M) \widehat{\otimes} \Lambda
$$

as follows. Let $h \in \Omega(M)$. We put

$$
\mathcal{S}_{\left(H_{\chi}, J_{\chi}\right) ; \alpha}^{\mathfrak{b}}(h)=\sum_{\ell=0}^{\infty} \frac{\exp \left(\int_{\alpha} \widehat{\mathfrak{b}}_{2}\right)}{\ell !} \operatorname{ev}+\infty !\left(\mathrm{ev}^{*}(\underbrace{\widehat{\mathfrak{b}}_{+}, \ldots, \widehat{\mathfrak{b}}_{+}}_{\ell}) \wedge \mathrm{ev}_{-\infty}^{*} h\right)
$$

where we use the correspondence

$$
\left(\mathrm{ev} ; \mathrm{ev}_{-\infty}, \mathrm{ev}_{+\infty}\right): \mathcal{M}_{\ell}\left(H_{\chi}, J_{\chi}^{H} ; *, z_{*}^{H} ; \alpha\right) \rightarrow E_{\phi_{H}}^{\ell} \times M^{2},
$$

and the above constructed CF-perturbation to define integration along the fibers. We define $\int_{\alpha} \widehat{\mathfrak{b}}_{2}$ as follows. Let $u \in \stackrel{\circ}{\mathcal{M}}_{0}\left(H_{\chi}, J_{\chi}^{H} ; *, z_{*}^{H} ; \alpha\right)$. It induces a map $\widehat{u}$ : $\mathbb{C} P^{1} \rightarrow E_{\phi_{H}}$. We put

$$
\int_{\alpha} \widehat{\mathfrak{b}}_{2}=\int_{\mathbb{C} P^{1}} \widehat{u}^{*} \widehat{\mathfrak{b}}_{2}
$$

It is easy to see that the integral depends only on $\alpha$ and is independent of the representative $u$.

Let $u \in \stackrel{\circ}{\mathcal{M}}_{0}\left(H_{\chi}, J_{\chi}^{H} ; *, z_{*}^{H} ; \alpha\right)$ and $p=\operatorname{ev}_{+\infty}(u)$. Then $\left[z_{p}^{H}, u\right] \in \operatorname{Crit}\left(\mathcal{A}_{H}\right)$. We put

$$
\mathcal{A}_{H}(\alpha):=\mathcal{A}_{H}\left(\left[z_{p}^{H}, u\right]\right)
$$

We then define

$$
\mathcal{S}_{\left(H_{\chi}, J_{\chi}\right)}^{\mathfrak{b}}=\sum_{\alpha} T^{-\mathcal{A}_{H}(\alpha)} \mathcal{S}_{\left(H_{\chi}, J_{\chi}\right) ; \alpha}^{\mathfrak{b}}
$$

Lemma 29.11. $\mathcal{A}_{H}\left(\left[z_{p}^{H}, u\right]\right)$ depends only on the homology class $\alpha$ but independent of its representative $u$. 
Proof. Recall the map $I_{+}: U_{2} \rightarrow U_{3}$ which was defined as $I_{+}(\tau, t, x)=\left(e^{-2 \pi(\tau-1+\sqrt{-1} t)},\left(\phi_{H}^{t}\right)^{-1}(x)\right)$. It is easy to see that $I_{+}^{*} \Omega=\omega$ where $\omega$ is the pull back of the symplectic form of $M$ to $U_{2}, U_{3}$ and $\Omega$ is as in Lemma 29.2

We have

$$
\int \widehat{u}^{*} \Omega=\int u^{*} \omega+\int H_{t}\left(z_{p}^{H}(t)\right) d t=-\mathcal{A}_{H}\left(\left[\left(z_{p}^{H}, w\right)\right]\right) .
$$

The lemma follows from Stokes' theorem.

Lemma 29.12. The map $\mathcal{S}_{\left(H_{\chi}, J_{\chi}\right)}^{\mathfrak{b}}$ satisfies

$$
\mathcal{S}_{\left(H_{\chi}, J_{\chi}^{H}\right)}^{\mathfrak{b}} \circ d=d \circ \mathcal{S}_{\left(H_{\chi}, J_{\chi}^{H}\right)}^{\mathfrak{b}}
$$

and so descends to a map

$$
\mathcal{S}_{\left(H_{\chi}, J_{\chi}^{H}\right), *}^{\mathfrak{b}}: H^{*}(M ; \Lambda) \rightarrow H^{*}(M ; \Lambda) .
$$

The lemma follows from Lemma 29.8(3), Stokes' formula (Theorem 34.8, FOOO11, Corollary 8.13]) and Composition formula (Theorem 35.7, [FOOO11, Theorem $10.20])$.

Theorem 29.13. $\quad$ (1) $\mathcal{S}_{\left(H_{\chi}, J_{\chi}^{H}\right), *}^{\mathfrak{b}}$ is independent of the family of compatible almost complex structures $J_{\chi}^{H}$ and other choices involved such as multisection.

(2) $\mathcal{S}_{\left(H_{\chi}, J_{\chi}^{H}\right), *}^{\mathfrak{b}}$ depends only on the homotopy class of the loop $t \mapsto \phi_{H}^{t}$ in the group of Hamiltonian diffeomorphisms.

(3) We have

$$
\mathcal{S}_{\left(H_{\chi}, J_{\chi}^{H}\right), *}^{\mathfrak{b}}\left(x \cup^{\mathfrak{b}} y\right)=x \cup^{\mathfrak{b}} \mathcal{S}_{\left(H_{\chi}, J_{\chi}^{H}\right), *}^{\mathfrak{b}}(y) .
$$

(4) Let $H_{1}, H_{2}$ be periodic Hamiltonians satisfying $\psi_{H_{1}}=\psi_{H_{2}}=$ identity. Then we have

$$
\mathcal{S}_{\left(\left(H_{1} \# H_{2}\right)_{\chi}, J_{\chi}^{H_{1} \# H_{2}}\right), *}^{\mathfrak{b}}\left(x \cup^{\mathfrak{b}} y\right)=\mathcal{S}_{\left(\left(H_{1}\right)_{\chi_{1}}, J_{\chi}^{H_{1}}\right), *}^{\mathfrak{b}}(x) \cup^{\mathfrak{b}} \mathcal{S}_{\left(\left(H_{2}\right)_{\chi}, J_{\chi}^{H_{2}}\right), *}^{\mathfrak{b}}(y) .
$$

We define the map $\mathcal{S}^{\mathfrak{b}}: \pi_{1}(\operatorname{Ham}(M, \omega)) \rightarrow H(M ; \Lambda)$ by

$$
\mathcal{S}^{\mathfrak{b}}\left(\left[\phi_{H}\right]\right)=\mathcal{S}_{\left(H_{\chi}, J_{\chi}^{H}\right), *}^{\mathfrak{b}}(1) .
$$

Here $H$ is a time-dependent Hamiltonian such that $\psi_{H}=1$. $\left[\phi_{H}\right]$ is the homotopy class of the loop in $\operatorname{Ham}(M ; \omega)$ determined by $t \mapsto \phi_{H}^{t} .1$ is the unit of $H(M ; \Lambda)$. (Note that 1 is also the unit with respect to the quantum cup product on $Q H_{\mathfrak{b}}(M ; \Lambda)$ with the bulk.)

The proof of Theorem 29.13 will be given in Subsection 29.2 for completeness' sake.

Corollary 29.14. $\mathcal{S}^{\mathfrak{b}}$ is a homomorphism to the group $Q H_{\mathfrak{b}}(M ; \Lambda)^{\times}$of invertible elements of $Q H_{\mathfrak{b}}(M ; \Lambda)$.

Definition 29.15. We call the representation

$$
\mathcal{S}^{\mathfrak{b}}: \pi_{1}(\operatorname{Ham}(M ; \omega)) \rightarrow Q H_{\mathfrak{b}}(M ; \Lambda)^{\times} .
$$

Seidel homomorphism with bulk. 
Remark 29.16. As mentioned before the homomorphism $\mathcal{S}^{\mathfrak{b}}$ is obtained by Seidel Se] in the case $\mathfrak{b}=0$ under certain hypothesis on the symplectic manifold $(M, \omega)$. Once the virtual fundamental chain technique had been established in the year 1996, it is obvious that we can generalize $\mathrm{Se}$ to arbitrary $(M, \omega)$. The generalization to include bulk deformations is also straightforward and do not require novel ideas.

Proof. We prove Corollary29.14assuming Theorem29.13 Let $\left[\phi_{H_{i}}\right] \in \pi_{1}(\operatorname{Ham}(M ; \omega))$. We have $\left[\phi_{H_{1} \# H_{2}}\right]=\left[\phi_{H_{2}}\right]\left[\phi_{H_{1}}\right]$. Then using Theorem 29.13 (3), (4) we have:

$$
\begin{aligned}
\mathcal{S}^{\mathfrak{b}}\left(\left[\phi_{H_{1} \# H_{2}}\right]\right) & =\mathcal{S}_{\left(\left(H_{1} \# H_{2}\right)_{\chi}, J_{\chi}^{H_{1} \# H_{2}}\right), *}^{\mathfrak{b}}(1) \\
& =\mathcal{S}_{\left(\left(H_{1}\right)_{\chi}, J_{\chi}^{H_{1}}\right), *}^{\mathfrak{b}}(1) \cup^{\mathfrak{b}} \mathcal{S}_{\left(\left(H_{2}\right)_{\chi}, J_{\chi}^{H_{2}}\right), *}^{\mathfrak{b}}(1)=\mathcal{S}^{\mathfrak{b}}\left(\left[\phi_{H_{1}}\right]\right) \cup^{\mathfrak{b}} \mathcal{S}^{\mathfrak{b}}\left(\left[\phi_{H_{2}}\right]\right) .
\end{aligned}
$$

Thus $\mathcal{S}^{\mathfrak{b}}$ is a homomorphism. It implies in particular that the elements of the image are invertible.

29.2. Proof of Theorem 29.13. The proof of Theorem 29.13 (1),(2) is similar to the proof of Theorem 7.7 and so omitted.

The proof of Theorem 29.13 (3),(4) is similar to the proof of Theorem 11.11 and proceeds as follows.

Let $\Sigma$ be as in Subsection 11.1 We also use the notations $h: \Sigma \rightarrow \mathbb{R}, \mathfrak{S} \subset \Sigma$ and $\varphi: \mathbb{R} \times((0,1 / 2) \sqcup(1 / 2,1)) \rightarrow \Sigma \backslash \mathfrak{S}$ etc. in Subsection 11.1. We define a $\Sigma$-parametrized family of almost complex structures $J^{H_{1}, H_{2}}$ by $J^{H_{1}, H_{2}}(\varphi(\tau, t))=$ $J_{t}^{H_{1} \# H_{2}}$. We assume that $\left(H_{1}\right)_{t}=\left(H_{2}\right)_{t}=0$ if $t$ is in a neighborhood of [0] $\in S^{1}=$ $\mathbb{R} / \mathbb{Z}$. Let $H^{\varphi}: \Sigma \times M \rightarrow \mathbb{R}$ be a function as in (11.6).

Definition 29.17. We denote by $\stackrel{\circ}{\mathcal{M}}_{\ell}\left(H^{\varphi}, J^{H_{1}, H_{2}} ; z_{*}^{H_{1}}, z_{*}^{H_{2}}, z_{*}^{H_{1} \# H_{2}} ; \alpha\right)$ the set of all pairs $\left(u ; z_{1}^{+}, \ldots, z_{\ell}^{+}\right)$of maps $u: \Sigma \rightarrow M$ and $z_{i}^{+} \in \Sigma$, which satisfy the following conditions:

(1) The map $\bar{u}=u \circ \varphi$ satisfies the equation:

$$
\frac{\partial \bar{u}}{\partial \tau}+J^{H_{1}, H_{2}}\left(\frac{\partial \bar{u}}{\partial t}-X_{H^{\varphi}}(\bar{u})\right)=0 .
$$

(2) The energy

$$
\frac{1}{2} \int\left(\left|\frac{\partial \bar{u}}{\partial \tau}\right|_{J^{H_{1}, H_{2}}}^{2}+\left|\frac{\partial \bar{u}}{\partial t}-X_{H^{\varphi}}(\bar{u})\right|_{J^{H_{1}, H_{2}}}^{2}\right) d t d \tau
$$

is finite.

(3) There exist $p_{-, 1}, p_{-, 2}, p_{+} \in M$ such that $u$ satisfies the following three asymptotic boundary conditions.

$$
\begin{gathered}
\lim _{\tau \rightarrow+\infty} u(\varphi(\tau, t))=z_{p_{+}}^{H_{1} \# H_{2}}(t) . \\
\lim _{\tau \rightarrow-\infty} u(\varphi(\tau, t))= \begin{cases}z_{p_{-, 1}}^{H_{1}}(2 t) & t \leq 1 / 2, \\
z_{p_{-, 2}}^{H_{2}}(2 t-1) & t \geq 1 / 2 .\end{cases}
\end{gathered}
$$

(4) The homology class of $u$ is $\alpha$, in the sense we explain below.

(5) $z_{1}^{+}, \ldots, z_{\ell}^{+}$are mutually distinct. 
Here the homology class of $u$ which we mention in (4) above is defined as follows. We put

$$
v(\tau, t)=\left\{\begin{array}{l}
\left(\phi_{2 H_{1}}^{t}\right)^{-1}(u(\tau, t)) \quad \tau \leq 0,0 \leq t \leq 1 / 2 \\
\left(\phi_{2 H_{2}}^{t-1 / 2}\right)^{-1}(u(\tau, t)) \quad \tau \leq 0,1 / 2 \leq t \leq 1 \\
\left(\phi_{H_{1} \# H_{2}}^{t}\right)^{-1}(u(\tau, t)) \quad \tau \geq 0
\end{array}\right.
$$

It defines a map $\Sigma \rightarrow M$ which extends to a continuous map $v: S^{2} \rightarrow M$. (Note that $\Sigma$ is $S^{2} \backslash\{3$ points $\}$.) The homology class of $u$ is by definition nothing but the class $v_{*}\left(\left[S^{2}\right]\right) \in H_{2}(M ; \mathbb{Z})$.

We denote by

$$
\left(\mathrm{ev}_{-\infty, 1}, \mathrm{ev}_{-\infty, 2}, \mathrm{ev}_{+\infty}\right): \stackrel{\circ}{\mathcal{M}}_{\ell}\left(H^{\varphi}, J^{H_{1}, H_{2}} ; z_{*}^{H_{1}}, z_{*}^{H_{2}}, z_{*}^{H_{1} \# H_{2}} ; \alpha\right) \rightarrow M^{3}
$$

the map which associates $\left(p_{-, 1}, p_{-, 2}, p_{+}\right)$to $\left(u ; z_{1}^{+}, \ldots, z_{\ell}^{+}\right)$. We also define an evaluation map

$$
\mathrm{ev}=\left(\mathrm{ev}_{1}, \ldots, \mathrm{ev}_{\ell}\right): \stackrel{\circ}{\mathcal{M}}_{\ell}\left(H^{\varphi}, J^{H_{1}, H_{2}} ; z_{*}^{H_{1}}, z_{*}^{H_{2}}, z_{*}^{H_{1} \# H_{2}} ; \alpha\right) \rightarrow M^{\ell}
$$

that associates to $\left(u ; z_{1}^{+}, \ldots, z_{\ell}^{+}\right)$the point $\left(u\left(z_{1}^{+}\right), \ldots, u\left(z_{\ell}^{+}\right)\right)$.

Lemma 29.18. (1) The moduli space $\stackrel{\circ}{\mathcal{M}}_{\ell}\left(H^{\varphi}, J^{H_{1}, H_{2}} ; z_{*}^{H_{1}}, z_{*}^{H_{2}}, z_{*}^{H_{1} \# H_{2}} ; \alpha\right)$ has a compactification $\mathcal{M}_{\ell}\left(H^{\varphi}, J^{H_{1}, H_{2}} ; z_{*}^{H_{1}}, z_{*}^{H_{2}}, z_{*}^{H_{1} \# H_{2}} ; \alpha\right)$ that is Hausdorff.

(2) The space $\mathcal{M}_{\ell}\left(H^{\varphi}, J^{H_{1}, H_{2}} ; z_{*}^{H_{1}}, z_{*}^{H_{2}}, z_{*}^{H_{1} \# H_{2}} ; \alpha\right)$ has an orientable Kuranishi structure with corners.

(3) The normalized boundary of $\mathcal{M}_{\ell}\left(H^{\varphi}, J^{H_{1}, H_{2}} ; z_{*}^{H_{1}}, z_{*}^{H_{2}}, z_{*}^{H_{1} \# H_{2}} ; \alpha\right)$ is described by the union of the following three types of fiber products.

$\mathcal{M}_{\# \mathbb{L}_{1}}\left(H_{1}, J^{H_{1}} ; z^{H_{1}} z_{*}^{H_{1}} ; \alpha_{1}\right){ }_{\mathrm{ev}_{+\infty}} \times_{\mathrm{ev}_{-\infty}, 1} \mathcal{M}_{\# \mathbb{L}_{2}}\left(H^{\varphi}, J^{H_{1}, H_{2}} ; z_{*}^{H_{1}}, z_{*}^{H_{2}}, z_{*}^{H_{1} \# H_{2}} ; \alpha_{2}\right)$ where the union is taken over all $\alpha_{1}, \alpha_{2}$ with $\alpha_{1}+\alpha_{2}=\alpha$, and $\left(\mathbb{L}_{1}, \mathbb{L}_{2}\right) \in$ $\operatorname{Shuff}(\ell)$.

$\mathcal{M}_{\# \mathbb{L}_{1}}\left(H_{2}, J^{H_{2}} ; z_{,}^{H_{2}} z_{*}^{H_{2}} ; \alpha_{1}\right){ }_{\mathrm{ev}+\infty} \times_{\mathrm{ev}_{-\infty}, 2} \mathcal{M}_{\# \mathbb{L}_{2}}\left(H^{\varphi}, J^{H_{1}, H_{2}} ; z_{*}^{H_{1}}, z_{*}^{H_{2}}, z_{*}^{H_{1} \# H_{2}} ; \alpha_{2}\right)$

where the union is taken over all $\alpha_{1}, \alpha_{2}$ with $\alpha_{1}+\alpha_{2}=\alpha$, and $\left(\mathbb{L}_{1}, \mathbb{L}_{2}\right) \in$ $\operatorname{Shuff}(\ell)$.

$$
\begin{aligned}
& \left.\mathcal{M}_{\# \mathbb{L}_{2}}\left(H^{\varphi}, J^{H_{1}, H_{2}} ; z_{*}^{H_{1}}, z_{*}^{H_{2}}, z_{*}^{H_{1} \# H_{2}} ; \alpha_{1}\right)\right) \\
& \mathrm{ev}_{+\infty} \times_{\mathrm{ev}_{-\infty}} \mathcal{M}_{\# \mathbb{L}_{1}}\left(H_{1} \# H_{2}, J^{H_{1} \# H_{2}} ; z_{*}^{H_{1} \# H_{2}}, z_{*}^{H_{1} \# H_{2}} ; \alpha_{2}\right)
\end{aligned}
$$

where the union is taken over all $\alpha_{1}, \alpha_{2}$ with $\alpha_{1}+\alpha_{2}=\alpha$, and $\left(\mathbb{L}_{1}, \mathbb{L}_{2}\right) \in$ $\operatorname{Shuff}(\ell)$.

(4) The (virtual) dimension is given by

$\operatorname{dim} \mathcal{M}_{\ell}\left(H^{\varphi}, J^{H_{1}, H_{2}} ; z_{*}^{H_{1}}, z_{*}^{H_{2}}, z_{*}^{H_{1} \# H_{2}} ; \alpha\right)=2 \ell+2 c_{1}(M)[\alpha]+2 n$.

(5) We can define a system of orientations on $\mathcal{M}_{\ell}\left(H^{\varphi}, J^{H_{1}, H_{2}} ; z_{*}^{H_{1}}, z_{*}^{H_{2}}, z_{*}^{H_{1} \# H_{2}} ; \alpha\right)$ so that the isomorphism (3) above is compatible with this orientation.

(6) $\mathrm{ev}_{-\infty, 1}, \mathrm{ev}_{-\infty, 2}, \mathrm{ev}_{+\infty}$, ev extend to strongly continuous smooth maps on $\mathcal{M}_{\ell}\left(H^{\varphi}, J^{H_{1}, H_{2}} ; z_{*}^{H_{1}}, z_{*}^{H_{2}}, z_{*}^{H_{1} \# H_{2}} ; \alpha\right)$, which we denote also by the same symbol. They are compatible with (3).

(7) $\mathrm{ev}_{+\infty}$ is weakly submersive. 
(8) The Kuranishi structure is invariant under the permulation of interior marked points.

We take a system of CF-perturbations on $\mathcal{M}_{\ell}\left(H^{\varphi}, J^{H_{1}, H_{2}} ; z_{*}^{H_{1}}, z_{*}^{H_{2}}, z_{*}^{H_{1} \# H_{2}} ; \alpha\right)$ that are transversal to 0 , compatible with (3) above and such that $\mathrm{ev}_{+\infty}$ is strongly submersive with respect to our CF-perturbations.

We define $\mathfrak{m}_{2 ; \alpha}^{\mathrm{cl}, H^{\varphi}}: \Omega(M) \otimes \Omega(M) \rightarrow \Omega(M)$ by

$$
\begin{aligned}
& \mathfrak{m}_{2 ; \alpha}^{\mathrm{cl} ; \mathfrak{b} ; H^{\varphi}}\left(h_{1}, h_{2}\right) \\
& =\sum_{\ell=0}^{\infty} \frac{1}{\ell !}\left(\mathrm{ev}_{+\infty}\right) !\left(\mathrm{ev}_{-\infty, 1}^{*} h_{1} \wedge \mathrm{ev}_{-\infty, 2}^{*} h_{2} \wedge \mathrm{ev}^{*}(\underbrace{\mathfrak{b}_{+}, \ldots, \mathfrak{b}_{+}}_{\ell})\right),
\end{aligned}
$$

where we use $\mathcal{M}_{\ell}\left(H^{\varphi}, J^{H_{1}, H_{2}} ; z_{*}^{H_{1}}, z_{*}^{H_{2}}, z_{*}^{H_{1} \# H_{2}} ; \alpha\right)$ and the evaluation maps thereon as a correspondence to define the right hand side.

We put

$$
\mathfrak{m}_{2}^{\mathrm{cl}, \mathfrak{b} ; H^{\varphi}}=\sum_{\alpha} e^{\alpha \cap \widehat{\mathfrak{b}}_{2}} T^{\alpha \cap \omega} \mathfrak{m}_{2 ; \alpha}^{\mathrm{cl} ; \mathfrak{b} ; H^{\varphi}} .
$$

It defines a chain map, since the contribution of the boundaries described in (3) above to the correspondence are all zero by the $S^{1}$ equivariance. Therefore $\mathfrak{m}_{2}^{\mathrm{cl}, \mathfrak{b} ; H^{\varphi}}$ defines a map

$$
\mathfrak{m}_{2}^{\mathrm{cl}, \mathfrak{b} ; H^{\varphi}}: H^{*}(M ; \Lambda) \otimes_{\Lambda} H^{*}(M ; \Lambda) \rightarrow H^{*}(M ; \Lambda) .
$$

Lemma 29.19.

is chain homotopic to

$$
\mathfrak{m}_{2}^{\mathrm{cl} ; \mathfrak{b} ; H^{\varphi}} \circ\left(\mathcal{S}_{\left(\left(H_{1}\right)_{\chi}, J_{\chi}^{H_{1}}\right)}^{\mathfrak{b}} \otimes \mathcal{S}_{\left(\left(H_{2}\right)_{\chi}, J_{\chi}^{H_{2}}\right)}^{\mathfrak{b}}\right)
$$

$$
\mathcal{S}_{\left(\left(H_{1} \# H_{2}\right)_{\chi}, J_{\chi}^{\left.H_{1} \# H_{2}\right)}\right.}^{\mathfrak{b}} \circ \cup^{\mathfrak{b}} .
$$

Proof. For $S \in \mathbb{R}$ we define $H_{S, \chi}^{\varphi}: \Sigma \times M \rightarrow \mathbb{R}$ by

$$
H_{S, \chi}^{\varphi}(\varphi(\tau, t), x)=\chi(\tau+S)\left(H_{1} \# H_{2}\right)_{t}(x) .
$$

Note that $J_{\chi}^{H}$ is the $\left(\mathbb{R} \times S^{1}\right)$-parameterized family of almost complex structures as in (29.6). For $S \in \mathbb{R}$ we define a $\Sigma$ parameterized family of almost complex structures $J_{S, \chi}^{H_{1}, H_{2}}$ by

$$
J_{S, \chi}^{H_{1}, H_{2}}(\varphi(\tau, t))=J_{\chi}^{\left(H_{1} \# H_{2}\right)}(\tau+S, t) .
$$

Let $\alpha \in \Pi_{2}\left(M ; H_{1} \# H_{2}\right)$.

Definition 29.20. For $S \in \mathbb{R}$, we denote by $\stackrel{\circ}{\mathcal{M}}_{\ell}\left(H_{S, \chi}^{\varphi}, J_{S, \chi}^{H_{1}, H_{2}} ; * *, z_{*}^{H_{1} \# H_{2}} ; \alpha\right)$ the set of all pairs $\left(u ; z_{1}^{+}, \ldots, z_{\ell}^{+}\right)$of maps $u: \Sigma \rightarrow M$ and $z_{i}^{+} \in \Sigma$, which satisfy the following conditions:

(1) The map $\bar{u}=u \circ \varphi$ satisfies the equation:

$$
\frac{\partial \bar{u}}{\partial \tau}+J_{S, \chi}^{H_{1}, H_{2}}\left(\frac{\partial \bar{u}}{\partial t}-X_{H_{S, \chi}^{\varphi}}(\bar{u})\right)=0
$$

(2) The energy

$$
\frac{1}{2} \int\left(\left|\frac{\partial \bar{u}}{\partial \tau}\right|_{J_{S, \chi}^{H_{1}, H_{2}}}^{2}+\left|\frac{\partial \bar{u}}{\partial t}-X_{H_{S, \chi}^{\varphi}}(\bar{u})\right|_{J_{S, \chi}^{H_{1}, H_{2}}}^{2}\right) d t d \tau
$$

is finite. 
(3) There exist $p_{-, 1}, p_{-, 2}, p_{+} \in M$ such that $u$ satisfies the following three asymptotic boundary conditions.

$$
\begin{gathered}
\lim _{\tau \rightarrow+\infty} u(\varphi(\tau, t))=z_{p_{+}}^{H_{1} \# H_{2}}(t) . \\
\lim _{\tau \rightarrow-\infty} u(\varphi(\tau, t))= \begin{cases}p_{-, 1} & t \leq 1 / 2, \\
p_{-, 2} & t \geq 1 / 2 .\end{cases}
\end{gathered}
$$

(4) The homology class of $u$ is $\alpha$, in the sense we explain below.

(5) $z_{1}^{+}, \ldots, z_{\ell}^{+}$are mutually distinct.

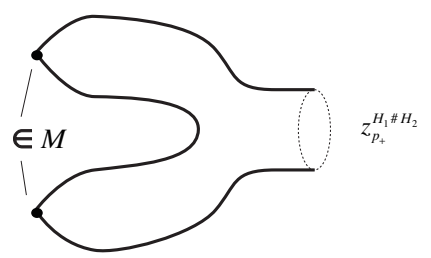

Figure 27. An element of $\stackrel{\circ}{\mathcal{M}}_{\ell}\left(H_{S, \chi}^{\varphi}, J_{S, \chi}^{H_{1}, H_{2}} ; * *, z_{*}^{H_{1} \# H_{2}} ; \alpha\right)$

Here the homology class of $u$ which we mention in (4) above is defined as follows. We consider $\Sigma \times M$ and glue $M$ at the two ends corresponding to $\tau \rightarrow-\infty$ by $\left(\phi_{H_{1}}^{2 t}\right)^{-1}$ $0 \leq t \leq 1 / 2$ and $\left(\phi_{H_{2}}^{2 t-1}\right)^{-1}, 1 / 2 \leq t \leq 1$, respectively. At the end corresponding to $\tau \rightarrow+\infty$ we glue $M$ but with twisting using the map $\phi_{H_{1} \# H_{2}}$ in the same way as the definition of $E_{H^{\varphi}}$. We then obtain $E_{H^{\varphi}}$. Actually this space together with projection to $S^{2}=\Sigma \cup\{3$ points $\}$ can be identified with $E_{\phi_{H_{1} \# H_{2}}}$. We define

$$
\widehat{u}(\tau, t)=((\tau, t), u(\tau, t)) \in E_{H^{\varphi}} .
$$

It extends to a continuous map $\widehat{u}: S^{2} \rightarrow E_{H^{\varphi}}$. The homology class of $\widehat{u}$ is well defined as an element of $\Pi_{2}\left(M ; H_{1} \# H_{2}\right)$.

By Theorem 29.9 we obtain $\widehat{\mathfrak{b}}_{2} \in H^{2}\left(E_{H^{\varphi}} ; \mathbb{C}\right)$ from $\mathfrak{b}_{2} \in H^{2}(M ; \mathbb{C})$.

We denote by

$$
\left(\mathrm{ev}_{-\infty, 1}, \mathrm{ev}_{-\infty, 2}, \mathrm{ev}_{+\infty}\right): \stackrel{\circ}{\mathcal{M}}_{\ell}\left(H_{S, \chi}^{\varphi}, J_{S, \chi}^{H_{1}, H_{2}} ; * *, z_{*}^{H_{1} \# H_{2}} ; \alpha\right) \rightarrow M^{3}
$$

the map which associate $\left(p_{-, 1}, p_{-, 2}, p_{+}\right)$to $\left(u ; z_{1}^{+}, \ldots, z_{\ell}^{+}\right)$. We also define an evaluation map

$$
\mathrm{ev}=\left(\mathrm{ev}_{1}, \ldots, \mathrm{ev}_{\ell}\right): \stackrel{\circ}{\mathcal{M}}_{\ell}\left(H_{S, \chi}^{\varphi}, J_{S, \chi}^{H_{1}, H_{2}} ; * *, z_{*}^{H_{1} \# H_{2}} ; \alpha\right) \rightarrow\left(E_{H^{\varphi}}\right)^{\ell}
$$

that associates to $\left(u ; z_{1}^{+}, \ldots, z_{\ell}^{+}\right)$the point $\left(\widehat{u}\left(z_{i}^{+}\right), \ldots, \widehat{u}\left(z_{\ell}^{+}\right)\right)$. We put

$\stackrel{\circ}{\mathcal{M}}_{\ell}\left(\right.$ para $\left.; H_{\chi}^{\varphi}, J_{\chi}^{H_{1}, H_{2}} ; * *, z_{*}^{H_{1} \# H_{2}} ; \alpha\right)=\bigcup_{S \in \mathbb{R}}\{S\} \times \stackrel{\circ}{\mathcal{M}}_{\ell}\left(H_{S, \chi}^{\varphi}, J_{S, \chi}^{H_{1}, H_{2}} ; * *, z_{*}^{H_{1} \# H_{2}} ; \alpha\right)$.

The above evaluation maps are defined on it in an obvious way.

We can define a compactification $\mathcal{M}_{\ell}\left(\right.$ para $\left.; H_{\chi}^{\varphi}, J_{\chi}^{H_{1}, H_{2}} ; * *, z_{*}^{H_{1} \# H_{2}} ; \alpha\right)$ of the moduli space $\stackrel{\mathcal{M}}{\ell}_{\ell}\left(\right.$ para $\left.; H_{\chi}^{\varphi}, J_{\chi}^{H_{1}, H_{2}} ; * *, z_{*}^{H_{1} \# H_{2}} ; \alpha\right)$ and a system of Kuranishi structures on it, that are oriented with corners. Its boundary is a union of the following 
five types of fiber products:

$$
\begin{aligned}
& \mathcal{M}_{\# \mathbb{L}_{1}}\left(H=0, J_{0} ; *, * ; \alpha_{1}\right) \\
& \mathrm{ev}_{+\infty} \times_{\mathrm{ev}_{-\infty, 1}} \mathcal{M}_{\# \mathbb{L}_{2}}\left(\text { para } ; H_{\chi}^{\varphi}, J_{\chi}^{H_{1}, H_{2}} ; * *, z_{*}^{H_{1} \# H_{2}} ; \alpha_{2}\right),
\end{aligned}
$$

where the union is taken over all $\alpha_{1}, \alpha_{2}$ with $\alpha_{1}+\alpha_{2}=\alpha$, and $\left(\mathbb{L}_{1}, \mathbb{L}_{2}\right) \in \operatorname{Shuff}(\ell)$.

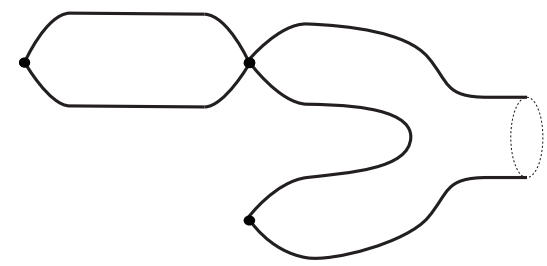

Figure 28. An element of (29.29)

$$
\begin{aligned}
& \mathcal{M}_{\# \mathbb{L}_{1}}\left(H=0, J_{0} ; *, * ; \alpha_{1}\right) \\
& \mathrm{ev}_{+\infty} \times_{\mathrm{ev}_{-\infty, 2}} \mathcal{M}_{\# \mathbb{L}_{2}}\left(\text { para } ; H_{\chi}^{\varphi}, J_{\chi}^{H_{1}, H_{2}} ; * *, z_{*}^{H_{1} \# H_{2}} ; \alpha_{2}\right),
\end{aligned}
$$

where the union is taken over all $\alpha_{1}, \alpha_{2}$ with $\alpha_{1}+\alpha_{2}=\alpha$, and $\left(\mathbb{L}_{1}, \mathbb{L}_{2}\right) \in \operatorname{Shuff}(\ell)$.

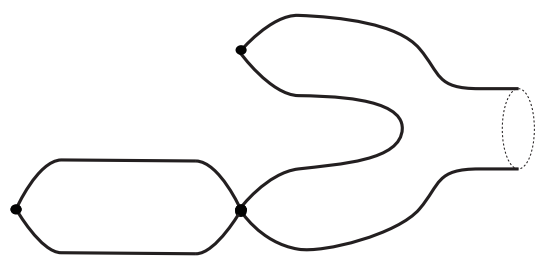

Figure 29. An element of 29.30

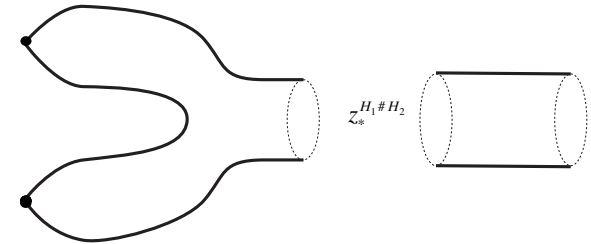

Figure 30. An element of 29.31)

$$
\begin{aligned}
& \mathcal{M}_{\# \mathbb{L}_{1}}\left(\text { para } ; H_{\chi}^{\varphi}, J_{\chi}^{H_{1}, H_{2}} ; * *, z_{*}^{H_{1} \# H_{2}} ; \alpha_{1}\right) \\
& \mathrm{ev}_{+\infty} \times_{\mathrm{ev}_{-\infty}} \mathcal{M}_{\# \mathbb{L}_{2}}\left(H_{1} \# H_{2}, J^{H_{1} \# H_{2}} ; z_{*}^{H_{1} \# H_{2}}, z_{*}^{H_{1} \# H_{2}} ; \alpha_{2}\right),
\end{aligned}
$$

where the union is taken over all $\alpha_{1}, \alpha_{2}$ with $\alpha_{1}+\alpha_{2}=\alpha$, and $\left(\mathbb{L}_{1}, \mathbb{L}_{2}\right) \in \operatorname{Shuff}(\ell)$.

$$
\mathcal{M}_{\# \mathbb{L}_{1}+3}^{\mathrm{cl}}\left(\alpha_{1}\right)_{\mathrm{ev}_{3}} \times_{\mathrm{ev}_{-\infty}} \mathcal{M}_{\# \mathbb{L}_{2}}\left(\left(H_{1} \# H_{2}\right)_{\chi}, J_{\chi}^{H_{1} \# H_{2}} ; *, z_{*}^{H_{1} \# H_{2}} ; \alpha_{2}\right) \text {, }
$$

where the union is taken over all $\alpha_{1}, \alpha_{2}$ with $\alpha_{1}+\alpha_{2}=\alpha$, and $\left(\mathbb{L}_{1}, \mathbb{L}_{2}\right) \in \operatorname{Shuff}(\ell)$.

$$
\begin{aligned}
& \left.\left.\mathcal{M}_{\# \mathbb{L}_{1}}\left(\left(H_{1}\right)_{\chi} ; J_{\chi}^{H_{1}} *, z_{*}^{H_{1}} ; \alpha_{1}\right)\right) \times \mathcal{M}_{\# \mathbb{L}_{2}}\left(\left(H_{2}\right)_{\chi} ; J_{\chi}^{H_{2}} ; *, z_{*}^{H_{2}} ; \alpha_{2}\right)\right) \\
& \left(\mathrm{ev}_{+\infty}, \mathrm{ev}_{+\infty}\right) \times\left(\mathrm{ev}_{\left.-\infty, 1, \mathrm{ev}_{-\infty, 2}\right)} \mathcal{M}_{\# \mathbb{L}_{3}}\left(H^{\varphi}, J^{H_{1}, H_{2}} ; z_{*}^{H_{1}}, z_{*}^{H_{2}}, z_{*}^{H_{1} \# H_{2}} ; \alpha_{3}\right)\right),
\end{aligned}
$$

where the union is taken over all $\alpha_{1}, \alpha_{2}, \alpha_{3}$ with $\alpha_{1}+\alpha_{2}+\alpha_{3}=\alpha$ and 'triple shuffle' $\left(\mathbb{L}_{1}, \mathbb{L}_{2}, \mathbb{L}_{3}\right)$ of $\{1, \ldots, \ell\}$.

Note that (29.29), (29.30), The ends of types (29.31) are the ends which appear while $S$ is bounded. (29.32) and (29.33) correspond to the case $S \rightarrow-\infty$ and 


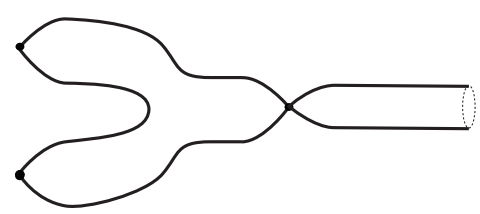

Figure 31. An element of 29.32

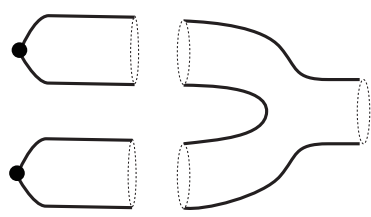

Figure 32. An element of (29.33)

$S \rightarrow+\infty$, respectively. Similarly as before the evaluation maps 29.27, 29.28) extend to this compactified moduli space, which we denote by the same notations.

We next take a system of $\mathrm{CF}$-perturbations on the moduli spaces

$$
\mathcal{M}_{\ell}\left(\text { para } ; H_{\chi}^{\varphi}, J_{\chi}^{H_{1} \# H_{2}} ; * *, z_{*}^{H_{1} \# H_{2}} ; \alpha\right)
$$

so that it is transversal to 0 and the evaluation map ev ${ }_{+\infty}$ is strongly submersive with respect thereto. Moreover we assume that it is compatible with the above description of the boundary. We remark that the first factor of (29.29), 29.30) and the second factor of (29.31) carry $S^{1}$ actions such that the isotropy group is finite.

To find an $S^{1}$ action on the second factor $\mathcal{M}_{\# \mathbb{L}_{3}}\left(H^{\varphi}, J^{H_{1}, H_{2}} ; z_{*}^{H_{1}}, z_{*}^{H_{2}}, z_{*}^{H_{1} \# H_{2}} ; \alpha_{3}\right)$ of (29.31) we map it to $\mathcal{M}_{\# \mathbb{L}_{3}+2}\left(H^{\varphi}, J_{0} ; \alpha_{3}\right)$, by sending an element

$$
\left(\mathbb{R} \times S^{1} \cup\{-\infty,+\infty\},\{-\infty,+\infty\} \cup \vec{z}^{+}, u\right) \in \mathcal{M}_{\# \mathbb{L}_{3}+2}\left(H^{\varphi}, J_{0} ; \alpha_{3}\right)
$$

to the element

$$
\left(\mathbb{R} \times S^{1} \cup\{-\infty,+\infty\}, \vec{z}^{+}, \bar{u}\right) \in \mathcal{M}_{\# \mathbb{L}_{3}}\left(H^{\varphi}, J^{H_{1}, H_{2}} ; z_{*}^{H_{1}}, z_{*}^{H_{2}}, z_{*}^{H_{1} \# H_{2}} ; \alpha_{3}\right)
$$

where $\bar{u}(\tau, t)=\phi_{H_{1} \# H_{2}}^{t}(u(\tau, t))$. The fibers of this map are the orbits of our $S^{1}$ action. Note the evaluation map $\mathcal{M}_{\# \mathbb{L}_{3}}\left(H^{\varphi}, J^{H_{1}, H_{2}} ; z_{*}^{H_{1}}, z_{*}^{H_{2}}, z_{*}^{H_{1} \# H_{2}} ; \alpha_{3}\right) \rightarrow$ $E_{H_{1} \# H_{2}}^{\# \mathbb{L}_{3}}$ is invariant under the $S^{1}$ action by definition.

Therefore we may take our CF-perturbations so that they are $S^{1}$ equivariant on those factors. Then the contributions of (29.29), (29.30), 29.31) become zero when we consider the correspondence of the moduli space $\mathcal{M}_{\ell}\left(\operatorname{para} ; H_{\chi}^{\varphi}, J_{\chi}^{H_{1} \# H_{2}} ; * *, z_{*}^{H_{1} \# H_{2}} ; \alpha\right)$.

Using the moduli space $\mathcal{M}_{\ell}\left(\right.$ para $\left.; H_{\chi}^{\varphi}, J_{\chi}^{H_{1} \# H_{2}} ; * *, z_{*}^{H_{1} \# H_{2}} ; \alpha\right)$ and the evaluation maps in the way similar to the way used for (29.22), we obtain an operator

$$
\mathfrak{H}:(\Omega(M) \widehat{\otimes} \Lambda) \otimes(\Omega(M) \widehat{\otimes} \Lambda) \rightarrow \Omega(M) \widehat{\otimes} \Lambda .
$$

Then $\mathfrak{H}$ satisfies

$$
\begin{aligned}
& d \circ \mathfrak{H}+\mathfrak{H} \circ d \\
& =\mathfrak{m}_{2 ; \alpha}^{\mathrm{cl} ; \mathfrak{b} ; H^{\varphi}} \circ\left(\mathcal{S}_{\left(\left(H_{1}\right)_{\chi}, J_{\chi}^{H_{1}}\right)}^{\mathfrak{b}} \otimes \mathcal{S}_{\left(\left(H_{2}\right)_{\chi}, J_{\chi}^{H_{2}}\right)}^{\mathfrak{b}}\right)-\mathcal{S}_{\left(\left(H_{1} \# H_{2}\right)_{\chi}, J_{\chi}^{H_{1} \# H_{2}}\right)}^{\mathfrak{b}} \circ \cup^{\mathfrak{b}} .
\end{aligned}
$$

In fact, the contributions of (29.32) and (29.33) correspond to the first and second term of the right hand side of this identity respectively. The proof of Lemma 29.19] is complete.

Theorem 29.13 (4) follows from Lemma 29.19

To prove (3) we apply (4) to the case $H_{1}=0, H_{2}=H$. Then using the fact that $\mathcal{S}_{\left(\left(H_{1}\right)_{\chi}, J_{\chi}^{H_{1}}\right), *}^{\mathfrak{b}}=i d$ we have

$$
\mathcal{S}_{\left((0 \# H)_{\chi}, J_{\chi}^{0 \# H}\right), *}\left(x \cup^{\mathfrak{b}} y\right)=x \cup^{\mathfrak{b}} \mathcal{S}_{\left(H_{\chi}, J_{\chi}^{H}\right), *}(y)
$$


in homology. Since $0 \# H \sim H$, we can prove $\mathcal{S}_{\left((0 \# H)_{\chi}, J_{\chi}^{H \# 0}\right), *}=\mathcal{S}_{\left(H_{\chi}, J_{\chi}^{H}\right), *}$ by using a homotopy between $0 \# H$ and $H$. Then (29.35) now implies Theorem 29.13 (3).

Therefore the proof of Theorem 29.13 is now complete.

29.3. Proof of Theorem 29.9, As we mentioned before, we did not use Theorem 29.9 in the definition of $\mathcal{S}_{\left(H_{\chi}, J_{\chi}^{H}\right)}^{0}$ or in the proof of Theorem 29.13 for the case $\mathfrak{b}=0$. We will use only that case in this subsection.

We consider the moduli space $\mathcal{M}_{1}\left(H_{\chi}, J_{\chi}^{H} ; *, z_{*}^{H} ; \alpha\right)$ for the definition of

$$
\mathcal{R}_{\left(H_{\chi}, J_{\chi}^{H}\right)}: \Omega(M) \widehat{\otimes} \Lambda \rightarrow \Omega\left(E_{\phi_{H}}\right) \widehat{\otimes} \Lambda
$$

as follows. Let $h \in \Omega(M)$. For each $\alpha$, we put

$$
\mathcal{R}_{\left(H_{\chi}, J_{\chi}^{H}\right), \alpha}(h)=\left(\mathrm{ev}_{1}\right) !\left(\mathrm{ev}_{-\infty}^{*}(h)\right)
$$

where we use the evaluation maps $\mathrm{ev}_{1}: \mathcal{M}_{1}\left(H_{\chi}, J_{\chi}^{H} ; *, z_{*}^{H} ; \alpha\right) \rightarrow E_{\phi_{H}}$ and $\mathrm{ev}_{-\infty}$ : $\mathcal{M}_{1}\left(H_{\chi}, J_{\chi}^{H} ; *, z_{*}^{H} ; \alpha\right) \rightarrow M$. We then take the sum

$$
\mathcal{R}_{\left(H_{\chi}, J_{\chi}^{H}\right)}=\sum_{\alpha} T^{-\mathcal{A}_{H}(\alpha)} \mathcal{R}_{\left(H_{\chi}, J_{\chi}^{H}\right), \alpha} .
$$

We consider the inclusion map $i_{\infty}: M \rightarrow E_{\phi_{H}}$ to the fiber of $\infty \in \mathbb{C} P^{1}$. By definition it is easy to see that $i_{\infty}^{*} \circ \mathcal{R}_{\left(H_{\chi}, J_{\chi}^{H}\right)}$ is chain homotopic to $\mathcal{S}_{\left(H_{\chi}, J_{\chi}^{H}\right)}$, where $\left(\phi_{H}\right)^{*}(\gamma(t))=\left(\phi_{H}^{t}\right)^{-1}(\gamma(t))$. Note that, if $\gamma(t)$ is a one-periodic orbit of $\phi_{H}^{t}$, $\left(\phi_{H}\right)^{*}(\gamma(t))$ is a constant. Therefore

$$
i_{\infty}^{*} \circ \mathcal{R}_{\left(H_{\chi}, J_{\chi}^{H}\right), *}=\mathcal{S}_{\left(H_{\chi}, J_{\chi}^{H}\right), *}
$$

in homology. It follows that for $h \in H^{*}\left(M ; \Lambda^{\downarrow}\right)$ we have

$$
\begin{aligned}
i_{\infty}^{*}\left(\mathcal{R}_{\left(H_{\chi}, J_{\chi}^{H}\right), *}\left(h \cup^{Q} \mathcal{S}\left(\widetilde{\psi}_{H}\right)^{-1}\right)\right) & =\mathcal{S}_{\left(H_{\chi}, J_{\chi}^{H}\right), *}\left(h \cup^{Q} \mathcal{S}\left(\widetilde{\psi}_{H}\right)^{-1}\right. \\
& =h \cup^{Q} S\left(\widetilde{\psi}_{H}\right) \cup^{Q} \mathcal{S}\left(\widetilde{\psi}_{H}\right)^{-1}=h .
\end{aligned}
$$

Thus

$$
h \mapsto \widehat{h}=\mathcal{R}_{\left(H_{\chi}, J_{\chi}^{H}\right), *}\left(h \cup^{Q} \mathcal{S}\left(\widetilde{\psi}_{H}\right)^{-1}\right)
$$

is a required section. The proof of Theorem 29.9 is complete.

\section{Spectral invariants And Seidel homomorphism}

In this section we study the relationship between the Seidel homomorphism and spectral invariants.

30.1. Valuations and spectral invariants. The next theorem is a straightforward generalization of the result Oh2, Theorem 4.3] and [EP1, Proposition 4.1].

Let $H$ be a time-dependent normalized Hamiltonian such that $\psi_{H}=i d$ and, let $\widetilde{\psi}_{H}$ be an associated element of $\pi_{1}(\operatorname{Ham}(M ; \omega))$.

Theorem 30.1. For each $a \in Q H_{\mathfrak{b}}(M ; \Lambda)$, we have

$$
\rho^{\mathfrak{b}}(H ; a)=\lambda_{q}\left(a \cup^{\mathfrak{b}} \mathcal{S}^{\mathfrak{b}}\left(\widetilde{\psi}_{H}\right)\right) .
$$

where $\lambda_{q}$ is as given in (4.6). 
Proof. The proof is similar to the proof of Theorem 9.1. Let $H_{k}$ be a sequence of normalized time dependent Hamiltonians such that $\psi_{H_{k}}$ are nondegenerate and $\lim _{k \rightarrow \infty} H_{k}=H$ in $C^{0}$ topology. We put

$$
F_{k, \chi}(\tau, t, x)=H_{k}(t, x)+\chi(\tau)\left(H(t, x)-H_{k}(t, x)\right): \mathbb{R} \times S^{1} \times M \rightarrow \mathbb{R} .
$$

We fix $J_{0}$ and define $J^{H_{k}}, J^{H}, J_{\chi}^{H_{k}}, J_{\chi}^{H}$ as in (29.5) and (29.6). Let $J_{k, \chi}$ be an $\mathbb{R} \times S^{1}$ parametrized family of almost complex structures such that

$$
J_{k, \chi}(\tau, t)= \begin{cases}J_{t}^{H} & \tau \geq 2 \\ J_{t}^{H_{k}} & \tau \leq-2\end{cases}
$$

Let $[\gamma, w] \in \operatorname{Crit}\left(\mathcal{A}_{H_{k}}\right)$ and $\alpha \in \Pi_{2}(M ; H)$.

Definition 30.2. We denote by $\stackrel{\circ}{\mathcal{M}}_{\ell}\left(F_{k, \chi}, J_{k, \chi} ;[\gamma, w], * ; \alpha\right)$ the set of all pairs $\left(u ; z_{1}^{+}, \ldots, z_{\ell}^{+}\right)$of maps $u: \mathbb{R} \times S^{1} \rightarrow M$ and $z_{i}^{+} \in \mathbb{R} \times S^{1}$ which satisfy the following conditions:

(1) The map $u$ satisfies the equation:

$$
\frac{\partial u}{\partial \tau}+J_{k, \chi}\left(\frac{\partial u}{\partial t}-X_{F_{k, \chi}}(u)\right)=0 .
$$

(2) The energy

$$
\frac{1}{2} \int\left(\left|\frac{\partial u}{\partial \tau}\right|_{J_{k, \chi}}^{2}+\left|\frac{\partial u}{\partial t}-X_{F_{k, \chi}}(u)\right|_{J_{k, \chi}}^{2}\right) d t d \tau
$$

is finite.

(3) There exists $p$ such that the following asymptotic boundary condition is satisfied.

$$
\lim _{\tau \rightarrow-\infty} u(\tau, t)=\gamma(t), \quad \lim _{\tau \rightarrow+\infty} u(\tau, t)=z_{p}^{H}(t) .
$$

(4) The homology class of $w \# u$ is $\alpha$, where \# is the obvious concatenation.

(5) $z_{i}^{+}$are distinct to each other.

The space $\stackrel{\circ}{\mathcal{M}}_{\ell}\left(F_{k, \chi}, J_{k, \chi} ;[\gamma, w], * ; \alpha\right)$ has a compactification $\mathcal{M}_{\ell}\left(F_{k, \chi}, J_{k, \chi} ;[\gamma, w], * ; \alpha\right)$, on which there exists a system of oriented Kuranishi structures with corners which is compatible at the boundaries. There exists a system of CF-perturbations of these Kuranishi structures so that the map $\left(u ; z_{1}^{+}, \ldots, z_{\ell}^{+}\right) \mapsto \lim _{\tau \rightarrow+\infty} u(\tau, 0)$ defines a strongly submersive map $\mathcal{M}_{\ell}\left(F_{k, \chi}, J_{k, \chi} ;[\gamma, w], * ; \alpha\right) \rightarrow M$ with respect to this CF-perturbation.

We use this in the way similar to the way how we did several times to define a map

$$
\mathcal{P}_{\left(F_{k, \chi}, J_{k, \chi}\right)}^{\mathfrak{b}}: \Omega(M) \widehat{\otimes} \Lambda^{\downarrow} \rightarrow C F\left(M, H_{k}, J ; \Lambda^{\downarrow}\right) .
$$

Here we identify $\Omega(M) \otimes \Lambda^{\downarrow}$ and the Floer chain module $C F\left(M, H, J ; \Lambda^{\downarrow}\right)$ of BottMorse type using the Hamiltonian loop $\left\{\phi_{H}^{t}\right\}$.

Lemma 30.3. $\mathcal{P}_{\left(F_{k, \chi}, J_{k, \chi}\right)}^{\mathfrak{b}} \circ \mathcal{P}_{\left(\left(H_{k}\right)_{\chi}, J_{\chi}^{H_{k}}\right)}^{\mathfrak{b}}$ is chain homotopic to b $\circ \mathcal{S}_{\left(H_{\chi}, J_{\chi}^{H}\right)}^{\mathfrak{b}} \circ$ b.

The proof is similar to the proof of Proposition 9.7 and is omitted.

Lemma 30.4.

$$
\mathcal{P}_{\left(F_{k, \chi}, J_{k, \chi}\right)}^{\mathfrak{b}}\left(F^{\lambda} C F\left(M, H_{k}, J ; \Lambda^{\downarrow}\right)\right) \subset \Omega(M) \widehat{\otimes} q^{\lambda+E^{-}\left(H-H_{k}\right)} \Lambda_{0}^{\downarrow} .
$$


The proof is similar to the proof of Lemma 9.3 and 9.20 and so omitted. Lemmas 30.4 implies

$$
\lambda_{q}\left(\mathcal{S}_{\left(H_{\chi}, J_{\chi}\right)}^{\mathfrak{b}}(a)\right) \leq \rho^{\mathfrak{b}}\left(H_{k} ; a\right)+E^{-}\left(H-H_{k}\right) .
$$

Taking the limit $k \rightarrow \infty$ we have

$$
\rho^{\mathfrak{b}}(H ; a) \geq \lambda_{q}\left(\mathcal{S}_{\left(H_{\chi}, J_{\chi}\right)}^{\mathfrak{b}}(a)\right) .
$$

We can prove the opposite inequality by using

$$
\mathcal{Q}_{\left(F_{k}^{\tilde{\chi}}, J_{F_{k}}^{\bar{\chi}}\right)}^{\mathfrak{b}}: C F\left(M, H_{k}, J ; \Lambda^{\downarrow}\right) \rightarrow \Omega(M) \widehat{\otimes} \Lambda^{\downarrow}
$$

that can be defined in a similar way as Definition 26.4. (See the proof of Proposition 26.10 also.)

By Theorem 29.13 (3) with $x=a, y=1$, we find that

$$
\mathcal{S}_{\left(H_{\chi}, J_{\chi}^{H}\right), *}^{\mathfrak{b}}(a)=a \cup^{\mathfrak{b}} \mathcal{S}^{\mathfrak{b}}\left(\widetilde{\psi}_{H}\right) .
$$

The proof of Theorem 30.1 is now complete.

Let $H$ be a time dependent periodic Hamiltonian such that $\psi_{H}=i d$. We do not assume that $H$ is normalized. Let $e \in Q H_{\mathfrak{b}}(M ; \omega)$ with $e \cup^{\mathfrak{b}} e=e$. We assume that $e \Lambda^{\downarrow}$ is a direct product factor of $Q H_{\mathfrak{b}}(M ; \omega)$.

Corollary 30.5. We put $e \cup^{\mathfrak{b}} \mathcal{S}^{\mathfrak{b}}\left(\widetilde{\psi}_{H}\right)=x e$ with $x \in \Lambda$. Then

$$
\zeta^{\mathfrak{b}}(H ; e)=\mathfrak{v}_{T}(x)+\frac{1}{\operatorname{vol}_{\omega}(M)} \int_{[0,1]} \int_{M} H_{t} d t \omega^{n} .
$$

Proof. We put

$$
\underline{H}_{t}=H_{t}-\frac{1}{\operatorname{vol}_{\omega}(M)} \int_{M} H_{t} \omega^{n} .
$$

It is a normalized Hamiltonian and $\psi_{\underline{H}}=\psi_{H}$. By Theorem 30.1 we have

$$
\rho^{\mathfrak{b}}(\underline{H} ; e)=-\mathfrak{v}_{T}\left(e \cup^{\mathfrak{b}} \mathcal{S}^{\mathfrak{b}}\left(\widetilde{\psi}_{H}\right)\right) .
$$

On the other hand,

$$
\rho^{\mathfrak{b}}(H ; e)=\rho^{\mathfrak{b}}(\underline{H} ; e)-\frac{1}{\operatorname{vol}_{\omega}(M)} \int_{[0,1]} \int_{M} H_{t} d t \omega^{n} .
$$

Therefore we have

$$
\begin{aligned}
\zeta^{\mathfrak{b}}(H ; e) & =-\left(\lim _{k \rightarrow \infty} \frac{\rho^{\mathfrak{b}}(k H ; e)}{k}\right) \\
& =-\lim _{k \rightarrow \infty} \frac{\rho^{\mathfrak{b}}(k \underline{H} ; e)}{k}+\frac{1}{\operatorname{vol}_{\omega}(M)} \int_{[0,1]} \int_{M} H_{t} d t \omega^{n} \\
& =\lim _{k \rightarrow \infty} \frac{\mathfrak{v}_{T}\left(e x^{k}\right)}{k}+\frac{1}{\operatorname{vol}_{\omega}(M)} \int_{[0,1]} \int_{M} H_{t} d t \omega^{n} \\
& =\mathfrak{v}_{T}(x)+\frac{1}{\operatorname{vol}_{\omega}(M)} \int_{[0,1]} \int_{M} H_{t} d t \omega^{n},
\end{aligned}
$$

as required. 
30.2. The toric case. In this section we generalize a result by McDuff-Tolman MT to a version with bulk and apply the result for some calculation. Our discussion here is a straightforward generalization of [MT]. (See also [EP3] for the general discussion for the Hamiltonian loops.)

Let $H$ be a time independent normalized Hamiltonian. We assume that $\psi_{H}=i d$. We put

$$
H_{\min }=\inf \{H(y) \mid y \in M\}
$$

and

$$
D_{\min }=\left\{x \in M \mid H(x)=H_{\min }\right\} .
$$

Since $D_{\min }$ is a connected component of the fixed point set of the $S^{1}$ action generated by $X_{H}$, it follows that $D_{\min }$ is a smooth submanifold. We assume that $D_{\text {min }}$ is of (real) codimension 2. We also assume the following:

Assumption 30.6. Let $p \in D_{\min }$ and $q \in M \backslash D_{\min }$ be sufficiently close to $p$. We consider the orbit $z_{q}^{H}(t)=\phi_{H}^{t}(q)$ and a disk $w:\left(D^{2}, \partial D^{2}\right) \rightarrow\left(M, z_{q}^{H}\right)$ which bounds $z_{q}^{H}$. If $w$ is sufficiently small, then

$$
\left[D_{\min }\right] \cdot w_{*}\left(\left[D^{2}\right]\right)=+1 .
$$

Let $\mathfrak{b}=\mathfrak{b}_{0}+\mathfrak{b}_{2}+\mathfrak{b}_{+}$as before.

Theorem 30.7. We have

$$
\mathcal{S}^{\mathfrak{b}}\left(\left[\phi_{H}\right]\right) \equiv T^{H_{\min }} e^{\overline{\mathfrak{b}}_{2} \cap D_{\min }} P D\left(\left[D_{\min }\right]\right) \quad \bmod T^{H_{\min }} \Lambda_{-}^{\downarrow} .
$$

Remark 30.8. In the case $\mathfrak{b}=0$ this is [MT, Theorem 1.9]. Our generalization to the case $\mathfrak{b} \neq 0$ is actually straightforward.

Proof. We start with the following lemma.

Lemma 30.9. If $\stackrel{\circ}{\mathcal{M}}_{0}\left(H_{\chi}, J_{\chi}^{H} ; *, z_{*}^{H} ; \alpha\right)$ is nonempty, then

$$
\mathcal{A}_{H}(\alpha) \leq-H_{\text {min }} \text {. }
$$

Proof. Let $u \in \stackrel{\circ}{\mathcal{M}}_{0}\left(H_{\chi}, J_{\chi}^{H} ; *, z_{*}^{H} ; \alpha\right)$. Then we have

$$
\begin{aligned}
\int u^{*} \omega & =\int \omega\left(\frac{\partial u}{\partial \tau}, \frac{\partial u}{\partial t}\right) d t d \tau=\int \omega\left(\frac{\partial u}{\partial \tau}, J_{\chi}^{H} \frac{\partial u}{\partial \tau}+\chi(\tau) X_{H}\right) d t d \tau \\
& \geq-\int \chi(\tau) \frac{\partial(H \circ u)}{\partial \tau} d t d \tau \\
& \geq-\int_{S^{1}} H\left(z_{p}^{H}(t)\right) d t+\int \chi^{\prime}(\tau)(H \circ u) d t d \tau \\
& \geq-\int_{S^{1}} H\left(z_{p}^{H}(t)\right) d t+H_{\text {min }} .
\end{aligned}
$$

Lemma 30.9 follows.

We remark that the equality holds only when

$$
\int \omega\left(\frac{\partial u}{\partial \tau}, J_{\chi}^{H} \frac{\partial u}{\partial \tau}\right) d t d \tau=\int\left|\frac{\partial u}{\partial \tau}\right|_{J_{\chi}^{H}}^{2} d t d \tau=0
$$

and so $\frac{\partial u}{\partial \tau}=0$. Therefore $u$ must be constant. Moreover since $u(\tau, t) \rightarrow z_{p}^{H}(t)$ as $\tau \rightarrow \infty$, the image of $u$ must lie in the zero locus of $X_{H}$. Thus 
Lemma 30.10. If the equality holds in Lemma 30.9. $\mathcal{M}_{0}\left(H_{\chi}, J_{\chi}^{H} ; *, z_{*}^{H} ; \alpha\right)$ consists of constant maps to $D_{\min }$.

Let $\alpha_{0}$ be the homology class such that Lemma 30.9 holds.

Lemma 30.11. The moduli space $\mathcal{M}_{0}\left(H_{\chi}, J_{\chi}^{H} ; *, z_{*}^{H} ; \alpha_{0}\right)$ is transversal and

$$
\operatorname{ev}_{1 \#}\left(\mathcal{M}_{0}\left(H_{\chi}, J_{\chi}^{H} ; *, z_{*}^{H} ; \alpha_{0}\right)\right)=\left[D_{\min }\right] .
$$

Proof. We consider $D_{\min } \times S^{2} \subset E_{\phi_{H}}$. Its tubular neighborhood is identified with a neighborhood of zero section in the line bundle $D_{\min } \times \mathcal{O}(-1) \rightarrow D_{\min } \times S^{2}$. Here we identify $S^{2} \cong \mathbb{C} P^{1}$ and $\mathcal{O}(-1)$ is a line bundle with Chern number -1 . (We use Assumption 30.6 here.) The moduli space $\mathcal{M}_{0}\left(H_{\chi}, J_{\chi}^{H} ; *, z_{*}^{H} ; \alpha_{0}\right)$ then is identified to the moduli space of the sections to the bundle. $D_{\min } \times \mathcal{O}(-1) \rightarrow S^{2}$. The lemma follows easily.

Theorem 30.7 now follows from Lemmas 30.9, 30.10, 30.11.

We now specialize Theorem 30.7 to the case of toric manifold. Let $(M, \omega)$ be a compact Kähler toric manifold. Then $T^{n}$ acts on $(M, \omega)$ preserving the Kähler form. Let $\pi: M \rightarrow P \subset \mathbb{R}^{n}$ be the moment map. Let $D_{j}=\pi^{-1}\left(\partial_{j} P\right), j=1, \ldots, m$ be the irreducible components of the toric divisor. As in Section 20 we have affine functions $\ell_{j}: \mathbb{R}^{n} \rightarrow \mathbb{R}$ such that $\partial_{j} P=\left\{\mathbf{u} \in P \mid \ell_{j}(\mathbf{u})=0\right\}$. We put $d \ell_{j}=\left(k_{j, 1}, \ldots, k_{j, n}\right)$ where $k_{j, 1}, \ldots, k_{j, n}$ are integers which are coprime. Let $S_{j}^{1}$ be a subgroup of $T^{n}$ such that

$$
S_{j}^{1}=\left\{\left[k_{j, 1} t, \ldots, k_{j, n} t\right] \mid t \in \mathbb{R}\right\} \subset T^{n}
$$

where we identify $T^{n}=\mathbb{R}^{n} / \mathbb{Z}^{n}$. We note that if we put $H=\ell_{j}$ then $\psi_{H}=$ identity. The next result is a corollary to Theorem 30.7. $S_{j}^{1}$ determines an element of $\pi_{1}(\operatorname{Ham}(M, \omega))$, which we denote by $\left[S_{j}^{1}\right]$.

Theorem 30.12.

$$
\mathcal{S}^{\mathfrak{b}}\left(\left[S_{j}^{1}\right]\right) \equiv T^{\mathrm{vol}(P)^{-1} \int_{P} \ell_{j}} e^{\overline{\mathfrak{b}}_{2} \cap D_{j}} P D\left(\left[D_{j}\right]\right) \quad \bmod T^{\mathrm{vol}(P)^{-1} \int_{P} \ell_{j}} \Lambda_{+} .
$$

Proof. We note that $\ell_{j}-\operatorname{Vol}(P)^{-1} \int_{P} \ell_{j}$ is the normalized Hamiltonian which generates $\left[S_{j}^{1}\right]$. (This is because the push out measure $\pi_{!}\left(\omega^{n}\right)$ on $P$ is the Lebesgue measure. Its minimum is attained at $D_{j}$. Therefore Theorem 30.12 follows from Theorem 30.7

Let $\mathbf{u}_{\mathrm{cnt}} \in P$ be the center of gravity and $e \in Q H_{\mathfrak{b}}(M ; \Lambda)$ the idempotent, which corresponds to $\mathbf{u} \in P$ by Theorems 20.18, 20.19 and Proposition 20.23. The same identity was established by Entov-Polterovich [EP3, Theorem 1.15] for general Hamiltonian loops, not just for the circle action, which dealt the monotone case with $\mathfrak{b}=0$.

\section{Theorem 30.13.}

$$
\mu_{e}^{\mathfrak{b}}\left(\left[S_{j}^{1}\right]\right)=\operatorname{Vol}(P)\left(\ell_{j}(\mathbf{u})-\ell_{j}\left(\mathbf{u}_{\mathrm{cnt}}\right)\right) .
$$

In particular, $\mu_{e}^{\mathfrak{b}}=0$ on the image of $\pi_{1}\left(T^{n}\right) \rightarrow \pi_{1}(\operatorname{Ham}(M, \omega))$ if and only if $\mathbf{u}=\mathbf{u}_{\mathrm{cnt}}$.

Proof. Let $\mathfrak{y}$ be the critical point of $\mathfrak{P O}_{\mathfrak{b}}$ that corresponds to $L(\mathbf{u})$. (Namely $\mathbf{u}(\mathfrak{y})=\mathbf{u}$.) By (20.35), Theorem 20.24, (20.39) and Theorem 21.3 we have

$$
i_{\mathrm{qm},(\mathfrak{b}, b(\mathfrak{y}))}^{*}\left(P D\left(\left[D_{j}\right]\right)\right)=\left[\frac{\partial \mathfrak{P} \mathcal{O}_{\mathfrak{b}}}{\partial w_{j}}\right] \equiv e^{\overline{\mathfrak{b}}_{j}} z_{j} \quad \bmod \Lambda_{+} .
$$


Here $z_{j}$ is as in 21.1. By Lemma 21.2

$$
i_{\mathrm{qm},(\mathfrak{b}, b(\mathfrak{y}))}^{*}\left(e_{\mathfrak{y}}\right)=1 \text {. }
$$

We put $b(\mathfrak{y})=\sum x_{i} e_{i}$ and $\partial \beta_{j}=\sum k_{j i} e_{i}$, where $e_{i}$ is a basis of $H(L(\mathbf{u}), \mathbb{Z})$. Then we get

$$
z_{j}(\mathfrak{y})=T^{\ell_{j}(\mathbf{u})} \prod_{i=1}^{n} \exp k_{j i} x_{i}
$$

Therefore we have

$$
i_{\mathrm{qm},(\mathfrak{b}, b(\mathfrak{y}))}^{*}\left(\mathcal{S}^{\mathfrak{b}}\left(\left[S_{j}^{1}\right]\right)\right) \equiv T^{\ell_{j}(\mathbf{u})-\operatorname{Vol}(P)^{-1} \int_{P} \ell_{j}} c \quad \bmod T^{\ell_{j}(\mathbf{u})-\operatorname{Vol}(P)^{-1} \int_{P} \ell_{j}} \Lambda_{+} .
$$

where $c \in \mathbb{C} \backslash\{0\}$. We note that $\operatorname{Vol}(P)^{-1} \int_{P} \ell_{j}=\ell_{j}\left(\mathbf{u}_{\mathrm{cnt}}\right)$.

Let us assume that $\mathfrak{y}$ is nondegenerate. Then using also the multiplicativity of $i_{\mathrm{qm},(\mathfrak{b}, b(\mathfrak{y}))}^{*}($ FOOO7, Theorem 9.1]) we have

$$
e_{\mathfrak{y}} \cup^{\mathfrak{b}} \mathcal{S}^{\mathfrak{b}}\left(\left[S_{j}^{1}\right]\right) \equiv T^{\ell_{j}(\mathbf{u})-\ell_{j}\left(\mathbf{u}_{\mathrm{cnt}}\right)} c e_{\mathfrak{y}} \quad \bmod T^{\ell_{j}(\mathbf{u})-\ell_{j}\left(\mathbf{u}_{\mathrm{cnt}}\right)} \Lambda_{+} .
$$

Therefore by Corollary 30.5 we obtain

$$
\mu_{e}^{\mathfrak{b}}\left(\left[S_{j}^{1}\right]\right)=\operatorname{Vol}(P)\left(\ell_{j}(\mathbf{u})-\ell_{j}\left(\mathbf{u}_{\mathrm{cnt}}\right)\right) .
$$

In the general case we recall that $\operatorname{Jac}\left(\mathfrak{P O}_{\mathfrak{b}} ; \mathfrak{y}\right)$ is a local ring and the kernel of the homomorphism $\operatorname{Jac}\left(\mathfrak{P O}_{\mathfrak{b}} ; \mathfrak{y}\right) \rightarrow \Lambda$ defined by $[\mathfrak{P}] \mapsto \mathfrak{P}(\mathfrak{y})$ is nilpotent. Therefore

$$
e_{\mathfrak{y}} \cup^{\mathfrak{b}} \mathcal{S}^{\mathfrak{b}}\left(\left[S_{j}^{1}\right]\right)=a e_{\mathfrak{y}}+b
$$

with $a \in \Lambda$,

$$
a \equiv T^{\ell_{j}(\mathbf{u})-\ell_{j}\left(\mathbf{u}_{\mathrm{cnt}}\right)} c \quad \bmod T^{\ell_{j}(\mathbf{u})-\ell_{j}\left(\mathbf{u}_{\mathrm{cnt}}\right)} \Lambda_{+}^{\downarrow}
$$

and $b$ is nilpotent. We use it to show

$$
\lim _{k \rightarrow \infty} \frac{\mathfrak{v}_{T}\left(e_{\mathfrak{y}} \cup^{\mathfrak{b}} \mathcal{S}^{\mathfrak{b}}\left(\left[S_{j}^{1}\right]\right)^{k}\right)}{k}=\ell_{j}(\mathbf{u})-\ell_{j}\left(\mathbf{u}_{\mathrm{cnt}}\right) .
$$

The proof of Theorem 30.13 is now complete.

Remark 30.14. Theorem 30.13 also follows from Theroems 21.1 and 25.1. 


\section{Part 7. Kuranishi structure and its CF-perturbation: summary}

This part is a brief summary of the notion of Kuranishi structure and its perturbation. There is nothing new in this part. We include this part only for the convenience of the reader. This is a summary which is not intended to include the full detail of the construction. We refer the reader to [FOOO2, Section A1], [FOOO4, Section 12], FOOO11 for the detail. (The reader will find that the detail is, in principle, mostly parallel to the standard theory of manifolds.)

\section{Kuranishi Structure And GOOd COORDinAte SYstem}

We first review a few basic terminologies of orbifolds. See [FOOO11, Section 15]. We remark that we only consider effective orbifolds.

\subsection{Orbifold.}

Definition 31.1. Let $X$ be a topological space. Its (effective) orbifold chart is a triple $(V, \Gamma, \phi)$ such that $V$ is a manifold, $\Gamma$ a finite group acting smoothly and effectively on $V$, and $\phi: V \rightarrow X$ is a $\Gamma$ equivariant continuous man 8 which induces a homeomorphism $\bar{\phi}: V / \Gamma \rightarrow X$ onto an open subset $U$ of $X$.

Let $(V, \Gamma, \phi)$ be an orbifold chart and $p \in V$. We put $\Gamma_{p}=\{\gamma \in \Gamma \mid \gamma p=p\}$. Let $V_{p}$ be a $\Gamma_{p}$ invariant open neighborhood of $p$ in $V$. We assume the map $\bar{\phi}$ : $V_{p} / \Gamma_{p} \rightarrow X$ is injective. (In other words, we assume that $\gamma V_{p} \cap V_{p} \neq \emptyset$ implies $\left.\gamma \in \Gamma_{p}.\right)$ We say such a triple $\left(V_{p}, \Gamma_{p},\left.\phi\right|_{V_{p}}\right)$ a subchart of $(V, \Gamma, \phi)$.

Let $\left(V_{i}, \Gamma_{i}, \phi_{i}\right)(i=1,2)$ be orbifold charts of $X$. We say that they are compatible if the following holds for each $p_{1} \in V_{1}$ and $p_{2} \in V_{2}$ with $\phi_{1}\left(p_{1}\right)=\phi_{2}\left(p_{2}\right)$.

(1) There exists a group isomorphism $h:\left(\Gamma_{1}\right)_{p_{1}} \rightarrow\left(\Gamma_{2}\right)_{p_{2}}$.

(2) There exists an $h$ equivariant diffeomorphism $\tilde{\varphi}: V_{1, p_{1}} \rightarrow V_{2, p_{2}}$. Here $V_{i, p_{i}}$ is a $\left(\Gamma_{i}\right)_{p_{i}}$ equivariant subset of $V_{i}$ such that $\left(V_{i, p_{i}},\left(\Gamma_{i}\right)_{p_{i}},\left.\phi\right|_{V_{i, p_{i}}}\right)$ is a subchart.

(3) $\phi_{2} \circ \tilde{\varphi}=\phi_{1}$ on $V_{1, p_{1}}$.

An orbifold structure on $X$ is represented by a set of orbifold charts $\left\{\left(V_{i}, \Gamma_{i}, \phi_{i}\right) \mid\right.$ $i \in I\}$ such that each two of the charts are compatible in the sense of (3) above and $\bigcup_{i \in I} \phi_{i}\left(V_{i}\right)=X$ is a locally finite open cover of $X$. The set of all orbifold charts compatible with a given representative of orbifold structure is said to be an orbifold structure.

Definition 31.2. Suppose that $X, Y$ have orbifold structures represented by $\left\{\left(V_{i}^{X}, \Gamma_{i}^{X}, \phi_{i}^{X}\right) \mid i \in I\right\}$ and $\left\{\left(V_{j}^{Y}, \Gamma_{j}^{Y}, \phi_{j}^{Y}\right) \mid j \in J\right\}$, respectively. A continuous map $f: X \rightarrow Y$ is said to be an embedding if the following holds.

(1) $f$ is an embedding of topological spaces.

(2) Let $p \in V_{i}^{X}, q \in V_{j}^{Y}$ with $f\left(\phi_{i}(p)\right)=\phi_{j}(q)$. Then we have the following.

(a) There exists an isomorphism of groups $h_{p ; j i}:\left(\Gamma_{i}^{X}\right)_{p} \rightarrow\left(\Gamma_{j}^{Y}\right)_{q}$.

(b) There exist $V_{i, p}^{X}$ and $V_{j, q}^{Y}$ such that $\left(V_{i, p}^{X},\left(\Gamma_{i}^{X}\right)_{p},\left.\phi_{i}\right|_{V_{i, p}^{X}}\right)$ is a subchart for $i=1,2$. There exists an $h_{p ; j i}$ equivariant embedding of manifolds $\tilde{f}_{p ; j i}: V_{i, p}^{X} \rightarrow V_{j, q}^{Y}$.

\footnotetext{
${ }^{8}$ The $\Gamma$ action on $X$ is trivial.
} 
(c) The diagram below commutes.

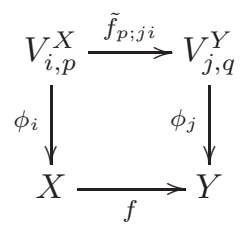

Two orbifold embeddings are said to be equal if they coincide set-theoretically.

A continuous map $f: X \rightarrow M$ from an orbifold to a manifold $M$ is said to be a smooth map if for each orbifold chart $(V, \Gamma, \phi)$ of $X$ the composition $f \circ \phi: V \rightarrow M$ is a smooth map between manifolds.

Definition 31.3. Let $(X, \mathcal{E}, \pi)$ be a pair of orbifolds $X, \mathcal{E}$ and $\pi: \mathcal{E} \rightarrow X$ a continuous map between their underlying topological spaces. An orbibundle chart of $(X, \mathcal{E})$ is $(V, E, \Gamma, \phi, \widehat{\phi})$ with $\mathfrak{V}=(V, \Gamma, \phi)$ an orbifold chart, $E$ is a finite dimensional vector space equipped with a linear $\Gamma$ action and $(V \times E, \Gamma, \widehat{\phi})$ is an orbifold chart of the orbifold $\mathcal{E}$, such that the diagram below commutes set-theoretically,

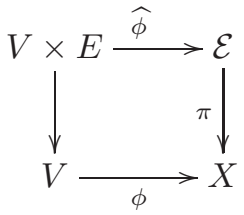

where the left vertical arrow is the projection to the first factor.

By modifying Definition 31.1 in an obvious way we can define the notion of subchart and then using it to define the structure of orbibundle on $(X, \mathcal{E}, \pi)$. (See [FO0011, Definition 15.17].)

We also say vector bundle on an orbifold instead of orbibundle.

The notion of embedding of orbibundle can be defined in the same way as Definition 31.2. (See [FOOO11, Definition 15.18].)

31.2. Kuranishi structure. We next define the notion of Kuranishi structure. Hereafter in this part, $X$ is always a separable metrizable space.

Definition 31.4. A Kuranishi chart of $X$ is $\mathcal{U}=(U, \mathcal{E}, \psi, s)$ with the following properties.

(1) $U$ is an orbifold.

(2) $\mathcal{E}$ is a vector bundle on $U$.

(3) $s$ is a smooth section of $\mathcal{E}$.

(4) $\psi: s^{-1}(0) \rightarrow X$ is a homeomorphism onto an open set.

Here a section of an orbibundle $\pi: \mathcal{E} \rightarrow X$ is an embedding $s: X \rightarrow \mathcal{E}$ (of orbifold) such that $\pi \circ s=$ the identity map.

If $U^{\prime}$ is an open subset of $U$, then by restricting $\mathcal{E}, \psi$ and $s$ to $U^{\prime}$, we obtain a Kuranishi chart which we write $\left.\mathcal{U}\right|_{U^{\prime}}$ and call it an open subchart.

The dimension of $\mathcal{U}=(U, \mathcal{E}, \psi, s)$ is by definition

$$
\operatorname{dim} \mathcal{U}=\operatorname{dim} U-\operatorname{rank} \mathcal{E} .
$$

Here rank $\mathcal{E}$ is the dimension of the fiber $\mathcal{E} \rightarrow U$.

We say that $\mathcal{U}=(U, \mathcal{E}, \psi, s)$ is orientable if $U$ and $E$ are orientable. An orientation of $\mathcal{U}=(U, \mathcal{E}, s, \psi)$ is a pair of orientations of $U$ and $\mathcal{E}$. (See FOOO2, Definition 
A1.17] for the precise meaning.) An open subchart of an oriented Kuranishi chart is oriented.

We call $U$ the Kuranishi neighborhood, $\mathcal{E}$ the obstruction bundle, $\psi$ the parametrization, and $s$ the Kuranishi map.

Definition 31.5. Let $\mathcal{U}=(U, \mathcal{E}, \psi, s), \mathcal{U}^{\prime}=\left(U^{\prime}, \mathcal{E}^{\prime}, \psi^{\prime}, s^{\prime}\right)$ be Kuranishi charts of $X$. An embedding of Kuranishi charts $: \mathcal{U} \rightarrow \mathcal{U}^{\prime}$ is a pair $\Phi=(\varphi, \widehat{\varphi})$ with the following properties.

(1) $\varphi: U \rightarrow U^{\prime}$ is an embedding of orbifolds. (See Definition 31.3)

(2) $\widehat{\varphi}: \mathcal{E} \rightarrow \mathcal{E}^{\prime}$ is an embedding of vector bundles over $\varphi$. (See Definition 31.4.)

(3) $\widehat{\varphi} \circ s=s^{\prime} \circ \varphi$.

(4) $\psi^{\prime} \circ \varphi=\psi$ holds on $s^{-1}(0)$.

(5) For each $x \in U$ with $s(x)=0$, the (covariant) derivative $D_{\varphi(x)} s^{\prime}$ induces an isomorphism

$$
\frac{T_{\varphi(x)} U^{\prime}}{\left(D_{x} \varphi\right)\left(T_{x} U\right)} \cong \frac{\mathcal{E}_{\varphi(x)}^{\prime}}{\widehat{\varphi}\left(\mathcal{E}_{x}\right)} .
$$

When $\mathcal{U}$ and $\mathcal{U}^{\prime}$ are oriented we say $\Phi$ is compatible with orientation if the isomorphism (31.3) induces an orientation preserving isomorphism.

Definition 31.6. For $A \subseteq X$, a Kuranishi neighborhood of $A$ is a Kuranishi chart such that $\operatorname{Im}(\psi)$ contains $A$. In case $A=\{p\}$ we call it a Kuranishi neighborhood of $p$ or a Kuranishi chart at $p$.

Definition 31.7. Let $\mathcal{U}_{1}=\left(U_{1}, \mathcal{E}_{1}, \psi_{1}, s_{1}\right), \mathcal{U}_{2}=\left(U_{2}, \mathcal{E}_{2}, \psi_{2}, s_{2}\right)$ be Kuranishi charts of $X$. A coordinate change in the weak sense (resp. in the strong sense) from $\mathcal{U}_{1}$ to $\mathcal{U}_{2}$ is a triple $\Phi_{21}=\left(U_{21}, \varphi_{21}, \widehat{\varphi}_{21}\right)$ with the following properties (1) and (2) (resp. (1), (2) and (3)):

(1) $U_{21}$ is an open subset of $U_{1}$.

(2) $\left(\varphi_{21}, \widehat{\varphi}_{21}\right)$ is an embedding of Kuranishi charts : $\left.\mathcal{U}_{1}\right|_{U_{21}} \rightarrow \mathcal{U}_{2}$.

(3) $\psi_{1}\left(s_{1}^{-1}(0) \cap U_{21}\right)=\operatorname{Im}\left(\psi_{1}\right) \cap \operatorname{Im}\left(\psi_{2}\right)$.

In case $\mathcal{U}_{1}$ and $\mathcal{U}_{2}$ are oriented $\Phi_{21}$ is said to be orientation preserving if it is so as an embedding.

Definition 31.8. A Kuranishi structure $\widehat{\mathcal{U}}$ of $X$ assigns a Kuranishi neighborhood $\mathcal{U}_{p}=\left(U_{p}, \mathcal{E}_{p}, \psi_{p}, s_{p}\right)$ of $p$ to each $p \in X$ and a coordinate change in weak sense $\Phi_{p q}=\left(U_{p q}, \varphi_{p q}, \widehat{\varphi}_{p q}\right): \mathcal{U}_{q} \rightarrow \mathcal{U}_{p}$ to each $p, q \in \operatorname{Im}\left(\psi_{p}\right)$ such that $q \in \psi_{q}\left(s_{q}^{-1}(0) \cap U_{p q}\right)$ and the following holds for each $p, q \in \operatorname{Im}\left(\psi_{p}\right), r \in \psi_{q}\left(s_{q}^{-1}(0) \cap U_{p q}\right) \cap Z$.

We put $U_{p q r}=\varphi_{q r}^{-1}\left(U_{p q}\right) \cap U_{p r}$. Then we have

$$
\left.\Phi_{p r}\right|_{U_{p q r}}=\left.\Phi_{p q} \circ \Phi_{q r}\right|_{U_{p q r}} .
$$

We also require that the dimension of $\mathcal{U}_{p}$ is independent of $p$ which we call the dimension of $\widehat{\mathcal{U}}$.

We replace $U_{p}$ by a smaller open subset and restrict $s_{p}$ etc. in an obvious way. We thus obtain an open substructure .

We remark that the equality (31.4) is the set theoretical equality of the maps involved.

We next define the notion of a good coordinate system. 
Definition 31.9. A good coordinate system of $X$ is

$$
\widehat{\mathcal{U}}=\left((\mathfrak{P}, \leq),\left\{\mathcal{U}_{\mathfrak{p}} \mid \mathfrak{p} \in \mathfrak{P}\right\},\left\{\Phi_{\mathfrak{p q}} \mid \mathfrak{q} \leq \mathfrak{p}\right\}\right)
$$

such that:

(1) $(\mathfrak{P}, \leq)$ is a partially ordered set. We assume $\# \mathfrak{P}$ is finite.

(2) $\mathcal{U}_{\mathfrak{p}}$ is a Kuranishi chart of $X$.

(3) $\bigcup_{\mathfrak{p} \in \mathfrak{P}} U_{\mathfrak{p}} \supseteq X$.

(4) Let $\mathfrak{q} \leq \mathfrak{p}$. Then $\Phi_{\mathfrak{p q}}=\left(U_{\mathfrak{p q}}, \varphi_{\mathfrak{p q}}, \widehat{\varphi}_{\mathfrak{p q}}\right)$ is a coordinate change in strong sense: $\mathcal{U}_{\mathfrak{q}} \rightarrow \mathcal{U}_{\mathfrak{p}}$ in the sense of Definition 31.7

(5) If $\mathfrak{r} \leq \mathfrak{q} \leq \mathfrak{p}$, then by putting $U_{\mathfrak{p q r}}=\varphi_{\mathfrak{q} \mathfrak{r}}^{-1}\left(U_{\mathfrak{p q}}\right) \cap U_{\mathfrak{p r}}$ we have

$$
\left.\Phi_{\mathfrak{p r}}\right|_{U_{\mathfrak{p q} \mathfrak{r}}}=\left.\Phi_{\mathfrak{p q}} \circ \Phi_{\mathfrak{q} \mathfrak{r}}\right|_{U_{\mathfrak{p q} \mathfrak{r}}} .
$$

(6) If $\operatorname{Im}\left(\psi_{\mathfrak{p}}\right) \cap \operatorname{Im}\left(\psi_{\mathfrak{q}}\right) \neq \emptyset$, then either $\mathfrak{p} \leq \mathfrak{q}$ or $\mathfrak{q} \leq \mathfrak{p}$ holds.

(7) We define a relation $\sim$ on the disjoint union $\coprod_{\mathfrak{p} \in \mathfrak{P}} U_{\mathfrak{p}}$ as follows. Let $x \in U_{\mathfrak{p}}, y \in U_{\mathfrak{q}}$. We define $x \sim y$ if and only if one of the following holds:

(a) $\mathfrak{p}=\mathfrak{q}$ and $x=y$.

(b) $\mathfrak{p} \leq \mathfrak{q}$ and $y=\varphi_{\mathfrak{q p}}(x)$.

(c) $\mathfrak{q} \leq \mathfrak{p}$ and $x=\varphi_{\mathfrak{p q}}(y)$.

Then $\sim$ is an equivalence relation.

(8) The quotient of $\coprod_{\mathfrak{p} \in \mathfrak{P}} U_{\mathfrak{p}} / \sim$ by this equivalence relation is Hausdorff with respect to the quotient topology.

Definition 31.10. Let $\widehat{\mathcal{U}}$ be a Kuranishi structure and $\widehat{\mathcal{U}}$ a good coordinate system of $X$. A strict $K G$-embedding of $\widehat{\mathcal{U}}$ to $\widehat{\mathcal{U}}$ assigns, for each pair $(p, \mathfrak{p})$ with $p \in X$, $\mathfrak{p} \in \mathfrak{P}$ satisfying $p \in \operatorname{Im}\left(\psi_{\mathfrak{p}}\right)$, an embedding of Kuranishi charts $\Phi_{\mathfrak{p} p}=\left(\varphi_{\mathfrak{p} p}, \widehat{\varphi}_{\mathfrak{p} p}\right)$ : $\mathcal{U}_{p} \rightarrow \mathcal{U}_{\mathfrak{p}}$ with the following properties.

If $\mathfrak{p}, \mathfrak{q} \in \mathfrak{P}, \mathfrak{q} \leq \mathfrak{p}, p \in \operatorname{Im}\left(\psi_{\mathfrak{p}}\right), q \in \operatorname{Im}\left(\psi_{p}\right) \cap \psi_{\mathfrak{q}}\left(U_{\mathfrak{p q}}\right)$, then the following diagram commutes.

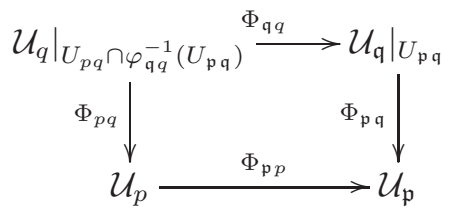

A $K G$-embedding of $\widehat{\mathcal{U}}$ to $\widehat{\mathcal{U}}$ is by definition a strict KG-embedding of an open substructure of $\widehat{\mathcal{U}}$ to $\hat{\mathcal{U}}$.

The notions of KK-embeding (embedding of a Kuranishi structure to another Kuranishi structure) and GG-embedding (embedding of a good coordinate system to another good coordinate system) are defined in a similar way. See FOOO11, Definitions 3.20 and 3.24].

The basic existence theorem of good coordinate system is the following.

Theorem 31.11. ([FO, Lemma 6.3], [FOO01, Lemma A1.11], [FOOO11, Theorem 3.10]) If $\widehat{\mathcal{U}}$ is a Kuranishi structure of $X$ then there exists a good coordinate system $\hat{\mathcal{U}}$ of $X$ with a KG-embedding $\widehat{\mathcal{U}}$ to $\hat{\mathcal{U}}$.

See [FOOO11, Section 11] for the proof. See FOOO13, for the detail of the argument to establish Definition 31.9 (7)(8). We can also prove its relative version. (See [FOOO11, Proposition 5.11 etc.].) 


\section{Strongly SMOOTH MAP AND FIBER PRODUCT}

\subsection{Strongly smooth map.}

Definition 32.1. Let $\widehat{\mathcal{U}}$ be a Kuranishi structure of $X$.

(1) A strongly continuous map $\widehat{f}$ from $(X, \widehat{\mathcal{U}})$ to a topological space $Y$ assigns a continuous map $f_{p}$ from $U_{p}$ to $Y$ for each $p \in X$ such that $f_{p} \circ \varphi_{p q}=f_{q}$ holds on $U_{p q}$.

(2) In the situation of (1), the map $f: X \rightarrow Y$ defined by $f(p)=f_{p}(p)$ is a continuous map from $X$ to $Y$. We call $f: X \rightarrow Y$ the underlying continuous map of $\widehat{f}$.

(3) When $Y$ is a smooth manifold, we say $\widehat{f}$ is strongly smooth if each $f_{p}$ is smooth.

(4) A strongly smooth map is said to be weakly submersive if each $f_{p}$ is a submersion.

We sometimes say $f$ is a strongly continuous map (resp. a strongly smooth map, a weakly submersive map) in place of $\widehat{f}$ is a strongly continuous map (resp. a strongly smooth map, weakly submersive), by an abuse of notation.

We can define similar notions for the good coordinate system by modifying the definition in an obvious way. (See [FOOO11, Definition 3.38].)

In [FOOO11, Proposition $6.49(2)]$ the next result is also proved.

Addendum 32.1. In the situation of Theorem 31.11, suppose there exists a strongly continuous map $\widehat{f}:(X, \widehat{\mathcal{U}}) \rightarrow Y$. Then we may choose a good coordinate system $\hat{\mathcal{U}}$ so that $\widehat{f}$ is induced by a strongly smooth map $\widehat{f}:(X, \widehat{\mathcal{U}}) \rightarrow Y$. If $Y$ is a manifold and $\widehat{f}$ is strongly smooth (resp. weakly submersive), we may choose $\hat{f}$ to be strongly smooth (resp. weakly submersive) also.

32.2. Fiber product. We next define a fiber product of Kuranishi structure. Note if $f: X \rightarrow Y$ and $f^{\prime}: M \rightarrow Y$ are continuous maps between topological spaces, their fiber product is a topological space defined by

$$
X_{f} \times_{f^{\prime}} M=\left\{(x, y) \in X \times M \mid f(x)=f^{\prime}(y)\right\} .
$$

Definition 32.2. For given $p_{i} \in X_{i}, i=1,2$, let $\mathcal{U}_{p_{i}}=\left(U_{p_{i}}, \mathcal{E}_{p_{i}}, \psi_{p_{i}}, s_{p_{i}}\right)$ be their Kuranishi neighborhoods. Then we define a Kuranishi neighborhood of $p=$ $\left(p_{1}, p_{2}\right) \in X_{1} \times X_{2}$ by $\mathcal{U}_{p}=\mathcal{U}_{p_{1}} \times \mathcal{U}_{p_{2}}=\left(U_{p}, \mathcal{E}_{p}, \psi_{p}, s_{p}\right)$ where

$$
\left(U_{p}, \mathcal{E}_{p}, \psi_{p}, s_{p}\right)=\left(U_{p_{1}} \times U_{p_{2}}, \mathcal{E}_{p_{1}} \times \mathcal{E}_{p_{2}}, \psi_{p_{1}} \times \psi_{p_{2}}, s_{p_{1}} \times s_{p_{2}}\right) .
$$

This system satisfies the condition of Kuranishi neighborhood (Definition 31.6.)

Suppose $q_{i} \in X_{i}$ and $q=\left(q_{1}, q_{2}\right) \in X_{1} \times X_{2}$. If $q \in \psi_{p}\left(s_{p}^{-1}(0)\right)$, then it is easy to see that $q_{i} \in \psi_{p_{i}}\left(s_{p_{i}}^{-1}(0)\right)$ for $i=1,2$. Therefore there exist coordinate changes $\Phi_{p_{i} q_{i}}=\left(\varphi_{p_{i} q_{i}}, \widehat{\varphi}_{p_{i} q_{i}}, h_{p_{i} q_{i}}\right)$ from $\mathcal{U}_{q_{i}}$ to $\mathcal{U}_{p_{i}}$. We define

$$
\begin{aligned}
\Phi_{p q} & =\Phi_{p_{1} q_{1}} \times \Phi_{p_{2} q_{2}}=\left(U_{p q}, \varphi_{p q}, \widehat{\varphi}_{p q}\right) \\
& =\left(U_{p_{1} q_{1}} \times U_{p_{2} q_{2}}, \varphi_{p_{1} q_{1}} \times \varphi_{p_{2} q_{2}}, \widehat{\varphi}_{p_{1} q_{1}} \times \widehat{\varphi}_{p_{2} q_{2}}\right) .
\end{aligned}
$$

This satisfies the condition of coordinate change of Kuranishi charts (Definition 31.7).

Then it is also easy to show that $\left(\left\{\mathcal{U}_{p_{1}} \times \mathcal{U}_{p_{2}}\right\},\left\{\Phi_{p_{1} q_{1}} \times \Phi_{p_{2} q_{2}}\right\}\right)$ defines a Kuranishi structure of $X_{1} \times X_{2}$ in the sense of Definition 31.8. (We note that effectivity of an 
orbifold is preserved by the direct product.) We call this Kuranishi structure the direct product Kuranishi structure.

Definition 32.3. Let $(X, \widehat{\mathcal{U}})$ be a space with Kuranishi structure and $\widehat{f}=\left\{f_{p}\right\}$ : $(X, \widehat{\mathcal{U}}) \rightarrow N$ a strongly smooth map, where $N$ is a smooth manifold of finite dimension. Let $f^{\prime}: M \rightarrow N$ be a smooth map between smooth manifolds. Assume $M$ is compact. We say $\widehat{f}$ is weakly transversal to $f^{\prime}$ on $X$ if the following holds. Let $(p, q) \in X \times_{N} M$ and $\mathcal{U}_{p}=\left(U_{p}, E_{p}, s_{p}, \psi_{p}\right)$ be a Kuranishi neighborhood of $p$. We then require that for each $(x, y) \in U_{p} \times M$ with $f_{p}(x)=f^{\prime}(y)$ we have

$$
\left(d_{x} f_{p}\right)\left(T_{x} U_{p}\right)+\left(d_{y} f^{\prime}\right)\left(T_{y} M\right)=T_{f(x)} N .
$$

Lemma 32.4. In the situation of Definition 32.3 we can define a Kuranishi structure on $X \times_{N} M$.

The proof is easy. In fact the Kuranishi neighborhood is obtained by the fiber product $U_{p} \times_{N} M$. See [FOOO11, page 34] for the detail. We call the Kuranishi structure obtained in Lemma 32.4 the fiber product Kuranishi structure and write:

$$
(X, \widehat{\mathcal{U}})_{f} \times_{N} M .
$$

Definition 32.5. Suppose $X_{i}(i=1,2)$ have Kuranihi structures $\widehat{\mathcal{U}}_{i}$ and the maps $\widehat{f_{i}}:\left(X_{i}, \widehat{\mathcal{U}}_{i}\right) \rightarrow N$ are strongly smooth. We say that $\widehat{f}_{1}$ is weakly transversal to $\widehat{f}_{2}$ if

$$
\widehat{f_{1}} \times \widehat{f_{2}}:\left(X_{1}, \widehat{\mathcal{U}_{1}}\right) \times\left(X_{2}, \widehat{\mathcal{U}_{2}}\right) \rightarrow N \times N
$$

is weakly transversal to the diagonal $N=\Delta_{N} \subset N \times N$ in the sense of Definition 32.3 .

Then we define the fiber product $\left(X_{1}, \widehat{\mathcal{U}_{1}}\right)_{f_{1}} \times_{f_{2}}\left(X_{2}, \widehat{\mathcal{U}_{2}}\right)$ by

$$
\left(X_{1}, \widehat{\mathcal{U}_{1}}\right)_{f_{1}} \times_{f_{2}}\left(X_{2}, \widehat{\mathcal{U}_{2}}\right)=\left(\left(X_{1}, \widehat{\mathcal{U}_{1}}\right) \times\left(X_{2}, \widehat{\mathcal{U}_{2}}\right)\right) \times_{N \times N} \Delta_{N}
$$

where the right hand side is as in (32.2).

\section{CF PERTURBATION AND INTEGRATION ALONG THE FIBER}

In this section we study integration of differential forms on the space with $\mathrm{Ku}$ ranishi structure or with good coordinate system.

\subsection{Differential form on the space with Kuranishi structure.}

Definition 33.1. A differential $k$ form $\widehat{h}$ of a Kuranishi structure $\widehat{\mathcal{U}}$ of $X$ assigns a differential $k$-form $h_{p}$ on $U_{p}$ for each $p \in X$ such that $\varphi_{p q}^{*} h_{p}=h_{q}$.

Differential form on a space with good coordinate system can be defined in the same way. (See [FOOO11, Definition 7.68].)

Definition 33.2. Let $\widehat{f}:(X, \widehat{\mathcal{U}}) \rightarrow M$ be a strongly smooth map to a manifold $M$ and $h$ a differential form on $M$. For $p \in X, \widehat{f}$ determines a smooth map $f_{p}: U_{p} \rightarrow M$ where $U_{p}$ is a Kuranishi neighborhood associated to $p \in X$ by $\widehat{\mathcal{U}}$. Then the family $\left\{f_{p}^{*} h \mid p \in X\right\}$ becomes a differential form of $(X, \widehat{\mathcal{U}})$. We call it the pull back and write $\widehat{f}^{*} h$.

The case of good coordinate system is similar.

Definition 33.3. Let $\widehat{h}=\left\{h_{p} \mid p \in X\right\}$ be a differential form on $(X, \widehat{\mathcal{U}})$. Then $\left\{d h_{p} \mid p \in X\right\}$ is also a differential form on $(X, \widehat{\mathcal{U}})$, which we write $d \widehat{h}$. The case of good coordinate system is similar. 
Let $\widehat{h}$ be a differential form on $(X, \widehat{\mathcal{U}})$. We want to integrate $\widehat{h}$ on $X . X$ is locally written as $s_{p}^{-1}(0)$, which can be singular. So to make sense out of this integration we need to perturb $s_{p}$ appropriately so that the zero set of the perturbed section becomes smooth. The notion of CF-perturbation (CF stands for 'continuous family') is defined for this purpose. Below we describe basic definitions of CF-perturbation and integration along the fiber of a differential form on a space equipped with good coordinate system or a Kuranishi structure together with CF perturbation. To obtain a global definition from the definition on each local chart, we need to check certain consistency or well-defined-ness of the definitions. We omit its explanation here since we describe its detail in [FOOO11, Section 7].

\subsection{CF-perturbation.}

Definition 33.4. Let $\mathcal{U}=(U, \mathcal{E}, \psi, s)$ be a Kuranishi chart of $X$. A $C F$-perturbation of $\mathcal{U}$ on an orbibundle chart $\mathfrak{V}=(V, \Gamma, E, \phi, \widehat{\phi})$ of $(X, \mathcal{E})$ consists of $\mathcal{S}=\left(W, \omega,\left\{\mathfrak{s}^{\epsilon}\right\}\right)$, $0<\epsilon \leq 1$, with the following properties:

(1) $W$ is an open subset of a finite dimensional vector space $\widehat{W}$ on which $\Gamma$ acts linearly. $W$ is $\Gamma$ invariant.

(2) $\mathfrak{s}^{\epsilon}: V \times W \rightarrow E$ is a $\Gamma$-equivariant smooth map for each $0<\epsilon \leq 1$, which depends smoothly on $\epsilon$. We sometimes regard $\mathfrak{s}^{\epsilon}$ as a family of sections $V \times W \rightarrow E \times V \times W$ of the trivial bundle $E \times V \times W \rightarrow V \times W$.

(3) For $(y, \xi) \in V \times W$ we have

$$
\lim _{\epsilon \rightarrow 0} \mathfrak{s}^{\epsilon}(y, \xi)=s(y)
$$

in compact $C^{1}$-topology on $V \times W$.

(4) $\omega$ is a smooth differential form on $W$ of degree $\operatorname{dim} W$ that is $\Gamma$ invariant, of compact support and

$$
\int_{W} \omega=1
$$

We assume $\omega=|\omega|$ vol where vol is a volume form of the oriented manifold $W$ and $|\omega|$ is a non-negative function.

For each $0<\epsilon \leq 1$, we denote the restriction of $\mathcal{S}$ at $\epsilon$, by $\mathcal{S}^{\epsilon}=\left(W, \omega, \mathfrak{s}^{\epsilon}\right)$.

Let $\mathcal{S}_{i}=\left(W_{i}, \omega_{i},\left\{\mathfrak{s}_{i}^{\epsilon}\right\}\right)(i=1,2)$ be two CF-perturbations of $\mathcal{U}$ on $\mathfrak{V}$. We say $\mathcal{S}_{2}^{\epsilon}$ is a projection of $\mathcal{S}_{1}^{\epsilon}$ if there exists a map $\Pi: \widehat{W}_{1} \rightarrow \widehat{W}_{2}$ with the following properties.

(1) $\Pi$ is a $\Gamma$ equivariant linear projection which sends $W_{1}$ to $W_{2}$ and satisfies $\Pi_{!}\left(\omega_{1}\right)=\omega_{2}$.

(2) For each $(y, \xi) \in V_{x} \times W_{1}$ we have $\mathfrak{s}_{1}^{\epsilon}(y, \Pi(\xi))=\mathfrak{s}_{2}^{\epsilon}(y, \xi)$.

Let $x \in X$. We write $\mathcal{S}_{1}^{\epsilon} \sim_{x}^{\prime} \mathcal{S}_{2}^{\epsilon}$ if a restriction of $\mathcal{S}_{1}^{\epsilon}$ to a neighborhood of $x$ is a projection of a restriction of the $\mathcal{S}_{2}^{\epsilon}$ to that neighborhood.

We denote by $\sim_{x}$ the equivalence relation generated by $\sim_{x}^{\prime}$.

We say $\mathcal{S}_{1}^{\epsilon}$ is equivalent to $\mathcal{S}_{2}^{\epsilon}$ and write $\mathcal{S}_{1}^{\epsilon} \sim \mathcal{S}_{2}^{\epsilon}$ if $\mathcal{S}_{1}^{\epsilon} \sim_{x} S_{2}^{\epsilon}$ at all $x$.

Definition 33.5. Let $\mathcal{U}=(U, \mathcal{E}, \psi, s)$ be a Kuranishi chart of $X$. A $C F$-perturbation of $\mathcal{U}$ is represented by $\left(\left\{\mathcal{S}_{\mathfrak{r}}^{\epsilon} \mid \mathfrak{r} \in \mathfrak{R}\right\},\left\{\mathfrak{V}_{\mathfrak{r}} \mid \mathfrak{r} \in \mathfrak{R}\right\}\right.$ ) (where $\mathfrak{R}$ is a finite set,) such that

(1) $\mathfrak{V}_{\mathfrak{r}}=\left(V_{\mathfrak{r}}, \Gamma_{\mathfrak{r}}, E_{\mathfrak{r}}, \phi_{\mathfrak{r}}, \widehat{\phi}_{\mathfrak{r}}\right)$ is an orbibundle chart of $(X, \mathcal{E})$ and the image $U_{\mathfrak{r}}$ of $\phi_{\mathfrak{r}}$ for various $\mathfrak{r}$ is an open covering of $X$. 
(2) $\mathcal{S}_{\mathfrak{r}}=\left(W_{\mathfrak{r}}, \omega_{\mathfrak{r}},\left\{\mathfrak{s}_{\mathfrak{r}}^{\epsilon}\right\}\right)$ is a CF-perturbation of $\mathcal{U}$ on $\mathfrak{V}_{\mathfrak{r}}$.

(3) For each $x \in U_{\mathfrak{r}_{1}} \cap U_{\mathfrak{r}_{2}}$, we require $\mathcal{S}_{\mathfrak{r}_{1}} \sim_{x} \mathcal{S}_{\mathfrak{r}_{2}}$.

Two such $\left(\left\{\mathcal{S}_{\mathfrak{r}}^{\epsilon}\right\},\left\{\mathfrak{V}_{\mathfrak{r}}\right\}\right)$ and $\left(\left\{\mathcal{S}_{\mathfrak{r}^{\prime}}^{\prime, \epsilon}\right\},\left\{\mathfrak{V}_{\mathfrak{r}^{\prime}}\right\}\right)$ are said to be equivalent if for $x \in$ $U_{\mathfrak{r}} \cap U_{\mathfrak{r}^{\prime}}^{\prime}$ the following holds.

$$
\mathcal{S}_{\mathfrak{r}}^{\epsilon} \sim_{x} \mathcal{S}_{\mathfrak{r}^{\prime}}^{\prime, \epsilon} .
$$

A CF-perturbation of $\mathcal{U}$ is by definition an equivalence class of this equivalence relation. We denote by $\mathfrak{S}=\left[\left\{\mathcal{S}_{\mathfrak{r}}^{\epsilon} \mid \mathfrak{r} \in \mathfrak{R}\right\},\left\{\mathfrak{V}_{\mathfrak{r}} \mid \mathfrak{r} \in \mathfrak{R}\right\}\right]$ the equivalence class of the representative $\left(\left\{\mathcal{S}_{\mathfrak{r}}^{\epsilon}\right\},\left\{\mathfrak{V}_{\mathfrak{r}}\right\}\right)$.

Let $\mathfrak{S}$ be a $\mathrm{CF}$-perturbation of $\mathcal{U}$. For each $x \in U$ and a sufficiently small orbibundle chart $\mathfrak{V}_{x}=\left(V_{x}, \Gamma_{x}, E_{x}, \phi_{x}, \widehat{\phi}_{x}\right)$, $\mathfrak{S}$ determines a CF-perturbation of $\mathcal{U}$ at $\mathfrak{V}_{x}$ up to the equivalence. We call an equivalence class a representative of the germ of $\mathrm{CF}$-perturbation $\mathfrak{S}$ at $x$

Definition 33.6. In the situation of Definitoin 33.4, we consider a CF-perturbation $\mathcal{S}=\left(W, \omega,\left\{\mathfrak{s}^{\epsilon}\right\}\right)$ of $\mathcal{U}$ on $\mathfrak{V}$.

(1) We say $\mathcal{S}$ is transversal to 0 if there exists $\epsilon_{0}>0$ such that the map $\mathfrak{s}^{\epsilon}$ is transversal to 0 on a neighborhood of the support of $\omega$ for all $0<\epsilon<\epsilon_{0}$. In particular

$$
\left(\mathfrak{s}^{\epsilon}\right)^{-1}(0)=\left\{(y, \xi) \in V \times W \mid \mathfrak{s}^{\epsilon}(y, \xi)=0\right\}
$$

is a smooth submanifold of $V \times W$ on a neighborhood of the support of $\omega$.

(2) Let $f: V \rightarrow M$ be a $\Gamma$ equivariant smooth map. We say $f$ is strongly submersive with respect to $(\mathfrak{V}, \mathcal{S})$ if $\mathcal{S}$ is transversal to 0 and there exists $\epsilon_{0}>0$ such that the map

$$
\left.f \circ \pi_{1}\right|_{\left(\mathfrak{s}^{\epsilon}\right)^{-1}(0)}:\left(\mathfrak{s}^{\epsilon}\right)^{-1}(0) \rightarrow M
$$

is a submersion on a neighborhood of the support of $\omega$, for all $0<\epsilon<\epsilon_{0}$. Here $\pi_{1}: V \times W \rightarrow V$ is the projection.

In the situation of Definition 33.5, we define transversality to 0 (resp. strongly submersivity) of CF perturbation $\mathfrak{S}=\left[\left\{\mathcal{S}_{\mathfrak{r}}^{\epsilon}\right\},\left\{\mathfrak{V}_{\mathfrak{r}}\right\}\right]$ on a Kuranishi chart as that of each of $\mathcal{S}_{\mathfrak{r}}^{\epsilon}$. We can easily prove that the definition does not depend on the representative of the $\mathrm{CF}$-perturbation $\mathfrak{S}$.

Definition 33.7. Let $\mathcal{U}=(U, \mathcal{E}, \psi, s), \mathcal{U}^{\prime}=\left(U^{\prime}, \mathcal{E}^{\prime}, \psi^{\prime}, s^{\prime}\right)$ be Kuranishi charts of $X$ and $\Phi=(\varphi, \widehat{\varphi})$ an embedding of Kuranishi charts : $\mathcal{U} \rightarrow \mathcal{U}^{\prime}$ in the sense of Definition 31.5.

Let $\mathfrak{S}=\left[\left\{\mathcal{S}_{\mathfrak{r}}^{\epsilon} \mid \mathfrak{r} \in \mathfrak{R}\right\},\left\{\mathfrak{V}_{\mathfrak{r}} \mid \mathfrak{r} \in \mathfrak{R}\right\}\right], \mathfrak{S}^{\prime}=\left[\left\{\mathcal{S}_{\mathfrak{r}^{\prime}}^{\prime, \epsilon} \mid \mathfrak{r}^{\prime} \in \mathfrak{R}^{\prime}\right\},\left\{\mathfrak{V}_{\mathfrak{r}^{\prime}}^{\prime} \mid \mathfrak{r}^{\prime} \in \mathfrak{R}^{\prime}\right\}\right]$ be $\mathrm{CF}$-perturbations of $\mathcal{U}$ and $\mathcal{U}^{\prime}$, respectively.

We say that $\mathfrak{S}, \mathfrak{S}^{\prime}$ are compatible with the embedding $\Phi$ if for each $x \in U$ we can find a representatives $\left(\mathfrak{V}_{x}, \mathcal{S}_{x}^{\epsilon}\right),\left(\mathfrak{V}_{x}^{\prime}, \mathcal{S}_{y}^{\prime, \epsilon}\right)$ of $\mathfrak{S}, \mathfrak{S}^{\prime}$ at $x, y=\varphi(x)$, respectively, such that the following holds. We put $\mathfrak{V}_{x}=\left(V_{x}, \Gamma, E_{x}, \phi_{x}, \widehat{\phi}_{x}\right), \mathfrak{V}_{y}^{\prime}=\left(V_{y}^{\prime}, \Gamma_{y}^{\prime}, E_{y}^{\prime}, \phi_{y}^{\prime}, \widehat{\phi}_{y}^{\prime}\right)$ and $\mathcal{S}_{x}=\left(W_{x}, \omega_{x},\left\{\mathfrak{s}_{x}^{\epsilon}\right\}\right), \mathcal{S}_{y}^{\prime}=\left(W_{y}^{\prime}, \omega_{y}^{\prime},\left\{\mathfrak{s}_{y}^{\prime}, \epsilon\right\}\right)$.

(1) $W_{x}=W_{y}^{\prime} \cdot \omega_{x}=\omega_{y}^{\prime}$.

\footnotetext{
${ }^{9}$ We can define a sheaf of CF-perturbations and germ makes sense using sheaf theory. See [FOOO11 Subsection 7.2].
} 
(2) The next diagram commutes.

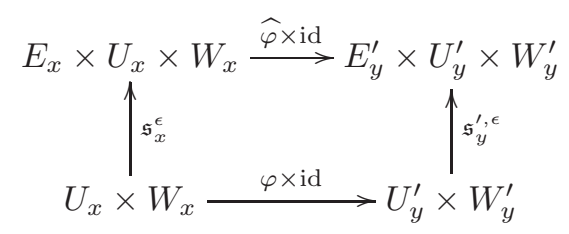

Definition 33.8. (1) Let $\widehat{\mathcal{U}}=\left((\mathfrak{P}, \leq),\left\{\mathcal{U}_{\mathfrak{p}} \mid \mathfrak{p} \in \mathfrak{P}\right\},\left\{\Phi_{\mathfrak{p q}} \mid \mathfrak{q} \leq \mathfrak{p}\right\}\right)$ be a good coordinate system of $X$. A $C F$-perturbation $\widehat{\mathfrak{S}}$ of $(X, \widehat{\mathcal{U}})$ assigns a CF-perturbation $\mathfrak{S}_{\mathfrak{p}}$ of $\mathcal{U}_{\mathfrak{p}}$ to each $\mathfrak{p} \in \mathfrak{P}$, so that whenever $\mathfrak{q} \leq \mathfrak{p}$, the restrictions of the corresponding CF-perturbations $\mathfrak{S}_{\mathfrak{p}}, \mathfrak{S}_{\mathfrak{q}}$ to the domain of $\Phi_{\mathfrak{p q}}$ are compatible with the embedding $\Phi_{\mathfrak{p q}}$ in the sense of Definition 33.7

(2) Let $\widehat{\mathcal{U}}=\left(\left\{\mathcal{U}_{p} \mid p \in X\right\},\left\{\Phi_{p q}\right\}\right)$ be a Kuranishi structure of $X$. A $C F$ perturbation $\widehat{\mathfrak{S}}$ of $(X, \widehat{\mathcal{U}})$ assigns a CF-perturbation $\mathfrak{S}_{p}$ of $\mathcal{U}_{p}$ to each $p \in X$, such that for any $q \in \psi_{p}\left(U_{p}\right), \mathcal{U}_{p}, \mathcal{U}_{q}$ are compatible with the embedding $\Phi_{p q}$ in the sense of Definition 33.7

Definition 33.9. (1) A transversality to 0 of the CF-perturbation on a good coordinate system or on a Kuranishi structure is defined in an obvious way based on Definition 33.6.

(2) Let $\widehat{f}:(X, \widehat{\mathcal{U}}) \rightarrow M$ (resp. $\hat{f}:(X, \widehat{\mathcal{U}}) \rightarrow M)$ be a strongly smooth map from a space with Kuranishi structure (resp. good coordinate system). We say $\widehat{f}$ (resp. $\widehat{f}$ ) is strongly submersive with respect to $\widehat{\mathfrak{S}}$ (resp. $\widehat{\mathfrak{S}}$ ) if it is so on each Kuranishi chart in the sense of Definition 33.6.

A key existence result of CF-perturbation is the following Theorem 33.11, To state Theorem 33.11 we need the next definition.

Definition 33.10. We say a good coordinate system $\widehat{\mathcal{U}^{\prime}}=\left(\left(\mathfrak{P}^{\prime}, \leq\right),\left\{\mathcal{U}_{\mathfrak{p}}^{\prime} \mid \mathfrak{p}^{\prime} \in\right.\right.$ $\left.\left.\mathfrak{P}^{\prime}\right\},\left\{\Phi_{\mathfrak{p}^{\prime} \mathfrak{q}^{\prime}}^{\prime} \mid \mathfrak{q}^{\prime} \leq \mathfrak{p}^{\prime}\right\}\right)$ is a shrinking of $\widehat{\mathcal{U}}=\left((\mathfrak{P}, \leq),\left\{\mathcal{U}_{\mathfrak{p}} \mid \mathfrak{p} \in \mathfrak{P}\right\},\left\{\Phi_{\mathfrak{p q}} \mid \mathfrak{q} \leq \mathfrak{p}\right\}\right)$ if the following holds.

(1) $\mathfrak{P}=\mathfrak{P}^{\prime}$.

(2) $\mathcal{U}_{\mathfrak{p}}^{\prime}$ is a restriction of $\mathcal{U}_{\mathfrak{p}}$ to a relatively compact open subset $U_{\mathfrak{p}}^{\prime}$ of $U_{\mathfrak{p}}$.

(3) $\Phi_{\mathfrak{p q}}^{\prime}$ is the restriction of $\Phi_{\mathfrak{p q}}$ to $\varphi_{\mathfrak{p q}}^{-1}\left(U_{\mathfrak{p}}^{\prime}\right) \cap U_{\mathfrak{q}}^{\prime}$.

Theorem 33.11. ([FOOO11, Theorem 7.49]) Let $\widehat{\mathcal{U}}$ be a good coordinate system of $X$ and $\widehat{\mathcal{U}^{\prime}}$ its shrinking.

(1) There exists a CF-perturbation $\widehat{\mathfrak{S}^{\prime}}$ of $\left(X, \widehat{\mathcal{U}^{\prime}}\right)$ transversal to 0.

(2) If $\hat{f}:(X, \hat{\mathcal{U}}) \rightarrow M$ is a weakly submersive and strongly smooth map, then we may take a CF-perturbation $\widehat{\mathfrak{S}}$ so that $\hat{f}$ is strongly submersive with respect to $\widehat{\mathfrak{S}}$.

Note in FOOO11 we use the notion of a support system and state Theorem 33.11 in a slightly different way. Here to shorten the description we use the notion of the shrinking in place of the support system.

We can prove a relative version of Theorem 33.11, See FOOO11, Proposition 7.52 and Lemma 7.53]. 
For the Kuranishi structure (instead of the good coordinate system) we have the following:

Theorem 33.12. For any Kuranishi structure $\widehat{\mathcal{U}}$ of $X$, we can find a Kuranishi structure $\widehat{\mathcal{U}^{\prime}}$ of $X$ and a KK-embedding $\widehat{\mathcal{U}} \rightarrow \widehat{\mathcal{U}^{\prime}}$ such that the following holds.

(1) There exists a CF-perturbation $\widehat{\mathfrak{S}^{\prime}}$ of $\left(X, \widehat{\mathcal{U}^{\prime}}\right)$ transversal to 0.

(2) If $\widehat{f}:(X, \widehat{\mathcal{U}}) \rightarrow M$ is a weakly submersive strongly smooth map, then we may take the CF-perturbation $\widehat{\mathfrak{S}^{\prime}}$ so that $\widehat{f}$ is strongly submersive with respect to $\widehat{\mathfrak{S}^{\prime}}$.

This theorem follows from Theorems 33.11, 31.11, Addendum 32.1] and [FOOO11, Lemma 9.9].

\subsection{Integration along the fiber.}

Definition 33.13. In the situation of Definition 33.4 we assume that $f: U \rightarrow M$ is a smooth map to a smooth manifold $M$. Let $\mathcal{S}=\left(W, \omega,\left\{\mathfrak{s}^{\epsilon}\right\}\right)$ be a CF-perturbation of $\mathcal{U}$ on $\mathfrak{V}=(V, \Gamma, E, \phi, \widehat{\phi})$. We assume $f$ is strongly submersive with respect to $\mathcal{S}$. Let $h$ be a smooth differential form on $U$ that has compact support in $\phi(V)$. Then we define a smooth differential form $f !\left(h ; \mathcal{S}^{\epsilon}\right)$ on $M$ for each $\epsilon>0$ by the equation (33.4) below. We call the differential form $f !\left(h ; \mathcal{S}^{\epsilon}\right)$ the integration along the fiber of $h$ with respect to $f, \mathfrak{S}$.

For any smooth differential form $\rho$ on $M$, we require $f !\left(h ; \mathcal{S}^{\epsilon}\right)$ to satisfy

$$
\# \Gamma \int_{M} f !\left(h ; \mathcal{S}^{\epsilon}\right) \wedge \rho=\int_{\left(\mathfrak{s}^{\epsilon}\right)^{-1}(0)} \pi_{1}^{*}(\tilde{h}) \wedge \pi_{1}^{*}\left(f^{*} \rho\right) \wedge \pi_{2}^{*} \omega .
$$

Here $\pi_{1}$ (resp. $\pi_{2}$ ) is the projection of $V \times W$ to the first (resp. second) factor, \# $\#$ is the order of the finite set $\Gamma$, and $\tilde{h}$ is the pull back of $h$ to $V$.

We remark that

$$
\operatorname{deg} f !\left(h ; \mathcal{S}^{\epsilon}\right)=\operatorname{deg} h+\operatorname{dim} M-\operatorname{dim} \mathcal{U} .
$$

The unique existence of such a differential form $f !\left(h ; \mathcal{S}^{\epsilon}\right)$ is an immediate consequence of the existence of integration along the fiber of a smooth form by a proper submersion.

Remark 33.14. Note that the right hand side of (33.4) depends on $\epsilon$. Therefore the differential form $f !\left(h ; \mathcal{S}^{\epsilon}\right)$ depends on $\epsilon$.

Definition 33.15. In the situation of Definition 33.5 let $\mathfrak{S}=\left[\left\{\mathcal{S}_{\mathfrak{r}}^{\epsilon} \mid \mathfrak{r} \in \mathfrak{R}\right\},\left\{\mathfrak{V}_{\mathfrak{r}} \mid\right.\right.$ $\mathfrak{r} \in \mathfrak{R}\}]$ be a CF-perturbation of $\mathcal{U}$ and $f: U \rightarrow X$ a smooth map. We assume $f$ is strongly submersive with respect to $\mathfrak{S}$. We consider the open covering

$$
U=\bigcup_{\mathfrak{r} \in \Re} U_{\mathfrak{r}}
$$

where $U_{\mathfrak{r}}=\phi_{\mathfrak{r}}\left(V_{\mathfrak{r}}\right)$. Let $\chi_{\mathfrak{r}}$ be a partition of unity subordinate to the covering (33.6). Let $h$ be a differential form on $U$ of compact support. We then define the integration along the fiber of $h$ with respect to $f, \mathfrak{S}$ by the following formula.

$$
f !\left(h ; \mathfrak{S}^{\epsilon}\right)=\sum_{\mathfrak{r} \in \mathfrak{R}} f !\left(\chi_{\mathfrak{r}} h ; \mathcal{S}_{\mathfrak{r}}^{\epsilon}\right) .
$$

Here the right hand is defined by Definition 33.13. The degree is given by (33.5). 
We can prove that the right hand side of (33.7) is independent of the choice of partition of unity $\chi_{\mathfrak{r}}$ as well as the representative $\left(\left\{\mathcal{S}_{\mathfrak{r}}^{\epsilon} \mid \mathfrak{r} \in \mathfrak{R}\right\},\left\{\mathfrak{V}_{\mathfrak{r}} \mid \mathfrak{r} \in \mathfrak{R}\right\}\right)$ of S. (FOOO11, Proposition 7.80].)

We next discuss the case of good coordinate system $\widehat{\mathcal{U}}=\left((\mathfrak{P}, \leq),\left\{\mathcal{U}_{\mathfrak{p}} \mid \mathfrak{p} \in\right.\right.$ $\left.\mathfrak{P}\},\left\{\Phi_{\mathfrak{p q}} \mid \mathfrak{q} \leq \mathfrak{p}\right\}\right)$ of $X$. We use the equivalence relation $\sim$ on the disjoint union $\coprod_{\mathfrak{p} \in \mathfrak{P}} U_{\mathfrak{p}}$, which is defined in Definition 31.9 (7) and denote by $|\widehat{\mathcal{U}}|$ the set of this equivalence class. This space is Hausdorff with respect to the quotient topology. Suppose $\widehat{\mathcal{U}}$ is a shrinking of $\widehat{\mathcal{U}}^{+}$. Then $|\widehat{\mathcal{U}}|$ is a subset of $\mid \widehat{\mathcal{U}}+$. We put the topology of $|\widehat{\mathcal{U}}|$ that is induced from the quotient topology of $\left|\widehat{\mathcal{U}^{+}}\right|$. Then $|\overrightarrow{\mathcal{U}}|$ is metrizable with respect to this topology. ([FOOO13, Proposition 2.11].) Hereafter we use this topology on $|\widehat{\mathcal{U}}|$. We take its metric $d$ and fix it. We remark that by construction of $|\widehat{\mathcal{U}}|$ we have natural embeddings $X \hookrightarrow|\widehat{\mathcal{U}}|, U_{\mathfrak{p}} \hookrightarrow|\widehat{\mathcal{U}}|$ so we may regard them as subsets

$$
X \subset|\widehat{\mathcal{U}}|, \quad U_{\mathfrak{p}} \subset|\widehat{\mathcal{U}}| .
$$

We first discuss the notion of partition of unity with respect to the covering $\left\{U_{\mathfrak{p}} \mid\right.$ $\mathfrak{p} \in \mathfrak{P}\}$ of $|\widehat{\mathcal{U}}|$.

Definition 33.16. A continuous function $g:|\widehat{\mathcal{U}}| \rightarrow \mathbb{R}$ is said to be smooth if the restriction of $g$ to any $U_{\mathfrak{p}}$ is a smooth function.

Definition 33.17. Let $K_{\mathfrak{p}} \subset U_{\mathfrak{p}}$ be a compact subset such that

$$
\bigcup_{\mathfrak{p} \in \mathfrak{P}} \psi_{\mathfrak{p}}\left(s_{\mathfrak{p}}^{-1}(0) \cap K_{\mathfrak{p}}\right) \supseteq X,
$$

and $\delta$ be a sufficiently small positive number.

$\mathrm{A}\left(\left\{K_{\mathfrak{p}}\right\}, \delta\right)$-partition of unity of $(X ; \widehat{\mathcal{U}})$ assigns $\chi_{\mathfrak{p}}$ to each $\mathfrak{p} \in \mathfrak{P}$, so that:

(1) $\chi_{\mathfrak{p}}:|\widehat{\mathcal{U}}| \rightarrow[0, \infty)$ is a smooth function.

(2) The support of $\chi_{\mathfrak{p}}$ is in the $\delta$ neighborhood of $K_{\mathfrak{p}}$ in $|\hat{\mathcal{U}}| 10$

(3)

$$
\sum_{\mathfrak{p} \in \mathfrak{P}} \chi_{\mathfrak{p}} \equiv 1
$$

holds on the $\delta$-neighborhood $\mathfrak{U}(X)$ of $X$ in $|\widehat{\mathcal{U}}|$.

Existence of a $\left(\left\{K_{\mathfrak{p}}\right\}, \delta\right)$-partition of unity of $(X ; \overrightarrow{\mathcal{U}})$ for sufficiently small $\delta$ is proved in [FOOO11, Proposition 7.67].

Definition 33.18. Let $(X, \widehat{\mathcal{U}})$ be a space equipped with an oriented good coordinate system and $\widehat{f}:(X, \widehat{\mathcal{U}}) \rightarrow M$ a strongly smooth map. Let $\widehat{\mathfrak{S}}=\left\{\mathfrak{S}_{\mathfrak{p}} \mid \mathfrak{p} \in \mathfrak{P}\right\}$ be a CF-perturbation of $(X, \widehat{\mathcal{U}})$ such that $\hat{f}$ is strongly submersive with respect to $\widehat{\mathfrak{S}}$. Let $\hat{h}$ be a differential form on $(X, \hat{\mathcal{U}})$. We take $K_{\mathfrak{p}}$ as in Definition 33.17 and a sufficiently small $\delta$. Let $\left\{\chi_{\mathfrak{p}} \mid \mathfrak{p} \in \mathfrak{P}\right\}$ be a $\left(\left\{K_{\mathfrak{p}}\right\}, \delta\right)$-partition of unity of $(X ; \widehat{\mathcal{U}})$.

Then the integration along the fiber of $\widehat{h}$ with respect to $\widehat{f}, \widehat{\mathfrak{S}}$ is defined by the formula

$$
\widehat{f} !\left(\widehat{h} ; \widehat{\mathfrak{S}^{\epsilon}}\right)=\sum_{\mathfrak{p} \in \mathfrak{P}} f_{\mathfrak{p}} !\left(\chi_{\mathfrak{p}} h_{\mathfrak{p}} ;\left.\mathfrak{S}_{\mathfrak{p}}^{\epsilon}\right|_{\left.\mathfrak{U}(X) \cap B_{\delta}\left(K_{\mathfrak{p}}\right) \cap U_{\mathfrak{p}}\right) .}\right.
$$

Here $B_{\delta}\left(K_{\mathfrak{p}}\right)$ is the $\delta$ neighborhood of $K_{\mathfrak{p}}$ in $|\hat{\mathcal{U}}|$. The degree is given by (33.5).

\footnotetext{
${ }^{10}$ We remark that this $\delta$ neighborhood is not contained in $U_{\mathfrak{p}}$ in general.
} 
Proposition 33.19. The right hand side of (33.10) depends on $\widehat{h}, \widehat{\mathfrak{S}}, \epsilon$ but is independent of other choices such as partition of unity $\chi_{\mathfrak{p}}, \delta$ and $K_{\mathfrak{p}}$ provided $\delta$ and $\epsilon$ are sufficiently small.

This is proved in [FOOO11, Proposition 7.80].

We next study the case of Kuranishi structure.

Addendum 33.1. In the situation of Theorem 31.11 suppose that we have a CFperturbation $\widehat{\mathfrak{S}}$ on $\widehat{\mathcal{U}}$. Then we may choose $\overrightarrow{\mathcal{U}}$ such that $\widehat{\mathfrak{S}}$ is induced from $\widehat{\mathfrak{S}} 11$

If $\widehat{\mathfrak{S}}$ is transversal to 0 then we may choose $\widehat{\mathfrak{S}}$ so that it is transversal to 0 .

Suppose in addition that we are in the situation of Addendum 32.1, that is $\widehat{f}$ : $(X, \widehat{\mathcal{U}}) \rightarrow M, \hat{f}:(X, \widehat{\mathcal{U}}) \rightarrow M$ are weakly submersive. We assume that $\hat{f}$ is strongly submersive with respect to $\widehat{\mathfrak{S}}$. Then we may choose $\widehat{\mathfrak{S}}$ so that $\widehat{f}$ is strongly submersive with respect to $\widehat{\mathfrak{S}}$.

See [FOOO11, Proposition $9.10(2)(3)$ and Proposition 6.49 (2)] for the proof. In case a differential form $\widehat{h}$ on $(X, \widehat{\mathcal{U}})$ is given we may choose a differential form $\widehat{h}$ on $(X, \hat{\mathcal{U}})$ so that $\widehat{h}$ is induced by $\hat{h}$. 12

Definition 33.20. Let $(X, \widehat{\mathcal{U}})$ be an oriented space with Kuranishi structure and $\widehat{f}:(X, \widehat{\mathcal{U}}) \rightarrow M$ a weakly submersive map. Let $\widehat{\mathfrak{S}}$ be a CF-perturbation of $(X, \widehat{\mathcal{U}})$ such that $\widehat{f}$ is strongly submersive with respect to $\widehat{\mathfrak{S}}$. Let $\widehat{h}$ be a differential form on $(X, \widehat{\mathcal{U}})$.

Then we define the integration along the fiber of $\widehat{h}$ by the formula:

$$
\widehat{f} !(\widehat{h} ; \widehat{\mathfrak{S}} \epsilon)=\widehat{f} !(\widehat{h} ; \widehat{\mathfrak{S}} \epsilon)
$$

where $\hat{f}, \widehat{h}, \widehat{\mathfrak{S}}$ are as in Addendum 33.1 and right hand side is defined by (33.10). The degree is given by (33.5).

Proposition 33.21. The right hand side of (33.11) depends on $\widehat{h}, \widehat{\mathfrak{S}}, \epsilon$ but is independent of other choices such as $\widehat{\mathcal{U}}, \hat{f}, \widehat{h}, \widehat{\mathfrak{S}}$ if $\epsilon$ is sufficiently small.

This is [FOOO11, Theorem 9.14].

\section{Stokes' THEOREM}

34.1. Normalized boundary. To describe Stokes' theorem we first discuss a few points on the boundary of manifold, orbifold and of Kuranishi structure. Let $M$ be a manifold with corner. We say a point $p \in M$ lies in the codimension $k$ corner of $M$ if $p$ has a neighborhood diffeomorphic to $[0,1)^{k} \times \mathbb{R}^{n-k}$ where $p$ corresponds to the origin. We denote by $\stackrel{\circ}{S^{k}}(M)$ the set of all the points which lie in the codimension $k$ corner of $M . S^{k}(M)$ has a canonical structure of a manifold of dimension $n-k$ (without boundary). Its closure in general does not have a structure of a manifold with corner. However we can easily show the next lemma.

\footnotetext{
${ }^{11_{\text {Namely }}} \mathfrak{S}_{p}$ and $\mathfrak{S}_{\mathfrak{p}}$ are compatible with the embedding $\Phi_{\mathfrak{p}, p}: \mathcal{U}_{p} \rightarrow \mathcal{U}_{\mathfrak{p}}$ in the sense of Definition 33.7 Here $\Phi_{\mathfrak{p}, p}$ is a member of KG-embedding $\widehat{\mathcal{U}} \rightarrow \widehat{\mathcal{U}}$.

${ }^{12}$ Namely $h_{p}$ is a pull back of and $h_{\mathfrak{p}}$ by the embedding $\Phi_{\mathfrak{p}, p}: \mathcal{U}_{p} \rightarrow \mathcal{U}_{\mathfrak{p}}$. Here $\Phi_{\mathfrak{p}, p}$ is a member of KG-embedding $\widehat{\mathcal{U}} \rightarrow \widehat{\mathcal{U}}$.
} 
Lemma 34.1. There exists an $(n-k)$ dimensional manifold with corner $\widehat{S}^{k}(M)$, such that the inclusion $\stackrel{\circ}{S}^{k}(M) \rightarrow M$ extends to a map $\pi: \widehat{S}^{k}(M) \rightarrow M$ such that

$$
\#\left(\pi^{-1}(p)\right)=\frac{\ell !}{k !(\ell-k) !}
$$

if $p \in \stackrel{\circ}{S^{\ell}}(M)$ for $\ell \geq k$.

Definition 34.2. We denote $\partial M=\widehat{S}^{1}(M)$, which we call the normalized boundary of $M$. We call it the boundary in case the meaning is clear.

We call $\widehat{S}^{k}(M)$ the normalized codimension $k$ corner of $M$.

Example 34.3. Let $M=[0, \infty)^{n}$. Then $S^{1}(M)$ is a disjoint union of $n$ copies each of which is identified with $(0, \infty)^{n-1}$. $\partial M$ is then a disjoint union of the closures of those $n$ copies. Namely it is a disjoint union of $n$ copies of $[0, \infty)^{n-1}$.

Definition 34.4. (1) An orbifold chart with corner of $X$ is a triple $(V, \Gamma, \phi)$ satisfying the same condition as Definition 31.1 except that this time $V$ may have a corner.

(2) We define the notion of the structure of orbifold with corner on $X$ using item (1) in the same way as Definition 31.1 .

(3) By taking the normalized boundary (resp. corner) of $V$ for each $V$ we can define the normalized boundary (resp. corner) of $X$, which we denote by $\partial X\left(\right.$ resp. $\left.\widehat{S}^{k}(X)\right)$. Then it itself carries the structure of an orbifold with corner. $\stackrel{\circ}{S}^{\ell}(X)$ is defined in the same way.

(4) If $X$ is an oriented orbifold with boundary then its normalized boundary $\partial X$ is also oriented.

(5) The notion of embedding of orbifold with corner is defined in the same way as Definition 31.10. Note we require $f\left(\stackrel{\circ}{S}^{\ell}(X)\right) \subseteq \stackrel{\circ}{S^{\ell}}(Y)$ for an embedding $f: X \rightarrow Y$ of orbifold with corner.

Definition 34.5. By considering the case when $U$ is an orbifold with corners in the definition of Kuranishi chart $\mathcal{U}=(U, \mathcal{E}, \psi, s)$, we define a Kuranishi chart with corners.

Using it in an obvious way we define Kuranishi structure and good coordinate system with corners.

The normalized boundary $\partial(X, \widehat{\mathcal{U}})$ (resp. normalized corner $\left.\widehat{S}^{k}(X, \widehat{\mathcal{U}})\right)$ of a space with Kuranishi structure with corners can be defined by considering normalized boundary (resp. corner) on each Kuranishi chart. We can define normalized boundary and normalized corner in the same way for the case of good coordinate system with corners in an obvious way.

Definition 34.6. Let $M_{i}$ be a manifold with corners, $f_{i}: M_{i} \rightarrow N$ a smooth map, for $i=1,2$. We say $f_{1}$ is transversal to $f_{2}$ if the restriction of $f_{1}$ to $\stackrel{\circ}{S}^{k}\left(M_{1}\right)$ is transversal to the restriction of $f_{2}$ to $\stackrel{\circ}{S^{\ell}}\left(M_{2}\right)$ for any $k, \ell$. Transversality to zero of the section on a manifold with corners is defined in the same way.

The case of orbifold with corners is treated in the same way. 
Various definitions of the transversality on various objects on Kuranishi structure and/or good coordinate system can be generalized to the case of Kuranishi structure and/or good coordinate system with corners.

Let $\widehat{f}:(X, \widehat{\mathcal{U}}) \rightarrow M$ be a map from a space with Kuranishi structure with corners to a manifold (without boundary). Then we can define its strong submersivity as above. (Namely we require the strong submersivity of the restrictions to all the normalized corners as well as interior.) It is then easy to see that if $\widehat{f}$ is weakly submersive then it induces a weakly submersive map $\widehat{S}^{k} \widehat{f}: \widehat{S}^{k}(X, \widehat{\mathcal{U}}) \rightarrow M$. The same holds for good coordinate system.

If $\widehat{\mathfrak{S}}$ is a CF-perturbation of $(X, \widehat{\mathcal{U}})$ then it induces CF-perturbation $\widehat{S}^{k} \widehat{\mathfrak{S}}$ of $\widehat{S}^{k}(X, \widehat{\mathcal{U}})$. The same holds for the case of good coordinate system. This construction preserves the transversality to 0 and the strong submersivity of maps.

\subsection{Statement of Stokes' theorem.}

Theorem 34.7. Let $(X, \widehat{\mathcal{U}})$ be a space equipped with a good coordinate system with corners and $\widehat{h}$ a differential form on it. Let $\widehat{\mathfrak{S}}$ be a CF-perturbation of $(X, \widehat{\mathcal{U}})$ and $\hat{f}:(X, \widehat{\mathcal{U}}) \rightarrow M$ a strongly smooth map. We assume $\hat{f}$ is strongly submersive with respect to $\widehat{\mathfrak{S}}$. Then we have the following equality:

$$
d\left(\widehat{f} !\left(\widehat{h} ; \widehat{\mathfrak{S}^{\epsilon}}\right)\right)=\widehat{f} !\left(d \widehat{h} ; \widehat{\mathfrak{S}^{\epsilon}}\right)+\widehat{f_{\partial}} !\left(\widehat{h_{\partial}} ; \widehat{\mathfrak{S}_{\partial}^{\epsilon}}\right) .
$$

for each sufficiently small $\epsilon>0$. Here $\widehat{f_{\partial}}, \widehat{h_{\partial}}, \widehat{\mathfrak{S}}_{\partial}$ are restrictions of $\hat{f}, \widehat{h}, \widehat{\mathfrak{S}}$ to $(\partial X, \partial \widehat{\mathcal{U}})$ respectively.

This is [FOOO11, Theorem 8.11]. (See also FOOO4 Lemma 12.13.) We remark that $d \hat{h}$ is defined in Definition 33.3 . For the Kuranishi structure we have the same statement.

Theorem 34.8. Let $(X, \widehat{\mathcal{U}})$ be a space equipped with a Kuranishi structure with corners and $\widehat{h}$ a differential form on it. Let $\widehat{\mathfrak{S}}$ be a CF-perturbation of $(X, \widehat{\mathcal{U}})$ and $\widehat{f}:(X, \widehat{\mathcal{U}}) \rightarrow M$ a strongly smooth map. We assume $\widehat{f}$ is strongly submersive with respect to $\widehat{\mathfrak{S}}$. Then we have the following equality:

$$
d\left(\widehat{f} !\left(\widehat{h} ; \widehat{\mathfrak{S}^{\epsilon}}\right)\right)=\widehat{f} !\left(\widehat{d h} ; \widehat{\mathfrak{S}^{\epsilon}}\right)+\widehat{f_{\partial}} !\left(\widehat{h_{\partial}} ; \widehat{\mathfrak{S}_{\partial}^{\epsilon}}\right) .
$$

for each sufficiently small $\epsilon>0$. Here $\widehat{f_{\partial}}, \widehat{h_{\partial}}, \widehat{\mathfrak{S}}_{\partial}$ are restrictions of $\widehat{f}, \widehat{h}, \widehat{\mathfrak{S}}$ to $(\partial X, \partial \widehat{\mathcal{U}})$ respectively.

This is [FOOO11, Proposition 9.26]. We remark that $d \widehat{h}$ is defined in Definition 33.3 .

The proof of Theorem 34.7 is similar to the proof of Stokes' theorem in manifold theory. Namely we use the partition of unity and show that it suffices to prove the equality on each chart. We then use Stokes' theorem in manifold theory to prove the equality on one chart. We omit the proof but refer the readers to [FOOO11, Section 8]. Theorem 34.8 follows from Theorem 34.7.

We consider the case when $M$ is a point. In this case the strong submersivity of $f:(X, \widehat{\mathcal{U}}) \rightarrow M$ with respect to $\widehat{\mathfrak{S}}$ is nothing but the transversality to 0 of $\widehat{\mathfrak{S}}$.

Let $\widehat{h}$ be a differential form of $(X, \widehat{\mathcal{U}})$ of degree $\operatorname{dim}(X, \widehat{\mathcal{U}})$. Then we define

$$
\int_{\left((X, \widehat{\mathcal{U}}), \widehat{\mathfrak{S}}^{\epsilon}\right)} \widehat{h}:=\widehat{f} !\left(\widehat{h} ; \widehat{\mathfrak{S}}^{\epsilon}\right) \text {. }
$$


Note in this case the right hand side is a differential 0 form on a point, which is nothing but a real number. (It depends on $\epsilon$.) We sometimes omit $\widehat{\mathfrak{S}^{\epsilon}}$ from the notation in case the choice of CF-perturbation is clear.

In case $\widehat{h}=\operatorname{dim}(X, \widehat{\mathcal{U}})+1$, (34.2) becomes the next formula:

$$
\int_{\left(\partial(X, \widehat{\mathcal{U}}), \partial \widehat{\mathfrak{S}}^{\epsilon}\right)} \widehat{h_{\partial}}=\int_{\left((X, \widehat{\mathcal{U}}), \widehat{\mathfrak{S}}^{\epsilon}\right)} d \widehat{h}^{.}
$$

If the obstruction bundles of $\widehat{\mathcal{U}}$ are all zero this Kuranishi structure gives an orbifold structure of $X$. In this case (34.4) is the standard Stokes' formula for orbifold. Thus Theorems 34.8 and 34.7 are generalizations of the standard Stokes' formula.

\section{Composition formula}

Another main result of the theory of integration along the fiber is the composition formula which we describe in this section. This is a version of Fubini's theorem on integration.

\subsection{Smooth correspondence and its perturbation.}

Definition 35.1. Let $M_{s}$ and $M_{t}$ be oriented compact smooth manifolds without boundary. A smooth correspondence from $M_{s}$ to $M_{t}$ is an object $\left((X, \widehat{\mathcal{U}}), \widehat{f}_{s}, \widehat{f}_{t}\right)$ such that:

(1) $(X, \widehat{\mathcal{U}})$ is a space with an oriented Kuranishi structure. (It may have boundary and corner.)

(2) $\widehat{f}_{s}:(X, \widehat{\mathcal{U}}) \rightarrow M_{s}$ is a strongly smooth map.

(3) $\widehat{f}_{t}:(X, \widehat{\mathcal{U}}) \rightarrow M_{t}$ is a strongly smooth and weakly submersive map.

Here $s$ and $t$ in the suffix stands for source and target, respectively.

Definition 35.2. A perturbed smooth correspondence from $M_{s}$ to $M_{t}$ is an object $\Xi=\left((X, \widehat{\mathcal{U}}), \widehat{\mathfrak{S}}, \widehat{f}_{s}, \widehat{f}_{t}\right)$ such that

(1) $\left((X, \widehat{\mathcal{U}}), \widehat{f}_{s}, \widehat{f}_{t}\right)$ is a smooth correspondence from $M_{s}$ to $M_{t}$.

(2) $\widehat{\mathfrak{S}}$ is a CF-perturbation of $(X, \widehat{\mathcal{U}})$ such that $\widehat{f}_{t}$ is strongly submersive with respect to $\widehat{\mathfrak{S}}$.

Definition 35.3. If $\left((X, \widehat{\mathcal{U}}), \widehat{f}_{s}, \widehat{f}_{t}\right)$ is a smooth correspondence then $\partial(X, \widehat{\mathcal{U}})$ together with the restriction of $\widehat{f}_{s}, \widehat{f}_{t}$ is a smooth correspondence, which we write $\partial\left((X, \widehat{\mathcal{U}}), \widehat{f_{s}}, \widehat{f}_{t}\right)$.

In the same way we can define the boundary of a perturbed smooth correspondence $\Xi$ which we write $\partial \Xi$.

Definition 35.4. Let $\Xi=\left((X, \widehat{\mathcal{U}}), \widehat{\mathfrak{S}}, \widehat{f_{s}}, \widehat{f_{t}}\right)$ be a perturbed smooth correspondence from $M_{s}$ to $M_{t}$. We define an $\mathbb{R}$ linear map

$$
\operatorname{Corr}_{\Xi}^{\epsilon}: \Omega\left(M_{s}\right) \rightarrow \Omega\left(M_{t}\right)
$$

by

$$
\operatorname{Corr}_{\Xi}^{\epsilon}(h)=\widehat{f}_{t} !\left(\widehat{f}_{s}^{*} h ; \widehat{\mathfrak{S}}^{\epsilon}\right) .
$$

Note the pull back $\widehat{f}_{s}^{*}$ is defined by Definition 33.2 and $\widehat{f}_{t}$ ! is defined by Definition 33.20. Here $\Omega\left(M_{s}\right), \Omega\left(M_{t}\right)$ are the vector spaces of all smooth differential forms on $M_{s}, M_{t}$, respectively. 
We remark that $\operatorname{Corr}_{\Xi}^{\epsilon}$ is a linear map and

$$
\operatorname{deg} \operatorname{Corr}_{\Xi}^{\epsilon}(h)=\operatorname{deg} h+\operatorname{dim} M_{t}-\operatorname{dim}(X, \widehat{\mathcal{U}}) .
$$

Note $d \widehat{f}_{t}^{*} h=\widehat{f}_{t}^{*}(d h)$. Therefore Stokes' theorem implies that:

$$
d \circ \operatorname{Corr}_{\Xi}^{\epsilon}=\operatorname{Corr}_{\Xi}^{\epsilon} \circ d+\operatorname{Corr}_{\partial \Xi}^{\epsilon} .
$$

35.2. Composition of smooth correspondences. We next define a composition of smooth correspondences.

Definition 35.5. Let $\left(\left(X_{21}, \widehat{\mathcal{U}_{21}}\right), \widehat{f}_{1 ; 21}, \widehat{f}_{2 ; 21}\right)$ and $\left(\left(X_{32}, \widehat{\mathcal{U}_{32}}\right), \widehat{f_{2 ; 32}}, \widehat{f_{3 ; 32}}\right)$ be smooth correspondences from $M_{1}$ to $M_{2}$ and from $M_{2}$ to $M_{3}$ respectively. Since $\widehat{f}_{2 ; 21}$ is weakly submersive the fiber product

$$
\left(X_{21}, \widehat{\mathcal{U}_{21}}\right)_{\widehat{f}_{2 ; 21}} \times{\widehat{f_{2} ; 32}}\left(X_{32}, \widehat{\mathcal{U}_{32}}\right)
$$

is well-defined. We write it $\left(X_{31}, \widehat{\mathcal{U}_{31}}\right)$. Then it is easy to see that $\widehat{f}_{1 ; 21}$ (resp. $\left.\widehat{f}_{3 ; 32}\right)$ induces $\widehat{f}_{1 ; 31}:\left(X_{31}, \widehat{\mathcal{U}_{31}}\right) \rightarrow M_{1}$ (resp. $\left.\widehat{f_{3 ; 31}}:\left(X_{31}, \widehat{\mathcal{U}_{31}}\right) \rightarrow M_{3}\right)$. We can use weakly submersivity of $\widehat{f}_{2 ; 21}, \widehat{f}_{3 ; 32}$ to show that $\widehat{f}_{3 ; 31}$ is weakly submersive 13 We thus obtain a smooth correspondence

$$
\left(\left(X_{31}, \widehat{\mathcal{U}_{31}}\right), \widehat{f}_{1 ; 31}, \widehat{f}_{3 ; 31}\right) .
$$

We call it the composition of $\left(\left(X_{21}, \widehat{\mathcal{U}_{21}}\right), \widehat{f}_{1 ; 21}, \widehat{f}_{2 ; 21}\right)$ and $\left(\left(X_{32}, \widehat{\mathcal{U}_{32}}\right), \widehat{f}_{2 ; 32}, \widehat{f}_{3 ; 32}\right)$.

Definition 35.6. Suppose we have two perturbed smooth correspondences $\Xi_{21}=$ $\left(\left(X_{21}, \widehat{\mathcal{U}_{21}}\right), \widehat{\mathfrak{S}_{21}}, \widehat{f_{1 ; 2}}, \widehat{f}_{2 ; 21}\right)$ and $\Xi_{32}=\left(\left(X_{32}, \widehat{\mathcal{U}_{32}}\right), \widehat{\mathfrak{S}_{32}}, \widehat{f_{2} ; 32}, \widehat{f_{3 ; 32}}\right)$ from $M_{1}$ to $M_{2}$ and from $M_{2}$ to $M_{3}$. We consider the composition $\left(\left(X_{31}, \widehat{\mathcal{U}_{31}}\right), \widehat{f}_{1 ; 31}, \widehat{f}_{3 ; 31}\right)$ of $\left(\left(X_{21}, \widehat{\mathcal{U}_{21}}\right), \widehat{f}_{1 ; 21}, \widehat{f}_{2 ; 21}\right)$ and $\left(\left(X_{32}, \widehat{\mathcal{U}_{32}}\right), \widehat{f}_{2 ; 32}, \widehat{f}_{3 ; 32}\right)$. Using strong submersivity of $\widehat{f}_{1 ; 21}$ with respect to $\widehat{\mathfrak{S}_{21}}$, we can easily construct the fiber product of two CFperturbations

$$
\widehat{\mathfrak{S}_{21}}{\widehat{f_{2 ; 21}}}_{\widehat{f}_{2 ; 32}} \widehat{\mathfrak{S}_{32}}
$$

which we denote by $\widehat{\mathfrak{S}_{32}} 14$ Then using strong submersivity of $\widehat{f}_{1 ; 21}, \widehat{f}_{3 ; 32}$ with respect to $\widehat{\mathfrak{S}_{21}}, \widehat{\mathfrak{S}_{32}}$, we can show that $\widehat{f}_{3 ; 32}$ is strongly submersive with respect to $\widehat{\mathfrak{S}_{32}} 15$

We thus obtain a perturbed smooth correspondence

$$
\left(\left(X_{31}, \widehat{\mathcal{U}_{31}}\right), \widehat{\mathfrak{S}_{31}}, \widehat{f}_{1 ; 31}, \widehat{f}_{3 ; 31}\right),
$$

for each sufficiently small $\epsilon>0$. We call it the composition of $\Xi_{21}$ and $\Xi_{32}$ and write

$$
\Xi_{32} \circ \Xi_{21}
$$

\footnotetext{
${ }^{13}$ See [FOOO11, Lemma 10.18 (2)] for its proof.

${ }^{14}$ See [FOOO11, Definition 10.13 (2)] for its proof.

${ }^{15}$ See [FOOO11 Lemma $\left.10.14(2)\right]$ for its proof.
} 


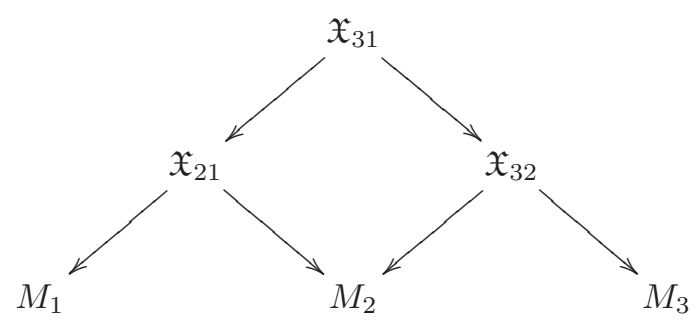

35.3. Statement of Composition formula. Now the composition formula is:

Theorem 35.7. Let $\Xi_{21}$ (resp. $\Xi_{32}$ ) be a perturbed smooth correspondence from $M_{1}$ to $M_{2}$ (resp. from $M_{2}$ to $M_{3}$ ). Then we have

$$
\operatorname{Corr}_{\Xi_{32}}^{\epsilon} \circ \operatorname{Corr}_{\Xi_{21}}^{\epsilon}=\operatorname{Corr}_{\Xi_{32} \circ \Xi_{21}}^{\epsilon}
$$

for each sufficiently small $\epsilon>0$.

Theorem 35.7 is [FOOO11, Theorem 10.20]. (See also FOOO4 Lemma 12.15.)

We consider the case $M_{2}$ is a point. Let $h_{1}$ and $h_{3}$ be differential forms on $M_{1}$ and $M_{3}$ of degree $\operatorname{dim}\left(X_{21}, \widehat{\mathcal{U}_{21}}\right)$ and $\operatorname{dim}\left(X_{32}, \widehat{\mathcal{U}_{32}}\right)$, respectively. Then we have

$$
\int_{M_{3}}\left(\operatorname{Corr}_{\Xi_{32}}^{\epsilon} \circ \operatorname{Corr}_{\Xi_{21}}^{\epsilon}\right)\left(h_{1}\right) \wedge h_{3}=\int_{\left(\left(X_{21}, \widehat{\mathcal{U}_{21}}\right), \widehat{\mathfrak{S}_{21}}\right)} \widehat{f}_{1 ; 21}^{*} h_{1} \times \int_{\left(\left(X_{32}, \widehat{\mathcal{U}_{32}}\right), \widehat{\mathfrak{S}_{32}}\right)} \widehat{f_{3 ; 32}^{*} h_{3}} \text {. }
$$

On the other hand

$$
\int_{M_{3}} \operatorname{Corr}_{\Xi_{32} \circ \Xi_{21}}^{\epsilon}\left(h_{1}\right) \wedge h_{3}=\int_{\left(\left(X_{21}, \widehat{\mathcal{U}_{21}}\right) \times\left(X_{32}, \widehat{\mathcal{U}_{32}}\right), \widehat{\mathfrak{S}_{21}} \times \widehat{\mathfrak{S}_{32}}\right)} \widehat{f}_{1 ; 21}^{*} h_{1} \times \widehat{f}_{3 ; 32}^{*} h_{3} .
$$

Thus in this case (35.4) is a generalization of Fubini's theorem.

To prove Theorem 35.7 we use the partition of unity to reduce it to one chart. Then we can use Fubini's theorem to prove it. See [FOOO11, Section 10]. 


\section{REFERENCES}

[Aa] J. F. Aarnes, Quasi-states and quasi-measure, Adv. Math. 86 (1991), 41-67.

[ASc1] A. Abbondandolo and M. Schwarz, On the Floer homology of cotangent bundles, Comm. Pure Appl. Math. 59 (2006),254-316.

[ASc2] A. Abbondandolo and M. Schwarz, Floer homology of cotangent bundles and the loop product, Geom. Topol. 14 (2010), no. 3, 1569-1722.

[AFOOO1] M. Abouzaid, K. Fukaya, Y.-G. Oh, H. Ohta and K. Ono, Quantum cohomology and split generation in Lagrangian Floer theory, in preparation.

[AFOOO2] M. Abouzaid, K. Fukaya, Y.-G. Oh, H. Ohta and K. Ono, Homological Mirror symmetry between toric A-model and Landau-Ginzburg B-model, in preparation.

[ASe] M. Abouzaid and P. Seidel, An open string analogue of Viterbo functoriality, Geom. Topolo., 14 (2010) 627-718.

[AM] M.l Abreu and L. Macarini, Remarks on Lagrangian intersections in toric manifolds, Trans. Amer. Math. Soc. 365 (2013), no. 7, 3851-3875, arXiv:1105.0640.

[Al] P. Albers, On the extrinsic topology of Lagrangian submanifolds, Int. Math. Res. Not. 38 (2005), 2341-2371.

[Au] D. Auroux, Mirror symmetry, T-duality in the complement of an anti-canonical divisor, J. Gokova Geom Topol. 1 (2007), 51-59.

[Ba1] A. Banyaga, Sur la structure du groupe des difféomorphismes qui préservent une forme symplectique, Comment. Math. Helvetici 53 (1978) 174-227.

[Ba2] A. Banyaga, The Structure of Classical Diffeomorphism Groups, (1997) Kluwer Academic Publishers, Dordrecht.

[BEP] P. Biran, V.M. Entov, L. Polterovich, Calabi quasi-morphisms for the symplectic ball, Commun. Contemp. Math. 6 (2004), 793-802.

[BC] P. Biran, O. Cornea, Rigidity and uniruling for Lagrangian submanifolds, Geom. Topol. 13 (2009), no. 5, 2881-2989.

[Bor] M. S. Borman, Quasi-states, quasi-morphisms, and the moment map, Int. Math. Res. Not. IMRN 2013, no. 11, 2497-2533, arXiv:1105.1805.

[Ca] E. Calabi, On the group of automorphisms of a symplectic manifold, Problems in Analysis (Lectures at the Sympos. in Honor of Salamon Bochner, Princeton Univ., Princeton NJ. 1969) Princeton University Press, New Jersey, 1970, pp 1-26.

[CL] K. Chan and S.-C. Lau, Open Gromov-Witten invariants and superpotentials for semifano toric surface, International Mathematics Research Notices, rnt050, 31 pages. arXiv: 1010.5287.

[Cho] C.-H. Cho, Non-displaceable Lagrangian submanifolds and Floer cohomology with nonunitary line bundle, J. Geom. Phys. 58 (2008), 1465-1476, arXiv:0710.5454.

[CO] C.-H. Cho and Y.-G. Oh, Floer cohomology and disc instantons of Lagrangian torus fibers in Fano toric manifolds, Asian J. Math. 10 (2006), 773-814.

[CZ] C. Conley and E. Zehnder, Morse-type index theory for flows and periodic solutions for Hamiltonian equations, Comm. Pure Appl. Math. 37 (1984), 207-253.

[D] T. Delzant, Hamiltoniens périodiques et images convexes de lápplication moment, Bull. Soc. Math. France 116 (1988), 315-339.

[ElP] Y. Eliashberg and L. Polterovich, Symplectic quasi-state on the quadratic surface and Lagrangian submanifolds, preprint, arXive:1006.2501.

[E] M. Entov, K-area, Hofer metric and geometry of conjugacy classes in Lie groups, Invent. Math. 146 (2001), 93-141.

[EP1] M. Entov and L. Polterovich, Calabi quasi-morphism and quantum homology, Int. Math. Res. Not. 2003, no. 30, (2003) 1635-1676.

[EP2] M. Entov and L. Polterovich, Quasi-states and symplectic intersections, Comment. Math. Helv. 81 (2006), 75-99.

[EP3] M. Entov and L. Polterovich, Rigid subsets of symplectic manifolds, Compositio Math. 145 (2009), 773-826.

[Fe] M. Fekete, Über die Verteilung der Wurzeln bei gewissen algebraischen Gleichungen mit ganzzahligen Koeffizienten, Math. Z. 17 (1923), no. 1, 228-249.

[Fl] A. Floer, Symplectic fixed points and holomorphic spheres, Commun. Math. Phys. 120 (1989), 575-611. 
[FH] A. Floer and H. Hofer, Symplectic homology. I. Open sets in $\mathbb{C}^{n}$. Math. Z. 215 (1994), $37-88$.

[Fu1] K. Fukaya, Floer homology for families - a progress report, Integrable Systems, Topology, and Physics (Tokyo 2000), Contemp. Math. 309 (2002), 33-68.

[Fu2] K. Fukaya, Application of Floer homology of Langrangian submanifolds to symplectic topology, Morse theoretic methods in nonlinear analysis and in symplectic topology, 231-276, NATO Sci. Ser. II Math. Phys. Chem., 217, Springer, Dordrecht, 2006.

[Fu3] K. Fukaya, Cyclic symmetry and adic convergence in Lagrangian Floer theory, Kyoto J. Math. 50 (2010) 521-590, arXiv:0907.4219.

[FOOO1] K. Fukaya, Y.-G. Oh, H. Ohta and K. Ono, Lagrangian intersection Floer theoryanomaly and obstruction, I, AMS/IP Studies in Advanced Mathematics, vol 46-1, Amer. Math. Soc./International Press, 2009. MR2553465.

[FOOO2] K. Fukaya, Y.-G. Oh, H. Ohta and K. Ono, Lagrangian intersection Floer theoryanomaly and obstruction, II, AMS/IP Studies in Advanced Mathematics, vol 46-2, Amer. Math. Soc./International Press, 2009. MR2548482.

[FOOO3] K. Fukaya, Y.-G. Oh, H. Ohta and K. Ono, Lagrangian Floer theory on compact toric manifolds I, Duke Math. J. 151 (2010), 23-174.

[FOOO4] K. Fukaya, Y.-G. Oh, H. Ohta and K. Ono, Lagragian Floer theory on compact toric manifolds II; Bulk deformations, Selecta Math. New Series, 17 no. 3, (2011), 609-711.

[FOOO5] K. Fukaya, Y.-G. Oh, H. Ohta and K. Ono, Anti-symplectic involution and Floer cohomology, Geom. Top. (to appear), arXiv:0912.2646.

[FOOO6] K. Fukaya, Y.-G. Oh, H. Ohta and K. Ono, Toric degeneration and non-displaceable Lagrangian tori in $S^{2} \times S^{2}$, Int. Math. Res. Not. IMRN 2012, no. 13, 2942-2993.

[FOOO7] K. Fukaya, Y.-G. Oh, H. Ohta and K. Ono, Lagragian Floer theory and mirror symmetry on compact toric manifolds, Astérisque, 376, 2016, Société Mathématique de Franc.

[FOOO8] K. Fukaya, Y.-G. Oh, H. Ohta and K. Ono, Lagrangian Floer theory on compact toric manifolds: survey, Surveys in Diff. Geom. vol 17, In memory of C. C. Hsiung, International Press, Boston, MA, 2012, 229 - 298.

[FOOO9] K. Fukaya, Y.-G. Oh, H. Ohta and K. Ono, Displacement of polydisks and Lagrangian Floer theory, J. Symplectic Geom. 11 (2013), no. 2, 231-268.

[FOOO10] K. Fukaya, Y.-G. Oh, H. Ohta and K. Ono, Technical details on Kuranishi structure and virtual fundamental chain, preprint 2012, arXiv:1209.4410.

[FOOO11] K. Fukaya, Y.-G. Oh, H. Ohta and K. Ono, Kuranishi structure, pseudo-holomorphic curve, and virtual fundamental chain: Part 1, arXiv:1503.07631.

[FOOO12] K. Fukaya, Y.-G. Oh, H. Ohta and K. Ono, Exponential decay estimate and smoothness of the moduli space of pseudoholomorphic curves, submitted, arXiv:1603.07026.

[FOOO13] K. Fukaya, Y.-G. Oh, H. Ohta and K. Ono, Shrinking good coordinate systems associated to Kuranishi structures, J. Symplectic Geom. 14 (2016), no. 4, 1295-1310.

[FO] K. Fukaya and K. Ono, Arnold conjecture and Gromov-Witten invariant, Topology 38 (1999), no. 5, 933-1048.

[Ful] W. Fulton, Introduction to Toric Varieties, Annals of Math. Studies, 131, Princeton University Press, Princeton, 1993.

[Gr] M. Gromov, Pseudoholomorphic curves in symplectic geometry, Invent. Math. 82 (1985) 304-374.

[Gu] V. Guillemin, Kähler structures on toric varieties, J. Differ. Geom. 43 (1994), 285-309.

[GLS] V. Guillemin, E. Lerman and S. Sternberg, Symplectic Fibrations and Multiplicity Diagrams, Cambridge Univ. Press, Cambridge, 1966.

$[\mathrm{H}] \quad \mathrm{H}$. Hofer, On the topological properties of symplectic maps, Proc. Royal Soc. Edinburgh 115 (1990), 25-38.

[HS] H. Hofer and D. Salamon, Floer homology and Novikov rings, The Floer memorial volume, 483-524, Progr. Math. 133, Birkhäuser, Basel, 1995.

[HZ] H. Hofer and E. Zehnder, Symplectic Invariants and Hamiltonian Dynamics, Birkhäuser, Basel, 1994.

[Iri1] H. Iritani, Convergence of quantum cohomology by quantum Lefschetz, J. Reine Angew. Math. 610 (2007), 29-69.

[Iri2] H. Iritani, An integral structure in quantum cohomology and mirror symmetry for toric orbifolds, Adv. in Math. 222 (2009), 1069-1079. 
[Ka] M. Kawasaki, Superheavy Lagrangian immersion in 2-torus, preprint, 2014, arXiv:1412.4495

[Ku] A.G. Kushnirenko, Polyédres de Newton et nombre de Milnor, Invent. Math. 32 (1976) $1-31$.

[LMP] F. Lalonde, D. McDuff and L. Polterovich, Topological rigidity of Hamiltonian loops and quantum homology, Invent. Math. 135 (1999) 369-385.

[Man] Y. Manin, Frobenius manifolds, quantum cohomology, and moduli spaces, American Mathematical Society Colloquium Publications, 47. American Mathematical Society, Providence, RI, 1999. xiv+303 pp.

[MS] D. McDuff and D. Salamon, Introduction to Symplectic Topology, 1995, Clarendon Press, Oxford.

[MT] D. McDuff and S. Tolman, Topological properties of Hamiltonian circle actions, Int. Math. Res. Pap. 2006, 72826, 1-77.

[Mo] A. Monzner, Partial quasi-morphisms and symplectic quasi-integrals on cotangent bundles, Dissertationzur Erlangung des Grades eines Doktors der Naturwissenschaften, 2012.

[MVZ] A. Monzner, N. Vichery, F. Zapolsky, Partial quasi-morphisms and quasi-states on cotangent bundles, and symplectic homogenization, J. Mod. Dyn. 6 (2012), no. 2, 205-249.

[NNU1] T. Nishinou, Y. Nohara, and K. Ueda, Toric degenerations of Gelfand-Cetlin systems and potential functions, Adv. Math. 224 (2010), no. 2, 648-706.

[NNU2] T. Nishinou, Y. Nohara, and K. Ueda, Potential functions via toric degenerations, Proc. Japan Acad. Ser. A Math. Sci. Volume 88, Number 2 (2012), 31-33, (Its longer preprint version is arXiv:0812.0066.)

[Oh1] Y.-G. Oh, Symplectic topology as the geometry of action functional II, Commun. Anal. Geom. 7 (1999), 1-55

[Oh2] Y.-G. Oh, Normalization of the Hamiltonian and the action spectrum, J. Korean Math. Soc. 42 (2005), 65-83.

[Oh3] Y.-G. Oh, Chain level Floer theory and Hofer's geometry of Hamiltonain diffeomorphism group, Asian J. Math. 9 (2002), 579-624.

[Oh4] Y.-G. Oh, Construction of spectral invariants of Hamiltonian paths on closed symplectic manifolds, in "The Breadth of Symplectic and Poisson Geometry", Prog. Math. 232, 525 - 570, Birkhäuser, Boston, 2005.

[Oh5] Y.-G. Oh, Spectral invariants, analysis of the Floer moduli space, and geometry of the Hamiltonian diffeomorphism group, Duke Math. J. 130 (2005), 199-295.

[Oh6] Y.-G. Oh, Lectures on Floer theory and spectral invariants of Hamiltonian flows, Morse theoretic methods in nonlinear analysis and in symplectic topology, 321-416, NATO Sci. Ser. II Math. Phys. Chem., 217, Springer, Dordrecht, 2006.

[Oh7] Y.-G. Oh, Floer mini-max theory, the Cerf diagram, and the spectral invariants, J. Korean Math. Soc. 46 (2009), $363-447$.

[Oh8] Y.-G. Oh, The group of Hamiltonian homeomorphisms and continuous Hamiltonian flows, pp 149 - 177, Contemp. Math., 512, Amer. Math. Soc., Providence, RI, 2010.

[Oh9] Oh, Y.-G., Symplectic Topology and Floer Homology II, New Mathematical Monographs 29, Cambridge University Press, Cambridge, 2015.

[OM] Y.-G. Oh, S., Müller, The group of Hamiltonian homeomorphisms and $C^{0}$ symplectic topology, J. Symp. Geom. 5 (2007), $167-219$.

[OZ] Y.-G. Oh and K. Zhu, Floer trajectories with immersed nodes and scale-dependent gluing, J. Symp. Geom. 9 (2011), 483-636.

[Os1] Y. Ostrover, A comparison of Hofer's metrics on Hamiltonian diffeomorphisms and Lagrangian submanifolds, Commun. Contemp. Math. 5 (2003), 803-811.

[Os2] Y. Ostrover, Calabi quasi-morphisms for some non-monotone symplectic manifolds, Algebr. Geom. Topol. 6 (2006), 405-434.

[On] K. Ono, On the Arnold conjecture for weakly monotone symplectic manifolds, Invent. Math. 119 (1995) 519-537.

[Piu] S. Piunikhin, Quantum and Floer cohomology has the same ring structure, preprint, 1994. 
[PSS] S. Piunikhin, D. Salamon and M. Schwarz, Symplecitc Floer-Donaldson theory and quantum cohomology, In C.B. Thomas, editor, Contact and Symplectic Geometry, vol 8 of Publ. Newton Institute, pp. 171-200, Cambridge Univ. Press, Cambridge, 1996.

[PS] G. Polya and G. Szegö, Problems and theorems in Analysis, Vol 1, Springer-Verlag, Berlin, 1972.

[P] L. Polterovich, The Geometry of the Group of Symplectic Diffeomorphisms, Lectures in Math. ETH Zürich, Birkhäuser, Basel, 2001.

[RT] Y. Ruan and G. Tian, Bott-type symplectic Floer cohomology and its multiplicative structure, Math. Res. Lett. 2 (1995) 203-219.

[SW] D. Salamon and J. Weber, Floer homology and the heat flow, Geom. Funct. Anal. 16 (2006), 1050-1138.

[SZ] D. Salamon and E. Zehnder, Morse theory for periodic solutions of Hamiltonian systems and the Maslov index, Comm. Pure Appl. Math. 45 (1992), no. 10, 1303-1360.

[Sc1] M. Schwarz, Cohomology operations from $S^{1}$-cobordisms in Floer homology, Ph.D. thesis, Swiss Federal Inst. of Techn. Zürich, Diss ETH No. 11182, 1995.

[Sc2] M. Schwarz, On the action spectrum for closed symplectically aspherical manifolds, Pacific J. Math. 193 (2000), 419-461.

[Se] P. Seidel, $\pi_{1}$ of symplectic automorphism groups and invertibles in quantum homology rings, Geom. Funct. Anal. 7 (1997), no. 6, 1046-1095.

[Si] J.-C. Sikorav, Some properties of holomorphic curves in almost complex manifolds, Holomorphic curves in symplectic geometry, ed. M. Audin, Progress in Math. 117, 165-190 (1994) Birkhäuser, Basel.

[Sw] M. E. Sweedler, Hopf Algebras, Benjamin, New York, 1969.

[Us1] M. Usher, Spectral numbers in Floer theories, Compos. Math. 144 (2008), 1581-1592.

[Us2] M. Usher, Boundary depth in Floer theory and its applications to Hamiltonian dynamics and coisotropic submanifolds, Israel J. Math 184 (2011), 1-57.

[Us3] M. Usher, Duality in filtered Floer-Novikov complexes, J. Topol. Anal. 2 (2010), no. $2,233-258$.

[Us4] M. Usher, Deformed Hamiltonian Floer theory, capacity estimate, and Calabi-quasimorphisms, Geom. Topol. 15 (2011), 1313-1417.

[Vi1] C. Viterbo, Symplectic topology as the geometry of generating functions, Math. Ann. 292 (1992), 685-710.

[Vi2] C. Viberbo, Functors and computations in Floer homology and applications, Part I, Geom. Funct. Anal., 9 (1999) 985-1033.

[WX] W. Wu and G. Xu, Gauged Floer homology and spectral invariants, preprint, 2015, arXiv:1506.03349. 


\section{INDEX}

$(V, \Gamma, E, \phi, \widehat{\phi}), 193$

$(V, \Gamma, \phi), 192$

$B V, 16$

$C F(M, H), 33$

$C F\left(M, H ; \Lambda^{\downarrow}\right), 21$

$C F\left(M, H ; \Lambda_{\mathfrak{b}}^{\downarrow}(M)\right), 46$

$C F_{0}\left(M, H ; \Lambda^{\downarrow}\right), 25$

$C F_{1}\left(M, H ; \Lambda^{\downarrow}\right), 25$

$E(H ; Y), 98$

$E V, 17$

$E^{+}(H), 34$

$E^{+}(H ; Y), 98$

$E^{+}(H ; \mathcal{L}(Y)), 150$

$E^{-}(H), 34$

$E^{-}(H ; Y), 98$

$G$-gapped, 40

$G$-set, 31

$G^{\prime}, 49$

$G(M, \omega), 46$

$G(M, \omega, \mathfrak{b}), 46$

$G W_{\ell}, 38$

$G W_{\ell}\left(\alpha: h_{1}, \ldots, h_{\ell}\right), 38$

$G_{0}(M, \omega), 46$

$G_{0}(M, \omega, \mathfrak{b}), 40,46$

$G_{0}(\mathfrak{b}), 40$

$H F\left(\left(L, \mathbf{b}^{(1)}\right),\left(L, \mathbf{b}^{(0)}\right) ; \Lambda_{0}\right), 96$

$H F_{*}\left(H, J ; \Lambda^{\downarrow}\right), 27$

$H F_{*}^{\mathfrak{b}}\left(M, H, J ; \Lambda^{\downarrow}\right), 44$

$H F_{T}\left((L, \mathbf{b}) ; \Lambda_{0}\right), 114$

$K_{2}(M), 21$

$Q H^{*}(M), 18$

$Q H_{\mathfrak{b}}^{*}(M), 39$

$\Delta^{n-1}, 90$

$\Delta_{\text {decon }}, 17$

$\Delta_{\text {shuff }}, 17$

$\Lambda(G)$ vector subspace, 50

$\Lambda\left\langle\left\langle y, y^{-1}\right\rangle\right\rangle^{\stackrel{\circ}{P}}, 118$

$\Lambda_{\mathfrak{b}}(M), 47$

$\Lambda(M), 33$

$\Lambda\left\langle\left\langle y, y^{-1}\right\rangle\right\rangle^{P}, 118$

$\Lambda^{\downarrow}(M), 32$

$\Lambda^{\downarrow}(G), 50$

$\Lambda^{\downarrow}\langle\langle s\rangle\rangle, 158$

$\Lambda_{+}^{\downarrow}(G), 49$

$\Lambda_{0}^{\downarrow}(G), 49$

$\Lambda_{\mathfrak{b}}^{\downarrow}(M), 46$

$\Omega_{*}(M), 18$

$\Phi=(\varphi, \hat{\varphi}), 194$

$\Pi_{2}(M ; H), 174$

$\|H\|, 34$

$\|\widetilde{\phi}\|_{U}, 73$

$\chi, 27$

$\cup^{\mathfrak{b}}, 39$

$\partial_{(H, J)}^{\mathfrak{b}}, 43$ $\delta^{\mathbf{b}^{(1)}, \mathbf{b}^{(0)}}, 95$

$\ell_{j}, 111$

b, 18,44

$\mathfrak{H}_{H \varphi, J \varphi}^{\mathfrak{b}}\left(a_{1}, a_{2}\right), 70$

$\mathfrak{m}_{2}^{\mathrm{cl}}, 60$

$\mathfrak{m}_{2}^{\mathrm{cl}}, 64$

$\mathfrak{m}_{2 ; \alpha}^{\mathrm{cl} ; \mathfrak{b} ; H^{\varphi}}, 182$

$\mathfrak{m}_{k}^{\mathbf{b}}, 94$

$\mathfrak{n}_{(H, J) ; \ell}\left([\gamma, w],\left[\gamma^{\prime}, w^{\prime}\right]\right)\left(h_{1}, \ldots, h_{\ell}\right), 42$

$\mathfrak{n}_{(H, J)}^{\mathfrak{b}}\left([\gamma, w],\left[\gamma^{\prime}, w^{\prime}\right]\right), 43$

$\mathfrak{n}_{(H, J)}^{\mathfrak{b}}\left(\llbracket \gamma, w \rrbracket, \llbracket \gamma^{\prime}, w^{\prime} \rrbracket\right), 43$

$\mathfrak{q}_{\ell, k ; \beta}^{H, S \geq 0}, 108$

$H ;[\gamma, w], 103$

$\mathfrak{q}_{\ell, k ; \beta}, 103$

$\mathfrak{q}_{\ell, k ; \beta}^{\text {para }}, 169$

$\mathfrak{q}_{\ell, k ; \beta}, 90$

$\mathfrak{q}_{\ell, k ; \beta}^{T}\left(\mathbf{p} ; h_{1}, \ldots, h_{k}\right), 114$

$\mathfrak{v}_{T}, 16$

$\mathfrak{v}_{q}, 16$

$\mathfrak{v}_{P}, 117$

$\mathfrak{v}_{\mathbf{u}}, 117$

$\mathfrak{g w}_{2 ; \alpha}^{\mathrm{cl}}, 69$

$\mathfrak{P O}, 95$

$\mathfrak{P O}_{\mathfrak{b}}^{\mathbf{u}}, 117$

$\mathfrak{k}_{\mathfrak{b}_{\mathfrak{b}}}, 120$

$\lambda_{H}, 22,46$

$\lambda_{q}, 34,49$

$[\gamma, w], 20$

$\llbracket \gamma, w \rrbracket, 21$

$\mathcal{A}_{H}, 7$

$\mathcal{H}_{(\mathcal{G}, \mathcal{J})}^{\mathfrak{b}}, 56$

$\mathcal{M}\left(H, J ;[\gamma, w],\left[\gamma^{\prime}, w^{\prime}\right]\right), 24$

$\mathcal{M}\left(H_{\chi}, J_{\chi} ; *,[\gamma ; w]\right), 28$

$\mathcal{M}_{\# \mathrm{E}_{1}}\left(0, J_{0} ; *, * ; C\right), 45$

$\mathcal{M}_{\ell}\left(F^{\chi}, J^{\chi} ;[\gamma, w],\left[\gamma^{\prime}, w^{\prime}\right]\right), 53$

$\mathcal{M}_{\ell}\left(H, J ;[\gamma, w],\left[\gamma^{\prime}, w^{\prime}\right]\right), 41$

$\mathcal{M}_{\ell}\left(H^{\varphi}, J^{\varphi} ;\left[\gamma_{1} ; w_{1}\right],\left[\gamma_{2} ; w_{2}\right],\left[\gamma_{3}, w_{3}\right]\right), 61$

$\mathcal{M}_{\ell}\left(H^{\varphi}, J^{H_{1}, H_{2}} ; z_{*}^{H_{1}}, z_{*}^{H_{2}}, z_{*}^{H_{1} \# H_{2}} ; \alpha\right)$, 180

$\mathcal{M}_{\ell}\left(H_{S}^{\varphi}, J_{S}^{\varphi} ; * *,[\gamma, w]\right), 66$

$\mathcal{M}_{\ell}\left(H_{\chi}, J_{\chi}^{H} ; *, z_{*}^{H} ; \alpha\right), 175$

$\mathcal{M}_{\ell}\left(H_{\chi}, J_{\chi} ; *,[\gamma, w]\right), 44$

$\mathcal{M}_{\ell}\left(H_{\tilde{\chi}}, J_{\tilde{\chi}} ;[\gamma, w], *\right), 151$

$\mathcal{M}_{\ell}\left(\right.$ para $\left.; *,\left[\gamma^{\prime}, w^{\prime}\right]\right), 56$

$\mathcal{M}_{\ell}\left(\right.$ para $\left.; H^{\varphi}, J^{\varphi} ; * *,[\gamma, w]\right), 66$

$\mathcal{M}_{\ell}\left(\right.$ para $\left.; H_{\chi}^{\varphi}, J_{\chi}^{H_{1}, H_{2}} ; * *, z_{*}^{H_{1} \# H_{2}} ; \alpha\right), 183$

$\mathcal{M}_{\ell}\left(\right.$ para $\left.; H_{\chi}, J_{\chi} ; *, * ; C\right), 154$

$\mathcal{M}_{\ell}^{\mathrm{cl}}\left(\alpha ; J_{0}\right), 38$

$\mathcal{M}_{k+1 ; \ell}(H, J ;[\gamma, w], L ; \beta), 100$

$\mathcal{M}_{k+1 ; \ell}\left(H^{S}, J ; *, L ; \beta\right), 106$

$\mathcal{M}_{k+1 ; \ell}(L(\mathbf{u}) ; \beta), 113$

$\mathcal{M}_{k+1 ; \ell}(L(\mathbf{u}) ; \beta ; \mathbf{p}(\mathcal{U})), 165$

$\mathcal{M}_{k+1 ; \ell}(L ; \beta), 91$

$\mathcal{M}_{k+1 ; \ell}($ para $; H, J ; *, L ; \beta), 106$ 
$\mathcal{P}_{(F \chi, J \chi)}^{\mathfrak{b}}(\llbracket \gamma, w \rrbracket), 55$

$\mathcal{P}_{\left(F_{k, \chi}, J^{k, \chi}\right)}^{\mathfrak{b}}, 187$

$\mathcal{P}_{\left(H_{\chi}, J_{\chi}\right), *}^{\mathfrak{b}}, 44$

$\mathcal{P}_{\left(H_{\chi}, J_{\chi}\right), *}^{\mathfrak{b}}, 46$

$\mathcal{P}_{\left(H_{\chi}, J_{\chi}\right)}(h), 30$

$\mathcal{Q}_{\left(H_{\tilde{\chi}}, J_{\tilde{\chi}}\right), *}^{\mathfrak{b}}, 151$

$\mathcal{S}=\left(W, \omega,\left\{\mathfrak{s}^{\epsilon}\right\}\right), 198$

$\mathcal{S}^{\mathfrak{b}}, 179$

$\mathcal{S}_{\left(H_{\chi}, J_{\chi}\right)}^{\mathfrak{b}}, 178$

$\mathcal{U}=(U, \mathcal{E}, \psi, s), 193$

$\mu_{H}, 21$

$\mu_{e}^{\mathfrak{b}}, 9,87$

$\mu_{L}(\beta), 90$

$\pi_{2}(\gamma), 20$

$\pi_{2}\left(\gamma, \gamma^{\prime}\right), 23$

$\rho(\mathfrak{x}), 33$

$\rho(\widetilde{\psi} ; a), 48$

$\rho^{\mathfrak{b}}(H ; a), 47$

$\rho_{L}^{\mathbf{b}^{(1)}, \mathbf{b}^{(0)}}(x), 96$

$\mathbf{x}_{c}^{n ; i}, 91$

Cal, 72

$\mathrm{Cal}_{U}, 72$

$\operatorname{Corr}\left(\mathcal{M}_{3+\ell}^{\mathrm{cl}}\left(\alpha ; J_{0}\right)\right), 69$

$\operatorname{Corr}_{\Xi}^{\epsilon}, 206$

$\operatorname{Crit}\left(\mathcal{A}_{H}\right), 20$

$\operatorname{Crit}\left(\mathfrak{P O}_{\mathfrak{b}}\right), 121$

$\operatorname{Ham}_{\text {nd }}(M, \omega), 14,47$

$\operatorname{Jac}\left(\mathfrak{P O}_{\mathfrak{b}} ; \Lambda\right), 120$

$\operatorname{Jac}\left(\mathfrak{P O}_{\mathfrak{b}} ; \mathfrak{y}\right), 121$

$\operatorname{Perm}(k), 17$

$\operatorname{Per}(H), 20$

Shuff $(\ell), 41$

$\operatorname{Spec}(H ; \mathfrak{b}), 46$

$\underline{0}, 35$

$\underline{H}(t, x), 32$

$\widehat{f}^{*} h, 197$

$\widehat{\otimes}, 30,64$

$\widehat{S}^{k}(X), 204$

$\widehat{\mathcal{M}}_{\text {weak,def }}^{T}\left(L(\mathbf{u}) ; \Lambda_{+} ; \mathbf{b}^{(0)}\right), 115$

$\widehat{\mathcal{M}}_{\text {weak,def }}\left(L ; \Lambda_{0}\right), 95$

$\widehat{\mathcal{M}}_{\text {weak,def }}\left(L(\mathbf{u}) ; \Lambda_{+} ; \mathbf{b}^{(0)}\right), 115$

$\widehat{\mathcal{U}}=\left(\left\{\mathcal{U}_{p}\right\},\left\{\Phi_{p q}\right\}\right), 194$

$\widehat{\operatorname{Per}}(H), 21$

$\widetilde{\operatorname{Ham}}(M, \omega), 7$

$a_{H}^{\mathfrak{b} ; \mathfrak{b}}, 47$

$e(\widetilde{\psi} ; Y), 98$

$e^{+}(\widetilde{\psi} ; Y), 98$

$e^{-}(\widetilde{\psi} ; Y), 98$

$i_{\mathrm{qm}, \mathrm{b}}^{T}, 115$

$i_{\mathrm{qm}, \mathbf{b}}^{*}, 97$

$i_{\mathrm{qm},(\mathfrak{b}, b)}^{T, *}, 121$

$\mathcal{H}^{2 k}, 112$
$\widehat{\mathcal{U}}=\left((\mathfrak{P}, \leq),\left\{\mathcal{U}_{\mathfrak{p}} \mid \mathfrak{p} \in \mathfrak{P}\right\},\left\{\Phi_{\mathfrak{p q}} \mid \mathfrak{q} \leq \mathfrak{p}\right\}\right)$, 195

action functional, 7, 36

action spectrum, 31

big quantum cohomology, 38, 40

bulk deformation

filtered $A_{\infty}$ algebra, 94

Hamiltonian Floer homology, 40-46

quantum product, 39

Calabi homomorphism, 72

CF-perturbation, 68

good coordinate system, 200

Kuranishi structure, 200

on a Kuranishi chart, 198

on an orbifold chart, 198

transversal to 0

on one orbifold chart, 199

closed-open map, 96

compatible with the embedding

CF-perturbation, 199

completion of Laurent polynomial ring, 118 composition

perturbed smooth correspondences, 207

smooth correspondences, 207

composition formula, 208

concatenation

Hamiltonian, 14

Conley-Zehnder index, 21

coordinate change

compatible with orientation, 194

Kuranishi chart, 194

coproduct

deconcatenation, 16, 91, 94

shuffle, 16, 91

defect, 12,126

deformed quantum product, 39

differential form

of Kuranishi structure, 197

dimension

of Kuranishi chart, 193

of Kuranishi structure, 194

displaceable subset, 72

duality, 83,85

embedding

of Kuranishi charts, 194

of orbifolds, 192

orbibundle, 193

equal

to maps between orbifolds, 193

equivalent

equivalence of $\mathrm{CF}$ perturbation on a

Kuranishi chart, 199

fiber product, 197

fiber product Kuranishi structure, 197 
fiber product of CF-perturbations, 207

filtered dual complex, 82

filtration, 22

Floer boundary map, 23-27

Floer homology, 27

Lagrangian submanifold, 96

of periodic Hamiltonian system, 27

fragmentation norm, 73

GG-embedding, 195

good coordinate system, 195

good coordinate system with corners, 204

Gromov-Witten invariant

definition, 38

Hamiltonian

homotopic, 32

nondegenerate, 21

normalized Hamiltonian, 8

heavy subset, 97

$\mu$-heavy, 10, 99

$\zeta$-heavy, 10, 97

Hirzebruch surface, 128

Hochschild cohomology

of filtered $A_{\infty}$ algebra, 146

Hofer norm, 34

Hamiltonian, 34

Hamiltonian diffeomorphism, 36

medium, 36

negative part, 34

positive part, 34

homotopic

Hamiltonian, 32

integration along the fiber on Kuranishi chart, 201

one chart, 201

good coordinate system, 202

Kuranishi structure, 203

Jacobian ring, 120

Kähler toric manifold, 111

KG-embedding, 195

strict, 195

KK-embeding, 195

Kodaira-Spencer map $\mathfrak{k}_{\mathfrak{b}}, 120$

Kuranishi chart

coordinate change in the strong sense, 194

coordinate change in the weak sense, 194

embedding of Kuranishi charts, 194

Kuranishi chart, 193

Kuranishi chart with corners, 204

Kuranishi neighborhood of $A, 194$

open subchart, 193

Kuranishi map, 194

Kuranishi neighborhood, 194

Kuranishi structure direct product, 197

fiber product, 197

Kuranishi structure, 194

Kuranishi structure with corners, 204

Lagrangian Floer cohomology, 96

Lagrangian version of spectral numbers, 96

level function, 22, 46

map from Kuranishi structure

strongly continuous map, 196

strongly smooth, 196

strongly submersive, 200

weakly submersive, 196

Maslov index, 90

moment map, 111

nondegenerate

Hamiltonian, 14

normalization

Hamiltonian, 32

normalized boundary

good coordinate system, 204

Kuranishi structure, 204

manifold, 204

orbifold, 204

normalized corner

good coordinate system, 204

Kuranishi structure, 204

manifold, 204

orbifold, 204

obstruction bundle, 194

open substructure, 194

open-closed Gromov-Witten theory, 90

operator $\mathfrak{q}, 90,147$

operator $\mathfrak{q}^{T}, 114$

orbibundle, 193

orbibundle chart, 193

orbifold

compatible (orbifold chart), 192

embedding of orbifolds, 192

subchart, 192

orbifold structure, 192

orbifold with corner, 204

oribfold chart, 192

orientable

Kuranishi chart, 193

orientation

Kuranishi chart, 193

orientation preserving

of coordinate change, 194

pants product, 59, 60

parametrization map, 194

partial quasi-morphism, 74

partial symplectic quasi-state, 8,72

spectral partial quasi-state, 73

partition of unity 
good coordinate system, 202

perturbed Cauchy-Riemann equation, 7, 23

perturbed smooth correspondence, 206

Piunikhin isomorphism, 85, 105, 151

multiplicative property, $65-70$

with bulk, 44

Poisson bracket, 58

potential function, 95, 116

critical point, 120

critical value, 134

leading order term, 124

pseudo-isotopy, 163

of filtered $A_{\infty}$ algebra, 162

unital, 163

pull back of a differential form by strongly smooth map, 197

quasi-morphism

construction, 87

Calabi quasi-morphism, 87, 128, 131, 139

spectral quasi-morphism, 9

Seidel homomorphism with bulk, 172

shrinking, 200

shuffle, 15, 41

smooth, 202

smooth correspondence, 206

smooth map

from orbifold to a manifold, 193

smoothing, 164

spectral displacement energy, 77

spectral invariant, 7,47

$C^{0}$-Hamiltonian continuity, 52, 57

axiom, 34

definition, 33

homotopy invariance, 47, 57-59

with bulk, 47, 75

spectral partial quasi-state, 9

standard basis, 50, 149

Stokes' theorem, 205

strongly continuous, 41

strongly submersive, 93

strongly submersive (w.r.t.

CF-perturbation)

on one chart, 199

strongly transversal, 199

superheavy subset, 97

$\mu$-superheavy, 10, 99, 146

$\zeta$-superheavy, 10, 97

Sweedler's notation, 90

symbol, 50

time-reversal

Hamiltonian, 14

toric degeneration, 137

toric divisor, 111

transversality in the case of manifold with

corners, 204 universal Novikov field, 15

universal Novikov ring, 15

Usher's spectrality lemma, 48-51

valuation, 16,20

$\mathfrak{v}_{T}, 16$

$\mathfrak{v}_{q}, 16$

vector bundle, 193

weakly submersive, 41

weakly transversal, 197

weakly transversal to a smooth map, 197

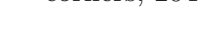


Simons Center for Geometry and Physics, State University of New York, Stony Brook, Ny 11794-3636 U.S.A., Center for Geometry and Physics, Institute for Basic Sciences (IBS), 77 Cheongam-ro, Nam-gu, Pohang, Korea

E-mail address: kfukaya@scgp.stonybrook.edu

Center for Geometry and Physics, Institute for Basic Sciences (IBS), 77 Cheongamro, Nam-gu, Pohang, Korea, Department of Mathematics, Postech, Pohang, Korea

E-mail address: yongoh1@postech.ac.kr

Graduate School of Mathematics, Nagoya University, Nagoya, Japan

E-mail address: ohta@math.nagoya-u.ac.jp

Research Institute for Mathematical Sciences, Kyoto University, Kyoto, Japan

E-mail address: ono@kurims.kyoto-u.ac.jp 